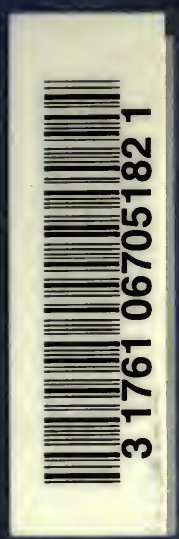



Digitized by the Internet Archive in 2007 with funding from Microsoft Corporation 



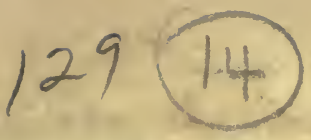

1827.

\title{
MEDICAL WORKS
}

PUBLISHED

\section{BY THOMAS AND GEORGE UNDERWOOD,}

\author{
32, FLEET-STREET.
}

\section{DR. PARIS ON DIET.}

A TREATISE on Diet: with a view to establish, on practical grounds, a System of Rules for the Prevention and Cure of the Diseases incident to a disordered state of the DIGESTIVE FUNCTIONS. By J. A. Paris, M.D. F.R.S. Fellow of the Royal College of Physicians, \&c. \&c. 8vo. 10s. 6d. New Edition.

\section{BLANE'S MEDICAL LOGIC.}

ELEMENTS OF MEDICAL LOGIC, or Philosophical Principles of the Practice of Physic. Third Edition, greatly enlarged. By Sir Gil Bert Blane, Bart., F.R.S.S. Physician to the King. The additions to this edition are such as to render the work a general and comprehensive compendium of Medical Science. 8vo. 10s. 6d.

\section{BY THE SAME AUTHOR,}

SELECT DISSERTATIONS on several subjects of MEDICAL SCIENCE ; Now first collected, with Alterations and Additions; together with several new and original Articles. 8vo. 12s.

\section{DR: PHILIP ON INDIGESTION.}

A TREATISE ON INDIGESTION AND ITS CONSEQUENCES, called Nervous and Bilious Complaints, with Observations on the Organic Diseases in which they sometimes terminate. By A. P. W. PhILIP, M. D. New Edition, with Additions. 8vo.9s.

BY THE SAME AUTHOR,

ON the MORE PROTRACTED CASES of INDIGESTION. 8vo. 3s. 6d.

AN EXPERIMENTAL INQUIRY into the LAWS of the VITAL FUNCTIONS. The present Edition presents a popular View of the Functions of Animal Life, a subject hitherto confined to the Members of the Medical Profession. 8vo. 10s. 6d. The Third Edition.

A TREATISE ON FEBRILE DISEASES ; including the various Species of Fever, and all Diseases attended with Fever. Fourth Edition. 2 vols. 8vo. $£ 1.4 \mathrm{~s}$. Each Volume sold separately.

\section{THOMSON'S CONSPECTUS.}

A CONSPECTUS of the PHARMACOPEEIAS OF THE LONDON, EDINBURGH, AND DUBLIN COLLEGES OF PHYSICIANS. By ANThoNy ToDd Thomson, M.D. New Edition, corrected and greatly improved; with an Appendix on Poisons; a Selection of Extemporaneous Prescriptions; and an Analysis of Mineral Waters, \&c. \&c. 18mo. $5 \mathrm{~s}$.

\section{NEW FRENCH REMEDIES.}

FORMULARY for the PREPARATION and MODE of EMPLOYING several NEW REMEDIES, namely Morphine, Iodine, Quinine, Cinchonine, the Hydrocyanic Acid, Narcotine, Strychnine, Nux Vomica, Emetine, \&c. \&c. \&c. Translated from Magendie's new and greatly enlarged edition, with Notes, \&c. 12 mo. 
GRAY'S SUPPLEMENT To THE PHARMACOPCEIA. A SUPPLEMENT to the PHARMACOPEIA; being a TREATISE on PHARMACOIOGY in general; including the Drugs and Compounds which are used by Practitioners of Medicine, also those which are sold by Chemists, Druggists, and Herbalists, for other purposes; with a Collection of the most useful Medical Formula; an Explanation of the Contractions used by Physicians and Druggists; and a very copious Index, English and Latin, of the Names by which the Articles have been known at different periods. By SAMUEx FreDERICK GRAY. Fourth Edition, improved and greatly enlarged, including the New French Remedies. 8vo. 14s. The present Edition contains a large collection of the most approved IIorse and Cattle Medicines, and Perfumery.

\section{BY THE SAME AUTHOR,}

ELEMENTS OF PHARMACY, and of THE CHEMICAL HISTORY OF THE MATERIA MEDICA; Containing an Explanation of the Chemical Processes of the London Pharmacopoia, the Chemical History of the several Articles of the Materia Medica of the I condon Pharmacopœia, and of some other Articles that have come into use since its publication; illustrated by Figures. The whole intended as a Companion to the Author's General Treatise of Pharmacology. In 8vo. 10s. 6d.

\section{DR. HARRISON ON SPINAL DISEASES.}

PATHOLOGICAL and PRACTICAL OBSERVATIONS on SPINAL DISEASES. Illustrated with Cases and Engravings. Also An Inquiry into the Origin and Cure of Distorted limbs. By EDW ARD HAR RISON, M.D. F.R.A.S. Ed., formerly President of the Royal Medical Society and Royal Physical Societies of Edinburgh, etc. etc. Price 21s.

\section{ECONOMISING FUEL AND PREVENTING SMOKE.}

THE THEORY AND PRACTICE OF WARMING and VENTILAT. ING PUBLIC BUILDINGS, DWELLING-HOUSES, and CONSER. VATORIES; including a description of all the known varieties of Stoves, Grates, and Furnaces, with an examination of their comparative advantages for Economising Fuel, and Preventing Smoke. By an ENGINEer. Illustrated by numerous Copper-plates and Wood Engravings. In 8vo. 18s.

\section{DR. SMITH ON FORENSIC MEDICINE.}

THE PRINCIPLES of FORENSIC MEDICINE, Systematically arranged and applied to British Practice; intended for the use of Magistrates, Coroners, Barristers, Medical Practitioners, and Jurymen. New Edition, greatly enlarged and improved. By JoHn Gondox SMrth, M.D. 8vo.16s.

\section{BY THE SAME AUTHOR,}

AN ANALYSIS of MEDICAL EVIDENCE; comprising Directions for Practitioners, in the View of becoming Witnesses in Courts of Justice; and an Appendix of Professional Testimony. 8vo. 12s.

\section{SIR ASTLEY COOPER'S LECTURES.}

THE LECTURES of SIR ASTLEY COOPER, Bart, F.R.S. Surgeon to the King, \&c. \&c. on the PRINCIPLES and PRACTICE of SURGERY; with additional Notes and Cases. By Frederick TYRREcL, Esq. Surgeon to St. Thomas's Hospital, and to the London Ophthalmic Infirmary. Vol. III. 10s. 6d. Vols. I. and II. 8vo. 10s. Gd. each.

\section{COOPER ON DISLOCATIONS.}

A TREATISE on DISLOCATIONS and on FRACTURES of the JOINTS. By SiR Astrey Cooper, Bart., F.R.S., Surgeon to the King, \&c. \&c. \&c. New Edition, 4to. 


\section{PRICHARD ON THE NERVES}

A TREATISE on DISEASES of the NERVOUS SYSTEM. Vol I. 8vo. 12s.

\section{DANIELL ON METEOROLOGY.}

METEOROLOGICAL ESSAYS and OBSERVATIONS ; embracing, among others, the following important subjects-On the Constitution of the AtmosphereOn the Climate of London-On the TRADE WINDS, considered with regard to Mr. Daniell's Theory of the Constitution of the Atmosphere. By CAPT. BASIL HaLI, R.N. F.R.S.-On EVAPORATION, as connected with Atmospheric Phenomena-On CLIMATE, considered, with regard to Horticulture-On the OSCILLATIONS of the BAROMETER_On the GRADUAL DETERIORATION of BAROMETERS and the MEANS of PREVENTION-On the HORARY OSCILLATIONS of the BAROMETER, \&c. \&c. With Plates of Instruments, Diagrams, and Linear Tables. Second Edition, improved and enlarged. By J. Frederick DaNiel , F.R.S. 8vo. 16s.

\section{DR. ARNOT'T'S ELEMENTS OF PHYSICS.}

ELEMENTS of PHYSICS, or NATURAL PHILOSOPHY, General and Medical, explained independently of Technical Mathematics. By N. AnNotT, M.D. of the Royal College of Physicians. In One Volume 8vo. with numerous Engravings on Wood. 11. 1s.

\section{DISEASES OF THE SKIN.}

A PRACTICAL TREATISE on DISEASES of the SKIN, arranged with a view to illustrate the Constitutional Causes of these Diseases, as well as their local Character. New Edition, improved, by Samuex Plumbe, Member of the Royal College of Surgeons of London, \&c. \&c. 8vo. 14s, with two beautifully coloured Engravings.

\section{DR. PARRY'S MEDICAL WRITINGS.}

COLLECTIONS from the UNPUBLISHED MEDICAL W RITINGS of the late C. H. PARrY, M.D., \&c. 2 vols. 11. 12s.

BY THE SAME AUTHOR,

ELEMENTS of PATHOLOGY and THERAPEUTICS. Second Edition, royal $8 \mathrm{vo}$., $14 \mathrm{~s}$.

An INTRODUCTORY VOLUME to the ABOVE COLLECTIONS, by Charles H. Parit, M.D., F.R.S. 8vo. 10s.

\section{ANNESLEY ON DISEASES OF INDIA.}

SKETCHES of the most PREVALENT DISEASES of INDIA, comprising a Treatise on the Epidemic Cholera of the East, Statistical and Topographical Reports of the Diseases in the different divisions of the Army under the Madras Presidency, and Practical Observations on the effects of Calomel on the Alimentary Canal. Illustrated by Tables and Plates. By James Ansescey, Esq., Madras Medical Establishment. 8vo. with coloured Plates. 18s.

\section{COOPER'S SURGICAL DICTIONARY.}

A DICTIONARY of PRACTICAL SURGERY, comprehending all the most Interesting Improvements from the earliest times down to the Present Period; an Account of the Instruments and Remedies employed in Surgery; the Etymology and Signification of the principal Terms; and numerous references to $\Lambda$ ncient and Modern Works, forming a "Catalogue Raisonné" of Surgical Literature. The Fifth Edition, corrected and enlarged. By SAMUEL CoOPER, Surgeon to the Forces, \&c. \&c. \&c. 8vo. 27s. 


\section{CURTIS ON THE EAR.}

A TREATISE on the PHYSIOLOGY and DISEASES of the EAR, with the most approved modes of treatment. By J. H. CuR Tis, Esq., Surgeon-Aurist to the King. The present Edition is intended not only for the profession, but also for the use of Deaf Persons ; it contains much new and important information on Otitis, Otorrhoea, Nervous Deafness, and Cases of Deaf and Dumb. Fourth Edition, 8vo. 7s. 6d.

BY THE SAME AUTHOR,

A NEW and IMPROVED MAP of the EAR; the Subjects taken from Anatomical Preparations in the possession of the Author. Designed chiefly for the, Use of Pupils. Coloured, 6s.

CASES ILLUSTRATIVE of the DISEASES of the EAR; with Practical Remarks relative to the DEAF and DUMB. 8vo. 3s.6d.

A CLINICAL REPORT of the ROYAL DISPENSARY for DISEASES of the EAR, with remarks on the objects and utility of the Institution.

\section{ON THE TREATMENT OF FRACTURES.}

A SYLLABUS of the LECTURES delivered by Mr. AMESBURY, on the above subjects; containing a Description of the Modes of Applying the Apparatuses, which he has invented for the Cure of Fractures, Stiff Joints, and for the removal of Deformities; illustrated by Twelve Plates, and a short Description of a Case under each head. 8vo. 12s.

\section{EARLE'S SURGERY.}

PRACTICAL REMARKS on FRACTURES at the UPPER PART of the THIGH, and particularly Fractures within the Capsular Ligament. Observations on Fractures of the Olecranon.-Description of a new Apparatus for securing the Upper Extremity in injuries of the Shoulder-joint and Scapula-By HENRY EARLE, F.R S. Assistant Surgeon to St. Bartholomew's Hospital. 8vo. 8s.

\section{DR. YOUNG ON CONSUMPTIVE DISEASES.}

A PRACTICAL and HISTORICAL TREATISE on CONSUMPTIVE DISEASES, deduced from original Observations, and collected from $A$ uthors of all Ages. By Thomas Young, M.D. 8vo. 12s.

\section{DIABETES AND DROPSIES.}

A PRACTICAL TREATISE on DIABETES, with Observations on the TABES DIURETICAE, or URINARY CONSUMPTION, especially as it occurs in Children; and on Urinary Fluxes in General. By ROBERT VeNABLEs, M.D. Physician to the Henley Dispensary. 8vo. 7s. 6d.

\section{BY THE SAME AUTHOR,}

CLINICAL REPORT on DROPSIES; with Observations explanatory of their Pathology and Therapeutics: with an Appendix, on the Theory and Treatment of Organic Diseases in general. 8vo. 8s.

\section{DR. THOMAS ON DIGESTIVE ORGANS.}

PRACTICAL OBSERVATIONS on CHRONIC AFFECTIONS of the DIGESTIVE ORGANS, and on BILIOUS and NERVOUS DISORDERS, \&c. \&c. \&c. By John Tromas, M.D. Physician at Cheltenham, one of the Physicians to the Cheltenham Dispensary. 8vo. 8s. a Third Edition, with considerable Additions.

\section{PRING'S PA'THOLOGY.}

AN EXPOSITION of the PRINCIPLES of PATHOLOGY, and of the 'TREATMEN'T of Diseases. By Daniel Pring, M.D. 8vo. 14s. 
DR. SUTTON ON GOUT, \&c.

TRACTS on DELIRIUM TREMENS and on the GOUT. By Thomas Sut ton, M.D. 8vo. 7s.

\section{DISTORTIONS OF THE SPINE.}

PRACTICAL OBSERVATIONS on DISTORTIONS of the SPINE, CHEST, and LIMBS; together with Remarks on Paralytic and other Diseases connected with impaired or defective motion. By WILLIAM TILLEard WARD, F.L.S. Member of the Royal College of Surgeons of London, \&c. \&c. 8vo. 7s.

\section{DENTAL SURGERY.}

PRINCIPLES of DENTAL SURGERY, exhibiting a new method of treating the Diseases of the Teeth and Gums, especially calculated to promote their health and beauty; in two Parts, by LeoNarD Koecker, Surgeon Dentist, 14s.

\section{DR. BARRY ON THE VENOUS BLOOD.}

EXPERIMENTAL RESEARCHES on the INFLUENCE of ATMO. SPHERIC PRESSURE upon the Venous Circulation, Absorption, and the Prevention and Cure of Hydrophobia, and the Symptoms arising from every Species of Poisoned Wounds. By D. BArry, Member of the College of Physicians of London, \&c. 8vo. 7s.

\section{HUTCHISON's SURGERY.}

PRACTICAL OBSERVATIONS in SURGERY : more particularly as regards the NAVAL and MILITARY SERVICE. Illustrated by. Cases, and various official documents. Second edition, considerably enlarged, by ALEXANDER Copland Hutchison, late Surgeon to the Royal Naval Hospital at Deal, \&c. \&c. 8vo. 12s.

\section{DISEASES OF THE CHEST.}

ORIGINAL CASES, with Dissections and Observations, illustrating the Use of the STETHOSCOPE and PERCUSSION in the Diagnosis of DISEASES of the CHEST; also Commentaries on the same subjects, selected and translated from A venbrugger, Corvisart, Laennec, and others. By John Forbes, M. D. Physician to the Chichester Dispensary. 8vo. 10s. 6d. with Plates.

\section{DISEASES OF THE EYES.}

A REVIEW of the different MODERN OPERAT:ONS performed on the EYES, for the Restoration of lost, and the Improvement of imperfect Vision; also a full account of the various Structures and Diseases of the Eyes, and their appendages. By William Cleobury, Member of the Royal College of Surgeons, London; and one of the Surgeons to the Radcliffe Infirmary, Oxford. 8vo. 10s. 6d.

\section{BY THE SAME AUTHOR,}

A FULL ACCOUNT of the SYSTEM of FRICTION, as adapted and pursued with the greatest success in cases of Contracted Joints, \&c. By JoHN Gros-' VENOR, Esq. of Oxford. Third Edition, 7s. 6d.

\section{LONDON DISSECTOR.}

THE LONDON DISSECTOR; or, System of Dissection practised in the Hospitals and Lecture Rooms of the Metropolis : explained by the clearest Rules, for the Use of Students, comprising a description of the Muscles, Vessels, Nerves, and Viscera of the Human Body, as they appear on Dissection; with Directions for their Demonstration. By James Scratch LEX, Surgeon to the Royal Regiment of Artillery, and to the Corps of Royal Engineers. Seventh Edition, price 6s. 


\section{LONDON PRACTICE OF MIDWIFERY.}

LONDON PRACTICE of MIDWIFERY; or, A Manual for Students: being a complete Course of Practical Midwifery; in which are included the Treatment of Lying-in Women, and the Diseases of Children. A new Edition, 12 mo. $6 s$.

\section{HOLBROOK ON HYDROCELE AND BRONCHOCELE.}

PRACTICAL OBSERVATIONS on HYDROCELE, with a view to recommend a new Mode of Operating for that Disease. To which are added, SOME PRACTICAL OBSERV ATIONS On BRONCHOCELE, and the INFLAM MATION of the MAMMA. By James Holbroox, Member of the Royal College of Surgeons. 8 vo. 4s. 6d.

\section{CARTER ON HOSPITALS.}

A SHORT ACCOUNT of some of the PRINCIPAL HOSPITALS of FRANCE, ITALY, SWITZERLAND, and the NETHERLANDS ; with Remarks upon the Climate and Diseases of those Countries. By H. W. CarTer, M. D., one of Dr.Radcliffe's Travelling Fellows from the University of Oxford. 8vo. 8s.

\section{EFFECTS OF CLIMATE ON CONSUMPTION.}

MEDICAL NOTES on Climate, Diseases, Hospitals, and Medical Schools in France, Italy, and Switzerland; comprising an Inquiry into the Effects of a Residence in the South of Europe, in Cases of Pulmonary Consumption, and illustrating the present state of Medicine in those countries. By JAMEs Crark, M. D. Resident Physician at Rome. 8vo. 7s.

\section{BARON ON 'TUBERCULOUS DISEASES.}

ILLUSTRATIONS of the INQUIRY respecting TUBERCULOUS DIS. EASES. By Joun BARon, M.D., F. R. S. This Work shows, in a particular manner, the progress of Tubercles in the Lungs. 8vo. with coloured plates, 15s.

\section{BEW ON TIC DOULOUREUX.}

OPINIONS on the CAUSES and EFFECTS of the DISEASE denominated TIC DOUloureUX, with Cases and Engravings. By Charles BEw, Surgeon Dentist to his Majesty. 8vo. 78. 6d.

\section{JAMES ON INFLAMMATION.}

OBSERVATIONS on some of the GENERAL PRINCIPLES, and on the PARTICULAR NATURE and TREATMENT of the different specics of INFLAMIMATION. By J. H. JAsies, Surgeon to the Devon and Exeter Hospital. 10s. 6d.

\section{DR. THOMAS'S PRACTICE OF PHYSIC.}

The MODERN PRACTICE of PHYSIC, exhibiting the Character, Causes, Symptoms, Prognostics, Morbid Appearances, and improved Method of treating the Discases of all Climates. By RовERT Tho MAs, M.D. Eighth Edition, revised, and considerably enlarged, by an addition of much new and important matter, the Prescriptions having been altered in conformity to the last Pharmacopoeia of the London College of Physicians. 18s.

\section{DR. THOMAS'S DOMESTIC MEDICINE.}

THE WAY to PRESERVE HEALTH, INVIGORATE a DELICATE CONSTITUTION, and attain an ADVANCED AGE : with a Treatise on Domestic Medicine, divested of Profersional Terms. By Robert Thomas, M. D. 8vo. 158. 
DR. RAMSBOTHAM ON MIDWIFERY.

PRACTICAL OBSERVATIONS IN MIDWIFERY, with a Selection of Cases, by John Ramsbotham, M.D. 8vo. 10s. 6d. Part I.

\section{HOOPER's QUINCY'S LEXICON.}

QUINCY's LEXICON MEDICUM. A New Medical Dictionary; containing an Explanation of the Terms in Anatomy, Physiology, Practice of Physic, Materia Medica, Chemistry, Pharmacy, Surgery, Midwifery, and the various Branches of Natural Philosophy connected with Medicine ; selected, arranged, and compiled from the best Authors. By Robent Hooper, M. D. 'Large 8vo. New Edition, 11. 7s.

\section{THE ANATOMIST's VADE MECUM.}

THE ANATOMIST'S VADE MECUM. Containing the Anatomy, Physiology, Morbid Appearances, \&c. of the Human Body ; the Art of making Anatomical Preparations, \&c. By Rober t Hooper, M. D. New Edition, 12mo. 8s.

\section{THE SURGEON'S VADE MECUM.}

THE SURGEON'S VADE MECUM. Containing the Symptoms, Causes, Diagnosis, Prognosis, and Treatment of Surgical Diseases; accompanied by the modern and approved Methods of Operating; a select Formulæ of Prescriptions, and a Glossary of Terms. By Robert Hooper, M.D. New Edition, with Plates, 12mo. 8s.

\section{THE PHYSICIAN'S VADE MECUM.}

THE PHYSICIAN'S VADE MECUM. Containing the Symptoms, Causes, Diagnosis, Prognosis, and Treatment of Diseases ; accompanied by a Select Collection of Formulæ, and a Glossary of Terms. By Robert Hooper, M. D. New Edition, 12mo. 78 .

\section{HOOPER'S EXAMINATIONS.}

EXAMINATIONS in ANATOMY, PHYSIOLOGY, PRACTICE of PHYSIC, SURGERY, MATERIA MEDICA, CHEMISTRY, and PHARMACY ; for the Use of Students who are about to pass the College of Surgeons, Medical and Transport Boards. By Robert Hooper, M. D. New Edition, much enlarged, $12 \mathrm{mo} .5 \mathrm{~s} .6 \mathrm{~d}$.

\section{HASTINGS ON THE LUNGS.}

A TREATISE on INFLAMMATION of the MUCOUS MEMBRANE of the LUNGS. To which is prefixed an Experimental Inquiry respecting the Contractile Powers of the Blood Vessels, and the Nature of Inflammation. By CHARL Es Hastings, M. D. 8vo. 10s. 6d.

\section{UNDERWOOD ON DISEASES OF CHILDREN.}

A TREATISE on the DISEASES of CHIIDREN; with Directions for Management of Infants from the Birth. By the late Dr. UNDERwood. Eighth Edition, revised, with Notes and Observations, by S. MERrimas, M. D., F. I. S. \&c. 8vo. 16s.

\section{WARE ON THE CATARACT.}

OBSERVATIONS on the CATARACT and GUTTA SERENA. By JAMEs WARE, Esq. Third Edition, 8vo. 10s. 6d.

$$
\text { BY THE SAME AUTHOR, }
$$

REMARKS on the OPHTHALMY, PSOROPHTHALMY, and PURULENT EYES of NEW BORN CHILDREN. Fifth Edition, 8vo. 10s. 6d.

OBSERVATIONS on SEVERAL DISEASES of the EYE, and Remarks on the Introduction of the Male Catheter, and on the Treatment of Hæmorrhoids. 8vo. 8s. 


\section{DR. LUCAS ON INFLAMMATION.}

ON the PRINCIPLES of INFLAMMATION and FEVER. By C. E. LuCAS, M.D. 8vo. 8s.

\section{JOHNSON ON TROPICAL CLIMATES.}

THE INFLUENCE of TROPICAL CLIMATES, more especially the Climate of India, on European Constitutions; the principal Effects and Diseases thereby induced; their Prevention or Removal; and the means of preserving Health in Hot Climates, rendered obvious to Europeans of every capacity. By James Johnson, M. D. New Edition, 8vo. 36s.

BY THE SAME AUTHOR,

AN ESSAY on MORBID SENSIBILITY of the STOMACH and BOWELS, as the proximate Cause, or characteristic Condition of Indigestion, Nervous Irritability, Mental Despondency, Hypochondriacism, and many other Ailments, with an improved Method of 'Treatment, Medicinal and Dietetic. To which are added, Observations on the Diseases and Regimen of Invalids, \&c. Third Edition, enlarged. 6s.

A TREATISE on DERANGEMENTS of the LIVER, DIGESTIVE ORGANS, HEART, and NERVOUS SYSTEM. New Edition, 8vo. 8s. 6d.

The INFLUENCE of CIVIC LIFE, Sedentary Habits, and Intellectual Refinement, on Human Health and Human Happiness. 8vo. 3s.6d.

\section{PARIS'S PHARMACOLOGIA.}

PHARMACOLOGIA : comprehending the Art of Prescribing upon Fixed and Scientific Principles ; together with the History of Medicinal Substances. By J. A. Par Is, M. D. In 2 vols. 8vo. Sixth Edition, considerably enlarged, 11. 5s. This edition contains, for the first time, the Medicinal Dynameter, or revolving scale, by which the absolute and relative strength of any quantity of a medicinal compound, as well as its several equivalents, may be immediately found, by bringing the substance in question to the figure representing its dose.

\section{LONDON MEDICAL REPOSITORY.}

LONDON MEDICAL REPOSITORY; MONTHLY JOURNAL AND REVIEW. Lately conductcd by Dr. Burrows, Dr. Uwins, and Dr. A. T. Thomson, and now edited by Dr. Copland, Dr. Darwall, and Dr. Conolly. Consisting of Original Communications on Medical Subjects-Reviews of New Books -Selections from Foreign Medical Works-Medical and Physical IntelligenceI ist of New Publications, \&c. \&c. Published in Monthly Numbers, at 2s.6d. each.

UNDERWOOD'S MEDICAL CATALOGUE FOR $182 \%$.

A CATALOGUE of an EXTENSIVE COLLECTION of BOOKS in Anatomy, Medicine, Surgery, Midwifery, Chemistry, Botany, \&c. Price ls.

\section{In the Press.}

A TREATISE on those DISEASES which are directly or indirectly connected with INDIGESTION ; comprising a Commentary on the Principal Ailments of Children. By David Úwins, M.D.

A TREATISE on the NATURE and CURE of HOOPING COUGH and CROUP. By JAMES CoPLAND, M. D.

A TRANSLATION of the New Edition of LAENNEC on DISEASES of the CHEST. By John Forbes, M. D. Physician to the Chichester Dispensary. 


\section{WOR K S}

WILLIAM CULLEN, M.D. 



\title{
WOR K S
}

OF

\section{WILLIAM CULLEN, M.D.}

PROFESSOR OF THE PRACTICE OF PHXSIC IN THE UNIVERSITY OF EDINBURGH:

CONTAINING HIS

PHYSIOLOGY, NOSOLOGY, AND

FIRST LINES OF THE PRACTICE OF PHYSIC:

WITH NUMEROUS EXTRACTS FROM HIS MANUSCRIPT PAPERS,

AND FROM HIS TREATISE OF THE MATERIA MEDICA.

\author{
EDITED BY
}

JOHN THOMSON, M.D. F.R.S. L. \& E.

LECTURER ON THE PRACTIE OF PHYSIC, CONSULTING PHYSICIAN TO THE NEW TOWN DISPENSARY, AND LATE REGIUS PROFESSOR OF MHITAKY SURGERY IN THE UNIVERSITY OF ENINBURGH.

IN TWO VOLUMES.

VOL. I.

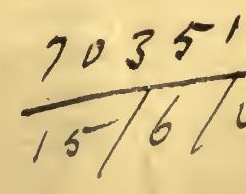

WILLIAM BLACKWOOD, EDINBURGH: AND

T. \& G. UNDERWOOD, LONDON.

MIDCCCXXVII. 



\section{CONTENTS.}

Institutions of Medicine

Physiology

Sect. I.-Of the Simple Solid.

Sect. II.-Of the Nervous System.

General View of the Nervous System........................... 14

Chap. I.-Of Sensation........................................................ 28

Chap. II.-Of the Action of Moving Fibres................... 63

Chap. III._Of the Functions of the Brain................... 102

Sect. III.-Of the Circulation of the Blood.

Chap. I.-Of the Course of the Blood.............................. 158

Chap. II._Of the Powers moving the blood................... 161

Chap. III.-Of the Laws of the Circulation..................... 163

Chap. IV.-Of Respiration................................................ 168

Sect. IV.-Of the Natural Functions.

Chap. I.-Of Digestion......................................................... 172

Chap. II.-Of Animal Blood............................................... 192

Chap. III.-Of Secretion................................................. 206

Chap. IV._Of Nutrition.................................................. 209

Of Temperaments ......................................................... 214 
Lectures Introductory to the Counse on the PracTICE of PHysic............................................................................. 363

History of Medicine........................................ 365

Method of Study.......................................... 415

Plan of Course............................................ 440

On Nosology .......................................... 444

First Lines of the Practice of Physic.

Preface.................................................... 467

Introduction............................................ 471

Part I._Of Pyrexiæ...................................... 479

Book I.-Of Fevers.

Chap. I.-Of the Phenomena of Fevers.......................... 480

Chap. II.-Of the proximate cause of Fevers ............... 490

Chap. III.-Of the difference of Fevers, and its causes 506

Chap. IV._-Of the remote causes of Fever.................... 540

Chap. V.-Of the prognosis of Fevers........................... 561

Chap. VI. - Of the method of cure in Fevers................. 598

Sect. I.-Of the cure of continued Fevers ................. 599

Sect. II.-Of the cure of intermittent Fevers............ 671 


\section{STUDENTS IN THE MEDICAL SCHOOL OF EDINBURGH.}

\section{Gentremen,}

I beg leave to dedicate to you this edition of Dr. Cullen's text-books on Physiology, Nosology, and the Practice of Physic. In preparing it for the press, I have had two objects chiefly in view; first, to furnish you with such extracts from Dr. Cullen's MS. papers, as seem to throw additional light on the subjects of which these books treat; and, secondly, to put the public in possession of documents that appear to me to establish Dr. Cullen's claims to originality for observations and doctrines, which, under various modifications, have been repeatedly brought forward since his time, and made the bases of new theories or systems of Medicine.

The Outlines of the Physiology have been prefixed to this edition, and illustrated in some parts with Dr. Cullen's own commentaries, in the belief that they are less generally known than his other writings, and that a knowledge of the view which they exhibit of the Nervous system, is necessary to a proper understanding of some of the doctrines contained in his First Lines of the Practice of Physic.

The Synopsis Nosologiæ Methodicæ, has been reprinted from the edition published by Dr. Cullen in 1784, with the omission only of the abridgment which he has given of the nosological systems of his predecessors and cotemporaries.

The Lectures introductory to the Course on the Practice of Physic, now published for the first time, have been printed from 
copies of them corrected in Dr. Cullen's hand-writing. To these Lectures I have restored a portion of the History of Medicine, which Dr. Cullen had transferred to his preface to the First Lines.

Besides numerous additions made to the First Lines of the Practice of Physic, from Dr. Cullen's MS. Lectures, I have introduced various passages from the Treatise of the Materia Medica, published by him in two volumes quarto in 1789. The passages from the MS. Lectures are distinguished from the original text by inverted commas; and those taken from the Materia Medica by the addition of the letters M.M.

In presenting you with these elementary works in their present form, I am well aware that the science of medicine has made great advances since they were first produced; advances which require corresponding changes in the manner in which this science should now be taught. But I know of no general work on the Practice of Physic, hitherto published in this country, calculated to supersede Dr. Cullen's writings as textbooks; and certainly none which can bear a comparison with them in the extent and variety of the medical information which they contain; in the model which they afford of distinct and comprehensive definitions and histories of diseases; and in the talext which they display for the accurate discrimination and simple generalization of the results of experience.

\section{I am,}

Gentlemen,

Your obedient Servant,

JOHN THOMSON, M.D.

80, George Street,

Edinburgh, 1st Nov. 1827. 


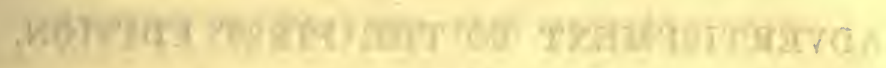
$\min$

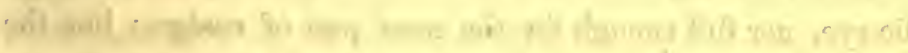

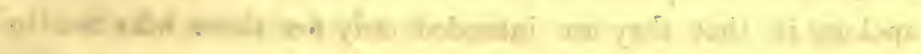

\section{INSTITUTIONS OF MEDICINE.}

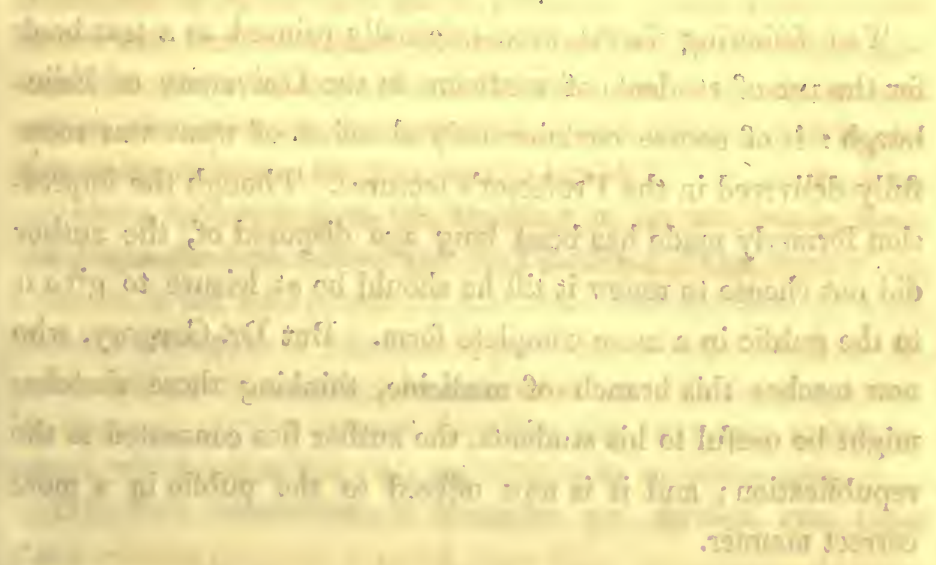




\section{ADVERTISEMENT TO THE FIRST EDITION,}

1772.

THE following sheets are neither correct enough for the public eye, nor full enough for the most part of readers; but the apology is, that they are intended only for those who are to hear them fully explained.

\section{ADVERTISEMENT TO THE SECOND EDITION,} 1777.

THE following sheets were originally printed as a text-book for the use of students of medicine in the University of Edinburgh : it of course contains only sketches of what was more fully delivered in the Professor's lectures. Though the impression formerly made has been long ago disposed of, the author did not choose to renew it till he should be at leisure to give it to the public in a more complete form. But Dr. Gregory, who now teaches this branch of medicine, thinking those sketches might be useful to his students, the author has consented to the republication; and it is now offered to the public in a more correct manner. 


\section{INSTITUTIONS OF -MEDICINE.}

I. Medicine is the art of preventing and of curing diseases."The common language is that, 'Medicine is the art of preserving health and of curing diseases;' but I have said 'the art of preventing diseases ;' for although I do not deny that the preserving of health is the object of a physician's care, yet I maintain that there is truly no other means of preserving health but what consists in preventing diseases. Every other idea is false, and has led to a superfluous, very often a dangerous practice. I say, that health being properly understood, we cannot add to it, nor increase its powers. There is never room for our art, but when there is some defect in the constitutionsome bias and tendency towards disease ; and it is only by preventing this tendency, by correcting these defects, that is, by preventing disease, that we preserve health.

"For a long time past, the teaching of physic has been divided into the Institutions and the Practice. What we call the Practice, is the art applied to particular diseases and persons. But the moșt part of physicians have been of opinion that"-

II. Before considering the application of this art to particular diseases, certain general doctrines are necessary to be premised, which are called THE INSTITUTIONS OF MEDICINE.

III. The Institutions of Medicine are divided into three parts :-

The first treats of life and health.

The second delivers the general doctrine of diseases.

The third delivers the general dactrine concerning the means of preventing and curing diseases.

"The first discovers to us the mechanism of the human body; the second treats of the manner in which that same 
mechanism is disordered and changed when disease prevails; and, from a comparison of these two, we see what changes must be produced in order to restore the health that is lost. This gives us what we call our indications; and the third part delivers the general doctrine of the means by which the indications can be answered. I think it is obvious that, as in physic the whole of our consideration resolves itself into these three subjects-health, disease, and remedy, there can be no more.

"The branch which treats of health has been named the Physiology; that which treats of disease, the Pathology,-by every writer. With regard to the branch which treats of remedy, there is some doubt in giving it a name, but, without hesitation, I call it Therapeutics. Some of you may observe that this term has been confined almost entirely to the study of remedies, as means employed for curing diseases; and that the means of preventing diseases have been supposed to constitute another branch. I have told you that there is no art of preserving health, but in so far as we prevent diseases by preventing the defects or errors that have a tendency to disease, as they exist only in their cause before they produce their effects. But this is really and truly removing a disease; and the means of accomplishing it are no other than the remedies we employ in curing them, so that they may be comprehended under the title of Therapeutics. I have omitted the Hygiene as a separate title, not because it is not an object of the physician's art, but because, as it consists entirely in the pointing out and in the avoiding the remote causes of disease, whether predisponent or occasional, a part of it will be found in the Pathology, and the other part of it in the Therapeutics.

"With regard to Semeiotics, which has formed one of the five parts into which the Institutions of Medicine have been divided, from the time of Galen to that of Boerhaave, I would observe that this part is no other than a repetition of what is delivered in that part of Pathology which explains the symptoms of diseases, and is therefore termed the Symptomatology." 


\section{INSTITUTIONS OF MEDICINE}

\section{PART I.}

\section{PHYSIOLOGY.}

IV. THE doctrine which explains the conditions of the body and of the mind necessary to life and health, is called Prysiology, or the Doctrine of the Animal Economy.- I I mean that Physiology considers the matter of which the body is formed in its mixt, in its aggregation, and, especially, in its organization or mechanism. With regard to the conditions of the body, Physiology considers every thing that natural philosophy, chemistry, or anatomy teaches with regard to it. But you are to observe that philosophy, chemistry, and anatomy consider the state of the body, and its several parts, abstracted from its several effects. The business of Physiology is only to explain the conditions which these sciences point out as applicable to. the exercise of the functions of the body.

"I have added here a particular in my Physiology that is not common-? and of the mind ;' and some persons may think that this is hardly done with propriety. However the condition of the mind may ultimately arise, we often do sec conditions of mind arise, that we cannot trace to a corporeal cause; while, at. the same time, they may produce very considerable effects upon the bodily state; so that it was necessary. to say that Physi- 
ology referred to the conditions of the mind, as well as to those of the corporeal part. So far from being able to neglect the mind, the most considerable functions are connected with particular operations, and a particular state of our immaterial part; and, indeed, I find that the conditions of the human mind must engage our attention more than they have done hitherto. Some, indeed, have thought that I have gone too far in introducing a great deal of metaphysics into my course ; but unless the history of the operations of the human mind is to be considered as such, they are mistaken; and I resolve to go no further than I can point out these operations as referring to the state of the body."

V. The functions of the animal economy are many and various, and so complicated with each other, that it is difficult to find the most proper order in which they may be delivered."By looking into the works of physiologists, you will see abundant proofs of this, and it will be easy to find out the reason of it :-The functions are complicated, and mutually dependent upon one another. Many of the functions may be considered as mutually the causes and effects of each other. Thus, the action of the heart cannot subsist without the power and energy of the brain; nor can the energy of the brain subsist long without the action of the heart."-That, however, seems the best, which considers them, as nearly as may be, according to the series of causes and effects.- "I say that, as nearly as is possible, that organ and function should be considered first, the knowledge of which is necessary to understand what is to come after. We should take the several matters with some view to their dependence on one another, and should suppose nothing known that has not been previously explained. I say as nearly as possible, for it is impossible to do this entirely."

VI. Upon this plan we shall treat-

1. Of the solid matter, of which a great part of every organ of the body consists.- "We cannot consider a function without considering its organ; and we cannot consider a single organ without considering in general the solid matter of which it is formed.

"6 The most remarkable organs are the muscular parts. A very small acquaintance with the body shews that there is hard- 
ly any sort of motion, connected with the exercise of the functions, which is not performed by muscles, or muscular fibres, so that they must be a very fundamental consideration in the animal economy. When we trace the matter further, a little anatomy must teach us that there is not a muscular fibre which has not a connexion with nerves; and this connexion with nerves is necessary to the action of the muscular parts-for compressing or cutting through these nerves destroys the action of the muscles which are connected with them; and the muscles must not only be connected with these nerves, but, through them, with the brain; for the nerve being cut through in any part of its course, the action of the muscle is destroyed. And when we consider what it is in the brain with which the muscles are thus connected, we find that by much the greater part of the muscles of the body do not stir unless there is a certain operation of the mind that we call the will, which excites and directs the action of almost every muscular part. We may trace the matter still farther :-The will may be primary and independent, but, at the same time, we can, for the most part, very clearly perceive that the will arises in consequence of sense. Unless I see you before me, or unless I at least imagine you present before me, I will not open my mouth to speak. It is the sight of some agreeable object that determines my will to put forth my hand, and bring the object nearer; and it is the feeling of something disagreeable that must direct me to put the object from me: so that, in most cases, the will depends upon sense. In the next place, we may inquire what sense is, and how it arises; and we can perceive that it depends upon the extremities of certain nerves expanded upon particular parts of the body, and fitted to receive the impulses of external bodies, which, acting upon these extremities, give what we call sensation; and this, at last, produces will. Further, there is an impulse upon certain extremities; but these again are connected with a course of nerves which ultimately end in the brain;-for, if the nerve between the brain and organ of sense be cut through, or otherwise destroyed, all sense ceases. In the fundamental operations of the human body, therefore, the nerves, and the parts connected with them, are especially concerned; and as 
it is the brain and the nerres, thus connected, that we call the nervous system, I think it is with some propriety that I have allotted my second section to treat"-

2. Of the nervous system, in which the motions of the body for the most part begin, and upon which the motions produced in it chiefly depend.- "We consider this as it is acted upon by other bodies, or as it acts upon these."

3. Of the motion and circulation of the blood; and of the several organs and actions employed in supporting it.- "For a long time past-since the discovery of the circulation of the blood-most physicians have viewed this as the function upon which all the rest depend; and perhaps there is not one entirely independent of it. But the action of the heart depends upon the nerves, and these upon the brain, so that the heart depends upon the energy and action of the brain. The brain, on the other hand, would not long be capable of its functions, if it were not for the circulation of the blogd. The consideration of the circulation of the blood, which must be taken abstractedly from the nature of the fluids, naturally leads to the consideration of these; and whilst a considerable quantity is necessary to be present, there is a daily and considerable waste. For we find by experiments that the body loses of its weight, and especially by a waste of the fluid parts. We also perceive another demand for a quantity of fluids. The body, from a small beginning, rises to a considerable bulk, and all this in a certain course of life, which must be effected by the addition of a quantity of matter to it; and we can find no other source for supplying this growth, but the matters taken into the mouth, which common observation presumes to be destined for this purpose, and calls aliments. But they are not of the nature of the body, and must be converted into the same nature by the body itself. So that in considering the body, as supplied and supported by the aliments, we must consider the functions necessary to convert the aliments into the nature of the animal fluids : therefore, we come next to treat" -

4. Of the functions employed in supporting and repairing the several solid and fluid matters of the body ; and, on this occasion, of the nature of the several fluids themselves. 
5. Of the organs employed in receiving and modifying the impressions of external bodies necessary to sensation; and of their several functions.

6. Of the motions of the whole body, or of its several parts, which depend on the action of muscles, and not before explained. - "Under these six titles we will, I think, exhaust the whole of the functions of animal bodies that are in common to every animal. But the human species, in particular, is distinguished by sexes, which have each of them somewhat peculiar to themselves. We therefore proceed to treat" -

7. Of the functions peculiar to the sexes; and of generation. - Physiologists have referred the functions to three classes or heads:-The Vital, the Natural, and the Animal Functions. We find some difficulty in referring the different particulars to these heads; but, in the main, it is an obviously just division. The vital functions are those that are more immediately necessary to life, and without the exercise of which, life could not be supported a few moments. With regard to these there have been some differences of opinion. Since the discovery of the circulation of the blood, physiologists have been disposed to consider the action of the heart, and the function of respiration, in this light; but as the action of the heart itself is dependent on the energy of the brain, this must be considered as a vital function, and the primary one. These, then, form our three first sections. The natural functions are necessary to life; but they are not so constantly and necessarily required. A man may fast for a length of days without dying, though this continued, to be sure, would destroy him; and if the aliment is not properly transmitted to the blood-vessels, the same thing will ensuc as if the thoracic duct were cut through. They are therefore next in point of necessity to the vital, and form our fourth section. Both the vital and the natural functions are necessary to the being and subsistence of the body. But there is a certain set of functions for which the body is made and supported, and which form our connexion with the rest of the universe-by which we act upon other bodies, and by which other bodies act upon us:- - these have bcen called animal functions; and the foundation of the whole of them is to be deduced from the nature of the nervous system. These animal 
functions reduced to the two heads of Sense and Motion, will be explained in our second section. But it would be improper to enter there on the consideration of the exercises of the particular senses and motions, and therefore we have reserved them for the fifth and sixth sections.

" Hitherto I have been speaking of the preparation that is necessary to the understanding of certain parts. With regard to the whole, the human body is a machine that must be governed by the laws of matter and motion that affect every part of nature; and, therefore, to the understanding of it, some of the principles of natural philosophy are very necessary; and I do not know where gentlemen have a better opportunity of studying these, in the most fundamental manner, than in this university. The knowledge of chemistry, so far as it considers the nature of the body and of the fluids, will be likewise necessary; but especially the knowledge of anatomy must every where accompany our present study; and I must suppose you all instructed in that respect, for if you are not, it will be impossible to supply it."

SECT. I.

OF THE SIMPLE SOLIDS.

VII. The solid parts of the body seem to be of two kinds : one whose properties are the same in the dead as in the living, and the same in the animate as in many inanimate bodies; the other, whose properties appear only in living bodies. In the last, a peculiar organization, or addition, is supposed to take place; in opposition to which, the first are called the SIMPLE solids. Of these only, we shall treat here; and of the others, which may be called vital solids, being the fundamental part of the nervous system, we shall treat under that title in the following section.

VIII. The simple solids are suited to the purposes of the animal economy by a certain force of cohesion, joined with a certain degree of flexibility and elasticity.

IX. These properties of the simple solids, in different parts of the body, in different bodies, and on different occasions in the same body, are necessarily in different degrees; and this seems 
to depend upon the difference of the mixture, aggregation, or organization of the solid.

$X$. The matter of the simple solid every where, except in the bones, appears to be an homogeneous aggregate; and there is no proper evidence of its being formed of certain parts naturally discrete and incoherent, which are cemented by others of a different nature.

XI. Of the simple solid considered as an homogeneous aggregate, the integrant parts are a mixt, which seems to be nearly of the same kind in all the different parts of the human body, and perhaps in most of the parts of every animal. So far as we yet know, the variety of it is very inconsiderable.

XII. This, which may be called the ANIMAL MIXT, is found, by chemical experiment, to be considerably different from every kind of vegetable or fossil matter; but the same experiments hardly teach us any thing exact or useful with respect to the constituent parts of this mixt.

XIII. The only particular relative to this which we exactly know, is, that the animal mixt is formed of water, and of some other matter concreting with it ; that, on different occasions, the state of it is varied by the proportion which the water bears to the other concreting matter; and that, especially by a different proportion in this respect, the simple solid differs in its force of cohesion, flexibility, and elasticity (VIII.)

XIV. The proportion of water to the other matter in the animal mixt of different persons, seems to depend, in the first place, upon the nature of the original stamina in each; as the different state of simple solids, which appears early to distinguish sex and-temperament, continues respectively the same through the whole of life, even though the different persons are under the same external circumstances.

$\mathrm{XV}$. But, in every particular person, that proportion is constantly changed by the progress of life; and this happens more or less as other causes concur.

XVI. The causes that can affect the mixture of the simple solid, are either the state of the nutritious fluid conveyed by the ordinary channels, or some matters from without insinuated into the solid.

XVII. The state of the nutritious fluid may be varied by 
the quantity and quality of the aliment taken in, by the powers of concoction and assimilation, by the circumstances of application and concretion, or by certain preternatural matters carried along with it.

XVIII. The external matter that may be insinuated into the simple solid, is various, but, for the most part, is only aqueous moisture in greater or less quantity.

XIX. That these several causes may affect the proportion of water in the simple solid, and thereby give a different state of it, is sufficiently obvious. That the same causes may also affect the other concreting matter, we can, in general, perceive to be possible; but in what manner, or upon what occasions they do so, is not easily discerned.

XX. The properties of the simple solid (VIII.) may be also varied by its state of aggregation; and this again may be varied, 1. By the temperature of the atmosphere to which the body is long exposed. 2. By the pressure, external or internal, which is applied to the solid. 3. By the degree of extension of the solid beyond its natural state, which, in every living body, is given more or less to every part of the soft or flexible solids; and, lastly, by the motion or rest to which the solid is accustomed.

XXI. The properties (VIII.) of the solid parts are also: varied by the state of their organization. This every where depends upon an arrangement of fibres, the state of cellular texture, or upon a texture of vessels; and, therefore, to explain the different states of organization, it will be enough to mention the causes of the differences which occur in these fundamental parts.

XXII. Fibres may differ in size by the several causes (XIV.-XXI.) affecting the mixture and aggregation of the matter of which they are formed, and by these causes alone; but how far the organization of any part depends upon an arrangement of fibres, we cannot distinctly perceive; and, if it does, we cannot perceive that the state of such parts differs otherwise than by the state of the cellular texture every where interposed between the supposed fibres.

XXIII. The state of cellular texture is a most important circumstance in all organized parts; and it may be varied by many different causes. 1. The texture may be more dense, and therely firmer, as it has been more pressed by the actions of 
life or external force; by which means especially, it is changed in the progress of life. 2. The cellular texture may be increased in bulk, and rendered firmer by a new growth taking place in it, as frequently happens in membranes which are slowly and gradually stretched out. 3. The same texture may become weaker by some part of it being eroded by acrid matters generated in the body, or externally applied. 4. It is analogous to this, that when any part is sustained by several layers of cellular texture or membranes, such support is weakened by one or more of these layers being cut through; and the same weakness is induced when any external compression, which, for some time, had been applied, is taken away. 5. 'The state of the cellular texture is varied by the matter contained in its cells, which is sometimes a matter concreting into a solid mass, and sometimes a preternatural quantity of an aqueous inelastic fluid. The bones formed in the first manner, may again become soft by the hardened matters being dissolved and reabsorbed. 6 . When the mobility of parts on one another depends upon the extent of cellular texture connecting them, that mobility is diminished or destroyed by a great part of the cellular texture being eroded or cut away, and the remaining parts being united together; so that the parts are now connected by a shorter portion of cellular texture than before. 7. Parts naturally separate may lose their mobility by being joined together by a cellular texture formed between them, as happens when any two surfaces are for some time kept closely applied to each other.

XXIV. In so far as a solid part is formed by a texture of vessels, its properties (VIII.) may be varied by the different states of these vessels; which, 1. may be more or less full of fluids. 2. They may be changed into a solid mass, by the fluid, contained and stagnating in them, concreting into a solid. 3. They may be changed into a solid, if the fluids that should pass through them are intercepted, and the cavity is filled with a cellular texture; or, 4 . They may be changed into a solid, if, by collapse or pressure, the sides of the vessels are applied to each other and concrete together.

XXV. The Pathology of the simple solids cannot be properly separated from their Physiology; and, therefore, many different states of these solids, though such as are always mor- 
bid, are mentioned above. We think it also proper to subjoin here a short view of the whole of that Pathology.

XXVI. The diseases of the simple solids are-

I. Those of the naturally soft parts.

1. Debility with flexibility.

Debile tenerum, gracile, Gaub. Pathol. 161. 1.

Debile tabidum, Gaub. ibid.161.2.

2. Debility with fragility.

Debile fissile, Gaub. 161. 3.

3. Laxity.

Debile laxum, flaccidum, Gaub. 160. 1.

4. Flaccidity.

Debile iners, Gaub. 160. 2.

5. Rigidity diminishing flexibility.

Rigidum tenax, Gaub. 165. 1.

6. Rigidity destroying flexibility.

Rigidum durum, Gaub. 165. 2.

II. Those of the naturally hard parts.

1. Flexibility.

Debile flexile, Gaub. 160. 3.

2. Fragility.

Fragile spongiosum, Gaub. 161. 4.

Fragile vitreum, Gaub. 165. 3.

Of all these morbid affections, we suppose the remote and proximate causes may be understood from what is delivered above, (XIV.-XXIV.)

SECT. II.

OF THE NERVOUS SYSTEM.

XXVII. The nervous system, as the organ of sense and motion, is connected with so many functions of the animal economy, that the study of it must be of the utmost importance, and a fundamental part of the study of the whole economy.

A general view of the Nervous System.

XXVIII. The nervous system consists of the medullary substance of the brain, cerebellum, medulla oblongata, and 
spinalis; and of the same substance continued into the nerves, by which it is distributed to many different parts of the body.

XXIX. The whole of this system seems to be properly distinguished into these four parts.

1. The medullary substance contained in the cranium and vertebral cavity, the whole of which seems to consist of distinct fibres, but without the several fibres being separated from each other by any evident enveloping membranes._- "This (the fibrous structure of the medullary substance) is a necessary part of anatomy. It may not be evident in many parts of that substance : the arrangement, however, is more evident in the brain of some animals than in the human brain; but by boiling, and thereby hardening the brain, it becomes evident in every one. Also, in certain cases of hydrocephalus, where the small portions are separated by the water, the fibrous structure appears. I say, "the whole seems to consist of distinct fibres, but without the several fibres being separated from each other by any evident enveloping membranes ;' but there is a suspicion that they are kept distinct by some substance which may be considered as an enveloping membrane."

Note.-When we speak of functions, which are or may be in common to every part of this portion of the nervous system, we shall speak of the whole under the title of the BRAIN; but, when it is necessary to distinguish the particular parts, we shall take care to avoid ambiguity.

2. Connected with one part or other of No. 1, are the NERVES; in which the same medullary substance is continued, but here more evidently divided into fibres, each of which is separated from the others by an enveloping membrane derived from the pia mater.

3. Parts of the extremities of certain nerves (2.) in which the medullary substance is divested of the enveloping membranes from the pia mater, and so situated as to be exposed to the action of certain external bodies, and perhaps so framed as to be affected by the action of certain bodies only: these we name the sENTIENT EXTREMITIES of the nerves.

4. Certain extremities of the nerves (2.) so framed as to be capable of a peculiar contractility, and, in consequence of their 
situation and attachments, to be, by their contraction, capable of moving most of the solid and fluid parts of the body. These we name the Moving extremities of the nerves: they are commonly named MoviNG or MUSCULAR FIBRES.

Note. - That the muscular fibres are a continuation of the medullary substance of the brain and nerves, has not been shewn by the anatomists, nor universally admitted by the physiologists; but we now suppose it, and hope, afterwards, to render it sufficiently probable.

Are the ganglions of the nerves to be considered as a part of the nervous system distinguished by a peculiar function?- " A fifth part of the nervous system might be mentioned, viz. the portions of the nerves formed into ganglions. It is ascertained that they are a distinct part; but my question is, Whether they are to be distinguished by a peculiar function? They may deserve to be considered even in that light as a distinct part; but this is not so well ascertained; and the present opinion is, that their functions are only modifications of the common properties of the nerves, without any distinct property or function."

XXX. These several parts of the nervous system are every where the same continuous medullary substance which we suppose to be the vital solid of animals, so constituted in living animals, and in living systems only, as to admit of motions being readily propagated from any one part to every other part of the nervous system, so long as the continuity and natural living state of the medullary substance remains.

Note--It is observed, that the compression of any part of the medullary substance prevents the communication of motion between the parts that lie on different sides of the part compressed; and it is probable there are other causes besides compression, which may also affect the medullary substance, so as to interrupt in it the communication of motion; but they are not distinctly known.- " Cold may be one of them ; and putrefaction, or a peculiar matter arising from the putrid state, may be another."-In the mean time, we use the expression that a nerve, or other portion of the nervous system is free, to denote its being free not only from compression, but from every other supposed cause interrupting the communication of motion. 
The condition fitting the medullary substance for having motion propagated in it, we suppose to be the presence of a certain fluid, which we therefore name the nervous fluid, without meaning, however, at present to determine any thing with regard to its source, nature, or manner of acting.- "It has been questioned. among physicians whether or not this communication of motion along the nerves does take place; but there are few of opinion that it does not, and it is almost universally admitted that there is a continuation of motion from the origin to the extremities, and in the contrary direction. You probably know, that it is disputed what is the foundation of this communication of motion, what it is that fits the nervous system for it. Some suppose that the nerve is an elastic chord, and that the communication is by a tremor, as in other elastic bodies; while others believe that there is a nervous fluid moving from the brain to the extremities, and that by this fluid a motion may be propagated from the extremities of the nerves to the brain: a third supposition is made by Sir Isaac Newton, who imagines that the nerves may be considered as solid capillamenta, and that $a$ fluid adhering to these is fitted to communicate the motions from one part to another.

"Now to avoid determining any thing with regard to these opinions, I have used the term of nervous power; but as this is a little ambiguous, I choose to express it by nervous fluid; not that I suppose, with Dr. Boerhaave, that the brain is an excretory, and that a fluid is secreted from it: I mean nothing more than that there is a condition of the nerves which fits them for the communication of motion. But I defer the consideration of these opinions for the present, and perhaps ad Grocas calendas; but nothing shall be rested upon the nervous fluid, it shall be considered merely as a power fitted for communicating motions."

XXXI. In the living man there is an immaterial thinking substance or MIND constantly present; and every phenomenon of thinking is to be considered as an affection or faculty of the mind alone. But this immaterial and thinking part of man is so connected with the material and corporeal part of him, and particularly with the nervous system, that motions excited in this give occasion to thought ; and thought, however occasioned, 
gives occasion to new motions in the nervous system. This mutual communication or influence we assume with confidence as a fact; but the mode of it we do not understand, nor pretend to explain; and therefore we are not bound to obviate the difficulties that attend any of the suppositions which have been made concerning it.

"I I have added, ' of the mind alone;' but there is some little ambiguity with respect to this, therefore I would take it as at first-' of the mind,' and say that thought must have for its cause an immaterial substance, and that, at least ultimately, every phenomenon of thinking is to be referred thereto. A demonstration of this proposition would lead me into a very long digression, which is not much connected with this subject, and the discussion might appear subtile and difficult. I am convinced that this proposition has been demonstrated by divines and metaphysicians, to whom therefore I must trust, and refer you for farther satisfaction; and I shall have occasion immediately to refer you to a physician for the proofs of it. But while we agree with mankind in general, and with philosophers, with regard to this fact, we observe, in the next place, that 'this immaterial and thinking part of man is so connected.with the material and corporeal part of him, and particularly with the nervous system, that motions excited in this give occasion to thought; and thought, however occasioned, gives occasion to new motions in the nervous system.' II need not illustrate this further, as it is so universally understood and agreed upon in every system that thinking or thought arises from certain changes in the state of the body; and it is as evident that certain modifications of thought, however they arise, do produce motions in the material or corporeal part; and I say that 6 this mutual communication or influence we assume with confidence as a fact.' 'There is not in nature, seemingly in the acknowledgment of all philosophers, a greater mystery than this mutual action of the soul and body upon one another; philosophers have at least talked about the matter, and there are three very celebrated systems with regard to it.

"The system of Aristotle, which is adopted by many moderns, is called the system of the physical influx. Des Cartes has proposed another, which is known by the name of the system 
of occasional causes; and Leibnitz has proposed a third, which supposes the existence of a pre-established harmony. You may consult these; but I do not say a word of them, because when I have considered them as well as I can, I cannot perceive that they have the least effect or influence in explaining any thing: they do not admit of any application, either in physic or in any other part of science, that I see. With regard to the mode of this mutual influence, it will be allowed that as you adopt one or other of these hypotheses, it may affect religious belief, but it can have no effect in physic, so that it is not my province to consider them. But I must say, that the mutual influence being supposed and granted, physicians have differed very much with respect to the degree and extent of that influence, and therefore I am obliged to take some notice of these opinions, though they truly have not such influence as has been imagined, nor is it necessary to adopt either the one or other opinion upon the subject. There might be certain philosophers who contradicted our general proposition, and believed that there was no immaterial part of man nor any soul, but supposed the whole phenomena, even those of thought, to depend upon certain circumstances of matter and motion, and, therefore, that the whole of the phenomena of the human body, even those of thinking, are a matter of pure mechanism, and arise from mechanical necessity. This is the system of the materialists; and there have some defenders of such a system appeared lately; but their sect is very narrow, and there are few of such an opinion; and, however those of our profession may have been suspected of dangerous opinions, the physicians have been generally sufficiently orthodox upon this point, and they very universally admit the presence of a soul. My late colleague, Dr. Whytt, has, I think, with great strength of argument, shown that the phenomena, even of the body itself, cannot be explained but upon the supposition of a soul as a sentient principle. It is to this writer that I proposed to refer you; as he considers the argument with a view to the animal economy and to physic, he is the proper authority to be consulted for the demonstration of the immateriality of the soul in the economy while in a living state. But I must explain more particularly his opinion, and what has been the common 
one of physicians also. Though they maintain the presence of a soul-and I think it necessary to the motions that occur in our material part,-yet they are far from considering that there is any thing left to it as a rational soul, but that the mutual influence of the soul and body takes place by what may be called a physical necessity; that there is nothing arbitrary in the power of the soul. And Dr. Whytt, after having occasion to observe several instances of the influence of the mind upon the body, says, 'Nor can we consider the mind as acting either ignorantly or perversely, when it sometimes excites such motions in the body as increase its own pain, and in the end prove more hurtful than beneficial: for these motions do not proceed, as the followers of Stahl have imagined, from any rational views in the mind, or a consciousness that the welfare of the body demands them, but are an immediate consequence of the disagreeable perception which excites it into action.- $-(O n$ Nervous Disorders ; Works, p. 520.)-He is more explicit still in his Treatise upon Vital Motions, where he considers the share which the mind has in producing motions: "The mind in carrying on the vital and other involuntary motions, does not act as a rational, but as a sentient principle, which, without reasoning, is as certainly determined by an ungrateful sensation or stimulus affecting the organs, to exert its power in bringing about these motions, as is a scale which, by mechanical laws, turns with the greatest weight.'-(Works, p. 152.)-This is establishing, in the strongest manner, a physical necessity in this communication between the mind and body; and I say, indeed, that this has been the most common opinion among physicians. I shall quote only two, but these, however, of the greatest authority. Dr. Boerhaave speaks in this manner; see his Institutions, $\$ 27$. where he has these words, - 'Homo constat mente et corpore unitis. Quorum utrumque naturâ ab altero differt. Adeoque vitam, actiones, affectiones diversas habet;' but this is what you are to take notice of- 'Tamen ita se habent inter se, ut cogitationes mentis singulares determinatis corporis conditionibus semper jungantur et vicissim.'-In short, the soul and body are distinct substances; but they are so united, that thought is constantly connected with certain states of the body, and, on the contrary, that these states produce a 
certain state of thought. In $\S 696$. of the same Institutions, where he defines a disease in general: 'Status corporis viventis tollens facultatem exercendæe actionis cujuscunque vocatur morbus ;' he adds, in view of some opinions that were then prevailing - ${ }^{6} \mathrm{Nec}$ mentio animae facta in hac definitione, quia corporis statum determinatum idem animæ status individue comitatur.'-The other authority, which you can more conveniently consult, is Dr. Haller : in $\$ 570$. of his Primæ Lineæ Physiologiæ, after having proved the existence of a soul in the body, he adds these words-' Et tamen hre anima, adeo diversa a corpore, arctissimis cum eo ipso conditionibus religatur; quæe et cogitari cogatur ad eas species, quas corpus ipsi repræsentet; et absque cerebri corporeis speciebus non videatur aut meminisse posse, aut judicare; et cujus denique voluntas in corpore vel causa, vel occasio velocissimorum maximorumque motuum est.' Now these passages, from Whytt, Boerhaave, and Haller, shew what is the most common system among physicians, that though they think the soul and body to be two distinct substances, yet, during their connexion in a living state, they act upon one another by a physical necessity. Dr. Boerhaave explains it, and also Dr. Haller:- 'Anima cogitari cogatur ad eas species quas corpus ipsi repræsentet ;' and that the state of the mind produces some corresponding state of the body. Very different is an opinion which has prevailed for the greater part of a century, the author of which may be considered to be Dr. Stahl. He and his followers not only maintain that the motions have their source in the power of the soul-so far Dr. Whytt agrees-but they go farther, in asserting that the soul is not necessarily determined by the state and condition of the body itself, but that the soul merely as present, and as perceiving what is going on, and what motions are conducive to life and health, excites one kind of motion, and puts a stop to another. The principal difficulty that occurs to them is, that the effects of the soul's motions, as Dr. Whytt alleges, are sometimes pernicious to the system. But in these cases they only suppose that the soul is not perfectly regular, that it is not perfectly wise, and so may mistake its purpose or omit the effecting a cure, and that thus many phenomena of the body may occur, independently of any mechanical condition of the body itself, 
merely from the state of the soul, as more or less intelligent or attentive, all which you will very readily understand.I have to mention one other opinion which is of a mixed kind, it is that of Dr. Gaubius. How far he is different from Boerhaave, I do not say; but he seems to be different : he allows, with Boerhave, that hardly any motions occur in the mind that have not a corresponding motion in the corporeal parts, so in $\$ 523$. of his Institutiones Pathologio, he says-'Mens nimirum corpori suo, dum vita viget, intime implicata est : ut difficulter concipere liceat tam exquisite puram ejus operationem quæ prorsus nihil corpores mutationis quoquo modo sibi innexum habeat,' \&c.-That is going near to assert the notion of Boerhave. But in the following paragraph, towards the end, Dr. Gaubius, as apprehending in some measure, or fearing-without ground, I think-the consequences of the Boerhaavian opinion, or, if you will, by way of explaining it, gives these words: - Agnoscendum tamen videtur, aliquod menti in eam concessum esse imperium, quo excitare silentem pro lubitu possit ad agendum tam in universo corpore, quam in singulari parte : perinde ut musculi etiam arbitrio parent.'-He says, that for the most part, indeed, we find a strict connexion between the state of the mind and body; but he thought that the 6 individue comitatur' was too strong, therefore he thought the mind might excite motions, or cease from its usual actions very much of itself. Now these are different opinions with respect to the share which the soul has in the action of the economy: I shall hereafter give my reasons for rejecting some of these and admitting others; but several matters must be explained before we consider them fully. It may, however, be necessary at present to say, that $I$ am equally remote from the materialists on the one hand, and the Stahlians on the other; and, if I were to choosc, I would takc the opinion of Dr. Gaubius; but even that is not necessary to our system of physic; nay, I think it. will disturb it, and confound it upon several occasions. I think it was not nccessary to make such an addition, for even Dr. Stahl truly acknowledges that his views are not necessary to the system of physic; so in the preface which Dr. Stahl has put to Juncker's Conspectus Medicino, after he has endeavoured to prove and ascertain-'Quod vera causa efficiens (directionum 
illarum quas pro pure medico scopo, pro efficiente causa agnoscere sufficere poterat) sit in Homine ipsa Anima'-he adds, - Agnosco quod hac propositione, utpote non tam ad medicam quam physicam anthropologiam plane supersedere potuissem.' -We shall show this more at large hereafter; but I say that the opinion of Dr. Stahl is not only superfluous with regard to physic, but it is somewhat incompatible with the study and practice of it : : for if we do not suppose that the causes acting upon the human body produce their effects from a physical necessity, we can neither judge of the effects of the causes of disease, nor of the operation of remedies. To this purpose I shall mention a passage from Dr. Gaubius.. Where he enumerates the causes of sleep, (Institut. Pathol. $\$ 759$.) he gives some as depending upon the corporeal parts; he adds a fourth cause, but very properly with a forsan: that one cause of sleep may be, that the soul is either tired or is too lazy, and therefore animalibus organis relaxatis, lubens ab opere feriatur; but if we are to admit of the possibility of such causes, I refuse that we can distinguish when the material part, or when the capricious whim of the soul is the cause, and we are done with studying physic, with attaching any importance to the causes we have observed, or to the opcration of remedies; for all these may be varied by things out of our reach. The Stahlians have spoken of causes; and are willing to admit of such ; causes, however, must be physical and not arbitrary; in short, whatever I say in joining with Dr. Gaubius, with regard to the soul having a power of beginning motion, yet in matters of physic we must entirely abstract from it; and, therefore, whatever is in that, we must still keep to the language of. Whytt, Boerhaave, Haller, and the greater part of the other writers upon the subject. It is true, the language of Boerhaave and the others will seem to be the same with the language of the materialists, but a very little explanation will always shew the difference. $\mathbf{I}$, in using their language, will seem to talk as a materialist; and very unhappily some persons have understood me so. I have however particularly guarded against this."

XXXII. The phenomena of the nervous system occur commonly in the following order. The impulse of external bodies acts upon the sentient extremities of the nerves; and 
this gives occasion to perception or thought, which, as first arising in the mind, we term sensation. This sensation, according to its various modifications, gives occasion to volitron, or the willing of certain ends to be obtained by the motion of certain parts of the body; and this volition gives occasion to the contraction of muscular fibres, by which the motion of the part required is produced.

"Metaphysicians have divided the operations of the mind into three parts, viz. perception, intellect, and will; and if the term thought is applied, it is to the intellect; but there is no other general term for the whole of these operations but that of thought. Perception is the first beginning of all our mental operations: I say then, that perception or thought, as first arising in the mind, we term sensation. When I was in the garden, and perceived a flower, I did so with a perception of its being an object before me; but now that it is removed, I can recollect the image of its colour, shape, \&c. but that is, at the same time, with an opinion that the object is not before me now. There are two different conditions, therefore, of the perception of yesterday and of to-day. The whole of perception has been long spoken of among metaphysicians under the general term of idea. It was Mr. Hume who first thought of distinguishing between the first perception arising, and that depending upon recollection or memory : he called the first an impression and the other only an idea; but the term impression does not convey all we would wish; I, therefore, have, with Dr. Haller, employed for a perception as it first arises, what I strictly call sensation; and recollected perceptions I term ideas. This sensation, according to its various modifications, that is, as it is agreeable or disagreeable, gives the one or other mode of volition, desire, or aversion. There are some subtile doctrines insinuated towards the latter part. I do not say that volition wills the contraction of muscular fibres; we are not conscious of any such thing; but I say, that volition is the willing of certain ends or purposes to be obtained by the motion of certain parts of the body, as in the example I adduced-the plucking of a flower, and the bringing it nearer, we attend very often to the motions we employ, but not at all to the muscles that perform these," 
Note.-This is an example of the most ordinary case; but we do not mean to say it is the only case of communication between the different parts of the nervous system.- "I do not assert that every communication between the parts of the nervous system is with the interposition of both sensation and volition; for many cases occur in which we are conscious of neither ; it may be merely by a mechanical communication, in consequence of the peculiar organization of all the several parts of the nervous system."

XXXIII. As the impulse of bodies on the sentient extremities of a nerve does not occasion any sensation, unless the nerve between the sentient extremity and the brain be free (XXIX. 3.); and as, in like manner, volition does not produce any contraction of muscles, unless the nerve between the brain and muscle be also free, we conclude, from both these facts, that sensation and volition, so far as they are connected with corporeal motions, are functions of the brain alone ; and we presume, that sensation arises only in consequence of external impulse producing motion in the sentient extremities of the nerves, and of that motion's being thence propagated along the nerves to the brain; and, in like manner, that the will operating in the brain only, by a motion begun there, and propagated along the nerves, produces the contraction of muscles.

"Sensation and volition, as far as they are connected with corporeal motions, are functions of the brain alone. This is put here with a view to a controversy, which has long subsisted in the schools of physic, viz. What is the seat of the soul? With many it is considered as diffused over the whole system, so that it perceives in the eye, and operates in the muscles; while others say that it is immediately and only connected with the brain, which has therefore been called the sensorium commune. Another statement of the question is, whether the soul occasionally acts in every part of the body, or if there is a sensorium commune with which alone it is connected? I maintain that there is a sensorium commune, and that this is the brain taken in its largest extent, the cerebrum, cerebellum, \&c. and I have concluded this from the facts. alleged. This may be illustrated with regard to both sensory and motory nerves, but it is clearest with respect to the latter. If you apply a ligature upon such a 
nerve, it has the effect of interrupting all power of will over the muscle. We might indeed suppose that the brain has nothing to do with this, and that it is owing to a superior portion of the nerve; but the contrary appears when the ligature removed to a part nearer the brain has the same effect. But the experiment may be diversified. We know that besides the power of the will there are other powers which excite the contraction of the muscles, and which should have been described in XXXII. when I said that the example adduced (sensation and volition) is not the only way of communication between the parts of the nervous system. Thus, the pricking of a needle between the ligature on the nerve and its extremity produces a contraction, while the will has no such power; but if we carry a puncture between the ligature and brain we can produce no such action of the muscle. If a ligature or compression be applied to a nerve of sense - of the latter of which we have frequent instances in the optic nerves-the impression, of light for instance, does not produce its peculiar sensation; but if we can go beyond the ligature, and apply a puncture between it and the brain, this will occasion a sensation, whilst there is no sensation by any application between the ligature or compression and the organ. 35.66 This, I think, points out clearly that, from the part on which the impression is made, some motion is excited, and that this can be carried to the other: extremity of the nerve. I say, therefore, 'we presume that sensation arises only in consequence of external impulse producing motion in the sentient extremities of the nerves, and of that motion's being thence propagated along the nerves to the brain.' It is in consequence of its arriving in the brain only that sensation is produced; so that sensation is a function of the brain alone: the soul perceives in the brain only, and not in the sentient extremities."

XXXIV. From what is now said, we perceive more distinctly the different functions of the several parts of the nervous system, as distinguished in XXIX.-1. The sentient extremities (XXIX. 3.) seem to be particularly fitted to receive the impressions of external bodies; and, according to the difference of these impressions, and of the condition of the sentient extremity itself, to propagate along the nerves motions of a determined kind, which, communicated to the brain, give occasion 
to sensation. 2. The brain (XXIX. 1.) seems to be a part fitted for, and susceptible of those motions with which sensation, and the whole consequent operations of thought are connected, and thereby is fitted to form a communication between the motions excited in the sentient, and those, in consequence, arising in the moving extremities of the nerves, which are often remote and distant from each other 3 . The moving extremities (XXIX. 4:) are so framed as to be capable of contraction, and of having this contraction excited by motion propagated from the brain, and communicated to the contractile fibre.- "It has been disputed what connexion the moving extremities of muscular fibres have with the brain, and the causes of their contractility in consequence of this. It is true, that a muscular part, being cut away from the body and from all mechanical communication with the brain, will still retain its peculiar contractility for some time. This has given occasion to many to believe that the power of contractility has no dependence upon other parts of the nervous system, but is something inherent in the muscular fibre itself, which has been called a vis insita. But without here making any distinctions between the vis insita and the vis nervea, it is only to the last that $\mathrm{I}$ : here refer." -4. The nerves, more strictly so called (XXIX. 2.), are to be considered as a collection of medullary fibres, each enveloped in its proper membrane, and thereby so separated from every other as hardly to admit of any communication of motion from any one to the others, and to admit only of motion along the continuous medullary substance of the same fibre, from its origin to the extremities, or contrariwise.

$\mathrm{XXXV}$. From this view of the parts of the nervous system, of their several functions and communication with each other, it appears, that the beginning of motion in the animal economy is generally connected with sensation; and that the ultimate effects of such motion are chiefly actions depending immediately upon the contraction of moving fibres, between which and the sentient extremities, the communication is by means of the brain. Wherefore, in studying the nervous system, we judge it proper to consider, 1. Sensation, and, with that, the general function of the sentient extremitics. 2. The action of the moving fibres. 3. The function of the brain. In considering these three heads, 
the function of the nerves, more strictly so called, will, of course, be sufficiently explained.

CHAP. I.-OF SENSATION.

XXXVI. Our sensations may be considered as of two kinds: 1. Those which arise from the impulse or impression of external bodies, which we therefore name sENSATIONS OF IMPRESSION."I I perhaps am not very strict and accurate in the use of terms, in saying from impulse and impression. To obviate this difficulty, I say that when I use the term impression, it applies to all the several modes of giving or exciting motion." -2 . Those which arise from the mind's being conscious of its own action, and of the motions it excites; and these we name SENSATIONS OF CONSCIOU SNESS.

\section{Sensations of Impression.}

XXXVII. The sensations of impression are very various; but have been generally referred to five heads or kinds, commonly called the five senses; that is, those of sight, hearing, smell, taste, and touch.

I XXXVIII. The first four of these are each of them properly considered as forming one genus of sensations:-1. As the particular sensations comprehended under each head (XXXVII.), though very various, are, however, perceived to have somewhat common to all of them. 2. As those of the same genus all arise from impressions made upon one part of the body only, and that of a peculiar organization. 3. As those of the same genus all arise from the action of external bodies of one kind only, or of one and the same quality, by means of which they act upon our organs.

XXXIX. No such characters concur in establishing one genus of the sensations referred to the fifth head of touch, which are various in all those respects (XXXVIII.); and physiologists seem to have referred, to this head of touch, every sensation that does not manifestly belong to the other four; and, among the rest, many of the sensations of consciousness. It might perhaps be useful to distinguish into genera the se- 
veral sensations referred to touch; but it is not necessary to be done here.

From certain sensations referred to touch, it appears, that not only the extremities (XXIX. 3.), but that every part of the nervous system (XXVIII.) is sentient with respect to certain impressions.

"When mankind found that they could not generalize touch, they brought it under the general notion of feeling, and thereby united a great variety of sensations; but there is no connexion between the sensations, $e$. $g$. of hardness or asperity, and of cold and heat; and we cannot refer those to any one part of the body. It is true, that the prominent nervous papillæ are the peculiar means of perceiving the roughness or smoothness of bodies; but it is only in this respect that we can say there is an organ of touch. The general sense of impulse, the action of a sharp pointed body may take place upon the end of a nerve in the skin, but we may also apply it to any internal part where there are no papillæ so modified, for it will give a sensation applied in any part of the course of a nerve. In short, of almost all parts of the body, external or internal, there are few that are not sensible to the impression of a sharp-pointed body; it is the same I think with respect to heat and cold,-the organ of which we can conceive to be placed in the skin no further than as it is external, for if any other part is laid bare, it is also affected by heat and cold. We cannot say, therefore, that touch is occasioned by any particular qualities of bodies; these are prodigiously diversified, so that there is no foundation for making a genus under the title of touch, any farther than as it comes under the title of feeling or perception. I say that mankind have referred to the head of touch every sensation that does not manifestly belong to the other four. It is particularly curious to observe, with regard to the other four, that while they are the organs of peculiar sensations, they are also the organs of touch, as in the stricter view they are organs fit for receiving mechanical action; 'as when the eye is pained, or the ear is uneasy with the intensity of light or sound; or when the nose is affected with pungent odours, or the tongue with acrid tastes. I consider these as modes of touch; and we shall find that not only a variety of sensations of impression, but, among the rest, many. 
of the sensations of consciousness are thus referred to touch, and confounded with the sensations of impression."

\section{Sensations of Consciousness.}

XL. The sensations of consciousness may be referred to the following heads:-1. Those of apperception, by which we are in general conscious of thinking, of perceiving, judging, and willing, and thereby of our existence and identity. 2. The sensations arising from the particular state of thinking, as perception, memory, and judgment, are more or less clear, ready, or exact. 3. The sensations arising from the particular state of volition, and its various modes. 4. The sensations arising from the general state of action, as vigorous or weak, easy or difficult. - "An example of this is, the sense of alacrity and of weight in moving the limbs; also, the sense of lassitude. I find that I cannot move my limbs with the same force as I used to do; I find that $I$ cannot continue their action so long as $I$ have been able to do; I find a sense of weight and uneasiness in endeavouring to do it. This is; plainly, merely a sense of the affection of the mind, and without any thing which can be called external impression." -5. The sensations arising from particular actions, or a consciousness of the actions excited, and of the motion of the different parts of the body._-66 These, also, probably take place with regard to all the internal functions. The action of the heart and arteries, for instance, is in general more obscurely, but sometimes very clearly perceived, as also the state of respiration, or the action of the lungs; still more evidently the actions of the stomach and intestines, which we cannot consider, with any propriety, without having thus distinguished the sensation of consciousness. Still, I own that this head will admit of most doubt, whether these sensations belong to those of consciousness, or to those of impression. I am willing to allow that they are sensations of impression, in so far as the action of the muscles overstretches certain parts ; but sometimes they are without any such impression, and we are conscious only of the degree to which our motions are exerted. Thus, I judge of the distance of a body, which I measure by stretching out my arm to come into contact with it, which is only a consciousness 
of the degree of motion excited; but I am not conscious of the several muscles which are put into motion to produce this action. I am sensible of these only when they are exerted with some unusual violence, or with spasmodic contraction. Internal motions also may come to be perceived when they are performed with a spasmodic force. Thus, we take no notice of the action of our heart, unless when any cause excites it in an unusual manner, which we call a palpitation; or if there is any interruption or intermission in the pulse, which many persons can mark in the heart.' And so with respect to respiration, the usual train of which we are not conscious of willing, or directing, but, when it is performed with unusual frequency of force, we are." 6. The sensations arising from the diminution or absence of impressions.- "I will not say that darkness is visible, but it is a positive sensation. The sensation of darkness or blackness is the absence of impression; it takes place when no rays of light fall on our eyes, so that the sensation of consciousness may be here separated from that of impression."

Under each of these heads a great number of particular sensations are comprehended, but not necessary to be farther specified here.

\section{Laws, or general Circumstances of Sensation.}

XLI. Of the four first genera (XXXVII.), the sensations arising give no indication of the nature of the bodies acting on our organs, or of the mode of their action ; and, when we otherwise learn these circumstances, we can perceive no necessary connexion between them and the sensations which they produce. But, from certain sensations of touch and consciousness, we acquire the notions of solid figure, of motion, impulse, impenetrability, and the communication of motion, and consider the sensations as exactly correspondent to the circumstances of external bodies. At the same time, as we know of no other action of bodies on each other but that of impulse; and as, in the case of the sensations of the first four genera, we learn that an impulse takes place, we have comprehended the whole under the title of sensations of impression, and consider all of them as perceptions of impulse.- "Whoever has the smallest tincture 
of metaphysics will know the distinction pointed at here between the qualities of bodies as primary and secondary. Those we call primary which we perceive by the sense of touch, viz. solidity, motion, \&c., which are inseparable from the idea of body, and upon which all the secondary are founded. Whether these distinctions be well or ill founded, it is not my business to inquire; but it is agreed among all philosophers, that, with regard to the first four senses, we have no notion of the nature of the bodies acting upon them; we can perceive no necessary connexion between these bodies and the sensations that they produce, as, why the tremor of bodies produces sound, or the impulse of light the sensation of vision, far less why a refrangibility of the rays of light produces red, \&c.; for all that we can perceive, this might have been inverted, and what now occasions the sensation of red, might have occasioned that of blue. I mean to take hold of the fact, that we see no necessary connexion between the operation of bodies, and the sensations they produce, so that we must not be very rash in concluding from sensations as to the nature of the actions occasioning them. But, though it is true that such are our sensations of certain modes of touch, that we have the idea of body, of an extended solid from them, I would not have this applied to all the sensations which we receive from touch. Thus, the appending a weight to my leg as a mechanical power to resist my moving so easily, gives a sensation of weight; but, at the same time, that very sensation can arise without any weight appended, and entirely from a particular state of the nervous system; it is merely a sense of resistance to the motion of the nervous power in the crural nerve. I have no doubt that all pain arises from the force of mechanical impulse; but if a man, in the gout for example, has a sensation as if a dog was gnawing at his toe, or as if a chord was constricting it, we must not infer that in this case, the pain arises from analogous modes of action."

XLII. To produce any sensation of impression, a certain force of impression is necessary; and, from a lesser force, no sensation arises. The degree of force is likewise so limited, on the other hand, that, in a high degree, it destroys the organ ; and, in degrees approaching to this, a general sensation of pain, rather than the sensation of any particular object, is produced. 
- This may be illustrated. Suppose we had a scale marking the different degrees of force from 1 to 100, as in the case of heat,-I might say that till the degree of force rises to 20 , no sensation is produced; and that at the degree of 100 the organ is destroyed; and at $\mathbf{8 0}$ the force produces the undetermined sensation of pain; and, in this way, our sensations are limited between the force of 80 and that of 20 of such a scale. When I say that a high force of impression sometimes entirely destroys the organ, I illustrate it by an intense sound breaking the membrane of the ear, and by a very powerful acrimony, or a great degree of heat or cold, destroying the mixture or texture of our solids. There is, however, a degree of force which destroys sensibility without destroying the mixture or texture of the organ. We cannot perceive in what manner it has acted upon the texture or mixture, but we are sensible that, when the stupor is recoverable, it has merely changed that state of the organization upon which sensation depends. Thus, a certain intensity of sound is not sufficient to destroy the organ of the ear, but only to give a stupor for some time; and thus the eye may, by the action of a strong light, be rendered insensible to light, or to any object. I remember a country schoolmaster who had been a menial servant to a professor of mathematics, and who wishing to maintain some reputation as an astronomer, would view an eclipse with a tube without glasses : he was struck blind for three months. In like manner, a certain percussion on the elbow will give a stupor to the whole arm; and it is in this way that we explain the effects of the stroke of the torpedo, and those of electricity. A concussion of the brain also produces an absolute insensibility for a time; but it soon appears that it has not destroyed the organization:"

XLIII. Within these limits, however, our sensations are not exactly correspondent to the force of impression, nor do they make any exact estimate of that force. Usually, sensation is relative to the change that is produced in the nervous system; and a sensation proves strong or weak only as it is stronger or weaker than that which had immediately preceded it, or than that degree of force to which the nerves had been immediately before accustomed. For this reason too, the limits (XLII.) are very variable. - "Suppose the impression to have a force of 50, it is possible that, in different circumstances, it may appear

voL. I. 
either strong or weak. If the force of impression, which is at 40 , is increased to 50 , it shall appear strong; but if one at 60 is succeeded by one at 55, this will appear weak to us. I give the following experiment in illustration :-

"I take it for granted that the sensations of heat and cold arise merely from different degrees of heat. If I take a vessel with water, heated to $40^{\circ}$, another heated to $60^{\circ}$, and a third heated to $50^{\circ}$; and if I put my two hands into the water at $40^{\circ}$ and at $60^{\circ}$, I shall have no comparison in feeling either remarkably cold or hot; buit if $\mathbf{I}$ take the hand immersed into the water at $60^{\circ}$, and put it into that at $50^{\circ}$, it will appear cold; but the other hand, removed into the same water, will feel hot. Thus, heat and cold are felt accordingly as the single hand was accustomed to a greater or less degree of heat.

"In the same manner, M. du Fay, of the French academy, being employed in ascertaining the property of the various precious stones of shining in the dark, after having been exposed to light, found that the most certain way of examining them, was to have them placed in his chamber after having been in the light, while his chamber was perfectly darkened. Upon awaking from his sleep, he, in this way, found many luminous which would not appear so in the middle of the day. When he disposed his stones, he did so in a position which required the admission of some light; and he perceived that his eyes were not sufficiently sensible after being exposed to this light. He tied up one eye, therefore; and, having arranged the stones, he darkened the chamber. He then perceived no light with the eye which had been open; but with the other, which had been closed, he perceived the lights of the stones.

"I shall add another illustration from my own observation. If $I$ sit in a chamber enlightened by one window, and am intent on the image of that window, upon shutting my eye the image still remains as before; but now the window partitions remain enlightened and the panes of glass dark. I know that this succeeds with some other persons; and indeed the remaining of the image is owing to this-that the light still comes through my eyelids; but now the parts which had been in the strong light have their sensibility diminished, and those which had been exposed to the image of the partitions have their sensibil- 
ity remaining-the parts of the image received by the former appear dark, and those received by the latter are more light."

XLIV. Different sensations do not always imply a different kind of action in the bodies producing them; for sometimes different sensations arise merely from a different degree of force in the same kind of action, as is manifest in the case of heat and cold.

XLV. To sensation from impression, a certain duration of impression is necessary.

XLVI. The mind's resting for some time upon one sensation, is called Atrention. This, like the duration (XLV.), is necessary to give an impression its full effect.

XLVII. The mind seems to be determined to attention by the force of impression; by the pleasure or pain arising from it; by the degree of emotion or passion produced by these ; and, lastly, by the emotions being more or less related to the person feeling.

XLVIII. If the force and duration of impression, and the attention of mind, are all in the due degree, the sensation often remains for some time after the impression or action of the external body has ceased.- " This has been noticed by philosophers; and is, by Sir Isaac Newton, illustrated by the following common experiment:- If I give a circular motion to the end of a stick made red hot in the fire, it appears as one circular fire. There certainly is a succession of sensations; but each of the impressions remains so long that it is succeeded by another, so that we only observe one single circle of fire."

XLIX. The mind admits of, or can attend to, one sensation only at one time._- This, I think, is universally agreed upon by metaphysicians; they have demonstrated, it, and I leave it to their proof. Thus, if a number of impressions quickly succeed one another, the first received is very often obliterated by the succeeding; and when our bodies are exposed to two impressions in the different parts, we attend only to one of them-to the strongest. So if my eyes are occupied upon any object that draws my attention, I can give very little heed to what is said; and if I am very attentive in hearing, and my attention is fixed to that, I am insensible to impressions made upon my eyes or other organs. So it is an old observation in 
physic, that of two impressions giving pain, the stronger obliterates the weaker. According to Hippocrates, the corporeal impression is equally made, whether the mind is attentive or not; but the mind can attend to one sensation only at a time. The difficulty that occurs here, arises from the power the mind has of making so very surprisingly quick transitions from one sensation or idea to another; so that it is difficult to perceive that we do not attend to several at once-But considering volition as a subject of sensation, the difficulty is still greater. If there are a certain number of motions under the power of the will-and volition arises from sensation, and, if we are conscious both of the sensation and the volition,-if this is necessary, as the Stahlians suppose, we attend to many volitions at once, for we make at once a number of motions that may be called voluntary. Thus, I am speaking, moving my eyes and my handswhich you may think I do too much-and many other motions are going on at the same time. It would appear, therefore, that the mind does attend to more than one sensation at a time; but I shall solve that difficulty by and by."

L. 'Though the mind admits but of one sensation at one time, several impressions may act at the same time, if they be such as can unite in producing a single sensation; and such is the case of many of the impressions which produce the particular sensations of the same genus, as in those especially of colour, sound, smell, and taste._- A sound and a colour, presented at the same time, cannot unite in one sensation; and if the one is observed, the other must be neglected. But different sounds or colours can very readily unite in producing one sensation: thus, rays of light being thrown on surfaces suited to reflect portions of them, some will give a yellow colour, others will, in the same circumstances, give the sensation at least of a blue colour; but both, yellow and blue, being by means of a prism thrown together upon the same spot, we find that the effect of these two impressions acting together, is to give the sensation of green. So there are certain tones in sound, which, sounded upon different instruments, or on different chords of the same instrument, will unite, and give the expression of one single sound: this we call a concord. The same applies to the union of tastes and smells."

I.I. In each of these genera, many impressions, which sepa- 
rately produce particular species, can unite in producing a single sensation, which is always a neutral, or one different from either of the separate sensations.

LII. This union of impressions may take place, either when the impressions are exactly synchronous, or when the one succeeds the other before the sensation of the first (XLVIII.) has ceased.

LIII. Though the motion excited in the sentient extremities, by impression, remains some time, as in XLVIII., it must be supposed to become continually weaker, till at length it ceases altogether, and with it the sensation.

LIV. The same impression soon repeated does not produce the same strength of sensation as before. Hence, all new impressions are, coteris paribus, strongest ; and moderate impressions frequently repeated produce no sensation, unless their force is considerably increased.

LV. Actions, which at first produced a sensation of consciousness, as accompanied with volition, come, by repetition, to be performed without any sensation; or they produce it only when they are performed with uneasiness, pain, or unusual force.

LVI. Impressions being given, their effects in producing sensation are different in different persons, and in the same person at different times. This must arise from some difference in the state of the bodies acted upon, which may perhaps be referred to the following heads:-1. The state of the common teguments, or other parts interposed between the impressing body and the medullary substance of the sentient extremity.- "No where do external bodies come in contact with medullary substance without passing through other substances. Thus, in the case of touch, the cuticle constantly intervenes; there is something analogous in the tongue, there is a membrane in the nose, the membrana tympani in the ear, and the various coats and humours of the eye, through which the light passes before actually striking the medullary substance. Now it is obvious that these intervening substances may be, in different conditions, fitted to transmit, more or less easily, the external impulse, or entirely to interrupt it. In many cases the loss of sense is entirely to be imputed to the imperfections of these media or in- 
terposed substances; in other cases there may be a singular tenuity of the cuticle, \&c. to which may be imputed an increased sensibility."-2. The different state of the medullary substance of the sentient extremities, as given to it in the original stamina. - Whenever we observe any state of the functions, which is respectively the same throughout the whole of life, and continues so under a considerable variety of external circumstances, we say it depends upon the whole habit-upon the constitution; and this carries us up to the state of the original stamina. It is acknowledged by all physicians, that there is a difference of sensibility which runs through the whole of life in one sex more -than in the other. It is proper to be observed that this gives a seminium or predisposition to disease; and when this is excessive, it has a considerable share in the most remarkable affections of the nervous system. We would be curious to inquire in what consists this constitution of the original stamina, which remains in its effects through the whole of life: we have little hopes, however, of solving this problem, and would wish to limit it, and inquire, whether it consists in the animal solid part of every medullary fibre, or in the different conditions of what we suppose an inherent fluid there. It is probable that these two are connected, but we are disposed to believe that it is in the state of the simple solid substance."--3. The different state of tension in the medullary substance of the sentient extremities, as given to it by the state of the bloodvessels constantly connected with it._" This is a point of some curiosity, and has not been hitherto noticed by any of our physiologists. In considering (XLVIII.) the continuance of sensations after the action of the external body has ceased, I alleged that the motion excited in the sentient extremity must be of the oscillatory or vibratory kind, somehow depending upon an electric tremor. But if there is any truth in this, a certain degree of tension is necessary to these oscillations; so that it is possible that the state of the sentient extremities may be varied by different states of tension. The difficulty is to understand how this state of tension is given to them; and I think it must be referred to the state of the blood-vessels which accompany them. Nothing is more remarkable than the uncommon number of blood-vessels intermixed with the sentient 
extremities. We find from the centre of the optic nerve a considerable artery arising, which is distributed in a very singular ramification, so that it would appear that a portion of an artery accompanies every separate fibril of the retina. In like manner, we know that blood-vessels accompany the auditory nerves in their expansion in the vestibulum, cochlea, \&c.; in the nose also a numerous plexus of blood-vessels is every where intermixed with the nervous extremities: hence hæmorrhagies are so easily produced there. With regard to the tongue and the skin, it is no less remarkable. In every separate papilla anatomists have traced an artery immersed into its substance, and ramified in its extremity to a great degree of subtlety. Now, admitting the fact that arteries every where accompany the nervous extremities, we further know, that every artery is in a state of tension, $i . e$. it is filled to a dimension beyond what it would assume if left to itself. But this tension of the bloodvessels is varied upon different occasions; and therefore the tension which they give to the nervous fibres must be also various. There is no doubt that such a tension is produced, but it remains to be proved that it produces this effect: but that is not more difficult. The sensibility is occasionally increased in the retina; but in no instance so remarkably as in the case of inflammation of the eye, especially in those inflammations which affect the arteries in the posterior part of the eye, in consequence of which, the sensibility is often increased to a surprising degree. The sensibility of my eye at present is so moderate, that I can destroy it entirely by shutting the eyelids; and if in some persons this is not sufficient, they can, by the application of a certain number of cloths, be made insensible to the presence or absence of light. But I have known cases of ophthalmia, in which the light could not be borne, even when six or eight folds of linen covered the eye, and the curtains of the bed were drawn round in a dark chamber. The patients would be sensible to the putting aside the curtain; and this is only to be explained in this way: that the inflammation is attended with an overdistention of the arteries, which increases the sensibility of the nerves by increasing the tension in the manner we have explained. In other cases, those of headaches approaching to a phrenitic affection, the sensibility both of ears and eyes is so 
increased that they cannot bear the smallest degree of light or noise."-4. The state of the same medullary substance, as affected by heat or cold.- "A certain degree of heat is necessary to the subsistence of the body in the living state; but if life consists especially in a certain condition of the nervous system, it will follow that heat has indeed the power of giving that condition to the nervous system upon which the functions of life, and perhaps sensation, necessarily depend. Hence, according to the different degrees of heat applied to our nerves, they are more or less exquisitely sensible; and I have no doubt that this is the just solution of the ordinary fact of the greater sensibility of men inhabiting warmer climates, than that of men inhabiting cold climates. But if there is any doubt with regard to the effects of heat, there is none with regard to the effect of cold. We have no fair instance of the effect of cold on the retina and auditory nerve; but with regard to the nerves more exposed to surrounding bodies, we know most certainly, that a certain degree of cold destroys the feeling altogether, and considerably affects the sensibility of the gustatory and olfactory nerves. I say, therefore, the sensibility is according to the state of the medullary substance as affected by heat and cold. But however heat may be produced, it is probable that it is connected particularly with the blood, and is distributed to the different parts by the blood; for we find that upon subtracting the circulation, the heat ceases as soon as it would do in a body of the same bulk which has acquired it. The action of the arteries, therefore, which are joined to the nervous extremities, has the effect of giving not only the tension, but also the heat necessary to sensation." -5 . The state of it, as produced by former impressions (XLIII.-LIV:) - "We see this particularly in its power of diminishing the power of those impressions which are the cause of fear; and the courage of the sailor and soldier, for example, are to be explained in no other way than by their having the impressions so often repeated as to lose their force; - and we have instances of the same in other parts of human life. Let us consider what may give a general character of more timidity and intrepidity; we observe it in the difference between the inhabitants of a rude and uncultivated climate, and those of a civilized and polished nation. The in- 
habitants of the former are obliged to walk along the narrow brink of the precipice, to stem or pass the foaming torrent, and to encounter every vicissitude of the weather and season ; whereas, in the cultivated country, if the inhabitant is obliged to travel, roads are prepared for him ; if he passes by a precipice, it is guarded by a parapet; he crosses the river by a bridge; he is guarded against the more severe attacks of the weather; and is therefore liable to a great deal of timidity, while the other becomes hardy and bold. The contrast is equally remarkable in the case of the women, in a state of simplicity and in that of refinement. Among the more rude nations, they are obliged to take part in all the hardships of the men, and meet with less indulgence from the men, who are of less delicate feelings; while, in the refined state, care is taken that the winds of heaven shall not visit their faces too rudely, and they are carefully protected and guarded against every thing that may hurt or even shock them ; accordingly, the one will show timidity in excess, the smallest troubles will throw them into fits; while among the other such appearances are unknown. Thus we cannot keep things within their proper bounds, and readily run to excess, perhaps to the other extreme."-6. The state of the nerves along which the motion is propagated.- " I suppose that no sensation arises but in consequence of a motion propagated from the sentient extremity, along the course of certain nerves, to the brain. Compression as well as other causes may prevent that motion; and this may amoun to a total interruption, or only go the length of a more imperfect transmission. (XXX.)"-7. The state of the brain or sensorium.- "This is an analogous case to what we mentioned with respect to the nerves (6.) Where the brain is compressed, all sensation is destroyed or considerably diminished. But there is a state of the brain in sleep, which I think does not depend upon any compression, (see CXXVI.) but which prevents sensation notwithstanding the impression. This, therefore, is another cause which determines sensibility; and there are intermediate states between sleeping and waking, in which the brain is in different conditions with regard to sensation. Another view to be taken of this matter is this:-We find that a determination of blood to the brain seems to be necessary to its 
functions; for, by withdrawing that, as happens in the deliquium animi, the functions of the brain and sensations disappear. I think it probable that a certain tension is given to the substance of the brain by the blood determined in such quantity to its arteries, - so that the sensibility of the brain and whole nervous system connected with it, is increased by the increased impetus of blood in the brain; e.g. in phrenitis the sensibility is increased, because most of the organs of sense share in the increase of impetus which gives the phrenitis. This impetus may operate by giving more sensibility to the sentient extremities themselves, but also by increasing the general sensibility of the brain; for this occurs also when the increased impetus has not been extended to affect the sentient extremities. Gaubius, treating of this subject says, in his Institutions, ( $\$ 194$. - A mente corpus irritabilius reddi non magis probabile est, quam illam ab hoc incitari.' This is again the Stahlian system produced. I am: willing to admit the modification of Gaubius, (XXXI.) that the mind is ever the beginning of motion in the body; but we must, in physic, entirely abstract from this, for the supposition is admitted to no purpose; and if we ever lose sight of the body as the object of physic, and suppose an action of the mind independent of it,-an operation of the cause of which we cannot judge,-we are quite thrown out of our system. Gaubius says that the affection of the body by the mind is not more probable than that of the mind by the body. I say it is much less probable. In a thousand instances we see that the body moves the mind, but in no one case do we clearly see that the mind moves the body." -8 . The state of attention (XLVI. and XLVII.) - " Lastly, I have to add another cause: the sensibility of the sentient extremities may be varied by causes of excitement or collapse (see CXXX.) directly applied to them. Of the causes of collapse operating directly upon the sentient fibre, we have proofs in the action of sedative or narcotic substances applied to them. Thus opium acts upon every portion of the nervous system to which it is applied. I am uncertain whether any powers of excitement exist, as I cannot observe their operation or effect; and we are not sensible of any powers but those which depend upon tension acting upon the sentient extremities. There are, indeed, substances 
which excite the action of irritable fibres, and which we call stimulants; but they are only causes of motion, and not of greater mobility."

LVII. Different parts of the body are sensible, and sensible only by means of nerves present in them; but anatomy does not always determine certainly with regard to the presence or absence of nerves; and, therefore, the sensibility of several parts can be determined by experiment only; which, however, is also fallacious.

LVIII. Particular sensations arise from impressions on certain parts only:-1. Because the sentient extremities in these parts are so situated as to be exposed to the action of certain external bodies only. 2. Because the sentient extremities are connected with an organ that increases the force of the external agent, or modifies its action in the manner necessary to a determined impression. 3. Because the fibres of certain sentient extremities are, by their size or tension, fitted to be acted upon by certain external bodies only. 4 . Because certain sentient extremities are so constantly preserved in a certain state, as to render them more sensible to a change.

These circumstances determine the mode of impulse, but do not account for the sensation arising from it.

LIX. Different sensations are accompanied with different judgments concerning the bodies making impression, and the part of the human body upon which it is made. Some sensations are referred to bodies at a distance; others, to external bodies in contact; and others, to the feeling body itself.

LX. When sensations are referred to our own bodies, it is in three several ways:-1. They are most commonly referred to the part on which immediately the impression is made, and this; with regard to the external parts; very accurately; but, with regard to the internal, much less so : and commonly the sensations arising from internal parts, are referred to the incumbent external part, with some obscure distinction between superficial and more deep.- "We may observe this in the stitch of the side. The patient commonly does not know " whether it is external or internal, till he tries if it can be affected by external pressurc. In many cases we know that the pain is on the ex- 
ternal surface of the lungs, but the patient has no distinct feeling between this and the insular pain in the pleuritis spuria. The same is true with regard to the pain of the head, whether within the cranium or without; only, finding it affected by external pressure, we suppose it to be in the external parts. And, in like manner, with regard to the abdominal region: as there is here a succession of parts lying upon one another, we cannot from the sensation say which is the place. Thus, a pain in the pit of the stomach may arise from the stomach itself, or from the adjacent biliary duct, from the liver, the pancreas, \&c. With respect to feelings referred to internal parts, therefore, we can only-form our judgments from the affections of the functions accompanying them."-2. Sensations are sometimes referred, not to the part upon which the impression is immediately made, but to a distant more sensible part, to which a motion is propagated from the part impressed." This is a matter of much nicety, and shews the fallacy of the general rule, that sensations are referred to the part on which the impressions are made. It is sufficiently known, that from impressions made upon one part, motions may be propagated to other parts, as in the case of a very acute sound,- - such as that produced by a file, or by a bit of cork rubbed upon the table, giving an uneasy sensation in the teeth; and in the case of the stone in the bladder, which can only act upon the neck of the bladder, but the pain from which we perceive only at the point of the urethra. In like manner, a pain arising from a stone in the pelvis of the kidneys, or in the beginning of the ureters, propagates a motion along the course of the urethra from the neck of the bladder, and is exquisitely felt only at the orifice of the urethra. With regard to other instances there may be more doubt, but I will mention them :-When a rheumatic affection attacks the hip-joint, a feeling is commonly propagated downwards to the knee, where it shoots out in violent pain; the same feeling goes still downwards, and then a sharp pain is felt in the ankle. In some cases it may be a question whether or not the rheumatic affection is in the knee as well as in the hip-joint, or in the ankle as well as in the other two parts. But in many of these cases we have reason to believe that the rheumatic affection is confined to the hip-joint 
only. In the case of dysentery, the griping pains, which we can prove to be in the colon, give the sensation of tenesmus at the anus.-I have added what may be called another piece of theory, - that the sensations are not only referred to a distant part, but to a more sensible part. In some instances, I have no doubt of explaining it in this way:-The glans has indeed more sensibility than the neck of the bladder; and we can hence conceive why in the case of stone, or in gonorrhoea also, the pain is felt more especially at that sensible part. But in other cases, I cannot see that this should take place from the greater quantity of nerves, or from their being more fully expanded; in these cases the sensation depends upon another circumstance: not, strictly speaking, that the parts are more sensible, but that the motion is in certain places rendered more considerable. You must allow me the hypothesis here, that motions are propagated along the nerves, and that these are of the vibratory or oscillatory kind. Now I say, that if an oscillation is propagated. along a nerve, so long as the course of the nerve is perfectly free, the oscillation will not be accumulated; but if there is a stoppage, the oscillation will be accumulated there, and so its force considerably increased. In this way I explain the many instances of motions propagated till they arrive at a more sensible part, or to an extremity, where a stop is put to the oscillations, as in the joints. Thus, the rheumatic affection of the hipjoint is moved along the nerves; and we have an obscure sensation which does not amount to pain, till it comes to the extremity of the muscles, where they are fixed in the joints. Thus also in the phenomena of electricity, when I receive the electric shock in my two hands, it is felt most considerably in the wrists, elbows, . shoulders, \&c."-3. As sensations usually arise from impressions made upon the extremities of the nerves, and are referred to these, so impressions made on the nerves in their course, are sometimes referred to the extremities from whence they had commonly arisen.- " Here I have rather given the. fact than the explanation. But the fact is well known, that in persons who have suffered the amputation of a leg or arm, the sensations referred to the toes or fingers remain; so that in the case of explosions of guns, and such things as can affect the 
body strongly, the uneasy sensations are referred to the toes, \&c. and not to the only proper remaining feeling part."

LXI. The sensations of consciousness (XL. 1, 2.) are referred to the encephalon. So are those of XL. 3. if they are moderate ; but if more vehement, they are often referred to those parts in which their effects are exerted, as the heart and organs of respiration. The sensations (XL. 4 and 5.) are seldom with accuracy referred to particular parts, but indistinctly to a whole member. We are not conscious of the action of particular muscles, except when their contraction is spasmodic.

LXII. We are disposed to combine our sensations as united in one object; and thus form what are called COMPLEX IDEAS. LXIII. We compare our several sensations, and from thence acquire new sensations of RELATION.

LXIV. When sensations, formerly received, are again renewed by the same objects, it is, for the most part, with a consciousness of their having been formerly received; and this faculty we call REMINISCENCE.

LXV. Perceptions, formerly received, can be renewed without the presence or action of the object which formerly gave occasion to them; and if this is attended with the consciousness of a difference between the vividity of the two perceptions, and particularly of the absence of the original objects, such a renewed perception is called an IDEA ; and the faculty by which this renewal is made is called MEMORY.

LXVI. Perceptions, formerly received, can, without the presence of the original object, be renewed also in such a manner that the mind does not perceive any difference between the original and the renewed perception; and, therefore, such renewal is always attended with the persuasion of the presence of the object. The faculty by which such renewal is made, we call IMAGINATION more strictly.

LXVII. Reminiscence depends upon the force or frequent repetition of the former sensation.

LXVIII. Memory depends upon an association of perceptions, which is formed by their being frequently repeated immediately after each other; by their being parts of the same complex idea; and by their having relations marked. Memory 
is generally faithful to such associations; but it is more or less so in different persons, according to the number and importance of the relations marked, according to the frequency of the repetition of the sensations, and the marking of their relations; and according to the different states of the brain, very little known._- That the difference of memory depends upon the state of our corporeal part, is undoubted. Children are a remarkable instance of this : they do not soon acquire the faculty of memory. Haller has put it at five years; but, without ascertaining the precise period, we may say that it is only by the frequent repetition, and some advance in age, that they acquire any power of remembering. In like manner, every body in the decline of life, comes to be sensible of some failure of memory ; and before our intellectual operations are obliterated, this is announced by the failure of memory, which happens to every man universally, and much about the same period; so that in infancy and in old age, there is a state of our corporeal organs, which is not so well suited to the exercise of this faculty. We have also organic affections of the brain obliterating memory. Thus, there are instances of persons in fever losing every thing laid upon their brain, as Haller speaks, so that they had to betake themselves again to learn the use of letters. And nothing is so common as that the memory is obliterated in palsy, and similar affections; so that there can be no doubt that, however the difference of memory may be referred to mental causes, it is, in many more instances, a corporeal affection."

LXIX. Imagination seems always to depend upon internal causes - that is, upon causes acting in the brain.

LXX. Memory and imagination renew distinctly the ideas of seeing and hearing only. All others are renewed imperfectly, or not at all ; but all others may be associated (LXVIII.) with the sensations or ideas of seeing and hearing, so that these become signs of the others. - $"$ This is a proposition that may be referred to every man's own experience, whether he can recall the taste of any thing, as well as he can recall the image which he received by sight, or the sound which he received by hearing."-The memory, in renewing these signs, so far renews the idea belonging to them as to renew their several associations and relations; to renew, in some degree, the pleasure or pain 
which formerly attended the sensations themselves, and, particularly, to renew the emotions of mind, or motions of the body, which the sensations formerly produced.

LXXI. Most of our sensations, perhaps all of them, are either pleasant or painful.

LXXII. The words pleasant and painful are commonly generic terms, each of them comprehending a great many species which seem to require being assorted under several different genera. Thus, in the first place, our sensations may be divided into those we desire, and those we are averse to. Of those we desire, we may distinguish those which arise from qualities we refer to other bodies, from those we refer entirely to our own. The first may be named, more strictly, the AGREEABLE-the last, the PLEASANT. In like manner, of the sensations we are averse to, we may distinguish the DISAGREEABLE and the PAINFUt. But, farther, the last must be distinguished from that sense of aversion which accompanies certain sensations of consciousness, as the sense of debility, lassitude, difficulty, \&c.; and, particularly, from that which is referred obscurely to internal parts, and this we name ANxiety. These sensations may be called the UNEASY ; - and every one distinguishes this kind from that of the PAINFUL, more strictly so called. These last seem to be always sensations of impression, referred pretty accurately to a particular part.

There is thus a foundation for establishing different genera of the sensations we desire, and of those we are averse to; as also, for greater precision in the employment of terms. But the fixing the limits of these genera, and assorting the several species, may be still difficult; so that we cannot be certain of applying the terms every where with strict propriety.

LXXIII. The enumeration of the agreeable or disagreeable, and even of the pleasant sensations, would not be of much use here; and the enumeration of the uneasy and painful, though much more interesting, belongs to the Pathology. "(See page 54.)" However, we think it proper to deliver- here the few following propositions.

LXXIV. Sensation and action, within certain limits, are always desired ; and the want of sensation, or imperfect and indistinct sensations, are always uneasy. In action, of every kind, 
the sensations of debility and difficulty are also uneasy. ("See page 59.")

LXXV. In sensations of impression, their being pleasant or painful often depends on the degree of force in the impression, allowance being made for the sensibility of the system.

LXXVI. As impressions, by being repeated, produce weak sensations (LIV.), impressions, at first painful; may, by repetition, be changed into pleasant, and the pleasant into insipid and uneasy. Hence arises, with regard to moderate impressions, the pleasure of novelty, the desire of variety, and the desire of increasing the force of pleasant impressions.

LXXVII. There is a condition of impressions rendering them objects of desire or aversion, that cannot, with certainty, be referred to their force.- "6 For instance, it is not yet determined whether the fragrant and fetid smells differ merely by the force of impression; and the same applies to sweet and bitter tastes. I suppose, therefore, that there may be other circumstances determining the desire or aversion."-This condition we call the quality of impressions.

LXXVIII. Impressions are often rendered objects of desire or aversion, by combination, succession, and relation.

LXXIX. No sensations arise originally in the mind without a previous change in the state of the body.- "For this, I refer to Mr. Locke. The import of it is, that we have no innate ideas."

LXXX: Certain impressions, and certain states of the body, like to those which produce the sensations of conseiousness, may both of them act upon the nervous system without producing any sensation.- " I repeat it again, that the ordinary course of the communication of the different parts of the nervous system, is by sensation and volition; but this is not universally the case. In how many cases it was so at first, and the consciousness of it has been lost by repetition, I will not determine; but, I say, it is probable there are certain cases where there never was any sensation, as in the instance of a purgative; this perhaps a man takes for the first time of his life; he has a sensation of the effect, the contents being propelled towards the extremity of the rectum; he is sensible of them when there, but the previous operation he is absolutely insensible of. There are,

vol. I. 
certainly, innumerable operations of this kind going on in the body. Though certain substances stimulate the kidneys we are not sensible of it; and so with regard to the stomach; if we take a dose of ipecacuan, it has operated for some time upon the stomach before we have any sensation of nausea; this only follows an operation of which we are not conscious; and I imagine that it is an established law of our system, that certain states of our stomach operate upon distant parts of the body without our being conscious of the operation, or of the effects. Thus we have a fact from Van Swieten, of a person who, by taking a small quantity of crab's eyes, had an efflorescence upon the surface of the body, which disappeared upon the crab's eyes being thrown out of the stomach. Now, here is a communication that cannot be said to be attended with any degree of sensation; and there may be many more of the same kind.',

"I now proceed to consider the errors or depravities of sense which we cannot refer to the different degrees of sensibility. With regard to this subject, I think it may be supposed to consist in these three particulars-1. Our perception of things that do not exist, or our supposing matters to exist that have no real existence. 2. In our judgment of things really existing without us, our perceiving them otherwise than they really exist. 3. When the qualities of impression do not operate in the usual manner. But of each of these more particularly.

"6' 1 . The first of these is what we more strictly call imagina'tion; and, with regard to it, I have said in LXIX. that it seems to depend upon internal causes, $i . e$. upon causes acting in the brain. If I had qualified this with the word chiefly, I should have been correct; but as it stands it is not just, for there are imaginations that are false, which depend upon impressions made not only upon the brain, but upon other parts of the nervous system, and even upon the sentient extremities. I was once informed, by a gentleman of great veracity, of a singular occurrence to this purpose. A lady was attacked with a disorder of the hysteric kind; and she imagined she was surrounded with goblins and spectres, which followed her from one part of the room to another. Her physician and "surgeon were present in one of these fits, and they endeavoured 
to restrain her; and, very accidentally, in attempting to shut out the light, they found, that upon covering one eye the spectres disappeared. Now there is no sort of doubt that there is a constitution of the sentient extremities of the organ of the eye itself, which can give this false imagination; and we have some-slender analogy here, as upon shutting my eye and rubbing it, I have the appearance of lucid stars; so that, from a motion excited in the retina the imagination can be produced. The muscæ volitantes, also, which patients sometimes see floating in the air, in the shape of the darting of lightning, of lucid ribbons, \&c. and which in some persons are always descending, and in other cases traversing the eyes, depend upon a particular constitution of the organ of the eye, and often upon some degree of inflammation which we can cure by the proper remedies of inflammation applied to the part; there is no doubt, therefore, that these false imaginations can arise from external impressions. So in the case of the ear; a man imagines that he hears the sound of a bell, but from nobody else hearing it, he comes to learn that it is the tinnitus aurium, which does occur probably from mótions excited in the organ itself: and it is proper to observe, that like deceptions may arise with regard to the other organs. Thus we have perceptions of odours, which we have no reason to believe to exist; but we are liable to impute this to the fluids of the nose, and never impute it to the organs of smell, or to any motions made upon it. So with regard to the false taste of the mouth, we are ready to impute it to the saliva, or to matters exhaled from the stomach; but we have reason to believe that it may be entirely from a change of motion in the organ. I will add another illustration with regard to the power of imagination in exciting the same state of the part formerly made by an impression upon it. If you bring a feather to tickle a man's upper lip, you can throw him into convulsions; and if you have done so once or twice, you need only for the third time approach him with the feather; for before you come within an inch, the person feels the same tremor in his lip as before; so that from the imagination he has this false perception, that he thinks the feather touches his nose when it does not. So with regard to the case of touch, it is not necessary to speak of the case of pruritus, which may, 
in like manner, take place from the different condition of the nervous extremities, without any acrid matter applied. Another case which might be referred to this head, is that of the amputated limb, where the sersation is referred to a place which no longer exists; and all the other instances where sensations are referred to other places than those upon which the impression is made.-(See LX.)

" 2 . With regard to the second head of the errors of sensewhere the supposition of the external existence is well-founded, but we judge of it otherwise than it really is-if we admit the philosophy of Bishop Berkley, we say, that the whole of the supposition of external existence is purely ideal, that it is an operation of the mind, and that the supposition of body is altogether false. But even the sceptics, who do not carry matters so far, allege that we have no certainty that, in any one case, we do perceive external things such as they really are. We can condescend upon many instances of our mistaking in this respect, from which we might extend the possibility to the whole. But we are not to be disturbed in the least by any subtleties of this sort; it is enough that we unavoidably suppose and conclude the existence of body, and that we do distinguish it in different cases by our different senses. This is the unavoidable practice of the human mind; and it is enough to say that our perceptions are erroneous, where we perceive objects at one time in a different manner from what we do at almost every other, or where the perceptions of one sense do not correspond to those of another. Thus, if I see a rod bent when immersed in water, but upon applying my hand I feel it straight, I know that my perception of its being crooked was erroneous. In like manner, when the perception of a single man is different from that of a great number of others, this is called a false judgment with regard to real objects. We may observe, that the sceptics are almost quite in the right, when they observe that very constantly we make a false judgment in establishing the force of external impressions. In XLIII. I was at pains to explain this matter, that our perceptions are merely of the relative force. When these are as usual, our judgment may be said to be as it ought to be in directing what relates to our bodies; but, in another respect, our sense is er- 
reneous, in so far as our judgment depends upon a number of different circumstances in our own organs, each of which may be changed without our being aware of it, or perceiving it, and therefore we judge differently. The opticians will afford us many instances of this, with regard to the magnitude, distance, figure, and even number of objects. I need not insist upon the particulars, as they will readily occur to you in considering the subject. These are errors of sense, which depend upon changes in the state of the external object itself, and, therefore, we are not concerned with them; only when they are from a change in the state of our organs, they are to be studied in Pathology.

" 3 . We conceive it as an error of sense when we perceive the qualities of bodies in a very unusual manner, or when these qualities have a very unusual effect upon us. I say the qualities of bodies affect us in a very unusual manner, when our judgment of these qualities is totally changed; as when we perceive asafoetida to be an agreeable odour, and musk to be remarkably disagreeable; instances of both these are common, and are certainly contrary to the most general state of sensation among mankind; or when the small portion of the white of an egg, which is a bland mild substance, taken into a certain stomach produces violent pain and other disorders; this is more uncommon. Many of the singularities mentioned by physicians under the title of Idiosyncrasies, are of the same kind; they are to be considered as errors of sense; and have been called depravities of sense. Dr. Whytt mentions these depravities of sense as one of the principal causes of nervous diseases, (Works, p. 543.) Some very curious questions may be started; e. $g$. in the case of Idiosyncrasies of the stomach. It may be a question whether the effect depends upon the action of the substance that is thrown in, acting upon a peculiar condition of the nerves there, or if it depends. upon the substance thrown in occasioning a peculiar fermentation there, or modifying, in a peculiar manner, the usual fermentation of the stomach, and thereby producing a matter in it which would act upon every other stomach. Thus, with regard to the effects of honey when taken into some stomachs, a strong suspicion arises that they de- 
pend upon a peculiar modification of the ordinary fermentation; but we observe the same singular effects of qualities, where there is not the least room to think of any fermentation being concerned. Another question upon the subject is this: Supposing that the substance acts upon the nerves, and that the effects arise from the singular constitution of these, do they depend upon the constitution of the extremities, or upon some circumstance in the common sensorium? And a third question would be, in what the peculiar condition of the extremities or of the sensorium consists? with regard to which last, I can only say that we know nothing of the matter. I have said that we know of no necessary connexion between impression and sensation, and that, for aught we know, the substances that act upon our smell might have been made to give the idea of colour ; and it will be difficult to say when the condition is in the extremities or in the sensorium; we can only say that one is probable in one case and another in another case."

"Among the symptoms referred to the functions of sense, we mention here the more general sensations of pain, pruritus, restlessness, and uneasiness :-

" PaIN.-In XLII. I stated that those strong impressions which do not destroy the organ, give the general sensation of pain rather than the sensation of any particular object; and in XLIII. I explained that sensations are strong or weak in proportion to the force of impressions and the degree of sensibility taken together, and that the sensibility is not to be measured by any standard, but is relative to the preceding state of the nerve. Now, when pain arises in the internal parts of the body, where the body giving impression is not evident, we must inquire into the nature of these internal impressions : it would seem that we can judge of them only by analogy drawn from external impressions; these are reduced to two heads-the mechanical and the chemical, to which, accordingly, we shall, in the first place, refer all painful sensations.

" 1 . The mechanical impressions are very various, but may all be comprised under the three heads of extension, compres- 
sion, and division of continuity. The last two occur, I believe, as causes of internal pain; but they are much rarer occurrences than extension, the chief instance of which is the distention of the arterial system. This may produce pain by stretching or pressing upon nerves placed in the neighbourhood; but more probably the pain depends upon the distention of the muscular fibres of the arteries themselves. Thus we observe, that membranes, which do not seem sensible to pricking or cutting, are still sensible to this kind of distention; and in chronic rheumatism, where there is not the same distention of nerves as in inflammation and in acute rheumatism, there is a difficulty in explaining the violent pain, except by a state of the muscular fibres which does not easily admit of extension.

"We observe different modes of pain, and distinguish between the pressing, tearing, pricking, cutting pain, \&c., which, when external, are manifestly different, according to the mode of impression. Now, the like feelings, from internal causes, are supposed to arise from the same modes of impulse; but these I find it difficult to suppose; and I think it much more probable that the chief internal impulse is the distention of the vessels, even by fluids moving in them, or the stretching of the neighbouring nerves by this distention. These, by their difference of force, manner of repetition, and extent, may produce sensations resembling those from the different modes of external impulse. For our sensations are very arbitrary : the sense of touch from external impression, indeed, gives us an idea of the mode of impulse; but, in many other sensations, it is agreed that we can perceive no necessary connexion between the mode of impression and the sensation; and we must, therefore, not expect to be able to explain how far any sensation depends on any particular mode of impulse. Thus, we are at a loss to explain the sensations of acrimony, dryness, \&c. in the fauces producing thirst, and the sensation of hunger in the stomach, either mechanically or chemically. 'The same applies to many other sensations, and to the causes of pain arising from internal causes.

"2. Chemical impressions. In many cases we can perceive no motion of a body producing the pain: we are contented to comprehend such impressions under the name of chemical, be- 
cause they are connected with qualities which we distinguish as such. A tendency to a solution of continuity might be supposed to be the cause of such pain, as we perceive that the substances which most powerfully produce it, such as caustic alkalies, concentrated acids, and a few others, have the power of dissolving and destroying the texture of our solids; but I am very doubtful how far this will extend; for there are certainly substances which may excite motions in our sentient parts suffieient to give pain, but without any tendency to destroy the solid texture : thus, a very concentrated solution of common salt gives considerable pain, but I think no chemist has discovered that it has a tendency to destroy the solid substance of the body. I would say, therefore, that even caustics are painful, not because they dissolve the part, but because they excite those motions in the sentient extremities upon which pain depends. Heat, also, is painful or pleasing, according to the preceding temperature of the body, and in a degree which does not, in any measure, approach to a solution of continuity; and the pain from cold certainly cannot be so explained, as this has the contrary effect of condensing the substances to which it is applied.

"T There is a cause of pain, which, though it probably may be referred to a mechanical impression, deserves a particular consideration, viz. the pain arising from Spasm, or from a contraction, considerable in degree and in force, of the muscular fibre. Wherein this contraction, difficultly admitting of relaxation, consists, we cannot pretend to explain; but I would observe that it is a direct cause of pain in itself, even in a less degree than that which amounts to spasm, in as far as it takes off the flexibility of the muscular fibres, and puts them into the state in which they are in chronic rheumatism, and thereby renders them more sensible to the slighter degrees of distention, and hence more liable to the sources of pain. Both the strictly spasmodic state therefore, and the rheumatic contraction, may be combined with causes of distention, and modify the pain arising from them. This is evident in the case of colic, nephritic, and biliary pains. In colic the pain arises from spasmodic contractions; but these have the effect of including a certain quantity of air, in consequence of which the distention becomes considerable, and the constriction from spasm becomes more painful. In nephritic 
pains we have instances of stones of considerable size passing from the kidneys to the bladder without much pain, while on other occasions a very small stone will give the most exquisite pain. In the one case the stone induces an irritation and constriction, by which the pain becomes considerable upon a small distention.

"It is highly probable that spasmodic constrictions take place in the blood-vessels also, and have a considerable share in modifying the pains which, as I formerly mentioned, arise from distention, and in producing the various sensations of impulse which I noticed, (page 55.) You will now perceive that spasm probably has a greater share than has been imagined in concurring with, and modifying pains from distention. Thus, I refer the pain from inflammation to distention by an increased impetus in the part of the fluids, which are pushed on in greater quantity, and with a greater velocity than that which the vessels can admit; but it is probable that neither the pain nor the obstruction would arise, if a spasm did not concur with the increased impetus of the fluids.

"Another modification of pain which deserves notice, is that which arises in parts where no external impression is made, in consequence of impressions made upon distant parts, from which they are propagated. (See LX. 2.)

"Pruritus.-This symptom is very properly subjoined by Gaubius, who calls it sensatio dolori proxima. It is not pain, but approaches to it: thus, certain chemical acrimonies give only a pruritus, which arises to a certain degree of tickling; in a more concentrated state they produce smarting and pain. The sensation of pruritus, however, does not always infer chemical acrimonies; the vermes intestinales excite an itching at the nose, which is no otherwise to be explained than by an oscillation propagated along the whole alimentary canal and fauces, till it reaches the more sensible part of the nose, where the epithelium terminates. The same thing holds in the case of calculi, and in the ardor urinæ from gonorrhøea.

" To the head of pain, and especially that of pruritus, Gaubius adds a set of feelings, which belong to the appetites and propensities which I have enumerated in CXIX. 4. and 5. They are frequently sensations sui generis, very often of 
the pleasant kind; very often variable between the pleasant and the painful. Thus we can hardly say what sort of sensation excites the oscitatio and pandiculatio (yawning). Cough and sneezing are indeed attended by a tickling or pruritus; the alvine evacuations, and those of the urine, are sometimes excited by a sense of weight merely, sometimes by a sense of irritation or acrimony.

"Restlessness is a frequent attendant upon anxiety, as also upon pain and pruritus; but I consider it now as occurring without either. A man is sometimes restless without being able to assign any particular feeling as the cause. We perceive this especially when we are disposed to sleep, but cannot; where we suppose an irritation, without being able to ascertain it. I would observe that our muscular fibres are liable to motion, of which we do not take notice. In the muscles of animals recently killed, there is distinctly observed a kind of palpitation-an alternate oscillation of the fibres; and such a thing may go on in the living animal also. In my own leg I have seen parts palpitating in different places, when the action of the heart and of the pulse was steady and uniform. This was attended with a certain feeling of uneasiness; and this is, if I mistake not, the anxietas tibiarum, or, in vulgar language, the fidgets.

" The motion of our muscles is also attended with uneasiness under lassitude; this may be produced by other causes than labour or long exercise; and no posture or exercise of the muscles can be long continued without uneasiness. But there is hardly a posture of the body in which some muscles are not exercised. When a man lies on his back, some muscles are employed; still more so if he attempts to lie on his side: we cannot, therefore, lie long in any one situation, without uneasiness and shifting the posture: this, therefore, very frequently is the cause of restlessness.

6 Restlessness may also depend upon compression. It is well known that our parts suffer by the incumbent weight of our own bodies. Soft bodies, with some degree of elasticity, will obviate the effects of compression. Persons who have a soft cushion of their own fat, accordingly, will bear compression better than those who are emaciated. But, besides, the sensibility of the parts may be various; there is a state of the body 
when it is all over more sensible, so that the slightest touch gives uneasiness, which indeed is a frequent foundation of restlessness.

"Anxiety or Uneasiness. - As this term has been referred to the state of the mind, as well as to that of the body, it has occasioned some confusion; and, in order to distinguish it from other feelings, I say that it is a feeling accompanying different sensations of consciousness, which is not attended by any sense of the mode of impression, nor referred to any small or single limited part: thus it is referred to the head, the thorax, the abdomen, and the limbs in general; but with no accurate limits. This will clearly enough distinguish the present subject, only: we must remember-natura non facit saltum; so that it may be sometimes difficult to discern between pain, pruritus, and uneasiness. It has been usual to join the idea of inquietude and restlessness with uneasiness, as Gaubius also has done; and it is true that restlessness frequently attends uneasiness; but I say it is not always the case.

"Gaubius has insinuated that anxiety as arising in the body, is analogous to that arising in the mind from the fear of an impending evil (Patholog. 682. 686.); as if the danger was the foundation of the anxiety. This Stahlian notion is without foundation. A sickness at stomach is often without any sense of fear; or if, when it is new and unusual, it gives some fear, it is corrected by experience; and yet the sense of sickness remains as much as before. It would disturb our whole system to confound the anxiety in hypochondriacs, melancholics, and maniacs, with other causes of anxiety. Let us take the example of suffocation, which is the most favourable of any to Gaubius's doctrine. An infant, I believe, has no sense of fear; but every body, observing that people die for want of breath, concludes that suffocation is a dangerous matter; and it is no wonder that people are affected with fear, on feeling any considerable difficulty of breathing. There are asthmatics, however, who bear such difficulty of breathing without fear, and even with very little anxiety.

"Anxiety therefore may be totally abstracted from a sense of fear; and the mental and corporeal causes are certainly to be distinguished. We treat here of corporeal anxiety, and of the 
states of the body attended with a peculiar uneasiness. I find this not easy, but I have laid a foundation for it in LXXIV.

"The want of sensation is always uneasy, except when the mind has been fatigued or pained by previous sensations. This is the langueur of the French, which I think the English language has no term to express, - for weariness does not convey the idea; however, the English are said to have the feeling, which is said to be the occasion of their so frequently hanging themselves; but they express it by the Latin term toedium vitoe. The Scotch have a term which expresses this, viz. waff-(a man is waff, and does not know what to do with himself,)-plainly derived from the English word to wafe or stray.

"The sensations of debility and difficulty are also uneasy. We do not always distinguish between the two; as in debility we have the same sense of weight as from any external cause of difficulty in motion. Thus, in the case of compression of the sciatic nerve, we have the same sensation as from a real weight appended to the foot. The debility and difficulty gives the feeling of languor and lassitude, in opposition to that of alacrity and vigour of motion. This may depend upon causes affecting the brain, in consequence of which there is a languor over the whole system, or upon causes affecting the muscular fibres in other parts: in both ways it occurs in the beginning of fevers, especially in those of the nervous kind.

66 Uneasiness is also produced by a sense of difficulty in the exercise of the intellectual functions; as when the memory fails us, so that we cannot recall the associations of a name or date; or when we find that we cannot pursue the several relations which we had formerly marked, so that our judgment is at a stand; still further, when other ideas than those of the usual train obtrude themselves: all these are instances of confusion in the head, muddiness of the brain, and are the beginnings of delirium.

"Now these are the uneasy feelings referred to the head; others are referred to the thorax. The various states of difficulty of breathing, from whatever cause they proceed, are attended with peculiar uneasiness; sometimes from a difficulty and resistance to the passage of the blood through the lungis, sometimes from an unfitness of the external air, sometimes 
from a weakness or imperfection in the exercise of the organs of respiration. The anxiety referred to the thorax, also, frequently depends upon the diseased motion of the heart, which we may consider chiefly as of two kinds, arising either from a difficulty or resistance to its frequent evacuation, or from a debility in the action itself, as in the approach to a deliquium animi, which occasions a most exquisite uneasiness. But the motion of the heart may also depend upon a certain general resistance to its easy and free evacuations, by a constriction of the arterial system, when we find the pulse small and contracted. This I take to be the proper anxietas febrilis, as also that anxiety which precedes eruptions, and follows their sudden repulsion.

"The sense of uneasiness, or load at the stomach, depends either on the weakness of the stomach in its action, whence it occurs in some persons from every kind of ingesta, or upon the improper nature of the food presenting a difficulty to the action of the stomach. Under the first head we must consider that this uneasiness arises not only from the debility of the stomach in evacuating itself, but also from a resistance at the pylorus, straitened either by scirrhus or by spasm. Such a feeling is anorexia or want of appetite, which, increased, arises to a more formal anxiety, commonly known under the name of sickness, which, in many instances, gradually proceeds to nausea and vomiting.

"6esides the stomach, other viscera of the epigastric region may be the seat of the uneasiness or anxiety of which we speak. Thus, an anxiety referred to the epigastrium may depend upon a difficult passage of the blood through the system of the vena portarum, from which resistance Boerhaave imagined that the anxietas febrilis arises.

"In the abdominal region I would readily think of the uterus, which may, by the more or less free passage of the blood through its vessels, produce an uneasiness; and many of the feelings which belong to hysteric diseases may arise from this source; but although we may suppose this, we cannot point out how far or when it actually takes place. The chief seat of anxiety in the abdominal region is in the intestinal canal. Here it may be a question whether it may not arise from a state of de- 
bility; but this is not easily demonstrated; and the most evident cause of anxiety here is a flatulent distention, which may indeed be common to the whole alimentary canal, to the stomach and intestines; but certain portions of the intestines, such as the two great flexures of the colon, and more especially that in the left side, are more particularly liable to it; and, therefore, this uneasiness is most commonly seated in the left flexure of the colon. That it depends upon flatulent distention appears from this, that it is attended with Borborygmi, that it precedes the expulsion of the wind upwards and downwards, and is commonly relieved by this evacuation.

" I have now enumerated the chief species of uneasiness or anxiety : the consideration might be prosecuted to others with curiosity and perhaps with use; but where we cannot speak distinctly, we may rather be silent.

"I proceed to observe, that, in many instances, this uneasiness proves an excitement to action, or acts as an indirect stimulus; examples of which are readily found with regard to the heart, lungs, and stomach. With regard to the heart, Gaubius says (Pathol. 688.), 'imminens hinc circulationis suffocatio Naturam urget, ut motibus duplicatis non sine summa anxie'tate contra insurgat.' You understand that I consider this as a blind impulse, without any foresight of danger; the lungs are excited to a more full or frequent respiration, simply by the difference in their state, without our consciousness. In the stomach an exertion of the muscular fibres is still more frequently the consequence of a sense of uneasiness. The chief application which I would make here is this : as some circumstances of anxiety'were not explained, so the circumstances of anxiety, debility, and resistance, may operate in cases to which we do not attend,- - and upon that is founded LXXX. I apply this particularly to the theory of hæmorrhagy and inflammation, where merely a certain resistance to the passage of the blood, attended with a distention of the vessels of the part, produces the increased impetus attending hæmorrhagy and inflammation." 
CHAP. II.-OF THE ACTION OF MOVING FIBRES.

LXXXI. The moving fibres (XXIX. 4.), so far as yet known, are of one kind only, and the same every where, as in the most commonly known muscles. Hence, the terms moving and muscular fibres are of the same import.

"What a muscle is, is well known from anatomy. I suppose, also, that it will be very well known, that these muscles are composed of bundles of separate and distinct fibres, that the separation goes to a great degree of minuteness, and that the motion which occurs in the whole muscle, depends upon the motion of those fibres of which it consists. What we know with respect to muscular fibres in general, has been learned from observation and experiment upon these muscles; but it has been supposed that they are different in various animals, and in different parts of the body in the same animal; and I allow that this may perhaps be so, but so far as our observations, strongly supported by analogy, yet go, the presumption is, that they are every where of the same nature; and anatomists are united in this, that although in many cases we cannot distinctly discover the fibres, or their appearance, we may conclude as to the structure of the whole wherever there is irritability. I go on to use the term muscular fibre, because I suppose that it is the moving fibre every where, and that it is every where of the same nature; 'we can trace it to the alimentary canal, to the fibres of the bronchiæ in the lungs, and over the whole vascular system; and where we cannot trace it, we presume upon its being present from the properties, of which we find the several parts possessed."

LXXXII. A muscular fibre is supposed to have a peculiar organization, different both from that of the simple solid fibre, and from that of the medullary fibres in every other part of the nervous system; but wherein that peculiarity of organization consists is not yet ascertained.

LXXXIII. A' muscular fibre is endued with a contractility which is different from that of the simple solids, or of any inanimate elastics; especially in this, that the contraction of a muscular fibre is excited by causes which do not affect these others. 
For the contraction of a muscular fibre is excited by being extended; and a contraction is produced, whilst the stretching power continues to be applied. The same contraction is also excited by various applications, whose mode of action we do not perceive; but we know them to be such as do not affect inanimate elastics.

In respect of these causes by which it may be excited, the contractility of muscular fibres hath been called IRRITABILITY.

LXXXIV. The force of contraction in muscular fibres is often much greater than that of the causes exciting it.

LXXXV. The contractility of muscular fibres (LXXXIII.) appears especially in living bodies, ceases with life, or soon after, and is probably never produced but with life. Hence it is, that, by some writers, it is called the vital Power, and the muscular fibre endued with it the LIving solid. (Gaub. Patholog. 169.170.)

LXXXVI. The contractility (LXXXIII., LXXXIV., and LXXXV.) hath been supposed to belong to muscular fibres, independently of their connexion with the other parts of the nervous system; and, upon that supposition, it hath been called the vis insita. We shall call it the INHERENT Power. (Haller. Prim. Lin. 400.) - " I consider this contractility as occurring in living vegetables: to consider vegetable life may be as difficult as that of animals; but this contractility is the property of living bodies; and a vegetable is such, in as far as its functions are connected, and depend upon a whole.

"A muscle cut out of the body-and this experiment has been frequently made with the heart-for a long time shews itself possessed of moving powers : it shews its irritability, and, when its spontaneous motion has seemingly ceased, it may, by the prick of a needle, or other irritamenta, be made to renew its motion. Now, this power may reside entirely in the muscular fibre itself; and so Dr. Haller and others have supposed, and termed it the vis insita."

LXXXVII. The contraction of muscular fibres can be excited by applications made to other parts of the nervous system, as well as to the muscles themselves; and, as the effects of those applications made to the other parts of the nervous system can be prevented by ligatures made upon the nerves between the 
place of application and the muscle to be moved, it is concluded that the contraction of muscular fibres can be excited by a power communicated to them by a motion propagated along the nerves. This power is called the Nervous Power.

LXXXVIII. The nervous power (LXXXVII.) is commonly determined to motion by the will. This we suppose to act in the brain only (XXXIII.), and to depend upon sensation, and other modifications of thought; and this power, which is to be chiefly referred to the mind, and acts in the brain only, we name the ANimal power.- " This term, animal power, has not been common in our systems of physic; but if it is true, as we have alleged, that, in consequence of will or volition, a motion is commenced in the brain, and that this happens in consequence of other exertions of the nervous power, $e . g$. in consequence of impressions made upon different parts of the system, which can only act by the intervention of the brain ; if, in all the communications between the different parts, much depends upon the present state of the brain, we will not be in any difficulty in lodging a particular power there, of which we shall speak under the name of animal power, or energy of the brain.

"There is a necessity for considering the contractility of muscles as in part depending upon the inherent power, in part upon the nerves, and in part upon the brain. Hence, the terms inherent, nervous, and animal power."

LXXXIX. The facility with which the contraction of muscular fibres can be excited, and the force with which it can be performed, are to be distinguished. The first we name the yoBILITy, the last the vigour of muscular fibres. Both have been confounded under the name of Irritability.- "Irritability, taken strictly, implies every circumstance of the contraction of muscular fibres which can be produced by the action of other bodies. But there occurs a very considerable difference :certain persons are irritable to much weaker impressions in point of force," or in point of the quality, than others; hence we say they are more moveable than others; and this gives the Moвility. But we likewise know, that, from any cause applied, the force with which the muscular fibres of one person can be contracted is different from that which another vor. $\mathrm{r}$. 
can exert: for example, I cannot lift the tenth part of the weight which other persons can raise. Abstracting, therefore, from the powers exciting, the actions are performed with more or less strength; this is called vigour. The distinction between mobility and vigour is of use, because mobility is sometimes connected with the most remarkable states of weakness."

XC. The mobility and vigour of muscular fibres (LXXXIX.) can both of them be increased or diminished by various means. Whatever can excite the contraction of muscular fibres is called a sтimulus ; and, in general, the means of exciting contraction are called stimulant powfrs. The means of diminishing the mobility and vigour of muscular fibres are called SEDATIVE Powers. - " I thought it thus necessary to define stimulus, in order to take off the notion of its being considered as the action of a sharp-pointed body. We can excite the action of the heart by the prick of a needle, but we can also do it by blowing in air ; and we say that the influx of venous blood is the common stimulus to the action of the heart."

XCI. The inherent power ( $\mathrm{LXXXVI.)} \mathrm{is} \mathrm{supposed} \mathrm{to} \mathrm{be}$ more vigorous, moveable, and permanent in certain muscular fibres than in others._- It is well known that this inherent power is more permanent in certain animals, as frogs, lizards, tortoises, and the other amphibia, after what we consider as death has taken place; in these it remains longer than in the fibres of warm-blooded animals. In the same person also, at different times, the muscular fibres are more vigorous and moveable ; and it is obvious that these conditions of vigour, mobility, or duration, may be different in different parts of the same body. I chose the term supposed, however, because the experiments adduced in proof of this difference, are not absolutely decisive. With regard to the permanency, Haller thinks it greatest in the heart ; but the experiments of Huber lead to the conclusion, that the inherent power is not more permanent in the heart than in other parts ; for, if you open the abdomen first, and the thorax afterwards, the power appears longest in the heart; but, if you open the thorax first, and the abdomen last, it is lost sooner in the heart than in the intestines. The permanency, therefore, is doubtful. Some time after death a stimulus applied to the heart will 
renew its palpitations, and will not do the same when applied to other muscles; but that is not because the inherent power is more moveable, but because being extremely weak, it will not shew itself, if it has much to move. Now the muscles are attached to bones and other parts, on which account they cannot be raised so easily. But cut away a muscle from its attachments in a recently killed animal, or in one yet living, and it is as moveable, on the application of a pin, as the heart is. This circumstance, therefore, may give the appearance of greater mobility to the heart and some muscles. Again, mobility may be the consequence of habit. It is, to me, a doubtful question if the constitution of muscular fibres, taken singly, is so different as to produce a different state of vigour in them,-the force of muscles is according to the number of their fibres; and in no other circumstance can the vigour be varied but by repeated exercise."

XCII. The inherent power, or the contraction dependent upon it, can be excited by certain applications made either to the muscles themselves, or to the nerves connected with them; and, in either case, the effects of such application are so exactly the same, as to allow us to conclude that the matter in the nerves, and in the muscular fibres, is of the same kind.- "A very ingenious gentleman, Dr. Thomas Smith, a pupil of mine, has written an inaugural dissertation, (De Actione Musculari, Edin. 1767.) to demonstrate this very fact. He applied to muscles and to nerves every variety of powers, mechanical or chemical, which are supposed to act upon them. He employed a great variety of different substances in this way; and he could alternately, or in circumstances well suited for comparison, apply them to both nerves and muscles. And what was curious, he found that there are substances that we consider as stimulant, which, when applied, appear manifestly sedative; and that some other substances suspected to be sedative, when thus applied, are found to be stimulant, although that is a rare occurrence. The general result is, that the same matter applied to the muscle or nerve, constantly produces either contractions or insensibility, and shows either stimulant or sedative powers, and this equally, whether applied to the nerve or to the muscle itself; and in no one instance did any diversity appear. The experiments were nicely executed, and indeed they requir- 
ed a great deal of nicety and attention. Sometimes a stimulus, when first applied, excited a contraction, and after some time no longer had the same effect, because the application had destroyed the contractility in that portion; but on cutting it off, the same stimulus, applied to a fresh part, had the same effect. The same happened with some sedative applications, of the sedative effects of which he judged by this, that after an application of them, a known stimulus had not its usual effects. Now, from these experiments, thus frequently repeated and diversified, it appears that the matter, whatever it is, which thus stimulates or shows a sedative power, acts upon us in the same way, whether applied to the nerve or to the muscle; and this appears to me to be equal to the ordinary steps in a geometrical demonstration:-as $\mathrm{A}=\mathrm{C}$ and $\mathrm{B}=\mathrm{C}$, therefore $\mathrm{A}=\mathrm{B}$; if we constantly find the same effects, we at least presume that the passive matter is the same in both cases."

XCIII. The muscular fibres are sensible to various impressions, and are otherwise organs of the sensations of consciousness, (XL. 4. 5.) From this also, it is presumed, that the muscular fibres consist of the same matter which is the subject of sense in other parts of the nervous system, (XXXIX.)

XCIV. From XCII., XCIII., and other considerations, we think it probable, that the muscular fibres are a continuation of the medullary substance of the brain and nerves, as alleged, (XXIX.) - "So far the direct proof is to me in some measure satisfying; but many objections have been started to this opinion, and I here, in the next place, endeavour to remove them."

$\mathrm{XCV}$. Though the muscular fibres consist of the same kind of matter as that in the nerves, the latter shew no contractility, because they have not the peculiar organization (LXXXII.) of the former.- " Dr. Haller labours much to establish a difference between the nervous and the inherent power; and he employs an argument which he thinks decisive-that the nervous power depends upon life, and finishes with life; but that the inherent power remains after life has ceased. But I say"-

XCVI. The nervous power (LXXXVII.), and the inherent (LXXXVI.), may subsist for some time without any connexion of the nerves or muscles with the brain; and they subsist also in entire bodies for some time after life has 
seemingly ceased. Both powers, however, are seemingly of equal duration in these respects; and neither power seems to subsist long but in entire and living bodies.- " $\mathrm{It}$ is strange that Haller could overlook this. You may cut through a nerve belonging to a great number of muscles, such as the great crural nerve': this' having lost all its connexion with the brain, still, by the application of a stimulus to the lower portion, the same effect takes place as if it had remained entire. This experiment not only applies to living animals; but if you cut a muscle out of the body, with its nerve attached to it, and apply the same stimulus to the nerve, as has been done by Swammerdam, the same effects will follow as if you apply it to the muscle, so that both the nervous and the muscular power do remain for some time without any connexion with the brain. They subsist also in entire bodies for some time after life has seemingly ceased; and if we were to determine the circumstance of life, it would be by saying that life subsists only so long as there is a communication between the brain and other parts of the system ; for every other part almost may be taken away, and the parts attached to the brain seemingly have their entire functions remaining. But other circumstances, such as destroying the functions of the brain by compression, answer the same purpose as removing the parts; in that case also, the inherent as well as the nervous powers do remain. Both powers, however, are seemingly of equal duration in these respects. This conclusion is intended for Dr. Haller. Dr. Smith's experiments are quite decisive; he never found that the irritability remained longer in the muscle than the nervous power did in the portion of the nerve adhering to it. Another part of the conclusion is this; ' and neither power seems to subsist long, but in entire and living bodies.' Undoubtedly the irritability can subsist for some time separate, but there is no instance of its subsisting for any length of time. If, in a tortoise and some other animals, it subsists for some days-it is for some days only-and it gradually ceases. Every view of the animal economy leads us to consider it as a system, all the parts of which mutually depend upon one another for their several functions; and it will appear from what I shall say afterwards, that the brain is one of the most important parts of this system. Whatever modification it is under-whatever 
other powers there are-the brain seems to be the principal part of the nervous system; and its faculties, as we know, may remain when a limb, or several limbs, are entirely cut away. The conclusion, therefore, more formally is-"

XCVII. From what is said (XCII.-XCVI.), it is probable that the nervous and the inherent powers are somewhat of the same nature; and it is also probable, that in entire and living bodies, both the nervous and inherent powers have a constant dependence upon the animal (LXXXVII.)-" This animal power we suppose to be seated in the brain, and only there seated. Whether you consider it as a sentient principle; or a mechanical energy, it is probably the fundamental part of the system, without which the functions cannot long remain.

"So far I have laid a foundation for avoiding some false reasonings, and for introducing some which are more convincing with respect to this subject. I must still try to carry the same views a little farther; and there is not a problem we would wish more to solve than that of muscular contraction. This is a fundamental power in the system, and physiologists are commendable for having attempted to explain it in different shapes; but no one is yet allowed to have had success, -no view has been offered by anatomists and physiologists which is satisfying. But to approach nearer, we must first reject the false suppositions formerly made."

XCVIII. The contraction of muscular fibres does not depend immediately on the motion of the blood; as it subsists in many animals after all motion of the blood has ceased. - "There is no muscle that is not provided with a large number of blood-vessels, which are distributed to every portion of its fleshy belly, and closely accompany the moving fibres, and which, in the opinion of many, do enter into their composition. So it has been alleged that a muscle will become paralytic, or lose its power of motion, not only when its nerve, but also when its artery is tied; and it has been from this experiment concluded, that the influx of arterial blood is as necessary as the nervous power; but this supposition, and all the theories which have been founded upon it, are suspicious, when we observe that this is not exactly the fact, for the muscle. is rendered paralytic, not immediately, but a long time afterwards, 
and this probably arises from a stagnation of the fluids in the remaining arteries and beginning veins, which gives a rigidity to the parts. I say, therefore, the influx of blood is necessary as a condition to give the flexibility, tension, and warmth; but the contraction subsists in many animals, after all motion of the blood has ceased. The thorax of a frog being opened, and the great vessels cut through, although nothing like the motion of the blood is subsisting, yet the animal will hop about with alacrity and vigour for hours afterwards. If in the animals of warm blood this experiment does not succeed as readily, it amounts to this, that the power of contraction is more permanent in colder blooded animals."

XCIX. The contraction of muscular fibres does not depend on the inflation of vesicles, or other such analogous structure, as the shortening of the fibres in contraction is often greater than can take place in such structure.- "Leuwenhoek started this hypothesis of the vesicular structure, and the ingenious Hook has prosecuted it. They assumed that the fibre was like a string, with such vesicles, and that these vesicles had an elasticity by which they could be drawn out into their several figures, \&c. This view readily explained the contraction by the influx of a fluid ; for, suppose that the muscular fibre, and its several vesicles, are drawn out to their greatest extent, if you fill up these a contraction will be produced; and almost every hypothesis has supposed this, that the fibres are a portion of artery, and that the influx of blood dilates the cylindrical vessels, and brings them into a spherical figure. But no such structure will account for the contraction which really takes place. Thus, if you fill a cylinder in such a manner that it shall change into a spherical form, which is the greatest contraction possible, it cannot be diminished above one-third of its former length ; but many observations shew that our muscular fibres are contracted to a greater degree; as in the polypus, which can be contracted to one-twelfth part. We must, therefore, seek for some other structure or organization of muscular fibres. If you will look over the various hypotheses, you will find how many are cut off by the observations in these two paragraphs, with regard to which I am sufficiently confident. But I am by no means so 
much so with regard to what I am going to add, which must be ascertained as a fact, and more clearly demonstrated, before it admits of a particular application."

C. As the force of cohesion in the muscular fibres of living animals is much greater than in those of dead ones, it is probable from this, and other considerations, that the cause of muscular contraction is an increase only of that same power which gives the contractility of the simple solids, and of other inanimate elastics. (Haller, Prim. Lin. 407. 408.)

"I have frequently told you, gentlemen, that I am not to entertain you with hypotheses of my own, nor with the description of the hypotheses of others, - not frequently at least ; but I must say, that if hypotheses are confined to the closet, or even to the college, they are not without their use ; there is no philosopher that has not more or less dealt in them; and in these parts of our science in which we have not made much progress, we shall not go much farther without them, and we shall be tempted to do so according to the importance of the subject. The consideration of muscular motion, the theory of muscular contraction, is of no little importance; for if we knew it more exactly it would throw light upon every other part of our system: In XCVIII. and XCIX. accordingly I have given a discussion which relates to most of the hypotheses that have been offered, and in rejecting error we never lose our labour; and in C. I have made an approach-it is no more than an approach-to one of my own. I have in some measure adopted the view of Dr. Haller, by alleging that the cause of muscular contraction is an ' alacrior attractio elementorum fibræ, qua ad se invicem propius accedunt,' (Haller, Prim. Lin. 407.) ' an increase only of that same power which gives the contractility of the simple solids, and of other inanimate elastics.' Now, if the premises in the first part of that proposition could be very well proved, the conclusion which I have just now repeated would be readily enough admitted. But these premises are not yet established with sufficient clearness. Haller refers to one $\mathrm{Li}$ bertus (?) who wrote de mechanices absentia; and the principal proof which he adduces is, that muscular fibres, in living bodies, sustain a force which in the dead body yrould tear them in 
pieces. What the proofs are which this author adduces I cannot say, as I never, by any means, could obtain a sight of his dissertation ; I can only give the grounds of my conjectures. Borelli, and other authors upon this subject, have computed the force in living bodies; and we have experiments upon the force in dead animals by Hales and Muschenbroek; and from these compared, we find that the force in the living is vastly greater than that in the dead fibre. But these experiments have not yet been fairly established; and so I give it as a matter of conjecture to be further prosecuted; but, independently of this calculation, there is some probability in the theory that I have offered. It is the opinion of some of the greatest philosophers, particularly the English, with Sir Isaac Newton at their head, and of several of the foreign of the greatest rank, that the cohesion of bodies depends upon the presence of an elastic fluid, which is more rare in the pores of bodies, and more dense upon their surface, by which the parts are kept together; and upon this doctrine, which there is no difficulty in admitting, there is no difficulty in explaining the whole phenomena of inanimate elastics; and all that our theory requires, is to suppose that this subtile elastic fluid is by various means excited to a greater degree : we have some illustration of it in the case of electricity and magnetism, which are only modifications of this subtile fluid; and we need only suppose that this elastic fluid can be thus excited upon the nerves and muscular fibres of living animals, and that, in consequence of a certain degree of excitement, the nerves are rendered sentient; and that by some peculiarity of the muscular fibre, it can be accumulated in them and occasion muscular action. Having thus stated the matter, I acknowledge to you that there are difficulties with regard to it, in establishing the fundamental facts and applying them to the several phenomena; but what we call the fluid of the nerves, that upon which the living power, upon which both sense and motion depend, is manifestly an elastic fluid of one kind or other.

"I go on to add another circumstance to obviate some of the difficulties which might be offered. I say" -

If this is true, it will also explain why the force of cohesion in muscular fibres is greater than that of the medullary fibres in any 
other part of the nervous system, though both kinds of fibres, by XCIV., consist of the same kind of matter.- "Nothing appears so soft and tender, and so little coherent, as medullary fibres in every part of the nervous system, and it may appear difficult to suppose that they consist of the same kind of matter as muscles; but if these fibres are capable of having that fluid, which operates by increasing the force of cohesion, accumulated, it will show why these fibres show more cohesion than the medullary fibres do in any other part. I have been at pains to inculcate this point, that the matter in the medullary substance and in the muscular fibres, is of one and the same kind.

"Dr. Haller has taken a partiality for a doctrine of which he thinks himself the author, viz. that the vis nervea and the vis insita of muscular fibres are totally distinct powers. I have touched the matter before, but I have not taken notice of the arguments of Dr. Haller in favour of the contrary opinion; and $I$ am now in a condition to enter into the controversy with Haller. In his Prima Linece, $\S 403$, he establishes the vis nervea, and in $\$ 404$ he proceeds in this manner: ' $\mathrm{H}$. vis non est eadem cum vi insita '; and his reasons are- 'Forinsecus ad musculum advenit, in quo altera inquilina habitat'-the nervous power comes from without, the inherent power is constantly resident in the muscular fibre. Various hypotheses with regard to muscular motion have assumed the nervous power flowing in larger quantity into the muscular fibre; at the same time none of them have supposed that the muscular fibre is independent of the nervous power; but they always assumed that a portion of that power is constantly there, upon which its contractility depends, and that the excitement of the muscular fibre may be increased by an addition of more nervous power. It is, agreeably to the doctrine we have laid down, very probable that the excitement we speak of is in common to the medullary fibre every where, and that it may receive an addition from the nerves, without however being a different matter. But his other argument is, that the nervous power: ' $C$ um vita destruitur, a qua altera, per certa experimenta diu superest.' I have prepared for this argument by what I have said in XCVI. From the experiments of Dr. Smith, which I formerly quoted, it appears that the nervous power remains as long as the inherent power; that so long 
as we can irritate the muscular fibre to contraction by a stimulus of any kind applied to its fibres, so long we can excite the contraction, by applying the same stimulus to a portion of the nerve; and the power if shewn to proceed from an inch of nerve is equally nervous as if proceeding from the brain. I wonder that Dr. Haller missed this fact. The explanation is, that the power belongs to the medullary fibres, and therefore subsists so long as certain conditions subsist; what these are it is not necessary to determine, but they are the same in both. 'Subprimitur a vinculo nervo injecto.' This does not touch the question of the nervous power; only, because a nerve is tied, the animal power no longer exerts itself in the muscle. This does not affect the question, whether these two powers are one and the same, or different; only that a motion cannot be propagated after a ligature is applied. 'A laeso cerebro, a propinato opio.' This amounts to the same, and relates to the animal power, which cannot be in these cases propagated from the brain. 'Motus insitus ab iis omnibus nihil patitur.' This is not true; the motus insitus suffers from all these, and is affected by every affection of the brain, and even by its ordinary action. With regard to the ' propinato opio,' Dr. Haller, in contradiction to several other experimenters, is disposed to refuse that opium has the effect of destroying the inherent power; he maintains that opium properly acts only on the brain, and particularly rejects the commonly received notion of its power over the heart. You will find experiments to this purpose fully detailed and frequently repeated by Dr. Whytt, and our colleague, Dr. Monro, who show that it acts upon the brain, and thereby destroys the in herent power in the same manner as when it is applied to the muscular fibres themselves. Haller adds, 'et superest nervo revincto, resecto, in intestino de corpore revulso.' I have already said that this is no proof of the different nature of these powers; only the animal power is taken away. 'Viget in animalibus cerebro destitutis." Here we are engaged in an anatomical dispute with Dr. Haller, viz. whether any animals are without a brain. He complains that we suppose the existence of a brain where we do not see it; but he should admit that there are animals in which, from their transparency and minuteness, we are not allowed to discern the distinction of parts, and are 
prevented from perceiving a brain; and it is as unfair for hint. to refuse it as it is for us to suppose it. My friend, Dr. Smith, had an opportunity, by new experiments of his own, to ascertain a common origin of nerves in some of these animals; and even where this cannot be perceived, the same conclusion may be drawn from the observation of animals being possessed of certain senses which Haller allows to argue a brain; so that in several animals in which we cannot point out where a brain lies, yet the presence of eyes is a sufficient proof; to Smith it appeared that hardly any animal is cerebro destitutum. Haller adds his last particular-' Movetur quod sensu caret, et sentiunt corporis partes quæ sunt absque motu.' This only infers the slight supposition, that there is a peculiar organization which is necessary to show the contractile power of fibres, and that there will not be motion without this organization, so that the motion is not in the medulla cerebri, or in the course of the nerves, because this organization is wanting there. Take notice, that Haller has made a very strange supposition: he says that the muscular fibres are destitute of sense; but there is no part of the fleshy belly of a muscle which you can touch with the smallest point of a needle in a living animal, that will not give the sense of pain, and shew that it is an organ of sense. The only means which Haller has for accounting for this, is to say that the nerves are minutely distributed over the muscles; but this amounts to the same as the saying that the whole muscle consists of nervous matter, since there is not a point that is not sensible. The fact is, it cannot be demonstrated either one way or other; for we cannot trace the subdivisions of the nerves in the belly of the muscle, and show how it is made so large by them; but if I say that the extremities of nerves are the only sentient part of a muscle, and that they are present in every individual part of it, I say that it is as difficult to suppose that they can be thus distributed, as it is to suppose the other view, and the one is more obvious than the other; but what follows is absolutely false- 'Voluntas motum nervosum ciet et aufert, in insitum nihil potest.' That the will, that a power in the brain excites the nervous power, is true, but we shall see presently that it has also an influence upon the inherent power. So much with regard to this theoretical discussion; some of you may 
think it is too much, but if you consider how much it is connected with the other parts of our subject, you will not think so. I proceed to some of the laws and circumstances of muscular action, though we cannot arrive at the fundamental theory."

CI. In living and healthy animals, the muscular fibres have a constant tendency to contract; and this tendency we call their TONE, Or TONIC POWER.

"The constant tendency of muscular fibres to contract in living bodies, is well illustrated by the gaping of wounds in muscles; and many experiments show, that, whatever takes off the stretching powers, gives occasion to the shortening of muscular fibres. This constant tendency to contract we call their Tone, or Tonic Power. The term tone has been employed in a loose manner, but this is the strict and proper meaning to be affixed to it."

CII. The tonic power of muscular fibres necessarily supposes their being constantly in a state of extension beyond their natural or most contracted state; and in this state they are constantly kept (1) by the action of antagonist muscles, (2) by the weight of the parts they sustain, (3) by fluids distending the cavities they surround, and (4) by their connexion with such distended cavities, particularly the blood-vessels.

"If the muscles show this constant tendency to contract upon taking off the stretching powers, they must be constantly in an extended state, which I can show to be beyond their natural, and it is of some consequence to inquire by what means they are kept in this state. I cannot enumerate all the causes, but here certainly are the principal ones: (1) I say that they are constantly kept in this state by the action of antagonist muscles. In whatever manner we account for the original extension of muscles, which is probably from the growth of the bones, the fact is so, that the muscles are constantly in the extended state of which I speak, and that chiefly by their being provided with antagonists which have a contrary tendency; which appears from this, that, if you cut through a muscle, its antagonist is contracted. But these are manifestly not of equal strength; and, therefore, the one is sometimes more extended or contracted than the other, according to the position that is most favour- 
able for them. For the most part, accordingly, we find that certain muscles, not only act against their antagonists, but likewise (2) against the weight of the member they sustain ; and many muscles seem manifestly to be more numerous, and more large and powerful, because they have to support the weight of the part; -as the muscles of the lower jaw, the muscles of the temple, the Pterygoid muscles, which are more powerful than their antagonists that pull down the jaw ; and whenever these are considerably relaxed or weakened, as by the power of sleep, or by narcotic powers, as in the case of drunkenness, the jaw falls down of itself. Many more instances may be given of this : thus, the biceps muscle, in the present position of my arm, not only acts against the triceps extensor, but against the weight of my arm ; so that if an external power takes off this, the biceps is contracted, or at least considerably relaxed. The weight of the parts, therefore, has a share. But (3) their extension is kept up by fluids distending the cavities which they surround. This, I say, takes place in the whole arterial system. I am not to prove at present that the arteries have muscular fibres; but they are kept extended, I say, both in the systole and in the diastole of the heart, beyond their natural condition, by the quantity of blood in the arteries; and this is the foundation of the tension which is given particularly to every portion of muscular fibres in every part of the system, the distention of the arteries by the fluids they contain, and particularly as these are impelled by the force of the heart. So far we have no difficulty with respect to a great number of muscular fibres being kept in a state of distention, but we add also, (4) by their connexion with such distended cavities. Perhaps there is no muscular fibre that is not connected with parts surrounding cavities, which are upon occasion inflated or distended, but many cavities are alternately sodistended and again contracted to a considerable degree, and then the muscular fibres are hardly in an extended state; thus in the stomach, the fibres there are in a state of extension, but there are occasions when the stomach is contracted to a small capacity, and we might expect that they were in a particular state of contraction. But there is ground to believe that muscular fibres every where have another particular cause of distention, viz. in consequence of arteries every where accompanying 
them; and, as the sentient fibres of the nerves every where do require, for their oscillation, to be kept in an extended state, for which very purpose the arteries every where accompany them, so the muscular fibres are every where in the same condition. The blood-vessels in the bellies of muscles, and every where accompanying them, are very numerous, as is allowed by all anatomists ; and it appears evidently by injections, and microscopical observations, that every single muscular fibre has its artery connected with it; the principal trunks of the arteries in the belly of a muscle, run parallel to the muscular fibres, and there are every where such trunks interposed between them ; and these parallel arterial vessels have every where a communication, but they pass off at right angles. It was Dr. Hales who first pointed out this. Dr. Haller throws in several doubts with regard to it; but in the third edition of his Prima Linece; ( $\$ 398$. which is since the publication of his other work, he seems to have returned to the idea of Dr. Hales. In short, it appears that there is a most industrious and plentiful distribution of blood-vessels every where? among muscular fibres, but that they are arranged in a particular manner, which gives a certain degree of tension to the muscular fibres in every part; and that such as are not stretched by the fluids distending the arteries they surround, are yet no where left without some cause of distention from this particular connexion with the arterial system. By Sanctorius already it has been observed, 'quod pondus addit robur,' and we shall say that the tone or tension of the muscular fibres is much the same with their vigour, which will be easily understood from the proposition which follows :"-

CIII. As the distention of muscular fibres, by LXXXIII, proves a stimulus (XC.), we conclude, that the tonic power in them will, cateris paribus, be in proportion to the degree of tension (CII.)

CIV. If the inherent power, as in XCVII. is in dependence upon the nervous and animal powers-and these may be increased or diminished by various means-the tonic, as a part of the inherent power, must, in some measure, be in proportion to the state of the nervous and animal powers. - "The language here is, I hope, sufficiently clear; it expresses very much the same 
as what I said in XCVII., that both the inherent and nervous power have a constant dependence upon the animal power. The proofs $I$ have chiefly reserved for this place, where the proposition is of more importance; indeed it is a very leading one in the whole of our Pathology, and therefore I take so much pains in order to support it in differing from (I do not like the expression of confuting) Dr. Haller. I think proper now to illustrate this further, and to shew that the inherent power is in a constant dependence upon the nervous, or more properly upon the animal power.

"I shall give instances with respect to both the voluntary and involuntary motions: I begin with the involuntary, because in these especially it has been attempted to show the independence. It is alleged that the action is produced merely by the mechanical power of the stimulus acting upon the inherent power, independently of any action or energy of the brain or nerves; but, from considering the effects of the passions of the mind upon the state of action of the heart, I say that the passions which diminish the tone or action of the heart, are proofs that the energy of the brain is constantly necessary. Fear is allowed to weaken the action of the heart, as much as anger can increase it; and nobody alleges that the heart is exempt from the causes of fear. There is no doubt that it is one of the pathemata reprimentia or sedantia, and that it and every motion of the mind acts in the brain only, and acts by withholding that power which is otherwise constantly necessary; and when these operate so immediately upon the heart, it is a proof that the action of the heart depends upon some energy derived constantly from the brain. But we have another proof from the action of the heart being destroyed by a ligature upon the nerves. This fact is disputed by Haller; but he has mentioned all the experiments which shew that by putting ligatures upon the eighth pair, or par vagum, and by cutting through the intercostals, a cessation of the heart's action, and death itself, in a shorter or longer time, is constantly induced-in some of these experiments, very suddenly, and in others at no great interval; and in all of them the effect at last is that the action of the heart is entirely destroyed. With regard to these experiments, which shew that the destruction of the action of the heart succeeds slower in 
one case than in another; we can impute it to the manner in which the experiment was made, to their not cutting through all the nerves at once, and, as the heart is supplied with nerves from various sources, to some of these being left uncut which might support its action. But, in another way we remove the difficulty, by saying that it is true that the action of the heart may, in some degree, subsist after all such communication is destroyed; that there is an inherent power to this effect we readily allow; and in a living and entire animal, with a very: few nerves, or, if you will, with none at all, it may give some action; but this does not disprove that the nerves are constantly necessary ; for in these experiments the actions were disordered, the pulse became intermittent, and the slight palpitations which continue in the heart after it is separated from the nervous system, are not that vigorous action which is necessary to the functions of life. From all these considerations together, I think it should appear that some energy of: the brain is required even in the involuntary motions, where it appears to be less necessary, and where a renewed action does not indeed always concur, as in the voluntary muscles. I could make a like remark with respect to the stomach, where we cannot shew such proofs of the brain exciting or diminishing its action; we know that the stomach requires the constant action of the nerves, the constant energy of the brain; for by destroying its nerves it becomes paralytic immediately,-an effect which was commonly found to occur in the experiments made upon the heart by cutting the par vagum : the appetite was destroyed, the contents of the stomach stagnated and were variously corrupted, \&c. In certain diseases the same accident has happened. With regard to the voluntary muscles the matter is more clear: if the nerve belonging to a muscle is tied, it becomes paralytic, in so far as the will has no more any power over its motions. But Dr. Haller says, 'motus insitus superest nervo revincto' ( $(404)$. Stimuli applied will indeed renew its contraction; but these contractions are not so vigorous and full as when they are produced by the power of the will, i. e. by the animal power. From Haller's own experiments it is evident that the motion is considerably weakened in consequence of ligatures upon the nerves. He refers to two authors, Du Bois and Jausșerand, who found VoL. I. 
that the irritability of the muscle was at length lost, and that stimuli no longer had their effect. Haller would willingly doubt the accuracy of these experiments, but he is obliged to say, in the conclusion, that we must acknowledge that there is a diminution of the irritability by a ligature. Therefore the animal power is not only necessary to voluntary contraction, but even to the irritability, to the affection of the inherent power by stimuli. How soon do the passions of the mind enervate, weaken the tone, and give a certain insensibility to the action of every stimulus applied in order to move the muscles! We will hardly doubt that the ordinary inherent power is in some dependence upon the animal and nervous; which is, I think, confirmed by what Haller allows, that the effects of the inherent power, in consequence of stimuli applied to muscles, are remarkably weaker than those that are exerted by the force of the animal power. Here is an experiment for showing the force of the inherent power considered by itself : if I just now support my arm and bend the cubit in this manner, I find that the biceps muscle is contracted; it is fuller and a little firmer in its middle part than it is when in an extended state; but it is not so much so as when I make the flexion by internal force, and give an opportunity to the muscle to contract. When this contraction is only made by its inherent power it is still flaccid; but, taking away the support, and continuing the arm in that position by means of the animal power, the biceps immediately grows remarkably harder and fuller than it was before; and in every other experiment which we can make to this purpose the same appears. I would say that the vigour of the inherent power cannot be evident in a living animal, because we cannot be certain of its acting entirely without the nervous power; and with regard to the strength with which that muscle contracts, I can undertake to show that, in different conditions of the body, as it is weakened by disease, or various other causes, or as it is again increased by powers which have that effect, this very contraction will be perceived to be manifestly different; and that the tone of the inherent power is increased or diminished by causes acting upon the brain, and upon the brain alone, I shall have many occasions to prove. I shall give one illustration at present. In the case of mania, it is well known that men can exert a force vastly greater than any 
which they could have exerted in their ordinary health, and that not from a more violent passion or emotion of mind, but in every trifling exertion; even when in a calm and placid state their strength is greater, and it continues greater, when various means, as cold, hunger, and evacuations have been employed to weaken it ; and this can be referred to nothing but the brain, the seat of the disease. Thus, too, we see that fear, a general timidity, very much weakens the tone of every part; the muscles are so much enervated as to lose manifestly their ordinary powers.

"I conclude with suggesting a little piece of theory in confirmation of the whole. If I have insinuated that the nervous power is an elastic fluid, in common to the whole of the nervous system, such is the nature of elastic fluids, that they will constantly endeavour to restore themselves to an equilibrium; wherever there is a communication, every part constantly presses upon another, so that there must be a constant energy from each part, and more especially from the brain. But this is enough to prove that the inherent power will be, in all cases, in some measure in proportion to the state of the nervous and animal powers, and here is the application of it."

CV. If the tonic power of any muscular fibre depends more upon its state of tension (CIII.) than upon the state of the nervous and animal powers (CIV.), such fibres will be more affected by changes of the state of tension, than by changes in the state of the nervous and animal powers; and on the contrary, \&c.

"I have brought it to this, that the tonic power may depend upon two causes, which are to be considered separate: the one is merely the tension of the fibre, the other a certain power derived from the brain or animal power. These two generally concur; but they may be separate, and in different degrees. And in considering the causes of increase or diminution of tonic power, we may refer them partly to the one and partly to the other, as some depend more upon the state of tension, others more upon the state of the animal power."

CVI. The force of contraction, or the vigour of muscular fibres, will be always as the force of stimulus, and the vigour of the animal, nervous, and inherent powers taken together.

CVII. The mobility of muscular fibres (LXXXIX.) seems 
often to be increased by causes which weaken their vigour; and, therefore, it is induced by the diminution of tension, and by causes weakening the animal, nervous, or inherent powers."Mobility is evidently more remarkable in weaker bodies; as in children, in women, in persons weakened by various means, such as a diminution of tension, and other causes affecting the animal power."

CVIII. The ordinary contraction of muscular fibres is disposed spontaneously to alternate with a relaxation or extension of the same.

CIX. In the straight muscles, and in the heart, the alternate contractions and relaxations readily take place; and that though a stimulus is constantly applied; but in muscular fibres surrounding cavities, as in the alimentary canal, bladder of urine, \&c. the alternate motions do not appear, unless a portion of the fibres is cut out and separated from the rest.

CX. From a difference in the state of a muscle contracted by inherent power, while the member it sustains is moved by external force, and that of the same muscle contracted by the power of the will, we perceive that in the muscles there may be a state of relaxation without their extension.- " The experiment is that which I mentioned (CIV.) with regard to the. biceps muscle."

CXI. There is a state of the contraction of muscles that is not disposed spontaneously to alternate with relaxation, and in which too the fibres do not easily yield to extending powers applied. Such a state of contraction is called a SPASM.— "There is not a term now more frequent in our Pathology than that of spasm; it is as old as any writings in physic, and has been on many occasions employed with very different meanings, so that it is necessary to limit its sense. As the word was originally used to express contraction in general, every kind of contraction might be called a spasm or drawing together; but it is necessary to have different terms to express the different modifications or states of contraction. Where pathologists have used the term according to the ancient meaning, they have distinguished the spasmi into tonici and clonici: this, in my opinion, is more difficult language than that of spasm and convulsion, which last, taken generally, comprehends every other 
preternatural mode of contraction, except that which we call spasm, and which I have endeavoured to explain.

"The state of spasm, strictly so called, consists in two different circumstances; the one is the degree of contraction, which is greater than what happens in the ordinary exercise of our functions; the other is, that in this state the contraction does not follow the more general law of alternation with a relaxation, nor readily yields to extending powers applied; we may suppose these two circumstances are strictly connected together, and that the rigidity or inflexibility depends upon the degree of contraction. Possibly it does so; but-what I want to observe is, that there is a state of contraction short of spasm, which is attended with a resistance, or with some difficulty in admitting of distention,- - so that much less distention will give considerable pain. That such a state as this takes place upon occasions, I think we cannot doubt. I find it a common consequence of spasm. I have frequently an opportunity of observing this in myself:-I am liable to be affected with spasm; and when it is remitted, there is still a manifest rigidity in the muscles which were affected by it, so that they do not yield, as before, to distention by the power of their antagonists, or by extending powers being applied. The same takes place in strains. It is well known that any more violent exertion is very ready to throw the muscles into a spasmodic state, so that the parts are overstretched, in consequence of which they not only recover their natural state of contraction, but go further to the spasmodic, so as to retain a certain degree of rigidity for a long time after. It has been a mistake to view the case of strain as a case of flaccidity, and too great a degree of flexibility in the part; there is rather a state of rigidity and contraction in the part. We have several instances of this, in consequence of what may be called strains. Thus, when a man is stooping, and exerting a considerable force in lifting a weight, and has a sudden spasm coming to certain muscles in his back, it affects him with such a rigidity that he is not able to bend his body again. I say the same state constantly occurs in what we call chronic rheumatism; and as this is most commonly the sequel of the acute, it points out, that when the overstretching of the blood-vessels, which takes place in acute rheumatism, ceases, they fall into this state. 
of contraction, which is attended with a degree of rigidity ; and the impetus of the blood in the vessels overstretching their fibres, leaves them in the same state. But I will not touch this subject further, as I cannot explain what is the state of spasm strictly so called; but it leads us to this, that as there is a state of increased mobility, so a state of diminished mobility takes place to a morbid degree in our nervous fluid. Thus, in chronic rheumatism, and after strains and spasms, not only do the muscular fibres remain in a preternaturally rigid state, but we find the nervous fluid in them is now not moveable by the animal power, so that we cannot exert the further contraction, which the animal power is otherwise capable of doing."

CXII. When muscles are excited to contraction by preternatural causes, and are contracted with unusual velocity and force, and especially when the contractions, alternating with relaxation, are frequently and preternaturally repeated, such motions are called convulsions.

"The causes of spasm and convulsion may be referred to three heads: 1 . Whatever disturbs the ordinary measure and order in the exertions of the animal power-I say, that in all men custom has established a measure in the velocity and force of every action, and an order in every series of actions ; this has a certain latitude, which is different in different men, and in different actions, but in every man that latitude has its limits; and if the mind is urged beyond that established limit of velocity and order, it commonly loses its measure, and readily runs to the degree of convulsion and spasm ; this is sufficiently evident with regard to particular muscles : thus. I am frequently liable to spasm, and the cause is some degree of strain in the motion I excite. If I turn about short and more quickly than ordinary, I have the whole of that side of the shoulder and thorax affected with spasm; and in most violent exertions we readily throw the muscles into spasms. But still it is more remarkable with regard to the exertions of the animal power in the brain; there, all hurry in the emotion or passion does produce a general effort of epilepsy, and hence the effects of surprise. All strong impressions which are sudden, throw moveable systems into convulsion and spasm, or into delirium or irregularity in the motions of the animal power. An illustration, which, I think, is very 
much to the purpose, is this : persons who stammer, or suffer a considerable interruption from convulsive motions arising in speaking, can, nevertheless, sing quite easily without any interruption; which appears to be owing to this-that in singing, they have their ear to determine both the measure and order, and, therefore, they go on without any interruption; but when they exert the organs of speech by the power of their own will, they do so without any such measure. It is commonly from some accidental bashfulness or hurry, that they fall into stammering; and I am of opinion, that in a person who stammered, and was, at the same time, a very excellent mimic, the case would be the same in imitating the sound and voice of other people, as if he was singing; but in his own exertions he has no such means of governing himself. This explains that it is the loss of measure that throws us into convulsive and spasmodic motions.-2. Direct stimulants. Gaubius (\$ 744.) mentions, first, the mechanical and chemical acrimonies applied to the brain, either directly, or by the intervention of other parts. He next proceeds to the 'potentiæ dolorificæ,'-I would rather have said 'dolor magnus acutus.' When we are taking notice of pains producing convulsion and spasm, we observe that the effect of violent pain is different in so far as it produces sometimes convulsion and epilepsy, and at other times syncope. The one I would refer to the stimulant powers; and certainly if there are any direct stimulant powers, we may find them in pain; but when pain acts by producing syncope it seems to operate as a very strongly sedative power. Here, however, I would not rashly conclude that the effects are opposite to what they are in the former case, for they may be the same, only different in degree; and it may be made a question whether pain, producing syncope, is not an instance of violent excitement producing considerable collapse; and whether it is not like the opposite effects of electricity in exciting action, or in producing death. Gaubius then mentions the 'immodica repletio,' which deserves particular attention: I have endeavoured to shew that a certain fulness of the vessels of the brain is necessary to its excitement, and that a certain increase of that tension and fulness, therefore, is indeed a strong stimulus, which will continue the excitement, and prevent the coming on of the state of collapse; there 
is, accordingly, not a more frequent occasional cause of epilepsy than a little increase of fulness in the vessels of the brain. Gaubius applies this to the whole system; but the effect of which I speak, happens especially when there appears some increased impetus in the vessels of the brain; the fulness, as general over the system, is rather to be considered as a cause of mobility, which, however, has a share here. I need not add here that the effect of the determination to the brain in producing epilepsy appears from this, that convulsions often happen where we have proof of an increased impetus, and where there is a degree of inflammation, as in phrenitis. 3. Indirect stimulants: 'Immodica evacuatio.' The chief proof of this is taken from hæmorrhagy. Dr. Hales was much occupied in experiments which led him to bleed animals to death; and he constantly found that no animal died thus without being affected with epilepsy, of which our butchers have evidence every day. Another proof that epilepsy may be brought on by causes of debility, is, that nothing is more common than syncope, attended with convulsion, so that both these symptoms must be imputed to one and the same cause. But further, many sedatives most powerfully narcotic, and therefore most capable of inducing a state of collapse, particularly opium, do not kill without producing convulsion; and it is very probable that as poisons so universally have the effect of producing epilepsy and convulsive motions, more of them are of the sedative kind than has been imagined, though, at the same time, some of them may operate by their stimulant power and the inflammation they produce. With regard to the effects of odours, I do not positively determine how far they are sedative. I doubt if their effects in producing convulsions are owing to their sedative power ; and I am of opinion that they do so only in so far as they are disagreeable ; and that if musk, for instance, occasions convulsions, it is because it proves as disagreeable as the most foetid substance, so that it is the degree of the disagreeable sensation that has this effect. To this head I must refer the emotion or passion of fear, which is so frequently the cause of epilepsy, and which is to be explained, not only by its direct sedative effects, but from its disturbing, in consequence of the surprise, the measure of the animal power."

CXIII. If the contractions of muscles are exerted with un- 
usual force, and such contractions are often repeated, they in a short time become uneasy and,weaker; and though contractions are not exerted with unusual force, if they are often repeated, and for a long time, without an interval of rest, they also become uneasy and weaker.- " There is here, perhaps, a little inaccuracy ; the unusual force may be referred to the cause of convulsion; but I mean here, that if the contractions of muscles, by their ordinary causes, are exerted with a little more than ordinary force, though that is measured and directed by the will, if such are often repeated, under these circumstances they in a short time become uneasy; and though the contractions are made by natural causes, if they be often repeated, and for a long time without an interval of rest, they become uneasy and weaker, they give the sense of lassitude, and we cannot continue to exert the same force with which we set out in such exercise. Nothing is a more common observation than that the muscles are exposed to an uneasy sensation which we call fatigue, and manifestly become weaker: so men, used to labour for ten or twelve hours of the day, by habit are able to do with ease what would be fatiguing to us. But even these men, if they are obliged one day to protract their labour for more than that time, become fatigued; and if they continue to urge on their labour, they will fall down, perhaps, entirely powerless, or perhaps they will fall into a deliquium altogether. The former part of the proposition will be as obvious, viz. that though the exercise is not continued for any unusual length of time, if it is for some time carried on with unusual force, the same fatigue, lassitude, and debility consequently ensue. I presume that it would be the case with most of us, that were we to attempt the work of a labourer in digging ground, we not only could not continue it for any length of time, but as we are accustomed to exercise our motions with a given velocity and force, the fatigue would be sooner adduced by our excess in this respect. I wish I could here account for this, and explain upon what it depends ; but till we know much more of the nature of muscular motion, and of the connexions of the system, how the several parts co-operate, and how far the animal power concurs upon this occasion, we are entirely at a loss to say any thing of this matter; but I shall have 
occasion to recur to it, when we have discussed another law, which is of more importance."

CXIV. Within certain bounds, with respect to force, frequency, and duration, the contraction of muscles, by being repeated, is performed with more facility and force.- "There is not a man who has not bounds of that kind; he can repeat his actions for a certain number of times, and for a certain length of time, without any fatigue or debility; and when they go no farther, they have a contrary effect, - the contraction is performed with more facility and force. Hardly any body has entered upon the learning of a new exercise, who does not know that the first attempts are what we call awkward. There is a concurrence of various muscles and members, that do not readily at first accommodate themselves to perform the motions exactly: Only by repetition we acquire the command over certain muscles, and are enabled to unite and accommodate various motions to one and the same action; and there is nobody who has not observed, that whether the motions are more simple or complex, we are constantly improving in the facility of moving. Thus, in the management of a musical instrument, we find that our fingers have been associated in some motions only: we must break these associations, and they disappear by repetition; and the agility and surprising motions thus acquired, astonish every body who has not had the same experience.

"I have an observation of more consequence to make, viz. that somewhat of this kind takes place with regard to involuntary motions, under the power of stimuli of different kinds; these also, by repetition, are performed with more facility, so that they can be excited to the same contractions with the application, perhaps, of a tenth part of the force. If the exhibition of an emetic, or any other medicine, whose effect is to be muscular contraction, is frequently repeated, a less and less dose is found to be necessary. I have known a person who thought it necessary to vomit very frequently - sometimes every day, and at least twice a week. At first he required three grains of tartar emetic, but at length half a grain would not only excite full vomiting, but was liable to throw him into cramps and spasms; and if by any accidental interruption, he imagined that a larger dose 
would be necessary, the increase of half a grain had such effects. We have many instances of the same kind with regard to purgatives, of persons at first requiring a certain dose, but who in consequence of repetition are moved by a smaller dose. This effect will not be disputed; but it gives difficulty when contrasted with a law of sensation, by which we become more insensible in consequence of the same stimulus being frequently applied; and accordingly in the very case of the stomach, a circumstance arises from the frequency of the repetition, which I cannot determine. In a person; who is just now, by a small dose, excited to vomiting, if that is repeated the stomach sometimes becomes more insensible, and the dose must be increased; and this event, perhaps, is more frequent than the other; but they are both equally 'true, and perhaps no rule can be made to determine the application, as it depends upon a temperament, and perhaps an idiosyncrasy. But a curious question here arises:-Do stimulants, which produce facility of motion, and operate more upon motion than upon sense and will, act in diminished doses in consequence of repetition? And do the sedatives, on the contrary, which are considered as operating upon sense, require to be increased upon repetition? Thus we have an hundred instances of persons in the habit of taking opiates; and I never knew an instance of a person that could be made to sleep by a smaller dose than that he had taken before. But that is a question for your speculation.

"The doctrine we are speaking of has been as much taken notice of in morals as in medicine ; and it is a maxim, that custom increases our active, and diminishes our passive habits."

CXV. Are not the contractions of muscles produced by the action of the animal power, those which are more especially liable to become uneasy and weak by frequent repetition?"6 It might be naturally supposed that the lassitude or debility, which is the consequence of frequently excited and long continued action, may depend upon some circumstance in the nature of muscular fibres themselves. 3 Till we know more of the nature of their contraction we cannot say much with regard to this; for in most of the instances the animal power is concerned, as in the case of the organs of voluntary motion. I will give you a fact, which is curious as affecting this question; 
whether it has been frequently or accurately examined I will not determine. We have been frequently exercised in observing the motions of the heart, after it was cut out from the body. and freed from all connexion with the animal power. If you will allow the heart to remain without any impression, and preserve it in a pretty uniform temperature, it is found that its little palpitations will continue for perhaps an hour. Now, supposing that the heart thus left to itself unstimulated, will continue to move so long, but, as it has its little intermissions, becoming more languid; if, in these intervals, you prick it with a needle, it will renew its vibrations with more vigour; but if you do employ these stimuli, and frequently excite to a greater degree its contractions, it is alleged that it will finish sooner entirely than it would have done; so that if you keep frequently pricking at the heart with a needle, it will lose its irritability, and cease to move perhaps in half an hour. Here we suppose that it is excited with unusual force, and that gives a kind of lassitude or debility, analogous to what happens in voluntary motions; and if so, the circumstance belongs to the muscular fibre itself. But further I cannot go; and, upon the contrary, there are many circumstances that lead us to answer the above question in the affirmative, that the actions which depend upon the animal power only are liable to the debility which arises upon their repetition. What led to the question is a passage in Dr. Haller, which I wish he had proved a little further, (Primae Linea, \$ 119.)- 'Sed quaeri potest, \&c.'-'Why, when the most part of the muscles of the body are exposed to lassitude and debility, has the heart this singular property, that for many thousand times in a day, for, all the days of so many years of life, it goes on without discovering any marks of lassitude and debility?' It is perhaps of some use to consider this. I think it may be explained in this way, that the heart has its action excited without that occasional concurrence of the animal power which is necessary in the voluntary motions, that it depends upon some constant energy derived from the brain, which I have demonstrated. But it does not require that concurrence of animal power, which is necessary to voluntary motions, but not required in such as are never spontaneously omitted and usually carried on without the power of the will. The action of 
respiration, accordingly, continues as long as that of the heart. It is true that it is not so frequent as that of the heart; it is but as one to three or four; but it is prodigiously frequent; and certainly. the same question occurs :-Why does the ordinary action of respiration go on greatly longer than any other muscles of the body continue to perform their functions? It is because the respiration, as much as the heart, is without the concurrence of occasional animal power; which appears from hence: Let the respiration be with unusual force for some time,-as speaking above an hour together, or blowing upon a wind instrument, or being exercised in actions which require stronger inspirations, - and we will feel the effects of lassitude and debility; only habit and custom vary these. The lassitude or debility accordingly, may in some measure, depend upon the state of the muscular fibre itself, but it depends more upon the animal power; and this has this singular constitution, that it necessarily requires the alternate state of rest ; and I shall use the circumstance of sleep and watching, which are affections of the animal power, as an argument of its being peculiar to that power.

"As it was proper to consider the action of muscular fibres in the two views, of the Vigour and the Mobility of contraction in the state of health, it is necessary to consider the morbid state of the muscular fibre also, as it respects the vigour or the mobility ; and it will preserve the clearness of our ideas, if we fix particular terms to the excess and defect of each. The excess of vigour, therefore, I name THE INCREASED TONE; ; and the defect of vigour I call, with every body else indeed, DEBILITY ; - on the other hand, the excess of mobility I name IRRITABILITY, and the defect TORPOR.

"I begin with the state of vigour; and I may first inquire if this can be morbid in excess. The occasion of this will appear from a passage of Dr. Whytt, who says, (Works, p. 527,) - A greater degree of that power in the nerves which is necessary for motion, can only give more force and steadiness to the muscles when they all possess it in an equal degree : the increase, therefore, of this power, is hardly to be accounted a distemper ; it is never exerted, except in consequence of an effort of the will, of some affection of the mind, or of the action of 
some stimulus on the brain and nerves.' When the increase of vigour is equal over the whole system, and therefore suited to the general tenor of the economy, it is indeed not a disease, but the most perfect state of health. But I think Dr. Whytt has forgot to observe that if this state is in any respect partial, it certainly is a disease. It is not easy to establish an instance of this partial excess; but I think we have one in the phlogistic diathesis, which, I conclude, consists in an increased tone of the arterial system, and is certainly partial in relation to the general system, for commonly there is, at the same time, a diminished tone of the other muscles, particularly those of voluntary motion, and often those of the alimentary canal. But, even as affecting the whole system, the increase of vigour forms a predisposition to disease, as exposing persons to be acted upon more readily by occasional causes, and disposing them to inflammatory diseases, to the partial excess of vigour in the phlogistic diathesis, and, as combined with more or less mobility, to other diseases, as melancholia, hypochrondriasis, and mania. Dr Whytt (L. c. p. 528,) alleges that the increased power of the nerves never produces a disease without a concurrent occasional cause. '.. 'Thus,' - says he, ' a tetanus, or unusual spasmodic contraction of any muscle, is not owing to an increase of that power in its nerves which is necessary for muscular motion, but to an extraordinary exertion of it in consequence of some uncommon irritation or affection of the brain and nerves.' This is very true, but if we take the history of the cases of tetanus occurring in the West Indies, we find that men are more liable to the disease than women; and men, in proportion as they are more robust. It appears, therefore, that this increased vigour is a predisposition, and concurs in aggravating the disease.

"But if debility is concerned in so many instances in producing disease, we cannot consider it properly without at the same time considering its opposite; the increased vigour of the system. Here the term of strength, however, is applied to disease, which may appear a little uncouth, and may be exposed to cavil, as Dr. Whytt imagined; on this account I have employed the term of ' increased tone,' though I am not certain that even this is altogether free from objection. 
"I now proceed to seek for the causes of the different states of vigour : it may depend-

" 1 . Upon the state of the simple solid (XIV.-XXIV.). In animals of different sexes, and of different temperaments, the energy of the brain being given, the vigour of the body is correspondent, in some measure, to the density and rigidity of the simple solid.

"2. The bulk of the muscle. It is very generally observed, that coeteris paribus, the larger animals are stronger than the smaller; it has been supposed-indeed it is probable-that the number of fibres is the same in the muscles of each ; therefore, if we admit that supposition of the equality of the fibres, we shall find no particular advantage from the length, so that there must be somewhat in the manner in which the fibres are collected into the belly of the muscle that promotes the vigour of the whole. In men of the same stature the strength is manifestly as the bulk of the muscles; the painters know this very well, when they express their Hercules by the swelling they give to the several muscles; and nothing serves to prove this better, than that when exercise gives an increase of strength, it, at the same time; always gives an increase of bulk in the muscles. We had many years ago in this country a very strong man of the name of Topham; his feats of strength were mostly performed by his arms, and his legs were little employed. I, along with several anatomists, examined him; and we found that while his legs were soft, and rather resembled those of a woman, the bulk of every muscle in the arm was very remarkable and considerable:

" 3. The state of the tension of the muscular fibre. If we employ the analogy of the simple solid, we have sufficiently explained how far the tension gives a stronger degree of contraction; and the excitement being given, I would explain it in the muscular fibre in the same manner. But there is truly somewhat more here, for we know that this tension is capable even of exciting a contraction, while the extending power continues to be applied; we conclude, therefore, that tension actually increases the excitement for the time, and so strengthens the muscular fibres more in proportion than it does the simple solid. A circumstance which particularly deserves to be taken notice 
of here, is, that the tension operates in exciting contraction, even when the muscle is entirely separated from the body; and therefore we say it operates by increasing the inherent power, not merely by acting upon the whole system, and thereby upon the muscular fibre. There is this further circumstance to be observed, that a certain condition of the inherent power seems to be necessary to the action of the animal power upon it, and therefore do we find it so necessary to give a tension to these fibres which, by the power of the will, we would excite to contraction. I have had occasion to point out this, and to mention the use of a full inspiration; and the effect which the frown, the contracting the eyelid, \&c. has upon the muscles of. the diaphragm, is, I think, no otherwise to be explained, than by supposing that the tension of the whole is necessary to the tension of every single muscle. But we find that this is deter-s mined to be in a certain degree according to the power of habit and custom, which regulates the degree of tension that is necessary to the actions of every muscle. Thus, when any external means of tension, as the designed or accidental appendage of weights, which have been employed, and have become habitual, are taken away, the action is weakened. In various exercises, and in the practice of several arts, we find that it is necessary to the due and steady performance of the action, that the various tools or instruments should be of a certain weight. In another respect we observe the power of custom in regulating the degree of tension. The most part of our muscles are stretched upon bones, and by means of this contrivance the antagonists are made to balance one another. But this tension will be varied in every difference of the flexure of the joints to which the muscles belong, and, therefore, in every different posture of the body; but if we are habituated to perform any particular action in a given posture, that becomes afterwards necessary to. the performance of the same; and this will explain the difference. which takes place among different men in performing the same action, and how the most awkward posture for performing an action may be as necessary as the most proper. The principal means of tension in our system, is that which is given to the arteries by the blood propelled into them (see page 79); and this can be communicated to every fibre of the system. Hence the dif- 
ference of force in different men, according to the quantity and quality of their diet, and the reason why an English workman may do double the business that a Scotchman can do in the same time. There also appears here the effect of a full meal; for as the tension of one part depends upon that of every other, the tension of the stomach necessarily has an effect upon the whole system. It is upon this footing that evacuations weaken the system; that this depends upon the tension is evident from the fact; that evacuations from a particular part do weaken that part more than the other parts. In this way, probably, the evacuation by purging weakens the tone of the intestines; and in proportion as the evacuation is made by less stimulus, this effect will be the greater. I conclude the consideration of tension, which we have considered here simply as giving vigour ; the explanation of the same tension; as a cause of mobility, I must reserve till afterwards.

64 4: Causes of excitement or collapse (CXXX.), applied directly to the moving fibre itself. As in the case of sensibility (LVI.), the operation of these causes is sufficiently evident in the diminution of tone by sedatives and narcotics ; but their effects as causes of increased tone are not so clearly perceived, though a little more clearly than in the other case. In the operation of various stimulants, we perceive nothing more than their producing a contraction,-we do not perceive that they produce any more vigour or irritability ; but this being given, they produce a contraction. Still, it is in general probable; that there are powers of excitement as well as of collapse, and that among the stimulant powers there are some powers of excitement which act in regard to the moving fibre. But however it may be with the stimulants, there are other powers which increase the tone of muscular fibres; such are those we call astringents and tonics.

"To this head of causes of difference in vigour, I must refer the power of exercise also. The effects of this in giving strength and vigour in certain conditions, are pointed out in CXIV.; but these effects are attended with a change in the muscle itself, with a manifest increase of bulk in such muscles; exercise, therefore, acts directly upon the muscular fibre, as well as upon the whole system; but how it acts apon the muscular fibre is not clear. We car observe that it gives more facility in action, voL. I. 
that is to say, it admits of the action of the animal power more readily; and if this depends in any degree upon the afflux of a cestain matter, we must suppose that the change in the muscular fibre consists in a higher degree of excitement being produced; and from its being attended with an increased bulk, the excitement produced is also of a more permanent kind. On the other hand, exercise may be the caise of debility, for the circumstances of which you must consult CXIII., and reconcile that with CXIV. in the manner I formerly endeavoured to explain.

" 5 . The vigour of the moving extremities will be different, according to a difference in the state of the brain which may be communicated to them. It is necessary to attend to this. In the case of voluntary motion, we very speciously allege, that, in every contraction of the muscles, an action of the animal power, or energy of the brain, constantly takes place; but in what are called the involuntary motions, whether they are originally such, or are so in consequence of habit, we suppose that no such exertion of the animal power does occur, but that the contraction is in consequence of the stimulus acting upon the inherent power, which is agreeable to the common doctrine of Dr. Haller; but I have before pointed out what limits this requires. I have said that the involuntary muscles are still under the influence of the brain, and depend upon its constant energy; which we infer not only from various stimuli applied to the brain having considerable influence upon the action of every muscular fibre, but from the muscles being liable to palsy, in consequence of the compression of nerves. The inherent power, therefore, the vigour of muscular fibres, is greater or less according to the state of the brain communicating its power constantly or' occasionally; accordingly, in the case of mania, where the tone is increased to a considerable degree, or in the case of fever, where it is as remarkably diminished, we seek for the causes of both only in the present state of the brain.

"These are the causes that we can assign for the different states of vigour in our moving fibres; but $I$ have said that the moving fibres are also to be considered with regard to their mobility. We are, therefore, to consider the causes of more or less mobility (irritability and torpor; see p. 93.). These have oc- 
cupied pathologists much more than those of the former class; but the subject has been treated imperfectly in two respects.

"First: It has been common to consider every convulsive or spasmodic affection in an unusual degree, as an instance of increased mobility. But often, the mobility being given, the spasm or convulsion produced is merely the effect of the stimulus applied, which may be capable of producing it in any person, though more readily where there is a certain degree of mobility; and, in several instances, we find that these effects are more considerable in proportion to the increased tone and vigour of the system, as in the case of tetanus; so that it is false to consider these preternatural motions as always a mark or effect of mobility alone. Secondly: I think the case of mobility has been improperly considered, as being the same with sensibility ; this, I humbly allege, is the fault of Dr. Gaubius. I acknowledge that increased sensibility and increased mobility are very commonly conjoined, and increased sensibility is to be mentioned as the first cause of irritability ; but it is necessary to be observed, that increased sensibility is not the sole cause of it,-increased sensibility and mobility may be considered as different affections; an illustration of which I take from the power of custom with regard to impressions made upon the stomach, or other parts of the system, where the impression may be considered not only as giving sensation, but as producing motion.

"A second cause of irritability is a state of debility in the moving fibres. Children and women are more moveable than adult men; and debility is a cause of increased mobility. I have pointed out increased sensibility as conjoined in these cases, but certainly debility is also present, and therefore may have its share. There are many instances of persons weakened by occasional causes, as fatigue, evacuations, preceding diseases, \&c., where the debility is very evident, while the change in the sensibility is by no means so observable; and therefore I say that in the connexion of the 'delicata solidi compagis teneritas' with both sensibility and irrritability, the latter is more connected with debility than with sensibility.

"A third cause of irritability is a subtile and theoretical one; but you will excuse me in taking up a moment of your time in throwing it out; viz. such things as give excitement 
greater in elasticity than in density; certain stimulants, particularly the repetition of them, may operate in this way; and I think that to this head we may refer the power of tension in giving irritability. It has been the observation of every physician, that plethoric systems, in which there is the greatest tension, are always the most irritable; so that persons who are disposed to plethora, viz. such as are of the sanguine temperament, the female sex, and indeed both sexes about the time of their acmé, which is their most plethoric state, have the most irritable systems; however the theory of this is certainly difficult. I have said that tension gives a certain degree of vigour, and hitherto I have considered it in that view; vigour is very often opposed to increased mobility or irritability; if, therefore, they here co-exist, there must be some singular combination of circumstances. But I conclude that the fact is sufficiently certain, that plethoric habits are the most irritable, or that irritability is most evident where plethora is present, or in systems that are liable to hæmorrhagy; and, therefore, I have sometimes said that hæmorrhagic systems are the most moveable, so that the female sex, who are most liable to hæmorrhagy, are also more irritable, especially at the time of menstruation, when the most remarkable symptoms of mobility occur in the female sex; and from the same effects of a plethoric habit we have a clear explanation of the cause mentioned by Gaubius, the 'genus vitæ lautum sine exercitatione,' which is the most effectual in giving a plethoric state, and therefore in giving a remarkably irritable system.

" The fourth cause of irritability we find in the effects of $h a-$ bit. This operates in two ways, -1 . in consequence of the law mentioned in CXIV. which is, that the repetition of motion gives greater facility of motion, $i$. $e$. irritability. A matter of more curiosity which I have to add, is, that repeated motions often put on the appearance of spontaneity, when they are associated with sensations or motions, which, by the laws of our system; periodically return. In that way I particularly explain the daily revolutions in our system, and how various other circumstances will continue to recur, in consequence of an association with other things that do occur in that circle; and, with regard to Pathology, how several diseases, which depend upon a state of irritability, as epileptic, hysteric, asthmatic affections, become 
periodical. 'The power of habit, in giving such laws in producing periodical revolutions, is especially remarkable in diseases which are most certainly periodical, i.e. intermittent fevers; a proof of which we have in the direction which Celsus gives - 'diu meminisse oportet diem et horam;' it appears, by the most exact observations, that intermittents continue their disposition to recur at the usual period; that though they should cease for a fortnight together, when they recur, it is not only on the day, but at the hour they usually did; therefore, says Celsus, a patient ought to be long attentive not to admit, at these times, any occasional cause that might excite the disease. And I must add another observation that is of use and curiosity also, that such is the apparent spontaneity that takes place with regard to repeated motions, that the slightest associations serve, upon occasion, to renew them; and even the memory of them is sufficient to bring them back. When I mentioned the power of memory, and its being limited with regard to sensations, I said that we recall only those of sight and hearing with any exactness, and but a few of those of touch; but even those of smell and taste can be in some measure renewed; thus, a person who has taken ipecacuan and is vomited with it, cannot recall the particular taste of it ; but he can renew the sense of the disagreeable, the memory of the nausea, and that will in many persons arise to vomiting; and this explains what appears very marvellous, that persons can recall at pleasure both epileptic and hysteric fits. There has been a question with regard to this epilepsia simulata, whether it is real? I believe the fits are as real as the original ones, and are only simulated with regard to their cause, as we know to be the case where they can be cured by exorcisms. I have a sort of observation to make with regard to real associations bringing on a variety of convulsive affections, as in the case of fear, especially where it is produced by surprise; we should expect that the law of sensibility should take place here, and that the association should have less and less effect; but in some instances it continues for life, and seems to gain strength.2. Another case in which habit has the effect of giving more mobility, is a strictly uniform manner of life, the 'strictius regimen' of Dr. Gaubius; for, as new impressions are, cateris paribus, always the strongest, persons of the least experience will be most exposed 
to them, and will thereby be rendered the most irritable; so that the 'strictius regimen' gives the appearance of irritability.

"Fifthly: the different state of mobility of the muscular fibres, depends upon the state of the brain communicated to them; and here $I$ would repeat the same reasoning as I did in pointing out that sensibility and vigour are communicated from the brain ; as every part of the nervous system communicates, the condition of one part will be communicated to every other, and therefore the different states of mobility in the brain must affect the muscular fibres with the same state."

CHAP. III. OF THE FUNCTIONS OF THE BRAIN.

"I here employ the term BRAIN in the same general meaning I did before, as comprehending the whole medullary substance within the cranium and vertebral canal : the cerebrum, strictly so called, the cerebellum, medulla oblongata, and spinalis. It may be necessary to distinguish these parts in treating of some of the functions, but it is not necessary to do so throughout.

"The Stahlians and some of the physiologists bave taken a fancy that the soul has no seat, no particular part of the body with which it is connected more than the rest; but that it is coextensive with the nervous system, perceives in the organs of sense, independently of any communication with the brain, and operates in the muscles without any motions propagated from the brain to the muscles. But in saying this, they cannot but be stumbled by what I have added:"

CXVI. From the effects of ligatures made upon the nerves, and of the destruction of their continuity, it appears, that, in their entire state, motions may be communicated from the brain to the other parts of the nervous system, and from the latter to the former ; and, from the same experiments, it appears, that the brain (XXIX. 1.) is the organ of sensation and volition, and of the several intellectual operations intervening between these: all which is confirmed by the effects of the organic affections of the brain upon the intellectual faculties.

CXVII. The brain is thus the sensorium or corporeal organ more immediately connected with the mind; and, so far as a 
corporeal organ is employed, all the operations of thought, arising in consequence of sensation, are operations of the brain, and are modified by its various condition.-(Boerh. Inst. Med. 581 . -Haller, Prim. Lin. 570.-Gaub. Path. Med. 523,-See afterwards CXXII.)

"A A circumstance relative to physiology, to which I suspect you are not very attentive, is the history of human opinions, which is by many considered as of little importance. The confuting of opinions manifestly false, and long ago exploded, may indeed be considered as a very trifling labour; and, if I were to entertain you with the confutation of the chemical or Cartesian opinions entertained more than a century ago, I would employ your time very improperly; but even this is not so useless as has been supposed; for as we must take our facts from many authors, we must be acquainted with the speculative opinions which influenced them. But it is absolutely necessary that opinions which still subsist in the schools of physic-particularly if they are more especially fundamental with regard to system, and common to a great many different persons-should be considered here, and that I should enable you to judge of their truth or falsehood. I speak all this with a view to the Stahlian system, which within these forty years has occupied the minds of many physicians in Germany, and has spread more or less into every country of Europe. A very ingenious gentleman in this country, Dr. Porterfield, received this doctrine; and in England, Dr. Nichols, who also infected Dr. Mead his father-in-law, in his old age. It has made a considerable figure in France, in the hands of Sauvage, as it did formerly in the hands of Perrault; and I have shewn how far it has influenced Dr. Gaubius. The influence of this system every where tends to corrupt not only the theory, but likewise the practice of physic; and it has made us lose many excellent hands and many facts which we should otherwise have had. I own, that in making up this compendium of physiology, I thought that it was necessary to introduce a very full confutation; but $\mathbf{I}$ know that the subject is embarrassed by many metaphysical difficulties, and I now see that it occupies a greater part than I wish it to do, so that I shall give a shorter commentary upon it than usual. One step towards des- 
troying the Stahlian system in its foundation, is to establish that the whole medullary origin of the nerves is a sensorium commune. But I carry this further, and state that the brain is not only the organ of the soul, but that, in every motion of the soul, some corporeal motion of the brain accompanies it; and $I$ add further, 'and so far as a corporeal organ is employed, all the operations of thought, arising in consequence of sensation are, operations of the brain, and are modified by its various condition.' I mean to say that the soul acts by means of the brain, and that it does not act without the brain; for a full enunciation of which opinion I would refer you to several late writers, and, as it would appear, of the immaterialists. Boerhaave, in his Institutions ( $\$ 581$.), where he has been explaining perception and memory, says, "Verum omne hoc pendet tantum a simplici illâ conditione sensorii communis, quae est ibi mera tanţum mechanica dispositio.' He is more explicit in $\$ 586$, 'Quæenam est in corpore conditio, unde judicium affirmans, negans; ratiocinium, et methodus ordinate cogitandi pendet ?' He indeed states his opinion in the way of question; but, in putting the question, he certainly does suppose a corporeal condition.

"6 Boerhaave has maintained that the soul and body are two distinct substances, but that during our life in this world they are closely connected with one another:- Corporis statum determinatum idem animi status individue comitatur.' 'This is adduced merely in opposition to the Stahlians, who say that the soul may act separately and independently of the body. Haller's opinion, in his Prim. Lin. (\$578.) I mentioned formerly (XXXI.); he goes very near to the doctrine of $\mathrm{Mr}$. Bonnet, who has maintained that neither in this life, nor in the world to come, can our souls act without the body, and therefore he infers the necessity of the resurrection of the body.

"In CXVIII. I mean to insinuate a proposition which I am by no means so intent upon maintaining:-That upon many occasions the body acts without the soul, and even that in the nervous system there are many actions which may be mechanical."

CXVIII. As certain impressions act on the nervous system, without producing any sensation (LXXX.); and as, at the same time, there is hardly any communication between the dif: 
ferent parts of the nervous system, but by the intervention of the brain; it is from hence also probable, that the brain, by its organization, is fitted to propagate the motions arising in one part to the other parts of the nervous system; and, as these mechanical communications produce different effects, according to the state of the brain itself, we, upon the whole, conclude, that the brain is a corporeal organ susceptible of various conditions, and thereby of considerable influence in most of the phenomena of the nervous system.

CXIX. The action of the brain, in moving the several parts of the body, is excited by various causes, or by the same causes in different circumstances.

1. It is especially excited by the wILL, directing the motion of certain parts as means to an end.

As the motion of certain parts is adapted to various purposes, we are conscious of .willing these purposes, as they occasionally occur, and so far also of the motion of the parts concerned in them; but, where the motion of the parts is connected with one sensation, or a few only, the motions required follow. these sensations without our being conscious of specially willing them; and, unless we have continued the practice of adapting the motions to different purposes, we lose the power of doing so, and the motions become unavoidabiy connected with those sensations which, for a long time, had also given occasion to them. In most of the instances of what are called voluntary MoTrons, we are conscious of willing the end proposed, more than the motions excited; and, of the motions produced, we are conscious chiefly of those of a whole member, or of the general effect, and very little of the many particular motions that concur to produce it. We are never conscious of the particular muscles employed.

"Unless we have continued the practice of adapting the motions to different purposes, we lose the power of doing so. An example of this is afforded by the motions of the two eyes. That both their axes are directed to one individual point does not happen by any necessary law; because many children squint without any faulty conformation of the organ of the eye; but the sense of the advantages which arise from their uniform motion, occasions its being very generally employed, and we 
certainly lose the power of moving the eyes differently, which we originally had. But certain young persons, before the habit is very strong, if they find any use in separating the motion of their eyes, of directing them different ways, or of neglecting the one, have the power of squinting,-and in some it is absolutely voluntary. The motions of the eyelids also correspond; and such is the power of habit, that some cannot shut one eye and look with the other; but there is a use in sometimes directing the eye along a line, and for that purpose we can generally shut one; but there are few people who can apply that to either eye at pleasure. Unless, therefore, we preserve the practice of adapting our motions to their purposes, they become indispensably connected with those sensations alone which all along have given occasion to them; and we can see why the action of respiration is with as little consciousness as that of the heart, and as little in the power of the will.

"I say; ' in most of what are called voluntary motions, we are conscious of willing the end proposed more than the motions excited; and, of the motions produced, we are conscious chiefly of those of a whole member, or of the general effect, and very little of the many particular motions that concur to produce it.' To explain all this I must observe that human actions, and several motions of the body that are performed, may be considered either as rational or as instinctive. They are said to be rational, when, in consequence of a certain train of reasoning, one end or purpose is chosen in preference to another; and they are often also rational in choosing the means most fit to obtain the end, in preference to others. They are instinctive, on the other hand, in two respects; first, when they arise without any reasoning, in consequence of sensations producing a desire, without any other end than the gratification of that desire. Thus, in the case of our several appetites; it is not to nourish the body that a man eats, it is not from any reflection or reasoning that can arise, nor is it from the propriety of satisfying it, in order to nourish the body, but hunger consists in a blind impulse to gratify a desire. So the venereal appetite may be sometimes directed or guided; but it does not arise from any reasoning with respect to the propriety of propagating our species; and all such motions are properly instinctive with us, as they are in other 
species of animals. Motions are instinctive in another view, because though they may have an end, they are a consequence of the perception of that end, as in the former case the desire was. Sneezing, for example, is very often without any end proposed, and it frequently happens quite involuntarily; but upon other occasions there is a propensity, a certain desire of motion, without our foreseeing of what nature it will be ; it depends upon the degree of stimulus exciting it; we feel a sensation, and are uneasy to have certain effects produced; but these are not under our command, from which it is plain that we have not a choice of the means. We raise up our heads, make a full inspiration, and the whole face is thrown into a strong convulsion to produce the particular forcible expulsion of the air through the nostrils, and the concussion of the nostrils that thence arises; and here our motions are instinctive, and not under the power of reasoning or choosing ends and means. Further,-the passions of the mind are modes of our will, and are directed to ends; but the end is frequently vague, and still more so the means. Thus, a man in anger strikes the object of his wrath, and perhaps beats every thing in his way, and we might say he did not know what he did,-he has no choice of the means whereby he would gratify his passion.-But this is enough with regard to rational and instinctive motions. We call voluntary those in which we will the motions as means to an end; in many cases an end is in view, and we likewise choose means; but in the motions of our own bodies, which are to obtain the ends, there is a mixture of the instinctive; the means, the motions of the body, follow from a conception of the end, without our being conscious of willing the particular means. Wo can analyze many of our complex actions; but, in the common exercise of them, I cannot perceive that we have any such conception; $e . g$. when a person conceives the intention of throwing a stone to a great distance, he does it by a horizontal swing from his arm ; by degrees, without any reasoning, from the extension of the mechanical power, he acquires a great deal of address from his lever; he sets back one foot for the central gravity, lifts up the arm to its utmost in order to give the centrifugal force, and from that brings down his arm, with a great variety of other motions. But many persons. would not think. 
of analyzing this motion into all its parts, and I, perhaps, can do it slowly; but that is what we commonly do not ; and certainly thousands of mankind have no other conception but that of throwing the stone; and custom, more or less exercise, or experience, enables them to do it with more or less address and agility, without their willing any more than the effect."

2. The action of the brain is excited by the more general and vehement volitions named EMOTIONS and Passions. Upon occasion of these, the consciousness of willing the particular motions produced is always much less distinct, and in many cases is not at all perceived. Of the last kind are, particularly, most of the expressions of the passions in the countenance and gesture.

3. By the disposition of human nature to rmitation. This imitation is sometimes involuntary-often without consciousness; and the consciousness which takes place is often of the general purpose only, without that of the particular motions produced; or, at least, it is of these only as a general effect.- "So

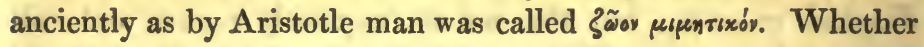
animals attain to the same power may be questioned; probably they do not by a great deal. We know the difficulty of resisting yawning, and we have many instances of imitation going farther. Upon this foundation, also, we explain the similarity of voice and gesture which prevails in families and countries. I know a person who learned to squint, who before that had no more power of squinting than I have: he was not conscious of squinting, but only of the general purpose of imitation. I have asked some exquisite mimics to analyze their motions, but they could not do it : they cannot tell us wherein the faculty of feature or gesture, which they are resolved to imitate, consists."

4. By APPETITES or desires, directed to certain external objects, and arising from sensation, without any reasoning directing to an end, at least, without any other end in the first instance but that of the gratification of the desire.- "Let us consider how far the motions here excited are to be considered as rational, voluntary, or instinctive ; take, for instance, the action of deglutition; we have an arbitrary power over this, -we will the action, but the execution of that will is as much of the instinctive kind as any other; but few persons know any thing of the velum, of the contraction of the glottis, or 
of the motions of the os hyoides, which is a principal means of thrusting the bolus into the pharynx; of all these actions most persons are as ignorant as they are of the motions of the stomach and the intestines; but what we will, is no more than this, to swallow over drink as well as solid food; and we not only do it from the appetite being present, but from the pleasure that meat and drink give to our palate. When a person has no appetite he may be persuaded to take food into his mouth, but he has a difficulty in swallowing it, which is not felt when it is swallowed with an appetite; he turns the morsel in his mouth more than an hundred times over, and still the fauces admit it most reluctantly; and frequently after onefourth of an hour's chewing it is rejected,-so that these motions are evidently instinctive, and strictly connected with the desire that excited them."

5. By certain propensities or desires to remove an uneasy or painful sensation, in consequence of which motions are excited, which are not directed to any external object, but confined to the body itself.

These motions are not foreseen; nor are we ever conscious of willing any thing but the general effect. Of this kind, the chief are the motions of sneezing, coughing, sighing, hiccuping, vomiting, voiding urine and fæces, yawning, stretching, (pandiculatio, ) and those motions of restlessness and inquietude which pain and uneasiness produce. Weeping and laughing are expressions of emotion and passion.- "I say that these motions are unforeseen; but of the truth of this proposition I am not certain. In these motions we at first do not know what will follow. As a sickness may take place without vomiting, I imagine that, in the first beginning of a nausea, if it was the first instance of our experience, we could not know that vomiting was to follow: it appears, therefore, that this motion is merely instinctive. But, independently of this, the instinctive nature of these motions is sufficiently proved by the other part of the proposition : for are we ever conscious of willing any thing but the general effect in sneezing, in coughing, and still more remarkably in hiccup, which is not yet explained, or even disputed about?

"With propensities, we are in danger of confounding several. 
passions : we have therefore said, that weeping and laughing are expressions of emotion and passion'; and in all these we are conscious of some volition or propensity."

In all these, as well as in the motions of No. 4., some volition is concerned, not only as they can often be prevented by another volition presenting itself, but, besides, as the several motions which occur in executing these propensities, are more or fewer, and more or less forcible, according to the vehemence of the propensity or effort. Very often the stimulus to these propensities is irresistible ; and, unless the peculiar stimulus is present, the motions cannot be produced by any volition.

6. By certain internal impressions arising from the exercise of the functions of the body itself, which produce no sensation, nor produce motions of which we are conscious except when exercised in an unusual manner. Such are the causes of the motions of the heart and arteries, of the organs of respiration, of the stomach, intestines, and perhaps of many other parts. With regard to most of these motions, it may be supposed that they are the mechanical effects of their several causes, acting upon the inherent power of muscular fibres (LXXXVI.); but it is sufficiently certain that they also depend upon an action of the brain ; and the effects of passions, as well as the effects of destroying or compressing the nerves of the organs concerned, are proofs of it.

The motions mentioned in this article are commonly supposed not to be accompanied with any volition of which we are distinctly conscious. This, perhaps, is not strictly true with regard to most of them; and, so far as it is, it may be imputed to that repetition which destroys consciousness, (LV.). But neither can we entirely adopt this explanation; as these motions, which are intended to follow one stimulus only, may be supposed to require no exercise of volition, as in the case of the heart, arteries, and alimentary canal, while the action of respiration, as adapted to various purposes, continues to be a voluntary motion.

7. By various occasional impressions of external bodies, and by various occasional states of the system, or of its particular parts, which excite motions not only in the parts to which the impressions are immediately applied, but also in distant parts 
on which they can operate only by the intervention of the brain. Some of these causes operate with, others without sensation or volition.

CXX. In all or any of these cases in which the action of the brain takes place, we cannot perceive the manner, that is, the mechanical means, by which the several causes produce their effects; and we perceive only an institution of our Maker, establishing a connexion between the several causes and the motions that ensue. At the same time we, for the most part, perceive, that the connexions established are suited to the purposes of the animal economy, and particularly to the purpose of supporting the system in a certain condition for a certain time, and of averting what might hurt or destroy it. This constitution of the animal economy, we call NATURE ; and every where in the economy we perceive the vires conservatrices and medicatrices natura, so justly celebrated in the schools of physic."There have been theories to explain the operation of the brain in our intellectual functions, but with no sort of success in their application. There have been other attempts to explain what might be viewed as mechanical communications by means of the brain, but this with as little success as the other. Thus, the tickling a man's side occasions laughter, and this may be said to be owing to the respiration being particularly affected; but tickling elsewhere has the same effect; and none can say why laughter is produced rather than coughing; nor car we assign the communication with the external skin, necessary to give such a modification of respiration as occasions laughter rather than coughing. When we consider sympathy, the communication by nerves is by no means established. I formerly said, that there is a peculiar connexion between the impressions made upon our sentient extremities, and the particular sensations that our sentient organs produce. So with regard to the whole of our volitions, there appears to be same. Why does a sensation of the fauces excite thirst, and not a sensation of dryness in any other parts? Why does a sense of ridicule produce laughter, or an object of grief weeping? For aught we know laughter might have expressed grief-and the contrary. But we must learn the facts; 'the modus operandi' is entirely without our reach.-' At the same time,' I say, 'we, for the most part, 
perceive, that the connexions established, are suited to the purpases of the animal economy ; and particularly to the purpose of supporting the system in a certain condition for a certain time, and of averting what might hurt or destroy it.'-Here is a particular which might admit of a very large and long commentary; but I purpose to give a very short one, because it is done to my hand in many other places. Philosophers have considered it as a proof of the existence of an intelligent and all-powerful Being governing the Universe, that they find a connexion of the several parts in it nicely adapted to their several ends, and this is adduced $a$ posteriori in proof of the existence of a Deity, by Ray, Derham, and Nieuwentyt. Their proofs are particularly taken from the human body; and the economy is evidently a system comprehending a great variety of single functions, and separate organs. We find the organs nicely accommodated to their functions, and these adapted to concur to the purposes of the economy. The tendency of all the functions is the general support of the body during a certain length of time; and while the course of life is exposed to a variety of accidents, there are as many powers for guarding against these injuries. Our passions also are more commonly intended to avert evil than to procure good. But whilst the philosophers have taken their illustrations from the external functions, physicians can go further, and show the nice adjustment and capacity of the heart, with its valves, for directing the motion of the blood; they can explain the alimentary canal, which takes in a promiscuous variety of aliment, converts it to the purposes of the economy by a nice assimilation, conveys it to the several places where the nutriment may be applied, obviates certain tendencies to degeneracy, throws out the noxious, and supplies the nutritious' parts. In short; upon the most minute consideration of the smallest internal functions, they will be found an illustration of this equal to that taken from the external functions, and from the various operations of moral causes which have been insisted upon. Physicians have at all times taken notice of this, that not only the body has a vis conser-. vatrix, but also a power of guarding against injuries, as in the case of noxious bodies taken into the stomach the action of vomiting is produced; and so in many other cases we guardagainst evil; and injuries having taken place, motions are ex- 
eited by which they are contracted, rendered innocent or expelled. This is what has been termed the vis medicatrix natura, but, as this is in every book, I do not think it necessary to insist upon it here. This constitution of the animal economy we have called NATURE, from the time of Hippocrates to the present; it is thus directed to the preservation, or to the remedying the faults of the system. We think it safer than any other term that has been applied-than the whimsical one of an Archaeus, of a Praeses systematis nervosi, or of the rational soul of Stahl, which is liable to dispute."

CXXI. It is in consequence of this constitution, that not only impulse, and other causes which may be supposed to produce motion, do accordingly excite motions in the animal economy; but that also many causes, which seem to diminish motion, do, however, produce an increase of motion in animal bodies. Thus, several passions which, in their first tendency, diminish motion, several propensities arising from debility and difficulty of action, the absence of usual impressions, evacuations, and other causes of relaxation, cold and narcotic powers, are all of them causes of considerable motions arising in the animal system.

"This proposition, if true, is to me one of the most curious and important in our system : that the animal economy has powers in itself, and, in consequence of its own peculiar constitution, resists and obviates various injuries, and not only so as to prevent their effects, but that when these are produced, the constitution remedies these very evils, and restores health. This, in general, to a certain extent, is universally. acknowledged. I shall speak in another place of its limits: I cannot go far in the theory; but this is an obvious reflection, that if the causes of the injuries are of themselves of an active nature, and do excite motions, we may imagine that in consequence of the constitution, these motions have a tendency to remove the effects of the injuries; : but it is difficult to find how any powers that diminish motion should prove active with regard to the system, and excite its latent powers. I give an example : opium, as proved by many experiments, has the power of diminishing sensibility' and irritability, and can go the length of extinguishing the vital powers altogether; but this same opium often acts as a stimulant, increasing the motion of

voL. I. 
the system;-perhaps this is not explained : the most common view is, that opium carries in its substance both a sedative and a stimulant power; but this is by no means clearly proved, and I think that another explanation may be given-that the stimulant power of opium arises from the vis medicatrix naturæ, from that power of the constitution by which it has a tendency to obviate any sort of injury applied to it, and can obviate the effects of these sedative and debilitating powers, as well as any others. Were our reasonings of this kind confined to opium, there might be some difficulty, but it appears to be the case with every sedative or debilitating power which we can apply ; thus, acids, according to direct experiments, are undoubtedly sedative powers, yet these are, in many instances, also stimulant; neutral salts, although for the most part sedative, are sometimes directly stimulant, and even the refrigerant are, at the same time, stimulant. It is proved especially in the operation of cold. It will be admitted that heat is the foundation of vital power, and that cold is of a directly contrary nature, and, if in a certain degree, will destroy life entirely. This is a matter not questioned; but within this degree, cold acts, for the most part, as a stimulant. How the same power of fire, if I may so speak, should, in its opposite degrees, be stimulant or sedative, is difficult to explain, except by taking the last effect as not depending upon the direct operation, but upon that condition of our economy by which it resists debilitating powers. I have formerly called it a reaction, but this term is not proper when applied to a body which is not an agent, as against an active power."

CXXII. As the mechanism of the brain, suited to its several functions, is not at all perceived (CXX.); and, at the same time, as very few of these functions are carried on without sensation and volition, it must appear from this and many other considerations, that the mechanism of the brain (CXVII.) would not be sufficient for the purpose, without being united with a sentient principle or mind that is constantly present in the living system.-- "This would require a good deal of explanation or detail, which I shall avoid, referring you to my late colleague Dr. Whytt, the whole of whose 'Essay on the Vital and Animal Motions' is to prove this. But we differ from other physicians with regard to the extent of the powers 
of the mind, or with regard to its manner of acting. I say" But, at the same time, it is with little probability alleged, that the administration of the corporeal functions is entirely directed by the mind acting independently of the body, and with intelligence perceiving the tendency of impressions, and exciting such motions as may favour the beneficial, or obviate the hurtful tendency, of all causes acting upon the body.- " This is a short account of the Stahlian system of which we have so often spoken. When a poison is thrown into the stomach, the common language of physicians is-that poison has the power of stimulating the stomach, and is thereby thrown out, and the further injury prevented. The language of the Stahlians is this-that poison has no peculiar power of exciting vomiting; but the soul, as soon as it perceives its being present, knowing the consequences, excites the stomach to throw it out. Now this is the mind acting independent of the powers of impression, and of the modifications of sensations, which, the Stahlians say, give only intelligence. These sensations, they maintain, do not necessarily excite the brain to the volition of an action in consequence: but the soul, perceiving the baneful or hurtful tendency, favours or resists the operation, and occasions all that follows after."

We are certainly conscious of no such administration. Many impressions have their effects without sensation or volition. In most cases where volition takes place, it is very general, with little consciousness of the motions excited, and none at all of the organs employed. The force of impression is every where absolute; and it is according to the force of impression, and other mechanical conditions of the system, that the motions excited prove either salutary or pernicious. The general principle, therefore, is ill founded; it is not necessary (Vide Stahl, Praef. ad Juncker. Consp. Med.); it can be of no use, and may be hurtful to the system of physic.

CXXIII. The action of the brain is often determined and regulated by custom and habit; that is, by laws established by frequent and uniform repetition. See above (XLIII. LIV. LV. LVI. 5. LX. 3. LXVIII. and LXX.) for the effects of custom on sensation, and CXIV. for one effect of it on the action of moving fibres. It is now to be observed, further, 1. That custom determines the degree of tension (CII. CIII.) ne- 
cessary to the action of muscular fibres. 2. That custom associates motions with sensations, which are not otherwise their causes; so that the renewal of the sensation, or of its idea, renews also the motion. 3. That custom associates different motions, so that they cannot be separately performed, though not originally nor necessarily connected; "as is observed in the childish play of rubbing the body with one hand and striking it with the other."-4. That custom determines the degree of force and velocity with which motions can be performed.- " Every action, especially where it is a matter of choice, we perform slower or faster ; thus, every man, in writing, falls into a manner (to use the language of painters); and such a degree of force and velocity comes to be a law, so that we can no more perform the same motion with a different velocity than we can work with tools of different weights." -5 . That custom determines the order of succession in associated motions, and the velocity with which they shall succeed one another.- " If a person has got a passage by heart, and, after a great interval of time, his memory is not so ready with regard to it, he may recover it, by having given to him a word or two at the beginning : he will then go on, but losing a little, must return to the beginning, and repeat the whole together. As the velocity of a motion is determined by custom, so the velocity with which motions succeed one another also is: a boy to repeat his lesson must do it as when at school; if we insist on his doing it more slowly, he cannot do it at all." -6 . That custom establishes the periodical return of certain sensations and motions, not originally necessary to the economy. 7. That custom fixes an exact period for the return of certain sensations and motions, which, by the laws of the economy, are disposed to return at intervals otherwise undetermined. These laws, which may be established by custom, are, many of them, with difficulty avoided, " though, for many different purposes, it is desirable to avoid them. We thereby preserve a greater latitude of health : at the same time, such is the constitution of our economy, exposed to vicissitudes, and to external things favouring these, and such is the uniformity in the business of life, that we must be subject to habits. They increase with the advance of life ; but it is desirable to preserve some latitude as long as we can; for, when 
habits are once established, any deviation from them is readily hurtful to the system." - They are often rigidly fixed, have a considerable influence on the action of the brain, and govern the revolutions of the animal system. Thus, any causes producing a deviation from the usual degree of force and velocity (No. 4.) are apt to destroy the measure of it altogether.- "6 I take as an example of this, the heart. In a man in health, and at rest, the motion of the heart is performed with wonderful regularity in point of size, strength, and quickness. Muscular motion exercised for any time, accelerates the motion of the blood towards the heart; and as the blood is propelled with greater velocity, it will accelerate the motion of the heart, and increase the frequency, fullness, and force of its contractions to a certain extent. Every person is accustomed to bear a certain degree of muscular exercise without much disturbance in the regularity of the heart's motion. But if a man runs fast, not being accustomed to do it, he will occasion a considerable irregularity in the contractions of the heart, which become convulsive, spasmodic, and are sometimes interrupted altogether."And, in like manner, causes producing a deviation from the usual order and velocity in the succession of motions (No. 5.) are apt to destroy the power of the mind in following that order, or in giving any measure to the several motions which should be performed; and perhaps, in this way, the effects of debility, of several passions, and of surprise, are, in some measure, explained.

CXXIV. The brain seems, by its constitution, to be disposed to the alternate states of rest and activity, as appears in the alternate states of -sleep and waking, which constantly take place in every animal; but wherein this constitution consists it is difficult to discover.- "In every animal, is perhaps too generally said: but in the first three classes, the Mammalia, Aves, and Amphibia, we are certain that sleep is universal. Every animal of these classes naturally has certain vicissitudes of sleep and waking, and, when in the state of health, once in twenty-four hours. Occasional deviations from this do not prevent our supposing a law of nature; the induction is very general. I need not add that these are states of rest and activity with regard to most parts of the system. The animal 
functions, at least those of sense and voluntary motion, are all at rest in sleep, though there are considerable exceptions; the vital functions go on, yet even they have less activity in this than in the other state. But it is to my purpose to notice that it is chiefly confined to those animal functions which depend upon the brain, and that it depends upon something fundamental in the constitution of the brain. The nature of this constitution is a problem which we would earnestly wish - to have solved; but we have little confidence in doing it. However, according to a maxim of which $I$ am very fond, initium sapientice est stultitia caruisse, our first step is to get out of the error with which mankind have been imbued. My first business, therefore, is to consider the solutions of this problem offered by others."

CXXV. The most common opinion is, that the brain is a secretory organ, which secretes a fluid necessary to the functions of the nervous system; that this fluid is alternately exhausted and recruited, and thereby gives occasion to the alternate states of sleep and waking. But this supposition is attended with many difficulties. 1. It is probable, that the nervous fluid existed in the animal embryo, before the action of the heart, or any secretory function, could take place. 2. In animals, which, during the winter, suffer a temporary death, such as bats, when, by heat, they are again restored to life, the vital power of the solids is restored before the fluidity of the blood. - "Van Swieten tells us, that in the wing of a bat, expanded so that it can be subjected to the microscope, a curious network of red lines is seen, which, in the torpid state of the animal, appear perfectly at rest. When the animal, brought into a chamber of a certain warmth, by degrees begins to shew the powers of motion and of life, it can be perceived that a motion arises in these vessels; the red lines appear broken into different parts, having an oscillation which is confined to very small bounds. By degrees the number of such moving pieces increases, and the size of each constantly diminishes till at last one entire fluid appears, moving with freedom before the eyes. From this experiment it manifestly appears that an oscillatory motion produced in the vessels restores the blood to its fluidity, and sets the animal in motion. When the heat is applicd, 
therefore, the vital power of the solid is restored before the fluidity of the blood, which is a proof of the existence of nervous power previous to any motion in our other fluids." 3. The nervous fluid subsists in the nerves and muscular fibres long after they are separated from the brain, and often when cut into many small parts. 4. Though it be true that the brain is a secretory organ, the fluid secreted may be destined to another purpose; and, so far as we understand that purpose, the fluid fit for it must be unfit for the purpose of sense and motion. 5. There is no appearance, in any part of the nervous system, of provision made for an occasional accumulation of the secreted fluid; nor is there any evidence of its actually taking place. 6. The phenomena of sleep and waking do not correspond with such a supposition; as sleep often takes place, when the secreted fluid must be copiously present, and waking can be protracted, when the fluid is exhausted much beyond its usual measure. 7. Both states are induced by many causes, which can hardly be supposed to act upon a secretion.- " The supposition of waking and sleep depending upon a recruited fluid is manifestly false and inconsistent; so that this doctrine is to be entirely rejected, and we must either give up the question altogether, or seek for another solution. One which has been offered, but which is by no means so commonly received or supported, is, that" -

CXXVI. A certain compression of the brain can produce a state of the system resembling sleep ; but that state is, in some respects, different from that of ordinary sleep: And it does not, by any means, appear, that natural and ordinary sleep depends upon any compression of the brain.-_" I say $a$ state resembling. sleep, for in some respects it is considerably different. With respect to the different states of excitability, we find that natural sleep is distinguished by this, that it can be removed by moderate stimuli, whereas this artificial sleep is not to be excited unless by removing the cause. At the same time I must say that I cannot insist upon that argument. There are certain states produced by the causes which induce natural sleep, but which are to such a degree, that the individuals are hardly more excitable than those in a state of stupor from compression. Thus, in a man dead drunk, where I suppose the sleep produced by a 
similar cause as that which gives natural sleep, the excitability is hardly more than in cases of compression. In an evening, when a person has been long in the erect posture, and the impulse of the blood to the head has been considerably weakened, yet an insuperable propensity to sleep comes on, with all the symptoms of empty vessels of the head, and a pale and flaccid countenance; so that there is no evidence that at this time the vessels of the brain are more full than upon any other occasion. When we consider also how often sleep can be brought on at. any time of the day by different causes, and its periodical returns fixed, there can be no foundation for supposing a compression either in' one case or other."

CXXVII. As it is therefore probable that sleep and waking do not depend upon a different quantity of the matter of the nervous fluid for the time present in the system (CXXV.), or upon any causes interrupting its motion, while the condition of the matter remains the same (CXXVI.), we are disposed to believe, that those states of sleep and waking depend upon the nature of the nervous fluid itself capable of becoming more or less moveable; that it is chiefly in the brain susceptible of these different conditions; and that, especially by its condition there, it has its more general effects on the whole system.

CXXVIII. This may perhaps be confirmed by considering the remote causes of sleep and waking: And it appears, that cold, the absence of impressions, attention to a single sensation, or to sensations that have no consequence in thought or action, the finished gratification of all vehement desires, sedative sensations and impressions, evacuations, relaxation, and any violent, frequent, or long continued exercise of the animal power, are all of them, severally or together, causes inducing sleep.

"Here I have enumerated all the remote causes of sleep, at least all those which have been assigned, and they are so many, that if they all apply, it will not be an exception to our opinion, even if some are overlooked. And first, with regard to cold.

"It is a fact ascertained in cold countries, that when cold induces death, it always does so by first inducing sleep. Now, if sleep is produced by cold, it is enough to say that cold is a power operating directly upon the nervous system. None will undertake to shew that cold causes death only by coagulating the 
fluids; for we have now many instances of the sleep induced by cold, where the waking state was quickly recoverable by moderate stimuli, so that there was no suspicion that the fluids were coagulated; and, upon the contrary, there are experiments upon such animals subjected to cold, which induced sleep ending in death, in which the fluids appeared to be in their common fluidity, and in motion as before, though perhaps more languidly and not equally extensively. I must further notice, that in animals which seem dead during the winter; as bats and swallows, I imagine that their fluids are at no time frozen, but that they merely. stagnate:when the motion is destroyed, and take their ordinary coagulation : indeed all these animals retreat to places to which the freezing cold never reaches, and they collect in such numbers that the mass is never affected to the freezing point. From the whole history of animal life constantly excited, and from its first beginnings reared to a certain degree of vigour, by a certain degree of heat, and from the check which eold produces on these risings of life, we can scarcely doubt that as heat produces life, by exciting the activity of the nervous system, so cold, its counterpart, induces a state of sleep by encroaching upon the same power.

“' ' The absence of impressions.' Lay a man upon a soft bed, remove the stimuli of light and noise, and if he does not think much, he will fall asleep at any hour of the day. The fact will not be refused; and the exceptions are, where the mind is put into a train of activity. Impressions being the beginning, are also the chief support of the activity of the whole of our nervous system; and it is from thence that all our sensations, and the actions depending upon them, do arise. It is necessary to this activity that these impressions should continue; on withdrawing, therefore, these causes of motion, the motion subsides, and sleep ensues.

' "'Attention to a single sensation.' This is a matter of common observation, that the hum of bees, the murmur of a distant stream, the lullaby of a nurse, will set a man asleep, as is finely pointed out in the lines of the poet :

" Hyblacis apibus florem depasta salicti

Sacpe levi somnum suadebit inire susurro."

VIRG. Ec. I. v. 55. 
The matter is well known, and the theory is extremely simple. The mind can apply but to one thing at once, so if it is attached to a single sensation, it is brought very nearly to the former state of the total absence of impressions. It is probable that not only sensations in general, but that indeed a variety and succession of impressions are necessary here ; if the intellectual operations, therefore, are for the most part suspended, a state near to that of the absence of impressions is induced; or if it is a sensation that has no consequence in thought or action, if it is a state with some uniformity succeeding another, and presents to us no reflections that lead to any train of intel. lectual operations, or if it leads to no desire or aversion, therefore to no action, it will operate in the same manner by reducing us nearly to a state of the absence of impression. The instances are matter of common observation; if a man endeavours to attend to a discourse pronounced in a language he does not understand, and with a sort of monotony, or, although the language is understood, if he is not engaged in pursuing the same train, it is truly to him a vox, et proterea nihil, sounds that lead to no action. Thus, what we call a dull sermon, is one that does not engage the intellectual operations of the hearers. Of the same kind are certain motions of the body which interrupt the train of thought; thus, the rocking of a cradle interrupts every intellectual operation, while external impressions are avoided. Persons carried along in an easy carriage on a smooth road arè very effectually induced to sleep; and if we turn a chicken's head under its wing, and give it two or three undulatory motions, we have removed the external impressions, and interrupted the internal operations, and the chicken falls asleep. These circumstances then are manifestly such as merely diminish the motions that otherwise should take place in our nervous system.

" "The finished gratification of all vehement desires." Aristotle has observed, omne animal post coitum triste; if the coition completes the gratification, it not only makes the animal 'triste,' but somnolentum also. I know that different explanations of this may be offered; we may fancy that the seminal fluid is of a singularly spirituous nature, and gives a particular waste of the animal spirits; but that is no ways founded, and every 
body at present is more ready to imagine that it is the effect of the pleasure that induces a relaxation of the system, and further takes off an irritation that had been considerable before. All sorts of gratifications thus induce some degree of relaxation, languor, and somnolency; but what I mean especially to mention here, is a full stomach. A brute animal no sooner fills his belly than he goes to sleep: man has the same disposition. It has been accounted for, agreeably to the theory I have mentioned, by its compressing the descending aorta, and thereby sending more blood to the head, and occasioning a compression of the head; but Dr. Haller positively refuses that in man the fullness of the stomach operates in this way; it evidently will not apply to those animals which are prone, and in which it may stretch the integuments, but cannot act upwards to compress the aorta; and it will hereafter appear from some considerations, which we shall use against compressions having any effect, that it must be somewhat in the sensation, somewhat in the satiety, somewhat in removing the irritation which had in some way acted upon the system.

"I go on to say, in the next place, that ' sedative sensations and impressions' are the cause of sleep. The sedative sensations, as fear and grief, are by our pathologists considered among the pathemata reprimentia, which have a direct tendency to diminish the motions of the system in general; that they are such I need not prove, but that they are causes of sleep is not so evident. But I know that they naturally induce sleep; it is a practice with nurses to frighten their children alittle, and commonly with the effect of laying them more quickly asleep; and I know more than one person who, when affected by an irrecoverable loss -as a mother for the loss of a child, where her grief can suggest no redress-readily fall asleep. I know several instances of such persons being disposed to sleep, and to sleep a great deal. With regard to our passions, I would observe, that there is such a variety that is is not easy to observe an uniformity, and this has been marked as a proof of their indifference; but I find that where they produced sleep, themind was thrown into such a melancholy state, as naturally produced this disposition to sleep; and this view of the matter agrees with our general plan; it suspends all the operations of the mind, it is an attention to one single ob- 
ject, so comes near to the absence of impressions.- Sedative impressions' I distinguish from sensations ; they very often produce no sensation: thus; opium, alcohol, mephitic air, applied to our bodies, induce a state of sleep; they are known to diminish the motions in general, and have got the appellation of sedatives. With regard to the chief of them all, opium, the question has been often put, quomodo opium facit dormire? and the variety of theories offered by the mechanical physicians has amounted to little more than that of the Galenists, quia habet in se facultatem dormifaciendi. It has been alleged by some, that opium coagulates, and by others, that it rarifies the blood; but we say, that opium produces its effect independently of the fluids and of their circulation. Whatever difficulties Dr. Haller has raised upon this subject, I say that the experiments of Alston, Whytt, and Monro; our colleagues, upon animals, after the circulation of the blood had ceased, are quite conclusive; that though opium acts slower, it most certainly does act, after all motion of the fluids have ceased; nay, that it acts upon every separate and detached part, even when the communication with the brain is destroyed, that it acts upon the inherent power, so that we need not discuss the matter whether it coagulates or rarifies the blood, as its direct operation is upon the nervous power, the mobility, sensibility, and irritability of which, it destroys in every particular part to which it is applied.

"I go on to add another cause- ' Evacuations.' That evacuations dispose to sleep has been commonly observed; they can go so far as to destroy the action of the brain, and produce deliquium animi, and therefore may induce sleep, or cause a cessation of the animal functions to a certain degree. These evacuations are so sudden in producing the deliquium animi, that they cannot act entirely by destroying the secretions, but by taking off the necessary tension of the brain. A certain degree of impetus is necessary to support its action, which ceases on drawing this off but a very little; and we know that the cessation of the action depends upon that cause, because, by the horizontal posture, we obviate or recover it ; so that it is owing to causes of a diminution of motion in the brain, and is not occasioned by the diminution of secretions there. Some would refer the whole ef- 
fect of evacuation to this head, perhapsnot improperly. I have said that the whole of animal fibres, those of the simple solid, and more especially those of the moving fibre, are under a constant state of tension, which gives irritation; and this is in a sort of balance over the whole system, so that if you diminish it in one part, you do it over the whole; so that by relaxing some particular portions, we relax the whole; and in this way we explain the effects of pediluvium, which is a means of inducing sleep, and our present mode of fomenting the lower extremities, which proves an evident cause of sleep upon many occasions. Perhaps this head is more general than may be imagined, and applies wherever we take off any irritation. The evacuations operate more upon the brain itself, but they also operate by the general relaxation of the system; and even the gratification of vehement desires, formerly mentioned, and the giving a certain satiety or complacency to the system, may be considered as relaxing or taking off the irritation that was formerly present.

"But I go to the last cause, and I say- 'Any violent, frequent, or long continued exercise of the animal power.' In CXIII. I have explained those circumstances of muscular action or contraction, which induce an uneasiness and state of debility in the muscles. I have only summed up those circumstances here more shortly, by saying-any violent and unusual exertion, frequently repeated ; or, independent of that, if continued beyond a certain time. The continued exercise of the animal power, with respect to particular muscles, induces uneasiness and debility ; so, with respect to the brain, it induces sleep. According to Haller-6 naturaliter somnus sequitur vigilium et laborem corporis humani.' 'It is this very circumstance, this peculiar cause of sleep, which deserves to be considered as the chief; it is this, however, that has supported the hypothesis which I have taken pains to refute, of a secretion, and of the action of the body depending upon that."

CXXIX. On the other hand, it appears, that a certain degree of heat, all sensations of impression, impressions analogous to those which produce sensation, all sensations which lead to thought and action, and the increased impetus of the blood in the vessels of the brain, are all of them causes favouring or inducing a state of waking.- " It is remarkable, that though 
physiologists have not neglected the causes of waking, they have been at much more pains to enumerate the causes of sleep. This seems paradoxical, because sleep would appear to be the natural state of the human body, and waking the unnatural one,-for I say, if I can keep off external impressions, there will be no action, but a state of death; whereas waking always depends upon external powers putting the system in motion, and producing, indeed, motions which contribute to support the same activity, but are all derived from some external influence.

"And first, ' a certain degree of heat.' This is in opposition to the first cause of sleep which I mentioned-viz. cold. I have explained the fact, that a certain degree of heat is necessary to animal life, and to the waking state : the limits of which, however, and the degree necessary, we cannot tell. We know that a certain degree of heat is an occasion of our waking, and prevents sleep; and that we procure sleep by cooling the body to a certain degree. But the heat going further disposes to sleep again; and going still further it induces waking. In order to discuss the last fact, I say that every strong impression is a cause of waking; but that heat does not by its stimulus act upon the nervous system, but upon the circulating system, increasing the impetus of blood in the vessels of the brain, which is one of the most powerful means of waking. When the heat does not amount to such a stimulus, it certainly gives a propensity to sleep: and I am not positive how this is to be explained. The external heat, with the concurrence of the generating power within the body, commonly supports a temperature of our bodies between $96^{\circ}$ and $100^{\circ}$. The generating power is in different circumstances, according to the external temperature. Whenever this is below $62^{\circ}$, the generating power is above its ordinary degree, in order to balance; but whenever the external temperature is above $64^{\circ}$, the generating power is constantly diminishing, - so that heat evidently is a means of inducing debility or a weaker motion in the generating power of heat, which, as I shall shew you, is in the nervous system.

" ' All sensutions of impression'-I am doubtful if I should not have said, all sensations-whether of impression or of consciousness. "But there are sensations of consciousness which are 
not stimulant, although others are. There is, however, no doubt of the fact, that every impression which produces a sensation, is a means of preventing sleep, and of causing waking; and you will easily recall what I said (CXIX.) with regard to this being the source of activity in the whole system.

"I have added-' impressions analogous to those which produce sensation.' This will be difficult to most readers, but it may be made easy. A great number of motions go on in the internal parts of our bodies, such as the dilatation of the heart by the venous blood, and the motion of food and air in the alimentary canal, which excite its action; and so, in the other organs of secretion also, there are a great many impressions going on that produce no sensations, of which we are no ways conscious, but which are causes of impulse; and many of these internal impressions, of which we are not conscious, are causes of waking. So persons who labour under a weakness of the stomach, as I have done for a great number of years past, know that certain foods, without their being conscious of it, prevent their sleeping. So I have been awaked a hundred times at two o'clock in the morning, when I did not feel any particular impression; but I knew that I had been awaked by an irregular operation in that organ, and I have then recollected what I took at dinner, which was the cause of it. Dr. Haller is liable to the same complaint; and, in his larger work especially, he gives the particulars of his own case, and to the same purpose that I have done, as he learned it from his own experience. It is very necessary to admit and observe these causes of waking. It was necessary to add, ' all sensations which lead to thought and action.' It is a sort of exception when I said that certain single sensations, or sensations which lead to no thought, are ever causes of sleep. I need not say any thing towards explaining this, viz. that it is sensations which especially lead to thought; for if they lead to no observation of their relations, or how far their relations may extend, they are, as with respect to certain sounds, vox, et proeterea nihil; and it is those sensations which lead to reflection, to intellectual operations, or those that are accompanied with desire and aversion, that especially lead to waking and prevent sleep. Thus there is a certain activity in dreaming which will produce the waking state. 
"Lastly, I mention 'the increased impetus of the blood in the vessels of the brain.' This I take to be the principal cause by which the active state of our system is kept up, and therefore by which the waking state of it is especially supported, and on many occasions produced. There is not a matter of more common experience in physic. No man can sleep while he can take notice of the throbbing or pulsation of the arteries of his temples; and when in fevers there is a constant state of waking induced, in nine cases out of ten it is accompanied with an increased impetus of the blood in the vessels of the brain. We know also that sleep is incompatible with the phrenitic state; and the most remarkable instances of that impetus is, in this and some other cases, from a resistance of the blood in the lower extremities, whence it is determined to the brain, so that nobody can sleep with cold feet. Among the causes inducing a morbid vigilium is enumerated the 'raptus humorum ad superiora.'

"To the action of the brain, as I have said, a certain degree of tension in its blood-vessels is necessary. In the case of deliquium animi which follows blood-letting, it has been supposed that a quantity of blood drawn away prevents the return of the due quantity to the heart; but considering the small quantity compared with that which should supply the heart, it is not likely to be owing to this ; and we observe that the deliquium does not happen while the ligature is still upon the arm, but in nine cases out of ten, takes place after the ligature is untied : the solution of which, therefore, is, that there is no derivation from the heart while the ligature was applied, for the resistance to the venous blood operates as much in keeping all the vessels full, as is sufficient to compensate the loss of the blood drawn off; and it is only when the ligature is taken off, that the blood can flow from the extreme arteries, in consequence of which there is a derivation by the subclavian, and by the carotid, and a subtraction of the quantity going to the brain. In most cases we can, by putting the body into a horizontal posture, effectually prevent that derivation from the vessels of the head. This effect of the erect and horizontal posture, in favouring or preventing this deliquium animi, shews that a certain fulness is necessary to the energy of the brain being exert- 
ed on the heart, and therefore to the activity of the whole system; and from experiments which shew that from such an affection of the brain, where the repletion of the ventricles is interrupted, deliquium animi, epilepsy, and other disorders do so certainly ensue, I am ready to conclude that not only the increased impetus is the cause of preternatural waking; but that a certain force is necessary to support the vital activity of the brain in all its different degrees.-I have thus gone over the several causes of sleep and waking as well as I can."

CXXX. As most of the causes (CXXVIII.) are evidently such as diminish motion in the brain, and those of CXXIX. are such as increase it, it is from thence probable, that the nervous fluid in the brain is truly capable of different states or degrees of mobility, which we shall call its states of ExcITEMENT and COLLAPSE, but without intending by these terms to express or determine any thing with regard to the nature of the nervous fluid, or wherein its different states consist.- " I take it for granted, that considering the weakness and manifest mistakes of the other hypotheses (CXXV. CXXVI.), you will readily think with me that the states of waking and sleep depend on something in the matter of the nervous fluid itself, on its having more or less mobility, and, in some cases, being capable of being moved with more ease and vigour, while in other cases it is unfit for being moved with either. Now, merely to avoid long expressions I shall choose shorter ones, and shall speak of the moveable state of the nervous fluid under the name of its exCITEMENT, and the deficiency or lesser degree of it I shall call its collapse. I had the hint of this term from Dr. Haller : now you must consider these words merely as terms employed to express what I take to be a matter of fact, and not as importing a matter of theory, or expressing any thing with regard to the nature of the nervous fluid or of its different states: whatever hypotheses I have fancied to myself, I consider them as hypotheses still, and I dare not trust you with them, unless you take them as they pass in my mind, and be very certain never to apply them in particular cases. However, I am willing to bestow a few minutes upon a lusus ingenii; if you carry it further, it is at your peril. This subject may receive some illustration in this way: There is seemingly dif-

voL. $\mathrm{I}$. 
fused over the whole of nature, a quantity of electric matter, which, however, in the ordinary state of most bodies, shows no disposition to a particular mobility in passing from one body to another, so that though it is present, it does not show any disposition to motion; but we can, by certain artifices, accumulate this electric matter in more considerable quantity upon the surface of certain bodies, in consequence of which it can be put in motion from one body to another, exhibiting the various phenomena of electricity; and it is agreed upon among philosophers to call this excitement, and to say that electricity is ex-. cited, and that such bodies are excited electrics; and all bodies may be so either by being excited themselves, or by having such bodies applied to them as are. So, in our medullary fibre, there is a fluid which was present in the germ, but was not excited; and it is in the excited state of this that I suppose life to consist, and when it is no longer excited in any degree, we call it the state of death ; and I can suppose, as in electricity, that it may exist in different degrees. Thus, sometimes I can take a stroke at a yard's distance from the surface of the body that is excited, and show that the electric fluid extends to such a distance; at other times we must come within a quarter of a yard, and at other times we must come still nearer; and so it can pass through various degrees till it is collapsed altogether. We can say that there is more excitement in one case than in another, and that the collapse may proceed to different degrees. So far with regard to the analogy that explains the terms. But there is another body in nature of a peculiar kind, viz. iron, which may probably have a quantity of magnetic power constantly present upon its surface. It does not show it to other iron, but we can give it its attractive and repelling powers; we can give it its polarity, \&c. Whether there are other bodies that are capable of this peculiar excitement I shall not say, but it is probable that the medullary fibre may be of that kind. I give another illustration that will perhaps approach nearer. It is now commonly enough supposed by philosophers, that the phenomena of the attraction and cohesion of bodies depend upon a fluid surrounding them: if there is any thing in this at all, it is capable of excitement in different degrees. Some bodies are, for the purpose of attraction and cohesion, always excited, 
others are only excited by particular artifices; so, taking the acid of nitre in any degree of concentration, and applying it to quicksilver, there will be an union; but if I take the concentrated acid of vitriol, and apply it to the quicksilver, in the present state of the air, they will lie in contact without the least mark of attraction, nor can the acid be made to act upon the mercury in the cold; but if you raise the temperature of the acid of vitriol to the boiling point, it acts as the other, and here the cohesion is increased or excited to a greater degree. These are some analogies which may, in some measure, illustrate the matter, but there must be a concurrent view, and a consideration of many other phenomena, before we can apply it more strictly or specially to our nervous system; and though I have thus thrown out a conjecture, which, every time I take a view of the nervous system, seems to me to approach nearer to probability, yet $I$ do not say that it will apply to the phenomena with any consistency."

CXXXI. This subject may be further illustrated, by observing that the excitement of the brain appears to be in very different degrees on different occasions. It seems to be greatest in certain maniacs endowed with uncommon strength, resisting most impressions, and with the utmost difficulty admitting sleep.- I say here, that the force or power producing excitement is the strongest, because the animal power is in a condition of resisting most powerfully all the causes that diminish motion, as the causes of sleep; so that I conclude from this, that an extraordinary degree of the power of excitement takes place. The fact is a matter of common observation : certain maniacs are not only endowed with greater strength than others of a similar condition are, but with vastly greater strength than they have had themselves at other times. We are apt to conceive the strength as residing in the muscles themselves, as a property belonging to them, but we not only cannot separate the consideration of the exertion of animal power from that of muscular contraction, but this is in proportion to the volition, to the exertion of animal power; and an uncommon degree of strength must imply an uncommon degree of excitement in the brain. I have added another circumstance, which is that of resisting the force of most impressions; the fact is certain that maniacs are likewise remarkable for resisting the power of impressions : thus, the 
same persons who otherwise would be vomited with one grain of tartar emetic, will now perhaps require ten to produce the same operation; and one that might have been laid asleep with half a grain of opium, will now take five. They will also sometimes resist the force of all impressions. There are maniacs, indeed, who. have an increased sensibility with regard to particular organs; but the opposite is commonly the case. What connexion there is between the diminution of sensibility and the increase of strength; may be difficult to explain; it is difficult in theory, and I do not attempt it, but the combination is frequent, and we say that it is analogous to the state of elastic chords : the more they are extended the greater is their force of contraction; at the same time, they are nearer breaking; and you will find somewhat analogous to this in the degree of density and elasticity of elastic fluids. Thus, in compressing the air, double the weight does not give double the compression; but it is in proportion to the degree of condensation, that the elastic power will show its force, and operate upon the body that is compressing it in order to remove it."

CXXXII. A lesser degree of excitement occurs in the ordinary state of waking men in health; when the excitement is total with respect to the functions of the brain, but readily: admitting of sleep.- - I I have been a little at a loss in the application of the terms of excitement and collapse. There is nothing more manifest than that the degree of excitement is very different upon different occasions; and if we take the lowest, every. higher degree than that must be called a degree of excitement; and if we take the highest degree, and consider the lower degrées that may take place while life still subsists, every lower may be called a degree of collapse; so the same ambiguity occurs here as with regard to the terms of heat and cold, which may be absolutely applied to the same individual state of bodies. Philosophers have not yet agreed where they should begin with applying the terms heat and cold, and so it may be with regard to the terms excitement and collapse; but I mean by this to settle the matter a little more exactly, and I would call those states, states of excitement, where the excitement is total with regard to the functions of the brain, where there is in every part the general exercise of sense and volition;-I know that there are 
many cases where there is a mixture of the excitement and collapse; but that I take to be the state of waking, when the whole of the functions of the brain can be exercised; and, upon the contrary, I would call that more strictly a state of collapse, in which these functions are for the most part suspended; and so upwards or downwards as it may occur."-This excitement may be considered as of two kinds, either as it respects the vigour, or as it respects the mobility of the system; and these different states of the brain are expressed in the body by strength or debility, alacrity or sluggishness; and, in the mind, by courage or timidity, gaiety or sadness. - "I formerly (LXXXIX.) took occasion to distinguish between these two states of vigour and mobility, the one expressing the force, the other the facility of motion. I own that in particular instances you will find a certain degree of slowness joined with strength, and great alacrity joined with debility : why that is so I cannot explain."

CXXXIII. A degree of collapse takes place in the case of natural sleep, when the collapse prevails so far as to suspend very entirely the exercise of the animal functions; and, though the exercise of the vital and natural continue, they are considerably weakened._- "The fact has been disputed; as some have alleged that these functions are rather invigorated: but I think this opinion is at present deserted; and De Gorter, in his Exercitationes de Somno et Vigilia, has proved that the vital and natural functions go on more slowly and weakly in sleep. You will find that Haller also has come to the same conclusion.Why is the action of the vital functions only weakened and not interrupted in ordinary sleep, as they are dependent upon the brain? Different theories have been proposed, such as the difference of origin of the nerves sent to the organs of the vital, from those of the animal functions, viz. that the latter are derived from the brain, and the former from the cerebellum; but at present anatomists are agreed that there is no such distinction. Haller thinks it enough to put it upon the force of habit, which will go some length, but it does not completely explain the matter. I have formerly prepared for an explanation which is quite easy : in the exercise of voluntary motion there is an increased excitement, however momentary, in the action of the brain, and a constant volition necessary a If, therefore, sleep 
consists in the suspension of the animal functions in the brain, it must suspend the voluntary actions which depend upon them. But as the action of the heart goes on without any particular excitement for the time, it will continue so long as life remains, depending upon the common energy. With this is connected the explanation of the fact, that the one action is attended with fatigue, and requires alternate rest, while the other does not, (CXV.)."

The partial collapse that may take place in the brain, discovers itself by the delirium which appears in a state that often occurs as intermediate between sleep and waking; and even in sleep, the collapse with respect to the animal functions takes place more or less entirely; whence the sleep is with or without dreaming, and the dreaming is more or less active._"See p.146."

CXXXIV. A still greater degree of collapse takes place in the case of syncope, in which it is so great as to suspend the exercise of the vital functions concerned in the circulation of the blood, notwithstanding the force of habit in these, and their being exposed to constant stimuli. Here the collapse may be very considerable; but there still remains some degree of excitement, while the brain can be acted upon by stimuli which act only on vital powers, and while its usual excitement is still recoverable by such stimuli.

"Syncope is understood to be either a very great diminution, or a total though temporary cessation of the heart's action. It may arise from various causes, which, however, may be reduced to two heads.-1. Those which directly act upon the heart itself, preventing the return of blood to the cavities to support its continued action; and these are the causes which physicians have looked for; but it is necessary to observe, that the action of the heart depends upon a constant energy of the brain; and we can perceive-2. Causes of syncope which first act upon the brain, and, by suspending its actions, upon the heart. Thus, when a fright, a sudden joy, and other emotions of the mind, produce a syncope, there is no doubt that these act upon the brain, diminishing its excitement so far as not to be sufficient to the vital functions of the heart, arteries, and organs of respiration."

CXXXV. If the collapse is still more complete and irrecoverable, it is the state of DEATH._ " I am not pleased with my 
expression " more complete:" it implies that the state of death, the entire, perfect, and complete collapse, is but a lesser degree of excitement; whereas this must be considered as different from any degree of excitement whatever."

CXXXVI. From what is now said of the excitement and collapse of the brain, it will appear that we suppose LIFE, so far as it is corporeal, to consist in the excitement of the nervous system, and especially of the brain, which unites the different parts, and forms them into a whole. But, as certain other functions of the body are necessary to the support of this excitement, we thence learn, that the causes of death may be of two kinds; one that acts directly on the nervous system, destroying its excitement; and another that indirectly produces the same effect, by destroying the organs and functions necessary to its support. - ${ }^{6}$ I have taken pains to prove (VI. 7.) that though the action of the brain is the proper vital function, it requires the external support of a certain degree of heat, and of the circulation of the blood-perhaps as one means of supporting the heat of the system; and also, that a certain degree of tension in the vessels of the brain, by means of the blood supplied by the action of the heart, is necessary to the energy of the brain. These are the causes which have been commonly attended to. In the system of Boerhaave, for instance, the circulation of the blood and respiration are the primary and fundamental functions; and where he has attempted to account for the causes of death in fevers and in wounds, he looks for those which interrupt the circulation of the blood in the brain. But many of the causes of death do not disturb the circulation, except in so far as they take away the excitement, and destroy the moveable nature of the nervous power. In Pathology, and in the prognosis of particular diseases, it is absolutely necessary to enter into the distinction of these causes. I call the one direct causes, those which act upon the nervous system directly; the other indirect causes, those which produce the same effect, but by destroying those organs which are necessary to the support of the excitement, viz. the whole system of circulation. These indirect causes have been explained in every system; but I think it necessary to point out the first class-the direct causes."-Of the first kind are chiefly the causes of sleep operating in a higher degree, as cold, sedative 
passions, poisons, and all causes of very violent excitement."With regard to cold, I have sufficiently explained myself (CXXVIII.), proving that it acts upon the nervous system, inducing sleep and even death, and that the stagnation of the fluids, and cessation of the action of the heart, are merely a consequence of its action upon the nervous system.-Of the sedative passions, fear may be considered as one, which, when it produces death, acts first upon the nervous system, and only secondarily upon the heart and its dependencies.-With regard to poisons, I have not placed the narcotics here; but whether these have that power or not, there are many poisons which, from the whole phenomena of their operations, undoubtedly act directly upon the nervous system.-I add another particular, which is not so obvious,' viz. ' all causes of very violent excitement.' Excitement is necessarily alternated with a corresponding degree of collapse (CXXX.), and the exercise of the animal powers necessarily induces the alternate state of rest, as in sleep. This I illustrate here by two causes of death : electricity and a sudden transport of joy. The first operation of electricity is upon the nervous system; its effects in stupifying and killing animals are in proportion to its being directed more immediately to the brain. The manner of its operation has been disputed. It has been supposed that the violence of the stroke breaks the tender structure of the brain; and $I$ allow that this may be sometimes the case, and there may even be marks of effused blood; but Dr. Priestley, who has particularly considered this subject, maintains, as the result of all his observations, that electricity kills without any evident tension or violence done to the organ of the brain. In the case of a sudden transport of joy, (see Medical Commentaries, vol. vi. p. 256.) no body can doubt that the operation is entirely upon the nervous power, upon the organ of the soul and of our emotions and our passions; and we must admit that merely by the force of violent excitement an entire and perfect collapse takes place, and therefore death is produced."

CXXXVII. This subject may receive still further illustration from considering the state of the other parts of the nervous system with respect to excitement and collapse. In the nerves strictly so called (XXIX. 2.), we do not know that the 
nervous fluid suffers any change, but what is exactly correspondent to its state in the brain and extremities; and therefore the only difference of the state of the nerves to be taken notice of, is, their being more or less free (XXX.).

CXXXVIII. In the sentient extremities of the nerves (XXIX. 3.) a difference of the state of the nervous fluid arises from the several causes mentioned (LVI. 2, 3, 4, and 5.), which give a different degree of sensibility; and it is probable, that these different states of the sentient extremities are analogous to the different degrees of excitement in the brain.

CXXXIX. The moving extremities, or muscular fibres (XXIX. 4.), may also be in a different condition with respect to excitement. It is probable that their constitution is such as to admit of a higher degree of excitement than any other portion of the nervous system ; and that upon this their contractility depenids. But, whatever is in this, we perceive very clearly, that the condition of muscular fibres may be varied by causes affecting their tonic power (CI.), or their vigour and mobility (LXXXIX.), and by the effect of custom (CXIV.); and it is probable, that the states produced by these causes are analogous to the different degrees of excitement in the brain (CXXX.); and in the sentient extremities (CXXXVIII.); and thus the several parts of the nervous system (XXIX.), as they consist of the same kind of matter (XCIV.), are also subject to similar conditions.

CXL. The beginning of motion in the nervous system is most commonly accompanied with sensation, and the force of this, in producing its several effects, is more or less, 1. According to the force (XLII.), quality (LXXVII.), and novelty (LIV.) of impression. 2. According to the sensibility of the sentient extremity and brain (LVI.). 3. According to the state of attention (XLVII.): These several causes often concur, frequently balance one another, and are always to be considered together.- " If a forcible impression is made upon a very sensible system, the effect is considerable. If the impression is less forcible, and the system less sensible, the effects will be much less. A weaker impression upon a very sensible system, will have considerable effect ; and a powerful one upon a less sensible system, may have very little." 
CXLI. The effect of sensation is commonly that of exciting the action of the brain; and this is generally according to the degree of volition produced under the different circumstances mentioned in CXIX.

CXLII. The action of the brain excited by volition or other causes, is determined to particular parts of the body most constantly by the connexions established in the system (CXX.), but also occasionally by acquired habits, or the greater mobility of certain parts._ " I know a person who lost a prodigious quantity of blood by - a wound of the arm, by which he was considerably weakened. Whatever emotions of the mind affected this person, the arm was seized with a considerable tremor; a weakness and mobility had been produced in this part which determined these effects. Thus, persons who have acquired a particular irritability and mobility of the stomach, are made to retch and vomit by slight emotions; and, in like manner, persons who have acquired a particular weakness of the bowels, are sent to their closet by every emotion of the mind. There are instances of mobility in certain parts; and whatever affects the system more generally, has its effect especially on that weak part."

"The different morbid states of the brain are not easily ascertained; but they are to be attempted, and we must say what we can regarding them; and I propose to do so by considering how it may be in different states affecting sensation and motion.

"66 I. With regard to sensation; the state of the brain giving sensibility in a greater or less degree is different: 1. As it is more or less free (XXX.). If from any cause the freedom of its motions is interrupted, no sensation can take place: we have frequent instances of this where the brain is under compression, the most frequent cause of this interruption, from external force or fluids effused in the ventricles or on the surface. But it is difficult to say what degree of compression is necessary to take away sensation and motion. Experiments have been made upon dogs and other animals, from which it appears that a strong and considerable degree of compression is necessary; but we have seen the loss of sense and motion in the human 
body when the compression appeared very partial; in several instances, where the effects did appear, the cause did not appear, and there are various instances of very inconsiderable tumours which produced palsy, apoplexy, and death.

" 2. As the brain is more or less excited (CXXX.).

" 3 . According to the different causes of attention (XLVII.).

"Sensibility may, however, be considered also with regard to its being erroneous or depraved. This consideration, I have said, (see p. 50.) may be reduced to three heads:-1. The perception of things which do not exist. When considering the states of the sentient extremities I have remarked that this sometimes depends upon them; but we may say that it more generally arises from a state of the brain. When the sensation is of a very simple kind, such as the starry light which arises from rubbing the eyes, the muscæ volitantes, or a simple tinnitus aurium, it may depend upon the state of the sentient extremities of the organ: but whenever the sensation-of the eye for example-is of a more complex kind, when we not only discern colours, but complex figures, \&c. few of these false representations depend upon the organ; thus also, when the false sensation appears in two or three organs, when we not only fancy that persons speak to us, but see them at the same time, the presumption is that such representations depend upon the brain. The inquiry how this state of the brain operates, is in a great measure out of our reach; but there are some circumstances which we may consider with regard to it. I have said (LXIV.-LXIX.) that memory and imagination differ in this, that though both renew combinations of our ideas, in the one case we are conscious of the absence of the object, while in the other we imagine that it is present. Now the common account is that this difference depends upon the vividness of the impression entirely : this supposition has some foundation, as in delirium there is commonly an increased impetus, tension, and excitement in the vessels of the brain; but in many instances of dreaming there is not the same foundation, as they are of the most placid and calm kinds. I have sought for another solution, therefore, and I think that imagination, or the perception of things which do not exist, is owing to this, that the brain does not admit of any communication 
between its several parts, and therefore does not admit of recovering the several relations which are necessary to determine the identity or presence of any object.

" 2. False judgments with regard to things really existing: Although most of these probably arise from the state of the organ (compare p. 52.), we consider them as depending upon the brain, when there is a want of steadiness in viewing objects, making us hesitate about their reality, which is owing to a considerable debility of the brain; or when, on the contrary, we see more vivid colours; as when in fevers the eyes throw a red colour over every object, or when a well known figure appears distorted, \&c.; also when the powers of reminiscence and memory have been impaired by causes operating upon the brain, in consequence of which persons feel a want of several circumstances necessary to ascertain the identity of the objects, and to give the proper complex idea (LXII.).

" 3. Of the unusual quality of impressions (comp. p. 53.) it is difficult to say when it depends on the sentient extremities, and when on the brain. We may, however, take this as a means of judging. Where it is a pure and single idiosyncracy, affecting one person and one part of the body, we believe that it depends on a certain modification of the sentient extremities of the part; but where it is connected with the whole temperament, with a certain disease, or is the immediate consequence of a disease, I should suspect a peculiar state of the brain; as -when to a hysteric woman a rose is disagreeable, and asafoetida particularly agreeable; and when this change of the agreeable and disagreeable, for instance, takes places constantly in a certain temperament.

- "II. To consider how the same state of the brain affects mo tion in the body, we shall, as before, treat of the states of vigour and of mobility (LXXXIX.); and, 1. With regard to the state of vigour and debility, (comp. p. 93.) I have endeavoured to prove that there is not a muscular fibre in the system, which does not for its power depend upon the sufficient vigour of the energy of the brain, and that without it the muscular actions are much more languid and weak. Now the vigour of the brain depends upon the state of the original conformation, or, according to the language which we use, upon the original sta- 
mina; upon the state of the simple solid (XIV.-XXIV.); upon exercise; upon the temperature to which men and 0 ther animals are accustomed, a degree of heat approaching to excess, relaxing and weakening the system, whereas a certain degree of cold invigorates and strengthens it; upon a certain. proportion of blood vessels in the system, and a fullness and tension of the blood vessels of the brain; and, lastly, upon certain stimulating powers, diet and wine, which produce more vigorous contraction.

"T The causes of debility of the brain are the converse of the causes of increased vigour now mentioned, viz. indolence, the excesses of heat and of cold, want of nourishment and evacuations, which diminish the state of tension in the whole system, and the absence of the usual stimuli. Where stimuli have, been daily applied, as in the case of wine, the system comes to depend upon them for the degree of its tension : their absence: therefore induces debility. Thus, in a person who has used the habitual stimulus of a dram, when that stimulus should be renewed a sense of debility, a tremor occurs, till the tension is again occasioned by the application of a dram to the stomach. There are causes of collapse also, such as certain sedatives, or as we call them poisons, which in like manner induce debility: Thus, a man intoxicated with wine or opium, has not his usual vigour, and though the quantity may not be sufficient to kill, him, it leaves the body under a considerable degree of debility. I could add here the causes of fatigue, although I cannot explain them (CXIII.). We may, perhaps, find a solution in this, that every unusual degree of excitement is followed by a like degree of collapse, and that these states induce one another (CXXX.). This leads to an important question in Pathology, viz. how the debility in fevers is occasioned, and how it it is continued? It may be referred to the head of poisons acting as. sedatives; and many circumstances lead to this supposition; but the most pure inflammatory fevers, produced without any, concurring contagion by the operation of cold, leave a state of debility subsisting in the system, in proportion to the degree of the fever, for a long time afterwards: and where contagion has been introduced as the first cause of debility, - when this has, been seemingly removed, and its symptoms have passed away, 
but the paroxysms have been continued, a debility is again induced which sometimes proves fatal. Any explanation of this debility must be the same as that of the debility induced by exercise, viz. that repeated excitements at length wear out the system.

"I I must add, that the same vigour and debility which affect the whole body, have also effects on the mind in producing the states of courage and timidity.

"2. The mobility of the brain (comp. p. 98.) is very different on different occasions; and will depend, 1 . On the various causes of sensibility formerly assigned; 2. On the state of reflex sensation. Most of our sensations are attended with pleasure or pain; and considering that these are again the immediate causes of desire and aversion, which, in their different modes, produce all the different states of volition, emotion, and passion, it is sufficiently evident how the actions depending upon them will give a greater or less degree of mobility; 3. Upon the intellectual operations, more purely considered; 4 . Upon the effects of habits, (see CXXIII.).

"I proceed to consider the symptoms indicating lesions of the intellectual functions or internal senses. I say there are two distinct cases of this, which I mark under the titles of fatuitas and delirium.

"1. Fatuity is an imbecility of thought and judgment: the sensations are imperfectly excited, and still more imperfectly retained; owing to the first, the relations of bodies are not marked; owing to the second, they are not recollected; so that there is a certain weakness in the exercise of thought, judgment, and of the will which depends upon judgment, that is to be distinguished from an error in these faculties. - With regard to this fatuity, we observe, that there exist two different cases of it, the fatuitas infantilis and the fatuitas senilis. As the affection occurs very constantly at these two periods of life, we imagine that it is connected with some peculiar state of the organ at those times. From some observations which show the state of the brain in these cases, we conclude, in general, that the $f a$ tuitas infantilis depends upon a certain humidity or flaccidity, and the fatuitas senilis upon a certain rigidity of the brain. 
" 2. I go on to consider the error of thought, judgment, or will, which is properly placed under the title of delirium. It may perhaps be considered as unnecessary to say what this state is, as no one can be supposed to know it except those who are or have been in the state themselves; still it may be useful to consider wherein it consists. Gaubius has doneso; heobserves thatdelirium appears in three different shapes (\$732.): 'Delirare dicitur, $(\mathbf{1}$, qui cum vigilat, somnianti tamen similis, ideas, sibi ab internis causis natas, ad res externas refert, percipitque adeo ac existimat præsentia esse, quæ absunt; tum, (2,) qui ideas undecunque ortas præpostere componit, disjungit, judicium ferens a communi sensu aberrans; et $(3$,$) qui præter rationem, vi morbi, alienos$ a suis mores induit, motibusque animi abstrahitur insolitis, effrenatioribus.'-You will readily perceive that the foregoing passage amounts to this: that the three states of delirium are false imagination, erroneous judgment, and irregular passion. The three operations of thought are perception, judgment, and will : and delirium is distinguished as it lies in one or other of these.

" The first state I have called false imagination, in compliance to common language; but in LXIX. I have said that we strictly call imagination the state in which the sensations, which usually arise from external objects, take place without the presence of these; and it is this that constitutes Gaubius's first kind of delirium. I hinted at the causes of this as far as we can prosecute them, (p. 50.), and I considered them as always internal impressions; but I mentioned my doubts whether some of the hallucinations may not sometimes depend upon the nerves of the organ itself.

" The second kind of delirium, error in judgment, is not quite so easily explained : you may observe, that thought depends upon memory, upon the power which we have of recalling the ideas of a great number of things which are not present; but memory depends upon an association of ideas, and it easily runs over that train of associations that it has been accustomed to. Thus, I come to a gentleman and tell him that he owes me five guineas; he hesitates, perhaps unwilling or willing to remember, and asks how do I owe you such a sum? I mention the persons that were present perhaps, the reason why he wanted it, \&c.; and, if he is an 
honest man, he is made to follow an association that he formerly made; and now he remembers the whole of the circumstances, and that it is justly demanded. It is in marking these relations, and judging of them as before, that the whole of our intellectual operations consist. So a man in his senses runs over the associations, takes up in course the several relations, applies the same judgment as before, and acts in consistency with his usual train; and this is what is called a man's having common sense. When, upon the contrary, a man does not recollect the relations that he had formerly established, we say that he is out of his senses. Thus also, if a man comes into a company, there are certain observances which custom has established, and his behaviour is suited according to the company he comes into ; - if it is a company of his usual merry companions, presently all their thoughts are of merriment, the laugh arises and the joke goes round; but if it is a company for business that he is to take a part in, he sits down seriously, speaks of the business in hand; and attends to what the other persons are engaged in; but if he were to treat the last company as he does the first, he would pass . for'a madman; thus too in the first company I supposed, he may speak of his amours or of his mistress; but if he finds the same. men among a number of ladies of honour and virtue, and he renews his discourse there, he would be thought a madman. So it is nothing but pursuing our ordinary train of associations, and marking their relations, and acting with the consistency that belongs to them, that constitutes.. what is called a sensible man; and delirium is where we do not follow our ordinary train, but, on the contrary, pursue one inconsistent with all our former established principles or notions. This delirium will accordingly depend upon some fault of memory chiefly; but this will be more considerable still, if not only the ordinary train of our associations is disturbed, but at the same time the imagination so far prevails as to obtrude upon the mind ideas which do not belong to the train which we are pursuing. Erroneous judgment accordingly consists chiefly in disjoining ideas which ought to be joined, or in joining those. which ought not to be joined. It may therefore be referred to. the heads of incoherence and inconsistence with regard to the proper connexion of ideas. But it is to be remarked, that there 
is, in this respect, in the marking of relations and in joining or separating ideas, a considerable difference among men in the state of health. The objects, indeed, about which men are conversant, are for the most part the same; and there is so much similarity in the operations of the human mind, that the same relations of ideas are marked, so that there is a sameness of judgment established among different men. We are accordingly most secure in ascertaining an error of judgment when there is a considerable deviation from the common sense of mankind; and such a deviation may then be considered as a disease. But still there is a certain latitude admitted in judgment. Men differ greatly from one another, without any of them being reckoned delirious. In accounting a man delirious, therefore, we must farther observe that he is deviating from his ordinary judgment, and from his usual train of thinking. This subject might be prosecuted with some curiosity : thus men speak of a weakness of mind without delirium; they explain errors in judgment by calling them mistakes and blunders ; they distinguish between the wrong-headed and those who are wrong in the head; they point out differences between whim, folly, and madness; \&c. These distinctions might have some curiosity and use; but they are matters of too much subtlety, and it is enough to leave them to common apprehension.

"The third manner in which delirium appears is in an erroneous will, that is, when emotions are not suited or proportioned to their objects; when they arise in a manner which is either uncommon to mankind in general or unusual to the person who is their present subject. With regard to this third kind, we may observe, that emotions and passions not proportioned to their objects, will readily follow when errors of judgment, the second state of delirium, exist. I have taken pains to show that the mind may be in different tones in this respect: that this tone may appear in gaiety and sadness, in irascibility and timidity, and that the causes of madness more frequently depend upon this tone of mind than even upon the errors of sense or judgment ; and it is sufficiently. evident, that irregular emotions or passions seldom occur, except when a particular tone of mind is joined to the false imagination and to the error in judgment. Thus, Dr. Sydenham observes, vor. I. 
that a paroxysm of the gout may often be called a paroxysmus irascendi; by which he insinuates that the body is so altered as to dispose the mind to irascibility, and that then the same objects are causes of anger or fear which would not have been so at any other time.

" This will go far in ascertaining the several species of delirium. We now go on to consider what may be its causes. Gaubius refers them to different states of the organization of the brain, in $\$ 737$, where he observes that they may be reduced to two heads, viz. 1. Those which weaken the sentient power by debilitating the tenor of the brain and the origin of the nerves; 2. Those which accumulate the blood in the vessels of the encephalon; 3 . Those which diminish the mobility of the sentient fibres; 4. Those which, by irritation, excite irregular, inordinate, and impetuous motions in the sensorium commune; 5. Those which destroy the regular motion of the medullary part by violence on the brain. I say these causes may be reduced to three heads, 1 . What may be strictly called a different state of organization of the brain (comprehending the first, third, and fifth head of Gaubius); 2. The different state of circulation of the blood (Gaubius's second); and, 3. The difference of stimulus (Gaubius's fourth head.).

" But I proceed to a more fundamental consideration of the state of delirium. The brain certainly is in different states in persons awake and in those who are asleep,-that is, in different states of thinking or of not thinking at all (CXXXIII.). I know, indeed, that this last proposition will be disputed by many who are fond of maintaining that the soul always thinks when we sleep. I refuse this; and allege, that the mind is absolutely at rest when the body is sound asleep : 'we perceive different degrees of mental operations in sleep, which are always proportioned to the degree of sleep; but in the most sound and perfect sleep we have no perception of the operations of the mind at all. But it is not necessary to enter into this discussion; it is enough to our purpose that the mind, in sleep, does not think with regularity, that it does not pursue its ordinary train, that it does not think to the purposes of life, that it approaches to delirium; and that, therefore, there is a considerable difference in the thinking of the mind when the body is 
awake and when it is asleep. The states of the brain corresponding to this difference, I have termed the states of excitement and collapse. I say, that in sleep the mind does not perceive its usual or ordinary train of thinking which is necessary to sound judgment. We have, in dreaming, false imagination, incoherence, and inconsistency ; but these are all the marks of delirium. The passage from waking to sleep also, or from sleeping to waking, is usually gradual. . Thus, when I find my friend just now asleep, his ears are open, and I get his eyes open; some time elapses before I can make him recollect who I am, or that I have made an appointment with him : he does not know me, or the purpose of our meeting together; but this is a matter of a few moments only, and is discussed by establishing the total excitement of the brain; and here there is no organic affection, but only one portion of the brain is in a state of collapse, while another portion of it is excited. This I cannot illustrate better than by the following very elegant account of the phenomena of sleep from Haller's Prim. Lin. $\S 580$. - Nocte redeunte sensim torpor percipitur in musculis longis, ineptitudo ad cogitationes severiores, amor quietis in animo et corpore. Tunc peculiariter vires corpus erectum tenentes laborant, et oculi nolenti clauduntur, et maxilla inferior pendet, et oscitationis necessitas ingruit, et caput antrorsum nutat, et objectorum externorum actiones minus nos adficiunt, et denique turbantur idex, et cogitationes, et delirium succedit, a quo in somnum non satis notus transitus est, quod tamen somnum semper præcedit.' $-V$ arious theories of dreaming have been given; some have supposed that it depends upon circumstances of our own bodies; others, that it is owing to the influence of spirits without us. With regard to the possibility of this last supposition I will not determine, but certainly, for the most part, it is owing to the state of our bodies; and we say with Haller, that it is in consequence of some stimulus that is more powerful in sleep, while from the ordinary causes of sleep, the collapse is very nearly entire, but the force of the stimulus can keep up the excitement in some portion of the brain, which runs more or less into some train of thought ; and so it is a dream, a delirium, and no other explanation is necessary to say why that may be in different de- 
grees-from the placid, quiet dream which is hardly recollected, to one that raises considerable emotions, that awakes us to certain functions, and gives occasion to somnambulism.

"We observe, accordingly, thát a state of delirium generally occurs between the states of sleeping and waking, one part of the brain being excited while another part is under collapse. Of this kind of delirium there will be two cases; one, while in sleep, the greater part of the brain being in a state of collapse, stimuli are applied, which excite only one part of the brain, in which intermediate state delirium will occur; the other, where, the person being awake, the powers producing sleep have acted and have affected certain parts of the brain only, from which cause the same unequal state of delirium must equally arise.

"No state can be more like common delirium than those conditions of dreaming; and we should consider both as always of the same nature, if dreaming had not become so common and familiar to us. See how Haller explains dreaming (Prim. Lin: $\S 578$,); and the same views in Gaubius ( $\$ 762$,), who also says that one part of the brain may be excited while another is under a collapse.

- $"$ This accordingly is the foundation of dreaming, and therefore of delirium also, according to a general principle-the want of an equal communication between the several parts of the brain ; and this may arise either from organic affections, or purely from some transitory difference in the states of excitement and collapse, connected with the states of sleep and waking. This opens to us a particular view; it does not leave the whole causes of delirium resting, as Gaubius has assigned, upon a more considerable change in the organization of the brain; and it shows that other powers occurring in diseases may produce collapse, and thereby lay the foundation of delirium. In some of the most violent instances, indeed, mania can be discussed, and the mind restored to its healthy state, almost as easily as sleep and dreaming are discussed and the waking state restored. Whoever has considered this subject will see its application in distinguishing two cases of delirium as depending upon the last mentioned causes, viz. that which arises from an uncommon degree of stimulus applied to an unequal portion of the brain, where the whole cannot be equally excited; and that 
in which the stimulus being as usual, delirium occurs in consequence of collapse taking place in a certain part. This is the distinction between the delivium ferox and delirium mite, which pathologists and practitioners have so constantly noticed, and which, in the case of fever, will have a very considerable influence upon our prognostic, as well as upon the conduct of our practice."

CXLIII. With regard to the connexions established in the system (CXX. CXXI.), it is to be observed, as of great consequence in pathology, that certain parts of the body which have a common function and constitution- "I should rather say a common function and peculiar constitution"-have thereby a peculiar relation to the brain, so as to be more liable to be affected by the different states of it, and, in their turn, by the difference of their condition to affect the brain. Such are, especially, the organs of voluntary motion; the alimentary canal, and especially the stomach ; the circulatory system, and particularly the extremities of the vessels on the surface of the body; the uterine and genital system in females; and some others to be mentioned in the Pathology. - "With regard to these, remember that the facts are certain, and therefore an object of our attention and curiosity; the reasons are doubtful: I do not pretend to explain them either certainly or completely, but only to make some approach.

"6 The 'organs of voluntary motion' have this in common, that their actions are in consequence of an action of the brain, which occasions, increases, and excites them, while the vital functions need only that general energy which is: constantly operating in the system. In consequence of this exertion of the animal power, the organs of voluntary motion are subject to lassitude and debility, and require a state of rest and of sleep. Hence also, various irritations of the brain, exciting its action, are especially exerted in convulsive motions of the organs of voluntary motion: hence palsy takes place in the same organs, and many of their diseases are explained by this their peculiar connexion with the brain.

"The 'alimentary canal, and especially the stomach,' are commonly affected by the different states of the brain; and the stomach again has more effect upon the brain than any other. 
part of the system. We will not pretend to explain completely what is the foundation of this singular connexion; but we can observe this, that no internal part of the system is so remarkably an organ of sensation as the stomach, that it not only expresses its own different conditions with regard to fulness, emptiness, the acrimony of the matter which it contains, \&c. but the general state of the system also, as more full or empty. The stomach must, therefore, have a more particular power in affecting the brain, as it is from the organs of sense that the beginning of all the motions of the system arises. When treating of the impressions of sensation as the causes of waking (CXXIX.), I mentioned analogous impressions, and the chief circumstance was their operation upon the stomach, which is sometimes with, and sometimes without sensation. This action is not so constant as that of the heart and organs of respiration, but differs in this, that it is subject to a very great variety ; for the difference between the action of a full stomach, and one almost empty, is very considerable; and it must be exposed to all the variety of intermediate states between its strongest and weakest action. For this it depends upon the energy of the brain, which indeed constantly operates, but must admit of some inequality, and must more especially affect this organ."

"We must not omit taking notice of one very general case of very great influence in almost the whole of the doctrines of the materia medica, as this particular sympathy is concerned in the operation of the most part of medicines, and explains the operation of many which is otherwise difficult to be understood.

6 6 This is the operation of medicines upon the stomach, from which motions are often propagated to almost every distant part of the human body, and peculiar effects produced in those parts, whilst the medicine itself is only in contact with the stomach.

"6 The stomach is the part by which the most part of substances introduced into the interior parts of the body generally pass; and it is endued with a peculiar sensibility, which renders it ready to be affected by every substance entering into it that is active with respect to the human body. Every thing, therefore, of this kind introduced into the stomach, operates al most always there, and for the most part only there. It is now, 
however, well known to physicians, that the most considerable instance of the sympathy mentioned above, is afforded by the stomach, so connected with almost every other part of the system, that motions excited there are communicated to almost every other part of the body, and produce peculiar effects in those parts, however distant from the stomach itself. This indeed is very well known; but that the effects of many medicines which appear in other parts of the body are entirely owing to an action upon the stomach, and that the most part of medicines acting upon the system act immediately upon the stomach only, is what has not been understood till very lately, and does not seem even yet to be very generally and fully perceived by the writers on the materia medica. It will, therefore, be proper here to say in what manner this doctrine may be established.

"First: That medicines shewing considerable powers with respect to the whole system, act especially or only on the stomach, will appear from all those cases in which the effects appear soon after the substance has been taken into the stomach, and before they can be supposed to have gone further into the body, or to have reached the mass of blood. Thus, Sir John Pringle, from the sudden operation of the Peruvian bark in preventing the paroxysms of intermittent fevers, properly concludes, that it cannot be by its antiseptic powers with respect to the fluids, but by a certain operation immediately upon the stomach. (See Diseases of the Army, Appendix, p. xxv.)

"Secondly: As medicines are commonly in the first place applied to the stomach, so all those of volatile, active, and penetrating parts, must immediately and especially act upon the stomach; and from this consideration, as well as from the suddenness of their effects which commonly appear, we may conclude their action to be upon the stomach only. Accordingly, I conclude that the action of the volatile alkali, and some other saline substances, is upon the stomach alone, and very rarely by any antiseptic powers with respect to the fluids,

"Thirdly: Though medicines do not to the taste or smell discover any volatile or active parts, yet if their effects depend upon the change which they produce in the state of the nervous power, it is hardly to be doubted that they operate only 
upon the sensible and irritable parts of the stomach. This I conceive to be the case of opiates and of most other narcotic powers, whose substance is known to remain in the stomach long after they have discovered their effects in the most distant parts of. the system.

"Fourthly: If there are medicines supposed to act only when they come in contact with the parts they are supposed to act upon, and if a certain quantity is necessary to be applied to these parts; and, further, if such medicines are either thrown into the stomach in small quantity, or are of a nature to be slowly dissolved there, so that they cannot be supposed in sufficient quantity to come in contact with the parts they are destined to act upon, whilst however their effects appear in these parts, it must, I think, be concluded, that these effects depend entirely upon the operation of these medicines upon the stomach. This, if I mistake not, applies to the case of most vegetable astringents, and perhaps to the fossil also, whose effects, and especially their sudden effects, upon distant parts of the system, can only be accounted for by their operation upon the stomach.

"Fifthly: Another circumstance leading us to suppose that medicines act immediately upon the stomach, and by their operation there, affect the rest of the system, is the consideration of all these cases where they affect the system very generally, while, at the same time, they act both suddenly and in small quantity, and therefore in circumstances which cannot allow us to suppose that they are conveyed in substance to the parts in which their effects appear. Thus, as has been above observed, medicines which act very generally upon the nervous system, or upon the particular parts of it remote from the stomach, cannot be supposed to be transferred in substance to the whole, or even to the particular parts of that system, and therefore must necessarily be supposed to act in the stomach only. Not only, however, with respect to the nervous system, but also with respect to the sanguiferous, any very general effects produced there, as, for example, a sweating excited universally over the whole body, can be produced no otherwise by internal medicines excepting by such as act on the stomach, and from thence communicate a stimulus to the heart and arteries. In many cases of increased evacuations, it is indeed pretty evident that the 
medicines exciting the evacuations are actually conveyed and applied to the secretories or excretories of the parts concerned; but this cannot possibly be supposed with regard to sweating; not only from the small quantity of medicine employed, but perhaps also from the nature of the excretion, which is certainly not depending upon glands and their excretories.

"Sixthly: Another circumstance inducing us to suppose medicines to act only on the stomach, is, that of their being capable of being changed by the assimilating powers of the stomach and intestines; for such medicines, if they act at all, must act immediately upon their entering into the stomach, or before they are are changed by digestion.

"6 It is true, with respect to vegetables, and also certain animal substances, it is often a certain portion of them only that can be subjected to our digestive powers, while the medicinal part of the same is hardly affected; and therefore it may be alleged, that their operation on the interior parts is not prevented by the powers of digestion. This indeed does certainly sometimes happen; but still, as digestion breaks down very entirely the texture of vegetables, and evolves the several parts of them more entirely than they were in the entire vegetable, it thereby gives them an opportunity of acting immediately upon the stomach, and even may thereby prevent their activity from reaching: beyond this organ.

"Seventhly: Another circumstance, which confines the operation of many medicines to the stomach, is their suffering a change there, if not by digestion at least by mixture.

"It appears to me very clear, that in all animals who take in a quantity of vegetable aliments, and therefore in man, there is an acid, and commonly in considerable quantity, very constant, ly present in the stomach. It is therefore probable, that all alkaline substances are more or less neutralized there; and that consequently, if they act at all as naked alkalines, they can act only upon the stomach before they are neutralized. It appears; however, that alkaline substances frequently prove powerful medicines with respect to the remote parts of the system; and I think it must be concluded that their effects must be imputed to their being changed into neutral salts in the stomach, and operating in the other parts of the system as neutrals only; or 
perhaps their operation may be that of their changing the nature of our fluids, by their abstracting a considerable portion of the acid which should have entered into the composition of these fluids." $-M . M$.

"I have added, the circulatory system, and particularly the extremities of the vessels on the surface of the body. Like the stomach, the action of the heart and arteries, and organs of respiration, depends upon the energy of the brain, but their actions are performed with no very great variety; they have a certain latitude, but it does not extend wide; and while the action of the brain very often shows its effects in the organs of voluntary motion, and in the alimentary canal, we know that the circulating system is much less regularly affected; only one set of causes, viz. those which produce fever, are remarkable for doing so. The organs of voluntary motion may be affected with vigour or debility, with alacrity or sluggishness, while the circulating system is not subject to the same states. If it is subject to them, however, we find them especially in the extremities of the vessels, every where, but more especially in those on the surface of the body. This has not been noticed formerly. I consider the arteries as elastic chords constantly in a state of tension between their two extremities; it is obvious that this tension is given by the fluids impelled by the concurrent action of the heart and larger vessels; this action is pretty steady, but not without some variety, at one time stronger and at another weaker; and the effects of the weaker action will be perceived more or less according to the distance of the arteries from the heart; it will be most considerable, therefore, in their extremities. There is a very frequent variety in the state of the extreme arteries, they are frequently now fuller, now emptier, sometimes greatly stretched out, at other times contracted in their length and diameter. Now the varied action of the heart, for some particular reasons, varies the state of these arteries more considerably on the surface of the body than in other parts. Every increased action of the heart and arteries operates more there, and produces sweat ten times for once that it increases any internal secretion. The extreme arteries of the surface are also connected with an organ of sense, and affected 
by impressions made upon that organ; and one of these, which surely has more effect than all the rest, is the change of temperature, which powerfully acts upon all our organs of sense, but to which the surface of the body is especially exposed. And it is remarkable that impressions made upon one part of the surface are very readily communicated over the whole. We are made to shiver by any part of the body touching a cold body, and heat is in the same manner communicated over the whole.

"6 ' The uterine and genital system in females.' Here I must adhere to the fact, without assigning the cause or particular purpose.

"6 These communications of motion have been called, in general, consent or sympathy. I need not tell you that they have been very much spoken of, as you will see in Dr. Hoffmann's Works, in the Tract.Med.de Sympathia of Dr. Rega, and in Dr. Whytt's Essay on Nervous Diseases. Every term which is like establishing a power exerted between bodies at a distance without-contact and motion, and therefore a term for an occult quality, is properly rejected: I would wish, therefore, that the terms sympathy and consent were rejected from our system. Our modern physiologists have sought for a mechanical communication by deriving the nerves of the parts from the same common fasciculus, or supposing that they have an origin in the brain near to one another, but this is to be rejected."

CXLIV. The communications of motion between the several parts of the nervous system which have been mentioned as instances of a particular sympathy between these parts, are very seldom to be explained by any contiguity or contact, either in the origin or course of the nerves of the communicating parts. - It seems to have been the intention of the economy, that the nerves tied up in the same common membrane should have no lateral communication, but that the nervous power should proceed along one single fibre to the brain, in order to produce the sensation, so that we might be able to refer the sensation to the place of impression, as otherwise there could be no distinct sensation; nor when the will determines the action of a single muscle, should we have any single separate action, but the action of all the separate muscles would follow, to which these 
nervous fibrils, so wrapped up, proceed. But such an accident seldom takes place; and there are many instances of nerves proceeding from the same origin without any such communication."-And more commonly they may be explained, by supposing the action of the impression to be general with respect to the brain; and that the affection of particular parts depends upon the causes of determination (CXLII. CXLIII.).- " I must illustrate this by an example. The sight of grateful food occasions the flow of saliva in a hungry person, and the appetite can be renewed by recollecting what was formerly grateful; and we know that when the appetite is very sharp, a flow of saliva from the mouth takes place. Now this is no instance of consent between the retina and the salivary glands; the affection of these glands is merely an expression of the appetite, and depends on the general causes of communication; for merely talking of very grateful food will make the mouth water."

When the actions of several parts, together or successively, are necessary to produce one effect, these concur, though the stimulus exciting the action of the brain is applied to one single part only; and commonly no other cause of communication can be assigned but the several motions being necessary to the execution of the volition, propensity, \&c. arising from the stimulus.- "Thus, by the laws of the economy, which we cannot further explain, certain impressions produce certain volitions and propensities, which, again, produce the action of certain muscles. I cannot say why my will to lift a weight with my hand determines the motion of the arm in a certain manner; but the motion is such that I cannot direct or even counteract it ; according to the degree of effort, it will produce contractions of my fingers, my wrists, and my shoulders, nay it will bring the whole body into concurrence. If my finger is pricked, it will have the same effect; but it is not that there is a necessary connexion between the nerve which was pricked, and the muscles in my back and trunk, but that the painful sensation gives me the volition of pushing away, which is necessarily attended by all these different motions of muscles. Now, in explaining vomiting, it is said that the stimulus applied to the stomach. touches nerves which have a sympathy with the diaphragm; 
but if the stimulus was not determined to produce the propensity of vomiting, this would not follow; so it may take place in consequence perhaps of an application of that stimulus to any one nerve of the system.

"I do maintain that you will find no other explanation of what may be strictly called sympathy and consent, but the accessary concurrence of certain actions to a volition or propensity, and the concurrence of several motions of the system produced in consequence of these volitions and propensities. Whether the connexion between the nerves of the teeth and those of the ear (see p. 44.), is an exception to what we have here said of lateral communications, I do not positively say. There is a communication between parts whose membranes are contiguous, such as the neck of the bladder and orifice of the urethra, between the intestines and abdominal muscles, the ear and glottis ; but there the vicinity of parts is to be taken into account. You will, I think, be able to distribute such instances in the manner I have pointed out, and be relieved from much confusion and appearance of mystery, which this language of sympathy has introduced into our system of physic."

CXLV. These are the chief facts and laws relative to the nervous system. The whole might perhaps be illustrated, and more exactly ascertained by a more particular inquiry into the nature of the nervous fluid; but we are not so confident in our opinion on this subject, nor of the application it will admit of, as to deliver it here.- I I do not say that this is the theory of the nervous system; I say that these are the facts and laws; and wherever, in some instances, you may think I have approached to theory, I would wish you to receive every particular I have delivered with great diffidence, till you are persuaded that the conclusion is established as a matter of fact. If you do so, there will be no danger of misapplication; you will be constantly enlarging the number of facts, and be much more fit for the application of them." 


\section{SECT. III.}

OF THE CIRCULATION OF THE BLOOD.

CXLVI. The circulation of the blood, by CXXIX., seems necessary to the excitement of the brain; and for this, as well as other reasons, it is, next after the function of the brain itself, the most important of the animal economy.

On this subject we shall consider, 1 . The course of the blood. 2. The powers by which the blood is moved. 3. The laws and general circumstances of the circulation. 4. Respiration as necessary to it.

On all these subjects we suppose the anatomy of the parts to be known.

CHAP. I. OF THE COURSE OF THE BLOOD.

CXLVII. Wounds and hæmorrhagies shew that, in living bodies, the blood is in constant motion, and flowing into one part from many others.

CXLVIII. In man, and other analogous animals which have once breathed for some time, the course of the blood is very constantly in the following manner.

From the left ventricle of the heart, the blood passes into the trunk of the aorta, and successively into the following vessels and cavities, viz. The branches of the aorta, the branches of the vena cava, the trunk of the same, the right auricle of the heart, the right ventricle of the same, the pulmonary artery, the pulmonary veins, the left auricle of the heart; from which last it passes into the left ventricle of the heart to return again into the same course as before.

From all this, it appears that in the arteries the usual course of the blood is from the heart towards the extreme branches of these; and that in the veins the course is in the contrary direction, from the extreme branches towards the heart.

CXLIX. The course of the blood through the cavities of the heart, as above described, is not in a continued stream, but alternately interrupted and free during the contraction and di- 
latation of these cavities, which alternately happen. Thus, while the left ventricle of the heart is in a state of contraction, the blood passes out of it into the aorta; but at the same time no blood passes into it from the left auricle, which is then dilated and filled by the blood flowing into it from the pulmonary vein. It is only when the ventricle is emptied by contraction, and consequently relaxed, that the blood passes into it from the auricle, urged by the contractions of the auricle and adjoining sinus venosus, which succeed immediately to that of the ventricle. During this contraction of the auricle and filling of the ventricle, no blood passes from the ventricle into the aorta, nor till a contraction of the ventricle succeeds in consequence of its being filled. The same circumstances take place with regard to the right ventricle and auricle of the heart, and precisely at the same times; for it appears, that the two ventricles of the heart are contracted and relaxed at the same time; and, in like manner, the two auricles.

CL. That the course of the blood, as described (CXLVIII. and CXLIX.), is its usual and constant course, appears from the inspection of the heart in living animals; from the situation of the valves of the heart; from the situation of the valves at the orifices of the aorta and pulmonary artery; from the situation of the valves of the veins; from the effects of ligatures made upon the arteries and veins; from the effects of hæmorrhagies of the veins; from observations with the microscope; and, lastly, from experiments of infusion and transfusion in living, and of injection in dead animals.

CLI. This course of the blood is, however, in some parts, and upon some occasions, a little changed. 1. In the penis, and some other parts, the blood does not pass from the extreme arteries immediately into continuous veins, but is poured out into an intermediate cellular texture, from which it is afterwards received by the extreme veins. 2. In the small branches of the aorta, the blood does not move constantly in a direction from the heart towards the extremities, but, in certain portions of them, is sometimes retrograde to that course. In this, it is favoured by the anastomoses frequent between the small vessels, which, at the same time, prevent this deviation from being considerable or durable, while the action of the heart continues. 
CLII. In the course of the venous blond there is also some variety. 1. In the small veins, the blood is liable to have a motion retrograde to its usual direction (CXLVIII.), as in the arteries (CLI. 2.). 2. The blood returning to the heart from most parts of the body, passes successively from smaller into larger veins, by a series of pretty regularly increasing vessels, till they form the vena cava entering the heart. But this is varied in the abdomen, where the veins carrying the blood returning from every viscus included in that cavity, except the kidneys and genitals, unite in forming the vena portarum, whereby they undergo a peculiar distribution. 3 . The veins returning the blood from the extreme arteries in the brain do not earry it to the heart by a series of regularly increasing vessels, but by the interposition of sinuses, into which the small veins issuing from the cortical part of the brain immediately pour their blood. 4. As the course of the blood through the vessels of the lungs is not at all times equally free, and, particularly, is considerably interrupted at the end of expiration, so at the same time it is also interrupted at entering the right ventricle of the heart; and this often occasions some regurgitation, or retrograde motion, in both the ascending and descending cava.

- CLIII. The whole of the fluids carried in the aorta to its extreme branches, are not returned again by the continuous veins to the heart, as, by secretory vessels, a part of thein is constantly carried out of the course above described. Some of these secreted fluids are thrown entirely out of the body, and others are poured into certain cavities; for various purposes of the economy; and some of these are again returned into the course of the circulation. Of these last, there is a peculiar fluid which, from the extremities of the arteries, is poured out in a liquid form, or exhaled in that of vapour, into, perhaps, every cavity and vacuity of the body: This, after having served the purpose of the effusion, seems intended to be regularly returned again into the course of the circulation; and, accordingly, in all the several cavities into which it had been effused, there are absorbents which again take it in. These do not carry the fluid immediately into the veins, but uniting form the vessels called LYMPHATICS, which, in their course, pass through 
the conglobate glands, and at length terminate either in the receptacle of the chyle, in the thoracic duct, or in the left subclavian vein; and, in this way, return the absorbed fluid into the course of the ordinary circulation.

CLIV. There are absorbent vessels, not only in the several cavities, but also on the external surface of the body, by which many extraneous matters may be introduced into it.

CLV. Most of the fluids secreted from the circulating mass, and poured into cavities, may be absorbed from these, and returned again by the lymphatics, as in CLIII., to the course of the circulation. But the same secreted fluids seem often to be returned also into the course of the circulation, by a regurgitation, or retrograde motion, in the excretory and secretory vessels.

CHAP. II.-OF THE POWERS MOVING-THE BLOOD.

CLVI. The chief power by which the blood is moved, and the circulation carried on, is the action of the heart, or its repeated contractions, occurring, as mentioned (CXLIX.). For this purpose the heart is a muscular part, the action of which may depend upon an inherent power stimulated by the dilatation of its cavities; but this inherent power requires the constant support of the nervous: and animal powers, and is often actuated by these.

CLVII. The contraction and relaxation of the heart, or, as these are called, its systole and diastole, are: necessarily alternate by the general law (CVIII.) affecting all muscles; and by the stimulus from the influx of venous blood being alternately applied and removed.

CLVIII. If we may be allowed to estimate the vigour of muscles by the number of their fibres, we must suppose the force of the heart to be very considerable; but it is very difficult to obtain any exact estimate of its absolute force. It is perhaps the relative force only that we are concerned to know.

CLIX. Do the arteries, by their contraction, contribute to promote the motion of the blood begun by the heart? They cannot do it by the elasticity of the simple solid in their structure, and can do it only by their being indued with a muscular. 
power, whereby they may, in their contraction, communicate to the moving blood more than was lost of the force of the heart in dilating them. That they are indued with a muscular contractility (LXXXII.) is probable, from the appearance of the muscular fibres in their structure; from their irritability appearing in the experiments of Verschuir ; from their becoming flaccid on tying the nerves belonging to them; from the motion of the blood being supported, when the force of the heart is considerably weakened; from the motion of the blood becoming languid, when the action of the arteries is destroyed; from the velocity of the blood in the extreme arteries being greater than was to be expected from the velocity of the blood issuing out of the heart, exposed to so many causes of retardation as constantly occur; and, lastly, from the velocity and impetus of the blood in different parts of the body, and at different times, being unequal, while the action of the heart continues the same.

It is probable, that the muscular fibres of the arteries become more irritable as the arteries are more distant from the heart.

CLX. The tone and action of the arteries, as a muscular part, may be increased by stimuli immediately applied to them, or by the increased force of the nervous and animal powers with regard to them; and they may be diminished by sedative powers applied, or by weakening the nervous and animal powers.

CLXI. There does not appear to be any oscillatory motion in the extreme arteries independent of the action of the heart.

CLXII. There does not appear to be any operation of capillary attraction in the extreme arteries; nor does there seem to be any occasion for such a power in any part of the arterial' system.

CLXIII. The power of derivation (Vis Derivationis Ill. Halleri, $\S 174$. ) in the sanguiferous system, seems to be no other than that which arises from the fulness of contractile vessels.

CLXIV. The motion of the blood in the arteries of any particular part, is promoted by the action of adjoining muscles.

CLXV. The blood in the vena cava and its branches, is moved by the action of the heart and of the arteries. These powers are assisted by the action of muscles, which, in their contraction, press the veins lying between their several fibres, and also, by the swelling of their whole mass, press the adjoin- 
ing veins. These veins are commonly provided with valves, which determine the effect of all pressure upon them, to be the motion of the blood towards the heart:

The great trunks, both of the vena cava and pulmonary vein, are provided with muscular fibres, and manifestly indued with muscular contractility.

CLXVI, In the absorbent vessels, the fluids are probably taken in by a capillary attraction.

CLXVII. In the lymphatic vessels, provided with numerous valves, which necessarily determine the motion of the contained fluid to be towards the heart, the fluid is moved by the pressure of the neighbouring muscles and arteries. But, further, as the lymphatics are remarkably irritable, it is probable that the fluid in them is moved by a peristaltic motion begun by the action of their absorbent extremities.

CLXVIII. The motion of the blood through the vessels of the lungs depends upon respiration, to be considered hereafter.

CHAP III.-OF THE LAWS OF THE CIRCULATION.

CLXIX. The velocity of the blood passing out of the left ventricle of the heart into the aorta, may be estimated from knowing the quantity of blood passing out at each systole, the area of the orifice of the aorta, and the time occupied by the systole; but none of these circumstances are exactly ascertained.

CLXX: As the blood moves onwards through the arteries, the velocity (CLXIX.) suffers a considerable retardation from several causes. 1. From the capacity of the arteries being enlarged as they are more distant from the heart. 2. From the frequent flexures of the arteries. 3. From the angles which the branches make with the trunks from which they arise. 4. From anastomoses. 5. From the viscidity of the blood. 6. From the friction of adhesion. 7. From the weight and rigidity of the parts surrounding the arteries.

CLXXI. The velocity (CLXIX.) and the causes of retardation (CLXX.) being given, the velocity of the blood in the arteries will be as the frequency of the systole of the heart. 
CLXXII. 'The frequency of the systole of the heart will be more or less, 1. As the blood in the veins is more or less quickly returned to either ventricle of the heart. 2. As the ventricles of the heart are more or less entirely evacuated at each systole. 3. As the muscular fibres of the heart are more or less moveable. 4. As the action of the nervous and animal powers are more or less increased with respect to the heart.

CLXXIII. As the arteries of a healthy body are always full, the blood thrown out of the ventricles into the arteries during the systole of the heart, can only find a place there by pushing on the blood with the velocity (CLXIX.), or by dilating the arteries; but as the resistances (CLXX.) prevent the blood from flowing with the velocity (CLXIX.), the blood thrown out of the heart must, in some measure, dilate the arteries, and thereby form what is called the PULSE.

CLXXIV. It appears, that, in the arteries, to a certain length, the blood moves faster during the systole than during the diastole of the heart; but, as the resistances and causes of retardation become greater in every portion of the arteries as it is more distant from the heart, so the acceleration of the blood during the systole of the heart 'must be greater in any portion of the arteries nearer the heart than in the next adjoining that is more distant ; and, so far as this takes place, a dilatation of the arteries will happen, even from a small quantity of blood thrown out of the ventricles.

CLXXV. As the resistances to the blood's motion in the blood-vessels increase with the distance from the heart, there may be a part of the sanguiferous system in which the motion of the blood will not be accelerated during the systole of the heart, and in which, therefore, no pulse can be discerned. This happens in the extreme branches of the aorta; and no pulse is ever observed in the extreme branches of the vena cava.

CLXXVI. The velocity and impetus of the blood, in the whole system of blood-vessels, will always be as the action of the heart and arteries taken together.

: CLXXVII. The velocity and impetus of the blood, in : any particular part of the system, will be, 1. As the part is more or less distant from the heart. 2: As the circumstances 
(CLXX.) take place more or less in the part. 3. As the gravity of the blood concurs with, or opposes its motion in the part. 4. As causes increasing or diminishing the action of the arteries of the part are applied or removed.

CLXXVIII. The quantity of blood distributed to any particular part of the sanguiferous system, will be greater or less according to the velocity and impetus of the blood in the part, by CLXXVII. and according to the resistances in other parts being increased or diminished by constriction, compression, ligature, position, relaxation, or aperture.

CLXXIX. The flexibility and contractility of the bloodvessels render the effects of all increase or diminution of resistance in any particular part most considerable in the nearest, and very little so in the more remote vessels of the system. By. this we are to judge of the celebrated doctrines of derivation and revulsion.

CLXXX. The quantity of blood distributed to the different parts of the system, is in different proportion at different periods of life.

1. The capacity and force of the heart, in proportion to the system of vessels, is greater at the beginning of life than at any after period. Till the body arrives at its full growth; the capacity of the vessels increases in greater proportion than that of the heart; but, from that period, the capacity of the vessels is constantly diminishing, while that of the heart suffers little change.

2. A greater quantity of blood is contained in the arteries, in proportion to that which is contained in the veins, at the beginning of life than at any after period. From the time that the body has arrived at its full growth, the quantity of blood contained in the veins, in proportion to that which is contained in the arteries, is constantly increasing.- ${ }^{6}$. This difference of the quantities of blood in the arteries and veins manifestly occurs in the ordinary progress of life; but it is also probable, that in some persons the same difference, to a certain degrec, takes place through the whole course of life, and gives a constant and considerable difference in the temperaments of men, as I shall mention more fully hereafter." $-M$. $M$.

3. The vessels of the head receive a greater quantity of blood 
in proportion to the rest of the system, at the beginning of life than at any after period.- "This certainly happens from the vessels of the head being, in respect of capacity and density, suited to that end; and consequently, in the first part of life, the blood is determined in a proportionally greater quantity into the vessels of the head than into other parts of the system; and it is sufficiently probable, that this proportion is greater as the animal is nearer to its origin, and continues greater till the body attains its full growth; after which, however, it continually decreases as the animal advances to that period when it may be supposed to cease." $-M$. M.

4. Any general increase of the action of the heart and arteries, determines the blood more copiously to the extreme arteries on the surface of the body than to those of the internal parts.

5. The equilibrium of the sanguiferous system, with regard to the distribution of the blood, may be changed by various causes (CLXXVII. CLXXVIII.); and these causes continuing to operate for some time, induce a habit which renders the changed distribution necessary to the health of the system. - When the body has arrived at its full growth, we very generally find a symmetry and exact proportion established in the size and bulk of the several parts which fall under our observation; and we may then suppose the distribution of the blood to be suited exactly to that proportion. This, indeed, takes place with great uniformity in the most part of men; but I still deem it possible that a disproportioned capacity in certain parts may occur in certain men, and subsist in them through the whole of life. Accordingly, it has been commonly observed, that men of large heads, and large in proportion to the length of their bodies, are more liable to a plethoric state in the vessels of the head, and to the diseases depending upon it. I have also remarked in several instances, that men having their feet and hands shorter than in the usual proportion to the rest of the body, were more liable to a plethoric state of the lungs.

- 66 This leads me to observe, that of the proportions of the capacities of the several parts of the body, influencing the distribution of the blood, one of the most considerable is, the difference of the capacities of the vessels of the lungs, and of those 
of the system of the aorta. It is especially discovered by the 'size of the thorax with respect to the other parts of the body; and this may be considered as occasioning a considerable difference in the constitutions of men. What effects it has in disposing to certain diseases is well known to physicians.

"Upon this subject of the distribution of the blood, it is particularly to be taken notice of, that there is a certain balance between the force of the heart and the resistance of the extreme vessels by which the perspiration is thrown out. It is probable that upon this the state of that excretion in different persons very much depends; and which perhaps may be illustrated by this, that the resistance of the extreme vessels seems, in some cases, to be so great as to diminish the perspiration, and in consequence the appetite. Accordingly, the circumstance of men of considerable bulk and tolerably full habit, having less appetite, and taking in less food, than others of the same bulk usually do, in my opinion, may be ascribed to the weakness of the heart with respect to the extreme vessels; and, on the other hand, we find men of a moderate size, and of a lean habit, take in very largely of food; which, I think, must be imputed to the force of the heart being in them great with respect to the resistance of the extreme vessels.

"With regard to the balance between the heart and extreme vessels, we cannot fail to remark, that although the interruption or diminution of perspiration is often owing to cold constricting the vessels, and increasing their resistance to the action of the heart, yet it is at the same time evident, that it frequently may be owing to the weakness of the heart not pushing the blood with due force towards the surface of the body, that this last is rendered more liable to be affected by cold. This disposition to be affected by cold may take place not only occasionally, as it may in most persons, but seemingly it subsists in some through a great part of life, and therefore may be considered as distinguishing the different conditions and temperaments of men."-M. M.

6. The lymphatic system is fuller in young persons than in old. 


\section{CIIAP. IV.-OF RESPIRATION.}

CLXXXI.-Respiration consists of the motion of inspiration, or the admission of air into the lungs, and of expiration, or the expulsion of air from the same, alternately happening.

- CLXXXII. Respiration takes place in man, and other analogous animals, soon after the infant is taken from the uterus of the mother, and is exposed to the air. After it has taken place for a little time, it is ever after necessary to the continuance of life, as it is absolutely necessary to the continuance of the circulation of the blood.

CLXXXIII.-The lungs are a hollow spongy mass, capable of confining air, and readily dilatable by it. By the windpipe they are open to the atmosphere; and they are so situated in the thorax that the air must enter into them, if the cavities of the thorax, in which they are placed, are enlarged. For, as there is no air in these cavities, and the external air cannot enter into them, the enlargement of the thorax must form a vacuum around the lungs, which the external heavy and elastic air will supply by entering into and dilating the lungs, while these do not allow the air to pass through them into the cavities of the thorax.

CLXXXIV. Inspiration therefore depends upon the enlargement of the capacity of the thorax; and this is performed chiefly by the contraction of the diaphragm. This, in its relaxed state, is suspended by the mediastinum, and its middle tendinous part is raised high in the thorax; wherefore, as this middle part, by the contraction of the muscular, is moved downwards, the thorax is thereby considerably enlarged.

CLXXXV. The capacity of the thorax is also enlarged by the motion of the ribs upwards, whereby the curvatures of opposite ribs are set at a greater distance from each other; and, by the same motion, the sternum is moved outwards, and set at a greater distance from the vertebre of the back. The motion of the ribs upwards is caused by the contraction of both layers of intercostal muscles. That the muscles called internal intercostals concur with the external in raising the ribs, appears from the situation of those muscles, from the greater mobility of the inferior ribs, from the inspection of those muscles 
in living animals, and from experiments imitating their action. In more violent and laborious inspirations, the raising of the ribs is assisted by many muscles attached to the ribs, and arising from the clavicle, humerus, scapula, and vertebræ of the neck or back.

CLXXXVI. By the enlargement of the thorax, a dilatation of the lungs is produced, in proportion to the bulk of air entering into them; but the dilatation may often be greater by the air that enters into the lungs being heated or rarefied; and the greatest distention of the lungs is obtained by a constriction of the glottis confining the air that has already entered into the lungs.

CLXXXVII. As inspiration, or the admission of air into the lungs, depends upon the enlargement of the thorax, the diminution of it must expel the air, or produce expiration. The capacity of the thorax is diminished, while the muscles dilating it are spontaneously relaxed by the elasticity of the ligaments connecting the ribs with the vertebro, and by the elasticity of the cartilages and ligaments connecting the ribs with the sternum; both which powers, commonly assisted by the weight of the ribs themselves, bring the ribs and the sternum into the position they were in before respiration. At the same time, the elasticity of the mediastinum draws the diaphragm upwards; and the contraction of the abdominal muscles both presses the diaphragm upwards and pulls the ribs downwards; and, in the last, they are assisted by the sterno-costal and infra-costal muscles. While these powers concur in diminishing the capacity of the thorax, the expulsion of the air from the lungs is assisted by the elasticity of the lungs themselves, and by the contraction of the muscular fibres of the bronchiae.

CLXXXVIII. These are the ordinary powers of expiration, which, depending upon the reaction of elastic parts, is performed slowly, and with little force; but when it is necessary to perform it with more velocity and force, some other and very powerful muscles, as the quadratus lumborum, sacrolumbalis, and longissimus dorsi, concur in pulling down the ribs; and, at the same time, the abdominal muscles, actuated by the animal power, are contracted with greater velocity and force than in spontaneous expiration. 
CLXXXIX. The situation of the blood-vessels of the lungs is such, that, in the contracted state of this viscus, these vessels must be much folded and straitened; and it appears, that, in the fœtus, where they are constantly in a contracted state, their capacity is not sufficient to transmit, in the time required, the whole of the blood returning to the heart by the vena cava; but, after respiration has been repeated for some time, by the dilatation of the lungs to a certain degree in inspiration, their blood-vessels are unfolded, lengthened, and enlarged, so as to be capable of transmitting the whole blood of the cava.

CXC. In the infant who has breathed for some time, the whole blood of the vena cava passes into the right ventricle of the heart, and from thence enters into the vessels of the lungs; but, in the contracted state of the lungs, which occurs at the end of expiration, the blood cannot be properly transmitted; and, for that purpose, an inspiration becomes absolutely necessary.

CXCI. It is, however, under a certain degree of inspiration only, that the blood is freely transmitted through the vessels of the lungs; for, if the inspiration is full and continued, so that the lungs are thereby much distended, we find that this state also interrupts the free passage of the blood, and renders expiration necessary.

Expiration becomes also necessary; because, perhaps, the air long retained in the lungs loses a part of its elasticity, and becomes thereby unfit to keep the lungs distended; but, more certainly and more especially, because, in an animal that has breathed for some time, there is a noxious vapour constantly arising from the lungs, which, if not dissolved by the air, and carried out of the lungs, proves pernicious to life.

CXCII. From what has been said, it appears that the alternate motions of inspiration and expiration are necessary to the circulation of the blood, and otherwise also to the health of the body; and it appears also, that the more frequent the alternate motions of respiration are, the more quickly is the blood transmitted from the right to the left ventricle of the heart.

CXCIII. We can now perceive also the causes exciting these alternate motions; and we find no occasion for supposing them to arise from any causes alternately interrupting the motions of 
the nervous fluid, or of the blood into the muscles concerned in these functions.

Inspiration, or the action of the muscles producing it, is excited, in all cases of general effort, to remove pain and uneasiness; and it is perhaps a propensity of this kind that gives the first beginning to respiration in the new-born infant exposed to several new and uneasy impressions.

For the continuance of respiration, inspiration is especially excited by the sense of uneasiness that attends any difficulty in the passage of the blood through the vessels of the lungs; but this uneasiness arises, in some measure, at the end of every expiration, and is much increased by any continuance of this state.

CXCIV. Expiration, in some measure, necessarily succeeds inspiration, by the spontaneous relaxation of the inspiratory muscles (CVIII.), while the elasticity of the membranes, ligaments, and cartilages, stretched in inspiration, brings back the ribs and diaphragm into their former situations; and the same effects are also produced by the action of the abdominal muscles, and of the muscular fibres of the bronchiae; both of which are stretched, and thereby excited in inspiration.

In the case of ordinary inspiration these causes are sufficient to produce a spontaneous expiration. But as it appears that a violent and long continued inspiration interrupts the passage of the blood through the lungs, this creates an uneasiness, and a propensity, which must produce a relaxation of the inspiratory, and excite a contraction of the expiratory muscles.

It is further to be supposed, that, in animals which have breathed for some time, custom has associated the several motions concerned both in inspiration and expiration; so that an irritation applied to any part of them, necessarily excites the whole; and it may also be supposed, that habit determines these motions regularly to succeed one another.

CXCV. In this manner (CXCIII. CXCIV.) respiration is continued for the general purposes of the animal economy; but the several motions of which it consists are also occasionally excited, and variously modified by the will, intending particular effects to be produced by these motions. They are also excited, and variously modified by certain emotions and passions, and give particular expressions of these. They are often excited 
also by imitations; and they are particularly excited by propensities to remove pain and uneasiness, which operate more frequently on respiration than upon any other function.

CXCVI. The consideration of the effects of respiration on the animal fluids is delayed till the nature of these fluids shall have been more generally considered.

\section{SECT. IV.}

OF THE NATURAL FUNCTIONS.

CXCVII. The animal body from a small beginning grows to a considerable size, and, at the same time, from the period of the birth, during the whole of after life, the body suffers, by various means, a daily and considerable waste.

CXCVIII. The increase of bulk, therefore, must be acquired, and the daily waste supplied, by matters taken into-the body, the most part of which, from the presumed purpose of them, we name ALIMENTS.

CXCIX. A great part of these aliments, as taken into the body, are of a different nature from the matter of the body itself, or at least are in such a state as not to be fit for being immediately applied to the purposes of it ; they must, therefore, be changed, and fitted to the purposes of the economy by powers within the body itself.

CC. The conversion, or assimilation of the aliments to the nature of the solids and fluids of the animal body-the farther changes of these fluids, for various purposes, by secretion-and the application of some part of them in nutrition, or in increasing the growth of the body, make what are called the NaturaI FUNCTIONS.

\section{CHAP. I.-OF DIGESTION.}

CCI. The term digestion is commonly employed to signify the function of the stomach alone in changing the aliments; but, in this chapter, we are to consider all the changes of these as 
they occur successively in the different stages through which the matters pass.

CCII. Animals are determined to take in aliment by the appetites of hunger and thirst.

CCIII. Hunger is an appetite depending upon a sensation referred to the stomach, and arising from a particular state of it.

This state seems to be, in some respect, the degree of emptiness, but, more especially, the state of contraction in the muscular fibres which emptiness gives occasion to. This state of contraction may also be excited by certain stimulants applied; but, more commonly, it depends upon, and is correspondent to the state of inanition, and therefore of contraction in the vessels of the skin emitting the matter of perspiration.- "I have here advanced an opinion which I believe to be new; and I will, therefore, allow you to receive it with such diffidence as you think proper; but I must observe that I find it necessary to have recourse to this opinion, because the ordinary accounts are not at all satisfactory. Haller supposes that the uneasy sensation of hunger is owing to the application of the opposite sides of the stomach to each other, and to the friction, or mutual attrition hence arising. But I doubt if ever the opposite sides of the stomach come to be applied to one another, so as to be exposed to any, such friction or attrition. . From every view of the stomach, in its natural state, it appears that its muscular fibres contract in such a manner as still to preserve the circular form of the stomach, and that the two opposite sides never fall flat on each other but are always kept at some distance. In men killed by long fasting, the stomach, instead of being a large bag, has been found of a small size, but still retaining its circular form ; so that no attrition could be produced. Moreover, in that state of the stomach no peristaltic motion is going on, nor will the alternate pressure of the diaphragm, which moves the.whole stomach, produce any attrition of its sides. Further, I say this friction could only take place between two dry surfaces, but this can never occur in the stomach, which is still filled in proportion to its capacity. with a considerable quantity of mucus, some of the relics of food, and the gastric fluid, which will prevent any friction. 
"As this common account, therefore, is not satisfactory, I believe that hunger is produced merely by the state of contraction of the muscular fibre, to which the emptiness of the stomach gives rise. This contraction gives a sensation of consciousness, which in cases of spasmodic contractions is very painful, and, though it does not amount to the same degree here, still may. be a cause of uneasiness, and sometimes of pain. Hence, in order to account for the sensation of hunger, it is only necessary to shew by what means this contraction is actually produced, and how it comes to be connected with a state of emptiness in the system.

"But I must first observe, that another account of this sensation has been given by imputing it to the operation of certain acrid matters upon the sensible coat of the stomach. I allow that acrid matters may occur in the stomach, especially in its most empty state, when they will also be applied in the greatest proportion to the surface of the stomach, which, towards the end of digestion, is diminished in extent. I also believe that these acrid matters have a share in exciting the appetite, because this effect can be produced by introducing analogous acrid matters, such as acids, into the stomach. But it is very improbable, that a state of acidity, or any other acrimony should be present in the stomach, regular in quantity and proportion, considering the varieties of aliment and of digestion; but hunger is very regular in its action, which leads me to believe that its return is not caused by those acrimonies. Supposing, however, that they have a share in producing hunger, it is still a question whether they act by giving a degree of pain, or whether they do not, in the first place, excite the state of contraction on which I say that appetite essentially depends.

" This view, therefore, will not disturb the general theory which I wish to establish, that hunger depends on a contraction of the muscular fibres of the stomach. I have to remark, that the above accounts are especially defective in not pointing out the state of appetite as connected with the general state of the system, and with the evacuations that daily occur and occasion the waste which requires to be daily supplied.

"It is certain that where the appetite is remarkably deficient, the evacuations also are diminished; and this is so suitable to 
the administration of the economy, that the appetite for taking in food is connected with the necessity for it, arising from the wants of the whole system. It is obvious that the appetite is particularly connected with the state of perspiration and the consequent inanition of the vessels on the surface. Exercise increases perspiration, and a sedentary life occasions its going on more slowly. In the same way, exercise a little greater than usual promotes, and a sedentary life diminishes the appetite. Sanctorius has ascertained by experiments; that the appetite is proportionally diminished by causes which diminish the perspiration. But it remains to explain how the perspiration is connected with the stomach-how in different degrees of emptiness of this organ the appetite will return under exercise, but; though the 'stomach is empty as usual, will be prevented by causes obstructing the perspiration. We can only say in general, that there certainly exists a particular connexion between the extreme vessels of the surface of the body and those of the stomach itself. We know that a loss of tone in the latter is not only attended by a loss of appetite, but may be productive of vomiting, which is again taken off by restoring the action of the vessels on the surface. We can therefore think of no other connexion than the state of tone of each. The contraction of the cutaneous vessels being increased, the fibres of the stomach will follow the same cause; and, e contrario, in proportion to the emptiness of these extreme vessels, a contraction will be occasioned in them, if they are not distended by fluids moving through them."

CCIV. Thirst is an appetite for liquids, (" perhaps hunger leads us to take in liquid too, ') which depends upon a sensation chiefly referred to the internal fauces, and arising from the dryness or heat of these parts; from acrimony applied to them, or existing in the fluids poured out there; from the putrescency or viscidity of the contents of the stomach; and from all increased evacuations.

"Hunger is a sensation absolutely referred to the stomach, but I say thirst is chiefly referred to the fauces, because I have doubts whether it always arises from a sensation felt there. It may arise from a sensation of the stomach; for every body distinguishes between a dryness of the mouth, which requires a 
frequent taking in of liquids in small quantity, and an inward thirst, which requires the copious deglutition of liquids. The dryness of the fauces alone will occasion thirst. The breathing an air which is more dry or filled with dust, will immediately occasion an absorption of the fluids and a dryness of the fauces. The 'heat of these parts' accompanies a heat of the body in general,-it operates partly by the sensation of heat, and partly by abstracting the fluids. Thirst is further increased by acrimony, that requires drink to allay the sensation which is raised, and by the putrescency or viscidity of the contents of the stomach. How the effect of the latter is produced, it is difficult to say, but I give it as a fact which we know, that veal, lamb, and chicken, produce thirst more readily than beef, mutton, or fowl. Thirst arises more particularly from all increased evacuations. Thus, sweating is certainly attended with thirst, which may perhaps in part be owing to the heat necessarily accompanying it, but in part also probably to the evacuation, which, by diminishing the secretion of the fauces, occasions a dryness there. But as this instance might be ambiguous, we mention diarrhoea and diabetes, diseases which are strongly marked with thirst; and, in like manner, an evacuation which is not constant in women, that of giving suck, when they drink more than at other times. It is not necessary that these evacuations should be considerable to produce this dryness; they are no sooner increased than the thirst arises. Such is the connexion established, that the deviations of the system should be obviated and corrected; and therefore nature has directed thirst to accompany all increased evacuations of the more liquid parts of the blood."

CCV. These appetites determine men to take in a great variety of solid and liquid matters, directed by instinctive likings and disgusts, in some instances corrected by experience.

CCVI. Of the matters chosen, it appears that some of them are suited to supply the matters of the fluids or solids of the body, and, therefore, properly named Aliment ; while others of them are suited only to improve the relish of aliment, or to obviate some deviations ready to happen in the business of digestion, and these we name coñuments. 
CCVII. The proper alimentary matters are animal or vegetable only.- "What are the proper aliments of the human species? In answer to this, we know in general from experience, that the human aliments are taken entirely either from other animals or from vegetables, and that no part of them, excepting water, is taken from the fossil kingdom. The substances employed are seemingly various; and in order to know the greater or less fitness of the individuals, it is necessary to consider in general how animal and vegetable matters are suited to give nourishment to the human body." $-M . M$.

CCVIII. The animal aliments seem to be so nearly of the same nature with the matter of the body itself, that, to be rendered fit for the purposes of the economy, they seem to require no other change but that of being rendered fluid.- " The most part of the matter taken from animals is so nearly of the same qualities with the matter of the human body, that there is little difficulty in supposing that the animal matters taken into the human body, as aliments, are perfectly well suited to this purpose, and require only the means of solution and mixture, with very little ehange of their qualities. It is true, indeed, that in many of the animal substances we take in, the likeness of qualities to those of the human body is not always exact and complete: but, in the mean while, all of them agree so much in the qualities which chiefly characterize the human fluids, that we may presume on their being a matter so nearly the same, that the former may be very well suited to supply the latter." $-M . M$.

CCIX. But the vegetable aliment is very different from the matter of the animal fluids or solids, and must therefore be changed into the nature of these by the powers (CXCIX.); and as many animals are nourished by vegetable aliment alone, and as, perhaps, all animal matters may be ultimately traced to a vegetable origin, it will appear, that, to account for the production of animal matters, it is especially, and in the first place, necessary to show how vegetable matter may be converted into animal._- "We may remark it to be highly probable, that all animal matter is originally formed of vegetable; beeause all animals either feed directly and entirely on vegetables, or upon other animals that do so. From hence it is probable, that all animal substances may be traced to a vegetable origin; and 
therefore if we would inquire into the production of animal matter, we must first inquire in what manner vegetable matter may be converted into animal? And this question relates especially to the human body; the nourishment of which is in a great measure immediately taken from vegetables.

"In attempting this, we shall find that the conversion mentioned is the effect of a peculiar power in the animal economy; which, it must be acknowledged, is by no means clearly or fully understood. We shall, however, make some steps towards understanding it better; and to this purpose there is one step absolutely necessary, which is, to determine amongst the seemingly great variety of vegetable matter, which is the kind that is especially, or perhaps only, fitted to be converted into animal ? Or if this question, as thus put, be too general, it may then be to determine, what are the vegetable substances chiefly fitted for being converted into the substance of the human body? Nothing is more evident than that every vegetable, or every part of any one vegetable, is not suited to this last mentioned purpose; and therefore it is necessary, both for the sake of the general question, and also for the particular purpose of the Materia Medica, to determine, as well as we are able, what vegetables, and what part of them, are most fit for the nourishment of the human body." $-M . M$.

CCX. If we consider the many different odours, tastes, and colours, which are to be observed in different vegetables, we should be ready to think that vegetable matter is of very great variety; but we know that the matter distinguished by its sensible qualities makes but a small part of the whole of any vegetable, and that, besides the matter peculiar to each, there is in most, perhaps in all, vegetables, a large proportion of common matter, which we presume to be the matter adapted, and that very universally, to the aliment of animals.- "In pursuing this inquiry, it is to be remarked, in the first place, that for the most part those vegetables are rejected from the list of aliments that are imbued with any strong odour or taste; and at least of the sapid, all except the acid and sweet are excluded. To this perhaps there are a few exceptions; as when the odorous or sapid part is in small proportion to the rest of the vegetable substance; when the odorous or sapid parts are such as pass 
quickly out of the body again by the excretions ; or when they are such as admit of their qualities being entirely changed by the powers of digestion in the first passages. Such exceptions, however, hardly affect the general doctrine; which is very much confirmed by this, that several vegetables which in their acrid state are unfit or even noxious, by being deprived of their acrimony by culture, by blanching, by drying, or by boiling, are rendered quite proper : and if there shall still be exceptions not to be accounted for in any of these ways, I would maintain that such acrid substances are admitted and taken in as condiments rather than as nutriments.

"This consideration of the exclusion of acrid matters from among our foods is to be applied in this manner. As the acrid, odorous, or sapid parts, seem for the most part to be the peculiar matter of particular vegetables, and to be even but a small portion of these, seldom diffused over the whole but deposited in certain parts of them only; and as this is more especially the case in those vegetables which are taken in as food; so we from thence conclude, that besides these peculiar matters, there is in the most part of vegetables a considerable quantity of matter, which, for reasons to be given hereafter, is manifestly in common to almost the whole of the vegetable kingdom. This we shall speak of as the common matter of vegetables, and having laid aside, as above, the peculiar, it is in the common matter that we are necessarily led to seek the vegetable substance that is suited to the nourishment of the human body.

"Whilst from this consideration it appears that a great portion of vegetables is of an alimentary quality, at the same time it is from daily experience evident, that certain vegetables contain a greater portion of this alimentary matter than others, and that certain parts of vegetables contain more of it than other parts of them." $-M$. $M$.

CCXI. It is this common matter of vegetables, therefore, that we are to consider here; and we think it may be considered as of three kinds only, that is, oily, saccharine, and what seems to be a combination of these two.-- In attempting this, it must, in the first place, be observed, that, contrary to what others have supposed, I cannot discern that any portion of matter is to be found existing in any vegetables directly fitted to 
supply the animal fluid. This, however, as we have already said, is seemingly, together with water, the foundation of all other fluids in animal bodies; and, particularly, that from which the nutritious matter applied to the increase of the solid parts, is, by the powers of the economy, formed and prepared. It is this animal fluid, therefore, that our vegetable food is to be converted into; and it seems to be a matter formed not from any one kind, but, by the powers of the animal economy, from various kinds of vegetable matter. Accordingly, when we are to say that certain parts of vegetables are alimentary, we mean only to say that they are matters fitted to enter into the composition of the proper animal fluid." $-M . M$.

CCXII. The oily matter of vegetables, which makes part of the aliment of animals, is without any sensible odour or taste, and is not only very nearly the same in the many different vegetables from which we take it, but is also in all of these so nearly akin to the oil which appears in animals; that it is not necessary to suppose any considerable change to be made upon the vegetable oil on its being taken into the bodies of animals.

1) "Here, however, it may be imagined, that oil enters into the composition of the animal fluid only as it is a part of farina, or as it happens to be mixed by nature with other vegetable matter ; and that, as it is taken in, whether from animals or vegetables in a separate state, it affords only the oily matter that is necessary to be constantly present in considerable quantity in the bodies of animals, not for their nourishment, but for certain other purposes of their economy.

2. "We cannot, however, enter into this opinion: for we are persuaded that even the oil which is taken into the body in the form of a pure oil, though entirely separate from other vegetable matter, does truly, in a large proportion, enter into the composition of the animal fluid; and that oil, therefore, may be considered, in the strictest sense, as a fundamental part of the human aliment.

"We are of this persuasion, how much soever neglected by physiologists, from the following considerations :-

" 1 st, We observe that oil, both from vegetable and animal substances, is daily taken in as part of diet by the people of all nations, and often in large quantity, without increasing obesity. 
Lt appears likewise that this oil does not remain separate from the other fluids of the alimentary canal, but is very accurately diffused in the chyle; which may be considered as a step towards a more intimate mixture.

" $2 d l y$, That such a mixture actually takes place, is very probable from this, that no chyle appears in the left ventricle of the heart, nor in the arteries and veins which carry the blood which has passed through that ventricle. If there be some instances of such appearance, which have been alleged, they are certainly, however, very rare, and probably morbid.

" $3 d l y$, Not only no chyle, but neither does any oil ever appear in any part of the mass of blood, nor ever in any part of the human body, till it appears in the cellular or adipose membrane, into which it is probably brought by a peculiar secretion. It has indeed been alleged, that oil has sometimes appeared on the surface of extravasated blood or serum; but in all such instances, we presume it to have been a preternatural appearance: for in the many hundred instances in which I have looked upon the human blood, I have never met with any appearance of that kind; and whilst oil is so constantly and copiously taken into the body, nothing can account for the absence of its appearance, but the supposition of its having undergone an intimate mixture with the other parts of the blood.

"Some physiologists have been fond of finding the red globules of the blood to be an oily matter; and in certain conditions these may appear to be inflammable : but a fluid readily, equally, and permanently diffusible in water, cannot be properly considered as an oil.

"A fourth consideration that leads us to suppose the oil taken in to be intimately mixed with the other parts of the animal fluid, and to make a considerable part in the composition of it, is this, that the oil, which is often copiously laid up in the adipose membrane of healthy animals; is again, upon various occasions, absorbed and taken into the course of the circulation. Some of these occasions are manifestly those states in which a great degree of acrimony.prevails in the mass of blood, as in scorbutic, syphilitic, hectic, and other such cases; and while it is highly probable that the purpose of such absorption is, by the 
oil to cover the acrimony of the animal fluid, it must prove at the same time that this admits of an intimate mixture with the oil.

"As the want of food is a principal occasion of the absorption mentioned, this affords a proof that such absorption is a means of supplying aliment, or at least of covering the acrimony which upon the want of aliment is ready to take place. Upon either supposition, it affords a proof that oil unites very intimately with the other parts of the blood: and, upon the whole, there can be little doubt that oil taken in, either in its separate or united state, is a part, and a considerable part, of the human aliment." $-M$. $M$.

CCXIII. It is the saccharine matter, and especially this when blended with oily matter in different proportion, that makes the greatest part of the common matter of vegetables, and is the chief part of the vegetable aliment of animals. It is this, therefore, that we have especially to consider here; and, as it lies in vegetables, it is different from the most part of animal matters in the following respects.

It is readily susceptible of a vinous and acetous fermentation, and spontaneously enters into the one or the other of these; and, without undergoing more or less of these, it perhaps never enters into a putrefactive fermentation.

The same matter treated by distillation, without addition, gives out always, in the first part of the distillation, an acid, and only afterwards a volatile alkali in small proportion.

The same vegetable matter, treated by calcination, leaves ashes, which contain a fixed alkali, and an earth, that is, or may be converted into a quick-lime.

CCXIV. In all these respects, the common matter of animals is considerably different.

This enters spontaneously into a putrefactive fermentation, and that without passing through the vinous or acetous: at least these are not to be distinctly perceived.

The same animal matter treated by distillation, gives out always, in the first part of the distillation, a volatile alkali in large proportion, and only afterwards, by a great force of fire, it gives out an acid. 
Animal matters, treated by calcination, leave ashes in which no alkali is to be found, and the earth is not calcareous nor convertible into a quick-lime by any means yet known.

CCXV. These differences are sufficiently marked; but it is proper to observe here, that the vegetable matter we treat of, by undergoing a putrefactive fermentation, is changed so as to acquire very exactly most of the characters of animal matter we have just now mentioned.

CCXVI. The aliment being thus considered, we proceed to consider the changes it undergoes after being taken into the animal body; but, first, of the course it passes through, and of the motions it is subjected to in its progress.

CCXVII. The aliment is taken into the mouth, and there the more solid parts of it are commonly subjected to a triture, or what is called manducation. At the same time a quantity of saliva, and of the other fluids of the mouth, with some portion of our drink, is intimately mixed with it, whereby the whole is reduced to a soft pulpy mass. In this state, by the action of deglutition, it passes through the fauces into the œsophagus, by which it is conveyed into the stomach.

.CCXVIII. Here the aliment is detained for some time, subjected to a constant agitation and some pressure, both by the contractions of the different parts of the stomach itself, and by the alternate pressure of the diaphragm and abdominal muscles. After some time, however, first the more fluid parts, and at length the most minute parts of the solid matter are pushed through the pylorus into the duodenum.

CCXIX. The matters received from the stomach into the duodenum pass on from thence successively through the several parts of the intestinal canal; and, in the whole of the course, the matters are still subjected to the alternate pressure of the diaphragm and abdominal muscles, and to the contractions of the intestines themselves.

CCXX. Through the whole course of the intestines, but especially the small, the more fluid part of the contents, and particularly the peculiar fluid we name chyle, is taken into the vessels named lacteals. These, from imperceptible beginnings on the internal surface of the intestines, unite into larger vessels laid in the mesentery, and convey the chyle, and what accom- 
panies it, first into the conglobate glands of the mesentery, and from thence to the receptaculum chyli, as it is called. From this the chyle passes by the thoracic duct into the left subclavian vein. In one or other part of this course of the chyle, the vessels carrying it are joined by lymphatics, returning the lymph from almost every part of the body.

CCXXI. The matters contained in the intestinal canal, not taken into the lacteals, are moved onwards in the course of the intestines, becoming by degrees of a thicker consistence, especially in the colon, where their motion is considerably retarded; but, at length, they are moved onwards to the extremity of the rectum, where their weight, bulk, and acrimony excite motions which throw them entirely out of the body.

CCXXII. This is the course of the alimentary matters, so far as they can be considered as any ways in a separate state. Of the motions of the several organs concerned in this course, we pass over those of manducation, deglutition, or others depending on the action of muscles, the functions of which are readily understood from a knowledge of their situation; and. we are here to consider only the motions of the alimentary canal itself.

CCXXIII. The motions in the osophagus depend upon: the action of its muscular fibres, which are chiefly those forming a chain and circularly surrounding it. This tube, by the morsel of food pushed into it by the action of deglutition, is necessarily dilated, and its circular fibres are thereby excited to a contraction. But as these fibres are successively dilated, so they are also contracted, and push on their contents through the several portions of the tube, alternately and successively dilated and contracted, giving the appearance of a vermicular motion, and what is commonly called peristaltic. This motion may be propagated either upwards or downwards, and the direction of it is in the one or the other way, as the motion happens to begin at the upper or lower extremity.

CCXXIV. The motion of the stomach is not so simple. Its muscular fibres are in like manner irritable by dilatation, and its circular fibres must therefore be in some measure subjected to a successive dilatation and contraction. But though the direction of such motions is from the left to the right, this does not 
immediately push the contents of the stomach into the intestines. It seems to be the purpose of the economy to detain the aliment for some time in the stomach; and, therefore, any considerable dilatation of the circular fibres, especially that which occurs in a full stomach, seems to have the effect of exciting the longitudinal fibres to a contraction, which draws the two orifices of the stomach nearer to one another. By this the pylorus is raised up, and rendered less easily passable, and probably, at the same time, the peculiar hand of circular fibres which - surround the pylorus, are more firmly contracted, and render it less pervious (Vide CXLIV.). From hence it is, that the direction of the peristaltic motion of the stomach is sometimes from the left to the right, and sometimes also the contrary way. It is, however, most constantly in the first manner, because it is commonly begun from the œesophagus; and because, when it is inverted, the resistances on the left from the blind sack of the stomach, from the higher situation of the cardia, and from the constriction of this by the diaphragm in inspiration, are commonly more considerable than the resistance at the pylorus. . The contents of the stomach, therefore, are at length pushed through the pylorus; in the first place, the more fluid contents, as these occupy the antrum pylori, while the more solid, having their air loosened by fermentation, are rendered specifically lighter, and float nearer the upper orifice. But at length, as the stomach is in any measure emptied, the pylorus is less raised, is more relaxed, and allows matters to pass more easily; and, at the same time, the empty stomach contracted more, is, especially towards the right extremity, contracted to such a degree, as to embrace the smallest solid matters now fallen down into it, and to push them through the pylorus.

This is an idea of the ordinary motions of the stomach; but they are, upon some occasions, subject to other modifications; as in eructation, rumination, and vomiting, which, however, as morbid, we reserve to be considered in the Pathology.

CCXXV. The motions of the intestines will be readily understood from what has been said of those of the osophagus. Any portion of the intestinal canal being dilated, will in consequence be contracted, and will urge on its contents in the same 
direction in which the motion was begun. But as the force here is gentle, and as, in the long course of the canal, there occur many flexures, different positions, and occasional irritations, it is obvious that resistances and stronger contractions may frequently occur here, to change the direction of the motion; accordingly we find it frequently changed, and directed from below upwards, in so far that the contents of the intestines frequently pass into the stomach. But the motions of the intestines are, however, most constantly directed from above downwards, both because they are commonly begun from the stomach, and because, when inversions do occur, there is commonly still so much resistance at the pylorus, and more especially at the valve of the colon, as to turn the direction again into its proper course. In the colon, from its position, structure, and the consistence of its contents, the progress of these is more slow and difficult, and it is therefore here assisted by the longitudinal fibres peculiarly disposed, so as by their contraction to contribute more to the dilatation of every succeeding portion of the intestine.

CCXXVI. The chyle is taken into the lacteals, and moved onwards in these in the same manner (CLXVII.) as the lymph is in the several lymphatics in other parts of the body, to which the lacteals are in structure and situation exactly similar.

CCXXVII. The course of the alimentary matters, and the motions by which they are carried on, being now explained, we return to consider the several changes which the aliment undergoes in this course.

CCXXVIII. In the mouth, if the aliment taken in be of a solid consistence, it is here, as we have said, subjected to a triture; and if our food is of a soft and moist kind, we are instinctively directed to take in along with it some dry matter, as bread, that the whole may be subjected more certainly to a complete manducation. By this our aliment is not only more minutely broken down, but is also intimately mixed with the liquids at the same time taken in, with the saliva and other fluids of the mouth, and with a quantity of air entangled by these viscid fluids.

CCXXIX. In this divided and moistened state, the aliment is taken down into the stomach, where it is farther dissolved; 
the vegetable matter of it begins to be changed to the nature of animal, and the oily parts of the whole begin to be united with the watery. But these changes by solution, assimilation, and mixture, require to be separately considered.

CCXXX. The solution here, as in other cases, may be assisted by the mechanical division of the solid matter, by the agitation of the dissolving mass, and by the application of heat; and, with these assistances, the solution must be performed by the application of a proper menstruum.

CCXXXI. The division of the solid is sometimes assisted by a previous cookery, and commonly by the manducation we have mentioned; but the human stomach does not seem by any mechanical powers to contribute to this. It gives only a moderate agitation, which, in any case, contributes little to mechanical division.

CCXXXII. The degree of heat applied here, being that of the common temperature of the human body, may assist the solution; but it is of no considerable power, and no assistance is got from any closeness of the vessel which occurs here. Upon the whole, the assistances applied here are not considerable, and the speedy solution that takes place must be chiefly owing to the power of the menstruum.

CCXXXIII. The menstruum that appears here is a compound of the liquid matters taken in, of the saliva, and of the gastric liquors; but in all or any of these, we do not readily perceive any considerable solvent power; nor, by any artifice in employing these out of the body, can we imitate the solutions performed in the stomach.- "What may be the power of the gastric menstruum, or the causes of its different power with respect to different substances, is not well ascertained; but we now know that it is different in different animals, insomuch that in many carnivorous animals it has little power with respect to vegetable matters; and that in phytivorous animals it has little power with respect to animal substances. (See Steven's De Alimentorum Concoctione, Edin. 1777.)

"Although in the human stomach the gastric menstruum seems commonly to have power with respect to both animal and vegetable matters, it is, however, probable, that upon different occasions its power is in a different degree with respect to these 
different substances; as it seems at certain times to dissolve the one more readily than the other. What this depends upon, or under what different modifications it may appear, we cannot at present venture to determine.

" At present it seems further necessary to remark, that with respect to perhaps every human stomach, the powers of it in general being given, there is a difference in the solution of different substances, arising entirely from the different texture of these. Thus it appears, that apple and melon are less readily soluble than strawberries and raspberries; that full grown cabbage is less soluble than cauliflower ; and a like difference may be observed with respect to many other vegetable substances, as we shall hereafter take notice of more particularly. In the mean time it may, in illustration of the whole, be in general observed, that in many vegetables there is a different solubility in the different parts of them; so that, in one and the same, while a certain part of them is entirely dissolved, another part of them passes off by stool in a very entire state. - Thus, as many fruits consist of a tender pulp enclosed in a firmer membrane or husk, so the solubility of the whole will depend upon the proportion of these parts; and as, in the maturation of fruits their pulp goes on increasing, while their membranes are constantly growing thinner and tenderer, so in many instances the solubility of fruits taken in an entire state will commonly be in proportion to their maturity.

"In illustration of this subject of the solubility of aliments, it may be remarked, that in so far as the arts of cookery render the texture of aliments more tender, it renders them in proportion more soluble in the stomach.

If ". At entering upon this subject, I should have observed, that we have a particular proof of the more ready or difficult solubility of different substances in the stomach. There are men who are occasionally, and many who are very frequently, liable to a rumination, or the bringing up, by an eructation, a part of the contents of the stomach. These parts are frequently somewhat entire portions of vegetable or animal matters, which are manifestly of a firmer, texture than the rest which had been taken down, and have not therefore been so readily dissolved. From the rarefaction of their air not entirely extricated, they 
float near to the upper orifice of the stomach, and are therefore most readily brought up. I have known several persons liable to this rumination, and from them have learned, that certain substances are more commonly brought up than others, and some at a longer time after eating than others; and both circumstances seem clearly to depend upon the different degrees of solubility in these substances." $-M . M$.

CCXXXIV. However, from what happens in the stomachs of certain animals, there is ground to presume that indeed in every one there is a peculiar solvent. But whether this be a menstruum dividing the solid into integrant parts, and thereby reducing it to a fluid state, or if the solvent here be a peculiar fermentative power, resolving matters more or less into constituent parts, is not clearly perceived.

CCXXXV. The latter is the most probable, as the circumstances of fermentation very constantly appear, and as the deviations which at any time appear in the course of digestion appear always to be an excess of fermentation, either acescent or putrefactive.

CCXXXVI. The business seems to us to proceed in this manner: The fluids of the stomach have the power of suddenly and powerfully loosening the fixed air of the alimentary matters, which is the first step towards putrefaction, and that which most effectually breaks down the texture, and perhaps the mixture of bodies. But we now know, that putrescent bodies are very powerful in exciting an acescent fermentation in vegetable substances, which the human stomach is hardly ever without; and that this acescency therefore, in the next place, very constantly succeeds, and an acid is produced in the stomach. This acidity makes the effects of the putrefaction disappear; and the acidity, in its turn, disappears also, probably by its being absorbed by, or united with the putrescent and oily matters here present ; and it is in this manner that we suppose that the animal fluid is produced, and daily renewed by the combination of a fresh portion of acid with putrescent fluids previously existing in the body. The daily production of acid in the human stomach, and its readily disappearing again, without showing any morbid effects, renders our doctrine sufficiently probable.

CCXXXVII. This is the assimilation of vegetables that I 
suppose to take place, and is begun in the stomach, but is not completed there; for we observe that the long retention of the alimentary matters in the stomach, whether from the insolubility of the matter, or from an obstruction of the pylorus, produces a greater degree of acidity; and, in general, the acidity which commonly prevails in the stomach does not disappear but in the after-course of the aliment.

CCXXXVIII. It is especially the bile, added to the matters which have passed from the stomach into the duodenum, that is fitted to cover the acidity which appeared in the stomach. It is probable, also, that the pancreatic and intestinal liquors contribute to the same effect; and it is perhaps for the same purpose that the lymph is constantly added to the chyle in its course. But, after all, we must rest in the general idea, and own that we do not know exactly how this matter proceeds, nor what the several fluids, added to the aliment in the different parts of its course, truly contribute to the changes of it.

CCXXXIX. It is probable however, that, by the mixture mentioned, the peculiar fluid which we name the chyle is produced; for, though it is certain that a variety of fluid matters may enter the lacteals, and accompany the chyle there, it is still probable that there is a peculiar fluid produced by the actions of the stomach and intestines, and such as becomes the principal ingredient in the animal fluids afterwards formed, that is strictly entitled to that appellation. This chyle does not appear in the stomach, but first in the duodenum, and more copiously still in the jejunum and first part of the ileum. It appears indeed in the whole of the ileum, cœecum, and colon, but in the last less copiously; all which shows that a particular mixture is necessary to it, and, at the same time, that it is not made at once, but successively in the course of the intestines.

CCXL. It remains to speak of the mixture of the oily with the watery parts of the aliment. This we cannot well explain; but it is of consequence to observe here, that such a mixture is actually made. It is evident that a large quantity of oil, in a separate state, is taken in as a part of our aliment, but at the same time no oil commonly appears in a separate state in the mass of blood; it must therefore be united with the other parts of the mass in the way of mixture. Hitherto, the physiologists 
have hardly mentioned any other means for this union of oil, but the application of viscid fluids; but these can occasion only a diffusion, and some means of mixture must necessarily be supposed. What these, however, are, we do not certainly know. They do not produce their effect in the first passages; for in the chyle, till it enters the subclavian vein, the oil appears to be only in a diffused state, and probably the perfect mixture is only made in the passage through the lungs.

CCXLI. It may be proper here to take notice of another matter which constantly enters into the mixture of animal fluids. This is air, which, by different means, can be extracted in considerable quantity from every kind of animal matter. What is properly the origin of this, when and where it is insinuated into the animal fluids, and by what means it is either fixed in these or loosened from them, are all questions not yet resolved, but perhaps necessary to be resolved; before we can speak with any confidence of the changes which the animal fluids undergo in different parts of the system. We can observe, in the mean time, that a quantity of air is always present in the chyle in a very loose state; that it becomes more fixed in the mass of blood after this has passed through the lungs; and that again, in the different secreted fluids, the air appears to be in some of them still fixed, and in others much more loose ; and it is probable, that all this has a particular relation to the production and properties of the different fluids of animals.

CCXLII. We have now followed the course of the aliments, so far as we can consider them as any ways in a separate state; but we do not perceive, that, in any part of this course, the proper animal fluids are entirely formed; and it is very justly supposed, that the proper mixture or assimilation is not finished till the chyle, mixed with the mass of blood, has undergone the action of the lungs, through the vessels of which it must almost immediately pass after entering the subclavian vein, and seemingly before it is applied to any of the purposes of the animal economy.

CCXLIII. What change the fluids undergo in passing through the lungs, or by what means the supposed changes are produced, after all that has been said, seems still to be very little known. 
The mechanical powers of pressure, commonly spoken of, do not in fact take place, nor are their supposed effects any ways consistent with sound philosophy ; and, on the other hand, it is very probable, that the changes produced are the effects either of chemical separation or mixture.

What has been supposed to be performed in this way by an absorption of air, or of a particular matter from it, is very uncertain in fact, and has led to a still more uncertain reasoning.

It is now certain, that a quantity of mephitic air, and perhaps some other matters, are constantly exhaling from the lungs of living animals, and are carried off by the atmospherical air alternately entering and issuing from the lungs. This is a pretty certain evidence that some change of mixture is going on in the fluids passing through the lungs; but from what particular portion of the fluids the mephitic air proceeds, or what is the effect of its separation, we know not; and indeed, as we have said before, what are the effects of the action of the lungs upon the state of the fluids, we are very uncertain. Upon the whole; we still know but little of the production or formation of the animal fluids ; and therefore, from the consideration of their formation, we have learned little of their nature; but we must now try to discover what we can of it, by examining these fluids as they are found already formed in the blood-vessels.

CHAP. II. -OF ANIMAL BLOOD.

" From the earliest accounts of physic with which we are acquainted, it appears, that from the most ancient times down to the present, physicians have been attached almost entirely to the study and consideration of the fluids; and from the supposed state of these, have endeavoured to explain the phenomena both of health and of sickness. In this, however, they appear to me to have been unfortunate; for, not to merition the imperfection and falsehood of the many speculations, both of Galenists and chemists, which have formerly prevailed on this subject, I would venture to assert, that the doctrine concerning the fluids is still the most imperfect part of our physiology. Every thing systematical that has been delivered upon it. till within these last forty years, may be totally disregarded; for it 
is only within the period now mentioned that we have acquired any distinct notions of a fundamental doctrine, or, in other words, of the state of aggregation in the mass of blood. Even with respect to this last, there still remains much doubt and obscurity; but, notwithstanding these difficulties, I shall endeavour to consider what may be said with regard to the state of the fluids." $-M$. $M$.

CCXLIV. The red fluid passing from the lungs to the left ventricle of the heart, and thence by the aorta and its branches to every part of the body, may be considered as a mass containing, either formally or materially, every part of the animal fluids, and may, therefore be properly called the common mass of blood. This term, however, must be strictly confined to the circulating fluids while they retain their red colour; for, when they lose this, it is always in consequence of some separation of parts. The same red fluid, indeed, as it is found in the veins, has also suffered some separation of parts; but as the blood in the veins is never entirely deprived of the whole of any matter that was present in the arteries, so we think the venous blood may still be considered as a part of the common mass.

CCXLV. This mass of blood we find to be an heterogeneous aggregate; and it will be proper to inquire into the several parts of this, before we employ any chemical trials for discovering the mixture of the whole, or of its parts.

CCXLVI. We discover the parts of this aggregate chiefly by the spontaneous separation of them, which takes place upon their being drawn out of the vessels of a living animal.

CCXLVII. The separation commonly proceeds ir this marner : immediately after the blood is drawn out, it exhales a sensible vapour, and, after some time, it is found by that exhalation to have lost a part of its weight, more or less, according to the degree of heat it is exposed to, according to the extent of surface by which it is exposed to the air, and probably also according to different conditions of the blood itself. The matter thus exhaling may be called the halitus or vapour of the blood:

CCXLVIII. Soon after the blood has been drawn out of the vessels it loses its fluidity, and the whole of it concretes into one soft gelatinous mass; but, after some time, there oozes out: from this mass a thin fluid, and as the separation of this protvol. $x$. 
ceeds, the máss contracts into a smaller bulk, and, in proportion, becomes more dense.

CCXLIX. This is the separation which almost always takes place, and has, at all times, been observed by physicians. The fluid part is called serum, and the thicker consistent part has been called cruor, but more properly the crassamentum.

CCL. Both parts seem homogeneous and simple, but are not. For, if the crassamentum taken from the serum be laid upon a linen cloth, and water is poured upon it, the water washes off a red coloured part, and carries it through the pores of the cloth, and there remains a whitish, consistent, but soft and tough mass, not to be further diminished or separated into parts by any ablution.

A like experiment shows always a like matter present in the mass of blood; and, upon several occasions, both while the blood remains within the vessels, whether of the living or dead body, and when it is drawn out of the vessels of the living, this matter spontaneously separates from the other parts of the blood. It is therefore a part constantly present in the blood. It is what Gaubius, after Malpighi, calls the fibra sanguinis. Mr. Senac names it the coagulable lymph, and we shall speak of it under the title of the gluten of the blood. When it appears upon the surface of the blood drawn out of the vessels of the living animals, it is called the inflammatory crust.

CCLI. When the blood is viewed with a microscope, whether as moving in the vessels of a living animal, or when out of the vessels remaining still fluid, there are certain parts of it which appear of a round figure, and also of a red colour, while the rest is almost colourless. The parts thus distinguishable by their figure, are called the red globules; andit appears, that the red colour of the whole mass depends upon the presence of these only. It is chiefly these parts which are washed off from the crassamentum in the experiment above-mentioned; and we now conclude that, besides the red globules, the gluten, and a portion of serum that happens to be entangled in the pores of the concreting mass, there is no other matter evident in the crassamentum.

CCLII. The serum is a transparent fluid of very little colour, and seemingly simple; but if it be exposed to a heat of 
$156^{\circ}$ of Fahrenheit's thermometer, it concretes into a firm and almost transparent jelly; and if this be cut into minute pieces, there exudes from it a thin colourless fluid of a saline taste. In proportion as this fluid is more carefully separated, the coagulated part becomes more insipid, and in all its properties resembles the gluten separated from the crassamentum. From, hence, we are ready to conclude, that the serum, as obtained by spontaneous separation, consists of a portion of gluten dissolved in a saline fluid, which we name the serosity.

CCLIII. From the whole that has been said, from CCXLIV. to CCLII., it appears that there are three distinct portions and kinds of matter in the common mass of blood; that is, red globules, gluten, and serosity. What other matters may also be there we shall consider afterwards; but, in the mean time, shall say a little more of each of the parts we have already mentioned.

CCLIV. The red globules have been considered as an oily matter, and from thence their distinct and globular appearance has been accounted for; but there is no direct proof of their oily nature, and their ready union with, and diffusibility in water renders it very improbable. As being microscopical objects only, they have been represented by different persons very differently. Some have thought them spherical bodies, but divisible into six parts, each of which, in their separate state, were also spherical; but other persons have not observed them to be thus divisible. To many observers they have appeared as perfectly spherical, while others judge them to be oblate spheroids or lenticular. To some they have appeared as annular, and to others as containing a hollow vesicle. All this, with several other circumstances relating to them, very variously represented, show some uncertainty in microscopical observations; and it leaves me, who am not conversant in such observations, altogether uncertain with respect to the precise nature of this part of the blood. The chemical history of it is equally precarious ; and, therefore, what has been hitherto said of the production, and changes happening to these red globules, we choose to leave untouched. We shall afterwards say something with respect to their general use in the animal system; and now we shall at- 
tempt fo explain the cause of some changes, which, in certain circumstances, appear in the colour of the whole mass of blood.

CCLV. We suppose that the red globules, when viewed singly, have very little colour, and that it is only when a certain number of them are laid one upon another, that the colour appears of a bright red; but this also hath its limits; so that, when the number of globules laid on one another is considerable, the colour becomes a darker red. Upon this supposition, the colour of the mass of blood will be brighter or darker as the colouring part is more or less diffused among the other parts of the mass; and we think this appears to be truly the case from every circumstance that attends the changes which have been at any time observed in the colour of the blood.

CCLVI. The gluten of the blood, from its resemblance, on the one hand, to the albumen ovi, and, on the other, to the matter of the solids of animal bodies, we consider as the principal part of animal fluids, as that which is immediately formed of the aliment taken in, and as that which is employed in increasing the growth of the solids, or in repairing their waste.

CCLVII. But it is well known that the animal fluids in general, and particularly the gluten, is prone to putrefaction; and that, even in the living body, if fresh aliment be not constantly taken in, and also if certain excretions which carry off putrescent matter be not constantly supported, a considerable putrefaction certainly takes place. From hence we are led to think, that some approach to putrefaction constantly takes place, even in the most healthy bodies; and that it appears especially in an evolution of saline matter, and that this, taken up by the water constantly present, forms the serosity. We suppose it is this which affords the vapour of the blood (CCXLVII.), and that it is the serosity dissolving a portion of the gluten which forms the serum that appears upon spontaneous separation (CCXLVII.).

CCLVIII. The saline matters impregnating the serosity, if we may judge from the analysis of urine, are of various kinds; but particularly, there is present an ammoniacal salt, now well known under the name of the essential salt of urine, which, if not originally formed, is at least most copiously evolved in animal fluids. 
CCLIX. These are conjectures concerning the parts of animal blood; and it remains to say in what proportion each of them is present in it. This will perhaps be always difficult; and in the mean time we can perceive, that many estimates formerly made could not be exact, as the several parts were not properly known; and, while judging chiefly from the appearances upon spontaneous separation, physicians were not aware how much these are affected by the circumstances of extravasation, and by those in which the blood is placed after being drawn out. There are not yet indeed experiments made to ascertain, with any exactness, the proportion of the several parts mentioned; but it is probable, that the red globules make a small part of the whole; that the gluten, if we consider both what is in the crassamentum and in the serum, is in much larger proportion, but that the watery portion is the largest of all, and at the same time that this has always a considerable quantity of saline matter dissolved in it.

"That the red globules may be in different proportions to the whole mass, we discern very clearly from șeveral diseases, in which their quantity is evidently and considerably diminished; but what may be their proportion in persons in health, or in what manner that proportion is connected with the other circumstances of health, is by no means ascertained by any proper experiments. From several observations on animals whose vessels are easily subjected to microscopical examination, it appears that the proportion of red globules is greater or lesser, according to the greater or lesser quantity of the aliment or nourishment the animal receives. It is very possible, therefore, that the quantity of them in the human blood may be affected by the same circumstances; but still this does not aid us in determining the question with respect to persons in health, who take in nourishment in proportion to their several bulks; and whether the effect of nourishment be determined by the quality, as well as by the quantity, is not ascertained. It appears to me, that, if at all, it is not affected much by the quality of nourishment, unless the difference of that quality be very considerable. It appears also, that the proportion of red globules is as great in the animals living entirely upon vegetable aliment, as it is in 
those who live entirely upon animal food, or as it is in man, who lives partly upon the one and partly upon the other.

" Many attempts have been made to estimate the proportion of the quantity of the crassamentum to that of the serum in the extravasated human blood; but hardly any of the experiments yet made afford a certain conclusion. The apparent proportion of the two masses is very fallacious; being very much varied by the circumstances which determine the concretion of the cruor to take place sooner or later, and by the time which is allowed to pass from the time of concretion to that at which the proportions are examined. It is now indeed well known, that these circumstances vary the separation which takes place; and it does not appear to me, that in any of the estimates which have been made, due attention has been given to the effect of those circumstances. When Dr. Haller, in his Prima Linea, ( $\$ 138$.$) gives his judgment:- ' In massa sanguinea media$ pars, et ultra, cruoris est. In robore valido serum minuitur ad tertiam partem, in febre ad quartam et quintam reducitur, in morbis a debilitate increscit;' I am persuaded that he had judged "entirely from the quantities that appear separated in ordinary blood-lettings, and had not attended to the different quantities that appear in these according to the different circumstances of the blood-letting. In cases of rheumatism, I have seen the crassamentum not equal to a third part of the serum surrounding it; and other cases, where the scrum did not separate from the crassamentum, to the amount of a fourth part of the whole mass; and from attending to the circumstances of the blood-letting, I have been able to foretel what, in twenty-four hours after, would be the condition of the separation. But even supposing we had a more exact estimate of the serum with respect to the crassamentum, or in other words, of the red globules and gluten taken together, it still remains undetermined what proportion these two last mentioned matters bear to one another; and consequently it is not yet ascertained what is the usual proportion of red globules in the blood of persons in. health, nor how far it may have a share in producing a peculiar temperament.

"With respect to the gluten of the blood, considered by. 
itself, it is equally difficult to ascertain its proportion either to the whole mass or to the several parts of it.

"Upon the whole of this inquiry into the state of the blood, with respect to its aggregation, or with respect to the state and proportion of the several parts which compose it as an aggregate, it seems not only to be uncertain how far these circumstances give a difference of temperament, but, on the contrary, it seems probable, that they never do so in any considerable degree.

"Notwithstanding all this, ever since chemical reasonings have been admitted into our physiology, that is, ever since the time of Paracelsus, physicians have considered our fluids as distinguished by the state of their chemical mixture, either in the whole of the mass of blood, or in the several parts which compose it as an heterogeneous aggregate. It is, however, in the latter view only, that is, with respect to the several parts, that we can chemically consider the subject; and here it will be readily acknowledged, that, till very lately, much frivolous, hypothetical, and false reasoning has prevailed in the chemical doctrines respecting the nature and state of our fluids. Even at present, physicians have hardly come to be sufficiently chaste in avoiding such hypothetical doctrines; and however confident they have been in their chemical reasonings, I am unable to find any thing either clear or certain upon the subject. Not to mention how little we have yet learned of the nature of vegetable or animal substances from their chemical analysis, it is enough to say here, that with respect to some parts of the mass of blood, it is by no means ascertained either that their chemical mixture is upon different occasions anywise changed, or what change is produced, or in what manner such change is effected. This may be very confidently asserted with respect to the red globules, the mechanical or chemical properties of which are neither of them clearly ascertained; and we neither know how they are formed or produced, nor in what manner they may be chemically changed.

"I cannot dismiss this subject without observing, that the supposition of a preternatural spissitude of the mass of blood, or as it may be expressed of a lentor, being a frequent cause of disease, has had a great share in almost all the modern systems 
of pathology; but I allege that it has been for the most part hypothetical ; and has hardly, so far as I know, in any case been ascertained as a fact. I am disposed to maintain, that the supposition is for the most part improbable. The functions of the economy, depending upon the constant motion of fluids through many narrow canals, require that those fluids should have a very great fluidity; and accordingly Nature has, for this purpose, provided, that a pure water should always make a very-great part of the animal fluids. It is also certain, that those parts whose particles might be disposed to unite together, and to form impermeable masses, are for the most part held in a state of solution, and in a very fluid state; or if there are certain parts which are only in a diffused state, these are in very small proportion to the entirely fluid parts; and while the heat and mobility of the whole continue, the cohesive matters are kept in a very minutely divided state, and diffused amongst the more fluid parts ; and there is not any evidence of their separation from those fluids but in consequence of stagnation. There is therefore little foundation for the supposition of a preternatural spissitude prevailing in the mass of blood, or of its proving commonly the cause of disease." $-M . M$.

CCLX. We would next put the question-By what means the parts of this heterogeneous mass are kept so equably diffused among one another, and the fluidity of the whole so constantly preserved? This we suppose to be done chiefly by motion and heat, and by the parts disposed to concrete being kept from the contact of any matters to which they might adhere more firmly than they do to the other parts of the blood. The diffused parts we suppose to be present only in those vessels in which a considerable degree of agitation is constantly kept up; and we suppose also that the heat always here present both diminishes the cohesion of the gluten, and increases the solvent power of the serosity. Experiments made with neutral salts seem to confirm the latter; and it is also probable that the same solvent power may be increased by a quantity of air that is constantly intermixed with the mass of blood while it remains in the vessels, and is under a constant agitation. It is supposed that an attention to these several circumstances will explain most of the cases of spontaneous separation that occur either in the liv- 
ing or dead body, within the vessels or without them; but the detail would be too long for this place.

CCLXI. We shall add here a few words on the use of this singular composition of animal blood which we have been considering.

It appears evidently, from many circumstances of the animal economy, that its functions require a system of vessels constantly filled and even distended ; but as, at the same time, these vessels must be open by a multitude of their extremities, if all the fluids were such as could pass by these extremities, the system could not be kept filled for a few minutes. It is necessary, therefore, that the fluids should be partly of such a size as that they cannot pass through the smaller vessels, and partly in a diffused state only, which has commonly the same effect. Hence it is, that the red globules, under the ordinary impetus of the heart and arteries, are strictly confined to certain vessels; and it is probable that, in the like circumstances, the diffused gluten does not go much farther. This serves to keep the larger vessels of the system constantly filled; but, on the other hand, the serosity being sufficiently fluid, might be supposed to run off by the many outlets open to it, and thereby to leave the fluids in the larger vessels of a consistence unfit to circulate. This, however, seems also to be obviated by the viscidity of the grosser parts of the blood, sufficient always to entangle so much of the more fluid as may be necessary to preserve the due fluidity of the whole.

CCLXII. The heat of the human body, supported by powers within itself, is probably the effect of the motion of the blood, and might have been treated of when we were considering that subject. But, as many persons suppose it to depend in some measure on the nature of the fluids, we have reserved it for this place, and here, perhaps, to say only, that the question concerning the cause of animal heat is not yet solved.

CCLXIII. The opinion of animal heat's being the effect of mixture, is to be little regarded, as the matters supposed to be mixed, the place in which the mixture is made, and the other circumstances relating to it, are equally hypothetical, and the whole is ill supported by any analogy.

CCLXIV. More speciously is animal heat supposed to be 
the effect of putrefaction, towards which there is certainly some approach in animal bodies; but the opinion is still very doubtful. For, first, the effect of any degree of putrefaction in producing heat is not well ascertained. Secondly : It is not supported by any analogy, that putrefaction, in the degree to which only it proceeds in living bodies, is capable of producing the heat appearing there. And, lastly, whatever is the degree to which putrefaction proceeds in living bodies, it does not appear that there is any increase of heat correspondent to the increase of putrefaction, and rather the contrary.

CCLXV. The suppositions either of mixture, or of putrefaction, as the cause of animal heat, are both of them rejected by this, that the generation of heat in animal bodies is manifestly dependent on another cause, that is, the motion of the blood. For the power of generating heat in any animal is not perfect, till the motion of the blood in it is fully established; and, when the generating power is established, we perceive the heat to be increased or diminished, as various causes increase or diminish the motion of the blood. In dying animals, the heat grows less as the motion of the blood grows less; and when at death this ceases altogether, the heat ceases also, commonly, at least, as soon after death as we can suppose a body of the same bulk to lose the heat it had acquired.

CCLXVI. This connexion between the heat and motion of the blood seems in general to be well proved; and, though it may be difficult to reconcile certain appearances to it, we would so far admit of the supposition as to inquire, in the next place, into the manner in which the motion of the blood may generate heat.

CCLXVII. On this subject the most common opinion is, that the heat is produced by the attrition of the particles of the blood upon one another, or of these on the internal surface of the vessels in which they move. But we cannot find any analogy to support either the one or the other supposition.

The attempt made to support the latter supposition, by endeavouring to shew that upon this the equality of heat in the different parts of the same body is well explained, deserves little regard, as it is founded on doubtful principles and mistaken facts. 
CCLXVIII. The equality of heat in the different parts of the same body seems to require the generating power to be very generally diffused over the whole; but it does not seem to require its being precisely equal in every part, as the interposition of pretty large vessels in every part of the body, and the speedy communication of the fluids from any one part to every other, will sufficiently account for the equality of heat, though the generating power should be in some measure confined to certain parts only.

However we take no notice of the suppositions which have been made of the generating power's being confined to certain small portions of the system only. These suppositions give no. relief in the general theory, and they are not supported by any particular evidence. The breathing animals are the warmest; but that they are warmer because they breathe, is not more probable than that they breathe because they are warmer.

CCLXIX. With respect to this theory, which deduces animal heat from the motion of the blood, we must own that it is. attended with several difficulties. It will be difficult to show, in so many animals of different age, size, and temperament, in which the degree of heat is nearly the same, that the motion of the blood, in all its circumstances, is also exactly the same; or to show, in the different animals in which the degree of heat is considerably different, that the motion of the blood in each is correspondent to the difference of heat. May it not be supposed that there is some circumstance in the vital principle of animals which is in common to those of the same class, and of like economy, and which determines the effect of motion upon, the vital principle to be the same, though the motion acting upon it may be in different circumstances?

CCLXX. In all we have hitherto said of animal fluids, we have considered the common mass of blood as consisting of three parts or three kinds of matter only; but many more have been supposed to be present in it; and we shall inquire upon what ground.

It is common to suppose that the aliment or the chyle formed of it is not perfectly assimilated in passing once only through the lungs; but that, for some time after such passage, it continues to circulatc with the blood under the same form and of 
the same qualities which it had when it first entered the subclavian, and particularly in this state to furnish the milk which is secreted in the breasts of females. There is, however, no proper evidence of the chyle's ever appearing in the blood-vessels, and the appearances of it alleged can be otherwise accounted for. The arguments, for the same opinion, which are drawn from the consideration of the secretion of milk, are embarrassed with many difficulties.

CCLXXI. It is probable that the animal fluid (CCLV. CCLVI.) is in a constant progress, and hardly for a moment stationary, or therefore uniformly the same over the whole of the common mass. Some part of it is that which was last formed, and therefore the nearest to the vegetable matter from which chiefly it was produced; while another part of it is that which has remained longest in the body, and is therefore the nearest to putrefaction. Between these two there may be several intermediate states, which, however, like the nearest shades of the same colour, are not distinguishable by our senses or experiments.

CCLXXII. Besides the difference of matter arising from the progress of the animal fluid, there have been other matters. supposed present in the common mass, and as commonly constituent parts of it. Such are a mucous matter, like to the mucous matter of vegetables; and a gelatinous matter, like to that which is extracted by decoction from the solid parts of animals. But there is no evidence of either being formally present in the mass of blood; and the supposition is founded on mistaken facts and false reasonings.

CCLXXIII. But it is proper to be observed here, that many extraneous matters may, by different ways, be introduced into the blood-vessels; and that many of the secreted fluids, sometimes very different from any thing that existed before in the mass of blood, may, by absorption or regurgitation, be again taken into the blood-vessels. But, with regard to all of these, whether extraneous matters or those produced in the body itself, it is probable that hardly any of them enter into the mixture of the animal fluid, and that they are only diffused in the serosity till they can be again thrown out of the bloodvessels by the readiest outlets. The oil of the adipose mem- 
brane is frequently, and perhaps necessarily reabsorbed, and seems to be, besides the lymph, the only reabsorbed matter which enters again into the mixture of the animal fluid.

"From the several remarks we have offered respecting the serosity, it clearly enough appears, that there is a portion of the mass of blood which is always in a saline and acrid state; and nothing has been more common among physicians than to suppose that an acrimony of the fluids is a frequent cause of disease.

"It is very possible that it may be so, and upon many occasions it certainly is so ; but it appears to me that the supposition has been too rashly and too frequently admitted, and that it has been for the most part purely gratuitous, without any proper evidence of it in fact. The possible species of acrimony have been little understood, and several of them altogether erroneously supposed. Others of them, though possibly occurring, have not been shown really to take place in any unusual quantity; and the phenomena adduced in proof of them may be commonly explained from other causes, and are certainly often produced by causes of a different and even contrary nature.

"With respect to the various acrimony which we have allowed to be constantly present in the serosity, it is probable, that upon different occasions, it may be in greater or lesser quantity; but for this very reason of its being constantly present, we must conclude that it does not stimulate the system very strongly. This happens partly from these acrids being constantly diffused in other mild fluids, partly from the arterial system not being sensible to such a stimulus, and partly from these acrids stimulating the secretory and excretory organs to a larger excretion, whereby they are by one or other excretion immediately thrown out of the body. From these considerations, I conclude that a spontaneous and noxious acrimony does not frequently arise; for the greatest part of mankind pass their lives without feeling any of the effects that might be imputed to it: and though there are some instances of its operation, these are very rare, and commonly in consequence of unusual and violent circumstances in which the body has been placed.

"With respect to acrid matters introduced into the body from without, there is no doubt that many of these are sufficiently 
powerful in disordering the system; but there are certainly also many thrown in that have no effect at all : for, not to mention the various precautions which nature has taken to prevent their reaching the mass of blood, I think it is only necessary to observe, that even when in the mass of blood, they are rendered innocent, by their being joined with, and diffused in the serosity, and with it soon thrown out by one or other of the excretions; so that some of the most acrid, as mercury and cantharides, show their effects only in the secretory or excretory organs.

" Upon the whole of this subject, therefore, I would conclude, that the supposition of an acrimony as the cause of diseases, has been too frequently admitted in our modern pathology; and that it ought not to be admitted, unless when the causes and existence of it are well ascertained.

"To conclude, I will not deny that the state of the fluids may have a share in distinguishing the different states of the body both in health and in sickness: but at the same time I must maintain, that we know little of the manner in which it may have this effect; that our theory of the human fluids is still very incomplete and imperfect; that, while in this condition, it has been employed too rashly and too largely in every part of the system of physic; and that we have little temptation to do this, as it is highly probable that the state of the fluids depends very much upon other circumstances of the constitution, which are more fundamental, and more powerful in determining the several conditions of it." $-M . M$.

CHAP. III.-OF SECRETION.

CCLXXV. After thus considering the parts of the mass contained in the red vessels, we must next consider the several fluids which appear in the other parts of the body.

CCLXXVI. All of these we suppose to be derived from the common mass, as they appear in vessels continuous with those of the common mass, and as their appearance ceases when the communication of the vessels containing them, with the sanguiferous vessels, is any how interrupted.

CCLXXVII. The fluids thus derived from the common 
mass seem to be produced in consequence of a certain structure, with perhaps some other condition in the extreme vessels through which the fluids pass; and a part having such a structure, is called a gland or secretory organ, the function of which, from the most obvious notion of the manner of it, is called secretion.

CCLXXVIII. The structure of the organ, and the manner of its function, seem to me for the most part unknown; at least, what we know or suppose with regard to the structure hardly in any case applies to the explanation of the function.

CCLXXIX. If it any how appeared that the several secreted fluids were all of them previously existent in the same forms in the mass of blood, it would not perhaps be difficult to explain what might be strictly called a secretion. But such previous existence does not appear ; for, except the matter of exhalation into the several cavities of the body, and the matter of urine and of perspiration, we find no proper evidence of any other secreted fluids present in the mass of blood. We cannot find there, either milk, mucus, or oil, and much less the appearance of many other fluids, which are only found after they have passed through certain organs.

CCLXXX. This being the case, the considerations of the physiologists with regard to the velocity of the blood, and other circumstances favouring the separation of the parts of a fluid which are only diffused among one another, deserve no attention. The effects of different apertures may go some length; but we can perceive their particular application only in the few cases of a simple separation. In most others there appears to be a change of mixture; but we perceive neither the precise changes that are made, nor the cause of them.

CCLXXXI. Till we can discover these more clearly, we may in the mean time observe, that the actions of the vessels of the secretory organ have a considerable share in determining both the quantity and quality of the secreted fluid, and that both very often are very little affected by the general state of the circulation, or by the different conditions of the mass of blood.

CCLXXXII. It would seem that no other secretion but those of perspiration and sweat are manifestly increased by the 
increased action of the heart and arteries (CLXXXI.), and that most of the other secretions are increased only by stimulants applied to their organs. These stimulants may be either such as are immediately applied externally or internally to the excretory, or perhaps to the secretory vessels, or they may be such as are applied to the sensorium, or to distant parts of the nervous system, which by the laws of the animal economy have a connexion with the organs of secretion. These stimulants, at the same time that they act in either of these ways on the secretory organs, for the most part have no sensible effect on the general state of the circulation of the blood.

CCLXXXIII. With respect to the influence of the condition of the common mass of blood upon the several secretions, we presume that the state of the quantity of the fluids in general will affect the quantity of every secretion; but the effects of the quantity of the whole mass are very remarkable only with respect to the secretions of perspiration, urine, and milk.

The qualities of the common mass may also be presumed to affect the several secretions; but the effect of these qualities appears most remarkable in the same secretions of perspiration, urine, and milk; and even in these the effect seems to depend upon the proportion of water more than upon that of any other matter in the common mass. With respect to the other secretions, we cannot perceive that any of them are increased by a particular matter present in the mass of blood, except it be such a matter as stimulates the secretory organ.

CCLXXXIV. The several secretions are frequently observed to affect each other mutually, so that the increase of one diminishes another, and vice versa. This seems to depend either upon a change of determination in the course of the blood (CLXXVIII.), or upon a change in the state of the fluidity of the common mass, or perhaps upon a connexion established between the different organs of secretion as parts of the nervous system ; and, except it be in the case of perspiration and urine, we cannot perceive that the effect of the state of one secretion upon that of another, depends upon an increase or diminution of any particular matter in the mass of blood.

CCLXXXV. After mentioning these generalities with respect to secretion, we should, perhaps, proceed in the next place 
to consider the application of them to the particular secretions, and also to consider more particularly the several secreted fluids; but we omit both these subjects, as we presume the former will be obvious from what is already said; and with respect to the latter, we have not yet a sufficient number of experiments to proceed any length in it.

CHAP. IV.-OF NUTRITION.

CCLXXXVI. Under this title we might consider how the matter both of the fluids and solids of the body is supplied; but, after what we have formerly said of the taking in and assimilation of the aliment, we have nothing now to add with respect to the supply of the fluids; and we therefore confine ourselves here to consider in what manner the solid parts obtain their increase of matter and growth, or have their occasional waste repaired.

CCLXXXVII. There is no doubt that the solids are formed of the fluid prepared from the aliment in the manner we have said; but it is required now to say what portion of the fluids is employed in nourishing the solids, by what channels the nourishment is conveyed to them, and, being applied there, how from fluid it becomes solid.

CCLXXXVIII. With regard to the first question, we have no doubt in asserting, that in oviparous animals, it is the albumen ovi that is employed in nourishing the chick; and we presume that it is an analogous fluid which is employed in nourishing the bird during the whole time of its growth. We think the analogy may be safely applied with respect to all animals, the solid matter of which is of the same kind with that of the oviparous.

CCLXXXIX. This analogous fluid we take to be the gluten of the blood, properly diluted and freed from any adhering saline matter.

CCXC. To determine in what manner this nutritious fluid is applied to the nourishment of the solids, it is necessary to consider, what are the simple fundamental solids of which all the others are formed.

CCXCI. It seems to be the opinion of the greater part of vol. I. 
modern anatomists, that the solid parts consist entirely of a cellular texture, of various density in the different parts; and, indeed, the structure of the greatest part of the solids is evidently of this kind. But, at the same time, it is also true, that a fibrous structure is to be observed almost everywhere in the body. It appears in the medullary substance of the brain and nerves, in the muscles and tendons, in the arteries, in the excretories of the glands, in the lymphatic vessels, in the alimentary canal, in the uterus and bladder of urine, in the ligaments, in most membranes, and it is to be seen in those membranes which are afterwards changed into bones, especially whilst this change is going on.

CCXCII. From this view of the universality of a fibrous structure in animal bodies, we are disposed to believe, that these fibres are the fundamental part of animal solids, that they are the primordial staminal part of animal bodies, and that the cellular texture is, for the most part, an accretion formed upon these fibres.

The consideration of the structure and growth of vegetables seems to illustrate and confirm this opinion.

CCXCIII. At the same time, from the fibrous parts (CCXCI.) being evidently, in most instances, parts of the nervous system, and from the gradual formation of the fœtus, in which the nervous system is first formed, we think it probable, that the whole of the fibres in the different parts of the body, are a continuation of the nerves; and this again will lead to the conclusion, that the nourishment of the soft and homogeneous solid everywhere is conveyed to it by the nerves.

CCXCIV. This supposes also, what is otherwise probable, that the cortical part of the brain, or common origin of the nerves, is a secretory organ, in which the gluten of the blood, being freed from all saline matter before adhering to it, becomes fit for the nourishment of the solids, and being poured in a sufficiently diluted state upon the organ of the nerves, it is filtrated along the fibres of these, and is thus conveyed to every staminal fibre of the system. We suppose, at the same time, that the medullary, or what may be called the solid matter of the nerves, is in the living body constantly accompanied with a subtile elastic fluid, which fits them for being the organs of sense 
and motion, and which, probably, is also the means by which the nutritious fluid is carried on in the substance of the nerves, from their origin to their extremities.

In what manner the nutritious fluid, thus carried to the several parts, is there applied, so as to increase the length of the nervous fibre itself, or to form a cellular texture upon its surface ; and in what manner, from fluid, it becomes solid, we cannot explain; nor can these particulars be explained upon any other supposition that has been formed with respect to nutrition.

CCXCV. It is probable that for a certain time, at its first beginning, the growth of animal bodies proceeds in the same manner as that of vegetables; but it is evident that, at a certain period in the growth of animals, a different economy takes place, and that afterwards the growth seems to depend upon an extension of the arteries in length and wideness by the blood propelled into them by the powers CLVI.-CLIX. It may be supposed that this extension of the arteries is applied to every fibre of the body, and that, by the extension of these, it gives an opportunity to the application and accretion of nutritious matter; to the growth, therefore, of the fibre itself, and to the growth of cellular texture on its surface. Perhaps the same extension of the arterial system gives occasion to the secretion of fluids, which, poured into the cellular texture already formed, according to the disposition of these fluids to concrete more or less firmly, gives the different degrees of density and hardness which appear in different parts of the body.

CCXCVI. By this extension of the arterial system, the several parts of the body are gradually evolved, some of them sooner, others later, as by the constitution of the original stamina, or after occurrences, they are severally put into the conditions CLXXVII. and CLXXVIII., by which they are more or less exposed to the impetus of the blood, and fitted to receive a greater quantity of it. But as the parts by these causes first evolved will increase the most in the density of their solid parts, they will therefore more and more resist their further growth, and, by the same resistance, will determine the blood with more force, and in greater quantity, into the parts not then so far evolved. Hence the whole system will be at length evolved, and every part of the solids will, in respect of density and re- 
sistance, be in balance with every other, and with the forces to which they are severally exposed.

CCXCVII. The extension of the arteries (CCXCV.) depends upon the resistances which occur to the free transmission of the blood through them, as in CLXX., and further, from a resistance in the veins. For, as a considerable portion of the blood, by CCLXI., does not commonly pass into the smaller branches of the arteries, but must pass very entirely into the veins, so these, by their capacity constantly diminishing as they approach nearer to the heart, and by their coats being of a density and firmness sufficient to prevent further dilatation, considerably resist the free passage of the blood from the arteries into them.

CCXCVIII. While these resistances continue, the arteries, and with them almost every fibre of the body, must be extended at every systole of the heart, and with this extension, the growth of every part will proceed; but, as every.part, by its receiving an addition of solid matter, becomes more dense and rigid; so it is less easily extended, and, perhaps, less readily receives an accretion of new matter than before. Hence it is, that the more the body grows, it admits of any additional growth more slowly; and, unless the extending powers increase in the same proportion with the increasing density of the solids, there must be a period at which these two powers will balance each other, and the growth will proceed no farther. But as it is evident that the bulk and weight of the heart, and probably, therefore, its force, does not increase with the increasing bulk of the body, and that the action of the heart is the principal extending power in the system, it is also plain, that the extending power does not increase in the same proportion with the increasing density of the solids; and, therefore, that these two powers will, at a certain period, come to balance each other.

CCXCIX. But not only is the force of the heart thus constantly diminishing with respect to the resistance of the arteries, but the force of the heart, though it were still subsisting, has, from other causes, less effect in extending the arteries. The blood is more confined to the arteries, and extends them further in proportion to the resistance in the veins, as in CCXCVII. ; and this resistance in the veins, and the extension of the arte- 
xies depending upon it, will be more or less, according to the respective density of these two sets of vessels. But it appears from the experiments of Sir Clifton Wintringham, that the density and firmness of the veins, with respect to their correspondent arteries, is much greater in young animals than in old; and thence it appears, that, during the growth of animals, the arteries are requiring an increase of density in a greater proportion than the veins are at the same time; and, therefore, that the resistance in the veins with respect to the arteries, must be constantly diminishing; that the veins will therefore receive a greater proportion of blood ; that in the same proportion the arteries will be less extended; and, lastly, that the diminished resistance in the veins, concurring with the diminished force of the heart, will the sooner bring the increasing rigidity of the arteries, and, therefore, of every fibre of the body, to be in balance with the extending powers; at least, so far as to prevent their producing any further growth.

CCC. This account of the change of the resistances in the arteries and veins, with respect to one another, is agreeable to phenomena, which shew that the arteries are larger, and contain more blood in proportion to the veins in young animals than in old; that arterial hæmorrhagies occur most frequently in young persons, and that congestions in the veins, with hrmorrhagies, or hydropic effusions depending upon them, occur most frequently in old age.

CCCI. It is probable, that the resistance both of arteries and veins goes on increasing, while the force of the heart is not increasing at the same time; but it appears also, that from the diminished force of the heart, and the compression which the smaller vessels are constantly exposed to from the distention of the larger, from the action of the muscles and other causes, the number of small vessels, and therefore the capacity of the whole system is constantly diminishing so much that the heart may still, for some time, be sufficient for the circulation of the blood. But, while the resistances in the vessels are constantly increasing, the irritability of the moving fibres, and the energy of the brain, are at the same time constantly diminishing; and therefore the power of the heart must at length become unequal to its task, the circulation must cease, and death ensue, 
CCCII. The unavoidable death of old persons is thus, in part, accounted for ; but it is, however, still probable, that the same event proceeds chiefly from the decay and total extinction of the excitement or vital power (CXXXVI.) of the nervous system, and that from causes very much independent of the circulation of the blood, and arising in the nervous system itself, in consequence of the progress of life. This seems to be proved by the decay of sense, memory, intellect, and irritability, which constantly takes place, as life advances beyond a certain period.

\section{OF TEMPERAMENTS.}

\section{(Extracted from the Treatise of the Materia Medica.)}

IN attending to the great number of circumstances in which the bodies of men may be different from one another, it is scarcely possible to enumerate every particular; but it has been at all times presumed, that a great number of these circumstances are commonly combined together in the same person; and that frequently one man shows a combination of circumstances not only different, but sometimes of an opposite kind to that of another. Such combinations, upon a particular supposition with respect to their causes, the ancients named temperaments; and the term has continued to be employed in the schools of physic from the most ancient to the present time.

Abstracting from all theory, we continue to employ the same term to denote a combination or concurrence of circumstances which happens in certain persons, but which in several respects is different from the combination that happens in certain others. Upon this footing, I believe the ancients distinguished what they called the different temperaments of men : for it is probable that at first they distinguished them by actual observation; but very soon they formed a theory with regard to them, from whence they formed appellations which have continued to be applied to them ever since. The appellations indeed have been continued, though the theories which laid the 
foundation of them have been long ago exploded; and the moderns have neither by observation extended the ancient distinctions, nor, though they have often attempted it, have they ever given, so far as I can judge, any happy explanation of the causes or foundation of the distinctions they have so generally adopted. I believe it will be generally allowed, that this part of medical doctrine is still in an embarrassed and undetermined state.

In treating the subject, philosophy would require that I should, in the first place, distinguish temperaments, by marking the external and observable circumstances which are found with some steadiness to be commonly combined together: but this I find a difficult task; and what my observation has not been so extensively applied to as to enable me to perform in the manner I would wish. I must therefore proceed in another way; and shall endeavour to consider those circumstances of the internal state of the human body which may give occasion to a difference in the state of the functions, and even in the external appearances which distinguish different men.

These circumstances may, I think, be referred to five general heads, according as they occur, 1st, In the state of the simple solids (See Physiology, VII.-XXVI.); 2dly, In the state of the fluids (ibid. CCXLIV.-CCLXXIV.); $3 d l y$, In the proportion of solids and fluids in the body ; 4thly, In the distribution of the fluids (ibid. CLXXX.); and, 5thly, In the state of the nervous power, (ibid. XXVII.-CXLV.)

OF PARTICULAR TEMPERAMENTS.

Temperaments, however, are not to be distinguished by attending to any one of these chief circumstances alone; for the state of any of these is commonly combined with a particular state of all the others; and it is only by a combination of the particular states of the chief circumstances in the same person, that the temperaments are to be properly distinguished. To explain this, we presume, that in any one person a particular state of the simple solid is pretty constantly combined with a particular state of the fluids, with a particular state of the dis- 
tribution and proportion of these, and all these with a particular state of the nervous system; and as such a combination may be formed in another person, but consisting in a difference of the particular states of each of the chief circumstances, this will give a different temperament in these two persons. So far, therefore, as we can find such combinations to be steadily formed in any particular person, we shall be able to assign his particular temperament.

It must, however, be acknowledged to be uncertain how far certain states of the chief circumstances of the economy are steadily connected together, and, therefore, how far we can extend our doctrine of temperaments to a great number of different men; but at the same time, it is only by presuming upon a certain steadiness of these combinations, that we can go any length in explaining the difference of temperament.

The ancients very early established a distinction of temperaments, which the schools of physic have almost universally adopted ever since, and which appears to me to be founded in observation. I am very much of opinion, that we can perceive a combination of a particular state of the chief circumstances of the economy to take place very steadily in certain persons, and thereby to form at least two of the temperaments assigned by the ancients. Accordingly, the circumstances in which these two temperaments seem to consist, we shall now endeavour to explain; and I shall hereafter consider how much farther we may proceed.

In doing this it will be proper, in the first place, to mark out the several external appearances that concur in the same person ; and from which concurrence taking place in many different persons, we are led to presume in these, one and the same combination or temperament.

Qne to be particularly mentioned, is that temperament which the ancients, and which physicians at all times since have distinguished by the appellation of the Sanguine. In this, the external appearances are the following: The hair soft, and never much curled, is of a pale colour, or from thence passing through different shades to a red; the skin is smooth and white; the complexion ruddy; the eyes commonly blue; the habit of 
the body soft and plump, after the period of manhood disposed to obesity, and at all times readily sweating upon exercise; the strength of the whole body is moderate; and the mind sensible, irritable, cheerful, and unsteady.

Before going further, it is necessary to observe, that as no exact measure can be had of the different degrees in which the qualities.we are to mention take place, I suppose a middle state very nearly ascertained by observation; and $I$ am unable to give any other measure of qualities than merely by marking them as below or above the middle state,

Upon this footing, I would explain the sanguine temperament as consisting in the following state of the several chief circumstances of the economy. I suppose the simple solids to be lax, the mass of blood to be of a moderate consistence, the red globules and serum to be in large proportion, and the serosity to be of moderate acrimony. I presume the heart to be active, and rather strong with respect to the system of bloodyessels; the quantity of blood in the arteries large with respect to that in the veins; and the quantity of fluids in the whole body large in proportion to the quantity of solid; the state of the nervous system to be sensible and irritable, but in every state readily changeable. This temperament is most exquisite from the time of puberty to that of manhood, but continues its character in some measure throughout the whole of life. This temperament is liable to hæmorrhagy, inflammation, and hysteria ; and with the ancients made the temperamentum calidum et humidum.

The other temperament distinguished by the ancients, which I can characterize most distinctly and explain most clearly, is that which has been very constantly named the Melancholic. In this, the external appearances are the following :The hair is hard, black, and curled; the skin is coarser, and of a dun colour, with a corresponding complexion; the eyes very constantly black; the habit of the body rather hard and meagre; the strength considerable; the mind slow, disposed to gravity, caution, and timidity, with little sensibility or irritability, but tenacious of all emotions once excited, and therefore of great steadiness. In this temperament, I judge the simple 
solids to be firm and dense; the mass of blood to be of a thicker consistence; the gluten abundant; the red globules and serum in moderate quantity, and the serosity more acrid; the heart rather torpid, but strong; the quantity of blood in the veins large with respect to that of the arteries; and the quantity of fluids in the whole system moderate in proportion to the solids; the state of the nervous system to be, as expressed above by the state of the mind, that is, less sensible and irritable, but strong and steady, and disposed to admit the reflex sensations of sadness and fear. This temperament is most completely formed in advanced life ; but the characters of it appear often very early. It is liable to melancholia, hypochondriasis, mælena, and hæmorrhois; and with the ancients made the temperamentum frigidum et siccum.

These are the two temperaments we can the most clearly distinguish; because they are almost in every respect the opposites of each other.

With respect to both, I think some illustration may be obtained, from considering what happens to every person both in the body and the mind during the progress of life. Of these changes I have already spoken pretty fully, when treating of the strength and weakness of the nervous system. From the circumstances there pointed out, it will appear, that those which chiefly determine to a sanguine temperament, occur especially in the first part of life; and that those which determine to the melancholic, as certainly occur in the after parts of it. Accordingly, from the effects we may conclude to the causes, especially when at the same time the existence of such causes is clearly ascertained; and therefore we may venture to asssert, that the changes which happen in the course of life do well illustrate the doctrine laid down respecting these two temperaments, the sanguine and the melancholic.

I have thus endeavoured to explain the different states of the human body, by referring them to two general states or temperaments, which not only serve to distinguish the most part of men through the whole of life, but also to distinguish the different sexes, and likewise the state of particular persons as they pass through the different ages of life. Our doctrine, 
therefore, will apply very extensively; but perhaps it may not seem to be very readily applicable to that great variety which would appear to take place in the human constitution.

With a view, therefore, to attempt some explanation of this variety, we shall remark, in the first place, that it may in some measure depend upon the two temperaments, which we have supposed chiefly to prevail, being seldom perfectly formed; or, in other words, upon the particular state of the circumstances in which they consist being seldom found in the most complete degree. For example, it is seldom that in the sanguine the simple solid is the most lax, or in the melancholic the most rigid that is compatible with health. There is reason to suppose, that from the medium state of density and firmness in the solid, there may be various intermediate degrees between the most lax upon the one hand, and the most rigid upon the other; and supposing that with each of these intermediate degrees, there is united a corresponding state of the nervous power, there may then be so many intermediate and seemingly varying temperaments, neither completely sanguine nor melancholic, though always approaching to the one or the other. This may, in some measure, explain the varieties in the temperaments of men, but it may be justly doubted if it will account for the whole.

It will therefore be proper, in the second place, to observe, that it is doubtful if the circumstances of the economy are always in the same proportion to one another that has been above supposed. For example, we have supposed that the density and the mobility of the nervous power are always in a certain proportion to one another: but this is not very certainly the case; and if we may suppose, as seems to be allowable, that in two persons, the density being equal, the mobility may be greater in the one than in the other; so that if this should happen, it will be obvious that it might give a more exquisite formation of the sanguine, or a more moderate state of the melancholic temperament. In this way it is possible, that with a certain degree of density greater than usual in the sanguine, there may be a mobility greater than in proportion to this; we shall then have a middle temperament between the sanguine and the melancholic, and perhaps what the ancients meant to denote by the title of 
Choleric; that is, of more strength than in the sanguine, and of more irritability than in the melancholic. It is possible also, that there may occur a simple solid more dense than usual in the sanguine, and at the same time, from a more humid state, of greater flexibility than in the melancholic; and if, along with these, there may be an analogous state of the medullary fibre, of less mobility and elasticity in proportion to the density, we shall then have that temperament which the ancients expressed by the title of Phlegmatic; that is, with less sensibility and irritability, but with more strength and steadiness than in the sanguine, and at the same time with more laxity and more mutability than in the melancholic.

In the whole of this discussion, we have considered the state of the nervous power as chiefly modifying the temperaments of men; and more readily enter into this supposition, because we presume the state of the nervous power to be almost always attended by a corresponding state of the simple solid, and that both these together pretty constantly modify the state of the fluids, both with respect to their quality, their proportion, and distribution.

Of these latter circumstances, however, constantly following the state of the simple solids and of the nervous power, I am by no means certain. As we observe, that at different periods of life there is a difference between the arteries and the veins as to the circumstances of density and capacity, so it is possible that some difference in these respects may be established in the original stamina, and may therefore, in some degree, run throughout the whole of life, and thus vary the state of the fluids. It is also possible, that there may be conditions of the original stamina determining a difference in the strength and activity of the heart with respect to the capacity of the blood-vessels; or, on the other hand, the state of the heart being given, there may be a difference in the density and resistance of the sanguiferous system. In all these cases, there may arise a difference in the quality, proportion, and distribution of the fluids, and, thereby, a further variety in the temperaments of men; and thus, perhaps, we may account for the difference of stature, bulk, and proportion, of the several parts of the body in different persons. 
All this might be illustrated more fully; but perhaps we have insisted long enough upon what may by many be thought perhaps too much depending upon conjectural reasonings. It no doubt may, in some respects, be liable to this imputation; but I should fain hope that it may serve to lay the foundation of speculations which must be pursued before we can, explain the important and therefore necessary doctrines concerning the temperaments of men.

\section{OF IDIOSYNCRASIES.}

The term Idiosyncrasies has been confounded with that of temperaments; but I mean here to express by it those conditions of certain persons whereby certain functions of the whole or of particular parts of the body are affected by applications made to them, very differently from what these functions are affected in others, and very differently from what they are in persons seemingly of the same general temperament.

Of these idiosyncrasies, the greater part of them seems to me to consist in a preternatural degree of the sensibility or irritability of certain parts of the system, or in a peculiar sensibility or irritability of the whole body, or in particular parts of it, with regard to certain applications, and to those only.

Of such idiosyncrasies, those that have been the most taken notice of are those which occur with respect to the effects of taste and odour. Tastes are of considerable variety, but they are reduced to certain classes and orders, in which the most part of men are so well agreêd, as to show that the operation is nearly the same in all of them. This certainly happens with respect to the simple sensation; but with respect to the reflex of agreeable or disagreeable, this is often considerably different in different persons, and shows that there is room here for an idiosyncrasy, which accordingly takes place ; and there are many instances of it in the records of physic.

The instances, however, of a peculiar aversion in particular persons to certain odours, are much more frequent. The records of physic are full of them; and examples of them are known almost to every body. The sensations arising from odour seem to be more various in different men than those arising from taste; so that mankind have hardly established any 
other distinction of the former than that of agreeable or disagreeable. Subdivisions have been attempted, but with no consent of mankind so as to be expressed with any precision in common language. The sensation, therefore, is probably much varied in different men, and gives room for idiosyncrasies, which accordingly appear without our being able to refer them to any particular classes or orders of odours; and the effects are not less remarkable by the operation of the same odour upon different persons, than by its being so powerful in its degree, producing syncope, hysteria, and epilepsy.

These peculiar effects of sensations are manifestly extended to the alimentary canal. In this, and particularly in the stomach, the sensibility is not correspondent to the general sensibility and irritability of the whole system; for there are instances of strong persons moved by very small doses of emetics; whilst, on the other hand, there are seemingly weakly persons who are not moved but by very large doses of the same.

There are instances of sensibility in the stomach that are peculiar to certain persons, and appearing in few others. But I must acknowledge, that with regard to several of these idiosyncrasies, it is not easy to determine whether their effects depend upon an impression made upon the nerves of the stomach, or upon a modification which these substances give to the fermentations and solutions that take place there. (See page 53.)

In any attempts to account for these peculiarities, it ought to be kept in view, that the stomach is not only affected by sensations depending upon impression, but likewise by those which depend upon consciousness, or a perception of the state of its own action; and that undoubtedly many of its sensations are of the latter kind.

It does not seem necessary to enter upon the consideration of the idiosyncrasies of the intestinal canal, as they are to be explained from the same degree of sensibility that may be peculiar here as in the stomach. What farther may arise from a peculiar state of the bile, or other fluids poured into the intestines, we cannot pretend to judge. The various state of the alvine excretion depends upon many different causes which there is no place for considering here; but it is most likely that some of 
these causes may be more considerable and peculiar in certain persons than in others, and give an idiosyncrasy in that respect. A torpor, or slower motion of the intestinal canal, is especially to be suspected.

We have thus endeavoured to mark out the various cases of idiosyncrasy; and although perhaps we may not have done it completely, yet it is hoped enough has been said to show, that in the employment of remedies a physician must be directed by the consideration of idiosyncrasies as well as by the general temperament.

In the case of any person, therefore, occurring to a physician for the first time as a patient, particular inquiry should be made respecting the idiosyncrasies which may prevail in his constitution; and if he himself should happen to have had no experience of the effects of particular applications, the idiosyncrasies of his parents should next be inquired after; for idiosyncrasies are very often hereditary.

We have thus attempted to point out the various states of the human constitution that may be found more constantly different in different persons; but it will be proper now to remark, that these constitutions may be variously modified by those circumstances of climate, diet, exercise, and the like, to which men may be exposed in the course of life, and which it is well known have a great power in changing the natural constitution into one not only very different, but perhaps even opposite. It is therefore well known that a physician, in practising upon the human constitution, either for the preserving of health or for curing diseases, must not only consider the temperaments and idiosyncrasies which nature has originally given to the constitution, but must also consider the accidental states of it, which may have been produced by the circumstances and manner of life.

It is, however, not my business here either to explain those various accidental states, or to assign their causes; although it might be proper enough to lay a foundation for that doctrine by explaining the powers of custom and habit in general, as I formerly endeavoured to do in my lectures on the Materia Medica. It does not, however, appear to me necessary to do it now, be- 
cause, for a pretty full information on this subject, I can refer to a Dissertation de Consuetudine, published some years ago by my son Dr. Henry Cullen; and another edition of which I hope he will soon give in a still more complete form in the English language. 


\title{
SY NOPSIS
}

\section{NOSOLOGIA METHODICA,}

\section{EXHIBENS \\ GENERA MORBORUM}

\section{PRACIPUA DEFINITA,}

ADDITIS SPECIEBUS

CUM HARUM EX SAUVAGESIO SYNONYMIS,

\author{
A \\ GULIELMO CULLEN.
}

EDINBURGI-MDCCLXXXV. 


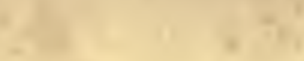

a

,

$11+10+10$

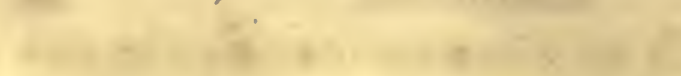

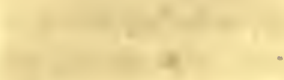

,

-

$$
-1+96 \times 2 \times
$$

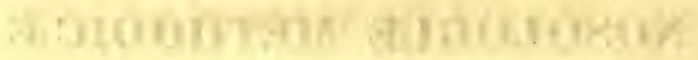

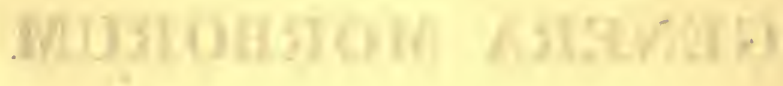

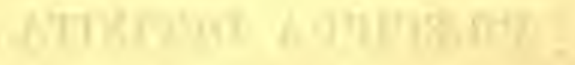

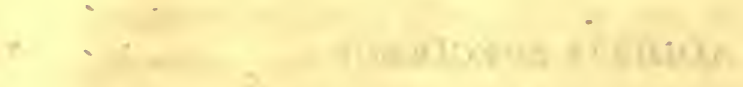

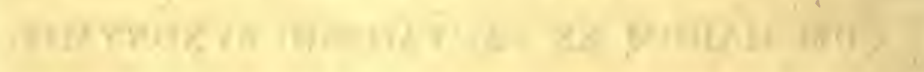
$+$

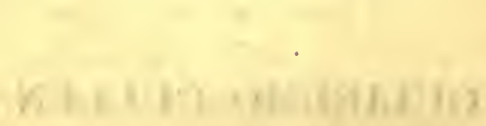

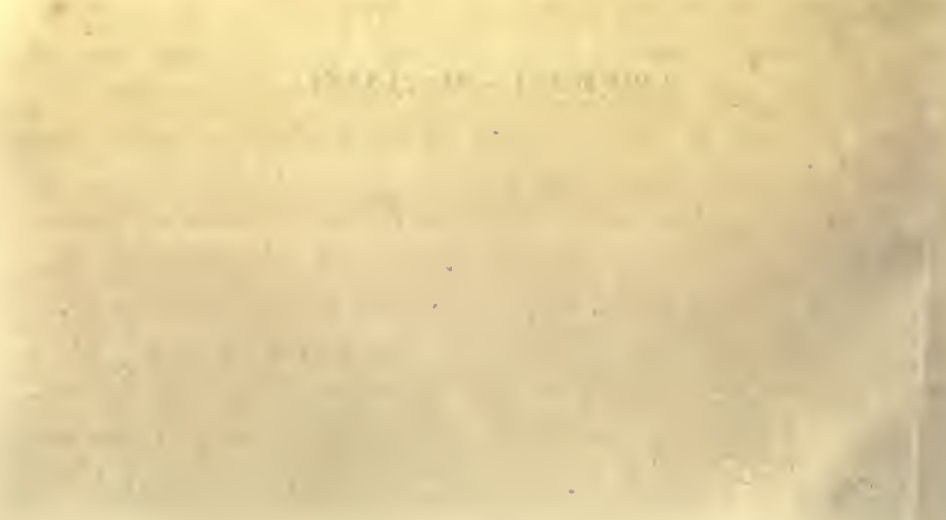




\section{PROLEGOMENA.}

RES est inter medicos satis nota, morbos quosdam natura sive causa proxima diversos, externa tamen facie, sive symptomatis, adeo similes esse, ut alii ab aliis difficillime internoscantur. Cum autem morbi natura diversi remedia diversa, quandoque etiam contraria, postulent, summi est momenti ut medicinam facientes morbum quemque, a quovis alio, certo dignoscant.

Dum medici vero, in ipso artis usu versati, de morbis distinguendis saepe incerti sint, merito queruntur, in scriptis de re medica non semper inveniri, quae hanc ambiguitatem solvere valeant. Saepe quidem observatum est, descriptiones, sive historias, quas vocant, morborum, in scriptis medicorum reperiundas, plerumque mancas et imperfectas esse; et nemo est, ut opinor, qui non facile agnoscat, historias morborum pleniores, magis exactas, et characteribus melius distinctas, quam quas adhuc habuimus, desideratas esse.

Putarunt quidem multi, medicos veteres Graecos et Romanos, in observandis et notandis morborum phaenomenis, diligentes et solertes fuisse, atque in horum scriptis historias morborum complures quae nobis utiles esse possint exstare. Mihi vero videtur, vel ineptam et superstitiosam antiquitatis venerationem, vel quandam eruditionis ostentationem, scriptis medicorum veterum in hac re aestimationem nimiam conciliasse. Mi- 
nime quidem dissimulandum, in scriptis illis quaedam quae morborum characteres vere delineant subinde reperiri. Quae autem ejusmodi ibi occurrunt, quantumcunque a recentioribus aestimata, parum profecto nos docuissent, et attentionem nostram penitus fortassis effugissent, nisi res eaedem nobis ex propria observatione prius innotuissent. Revera, neoterici libros veterum raro, ad primam rerum notitiam comparandam, evolvunt, sed plerumque, ut propria tantum inventa et observationes horum autoritate suffulciant, perscrutantur. Mihi quidem persuasum est, vel historias morborum plerasque, quae in scriptis veterum jam inveniuntur, ad morbos regionis a nostra plurimum diversae pertinere, ideoque nobis parum utiles esse; vel scripta eorum adeo mutilata et corrupta ad nos pervenisse, ut ad usus nostros parum conferre queant. Fatendum saltem, notitiam morborum ex scriptis veterum hauriendam multo minorem esse, quam qua opus est ad illam, de qua diximus, ambiguitatem eximendam.

Nec nisi sero admodum et lente ad hanc rem expediendam progressi sunt recentiores. Seculo enim decimo sexto, Galeni sequaces, in instauranda veterum doctrina, tanta cum laude versati, in horum scriptis explicandis et illustrandis fere solum occupati; parum omnino ad emendandam vel amplificandam morborum historiam contulerunt.

Nec ejusdem aevi medici chemici, Galenicorum adversarii acerrimi, in illa re plus fecerunt. Medicamentis enim inveniendis, et promiscue adhibendis, solum intenti, a morborum studio hominum animos prorsus avertebant.

Hinc quidem factum est, quod ab instauratis literis seculo decimo quinto, usque ad tempora Sydenhami, nullae fere morborum historiae plenae vel accuratae conscriptae sint. Sydenhamus vero, sagacitate et judicio quibus maxime pollebat, majorem in notandis et describendis morborum phaenomenis diligentiam adhibendam esse protinus sensit, et in hunc finem ad sedulam observationem se accinxit, qua plures tandem et meliores, quam antea quisquam, morborum descriptiones protulit.

Ad hujus exemplum, et ad normam methodi, per observationes et experimenta, philosophandi, tunc temporis invalescentis, evenit, ut ab eo tempore plures medici, in historiis morborum augendis et accuratius reddendis, operam collocaverint. 
A tempore igitur Sydenhami in hac re multum laboris nec frustra impensum est ; rem tamen minime adhuc absolutam esse censeo. Multa parum accurata, multa erronea, multa denique prorsus falsa, historiis morborum, quas jam habemus, inesse, novit quisque in his aliquantulum versatus; et novit etiam ex variis causis haec vitia subrepsisse. Scriptores enim, alii ad theorias sibi placentes stabiliendas, alii ad fidem remediis $\mathbf{a b}$ ipsis sive inventis sive multum laudatis conciliandam; vel veritatem, praeconceptis occaecati, non percipiebant, vel etiam fictis et falsis corrumpebant. Plures, re omni quovis modo mirabili capti, quod ipsi facile crediderunt, alios credere cupientes, rem omnem praeter modum amplificarunt. Plures denique, ut observationibus, hodie adeo expetitis, famam sibi compararent, historias in museo fictas pro veris tradiderunt. Haec omnia si specialius indigitarem, de imperfecta hactenus morborum historia satis clare constaret; sed haec omnia impraesentiarum praetermitto, et unum solummodo, in historiis morborum conscribendis, vitium, ad rem nostram maxime pertinens, indicatum volo.

Multi optimae etiam fidei medici, in morbis observandis diligenter versati, in historiis eorum conscribendis saepe nimium fecerunt. Non solum enim symptomata morbi cujusvis semper praesentia et inseparabilia, sed plura etiam, et cuncta quidem quovis tempore morbum comitantia, recensuerunt. Ita symptomata rarius, nec necessario cum morbo conjuncta, et plura omnino adventitia et fortuita, notarunt; dum simul illa inusitata et fortuita $a b$ usitatioribus et inseparabilibus distinguere penitus neglexerunt. Hinc, historiam morborum plenissimam reddere conantes, quae ad distinctionem cujusque ab alio quovis conferre possent, omiserunt, immo difficiliorem reddiderunt.

De hacce in historiis morborum redundantia saepe conquesti medicinam facientes, pathognomonica quae dicuntur desiderarunt; hoc est, ut notentur symptomata illa pauca, quae cuique morbo adeo propria sunt, ut ab illis solis quisque morbus ab alio quovis illico et certo dignoscatur. Hujusmodi autem pathognomonica in scriptis medicis nondum data, neque unicuique morbo assignata sunt; nec quantum video nisi per Nosologiam Methodicam, rite institutam, assignari queunt. 
Dudum quidem Sydenhamus et solers Baglivius, quo facilius et certius morbi distinguerentur, morbos omnes, per normam methodi botanicae, ad genera et species, appositis characteribus idoneis, referri voluerunt : id est, ut institueretur Nosologia Methodica. Hoc etiam consilium alii summae famae medici comprobarunt, et quisque, ni fallor, in artis usu occupatus factum fuisse vehementer optaret. Nihil tamen hujusmodi protinus tentatum fuit; an quidem ex fructu inde capiendo parum perspecto, an vero ex difficultate operis praevisa, non constat ; sed revera nihil omnino tentatum fuit, donec celeberrimus Franciscus Boissier de Sauvages, circa annum 1732, rem aggressus sit.

Quæ quidem, exeunte seculo decimo sexto, Felix Platerus in morbis secundum symptomata ordinandis conatus est, quodammodo huc referri possunt; conatus autem isti inconditi adeo et imperfecti erant, ut quenquam eosdem prosecuturum esse vix expectandum fuerit; et revera ante Sauvagesium nemo, quantum novimus, ulla ex parte prosecutus est. Haud dissimulandum quidem, Platerum Sauvagesio quaedam suggessisse; pauca tamen, nec fausta admodum, et quae nemini usui fuissent, nisi Sauvagesius, cum meliore spe, opus multo longius provexisset.

Ipse quidem Sauvagesius primum lente et titubanter progrediebatur; et, re iterum atque iterum tentata, non nisi post multam lectionem, et assiduam per triginta annos meditationem, Nosologiam methodicam suam, anno 1762 editam, protulit.

Interea, duo alia, in Nosologia methodica, tentamina facta sunt ; alterum a celeberrimo viro, et in distributione rerum methodica plurimum exercitato, Carolo a Linné ; alterum ab erudito Professore Gottingensi, Rudolpho Augusto Vogel. A neutro tamen, utpote vestigiis Sauvagesii presse nimis insistente, opus multum promotum fuisse videtur.

Cum de utilitate hujusmodi tentaminum, jamdudum mihi persuasum sit, simulac ad medicinae praxin docendam in hac alma Academia admotus fui, mei officii esse putavi alumnos nostros ad Nosologiae methodicae studium allicere; et, quo facilius hoc efficerem, libellos quotquot ad id conferre possent, hic edendos, et in discipulorum manus tradendos, curavi.

Ex opere Sauvagesii, aliis rebus non admodum utilibus referto, ea tantum quae ad genera et species morborum distinguen- 
das pertinerent excerpsi, et cum his Linnaei et Vogelii libellos integros conjunctos edidi.

Auctores hi aliquid laudis sine dubio merentur; quanquam enim eorum opera, rem ex toto confecisse minime videantur, nec multum utilitatis tyronibus inexpertis afferre queant; in arte tamen versatis, et in dignoscendis morbis aliquatenus exercitatis, nonnihil commodi exinde oriri possit.

Difficile quidem erit, hanc rem protinus perfectam reddere, nec, ut opinor, nisi tentaminibus repetitis fieri potest. Non solum igitur mihi licitum, sed mei muneris esse, opus hoc Nosologicum, quantum possem, promovere arbitratus sum; eoque consilio methodum quodammodo novam tentavi, et una cum supra dictis edidi. Hanc, etsi nequaquam omnibus numeris absolutam, prioribus tamen in quibusdam magis accuratam inventam fore spero.

Qui ante nos huic studio operam dederunt, minus consulto, ut mihi videtur, rem aggressi sunt; morborum enim species parum perspectas habentes, ad suprema classium et ordinum genera constituenda illico se accinxerunt. A natura vero, species solum datae sunt ; et generum constitutio est mentis humanae excogitatio, quae, donec species omnes bene notae et perspectae fuerint, fallax et incerta erit ; et in generibus quidem constituendis, nisi ad species semper respiciamus, labor noster vanus et futilis evadet.

Constitutio generum supremorum, in quovis rerum systemate, neutiquam adhuc absoluta est. Classes enim vel etiam ordines, omnino naturales, vel in systemate vegetabili, vel in animali, nondum ubique constitutae sunt. De fossilibus methodus etiam difficilior, et de morbis fortassis omnium difficillima erit. Videamus igitur quomodo hanc rem quam optime tractare possimus.

Cum res distinguendae, numero quam plurimae sint, tum ad investigationem, tum ad reminiscentiam, utilissimum et necessarium esse videtur, ut ad genera quaedam suprema' referantur; cum vero species rerum non admodum numerosae sint, et quarum sigillatim recensitarum reminisci, memoriae in plerisque hominibus vires non excedat, eas, summa solicitudine, ad classes et ordines referre vix opus esse videtur.

Ita Illustris De Buffon quadrupedia, quorum species numero paucae sunt, ad classes et ordines referre haud necessarium 
esse censuit, et quae in hunc finem alii tentarunt, frivola esse, et ad denominationem studiosos plurimum turbantem duxisse, putavit.

.. Illustri tamen et egregio viro prorsus assentire nequeo: Etiamsi enim institutio classium et ordinum morborum, neque pro investigatione eorum satis perfecta reddi queat, neque pro numero valde necessaria sit; quodammodo tamen tentandam, et ad majorem accurationem diligenter perducendam esse, puto. - Enimvero, si ab illusione quae ex characteribus classium et ordinum oriri queat, cavemus, et, ni fallor, cavere plerumque licet, etiam classium et ordinum institutionem, in multis ad pleniorem et magis exactam specierum distinctionem conferre posse contendo. Etsi enim ejusmodi institutionem, ubique certam et accuratam semper assequi haud liceat, ipsos tamen conatus ad illam assequendam magnopere ex re fore putarem, quippe qui ad disquisitiones utiles, tum in pathologia; tum in morborum historia, saepe obvenientes, subinde ducant. Certe, cum de natura morborum disquirere velimus, distinguendi sunt, tum: per symptomata cuique propria, tum per illa cum aliis quibusdam communia, quod nihil aliud est, quam morbos, uti alia quaecunque in rerum natura distingui debent, per genera et species distinguere, et hujusmodi distinctio generum, tum supremorum tum proximorum, notationem necessario exigit.

Quantacunque tamen ex instituendis morborum classibus et ordinibus utilitas provenire possit, certe fatendum est, Nosologos, huic rei praecipue intentos, successus optatos nondum obtinuisse; et ex hujus studii adeo imperfecti conspectu, ni fallor; accidit, quod totam rem Nosologicam alii despexerint, alii vero impossibilem judicaverint.

Qui impossibilem putant, certe falluntur. Distinctionem morborum aliquando difficilem esse, fatentur omnes; possibilem autem in plerisque esse, fateri etiam oportet; nam, si quis hoc negaverit, idem fecerit, ac si nullam esse artem medicam dixisset. Si revera tamen medici morbos a se invicem dignoscere possint, certe quibus indiciis id faciant, etiam dicere queunt. Haec autem indicia nulla alia esse possunt, quam quae morbum quemque per genus et speciem definiant, quae rursus per methodum Nosologicam, rite institutam, solummodo exponi queant. 
Distinctionem itaque morborum; hactenus saepe dubiam, per Nosologiam methodicam magis certam fieri posse, affirmare nullus dibito. Porro observandum, quoties hujusmodi distinctionem morborum exponere conati fuerimus, id commodi oriturum, quod hujus distinctionis vel defectus, vel errores, facillime percipientur, et cum percepti, vel ad observationes jam factas accuratius considerandas, vel ad observationes postea diligentius faciendas, ducent ; quin et plurimum valebunt, tum ad Nosologiam methodicam, tum ad distinctionem morborum, tandem perfectiorem reddendam.

Quamvis morborum characteres hactenus propositi saepe erronei, saepe deficientes sunt ; tentamina tamen ommia jam facta exhibere cupiens, in tertia Synopsis editione ex systemate symptomatico Cl. Michaelis Sagar, quae ad genera morborum definienda pertineant, excerpsi, et in hac quarta editione, ingeniosi Davidis Macbride, Tabulas Nosologicas addidi, et caeteris supra memoratis subjunxi. Haec autem tentamina omnia simul edita sunt, ut juxta posita facilius conferri possint, et ut collata vel optimos et maxime idoneos characteres indicent, vel ad erroneos corrigendos, et ad distinctiones magis exactas obtinendas, lectores ducant.

Hujusmodi studium plurimis medicis necessarium fore facile credo; nam morborum titulis inhaerentes, notiones parum accuratas et determinatas habent; et quantum video, ad magis exactas, nisi Nosologiae methodicae studio, duci vix possunt.

Ex is quae jam diximus, tum utilitatem tum necessitatem Nosologiae Methodicae manifestam fore spero; et simul patebit, quare antehac, et nunc etiam, eandem colere allaboravi. Proximum est, ut in hisce prolegomenis, quas in hoc studio regulas ipse observavi, et ab aliis observandas vellem, nunc exponam.

Prima et praecipua nostra cura fuit, ut morborum species in ipsis aegris praesentes detegerentur et dignoscerentur. Cum vero species nisi simul genus indicaretur, vix recte definiri possint; et cum genera plurima a Nosologis indicata unius tantum speciei sint, inde factum est, quod nostri etiam labores, in generibus praesertim distinguendis, impensi videantur. Ad species tamen semper respeximus; et ad eas dignoscendas, characteres generum a nobis datos úbique utiles fore speramus. 
In generibus morborum recensendis, pauciora, quam in aliis systematibus dicta sunt, recensui ; quod si recte feci, id studiosis multitudine rerum contemplanda facile turbatis imprimis commodum esse puto. Etiamsi enim numerum generum minuens aliquando erraverim, id tamen tuto tentatum fuisse censeo ; cum enim discipuli nostri genera facile et satis certo distinguenda recte perspexerint, illos genera forte omissa facile postea agnituros esse, credo.

Genera morborum vero apud nos, quam apud alios Nosologos, necessario pauciora sunt; quia plura, pro diversis ab aliis. habita et recensita, a nobis, utpote nulla, quantum nobis videtur, re diversa, sub uno titulo comprehensa sunt; et hujus rationes postea locis idoneis tradendae venient. Interea notandum, plura genera, quae primo intuitu omissa videantur, nequaquam ex oculis studiosorum sublata esse, cum generis illius, ad quod pertinere nobis videntur, uti synonyma recenseantur, et locum suum in indice adhuc retineant.

Deinde, genera morborum a nobis, quam ab aliis enumerata et definita, pauciora sunt; quia nulla, nisi idiopathica et primaria notanda esse duximus; dum plura ab aliis memorata, sympathica tantum sunt, sive symptomata nusquam per se existentia ; et neutiquam igitur pro morbis primariis agnoscenda. Ita Carphologia,Pandiculatio, Rigor, Sternutatio,Oscedo,Singultus, Stertor, Anxietas, Lassitudo, Stupor, Pruritus, Algor, et Ardor, a Sauvagesio, et alia ab aliis, pro generibus recensita, a nobis penitus omittuntur; et recte, ni fallor, nisi quot symptomata, totidem genera morborum, ponere velimus.

Denique, genera morborum apud nos pauciora sunt; quia, ex classibus Vitiorum et Deformitatum, plura a nobis omittuntur; vel quod morbi adeo leves sint, ut medicorum attentionem et curam non mereantur; vel quod congeniti et immutabiles medicorum artem penitus eludant. Ita Lentigo, Leuce, Cyasma, Seline, et Cicatrix, ex classe Vitiorum; et Phoxos, Gibber, Rhyssemata, Canities, Coloboma, Naevus, Monstrositas, Rhicnosis, Varus, Valgus, Leiopedes, Saniodes, Crypsorchis, Hermaphroditus, Dionysiscus, Galiancon, Galbulus, et alia ex classe Deformitatum apud Vogelium recensita, recte a nobis omissa putamus. Ejusmodi vitia et deformitates, fortassis in pathologia, sive historia generali mutationum quas quavis oc- 
casione subit corpus humanum, notari possent; Nosologiam autem methodicam is adeo frivolis onerare, vel eandem tam multis novis et inutilibus appellationibus turbare, minime idoneum videtur. Judicet itaque lector, quam merito gloriatur Cl. Sagar, se generum numerum auxisse.

Quod modo memorata omiserim, venia forsitan mihi facile dabitur. Sunt autem alii morbi, memoratu quidem digni, quos omissos non adeo facile condonabit studiosus. Hujusmodi omissiones agnosco, et quidem doleo; sed variae rationes, ut quidam morbi hic omitterentur, effecerunt. Primo, fieri potest, ut quidam observationem nostram penitus effugerint; dein, sunt alii satis noti, quibus, in nostro systemate, locus idoneus nusquam inventus est; denique, sunt alii quorum historia apud medicos adeo sit imperfecta, ut nec locus nec character idoneus iis assignari queat. Ne tamen ejusmodi genera penitus omitterentur, ad calcem operis, omissorum, quotquot noverim, catalogum subjunxi, ut posteri perspicaciores, ea diligentius perpendentes, characteres et locum iis assignent.

Eodem consilio, ne scilicet tyronibus contemplanda praeter necessitatem multiplicarentur, non genera solum, sed species morborum etiam ad minorem numerum redegi. Hoc magis periculosum videri potest, cum nullam speciem, quae revera existat, recte omittere liceat. Ne quidem ad recensionem specierum ipse accederem, 'difficultas operis me dubium, et timidum antehac diu cohibuit; et id tantum tentare ausus sum, ut species, a Sauvagesio recensitas, serie paulo luculentiore et emendatiore traderem: re autem amplius et curatius perpensa, species jam recensurus, numerum earum, apud Sauvagesium recensitarum, multum minuendum esse, arbitratus sum.

Solus quidem Sauvagesius, nam Sagarum nihil moror, in recensione specierum quicquid praestitit; et licet egregius vir, plurimis observationibus undique collectis, multum de re medica meritus sit; haud tamen dissimulandum, illum in multis graviter errasse, dum species revera easdem, sub diversa appellatione repetitas, pro diversis ponebat; et praesertim dum species sympathicas, tam saepe idiopathicis immiscebat, qua ratione earum numerum totum praeter modum auxit. Hos ut tollerem errores, quod potui feci, numerosis nempe apud Sauvagesium speciebus, recte, quantum egó judico, ad pauciores redactis. 
Quod ut efficerem, primo species plures, ut diversas a Sauvagesio recensitas, pro una eademque habui, et sub uno titulo posui; atque hujus rei rationes locis suis idoneis posthac tradendae sunt.

Deinde, cum species idiopathicas solum pro veris et genuinis 'enumerare velim, quotquot pro symptomaticis habere potui, totidem ex numero specierum apud Sauvagesium sustuli. Cum autem, in recensione specierum symptomaticarum aliquis usus sit, earum recensionem, seorsum tamen, denuo apposui.

Denique, alia et praecipua ratio, qua specierum, a Sauvagesio recensitarum, numerum minuendum esse censui, ea est, qua plures ab eo pro diversis positas, pro specierum varietatibus, tantum habui et enumeravi.

Hoc autem est, quod in Nosologia Methodica maxime difficile videtur, dicere nimirum, quid pro ipsa specie morbi, quid pro ejusdem speciei varietate tantum, habendum sit. Cum autem criteria illa, quae in Zoologia et Phytologia, ad species a varietatibus certius distinguendas adhiberi possint, in Nosologia Methodica nequaquam invenienda sint; varietates morborum a speciebus distinguere ubique difficilius erit. Quamobrem varietates plures recensere, tutissimum et fere necessarium esse duxi. Ejusmodi vero distinctionem, in artis usu utilissimam fore ratus, illam adhiberi ubique annisus sum; quod si non in omnibus, cum aeque certa cognitione, cum aliqua saltem verisimilitudine, diligenter quae sequuntur pensitatis, saepe fecisse videor.

Cum morbus aliquis, quo plures homines laborant, symptomata cujusdam speciei characteristica omnia et sola, in unoquoque ostendit; quantumvis haec in alio minus, in alio magis vehementia sint, hoc speciem diversam exhibere non putamus; et, in universum, morbos gradu solum differentes, non nisi ejusdem speciei varietates praebere censemus.

Tunc igitur de varietate a specie distinguenda quaestioni tantum locus est, cum in diversorum hominum morbis, vel ex solitis cujusvis speciei symptomatis, quaedam absint, vel iisdem quaedam addita sint.

Cum ex symptomatis solitis quaedam absint, quoties inter symptomata, quae magis, et ea quae minus, essentialia sunt distinguere liceat, ex horum absentia non nisi varietatem adesse concludimus. 
Cum vero symptomatis characteristicis alia quaedam addita sint, si addita pro symptomatis symptomatum, potius quam pro symptomatis causae haberi queant, varietatem tantum constituunt.

Denuo, cum symptomata addita, in specie data, prorsus insolita sint, et simul conditiones morbi praecipuas parum vel nihil mutare videantur, haec quoque varietatem tantum praebere censenda.

Cum morbi genus quoddam ex principiis diversis oriri queat, idem, ex diversitate principii, specie diversum evadere potest, nec semper tamen; nam, quotiescunque diversitas illa parva fuerit, et simul symptomata parum ab ea fuerint mutata, hoc etiam non nisi varietatem exhibere, putamus.

Morbi genus itidem, pro diversa ejus sede, diversae speciei fieri potest; quotiescunque vero sedes diversa, neque partis structurae, neque functionis ratione differat, hujusmodi sedis diversitas etiam pro varietate tantum habenda est.

Porro, cum de morbis invicem distinguendis hic agatur, observari vellem, res duas esse, quae ad similitudinem et affinitatem morborum in diversis hominibus indicandam, plurimum facere possint.

Altera est, quod principii quaedam similitudo, morborum inde in diversis hominibus genitorum similitudinem arguit; ita, quando morbi diversorum hominum, ex une eodemque principio oriantur; quando, etiam principium illud, ad morbum gignendum, in unoquoque necessarium sit; denique, quando idem principium, ubique fere ejusdem qualitatis et vis esse videatur, tum demum morbos, ex ejusmodi principio genitos, ejusdem vel simillimae naturae esse, judicare licet.

Hoc de morbis contagiosis plerisque valere mihi videtur. Ex his enim sunt, qui in pluribus hominibus ab eadem specifica contagione oriuntur; nec, in eorum aliquo morbus, nisi ab illa contagione applicata, oriri unquam observatur; et simul illius contagionis vires et qualitates, ubique fere, eaedem esse videntur. Quod ad proxime dictum spectat, id quibusdam dubium videri potest; sed cum ejusdem contagionis effectus, per secula plura jam observati, semper et ubique admodum similes sint, eam qualitate et viribus eandem esse, merito concludimus; et ob hoc pracsertim, quod effectus isti, quatenus diversi videantur, ple- 
rumque hominis infecti conditioni cuidam, potius quam conta gioni, qualitate vel viribus diversæ, tribui possunt.

Morbos, itaque, eadem contagione specifica ortos, simillimae naturae esse credendum ; ejusmodi morbos etiam vix, nisi unicam ejusdem generis speciem, praebere; immo, earum diversitatem quandam aliquando observatam, non nisi ejusdem speciei varietatem indicare, suspicandum est.

Hæc omnia, non solum de exanthematis contagiosis, sed etiam de plerisque febribus epidemicis, dicta velim. Hanc autem opinionem, de similitudine febrium epidemicarum proferens, a gravis auctoritatis viro Sydenhamo, hujusmodi febres specierum plurium esse statuente, dissentire cogor. Recte quidem, an secus, multum dubito; et posteris litem dirimendam relinquo, hoc simul praemonito, plurimum medicinam facientibus inter futurum, de alterutrius sententiae veritate sibi satisfacere, vel potius quos limites utrique sint ponendi, statuere.

Altera res, quae similitudinem morborum, in diversis hominibus, ostendere possit, est similitudo remediorum quibus sanantur. Similitudo quidem morborum in similitudine causae eorum proximae, qualiscunque sit, revera consistit; cum remedia vero morbis medeantur, tantum quatenus causas eorum proximas tollant, morbos, qui iisdem omnino remediis sanantur, ejusdem naturae esse oportet.

Hujusmodi ratiocinatio, tum ad phlegmasiarum missione sanguinis, tum ad febrium intermittentium cortice Peruviano sanatarum, similem naturam indicandam, plurimum certe facere potest. Hanc autem doctrinam, utcunque in praxi mediea aliquando utilem, fallacem tamen, neque in praxi, neque in Nosologia methodica, nisi caute admodum, adhibendam esse censeo. Apud vulgus medentium quidem, remedia eadem quibusque fere ejusdem generis, speciebus, et harum varietatibus adhiberi, et hinc genera plura nullo modo specie differre, videantur. Sed peritiores norunt, morbos, aliquo modo diversos, rarius quam putatur, ab iisdem prorsus remediis sanari: bene etiam norunt, ex promiscua remediorum adhibitione fieri, quod eadem remedia adeo saepe frustranea, sanationes saltem minus perfectac sint. Porro, norunt expertissimi, ad recte medendum, omnino opus esse, ut remedia, non solum generi sed speciei cuique; et saepe etiam varietatibus quibusdam, apprime sint accommodata. 
Ad artem medicam breviorem, et exinde faciliorem, reddendam, species morborum quascunque, a varietatibus suis distinguendas esse, arbitratus sum. Hanc tamen distinctionem in pluribus quodammodo incertam esse fateor, et, in Nosologia methodica, tutissimum fore puto, varietates morborum plerasque notare et recensere. Hoc itaque praestare ubique annisus sum, aliis sapientioribus, qui posthac huic operi se immiscere velint, relinquens, discriminationem magis certam statuere.

Exposita jam qua ratione morborum, tum generum, tum specierum, numerum minuere laboravi ; superest, ut quo modo eorum, quae admissi, characteres constituendi sunt, nunc exponam.

Imprimis igitur, notas externas, sensibus nostris facile obvias ubique selegi, neglectis, vel potius rejectis, de statu corporis interno conjecturis quibuscunque. Porro, ex notis quae sensibus sunt obviae, illas medici potius quam aegri sensibus percipiendas, semper praetuli ; quum tamen has, utcunque fallaces, penitus negligere vel omittere non liceat.

Secundo, ea symptomata pro notis characteristicis praecipue seligenda putavi, quae perpetuo cum morbo praesentia sunt, et hoc quidem semper annitendum esse puto. Cum vero plurimi morbi sub eorum decursu, aliam atque aliam omnino formam capiant, in his, ex serie rerum, et symptomatum sibi invicem succedentium, character saepe necessario petendus est.

In hac re autem tractanda, duplex quaestio exoritur. Prima est, An ex morbi principio characteris partem desumere liceat?

Ad hanc responderi possit, quod, etiamsi de principiis morborum apud medicos judicium saepe fallax, et prorsus falsum sit, nec temere idcirco ad morbos distinguendos adhibendum; cum eadem tamen aliquando satis certe nota sint, et facile observanda, hujusmodi principia in Nosologia, pro notis characteristicis recte adhiberi posse censeo.

Altera quaestio hic exoriens est, quatenus ex serie symptomatum, sub decursu morbi occurrentium, characterem ejus petere liceat? Ad hanc etiam possit responderi, quod, cum plures morbi, uti exanthemata et febres intermittentes, nisi post decursum aliquot dierum, ex serie symptomatum sibi invicem succedentium, nullo modo dignosci queant; ut hujusmodi morbi distinguantur, ex serie illa character necessario sumendus est. Characteres autem, non nisi post longum morbi decursum, for- 
tassis non nisi post morbum finitum, statuendi, nequaquam in Nosologia usurpari debent. Ita apud Ill. Linnæum Febrium continentium, quas vocat, characteres a diuturnitate totius morbi desumpti prorsus inepti sunt.

In constituendis morborum characteribus, regula tertia est, quod cum quisque fere morbus, ex plurium symptomatum concursu solum dignoscatur, ex iis pro notis characteristicis quot sufficiant; nec plura tamen adhibenda sunt. Priores Nosologi mihi videntur, in characteribus quos dederint, plerumque nimis breves et deficientes fuisse; et forsan mihi vitio verti potest, quod aliquando sim in iisdem nimis longus et redundans. Nusquam quidem volens fui redundans; ut vero essem copiosus ubique me conatum fateor, prudentissimum fore ratus etiam ad redundantiam appropinquare, quippe posteris multo facilius erit, quod nimium est tollere, quam quod deficiat supplere.

In characteribus denique morborum constituendis, quaeritur etiam, an ex defectu functionis cujuslibet, quae in sano unoquoque exercetur, vel ex absentia symptomatum, in aliis generibus vel speciebus apparentium, notas quasdam desumere liceat? Sauvagesius quidem defectus functionum, utpote nihil positivum indicantes, pro morbis noluit admittere; plures igitur, qui a Linnæo sub ordinc constrictoriorum, et a Vogelio sub classe epischesium, recensentur, penitus omisit.

Sed, pace tanti viri, dixerim, quanquam defectus, si strictius loqui velimus, nihil positivum indicet; defectum tamen functionis, quam exercet quisque sanus, statum corporis humani exhibere, quem ut distinguamus utilissimum est, atque etiam necessarium, et ejusmodi status pro morbo recte haberi potest. Porro, etiamsi in quibusdam defectus isti aliquando sine molestia vel morbi symptomate observentur; in plerisque tamen magnam molestiam, et symptomata plurima pariunt. In his igitur, defectum functionum, saepe symptomatum causam manifestam, pro morbo haberi licet, et ex ejusmodi defectu, in plurimis morbis horum notas characteristicas petere licet, et oportet. Sauvagesius ipse in hac re parum sibi constare videtur, Debilitatum classem instituens, et Amnesiam, Agrypniam, Ischuriam, et alia etiam tanquam genera recensens.

Quod notas ex absentia symptomatum, in aliis congeneribus apparentium, spectat; eas nequaquam bonas, et, ubicunque 
fieri potest, evitandas censeo. In pluribus vero vitari, quantum video, non possunt; nec a quovis Nosologo adhuc fuerunt vitatae.

Cum, secundum has regulas, characteres morborum constituendi sint; plurimum refert, eosdem sermone accurato et luculento exprimere; nec minus, morbum quemque apto nomine insignire.

Norunt eruditi, Ill. Linnaeum methodum plantarum perfectiorem reddidisse, eo, quod botanices linguam, usurpatis ubique vocibus maxime idoneis et definitis, magis accuratam effecit; nec quidem dubito quin delineatio morbi, ad instar delineationis plantae, a Linnaeo exhibitae, in Nosologia multum profutura sit. .In Nosologia autem, ac in botanice, non aeque necessarium esse videtur; tum quod tam multae rerum circumstantiae, in Nosologia, ac in botanice, notandae non, veniunt; tum quod symptomatologia, in quibuscunque fere Pathologiae generalis systematibus exhibita, plurimum in hac re jam praestitit. Fátendum quidem, symptomatologias vulgo prolatas, nec adeo plenas esse, nec adeo exactas, ac optandum esset; et, si pleniores fierent, atque ubique voces magis idoneae et accurate definitae usurpatae essent, ad Nosologiam perficiendam certe plurimum conferre posset. In hujusmodi vero tentamine cavendum est, ne distinctiones nimium subtiles et dubiae adhibeantur, uti ab auctore Observationum Clinicarum, quae Warsaviae annis 1767 et 1768 prodierunt, factum fuisse opinor. Ipse quidem hujusmodi nihil tentavi, tum quod plus exegisset, quam per longum tempus mihi suppetivit otii, tum quod mihi minus necessarium videtur, qui voces apud medicos usitatissimas, et in sensu vulgo recepto, ubique fere usurpandas curaverim.

Quod ad denominationem morborum attinet, in Classium, Ordinum, et Generum nominatione, eas, quantum potui, regulas observavi, quae in Linnaei Criticis-Botanicis, et in ejusdem Philosophia Botanica, ab ill. auctore traduntur.

Quando notiones novae sunt proferendae, etiam nova nomina imponenda sunt, et ejusmodi aliquando, parcius licet, adhibuimus. Cum vero nomenclaturam medicam, diuturno usu confirmatam, sine gravi causa mutare non liceat; ne factum esset ubique curavimus : quod tamen Ill. Linnaeum saepe, nulla ur-

vot.. I. 
gente necessitate, fecisse, et Vogelium appellationibus novis, frivolis, ineptisque, Nosologiam deturpasse, observavimus.

Ut clarior et certior habeatur morborum notitia, appellationibus selectis, synonyma, tum aliorum Nosologorum, tum auctorum maxime celebrium, ubique apposuimus. Hoc diligentius feci, ut inde norint studiosi, ex quibus praecipue scriptis, morborum scientia optime obtineri possit.

In recensendis specierum synonymis, appellationes, quibus Sagarus usus sit, omisi, tum quod ipse aliorum auctorum synonyma ubique omittens, nos de speciebus, quas indicare voluit incertos, saepe reliquit; tum quod, in recensione specierum, illum profecto minus peritum fuisse, et regulas Nosologiae methodicae optimas quasque neglexisse, nobis videtur.

Synonyma veterum medicorum adhuc omisi, non quod eorum scripta prorsus negligenda esse putaverim, sed quod ego, neque satis certo, neque cum fructu, synonyma eorum indicare potuerim. 
CLASS. I.-PYREXIÆ.

- ORDO I. FEBRES.

Sect. 1. Intermittentes.

1. Tertiana.

2. Quartana.

3. Quotidiana.

Sect. 2. Continuce.

4. Synocha.

5. Typhus.

6. Synochus.-Hectica.

ORDO II. PHLEGMASIE.

7. Phlogosis-Apostema. Gangrena.Sphacelūs.

8. Ophthalmia.

9. Phrenitis.

10. Cynanche.

11. Pneumonia.-Vomica. Empyema.

13. Carditis.

14. Peritonitis.

15. Gastritis.

16. Enteritis.

17. Hepatitis.

18. Splenitis.

19. Nephritis.

20. Cystitis.

21. Hysteritis.

22. Rheumatismus-Arthrodynia.

23. Odontalgia.

24. Podagra.

25. Arthropuosis.

ORDO III. EXANTHEMATA.

26. Variola.

27. Varicella.

28. Rubeola.

29. Scarlatina.

30. Pestis.

31. Erysipelas.

32. Miliaria.

33. Urticaria.
34. Pemphigus.

35. Aphtha.

ORDO IV. HEMORRHAGIE.

36. Epistaxis.

37. Hæmoptysis. Phthisis

38. Hæmorrhois.

39. Menorrhagia.

40. Catarrhus.

41. Dysenteria.

Class. II.-NEUROSES.

ORDO I. COMATA.

42. Apoplexia.

43. Paralysis.-Tremor.

ORDO II. ADYNAMIE.

44. Syncope.

45. Dyspepsia.

46. Hypochondriasis.

47. Chlorosis.

ORDO III. SPASMI.

48. Tetanus.

50. Convulsio.

51. Chorea.

52. Raphania.

53. Epilepsia.

54. Palpitatio.

55. Asthma.

56. Dyspnœa.

57. Pertussis.

58. Pyrosis.

59. Colica.

60. Cholera.

61. Diarrhoea.

62. Diabetes.

63. Hysteria.

64. Hydrophobía.

ORDO IV. VESANIE.

65. Amentia.

66. Melancholia.

67. Mania.

68. Oneirodynia.

* Classium institutionem primo a Sauvagesio datam, Linnaeus, Vogelius, et Sagarus, in omnibus fere, secuti sunt. Cum vero ex eorum classibus plures sint, uti Vitiorum, Anhelationum, Dolorum, et Fluxuum, neque naturales, nec quovis modo idoneae, ejusmodi institutionem sequi non potui. Aliam itaque simpliciorem, et in universum, ut mihi videtur, aptiorem, hic protuli. Haec fortasse etiam minus absoluta videri possit, quod inter morbos totius systematis, in primis tribus classibus recensitos, et illos partis singularis, sive Locales, in classe quarta collocatos, distinctio non ubique facilis erit. Hoc quidem, rarius licet, aliquando fieri posse concedimus. Sed nibil praestantius nobis nunc suppetit; et, ob rationes in Prolegomenis, pag. 227. allatas, de perfecta classium institutione non admodum solliciti sumus. 
Crass. III-CACHEXIæ. ORDO I. MARCORES.

69. Tabes.

70. Atrophia.

ORDO II. INTUMESCENTIE.

Sect.1. Adiposa.

71. Polysarcia.

Sect. 2. Flatuosa.

72. Pneumatosis.

73. Tympanites.

74. Physometra.

Sect. 3. Aquosa.

75. Anasarca.

76. Hydrocephalus.

77. Hydrorachitis.

78. Hydrothorax.

79. Ascites.

80. Hydrometra.

81. Hydrocele.

Sect. 4. Solider.

82. Physconia.

83. Rachitis.

ORDO III. IMPETIGINES.

84. Scrophula.

85. Syphilis.

86. Scorbutus.

87. Elephantiasis.

88. Lepra.

89. Frambœsia.

90. Trichoma.

91. Icterus.

Class. IV.-LOCALES.

ORDO I. DYSESTHESIE.

92. Caligo.

93. Amaurosis.

94. Dysopia.

95. Pseudoblepsis.

96. Dysecoea.

97. Paracusis.

98. Anosmia.

99. Ageustia.

100. Anæsthesia.

ORDO II. DYSOREXIA.

Sect. 1. Appetitus Erronei.

101. Bulimia.

102. Polydipsia.

103. Pica.

104. Satyriasis.

105. Nymphomania.

106. Nostalgia.
Sect. 2. Appetitus deficientes.

107. Anorexia.

108. Adipsia.

109. Anaphrodisia.

ORDO III. DYSCINESIE.

110. Aphonia.

111. Mutitas.

112. Paraphonia.

113. Psellismus.

114. Strabismus.

115. Dysphagia.

116. Contractura.

ORDO IV. APOCENOSES.

117. Profusio.

118. Ephidrosis.

119. Epiphora.

120. Ptyalismus.

121. Enuresis.

122. Gonorrhœa.

ORDO V. EPISCHESES.

123. Obstipatio.

124. Ischuria.

125. Dysuria.

126. Dyspermatismus.

127. Amenorrhœa.

ORDO VI. TUMORES.

128. Aneurysma.

129. Varix.

130. Ecchymoma.

131. Scirrhus.

132. Cancer.

133. Bubo.

134. Sarcoma.

135. Verruca.

136. Clavus.

137. Lupia.

138. Ganglion.

139. Hydatis.

140. Hydarthrus.

141. Exostosis.

ORDO VII. ECTOPIJE.

142. Hernia.

143. Prolapsus.

144. Luxatio.

ORDO VIII. DIALYSES.

145. Vulnus.

146. Ulcus.

147. Herpes.

148. Tinea.

149. Psora.

150. Fractura.

151. Caries. 


\section{CLASSIS I. PYREXIAE.}

Post horrorem pulsus frequens, calor major, plures functiones laesae, viribus praesertim artuum imminutis.*

Synonyma. Morbi febriles Auctorum.

\section{ORDO I. FEBRES.}

Praegressis languore, lassitudine, et aliis debilitatis signis, pyrexia, sine morbo locali primario.

Febres Auctorum, Sauv. cl. ii. Vog. cl. i. Sag. cl. xii.Morbi febriles critici Lin. cl. ii.

\section{SECT. I. INTERMITTENTES.}

Febres, miasmate paludum ortae, paroxysmis pluribus, apyrexia, saltem remissione evidente interposita, cum exacerbatione notabili, et plerumque cum horrore redeuntibus, constantes : Paroxysmo quovis die unicồ tantum.†

- Quod hic, et pluribus quae sequuntur locis, nobis forsitan vitio verti possit, quodammodo hic obiter excusare liceat. Ita, dici potest, pyrexias aliquando videri, quas nullus praecesserat horror, et pyrexias in quibus neque pulsus frequentior, neque calor major, quam in sanis esse solet; characterem ideo datum neque verum, neque ubique adhibendum. Istiusmodi pyrexias, rarissime licet, aliquando videri negare nolo ; sed, in characteribus classiun, ad species dignoscendas adhibendis, ut singulae classis notae in unaquaque specie conspiciantur, haud necessarium videtur, et sufficit, si earum pleraeque in specie quavis adsint.

Character idoneus quisque, plurium notarum sive symptomatum concursum notare debet, et male quidem cum veteribus Vogelius calorem auctum solum, et recentiores Sylvius et Cel. Boerhaavius pulsuum velocitatem solam, pro charactere pyrexiae, sive febris ut vocant, posuerunt. Certe, qui dicunt febrem adesse, etsi pulsus nihilo naturali frequentior sit, ex aliis quam velocitate pulsuum signis febrem adesse judicant; quod etiam plurium symptomatum notationem, in quovis charactere necessariam esse, os. tendit.

Porro, hic obi ter observari velim, pulsuum velocitatem solam, pyrexiam vel febrem adesse nequaquam semper indicare; cum a causis externis plurimis pulsus fit naturali velocior, sine morbo, sive quavis functionum laesione. Hisce de pyrexiae charactere quaestionibus, in charactere a nobis hic dato, quantum potuimus, prospectum est. Sed, in aliis classium et ordinum characteribus adeo felicem esse vix licet; et in characteribus plerisque, si ad species plerasque character recte adhiberi possit, paucas exceptiones non moror. Utilem plerumque fuisse mihi sufficit, ubique perfectum fore non spero.

† Qui perpenderit quae de febribus Remittentibus, quae videntur continuae, a Continuis, strictius dictis distinguendis, mox dicenda sunt, facile videbit quamobrem, tum intermittentium tum continuarum eharacterem antea datum, hic mutare nccessarium habui. 
Intermittentes Auctorum. Sauv. cl. ii. ord. 3. Linn. cl. ii. ord. 2. Vog. cl. i. ord. 1. Sag. cl. xii. ord. 3.-Remittentes Auct. S. cl. ii. ord. 2. Sag. cl. xii. ord. 2. *-Exacerbantes Lin. cl. ii. ord. 3.-Continuae Vog. cl. i. ord. 2. † -Continuae periodicae, Sennert. de febr. lib. ii. cap. 13. -Continuae remittentes, Boerh. Aph. 727.-Compositae ex acuta periodo, Junck. tab. 82.-Continuae remittentes, proportionatae, Torti. Therap. Spec. lib. v. cap. 1.-Continentes Mortoni, Exer. ii.

\section{GENUS I. TERTIANA.}

Paroxysmi similes intervallo quadraginta octo circiter horarum : Accessionibus meridianis.

Tertiana Auctorum, Sauv. g. 88. Lin. 16. Vog. 2. Sag. p. 704. Hoffm. tom. ii. p. 11. Stahl. de tertiana febris genium universum manifestante, Halae 1706. Ejusd. Casual. magn. cas. 21. Casual. min. cas. 96. Cleghorn's diseases of Minorca, cap. 3. Senac, de recond. febr. natura. Edinb. Med. Ess. vol. iv, art. 24.

Tertiana est vel,

\section{INTERPOSITA APYREXIA, QUAE}

A. Variat paroxysmi duratione.

$a$. Tertiana paroxysmis haud ultra horas duodecim extensis.

T. legitima, Sauv. sp. 1. Sennert. de febr. lib. ii. c. 18. Hoffm. tom. ii. p. 12.-T. vera, Clegh. Min. p. 140.

b. T. paroxysmis ultra horas duodecim extensis.

T. notha sive spuria, S. sp. 2. Sennert. de febr. lib. ii. c. 18. Clegh. Min. p. 140. Hoffm. tom. ii. p. 12.

B. Variat paroxysmorum recursu.

c. Tertiana quotidie revertens, paroxysmis inaequalibus, alternis similibus.

T. duplex, S. sp. 13. Sennert. de febr. lib. ii. c. 21 . Vog. gen. 12. Clegh. Min. p. 141.-Duplicana, Linn. 18.

* Febrium Remittentium, quasi a febribus Intermittentibus exquisitis prorsus differentium, ordinem separatum instituerunt Nosologi Sauvagesius, Linnaeus, et Sagarus; minus recte tamen, si mihi judicare fas sit. Remittentes enim, qui dicuntur, ex eodem principio, miasmate nempe paludum, ac Intermittentes, oriuntur; iisdem in locis, et eodem anni tempore, utraque simul epidemice grassatur; utraque iisdem prorsus remediis sanatur; et saepissime in eodem homine idem, qui videtur, morbus, nunc intermittentis nunc remittentis typum exhibet. Morbi idcirco, causis, sana tione, et typo simillimi, nec ad ordinem, nec ad sectionem diversam distrahendi erant.

+ Magis inconsulto adhuc Vogelius Remittentes omnes cum Continuis sociavit. Con. tinuae, quas vocamus, omnes quidem remissiones et exacerbationes ostendunt; sed a Remittentibus ad Intermittentes merito referendis, plurimum aliis multis differunt, ut mox dicetur. 
(TERTIANA.)

d. Tertiana alternis diebus revertens, paroxysmis eodem die binis.

T. duplicata, S. sp. 14. Jones, de febr. interm. p. ii. cap. 6. River. Cent. iv. obs. 16.

e. Tertiana quotidie revertens, paroxysmis altero die binis, altero unico tantum.

T. triplex, S. sp. 15. Clegh. Min. p. 142. Semitertiana Hoffm. ii. p. 40.-Semitertiana primi ordinis Galeni. Spig. de semitertiana, lib. ii. cap. 4.

$f$. Tertiana quotidie revertens, interposita remissione inter diem imparem et parem magis, inter parem et imparem minus, notabili.

Hemitritaeus, Celsi lib. iii. cap. 3.-Semitertiana, Clegh. Minorc. p. 143. *-Semitertiana secundi ordinis Galeni, Spig. 1. ii. c. 5.-Amphimerina hemitritaeus, S. sp. 8.-Amphimerina pseudo-hemitritaeus, S. sp. 9.*

C. Variat symptomatibus.

g. Tertiana affectibus soporosis stipata.

T. carotica, S. sp. 10. Werlhof. de febribus, et auctores ab eo allegati, p. 6.-T. hemiplegica, S. sp. 20. Werlhof. ibid.-Quotidiana soporosa, S. sp. 8. Car. Pis. obs. 175. 176.-Febris caput impetens, Sydenh. Ep. ad R. Brady.

$h$. Tertiana spasmis et motibus convulsivis stipata.

T. asthmatica, S. sp. 6. Bonet. Polyalth. vol. i. p. 250. -T. hysterica, S. sp. 8. Wedelii A. N. C. dec. i. an. ii. obs. 193.-Hysteria febricosa. S. gen. 135. sp. 8. A. N. C. dec. i. an. ii. obs. 193.-T. epileptica, S. sp. 16. Calderae Trib. med. p. 225. Lautter. Histor. med. bienn. cap. ii. cas. 2.-Quotid. epileptica, S. sp. 3. Edin. Ess. vol. v, part ii, art. 49.-Ecclampsia febricosa, S. gen. 133, sp. 17.-Epilepsia febricosa, S. gen. 134, sp. 9.-Tertiana tetanodes, Medicus Beobacht. Band i.p. 24.-Tetanus febricosus, S. gen. 122, sp. 10. Stork, Ann. Med. ii, p. 163.

i. Tertiana efflorescentia cutis stipata.

Tertiana petechialis, S. sp. 3. M. Donati, lib. iii, cap. 14 . Lautter. Hist. med. cap. ii, cas. 10. - Tertiana scorbutica, Wedel. A. N. C. dec. i, an. ii, obs. 193. -Tertiana urticata, S. sp. 22. Planchon. Journ. de Méd. 1765. Clegh. Minorc. p. 157. Tertiana miliaris, S. sp. 21. Walthieri de Med.German. apud Roncalli Europ. Med.p. 151.

* Fortassis hemitritaea quaevis ad remittentes relegari possit ; et, licet affines separare. nolui, limites non satis certe ponendos esse fateor. 
(TERTIANA.)

k. 'Tertiana phlegmasia stipata.

Tertiana pleuritica, S. sp. 4. Valesii in epid. lib. i, sect. 3. Lautt. Hist. Med. cap. ii, cas. 5, 9.-Pleuritis periodica, S. gen. 103, sp. 14.-Tertiana arthritica, S. sp. 5. Morton. Exerc. i, cap. ix, hist. 22. Lautt. loc. cit. cas. 19.

D. Variat aliis morbis complicata.

Tertiana scorbutica, S. sp. 9. Etmuller, prax. lib. i, sect. xvi, cap. 2. Timæi lib. viii, cas. 15.-Tertiana syphilitica, S. sp. 17. Deidier de morb. vener, sect. iv.-Tertiana verminosa, S. sp. 18. Stisser. in act. Helmstad. Lancis. de noxis palud. lib. ii, cap. 5 et 6 . Illust. Pringle Diseases of the army, p. 98. Ramazzini Const. epidem. rural. ann. 1690, sect. xi. Van den Bosch Const. epidem. verminos. ann. 1760 , \&c.

E. Variat ratione principii.*

Tertiana accidentalis, S. sp. 12. Sydenham, cap. 5.-Tertiana a scabie, S. sp. 11. Juncker, tab. 80. Hoffm. p. 12.

\section{INTERPOSITA REMISSIONE TANTUM. $†$}

Tritaeophya, S. gen. 85. Sag. p. 695.-Tritaeus, Linn. 21. -Hemitritaea, Linn. 23.-Tertianae remittentes et continuae Auctorum.-Tertianae subintrantes, proportionatae, subcontinuae, Torti Therap. special. lib. iii, cap. 1. -Tertiana subcontinua, S. sp. 19.-Quotidiana deceptiva, S. sp. 2.-Amphimerina semiquintana, S. sp. 24.Tritaeophya deceptiva, S. sp. 10.

Ex tertianis comitatis Torti loc. cit. praecipuae sunt :

i. Tertiana cholerica sive dysenterica, Tort. ibid. Lautter. Hist. med. cas. 6, 16, 17, 20. Morton, app. ad exerc. ii.

ii. Tertiana subcruenta sive atrabilaris Tort. ibid. Haec a Cleghornio nunquam visa est.

iii. Tertiana cardiaca Tort. ibid. Lautter. Hist. med. cas. 15, 18, 23.-Amphimerina cardiaça, S. sp. 5. - Tritaeophya assodes, S. sp. 6.-Febris continua assodes, Vog. 27.

* Nullum quidem febrium intermittentium principium sufficiens praeter miasma paludum agnoscimus; sed cum miasma hoc nón semper validum sit ad morbum arcessendum, nisi aliae potestates excitantes simul agere concurrant; has potestates excitantes pro parte principii hic admittimus, licet neutiquam morbum excitassent, si miasma paludum non antea applicatum fuisset.

+ Tertianae Remittentes variant aleo, tum typi tupn symptomatum ratione, et formae earum diversae adeo saepe in eodem lomine inter se commutantur, ut in iis denominandis nihil sere quod perpetuum sit efferri possit. Tertianas astem, quas comitatas dixit Torti, eas scilicet quae symptomate quodam peculiari et gravi comitantur, recensui ; dein, ut caetera ad hanc rem pertinéntia intelligantur, auctores plures qui de tertianis remittentibus optime scripserunt, et exempla earum praecipua exlibuerunt, enumeravi.

‡ Amphimerinas inter et Tritaeophyas, uti inter quotidianas et tertianas duplices, distinctio saepe difficilis. Sed quotidianas tertianis multo rariores, et oeconomiam animalem in febres typi tertiani maxime proclivem esse norunt experti; hine plures amphimerinas ad tertianas remittentes retuli. "Judicent autem autoptae," 
(TERTIANa.)

iv. Tertiana diaphoretica, Tort.-ibid.-Tritaeophya typhodes, S. sp. 4.-T. elodes, S. sp. 5.*-Febris continua elodes, Vog. 21.

v. Tertiana syncopalis Tort. ibid. Lautter, c. 11, 12, 13, 15, 16.-Tritaeophya syncopalis, S. sp. 1.-Amphimerina syncopalis, S. sp. 4.-A. humorosa, S. sp. 6.-Febris continua syncopalis, Vog. 29.

vi. Tertiana algida, Tort. ibid. Lautter, cas. 13-Amphimerina epiala, S. sp. 3. A. phricodes; S. sp. 7.-Tritaeophya leipyria, S. sp. 9.-Tertiana leipyria, S. sp. 23. Valcarenghi Med. ration. p. 18.-Febris continua epiala et leipyria, Vog. 19 et 24.

vii. Tertiana lethargica, Tort. ibid.-Tritaeophya carotica, S. sp. 7. Lautter, 1, 7, 14.-Tertiana apoplectica Morton, exerc. i, cap. ix, hist. 25.-T. soporosa Werlhof. de febr. p. 6.-Febris epidemica Urbevetana. Lancis. de noxis pal. effluv. lib. ii, cap. 3 .

Tertianarum remittentium exempla praecipua, nobis nota, sunt sequentia :

Causos Hippocratis. De Morb. Vulg. lib. iii, sect. 3. Tritaeophya causus, sp. 2.

Febris ardens, Boerhaavii, Aph. 738?

Remittens ardens sive causis, Macbride, p. 360.

Tertiana perniciosa, quae simulata tertiani circuitus effigie lethalis, et mille accidentibus periculosissimis implicata, existit. Lud. Mercatus de febribus, lib. vi.

Tertiana pestilens, P. Sal. Diversus de febri pestilent. cap. $\mathbf{x}$.

Tertiana maligna pestilens Riverii, lib. xvii, sect. ii, c. 1 , et sect. iii, c. 3 .

Morbus Hungaricus Lang. Lemb. lib. i, ep. 4. Sennert. de febr. lib. iv, cap. 14. Jordan. de pestis phæn. cap. 19.Languor Pannonicus Cober. obs. castr. Hung. i, obs. 6, \&c.-Amphimerina Hungarica, S. sp. 10.-Vide postea, notas ad Typhum.

Hemitritæus pestilens, Schenck obs. lib. vi. ex Corn. Gemma.

Febres pestilentes Ægyptiorum, Alpin. de med. Ægypt. lib. i. cap. 14.

Febris tertiana epidemia, Bartholin. Hist. anat. cent. ii, 56.

Febres epidemicæ Autumni ann. 1657 et 1658, Willis de febr. cap. 16.

Febris syneches epidemica ab. ann. 1658 ad 1664, et postea ab ann. 1673 ad 1691. Morton. app. ad exerc. ii.

* De typo febris sudatoriae Boyeri Meth. a suivre dans les malad. epidem. quam huc retulit Sauvagesius, ex ipsius Boyeri descriptione mihi non liquet, et ad typhum referri mallem. 
(Tertianá.)

Febres autumnales incipientes, Sydenh. de morb. acut. ad ann. 1661-1664, et in epist. ad H. Paman ad 1678, et seq.

Affectus epidemicus Leidensis, Fr. Sylvii prax. med. app. tract. $x$, et oratio de affectus epidem. causis.

Morbus epidemius Leidensis 1669, Fanois, diss. apud Haller. disp. tom. $\mathbf{v}$.

Tertianæ perniciosæ et pestilentes et febres castrenses epidemiæ Lancisi, de nox. palud. effluv.

Febres intermittentes anomalæ et mali moris, Hoffm. ii, p. 38.

Febris cholerica minus acuta, Hoffm. ii. p. 112.

Febris epidemica Leidens. ann. 1719. Koker apud Haller, disp. tom. v.

Amphimerina paludosa, S. sp. 19.

Febris paludum, illustr. Pringle Diseases of the Army, edit. 4ta, 8vo, p. 179.

Bononiensis constitutio hiemalis 1729, Beccari in A. N. C. vol. iii. obs. 48.

Amphimerina biliosa, S. sp. 22.

Remittens biliosa, Macbride, p. 360.

Febris Castrensis, illustr. Pringle loc. cit. p. 174.

Febris putrida epidemica, Huxham de aëre ad ann. 1729.

Febris biliosa Lausanensis, cl. Tissot. de febr. bilios.

Tritæophya Wratislaviensis, S. sp. 3. Hahn. epidemia verna Wratislav. in app. ad. A. N. C. vol. x.

Tritæophya Americana, S. sp. 12 ?

Febris anomala Batava. Grainger de febr. anomala.

Morbus Naronianus, Pujati de morb. Naron.

Febris continua remittens, Hillary's dis. of Barbadoes, variis annis, Lond. med. obs. iv. 24. v. 2.

Febris ann. 1772, et seq. ad urbem novam Avenionensem, Soc. Royale de Médecine i. Histoire, p. 213.

Febris intermittens irregularis Tolosæ 1772. Soc. Royale i. mém. p. 14.

Febris intermittens et remittens a l'Isle Jourdain, anno 1777, Soc. Royale ii. hist. p. 145.

Febris remittens Indiæ Orientalis, Lind. med. Edin. diss. inaug. 1768. Lond. med. obs. vol. iv. a. 12.

Febris critica et febr. biliosa æstatis, Rouppe de morb. navig.

Febris remittens regionum calidarum, cl. Lind. med. Haslar. Essay on the diseases of hot climates.

Tertiana remittens symptomatica est:

Tritaeophya lactea, S. sp. 8.-Febris lactea, Etmulleri Coll. consult. cas. 17 . 
G. II.-QUARTANA.

Paroxysmi similes intervallo septuaginta duarum circiter horarum: accessionibus pomeridianis.

Quartana Auctorum, Sauv. gen. 89. Lin. 17. Vog. 3. Est vel,

Sag. 711. Hoffm. ii. p. 23. Junck. tab. 81.

\section{INTERPOSITA APYREXIA.}

A. Variat typo.

a. Quartana paroxysmis quarto quoque die singulis; aliis diebus nullis.

Q. legitima S. sp. 1. Sydenham de morb. acut. cv.

b. Quartana paroxysmis quarto quoque die binis ; aliis diebus nullis.

Q. duplicata, S. sp. 4. Bonet.

c. Quartana paroxysmis quarto quoque die tribus; intermediis diebus nullis.

Q. triplicata, S. sp. 16.

d. Quartana quæ ex quatuor diebus tertium tantum a febre vacuum habet, paroxysmis quarto quoque die similibus.

Q. duplex, S. sp. 3. Vog. 13.

e. Quartana quotidie accedens, paroxysmis quarto quoque die similibus.

Q. triplex, S. sp. 5. Vog. 14. Barthol. hist. anat. c. i, 95.

B. Variat symptomatibus.

Q. cataleptica, S. sp. 7. Bonet. Polyalth. vol. i. p. 805.

Q. comatosa, S. sp. 15. Werlhof. de febr. C. Pisonis observ. de morbis a colluvie seros. obs. $166,167,168$, $169,171,172,173,174$.

Q. epileptica, S. sp. 8. Scholzii Cons. 379, 380.

Q. hysterica, S. sp. 10. Morton. pyret. exerc. i. c. ix. h. $10,11$.

Q. nephralgica, S. sp. 9.

Q. metastatica, S. sp. 17 .

Q. amens. S. sp. 12. Sydenham de morb. acut. c. v.

Q. splenetica, S. sp. 2. Etmuller. Coll. consult. cas. 25.

Q. hepatica, Macb. p. 354 .

C. Variat aliis morbis complicata.

Q. syphilitica, S. sp. 6. Plateri obs. 1. iii, pag. 676. Edinb. Essays, art. xlvii, obs. 8. 
(QUARTANA.)

Q. arthritica, S. sp. 11. Musgr. de arthr. symptom. cap. ix, h. 4 et 5.-Arthritis febrisequa, S. sp. 10.-Arthritis febricosa, S. sp. 10. Werlhof. de febr. Cockburn de morbis navigantium, obs. 19.

Q. scorbutica, S. sp. 14. Barthol. de med. Dan. diss. iv. Tim. 1. viii, cas. 18.

\section{INTERPOSITA REMISSIONE TANTUM.}

Tetartophya, S. gen. 85. Sag. 323. Lin. 22.-Quartana remittens Auctorum.

\section{Varietates sunt,}

Tetartophya simplex, S. sp. 1.*

Amphimerina semiquartana, S. sp. 23.

Tetartophya semitertiana, S. sp. 25.

Tetartophya maligna, S. sp. 6. Lautter. Hist. med. cas. 21. M. Donat. lib. iii., cap. 14. et M. Gatenaria Horst. lib. i. obs. 15.

Tetartophya carotica, S. sp. 4. Werlhof. de febr. Bianchi Hist. hep. pars. iii. const. ann. 1718, p. 751.

Tetartophya splenalgica, S. sp. $2 . \dagger$

Tetartophya hepatalgica, S. sp. 3. Car. Pis. in praefat. p. 33.

Amphimerina spasmodica, S. sp. 16.

Erratice. Ad Tertianam vel Quartanam Erraticas S. pertinere putamus $s_{+}^{+}$; et hic ideo earum varietates subjunximus.

Erratica quintana, S.sp. 1. Tulp. lib. iii. 52. Forest. lib. iii. obs. 43. - Ex quartana ill.jVan Swieten, comm. p. 505.

Erratica septana, S. sp. 2. Boerh. apud ill. Van Swieten, ibid. M. Don. 1. 3. 14.-Hebdomadaria Schenkii ex Gibalto.-Septimana, ill. Morgag. xlix. 36.

Erratica octana, S. sp. 3. Etmuller, prax. lib. i., § xv. cap. 2. Cyrilli in Etmuller. p. 187, 188, 365. Valles Controv. 1. v., c. 25. P. Sal. Div. in not. ad D. A. de Altomari, cap. xii. Zacut. Lus. P. M. lib. iii. obs. 34 .

* Quartanam Continuam morbum rarissimum esse, testantur scriptores medici omnes; sed illius exemplum ex Franc. Joelis operum tomo 3. citat Sauv. In loco autem citato, nihil ad rem pertinens inveni; sed tomi quinti pag. 63, haec verba proferuntur. Febris quartanae 'species veteres duas constituunt. Quartanam Continuam, (quae mihi septuagenario nunquam visa est, et ideo eam aut rarissimam aut nullam esse judico) et Interpolatam, quae frequentissima est.'

+ Huc febrem Fernelianam Raym. Fortis refert Sauv. et vult febrem ite a Forte dictam fuisse, quod ea mortuus sit ipse Fernelius. Sed quodammodo hic errasse Sauvagesius mihi videtur. Nam febres quasdam Fernelianas vocat Fortis, tom. ii, cent. ii. obs. 35 et 36, non quod e genere remittentium fuerint, sed quod visceris cujusdam, hepatis praesertim, labe comitatae sint; et mortuus quidem est Fernelius ex inflammatione lienis. Sed quod hic morbus quartanae typum unquam exhibuit, non dicitur a Plantio, qui in vita Fernelii, operibus ejus praefixa, de eo morbo narrat.

¥Vide Senacum de reeondita febrium natura, lib. i. cap, 1 , 
(QUARTANA.)

Schultzius in E. N. C. dec. I. ann. iv. et v. obs. 70. Arnold. de febr. Stomach. epid. sect. iv. apud Haller. dissert. pract. vol. v. De Haen de divis. febr. div. iv. p. 9.

Hemicrania lunatica, S. sp. 10.

Erratica nonana, S. sp. 4. Zac. Lus. ibid. decimana, S. sp. 5. Zac. Lus. ibid.

Ephemera dichomene, S. sp. 11. M. Don. lib. iii. 14. ex Gentili.

Erratica vaga, S. sp. 6. River. cent. iii. observ. 32 . Etmuller, lib. i. sect. xv. cap. 2.

\section{G. III.-QUOTIDIANA.}

Paroxysmi similes intervallo viginti quatuor circiter horarum : Paroxysmis matutinis.

Quotidiana Auctorum, S. gen. 87. Linn. 15. Vog. 1. Hoffm. 11, 33. Junck. tab. 79.

\section{INTERPOSITA APYREXIA.}

A. Variat solitaria.

$a$. Universalis: Quotidiana eadem hora matutina rediens.

Quotidiana simplex, S. sp. 1.-Quotidiana legitima, Sennert. de febr. cap. 18.

b. Partialis.

Quotidiana partialis, S. sp. 10. Cnoffel. E. N. C. dec. I. a. iii. obs. 205. Edinb. Med. Ess. vol. i. art. 31. vol. ii, art. 19.-Quotidiana cephalalgica, S. sp. 6. Morton Pyret. exerc. i. hist. 27. Van Swieten in Boerh. p. 534. Soc. Royale, ii mém. p. 38.-Cephalalgia intermittens, S. sp. 7.-Cephalaea febricosa, S. sp. 4.-Quotidiana ophthalmica, Morton, ibid. hist. 17. Van Swieten, ibid.-Ophthalmia febricosa, S. sp. 23.

B. Variat comitata.

Q. ischiadica, S. sp. 5. Edinb. Ess. vol. v. art. 49.-Ischias intermittens, S. sp. 1.-Q. nephralgica, S. sp. 7. Morton, ibid. hist. 28. - Nephralgia febricosa, S. sp. 13. Q. uretico-sputatoria, B. Scharf. E. N. C. dec. ii, a. ii, obs. 104.-Q. epileptica, S. sp. 4. Edinb. Ess. loc. cit. -Hysteralgia febricosa, S. sp. 9.

Vespertinæ vel symptomaticæ videntur sequentes :

Q. hysterica, S. sp. 3. catarrhalis, sp. 9. stranguriosa, sp. 11. 
(qUOTIDIANA.)

\section{INTERPOSITA REMISSIONE TANTUM.}

Amphimerina, S.gen. 84. Lin. 20. Sag. 321.-Quotidiana continua, Vog. 15.-Quotidianæ remittentes et continuæ Auctorum.-Amphimerina latica, sp. 1.-Febris continua lymphatica, Etmuller Coll. cons. cas. 32. River. obs. cent. i, ob. 57.-Amphimerina singultuosa, sp. 14.-Febris continua Lyngodes, Vog. 26.

Amphimerinæ S. cæteræ vel ad Tertianas pertinere, quorsum plures supra retuli, vel symptomaticæ et vespertinæ esse videntur. Hujusmodi sunt, .

Amphimerina catarrhalis, sp. 2. anginosa, sp. 12. tussiculosa, sp. 13. peripneumonica, sp. 15. variolosa, sp. 20. miliaris, sp. 11. arthritica, sp. 21.

De Amphimerina mimosa Bontii et phrenitica ejusdem, S. sp. 17, 18, non liquet.

\section{SECT. II.-CONTINUAE.}

Febres, sine intermissione, nec miasmate paludum ortae, sed cum remissionibus et exacerbationibus, parum licet notabilibus, perstantes : Paroxysmis quovis die binis. $\uparrow$

- Quae sine exacerbatione partiali, et sine accessu, non pluries quam semel vel bis in mense revertens, ad usque finem aegritudinis perseverat, Febrem Continuam esse,' dixit Sauvagesius ; et hujusmodi febres, cum scholis medicis plerisque, Continentes vocat Linnaeus. Hujusmodi autem febrem in quadraginta annorum praxi satis frequenti, vix unquam certo vidi, et fere semper in febribus, maxime continuis, exacerbationes et remissiones, etiam quotidie, satis manifestas observavi. Mecum quidem sentire videtur Vogelius, uti ex ejus continuarum charactere satis patebit; nec dissentit expertissimus De Haen, uti videre est in libro ejus de divisione febrium, Divis. iv. Schol. i. ; et apprime sententiam nostram confirmat Brendelius, verbis sequentibus: 'Omnes namque febres nostrae, acutae, et inflammatoriae, et exanthematicae, et malignae reliquaeve hujus generis, sunt continuae remittentes, et luculenta intensionum remissionumque spatia integrant.' Brendel. Opuscul. p. ii. diss. xi. sect. v.

Porro, etsi in Febribus quibusdam exacerbationes et remissiones parum notabiles, et perinde observatu difficiles sint, easdem tamen revera fieri censeo; et ob hoc praesertim, quod in febribus quibuscunque, ex paroxysmis pluribus manifesto constantibus, paroxysmus quisque, inter nycthemerae spatium, cursum suum, ex exacerbatione et remissione constantem, semper absolvat, ita ut in his rebus oeconomiam animalem circuitus diurni legi cuidam subjici videatur. Huic etiam in sanitate parere solet, et huic in febribus hecticis quibusque ad amussim subjicitur. Verisimillimum itaquevidetur, eundem circuitum, quem toties observavimus, in febribus quibuscunque locum habere; nec febrem quamcunque continentem revera in rerum natura dari.

+ Cum continuam quámque ex paroxysmis repetitis consistere ponimus, saepe ambiguum fieri possit, utrum febris data ex continuarum sit, an ex remittentium ordine, et ut rite determinetur, tum intermittentium, ad quas omnes auctorum remittentes pertinere putamus, tum continuarum, definitionem aliam quam antea nunc dedi. In plerisque casibus, ut opinor, definitiones nunc datae, satis facile et certo adhiberi possint. Sed sagacioribus quibusvis examinandum relinquo, an vel definitiones nostrae recte dentur, vel si quid rectius dari queat. Febrem quamque continuam ex paroxysmis quovis die binis constare credimus; cum autem in quibusdam, tum intermittentibus tum remittentibus, itidem paroxysmi duplicati videantur, ex illa nota sola febres continuae dignosci non possunt. Ex illa nota sola quidem ut dignoscantur nequaquam volui; nam in casu quovis ambiguo, vel a principio, vel a typo, vel ortu ab intermittentibus, remittentem quamvis cognosci posse puto. Videant porro in his rebus versati, annon a principio, saepe manifeato et maxime frequenti, contagione nimirum humana, febres continuae saepe certe cognosci possint. 
Continuae, Sauv. cl. ii. ord. i. Vog. cl. i. ord. ii. Sag. 666. Macb. p. 303. Boerh. 727.

Continentes, Lin. cl. ii. ord. i. Stahl. Cas. mag. 35. Cas. min. 87. Junck. 58. Sennert. de febr. lib. ii. cap. 2, et 10 .

\section{G. IV.-SYNOCHA.*}

Calor plurimum auctus; pulsus frequens, validus, et durus ; urina rubra; sensorii functiones parum turbatae.

Synochus, S. gen. 80. Lin. 12. Junck. 58.-Synochus sive febris acuta sanguinea, Hoffm. ii, 105.-Synochus, Vog. 16.-Continua non putris, Boerh. 729.-Ephemera, S. gen. 79. Boerh. 728. Junck. 57.-Diaria, Lin. 11.Febris inflammatoria Auctorum.

\section{Varietates sunt;}

Synocha plethorica, S. sp. 1. sanguinea, Sennert. de febr. lib. ii. c. 11.-Ephemera plethorica, S. sp. 1. a frigore, sp. 3. a calore, sp. 4.-Synochus pleuritica,'sp. 5. Febris pleuritica ann. 1765. Sydenh. sect. v. cap. 1, 5.-Synochus rheumatisans, S. sp. 7. Sydenh. sect. v. c. 2.-Synochus hiemalis, S. sp. 8. Sydenh. in P.S. ad tractat. de hydrope.

\section{Symptomaticae sunt :}

Ephemera nauseativa, S. sp. 2. a phlogosi, \&c. sp. 6.-Synocha dolorum, sp. 7. M. Tab. ord. i, gen. i. sp. 1, var. D.-Ephemera lactea, S. sp. 5, menstrua, sp. 8.-Synocha catarrhalis, sp. 5. M. Tab. ord. i, gen. 1, sp. i, var. E.-Synocha scorbutica, S. sp. 6. - Synochus scorbutica, sp. 13.-Synocha cephalalgica, sp. 8. M. Tab. ord. i, gen. i. sp. 2. var. A.

\section{G. V.-TYPHUS.}

Morbus contagiosus; calor interdum parum auctus; pulsus parvus, debilis, plerumque frequens ; urina parum mutata ; sensorii functiones plurimum turbatae; vires multum imminutae.

Typhus, Sauv. gen. 82. Sag. 677.

- Febrium continuarum divisio, a Galeni temporibus, in hunc usque diem, eadem fere usitata est, et vel ex putredinis gradu parum accurate definito, vel ex duratione morbi, desumpta fuit ; utraque tamen nota, posterior praesertim, qua praecipue usi sunt Nosologi Sauvagesius, Linnaeus, et Sagarus, manifesto inepta est. Vide Prolegomena, pag. 239. Aliam itaque divisionem a differentia symptomatum, et a natura morborum, quantum de ea judicare liceat, desumptam, institui ; secutus simul illam apud Britannos nunc maxime usitatam divisionem febrium continuarum in Inflammatorias et Nervosas. Has autem appellationes, utpote quodammodo theoreticas, vitavi. Nomina quibus usus sum apud medicos dudum recepta sunt; et si forsitan sensu vulgo recepto, in quo medici ipsi parum constantes fuerunt, iis non usus sum, parum curo, cum definitiones subjunctae ab errore quovis praecavere possint. 
(TYPHÜ.)

\section{Species sunt,}

A. Typhus (petechialis) plerumque cum petechiis.

Variat gradu :*

a. Typhus mitior.

Febris maligna hectica sive lues yะvewdns convulsiva, Willis de morb. convulsiv. cap. 8.†-Febris pestilens Fracastor. de morb. contag. lib. ii, cap. iv.-Febris pestilens sine charactere veneni Forest. lib. vi, obs. 26. Febris hectica pestilens Forest. lib. vi, obs. 32.-Febris nova anni 1685. Sydenham Sched. monitor.-Febris putrida nervosa Wintringh. Comm. Nosolog. ad annos 1720,1721 . - Febris lenta nervosa. Huxham on $\mathrm{Fe}$ vers, chap. 8. Edinb. Med. Ess. ii, 18. iv, 23. v, 48. Macb. p. 304. Soc. Royale, i mém. p. 23.-Febris contagiosa. Lind on Fevers and Infection, passim.Typhus nervosus, S. sp. 2.-Typhus comatosus, S. sp. 3. Tritaeophya typhodes Mangeti, S. sp. 11. Raym. Fort. de febribus, p. 24. M. 304.

b. Typhus gravior.

Febris pestilens, P. Sal. Divers. de febre pestilenti.-Febris pestilens Egyptiorum Alpin. de med. Fgypt. lib. i, cap. 14.-Typhus Ægyptiacus, S. sp. 6-Cephalitis epidemica, anno 1510 , S. sp. 6.-Febris pestilens maligna Sennert. de febribus, 1. iv, cap. 10.-Febris maligna pestilens River. lib. xvii, s. iii, c. 1.-Febris pestilens maligna, anno 1643. Willis de febribus, cap. 14. -Febris Senegallensis, Lond. med. obs. ii, 21.-Typhus carcerum, S. sp. 1.-Febris nautica pestilentialis, Huxham de aëre ad annum 1740.-Miliaris nautica, S. sp. g.-Febris putrida contagiosa in carceribus genita, Hüxham de aëre ad annum 1742.-Miliaris purpurata, S. sp. h.-Febris carcerum et nosocomiorum. Ill. Pringle, Diseases of the Army, p. 294. Ill. Van Swieten, Maladies des armées, p. 136. Soc. Royale, ii mém. p. 53. Ibid. iii mém. p. 45 et 55.-Typhus castrensis,

* Morbos gradu solum differentes nominibus diversis insignire nequaquam convenit; cum autem nuperis temporibus apud medicos usitatum sit, febrem quandam, quasi ab aliis quibusvis differentem, nomine Febris Nervosae appellare; buic opinioni quodammodo morem gerens, sub titulo Typhi Mitioris, variorum auctorum febres ad recentiorum nervosas aliquomodo referendas recensui. In hac re autem, cum limites neutiquam accurate ponend s sunt, me accuratum fuisse non dixerim.

Minus adhuc placuit, ad mentem vel veterum vel recentiorum inter febrium genera aliquod sub nomine Febris. Putridae recensere. In omni Typho humorum in putredinem proclivitatem adesse puto; sed vario tantum gradu adest; ita ut major minorve putredo speciem variare, nequaquam mutare, potest. Sufficiat sub titulo Typhi Gravioris eas, quae praesertim putridae adpellatae sunt, febres indigitasse, uti ex appositis variorum auctorum appellationibus satis patebit.

+ Hoc est exemplum, ni fallor, primum appellationis N\&upwinn, vel nervosae, febribus quibusvis datae; quam protinus Angli, non tamen nisi nuper admodum aliarum regionum medici, usurpati sunt. 
S. sp. 5.-Febris castrensis, quam vulgo cephalalgiam epidemicam vocant, Henr. Maii et $\mathrm{A}$. Ph. Koph. Diss. apud Hallerum, tom. v.-Febris Hungarica sive castrensis Juncker, 74. et plurium Auctorum.*-Febris castrensis Gallorum in Bohemia, anno 1742. Scrinci diss. apud Haller. tom v.-Febris petechialis, Sennert. lib. iv, c. 13 . River. prax. lib. xvii, sect. iii. c. 1 . Hoffm. ii, p. 84. Juncker, 73. Huxham on fevers, ch. 8. Ludwig. Inst. med. clin. no. 146. Schreiber von Erkenntniss und Cur der Krankheiten, p. 126. Monro, Diseases of military hospitals, p. 1.-Febris catarrhalis maligna petechizans, Juncker, 72. Hoffm. ii, 75. Eller de cogn. et cur. morb. sect. vi.-Febris putrida, Macb. p. 305.-Febris catarrhalis putrida 1768. De Martens obs. cap. 1.-Febris putrida biliosa 1769. De Martens, cap. 2.-Febris putrida nervosa 1770. De Martens, cap. 3.

Exempla febrium petechialium sunt sequentia :

Febris quam lenticulas, puncticula, aut peticulas vocant, Fracastorius de morb. contag. lib. ii, c. 6 .

peticularis Tridenti, anno 1591. Roboretus de febr. peticul.

- petechialis epidemica Coloniæ, anno 1672. Donckers, Idea febris petechialis.

_ petechialis epidemica, Posonii 1683, C. F. Lou in Append. ad A. N. C. vol. ii.

- petechialis epidemica Mutinæ, 1692. Raməzzini Const. Mutinensis, Oper. p. 187.

— maligna petechizans, anno 1698. Hoffm. ii, p. 80. petechialis Wratislaviæ, anno 1699. Helwich, Ephem. Germ. dec. iii, a. 7 et 8, obs. 132, p. 616.

epidemia Lipsiæ 1718. M. Adolph. A. N. C. iii, obs. 131, p. 296.

- endemica et epidemica Corcagiensis, anno 1708, 1718, et seq. Rogers, Essay on epidemic diseases.

- continua epidemica Corcagiensis, anno 1719 et seq.

M. O'Connel Observ. de morbis.

petechialis epidemica, Cremonæ 1734. Valcharenghi Med. ration. sect. iii.

petechizans Petropoli 1735, Weitbrecht. diss. apud Haller. tom. v.

- Morbum sive febrem Hungaricam ad tertianas remittentes, cum Sauvagesio et ill. Pringle, supra retuli; nec dubium est quin morbus Hungaricus dictus saepius sub forma remittentis apparuerit; sed simul constat febrem in castris Hungaricis primo ortam, et per milites inde redeuntes per totam fere Germaniam dispersam, ex genere continuarum fuisse (Vide Ruland. de morbo Hungarico, c. viii. quaest. 39), et plerumque eandem esse quam nunc in Nosocomiis quibusvis saepe exorientem bene novimus. Ad hunc locum igitur etiam apponendam esse putavi.

vol. I. 
(TYPHUS.)

Febris petechialis, annis 1740,1741 , in Hassia, Ritter, A. N. C. vol. vii, obs. 4.

- petechialis epidemica Vigorniæ et aliis Angliæ locis. Wall's Works, p. 337 .

maligna petechialis Rintelii 1741. Furstenau, A.N.C. vol. vii, obs. 5 .

- petechialis epidemica Silesiæ 1741 et seq. Brandhorst. diss. apud Haller, tom. v.

_ petechialis epidemica Viennæ 1757, Hasenohrl hist. med. cap. 2.

— petechialis epidemica Lipsiæ 1757, Ludwig. Adversar. tom. i, pars 1 .

- petechialis epidemica variis Germaniæ locis ab anno 1755 ad 1761 . Strack de morbo cum petechiis.

- epidemica maligna a Coutances 1772 et 1773 . Soc. Royale, i mém. p. 23.

petechialis nervosa a Dijon 1760 et 1761 . Mém. par Maret. 1775.

B. Typhus (icterodes) cum flavedine cutis.

Typhus icterodes, S. sp. 7.-Febris flava Indiæ Occidentalis. Warren, Malignant fever of Barbadoes. Hillary's Diseases of Barbadoes. Linning on the yellow fever of South Carolina. Edinb. phys. litter. essays, vol. ii. Mackittrick de febre flava Indiæ Occidentalis, Edinb. 1766.

Typhi etiam species esse videtur,

Ephemera sudatoria, S. sp. 7.-Ephemera Britannica, Caius de ephem. Brit.-Sudor Anglicus, Sennert. lib. iv. cap. 15.-Hydronosus, Forest. lib. vi, obs. 8.

An eodem pertinet:

Miliaris sudatoria, S. sp. e.-Febris sudatoria, La Suette Gallorum, Meyserey Méth. aisée, \&c. Ejusd. Maladies des Armées, 250. Boyer Methode à suivre dans les maladies épidémiques. Tessier, Soc. Royale, ii mém. p. 46.

De typho hysterico verminoso, S. sp. 4. exhaustorum, sp. 8. a manipuera, sp. 9. nobis non liquet.

\section{G. VI.-SYNOCHUS.*}

Morbus contagiosus. Febris ex synocha et typho composita, initio synocha, progressu et versus finem typhus.

- Cum plures febres nec inflammatoriae nec nervosae ex omni parte sint, neque idcirco vel ad Synocham vel ad Typhum facile referendae; genus Synochi, cujus typus hisce regionibus frequens conspicitur, hic inserui. Inter Typhum tamen et Synochum limites accuratos ponere non possum; et an revera pro diversis generibus habenda, vel positis diversis, utri eorum Synonyma auctoruin referenda sunt, dubito. Quae vero hic subjunguntur, a Sauvagesio species dictae, ad Synochum satis recte, ut mihi videtur, referri possunt. 
(sXNOCHUS.)

Synochus, S. gen. 81. Lin. 13.-Lenta, Lin. 14.-Phrenitis, Vog. 18.-Febris continua putrida, Boerh. 730.

Varietates ex Sauvagesio sunt :

Synochus sanguinea, sp. 1. Febris depuratoria, anno 1661 ad 1664. Sydenh. de morb. acut. - Synochus sudatoria, sp. 2. Febris continua epidemica, anno 1665 ad 1667. Sydenh. ibid.-Synochus soporosa, sp. 12. Febris continua epidemica, anno 1673. Sydenh. ibid.-Febris continua putrida annorum variorum. Wintringham Com. Nosolog.-Synochus ardens, sp. 6.-Synochus miliaris, sp. 14. - Synocha miliaris, sp. 6.

De synocho variolode, sp. 3. et synocha dysenteriode, sp. 4. nobis non satis liquet; et minus adhuc de Synocho anniversaria, sp. 9. spermatica, sp. 10. tarantata, sp. 11.-Complicata est Synochus scorbutica, sp. 13. ; et symptomatica est Synochus a scabie, sp. 15 .

\section{HECTICA.}

Febris quotidie revertens; accessionibus meridianis et vespertinis; remissione, rarius apyrexia, matutina; plerumque sudoribus nocturnis, et urina sedimentum furfuraceo-lateritium deponente.*

Hectica, S. gen. 83. Linn. 24. Vog. 80. Sag. 684. Med. trans. ii, 1 .

\section{Ex Sauvagesio species sunt :}

Hectica chlorotica, sp. 3. syphilitica, sp. 4. scrophulosa, sp. 5. a calculis, sp. 6 . hydropum, sp. 7 . verminosa, sp. 9. cachectarum, sp. 10. fluxuum, sp. 11.-Hectica infantilis, sp. 1 . charactere caret, et nobis vel rachitica, vel scrophulosa, vel verminosa videtur.-Hectica vespertina, sp. 2. vix pro morbo haberi potest.-De hectica lymphatica Baglivi, S. sp. 12. et hectica nervea cl. Lorry, S. sp. 13. nobis non liquet.

* Cum febrium in numerum, hecticam receperint Nosologi omnes, eam etiam cum charactere hic apponendam duxi. Ejusmodi autem febrem, cum nunquam nisi symptomaticam observaverim, in numerum idiopathicarum, quas solas enumerare licet, admittere nolui. In plerisque exemplis, hecticam revera symptomaticam esse, ex Sauvagesii speciebus, quas vocat, hic recensitis, satis manifestum fiet. Vide ipsius Sauvagesii Scholium, tom. i. pag. 319. 


\section{ORDO II.-PHLEGMASIAE.}

Febris synocha; phlogosis; vel dolor topicus, simul laesa partis internae functione; sanguis missus, et jam concretus, superficiem coriaceam albam ostendens.

Phlegmasiae membranosae et parenchymatosae, S. cl. iii. ord. i. ii. Sag. 605.*-Morbi febriles phlogistici, Linn. cl.iii.-Febres continuae compositae inflammatoriæ, Vog. Morbi acuti febriles, Boerh. 770.-Febres inflammatoriae, Hoffm. ii. 105. Junck. 61.

\section{G. VII._PHLOGOSIS. $†$}

Pyrexia, partis externae rubor, calor, et tensio dolens. Species sunt,

1. Phlogosis (Phlegmone) rubore vivido ; tumore circumscripto, in fastigium plerumque elevato, saepe in apostema abeunte; dolore saepe pulsatili.

Phlegmone Auctorum, Sauvag. gen. 15. Lin. 39. Vog. 351. Sag. 299. Macb. p. 418.-Inflammatio, Lin. 231. Boerh. 370. Junck. 20.

a. Variat forma.

Furunculus, S. gen. 18. Vog. 352. Sag. 23. Macb. p. 222.-Terminthus, Vog. 381.-Papula, Lin. 275. S. p. 6.-Varus, Vog. 436. Lin. 269. S. p. 7. Bacchia, Lin. 270. Gutta rosea, Sauv. gen. 4. Gutta rosacea, Vog. 437.

b. Variat sede. +

Hordeolum, S. gen. 27. Lin. 276. Vog. 434.-Otalgia, S. gen. 197. Lin. 44. Vog. 148. Dolor otalgicus, Hoffm. ii, 336.-Parulis, Vog. 362.-Mastodynia, S. gen. 210. Vog. 153.-Paronychia, S. gen. 21. Lin. 258. Vog. 345.-Arthrocace, S. gen. 78. Lin. 256. Paedarthrocace, Vog. 419. Spina ventosa, Boerh. 526.-Phimosis, S. gen. 22. Lin. 297. Vog. 348. Paraphimosis, Vog. 349.-Proctitis, Lin. 30. Proctalgia, S. gen. 214. Clunesia, Vog. 167.

* Haec phlegmasiarum in membranosas et parenchymatosas divisio prorsus inepta est ; primo enim, quod de statu partium internarum statuit, de quo in Nosologia statuere non licet; dein, quod partes membranosae et parenchymatosae non satis certo distinguuntur; et hinc Metritis, quam inter membranosas recenset Sauvagesius, a Linnaeo et Sagaro inter parenchymatosas collocatur; denique quod phlegmasiae quaedam, ut hepatitis, vel membranosae vel parenchymatosae esse possunt.

Nec magis idonea est ordinis phlegmasiarum musculosarum apud Linnaeum et Sagarum institutio, in quo neuter quidem satis accuratus est. Male enim phlegmone a Linnaeo musculosa dicitur, nec bene Cynanche plerumque membranosa, rarius musculosa, a Sagaro ad musculosas in universum refertur.

+ Pro nomine generis cujus species est Erythema, minus recte in priore editione usurpatum fuit phlegmone. Novum nomen necessarium nobis videbatur, et nihil aptius quam Phlogosis suppetehat.

¥ An sequentes pro totidem speciebus recenseri possint, judicent peritiores. 
(PHLOGOSIS.)

2. Phlogosis (Erythema) colore rubicundo, pressione evanescente; ambitu inaequali, serpente; tumore vix evidente, in cuticulae squamulas, in phlyctaenas vel vesiculas abeunte; dolore urente.

Erythema, S. gen. 11. Sag. 16.-Erysipelas Auctorum, Vog. 343. Macb. p. 421.-Hieropyr, Vog. 344.

a. Variat vehementia.

Anthrax, S. gen. 19. Lin. 272. Vog. 353. Sag. 24.-Carbo et carbunculus Auctorum, Macb. p. 423.-Erythema gangraenosum, S. sp. 7 .

b. Variat causa remota.

Erythema a frigore. Erythema pernio, S. sp. 4. Pernio, Lin. 259. Vog. 350.-Erythema ambustio, sp. 2. Erysipelas ambustio, sp. 4. Combustura, Lin. 245. Combustio, Boerh. 476. Encausis, Vog. 347.-Erythema ab acri alieno applicato, Erysipelas Chinense, sp. 7.Erythema ab acri inquilino, Erythema intertrigo, sp. 5. Intertrigo, Lin. 247. Vog. 502.-Erythema a compressione, Erythema paratrima, sp. 6.-Erythema a punctura, sp. 9. Erysipelas a vespis, sp. 19. Psydracia a vespis, sp. 2.

c. Variat complicata.

Erythema cum phlegmone, Erysipelas phlegmonodes Auctorum.-Erythema cum oedemate, Erysipelas symptomaticum, S. sp. 6.

Phlogosis sequelae sunt,

1. Apostema. Post phlogosin, remittentibus dolore et pulsatione, tumor albescens, mollis, fluctuans, pruriens.

Apostema, S. gen. 39. Sag. 55. Abscessus, Lin. 132. Vog. 354. Boerh. 387, 402. Macb. p. 414. Pustula, Lin. 274.

2. Gangraena. Post phlogosin, pars livens, mollis, parum sensibilis, saepe cum vesiculis ichorosis.

Gangraena, S. gen. 314. Lin. 233. Vog. 327. Sag. 203. Boerh. 338, 419. Macb.p. 415.

3. Sphacelus. Post gangraenam pars nigricans, flaccida, facile lacerabilis, sine sensu vel calore, et cum foetore carnis putridae; vitio celeriter serpente.

Sphacelus, Lin. 234. Vog. 328. Boerh. 419, 457.

G. VIII. OPHTHALMIA.

Rubor et dolor oculi; lucis intolerantia; plerumque cum lachrymatione.

Ophthalmia, S. gen. 196. Lin. 43. Vog. 341. Sag. 231. Junck. 24. Macb. p. 423.-Chemosis, Vog. 46.-Ophthalmites, Vog. 47.-Inflammatio oculorum, Hoffm. ii, 165 . 


\section{(OPHTHALMia.)}

Species et varietates sunt,

\section{IDIOPATHICAE.}

1. Ophthalmia (membranarum) in tunica adnata, et ei subjacentibus membranis, sive tunicis oculi.

a. Variat gradu phlogosis externae.

Ophthalmia taraxis, S. sp. 1. humida, sp. 8. chemosis, sp. 12. Erysipelatosa, sp. 7. pustulosa, sp. 6. phlyctaenodes, sp. 21.

b. Variat affectis tunicis internis.

Ophthalmia choroideae, S. sp. 13. tenebricosa, sp. 10.

2. Ophthalmia (Tarsi) cum tumore, erosione, et exudatione glutinosa tarsi palpebrarum.

Ophthalmia trachoma, S. sp. 4. et sicca, sp. 5.

\section{SYMPTOMATICAE.}

a. A morbo ipsius oculi.

Ophthalmia angularis, S. sp. 14. tuberculosa, sp. 3. trichiasis, sp. 2. cancrosa, sp. 15. a synechia, sp. 16. a lagophthalmo, sp. 17. ab elcomate, sp. 18. ab ungue, sp. 19. a corneae fistula, sp. 20. uveae, sp. 22.

b. A morbis aliarum partium vel totius corporis.

Ophthalmia metastatica, sp. 24. scrophulosa, sp. 9. syphilitica, sp. 11. febricosa, sp. 23.

\section{G. IX.-PHRENITIS.}

Pyrexia vehemens; dolor capitis ; rubor faciei et oculorum ; lucis et soni intolerantia; pervigilium; delirium ferox vel typhomania.

Phrenitis, S. gen. 101. Lin. 25. Sag. 301. Boerh. 771. Hoffm. ii, 131. Junck.63. Macbr.p.432.-Phrenismus, Vog. 45.-Cephalitis, S. gen. 109. Sag. 310.*-Sphacelismus, L. 32.-Siriasis, Vog. 34.

1. Phrenitidis idiopathicae speciem unicam hic ponere licet, et cujus synonyma sequentia esse putamus :

Phrenitis vera, S. sp. 1. Boerh. 771.-P. idiopathica, Junck. 63.-Cephalalgia inflammatoria, S. sp. 9-Cephalitis spontanea, S. sp. 3.-Cephalitis siriasis, sp. 4. -Siriasis, Vog. 34. - Cephalitis Littriana, S. sp. 5.

* Cum symptomata nulla dentur quae semper phlegmasiama cerebri a plegmasia membranarum ejus, sive meningum, certo distinguere possint; neque sectiones cadaverum distinctiones a Sauvagesio, Linnaeo, et Sagaro, adhibitas confirment, Cephalitidem, Sauv. gen. 109, Sag. gen. 310, et Sphacelismum, Lin. 32, pro generibus a Phrenitide diversis admittere nolui, et ea hic ideo, uti synonyma, sub titulo Phrenitidis posui.

Recte monet Vogelius signa phrenitidis, vel ut vocat phrenismi, hoc est, inflammationis cerebri aut membranarum ejus, admodum ambigua esse. 


\section{(PHRENITIS.)}

2. Phrenitides symptomaticae sunt,

Phrenitis synochi pleuriticae,S. sp. 2. P.synochi sanguineae sp. 4. P.calentura, sp. 11. P. Indica, sp. 12.-Cephalitis Egyptiaca, sp. 1. epidemica, ann. 1510, sp. 6. verminosa, sp. 7. cerebelli, sp. 8.-Phrenitis miliaris, sp. 3. variolosa, sp. 5. morbillosa, sp. 6.-Phrenitis a plica, sp. 8. aphrodisiaca, sp. 9. a tarantismo, sp. 14. hydrophobica, sp. 15.-Phrenitis a dolore, sp. 13.-Cephalitis traumatica, sp. 2.

\section{G. X.-CYNANCHE.}

Pyrexia aliquando typhodes; rubor et dolor faucium; deglutitio et spiratio difficiles, cum angustiae in faucibus sensu.

Cynanche, S. gen. 110. Linn. 33. Sag. 300.-Angina, Vog. 49. Hoffm. ii, 125. Junck. 30.-Angina inflammatoria, Boerh. 798.

\section{Species sunt,}

1. Cynanche (tonsillaris) membranam faucium mucosam, et praecipue tonsillas, tumore et rubore afficiens, cum febre synocha.

Cynanche tonsillaris, S. sp. 1.-Anginae inflammatoriae sp. 5. Boerh. 805.

2. Cynanche (maligna) tonsillas et membranam faucium mucosam afficiens tumore, rubore, et crustis mucosis coloris albescentis vel cineritii, serpentibus, et ulcera tegentibus; cum febre typhode et exanthematis.

Cynanche maligna, S. sp. 3.-Cynanche ulcerosa, S. var. a. Journ. de Méd. 1758.-Cynanche gangraenosa, var. b. Ibid. 1756.-Ulcera faucium et gutturis anginosa et lethalia, Hispanis Garotillo, Lud. Mercat. consult. 24. -Angina ulcerosa, clar. Fothergill, Account of the ulcerous sore throat, edit. 1751 ; Huxham on the malignant ulcerous sore throat, from 1751 to 1753 . - Febris epidemica cum angina ulcusculosa, Douglas Practical History, Boston, 1736. Lond. med. obs. i, 20.-Angina epidemica, Russel oecon. natur. pag. 105.-Angina gangraenosa, Withering's diss. inaug. Edinb. 1766.-Angina suffocativa, Bard's Inquiry, New-York, 1771.Angina maligna, Johnstone on the malignant angina, \&c. Worcester, 1799. Wall's Works, p. 58.

3. Cynanche (trachealis) respiratione difficili, inspiratione strepente, voce rauca, tussi clangosa, tumore fere nullo in faucibus apparente, deglutitione parum difficili, et febre synocha. 
(CYMANCHE.)

Cynanche trachealis, S. sp. 5.-Cynanche laryngea Auctorum, Eller de cogn. et curand. morb. sect. 7.-Anginae inflammatoriae S. sp. 1. Boerh. 801.-Angina latens et difficilis, Dodon. obs. 18.-Angina interna, Tulp. lib. i, obs. 51.-Angina perniciosa, Greg. Horst. observ. 1. iii, obs. 1 .

An haec Sauvagesii synonyma et sequentia ad eundem. morbum pertinent, videant experti ; sequentia autem ad morbum characteris dati manifesto pertinere videntur :

Suffocatio stridula, Scotis the Croup. cl. Home on the Croup.-Asthma infantum, Millar on the asthma and chin-cough.-Asthma infantum spasmodicum, Rush's dissertation, London, 1770.-Cynanche stridula, Crawford, dissert. inaug. Edinb. 1771.-Angina epidemica, anno 1743. Molloy apud Rutty's history of the weather. -Morbus strangulatorius, Starr, Phil. Transact. No. 495.*-Morbus truculentus infantum, Francof. ad Viae drum et in vicinia grassans, anno 1758. C. a Bergen A. nova N. C. tom. ii. p. 157.-Angina inflammatoria infantum, Russel, Oecon. nat. p. 70.-Catarrhus suffocativus Barbadensis, anno 1758. Hillary's Barbadoes, p. 134.-Angina polyposa, sive membranacea Michaelis, Argentorati, 1778, et Auctores ab ingenioso et erudito Michaele allegati.-Morbus anginae polyposae analogus. Soc. Royale ii. hist. p. 206.

4. Cynanche (pharyngea) cum rubore in imis praesertim faucibus; deglutitione maxime difficili, dolentissima; respiratione satis commoda, et febre synocha:

Cynanche pharyngea, S. sp. 6. Eller de cogn. et cur. sect. 7.-Anginae inflammatoriae S. sp. 4. Boerh. 804.

5. Cynanche (parotidea) cum tumore externo parotidum et maxillarum glandularum magno; respiratione et deglutitione parum laesis; febre synocha plerumque leni.

Cynanche parotidaea, S. sp. 14. Gallis Oreillons et Ourles, Tissot Avis au peuple, No. 166 . Encyclopédie, au mot Oreillons.-Angina externa, Anglis the Mumps, Russel, Oecon. natur. 114. Scotis the Branks. Catarrhus Bellinsulanus, S. sp. 4. - Osservazioni di Girol. Gaspari, Venez. 1731.-Osservaz. di Targ. Tozzetti, Raccolta prima, p. 176.

* An hic morbus ad Cynanchem malignam, an ad trachealem pertinet, mihi non certo constat, et saepius de earundem morborum apud plures auctores descriptionibus itidem incertus sum. 
(CYNANCHE.)

Cynanche purpuro parotidæa, S. sp. 15, vel ad malignam, sp. 3. vel ad Scarlatinam pertinere videtur.

Cynanchae symptomaticae sunt,

a. A causis externis.

Cynanche epidemica, S, sp. 2. Amphimerina anginosa, sp. 12. Febris anginosa, Huxham de aëre--Cynanche prunella, S. sp. 12. exanthematica, sp. 4. arthritica, sp. 10. hepatica, sp. 8. a dysenteria, sp. 13.

b. A causis externis.

Cynanche a deglutitis, S. sp. 9. mercurialis, sp. 11.Cynanche thymica, S. sp. 7, raro inflammatoria est, et vix hujus loci.

G. XI.-PNEUMONIA.*

Pyrexia; dolor in quadam thoracis parte; respiratio difficilis; tussis.

Febris pneumonica, Hoffm. ii, 137. Macb. p. 435.

Species sunt,

1. Pneumonia (peripneumonia) pulsu non semper duro, aliquando molli; dolore thoracis obtuso ; respiratione perpetuo difficili, saepe non nisi trunco corporis erecto exercenda; faciei tumidae colore purpureo; tussi plerumque humida, saepe cruenta.

Peripneumonia, S. gen. 112. Lin. 34. Vog. 51. Sag. gen. 311. Boerh. 820. Juncker 67 .

1. Peripneumoniae idiopathicae simplices.

Peripneumonia pura sive vera Auctorum S. sp. 1.Peripneumonia gastrica, S. sp. 11. Morgagn. de caus. et sed. epist. xx. art. 30, 31. +

* Ab omni aevo, in hunc fere diem, scriptores medici, et eos secuti Nosologi omnes, phlegmasiarum pectoris interni duo esse genera, peripneumoniam scilicet et pleuritidem, statuerunt. Recentiores autem ex sectione cadaverum didicerunt, veteres de sede, ideoque de differentia horum morborum saepe falso statuisse, et quem in pleura costali sedem habere veteres putabant, saepius in pleura pulmonem investiente situm fuisse invenerunt anatonici recentiores. Novimus, porro, quod si quidem morbi illi diversam aliquando sedem habuerint, id tamen per symptomata vix certo cognosci posse ; et, utcunque sit, morbos illos quatenus, quovis modo distinctos, revera maxime esse affines, plerumque simul conjunctos, et eosdem distinxisse ad usus medicinae parum omnino contulisse. Hisce igitur perpensis, phlegmasias pectoris interni non nisi genus unicum constituese, cum Hoffmanno expertissimo putamus ; quamobrem hujusmodi genus hic sub nomine Pneumoniae posuimus. Symptomata, quae pro characteristicis adhibuimus, cum quae in omni phlegmasia pectoris interni semper adsint, et in qualibet praecipua sint, ea recte characterem genericum constituere videntur.

An species diversae vere statuendae sint, anceps haereo. Sed, opinionibus et consuetudini medicorum aliquid concedere volens, peripneumoniae et pleuritidis quatenus, vel ex opinione medicorum vel ex propria observatione, eas distinguere liceat, characteres specificas dedi. Interim tamen observari velim, inter illos morbos, utcunque aliquando distinctos, limites accuratos plerumque non ponendos esse.

+ Ad Peripneumoniam, an ad Pleuritidem, potius pertinet haec varietas, mihi non certo constat. 
(PNEUMONIA.)

Variat gradu,

Peripneumonia catarrhalis, S. sp. 6.-Peripneumonia notha,* Sydenh. sect. 6, c. 4. Boerh. 867. Morgagni de caus. et sed. epist. xxi. 11-15.

II. Peripneumoniae idiopathicae complicatae febre. $†$

Peripneumonia putrida, S. sp. 2. $\ddagger$ ardens, sp. 3. maligna, sp. 4. typhodes, sp. 5. Amphimerina peripneumonica, sp. 15.

III. Peripneumoniae symptomaticae.

Peripneumonia arthritica, S. sp. 7. exanthematica, sp. 9. phthisicorum, sp. 8. hydrophobica, sp. 10. rachialgica, sp. 12.

2. Pneumonia (Pleuritis) pulso duro; dolore, plerumque lateris, pungente, sub inspiratione praesertim aucto ; decubitu in latus molesto ; tussi dolentissima, initio sicca, postea humida, saepe cruenta.

Pleuritis, S. gen. 103. Lin. 27. Vog. 50. Sag. 303. Boerh. 875. Junck. 67.-Paraphrenesis, S. gen. 102. Lin. 26.§-Paraphrenitis, Vog. 55. Boerh. 907.-Diaphragmitis, Sag. gen. 304 .

J. Pleuritides idiopathicae simplices.

Pleuritis vera S. sp. 1. Boerh. 875. Verna, princeps

* Peripneumoniam Notham a Vera gradu tantum differre opinor; ideoque uti varietas hic apposui In en quidem differre puto, quod in peripneumonia Notha inflammatio sit lenior, et effluxus humorum in pulmones copiosior, quam in Vera. Cum autem in Notha symptomata pyrexiae, doloris, dyspnoeae, et tussis, varia gradu et varia ratione conjuncta, adesse possint, minime mirandum si hujus morbi apud diversos auctores descriptiones parum uniformes sint, et necne morbus sub titulo peripneumoniae nothae a diversis auctoribus descriptus idem revera sit, dubia quaedam moverit doctissimus Morgagni. Perpensis tamen omvihus de hac re lietis, et cum observationibus pluribus apud ipsos aegros factis collatis, nihil duhito quin morbus a Sydenhamo et Boerhaavio, sub titulo Peripneumoniae nothae descriptus, unus idemque sit, nec ab eo morbum a Valsalva in Francisco Coralio, vel morbum in celebri Antonio Valisnerio ab ipso Morgagni observatum, re ipsa diversum fuisse puto. Vide First Lines of the Practice of Physic, \$ ceclxxvi.

+ Cum morhi diversi simul complicati sint, saepe, quis illorum pro morbo primario habendus sit dubitari potest; nec in hac re me ubique recte judicatum iri spondeo. In quibusdam mei ipsius judicio usus sum; in plerisque vero Sauvagesium secutus sum. Sufficere plerumque ratus ob oculos studiosorum complicationes istas quomodocunque ponere.

+ Sauvagesium, ut soleo, secutus, hanc et tres sequentes species quasi diversas recensui, cum tamen pro una eademque habere vellem.

\$ In morho qui vulgo Paraphrenesis vel Paraphrenitis, et rectius a Sagaro Diaphragmitis, appellatur, pleurae super diaphragma extensae inflainmationem adesse, et in ea morbum consistere agnoscunt medici omnes; non recte igitur uti genus a pneumonia diversum recensuerunt Nosologi.

Nec quidem pro diversa a pleuritide specie recenseri potest ; cum plerumque nulla alia praeter illa pleuritidis symptomata ostendit. Quae a plurimis huic morbo tributa sunt, risus nempo sardonicus, et delirium phreniticum, certe non semper adsunt. Primum quod 
morb. acut. pleuritis lib. i, cap. 2,3. Zeviani, della parapleuritide, cap. 3. Morgagn. de sed. et caus. morb. ep. xx, artic. 56, xxi, 45. Wendt de pleuritide, apud Sandifort. thes. ii.-Pleuritis pulmonis, S. sp. 2. Zevian. dell. parapleur. iii. 28, \&c.-Pleuripneumonia, pleuroperipneumonia, peripneumo-pleuritis Auctorum. Baronius de pleuripneumonia. Inl. Halleri opuscul. patholog. ob. 13. Morgagn. de sed. et. caus. epist. xx. et xxi. passim. Cleghorn Minorca, p. 247. Triller de pleuritide, aph. 1, 2, 3, cap. i, 8 . Huxham dis. on pleurisies, \&c. ch. 1. Ill. Pringle dis. of the army, p. 147.-Pleuritis convulsiva, S. sp. 13. Bianch. hist. hep. vol. i, p. 234.-Pleuritis hydrothoracica, S. sp. 15. Morgagn. de caus. et sed. xx, 34 .

Pleuritis dorsalis, S. sp. 3. Verna, p. iii, c. 8.

Pleuritis mediastini, S. sp. 4. P. Sal. Div. de affect. part. cap. 6. Friend. hist. med. de Avenzoare. Mediastina, Vog. 52.

Pleuritis pericardii, S. sp. 5. Verna, p. iii, c. 9.-Parapleuritis, Zeviani della parapleuritide.-Pleurodyne, parapleuritis S. sp. 19.

Paraphrenesis diaphragmatica, S. sp. 1. De Haen, rat. med. i, 7. iii, p. 31.-Paraphrenesis pleuritica, S. sp. 2. -P. hepatica, sp. 3.

De inflammatione mediastini, pericardii, et diaphragmatis, vide Morgagn. de caus. et sed. viii, 13 . xxi, 35, 36, 46. xlv, 16. Van Swieten ad Boerhaav. 913.

-II. Pleuritides complicatae.

a. Febre.

Pleuritis biliosa, S. sp. 7.-erysipelatosa, sp. 11. Pleuritis biliosa, Bianch. hist. hep. vol. i, p. 236. - putrida, S. sp. 9. Synochus pleuritica, sp. 5.-Pleuritis pestilens, sp. 16. -miliaris, sp. 20.

\section{b. Catarrho.}

Pleuritis catarrhalis, S. sp. 10.-Pleuritis lymphatica, Bianch. hist. hep. i, p. 233.

III. Pleuritides symptomaticae.

Pleuritis traumatica, S. sp. 6.-Pleuritis verminosa, S.sp. 8. Verna pleur. p. i. c. 1. p. iii. c. 11. Morgag. de sed. et caus. xxi, 43, 44.-Pleuritis stomachalis, Bianch. hist. 


\section{(PNEUMONIA.)}

hep. i, p. 232.-Pleurodyne verminosa, S. sp. 2.-Pleuritis lactea, sp. 17.-Pleuritis polonica, sp. 18.

Iv. Pleuritides Falsae.

Pleuritis hepatica, S. sp. 12. Panarol. Pentac. i, obs. 37.Pleuritis splenica, S. sp. 19.

Pneumoniae sequelae sunt,

1. Vourca.-Post pneumoniam, resolutione quadam non terminatam, dyspnoea et tussis perstantes, cum decubitu in latus sanum difficili, et febre hectica.

Vomica, Boerh. 835. Junck. 35.*-Pleurodyne vomica, S. sp. 21.

2. Eмpүema.-Post pneumoniam suppuratione terminatam, saepe post vomicam, remissio doloris, dum perstant dyspnoea, tussis, decubitus difficilis, et febris hectica, saepe cum sensu liquoris in pectore fluctuantis, et signis hydrothoracis.

Varietates ex Sauvagesio, licet non semper distinguendas, hic subjungimus :

Empyema a peripneumonia, S. sp. 1. a vomica, sp. 2. pleurae, sp. 3. mediastini, sp. 4. diaphragmatis, sp. 5. intercostale, sp. 6 .

\section{G. XIII.-CARDITIS.†}

Pyrexia ; dolor in regione cordis; anxietas ; spiritus difficilis; tussis; pulsus inaequalis; palpitatio ; syncope.

Carditis, S. gen. iii. Vog. 54.-Pericarditis, Vog. 53.

1. Idiopathica.

Carditis spontanea, S. sp. 1. Senac, Traité du coeur. l. iv, ch. 7. Meckel, mém. de Berlin, 1756.-Erysipelas pulmonis, Lomm. observ. lib. ii.

2. Symptomatica.

Carditis traumatica, S. sp. 2. Senac, ibid. ch. 6.

- Vómicam vocamus quam vomicam clausam appellant medici, et inde character noster. Characteres autem vomicae apud Linnaeum et Vogelium vomicam apertam tantum respiciunt; ideoque ad illos auctores hic non referimus.

+ Recte omnino dixit Vogelius, 'Carditidis symptomata fere eaden esse ut in peripneumonia, sed graviora,' et ita etiam sensisse videtur Linnaeus, cum apud illum neque Carditis, neque Pericarditis, inter morbos phlogisticos recensetur. Certe novimus, pericardium saepe inflammatum fuisse sine aliis praeter peripneumoniae signis. 
G. XIV.-PERITONITIS.

Pyrexia; dolor abdominis, corpore erecto auctus; absque propriis aliarum phlegmasiarum abdominalium signis.

Si signa darentur quibus dignosci queant sequentes, pro speciebus peritonitidis recenseri possent.

1. Peritonitis (propria) in peritonaeo strictius dicto, sive in peritonaeo, abdomen intus succingente.

Peritonitis, Vog. 62. Lieutaud. hist. anat. med. l. i, obs. 3. Raygerus apud eund. 1. i, obs. 341. Morgagn. de sed. lvii, 20.

2. Peritonitis (omentalis) in peritonaeo, per omentum extenso.

Epiploitis, S. gen. 106. Sag. 308.*-Omentitis, Vog. 61. -Omenti inflammatio, Boerh. 958. et Van Swieten Comm. Stork. ann. med. i, 132. Hulme on the puerperal fever.

3. Peritonitis (mesenterica) in peritonaeo, per mesenterium extenso.

Mesenteritis, Vog. 60.-Enteritis mesenterica, S. sp. 4.

G. XV.-GASTRITIS.

Pyrexia typhodes; anxietas; in epigastrio ardor et dolor, ingestis quibuslibet auctus; vomendi cupiditas, et ingesta protinus rejecta; singultus.

Gastritis, S. gen. 104. Lin. 28. Vog. 56. Sag. 306.Ventriculi inflammatio, Boerh. 951.-Febris stomachica inflammatoria, Hoffm. ii, 120.

I. Idiopathicae.

a. A causis internis.

1. Gastritis (phlegmonodea) dolore acuto, pyrexia vehementi. $†$

Gastritis legitima, S. sp. 1. Eller, de cogn. et cur. morb. sect. xi. Haller. obs. 14. hist. 3. Lieut. hist. anat. med. lib. i, 74.-Gastritis erysipelatosa, S. sp. 4. - Cardialgia inflammatoria, S. sp. 13. Tralles de opio, sect.ii, p. 231.

- Tum Epiploitis, tum Mesenteritis, ut Peritonitidis species hic recensetur, non quam quod ejusdem continuae membranae sint affectiones, quam quod nulla fere signa dari possunt, quibus a peritonitide, strictius dicta, dignosci queant.

+ Tum gastritis, tum enteritis duarum esse specierum, quarum una phlegmonodea, altera erysipelatosa dici potest, mihi ex pluribus observationibus satis certo constat ; et ejusmodi species itaque hic recensui, etsi fatendum sit, gastritidis erysipelatosae, et magis adhuc enteritidis erysipelatosae, signa saepe obscura et incerta esse. De his vero monere volui ut diligentius inquirant posteri.

f Quam hic gastritidem erysipelatosam dixit Sauvagesius, omnino phlegmonodeam esse mihi videt 
(GASTRITI8.)

b. A causis externis.

Gastritis a veneno, S. sp. 3.

2. Gastritis (erythematica) dolore, et pyrexia lenioribus, rubore erysipelatoso in faucibus apparente.

II. Symptomaticae.

Gastritis exanthematica, S. sp. 5-Gastritis herniosa, sp. 7. -De gastritide sternocostali, sp. 6. non liquet.

\section{G. XVI.-ENTERITIS.}

Pyrexia typhodes; dolor abdominis pungens, tendens, circa umbilicum torquens; vomitus; alvus pertinaciter stricta.

Enteritis, S. gen. 105. Lin. 29. Vog. 57. Sag. 307.Intestinorum inflammatio, Boerh. 959.-Febris' intestinorum inflammatoria ex mesenterio, Hoffm. ii, 170.

I. Idiopathicae species sunt,

1. Enteritis (phlegmonodea) dolore acuto, pyrexia vehementi, vomitu et alvo astricta.

Enteritis iliaca, S. sp. 1. colica, sp. 2. Boerh. 963.

2. Enteritis (erythematica) dolore et pyrexia lenioribus, sine vomitu, et cum diarrhoea.

II. Symptomaticae.

Enteritis flatulenta, S. sp. 3. enterocelica, sp. 5.

\section{G. XVII.-HEPATITIS.}

Pyrexia; hypochondrii dextri tensio, et dolor saepe pungens pleuritici instar, saepius obtusus; dolor ad claviculam et summum humeri dextri ; decubitus in sinistrum latus difficilis ; dyspnoea ; tussis sicca ; vomitus; singultus.*:

Hepatitis, S. gen. 113. Lin. 35. Vog. 58. Sag. gen. 312. Boerh. 914. Hoffm. ii, 14. Junck. 66. Macbr. p. 446.

Variat,

1. Acuta, signis in charactere dictis dignoscenda.

2. Chronica. Haec saepe nulla, quibus dignoscatur, signa praebet; aliquando tamen eandem adesse suspicari potest, ex hepatitidis causis quibusdam praegressis, ex sensu quodam plenitudinis et gravitatis in hypochondrio dextro, ex doloribus plus minusve pungentibus in eadem

* Inter symptomata hepatitidis, 'colorem faciei flavescentem,' posuit tum Sauvagesius, tum Sagarus, et hic etiam addit, 'urinas icteritias, biliosas, et serum sanguinis missi et crusta subflava,' sed haec symptomata bilis regurgitantis vel resorptae rectius a Linnaeo et Vogelio omissa fuisse censeo; nam ejusmodi symptomata non tantum non perpetua, sed etiam rarissima sunt. 


\section{(HEPATITIS.)}

parte subinde perceptis, ex dolore quodam a presso hypochondrio dextro, vel a decubitu in latus sinistrum sentito, et denique ex pyrexia leviori cum dictis signis subinde infestante.

Sauvagesii species sunt,

Hepatitis erysipelatosa, sp. 1.*-Hepatitis pleuritica, sp. 2. Bianch. hist. hep. tom. i. p. 388.-Pleuritis hepatica, sp. 12.-Hepatitis cystica, sp. 4.†-Hepatitis obscura, sp, 5 .

Pro specie male recensetur morbus secundarius:

Hepatitis suppurans, sp. 6. Cheston's pathol. inq. ch. iii sect. 2, et app. case 12.-Hepatalgia apostematosa, $\mathrm{S}$ sp. 7 .

\section{G. XVIII.-SPLENITIS.}

Pyrexia; hypochondrii sinistri tensio, calor, tumor, et dolor pressu auctus; absque signis nephritidis.

Splenitis, S. gen. 114. Lin. 36, Vog. 59. Junck. 67. Sag. 313. Macb. p. 448.-Lienis inflammatio, Boerh. 958, et ill. Van Swieten Comm.

Una tantum species recensetur,

Splenitis phlegmonodea, S. sp. 1. Forest, lib. xx. obs. 5, 6. De Haen, apud Van Swieten, p. 958.-Pleuritis splenetica, S. sp. 19.-Splenalgia suppuratoria, sp. 3.

\section{G. XIX.-NEPHRITIS:}

Pyrexia; dolor in regione renis, saepe ureteris iter sequens ; mictio frequens urinae, vel tenuis decoloris, vel ruberrimae; vomitus ; cruris stupor; testiculi ejusdem lateris retractio aut dolor.

Nephritis, S. gen. 115. Lin. 37. Vog. $65 . \quad$ Sag. 314. Species,

I. Idiopathica. Spontanea.

Nephritis vera, sp. 1 .

- De erythemate vel erysipelate, quod vocant, viscerum, minus recte sensisse medicos censeo; nam, me saltem judice, erythema non nisi superficies illas internas quas tegit epithelion et tunica villosa, epidermidi externae continua et analoga, afficere potest. $\mathrm{Nul}$ lum igitur erysipelas pulmonis, de quo scripsit Lommius, observ. lib. 2. agnosco; nec hepatitidem quandam a Sauvagesio hic erysipelatosam recte dictam esse puto, et hepatitidem quidem ex Amato hic descriptam revera phlegmonodeam fuisse credo.

† Quin ex contusione vel aliis causis oriri possit inflammatio cystidis felleae, vel ductus choledochi, nullus dubito ; sed quibus signis cognosci queat, nisi in uno casu, non novi. In ictero, scilicet, cum dolore in regione epigastrica acuto, a calculo, ut videtur, in ductu choledocho haerente, inflammationem duetus cystici saepe ortam fuisse, omnino censendum, et ne oriatur semper cavendum. 
(NEPHRITIS.)

II. Symptomaticae.

Nephritis calculosa, S. sp. 2--Nephralgia calculosa, sp. 1.- arenosa, sp. 2.-purulenta, sp. 7. Cheston's inq. ch. 2.-arthritica, sp. 3 .

G. XX-CYSTITIS.

Pyrexia; hypogastrii tumor et dolor; mictio frequens dolorifica, vel ischuria; tenesmus.

Cystitis, S. gen. 108. Lin. 31. Vog. 66. Sag. 309.Inflammatio vesicae, Hoffm. ii. 157 .

Species sunt,

1. A causis internis.

Cystitis spontanea, S. sp. 1.

2. A causis externis.

Cystitis a cantharidibus, sp. 2. traumatica, sp. 3.

G. XXI.-HYSTERITIS.

Pyrexia ; hypogastrii calor, tensio, tumor, et dolor ; os uteri tactu dolens; vomitus.

Hysteritis, Lin. 38. Vog. 63.-Metritis, S. gen. 107. Sag. gen. 315.-Inflammatio et febris uterina, Hoffm. ii. 156 .

Species sunt,

Metritis puerperarum, S. sp. 1. typhodes, sp. 2. lactea, sp. 3.

\section{G. XXII.-RHEUMATISMUS.}

Morbus ab externa, et plerumque evidente causa ; pyrexia ; dolor circa articulos, musculorum tractum sequens, genua et reliquos majores, potius quam pedum vel manuum articulos, infestans, calore externo auctus.

Rheumatismus, S. gen. 285. Lin. 62. Vog. 138. Boerh. 1490. Junck. 19.-Dolores rheumatici et arthritici, Hoffm. ii. 317.-Myositis, Sag. gen. 3 1.Lumbago, S. gen. 212 . Vog. 138. Sag. 169.-ISchias, S. gen. 213. Vog. 138. Sag. 170.-Pleurodyne, S. gen. 148. Vog. 138. Sag. 254. Macb. p. 511.

Species idiopathica,

Rheumatismus acutus, S. sp. 1. vulgaris, sp. 2.

Variat sede.

a. In musculis lumborum.

Lumbago rheumatica, S. gen. 212, sp. 1.-Nephralgia rbeumatica, S. sp. 4.

b. In musculis coxendicis.

Ischias rheumaticum, S. gen. 213, sp. 10. 
(RHeUmatismús.).

c. In musculis thoracis.

Pleurodyne rheumatica, S. gen. 148, sp. 3.-Pleuritis spuria, Boerh. 878.

\section{Species symptomaticae,}

Lumbago plethorica, S. sp. 3. Ischias sanguineum, sp. 2. Pleurodyne plethorica, sp. 1.-Rheumatismus hystericus, sp. 7. Ischias hystericum, sp. 3. Pleurodyne hysterica, sp. 6.-Rheumatismus saltatorius, sp. 8. Pleurodyne flatulenta, sp. 4. a spasmate, sp. 9.-Rheumatismus scorbuticus, sp. 4. Lumbago scorbutica, sp. 5. Pleurodyne scorbutica, sp. 11.-Ischias syphiliticum, sp. 7. Pleurodyne venerea, sp. 5.-Lumbago sympathica, sp. 13.-a mesenterii glandulis induratis, _a pancreate tumido, purulento, scirrhoso, putri,_ab induratis pyloro, vena cava, pancreate, - a rene scirrhoso, putrefacto,-ab abscessu circa vence cavce bifurcationem, - a vermibus intra renes.-Lumbago a saburra, sp. 8. Pleurodyne a cacochylia, sp. 7.-Rheumatismus saltatorius verminosus, sp. 8. Ischias verminosum, sp. 8. Pleurodyne verminosa, sp. 2.-Rheumatismus metallicus, sp. 10.-Lumbago a hydrothorace, sp. 14. pseudoischuria, sp. 16. ex aneurysmate, sp. 11. Pleurodyne ex aneurysmate, sp.10. a rupto oesophago, sp.20. rachitica, sp. 13.-Ischias a sparganosi, sp. 5. Pleurodyne catarrhalis, sp. 14. phthisica, sp. 8.- Rheumatismus necroseos; sp. 14.-Rheumatismus convulsivus, sp. 15. Ischias gravidarum, sp. 4. Lumbago partus, sp. 7 . a nisu, sp. 10.-Ischias a subluxatione, sp. 9. Rheumatismus dorsalis, sp. 11. Lumbago a satyriasi, sp. 15. Rheumatismus febricosus, sp. 9. Lumbago febrilis, sp. 4. Pleurodyne febricosa, sp. 15.-Rheumatismus miliaris, sp. 12. Lumbago miliaris, sp. 9. 'Pleurodyne miliaris, sp. 16.

\section{Rheumatismi sequela est,}

Arthrodrnia.* Post rheumatismum, nisum violentum, vel subluxationem; dolores artuum vel musculorum, 'sub motu praesertim, aucti, plus minusve fugaces, calore lecti vel alio externo levati; artus debiles, rigidi, facile,

- $\mathrm{Si}$, ut quidam statuunt, rheumatismus chronicus ab acuto, genere omnino diversus sit, nomen simplex, uti genus omne, habere oportet, et hujusmodi nec ineptum, ut puto, imposui. Porro, cum pro sequela rheumatismi acuti vel rheumatismi simpliciter dicenili, rheumatismum chronicum dictum semper habeo, et cum inter utrumque morbum saepe limites vix statuendi sint, ideoque pro diverso genere rheumatismum chronicum recensere vix potui; rheumatismus tamen chronicus exquisitus ab acuto natura adeo diversus evadit, et medelam adeo diversam exposcit, ut merito nomine prorsus diverso insigniri possit; et si quis pro genere etiam diverso ponere velit, per me profecto licet.

VOL. $I$. 


\section{(RHEUMATISMUS.)}

et saepe sponte frigescentes; pyrexia nulla; tumor plerumque nullus.

Rheumatismus chronicus Auctorum.

Lumbago et ischias aliquando morbi acuti, sed cum saepius chronici sint, plerumque ad hunc locum pertinent.

Lond. med. obs. iv, 5 .

\section{G. XXIII.-ODONTALGIA.*}

Rheumatismus vel arthrodynia maxillarum a carie dentium.

Odontalgia, S. gen. 198. Lin. 45. Vog. 145. Sag. gen. 157. Junck. 25. Macbr. p. 509.-Odontalgia sive rheumatismus odontalgicus, Hoffm. ii, 330 .

Varietates ex Sauvagesio sunt,

Odontalgia cariosa, sp. 1. scorbutica, sp.4. catarrhalis, sp. 3. arthritica, sp. 6 . gravidarum, sp. 2. hysterica, sp. 3. stomachica, sp. 9.

\section{G. XXIV.-PODAGRA.†}

Morbus haereditarius, oriens sine causa externa evidente, sed praeeunte plerumque ventriculi affectione insolita; pyrexia; dolor ad articulum, et plerumque pedis pollici, certe pedum et manuum juncturis, potissimum infestus; per intervalla revertens, et saepe cum ventriculi, vel aliarum internarum partium, affectionibus alternans.

Podagra, Vog. 175. Boerh. 1254-Febris podagrica, Vog. 69--Arthritis, S. gen. 183. Lin. 60. Vog. 139. Sag. 142. Macb. p. 487. Lond med. obs. vi, 20.-Dolor podagricus et arthriticus verus, Hoffm. ii, 339.-Dolores arthritici, Hoffm. ii, 317.-Affectus spastico arthritici, Junck. 46.

Recte forsitan Sauvagesius, more sibi insolito, hujus

- In priori Nosologiae methodicae nostrae editione, Odontalgiam pro rheumatismi specie habui, ac recensui ; sed, cum eam a rheumatismo alio quovis causam prorsus diversam, acre, scilicet, membranas sensibiles irritans, habere observaverim, pro genere, a rheumatismo diverso, etiam habere vellem, et hic itaque uti genus diversum posui.

Etiamsi odontalgiam a frigore et aliis irritamentis aliquando suscitari videtur, cariem dentium, diathesin phiogisticam inducentem, semper subesse puto. Sunt fortassis alii morbi, ab acri irritante orti, qui rheumatismum exacte satis referunt; sed de iis nobis non constat. Si tamen revera existeret, ad mentem clariss. D. Cottunnii, Ischias nervosa, rheumatismi ab acri nervos irritante orti exemplum aliud haberemus; sed neque phaenomena a Cottunnio relata, neque ratio medendi, neque sectiones cadaverum, ejusmodi morbum unquam exstitisse mihi probant; ideoque neque hic, neque alibi, Ischiadem nervosum Cottunnii inter rheumatismi species recensul.

+Arthritis nomen, utpote apud medicos ambiguum, rejeci, et podagru nomine, utpote typum morbi praecipuum notante, cum celebri Boerhaavio usus sum. 
(PODAGRA.)

morbi non nisi unicam esse speciem affirmavit; sed varietates sequentes notari possunt :

1. Podagra (regularis) cum inflammatione artuum satis vehementi, per aliquot dies perstante, et paulatim cum tumore, pruritu, et desquamatione partis, recedente.

Arthritis podagra, S. sp. 1. rachialgica, sp. 11.* aestiva, sp. 4.

2. Podagra (atonica) cum ventriculi vel alius partis internae atonia, et vel sine expectata aut solita artuum inflammatione, vel cum doloribus artuum lenibus tantum et fugacibus, et cum dyspepsia vel aliis atoniae symptomatis, subito saepe alternantibus.

Arthritis melancholica, S. sp. 6. hiemalis, sp. 2 . chlorotica, sp. 5. asthmatica, sp. 9.

3. Podagra (retrograda) cum inflammatione artuum subito recedente, et ventriculi vel alius partis internae atonia mox insecuta.

4. Podagra (aberrans) cum partis internae inflammatione, vel non praegressa, vel praegressa, et subito recedente, inflammatione artuum.

Conjungitur aliquando aliis morbis podagra.

Arthritis rheumatica, S. sp. 3. scorbutica, sp. 7. syphilitica, sp. 8.

Ad podagram nequaquam pertinere videntur sequentes :

Arthritis rachitica, S. sp. 13. Americana, sp.14. Bahamensis, sp. 15.

\section{G. XXV.-ARTHROPUOSIS.†}

Dolores artuum vel partium musculosarum, saepe post contusionem, profundi, obtusi, diuturni; tumor vel nullus, vel modicus, et diffusus; phlogosis nulla; pyrexia primum lenis, tandem hectica, et simul partis apostema.

Lumbago psoadica, S. sp. 6. Fordyce, Practice of Physic, part ii, p. 70. ' Cheston, app. case 4, et 5.-Lumbago apostematosa, S. sp. 12.-Lumbago ab arthrocace, sp. 17. Ischias ex abscessu, sp. 6.-Morbus coxarius, De Haen, Rat. med. vol.i, c. 32 .

- Ut solitus sum, Sauvagesii varietates, sive species quas vocat, hic appono; sed tum quod species ejus non semper bene definitae sint, tum quod podagra regularis et atonica a se invicem non semper prorsus disjungantur, in apponendis loco idoneo Sauvagesii speciebus, me ubique accuratum fore non spondeo.

+ Cum neque rheumatismum neque podagram in apostemna unquam abire credo, est hic morbus ab utroque certe diversus, ideoque pro genere peculiari recensendus. Utinam character ad eum a rheumatismo, arthrodynia, arthrocace, et phlogosi, distinguendum, ubique satis certo adhibendus, dari posset. 


\section{ORDO III.-EXANTHEMATA.}

Morbi contagiosi, semel tantum in decursu vitae aliquem afficientes; cum febre incipientes; definito tempore apparent phlogoses, saepe plures, exiguae per cutem sparsae.*

Exanthemata, Sag. c̀l. x.-Phlegmasiae exanthematicae, Sauv. cl. iii. ord. 1.-Morbi exanthematici, Lin. cl. i. ord. 2.-Febres exanthematicae, Vog. cl. i. ord. 2.

\section{G. XXVI.-VARIOLA.}

Synocha contagiosa cum vomitu, et, ex epigastrio presso, dolore:-Tertio die incipit, et quinto finitur eruptio papularum phlegmonodearum, quae, spatio octo dierum, in suppurationem, et in crustas demum abeunt, saepe cicatrices depressas, sive foveolas in cute, relinquentes.

Variola S. gen. 92. Lin. 3. Sag. gen. 290-Febris variolosa, Vog. 35. Hoffm. ii. 49.-Variolae, Boerh. 1371. Junck. 76.

Species sunt,

1. Variola (discreta) pustulis paucis, discretis, circumscriptione circularibus, turgidis; febre, eruptione facta, protinus cessante.

Variola discreta benigna, S. sp. 1.-Variolae regulares discretae, Sydenh. sect. iii, c. 2.-Variolae discretae simplices, Helvet. obs. sp. 1.-Variola discreta complicata, S. sp. 2. Helv. sp. 2.-Variolae anomalae, Sydenh. sect. iv. cap. 6. - Variola discreta dysenteriodes, S. sp. 4. Sydenh. sect. iv, cap. 1.-Variola discreta vesicularis, S. sp. 5.-Variola dișcreta crystallina, Mead de

* Quo charactere recte definiri, et quibus limitibus circumscribi debeat hic ordo, ad. modum incertum est. Si eruptiones enim a contagione specifica ortae, solae pro exanthematis, et morbis hujus ordinis, habendae sint ; certe eruptiones a nulla contagione specifica, sed a quodam febris statu solum, pendentes, inter exanthemata genuina nequaquam recensendae sunt. Ita Petechia a nobis omittitur, et forsitan tum Miliaria tum Aphtha omittenda esset. Dein, cum exanthemata pleraque contagiosa sint, et non nisi semel in decursu vitae aliquem afficiant, omnino idoneum videtur ut haec ab eruptionibus non contagiosis, quae saepe in decursu vitae aliquem afficiant vel afficere possint, rite distinguantur. Variola igitur et erysipelas minus recte sub eodem ordine collocantur. Denique, cum eruptiones in morbo quodam non semper ejusdem sint formae, nec semper, nec certo quovis morbi die, in illo morbo compareant, an ejusmodi morbus inter exanthemata recensendus sit, merito dubitari potest ; ideoque an pestis ad exanthematum, an ad febrium ordinem, referri debeat, dubito.

De hisce omnibus utiliter fortassis disputari potest; de iisdem vero certo statuere vix ausim; quod mihi tamen in Nosologia maxime idoneum esse videtur id ex charactere quem dedi satis patebit.

Cum autem ad characteres generum et specierum, ordinis character non omnino necessarius sit, hunc quodammodo neglexi, et hic morbos a Nosologis pro exanthematis habitos plerosque recensui. 
(VARIOLA.)

variol, cap. 2.-Variola discreta verrucosa, S. sp. 6. Mead ibid.-Variola discreta siliquosa, S. sp. 7. Friend oper. p. 358-Variola discreta miliaris, S. sp. 8. Helvet. obs. sp. 3.

2. Variola (confluens) pustulis numerosis, confluentibus, circumscriptione irregularibus, flaccidis, parum elevatis; febre post eruptionem perstante.

Variola confluens, S. sp. 9. Variolae regulares confluentes, ann. 1667. Sydenh. sect. ii. cap. 2. Variolae confluentes simplices, Helvet. obs. sp. 1.-Variola confluens crystallina, S. sp. 10. Variola Japonica, Kempfer. Vesiculae Divae Barbarae, C. Pis. obs. 149.* Variola confluens maligna, Helvet. obs. sp. 1.- Variola confluens cohaerens, S. sp. 11. Variola confluens maligna, Helvet. sp. 2.-Variola confluens nigra, S. sp. 12. Sydenh. sect. v, c. 4.-Variola confluens maligna, Helvet. sp. 3. Variola sanguinea, Mead de variolis, cap. 2.-Variola confluens corymbosa, S. sp. 13. Variola confluens maligna, Helvet. S. sp. 4.

G. XXVII.-VARICELLA.

Synocha. Papulae post brevem febriculam erumpentes, in pustulas variolae similes, sed vix in suppurationem euntes ; post paucos dies in squamulas, nulla cicatrice relicta, desinentes.

Varicella, Vog. 42.-Varicella lymphatica, S. sp. 1.-Anglis the Chicken-Pox, Ed. med. ess. vol. ii, art. 2, near the end. Heberden, Med. trans. art. 17.

\section{G. XXVIII. RUBEOLA.}

Synocha contagiosa cum sternutatione, epiphora, et tussi sicca, rauca.-Quarto die, vel paulo serius, erumpunt papulae exiguae, confertae, vix eminentes, et post tres dies in squamulas furfuraceas minimas abeuntes.

Rubeola, S. gen. 94. Lin. 4. Sag. 293.-Febris morbillosa, Vog. 36. Hoffm. ii, 62.-Morbilli, Junck. 76.

1. Rubeola (vulgaris) papulis minimis, confluentibus, corymbosis, vix eminentibus.

Rubeola vulgaris, S. sp. 1.-Morbilli règulares, Sydenh. sect. iv. cap. 5.-Lond. med. obs. iv. 19, 20.

Variat

$a$. Symptomatis gravioribus, et decursu minus regulari.

* Pro specie variolae, has vesiculas hic posuit Sauvagesius. Sed vesiculae a Pisone descriptae potius ad pemphigum pertinere mihi videntur. 
(RUBEOLA.)

Rubeola anomala, S. sp. 2.-Morbilli anomali, Sydenh. sect. v. cap. 3 .

b. Comitante Cynanche.

Rubeola anginosa, sp. 4. Edinb. essays, vol v, a. 2.

c. Comitante diathesi putrida.

Clar. Watson, Lond. med. obs. vol. iv, art. 11.

2. Rubeola (variolodes) papulis discretis eminentibus. Rubeola variolodes, S. sp. 3.* Scotis, the Nirles.

G. XXIX.-SCARLATINA.

Synocha contagiosa. Quarto morbi die, facies aliquantum tumens ; simul in cute passim rubor floridus maculis amplis, tandem coalescentibus, post tres dies in squamulas furfuraceas abiens ; superveniente dein saepe anasarca.

37 Scarlatina, S. gen. 98. Vog. 39. Sag. 294. Junck. 75. Species sunt,

1. Scarlatina ( simplex) nulla comitante cynanche.

Scarlatina febris, S. sp. 1. Sydenh. sect. vi, cap. 2. Soc. Royale, i. mém. p. 10. Macbr. p. 380.

2. Scarlatina (cynanchica) cum cynanche ulcerosa. $†$

Scarlatina anginosa, S. sp. 6. Withering on the scarlet fever.

De scarlatina porriginosa, S. sp. 3. Sydenh. sect vi. cap. 6. et Scarlatina variolode, S. sp. 5, nobis non liquet.

* Sauvagesium secutus, hunc morbum hic indicavi, etsi multum dubito an recte ad rubeolam referendus sit. Non solum enim forma papularum plarimum differt, sed, quod majoris momenti esse videtur, est plerumque absque symptomatis catarrhalibus, rubeolae adeo propriis.

+Spatio quadraginta annorum Scarlatinam sexies vel septies in Scotia epidemicam observavi, et in unoquoque exemplo morbus illius speciei erat quam Scarlatinam anginosam vocat Sauvagesius. Nos Cynanchicam appellavimus, ex eo quod ulceribus faucium internarum fere semper comitata fuit. Cum in plerisque aegris morbus cynanchem malignam plurimum, aliquando satis exacte referebat, nec unquam scarlatinam epidemicam in omnibus aegris simplicem esse viderim, saepe dubitavi annon scarlatina omnis sit cynanchica, et semper idem fere morbus ac cynanche maligna, supra inter Phlegmasias relata. Plures sutem rationes in banc sententiam ire non sinunt. Certe enim, testibus Sydenhamo perspicacissimo et aliis, scarlatina simplex, sive nulla comitante cynanche, aliquando observata fuit, et datur, itaque contagio quaedam specifica efflorescentiam cutis scarlatinam et hanc absque affectione quadam cynanchica, gignens. Quod, si aliquando etiam contagio sit, fauces afficiens, quae plerumque etiam scarlatinam gignat, hoc nihil praeterea mihi probare videtur, quam quod sit contagionum quarundam natura, ejusmodi ut simul tum fauces, tum cutem, afficere valeat. Cum tamen contagiones istae ita differre possint ut altera cutem, altera fauces magis et primario afficere proclivis sit, hanc differentiam inter scarlatinae et cynanchis malignae contagionem semper inesse puto. In morbo epidemico, quem pro Scarlatina habui, aliquot aegrotantium sine cynanche quacunque erant; et in is fere omnibus, quibus adfuit cynanche, haec benigna admodum, ulcera tonsillarum, parva tantum nec serpentia, et plerumque pus bonum fundentia, exhibuit, et morbus raro funestus fuit. In cynanche vero maligna aegrotantes omnes in ipso morbi ingressu, cynanche correpti sunt, et haec fere semper ulcera maligna, foeda, serpentia, gangraenosa, ostendebat. Morbus porro, putredinis et debilitatis indicia plurima prodens, plerumque exitialis evasit. 
G. XXX.-PESTIS.

Typhus maxime contagiosa, cum summa debilitate.* Incerto morbi die eruptio bubonum vel anthracum.

Pestis, S. gen. 91. Lin. 2. Junck. 78.-Febris pestilentialis, Vog. 33. Hoffm. ii. 93.

Variat gradu,

Pestis benigna; S. sp. 2. Pestis Massiliensis class. 3. Traité de la peste, pag. 41.-Ejusdem pestis, cl. 5. Traité, p. 228. Pestis remittens, S. sp. 9.-Pestis vulgaris, S. sp. 1.-Pestis Massil. cl. 2. Traité, p. 38.-Ejusd. cl. 2, 3, et 4ta. Traité, p. 225, \&c.-Waldschmidt, de peste Holsatica, apud Halleri diss. pract. tom. v.Chenot de peste Transylvanica 1755, 1759. De Haen, Rat. med. pars xiv.-Car. de Mertens de peste Moscouense 1771. Samoilowitz Mémoire sur la peste de Moscou. 1774. 'Ejusd. lettre sur l' expérience des frictions glaciales pour la guérison. de la peste. Ejusd. mém. sur l' inoculation de la peste.-Unterricht vors gegen die Pest. Dantzig 1770.-Murati Del Governo della peste. Brescia 1721 .

Pestis Agyptiaca, S. sp.11. Alpin. de med. Agypt:

Pestis interna, S. sp. 3. Pest. Massil. cl. i. Traité, p. 37, 224.

Species dubiae sunt,

Pestis sporadica, S. sp. 4. carbunculosa, sp. 5. Siamaea, sp. 8. scorbutica, sp. 10.

\section{G. XXXI.-ERYSIPELAS.†}

Synocha duorum vel trium dierum, plerumque cum somnolentia, saepe cum delirio.-In aliqua cutis parte, saepius in facie, phlogosis erythema, (gen. vii. sp. 2.)

Erysipelas, S. gen. 97. Lin. 10. Sag. gen. 296.-Febris erysipelacea, Vog. 68. Hoffm. ii. 98.-Febris erysipelatosa, Macb. p. 382.

\section{Species sunt,}

1. Erysipelas (vesiculosum) erythemate, rubedine serpente, latum spatium occupante, et locis ejus quibusdam in vesiculas magnas abeunte.

* De charactere pestis, lites inter medicos saepe ortae sunt, nec facile dirimendae ita ut character quibuscunque pestis casibus adhibendus dari possit; sed sufficiat characterem dedisse qui in plerisque adhiberi queat.

+Vox Erysipelas tum pro phlogosi erythemate, tum pro febre erysipelacea, a scriptoribus medicis usurpatur, sed recte Sauvagesius vitium cutaneum, quod nullam febrem, nisi symptomaticam, sibi junctam habeat, erythema appellari, et erysipelas, tantum febris illa exanthematica, quam erythema sequitur, vocari velit.

Minus recte forsitan III. Linnaeus erysipelas pro protypo exanthematicorum habuit; inter exanthemata enim, an recte, recensétur ipsum erysipelas dubitari potest. 
(ERYSIPELAS.)

Erysipelas rosa, S. sp. i. Sennert, de febr. lib. ii. c. 15.Febris erysipelatosa, Sydenh. sect. vi. cap. 5.-Erysipelas typhodes, S. sp. 2.* pestilens, sp. 5.† contagiosum, sp. 9.

2. Erysipelas (phlyctaenodes $\ddagger$ ) erythemate ex papulis pluribus, trunci corporis partes praecipue occupantibus, et protinus in phlyctaenas, sive vesiculas parvas, abeuntibas.

Erysipelas Zoster, S. sp. 8.-Zona, Anglis the shingles, Russel de tabe gland. p. 124. hist. 35.-Zona ignea, Hoffm. app. tom. iii. p. 426.-Herpes Zoster, Sp. sp. 9.

Symptomaticum ;

Erysipelas a veneno, S. sp. 3.

Caeterae Sauvagesii species ad erythema pertinent.

\section{G. XXXII.-MILIARIA.§}

Synochus cum anxietate, frequenti suspirio, sudore olido, et punctionibus cutis.-Incerto morbi die erumpunt papulae rubrae, exiguae, discretae, per totam cutem, praeter faciem, crebrae, quarum apices, post unum vel alterum diem, pustulas minimas, albas, brevi manentes, ostendunt.

Miliaria, Lin. 7.-Miliaris, S. gen. 95. Sag. gen. 295. Febris miliaris, Vog. 37.-Febris purpurata rubra et alba miliaris, Hoffm. ii, 68.-Febris purpurea seu miliaris, Junck. 75.-Germanis der Friesel. God. Welsch. hist: med. de novo puerperarum morbo, qui der Friesel dicitur, Lips. 1655.

De hoc morbo scriptores praecipui sunt,

* Haec non nisi varietas erysipelatis vesiculosi gradu solum differens, esse videtur.

+ Haec et sequens non nisi febres cum erythemate symptomatico videntur. Hic tamen notandum est, erysipelas saepe aliquid putridae et gangraenosae indolis prae se ferre ; nec dubito quin erysipelas, quod in hac regione, plerumque diathesi phlogistica comitatur, aliquando, praesertim in regionibus calidioribus, cum diathesi putrida, adsit; et forsitan duas erysipelatis species, inflammatoriam nempe et putridam, indicare potuissemus; sed de posteriori non nobis satis constat.

$\mp$ An haec species ad idem cum erysipelate vesiculoso genus recte referatur, dubito ; sed judicent periti.

\$ Inter medicos, speciatim Viennenses, de indole morbi miliaris nuper acriterdisputatum est; et imprimis an unquam idiopathicus, an vero semper symptomaticus sit, certatim quaeritur. Quod nunquam idiopathicus sit, praeter opinionem medicorum, a medio seculi decimi septimi in hunc fere diem, omnium, et contra sententiam medicorum hujus aevi quorundam spectabilium, affirmare non ausim; sed cum experientiam in hac re saepe fallacem, et medicos plerosque imitatorum servum pecus fuisse noverim, dubitare cogor; et, uncunque sit, morbum miliarem plerumque symptomaticum fuisse, ex observatione propria, per multos annos frequenti, certo novi. Nunquam contagiosum, nec manifesto epidemicum, quibusdam licet temporibus solito frequentiorem, vidi. Morbis febrilibus quibuscunque, tum infiammatoriis tum putridis, aliquando adjungitur; in nullis tamen, nisi regimine calido et sudoribus praeeuntibus, or tum, et in pluribus, regimine temperalo, et sudoribus vitatis, morbum, alias expectandum, prorsus vitatum observavi. 
Hamilton, de febr. miliar. 1710. Fontanus, de febr. mil. 1747. Allioni de miliar. 1758. Fordyce, de febr.mil. 1748. Fischer de. febr. mil. 1767. De Haen, de divis. febr. 1760. et in Ratione med. passim. Matt. Collin ad Baldinger de mil. 1764. Baraillon sur la fièvre miliaire, Soc. Royale, i. mém. p. 193. ibid. ii. hist. p. 198.

Gastellier sur la fièvre miliaire des femmes en couche, Montargis 1779. Varnier sur la fièvre miliaire de Normandie, Soc. Royale, iii. mém. p. 281.

Ad idiopathicam, si qua datur, pertinent,

Miliaris benigna, S. sp. 1. maligna, sp. 2. recidivans, sp. 3. Germanica, sp. 5.

Symptomaticae manifesto sunt,

Miliaris Boia, S. sp. a. Britannica, sp. $i$. nova febris, Sydenh. Sched. monit. S. sp. $d$. sudatoria, sp. $e$. nautica, sp. $g$. purpurata, sp. $h$. lactea, sp. $c$. puerperarum, sp. $k$. scorbutica, sp. $l$. critica, sp. $b$.

\section{G. XXXIII.-URTICARIA.*}

Febris amphimerina. $†-D i e$ secundo rubores maculosi, urticarum puncturas referentes, interdiu fere evanescentes, vespere cum febre redeuntes, et post paucos dies in squamulas minutissimas penitus abeuntes. +

Febris urticata, Vog. 40.-Uredo, Lin. 8.-Purpura urticata, Junck. 75.-Scarlatina urticata, S. sp. 2.-Erysipelatis species altera, Sydenh. sect. vi. cap. 6.-Febris scarlatina, et febris urticata, Meyserey, Mal. des armées, 291. et seq.

In quibusdam corporis partibus quasi arte excitatum aliquoties novi. Denique, cum contagionum specificarum plerarumque indolem materia, si qua datur, miliaris nequaquam imitetur, tum quod certo morbi die eruptionem non efficiat, tum quod non semel tantum, sed saepius in vitae decursu, hominem afficiat. De hujus igitur materiae natura specifica, vel ad morbum quemvis idiopathicum gignendum apta, valde dubito. In hac re mecum sentientem experientissimum et peritissimum Carolum White habere mihi gratulor. Vide White on the Management of Lying-in Women.

Cum autem in hac re me falli potuisse, facile agnosco, ne tamen alios simul fallerem, miliariam inter exanthemata hic recensui, et characterem ejus, quem quivis, hunc morbum pro idiopathico habens, dare voluerit, exhibui; peritis quibusvis hanc rem certius dijudicandam relinquens.

* Est morbus ab Anglis the Nettle rash dictus, qui quibusdam Urticaria audit; sed morbus ille, the Nettle rash, ut ab eruditissimo viro G. Heberden, in Coll. Reg. Med. Lond. Actis describitur, et qualem saepius ipsi vidimus, est ab Úrticaria nosologorum omnino diversus, utpote sine febre chronicus, et ad Impetigines fortassis referendus.

+ Hanc in priori editione contagiosam dixi ; sed ex propria experientia contagiosam fuisse non novi, et quo auctore contagiosam antea dixi, nunc non invenio.

‡ Ad aliorum potius quam meipsius mentem hic character conscriptus est; nam hunc morbum raro vidi, et nunquam epidemicum, vel regulariter, ut bic describitur, decurrentem, observavi.

VOL. I. 


\section{G. XXXIV.-PEMPHIGUS.}

Typhus contagiosa.-Primo, secundo, aut tertio morbi die, in variis partibus vesiculae, avellanae magnitudine, per plures dies manentes, tandem ichorem tenuem effundentes.

Pemphigus, S. gen. 93. Sag. 291.-Morta, Lin. 1.-Febris bullosa, Vog. 41.

\section{Species ex Sauvagesio sunt,*}

Pemphigus major, S. sp. 1.-Exarthemata serosa, C. Pison. obs. 150.-Febris pemphygodes, Eph. Germ. dec. i. ann. viii, obs. 56 .

Pemphigus castrensis, S. sp. 2.-Febris syneches, cum vesiculis per pectus et collum sparsis, Morton. app. ad exerc. ii.

Pemphigus Helveticus, S. sp. 3.-Langhans in Act. Helvet. vol. 2. p. 260. et in Beschreibung des Siementhals, Zurich, 1753.-Febris Vesicatoria, Macbr. p. 389.

De Pemphigo Indico, S. sp. 4. et Pemphigo Brasiliensi, sp. 5. non liquet.

\section{G. XXXV.-APHTHA.}

\section{Synochus. $†$ Lingua tumidiuscula; linguae et faucium color purpurascens; escharae in faucibus et ad linguae margines primum comparentes, os internum totum de- mum occupantes, albidae, aliquando discretae, saepe co-}

- Cum Pemphigum, quem pro exanthemate habere possim, nondum ipse viderim, et in scriptis medicorum de ejusmodi morbo paucissima tantum invenerim ; quae in Nosologia nostra de eo dicenda essent, omnia fere ex Sauvagesio necessario depromenda erant. Eum itaque secutus sum; sed hunc morbum lubenter ipse prorsus omisissem, cum omnia fere de eo diuta, dubia, obscura, et ambigua mihi videntur. Quae ex sui ipsius aut Caroli Pisonis observatione habet Sauvagesius, omnino credere fas est ; quae autem ex Christophori Seligeri, tenuis certe judicii hominis, observatione duxit, parum attentione digna mihi videntur. Quae porro ex D. Thierry relatione adducuntur meam fidem omnino superant: Nimirum, 'quod in morbo admodum exitiali acetum bezoarticum, a medico quodam datum, omnes salvos fecit, interea dum vix unus, aliis medicis commissus, mortem evadebat.' Denique, de morbo admodum ambiguo a D. Langhans in Actis Helveticis descripto, donec aliae observationes rem elucidaverint, nihil concludere vellem. Nihil aliud, fortassis, quam cynanche maligna fuit ille morbus, et, ad summum, quod pemphigus omnis morbus symptomaticus sit, satis verisimile videtur. Paulo postquam haec impressa sunt, Collega noster eximius Franciscus Home mihi hominem leviter febricitantem ostendit, cui primum in brachiis, et successive demum in toto corpore, vesiculae magnitudinis avellanae obortae sunt, et post duos tresve dies effuso humoris serosi pauxillo collapsae sunt. Haec febris autem nullam indolem vel typum peculiarem monstrabat et cito disparuit nequaquam contagiosa.

+ Au aphtha inter exanthemata recensenda sit, dubito. Aphtha enim quaeque fere quam ego vidi sine febre fuit; et si quando cum aphtha infantum febris adfuit, haec fere semper aphthae" supervenerat. A phtha adaltorum quidem saepe febri supervenit. Sed, febribus diversarum specierum plerumque sub earum finem jungitur, nec, quantum novi, febris quaevis aphthae propria, a medicis nota, vel quidem dicta fuit. 
(Aтнтна.)

alescentes, abrasae cito renascentes, et incerto tempore manentes.

/ Aphtha, S. gen. 100. Lin. 9. Sag. 298. Boerh. 978. Hoffm. ii. 478. Junck. 137.-Febris aphthosa. Vog. 44 Speciem idiopathicam unicam tantum novi :

Aphtha infantum.

Aphtha lactucimen, sp. 1.

Symptomaticae videtur,

Aphtha febrilis, S. sp. 2. maligna, sp. 3.

Manifesto symptomaticae sunt,

Aphtha syphilitica, S. sp. 4. scorbutica, sp. 5.

\section{Exanthema symptomaticum est}

\section{Purpura.}

S. gen. 96. Sag. gen. 292.*-Petechia, Lin. 5.

\section{ORDO IV. HAEMORRHAGIAE. $\uparrow$}

Pyrexiae cum profusione sanguinis absque vi externa ; sanguis missus ut in phlegmasiis apparet.

Haemorrhagiae, Vog. cl. ii. ord. i. Hoffm. ii. 194. Junck. 5.-Sanguifluxus, S. cl. ix. ord. i. Sag. cl. v. ord. i.

\section{G. XXXVI.-EPISTAXIS. +}

Capitis dolor vel gravitas; faciei rubor; profusio sanguinis e naribus.

- Cum in quibusvis febribus, vel intermittentibus vel continuis, in quibusvis etiam exanthematis et profluviis modo in iis, morbis sit quaedam ad putredinem proclivitas, appareant petechiae; has pro efflorescentia symptomatica, potius quam pro exanthemate idiopathico, habere volui.

+ Classem Fluxuum apud Sauvagesium et Sagarum, vel Profluviorum apud Vogelium, vel morborum evacuatoriorum apud Linnæum, cum illa morbos, tum facie, tum natura, omnino dissimiles conjunxerit, minime naturalem esse, nec proinde idoneam putavi. In nostram, igitur, Nosologiam ejusmodi classem admittere nolui, et morbos in ea recensitos aliorsum referendos esse censui. Illi, quos pyrexia quædam semper comitatur, sub classe Pyrexiarum certe recensendi sunt; et hic igitur hæmorrhagias Activas quas vocant posui. Hoffmannum sequor, qui mox post febres de ejusmodi hæmorrhagiis tractavit, et in præfatione rationem reddidit; quod, scilicet, 'Probe consideratis phænomenis, et paulo pensiculatius subductis rationibus, sanguinis eruptiones iisdem propemodum, utut, non satis completis motibus quibus febres oriuntur, progignuntur.'

\# Recte tum in Criticis Botanicis, tum in Philosophia Botanica, No. 233. monuit Linnaeus, "Nomina generica, cum classium et ordinum naturalium nomenclaturis communis, omittenda esse.' Contra hanc regulam tamen ipse peccat, cum vocabulo haemorrhagia, classis vel ordinis naturalis appellatione satis frequentur usitata, pro nomine generico utatur. Nec quidem, cum erraverit, ipse Hippocrates imitandus sit. Hujus generis itaque nomen mutandum erat, et a Vogelio quod hic usurpatum est desumpsi. 
(EPISTAXIS.)

Epistaxis, Vog. 82.-Haemorrhagia, S. gen. 239. Lin. 173. Sag. 174.-Haemorrhagia narium, Hoffm. ii. 196. Junck. 6 .

1. Idiopathica.

Haemorrhagia plethorica, S. sp. 2. Hoffm. ii. 198.

Variat ratione aetatis.

a. Epistaxis (juniorum) cum signis plethorae arteriosae.

b. Epistaxis (senum) cum signis plethorae venosae.

2. Symptomaticae.

a. A causis internis.

Haemorrhagia febrilis,. S. sp. 3.-critica, sp. 4. Hoffm. ii. 198.-insalubris, sp. 5. Hoffm. ibid.-in chronicis, sp. 6.

b. A causis internis.

Haemorrhagia passiva, sp. 1.-a hirudine, sp. 7 .

\section{G. XXXVII.-HAEMOPTYSIS.}

Genarum rubor; molestiae aut doloris, et aliquando caloris, in pectore sensus; dyspnoea ; titillatio faucium ; tussis aut tussicula sanguinem floridum, saepe spumosum, rejiciens.

Haemoptysis, S. gen. 240. Lin. 179. Vog. 84. Sag. gen. 175. Junck. 8.-Haemoptoe, Boerh. 1198.-Sanguinis fluxus ex pulmonibus, Hoffm. ii. 202.

Species idiopathicae sunt.

1. Haemoptysis (plethorica) nulla vi externa applicata, neque praegressa tussi aut evacuationis solitae suppressione.

2. Haemoptysis (violenta) a vi externa applicata.

Haemoptysis accidentalis, S. sp. 1. habitualis, sp. 2. traumatica, sp. 12.

3. Haemoptysis (phthisica) post tussim cum macie et debilitate diuturnam.

Haemoptysis phthisica, S. sp. 9. Haemoptysis ex tuberculo pulmonum, sp. 10. Haemoptoe, Lond. med. obs. iv, $16,18$.

4. Haemoptysis (calculosa) rejectis simul moleculis calculosis plerumque calcariis.

Haemoptysis calculosa; S. sp. 14.

5. Haemoptysis (vicaria) post evacuationis solitae suppressionem.

Haemoptysis catamenialis, S. sp. 4. periodica, sp. 5 . 
(HAEMOPTYSIS.)

Symptomaticae sunt,

Haemoptysis pneumonica,-catarrhalis, S. sp. 8. peripneumonica, Laelii a Fonte.

Haemoptysis exánthemática,-variolosa, S. sp. 7.-rubeolosá.

Haemoptysis hydropica,-ascitica, S. sp.15. hydrothoracica.

Haemoptysis cachectica,--splenetica, S. sp. 16.

Ineptae et spuriae sunt,

Haemoptysis a diapedesi, S. sp. 3. - a sphacelo pulmonum, sp. 11.-scorbutica, sp. 6.-Helvigiana, sp. 17.-a hirudine, sp. 13.

Haemoptyseos sequela est

PHTHISIS-Corporis emaciatio et debilitas, cum tussi, febre hectica, et plerumque expectoratione purulenta.

Phthisis, S. gen. 276. Lin. 208. Vog. 319. Sag. 101. Junck. 33.-Phthisis pulmonalis, Boerh. 1196.-Affectio phthisica, sive tabes pulmonalis, Hoffm. iii, 284. Lond. med. obs. iv. 18,23. Vog. 33.-Simmons on consumptions, 1780 .

Species sunt,

1. Phthisis (incipiens) sine expectoratione puris.

Phthisis incipiens, Morton. Physiolog. lib. ii, c. 3.-Phthisis sicca, S. sp. 1.

2. Phthisis (confirmata) cum expectoratione puris.

Phthisis confirmata Auctorum. humida, S. sp. 2.

Variat utraque species,

a. Ratione causae remotae.

Phthisis haemoptoica, S. sp. 6.-a peripneumonia, sp. 11. asthmatica, sp. 5. - calculosa, sp. 7.- exanthematica, sp. 14. - a febribus, sp. 13. - rheumatico-arthritica, sp. 12. chlorotica, sp. 10.-icteritia, sp. 15. Schacht. de phthisi icterit. 1724.-hypochondriaca, sp. 16. - scrophulosa, sp. 3.-chylosa, sp. 17. - scorbutica, sp. 4. - syphilitica, sp. 8. -a plica, sp. 19.-a metastasi puris, sp. 9.

b. Ratione fontis purulentae.

Phthisis a vomica, S. sp. 18. cellularis, sp. 20. De Haen, Rat. medendi, vol. i, cap. 7, 11. Ed. Leid.*

\section{G. XXXVIII.-HAEMORRHOIS.}

Capitis gravitas vel dolor; vertigo; lumborum dolor; dolor ani ; circa anum tubercula livida dolentia, e quibus ple-

- Utrum, de hoc fonte puris, recte an secus judicaverit Haenius, hujusmodi species vix in Nosologia locum habere potest, cum a signis quibusvis externis dig. nosci non queat. 
rumque profluit sanguis, qui aliquando etiam, nullo tumore apparente, ex ano stillat.

Haemorrhois, Sauv. gen. 217. Lin. 192. Sag. 182. Macb. p. 481.-Haemorrhoidalis fluxus, Hoffm. 219.-Haemorrhoides, Junck. 11 et 12.-Leucorrhois, Vog. 112.Proctalgia haemorrhoidalis, Macb. p. 540.

Species sunt,

1. Haemorrhois (tumens) externa a mariscis.

Marisca, S. gen. 35. Sag. gen. 37.

Variat,

a. Cruenta.

Haemorrhois moderata,S. sp. 1. Haemorrhoides ordinatae, Junck. 11.-Haemorrhoides nimiae, Junck. 11. Haemorrhois immodica, S. sp. 2. Haemorrhoides excedentes, Alberti de haemorrhoid. p. 179.-Haemorrhois polyposa, S. sp. 3.

\section{b. Mucosa.}

Haemorrhoides decoloratae, albae, et mucidae, Junck. 13. Alberti, pag. 248.-Proctalgia intertriginosa, Macb. p. 541.

2. Haemorrhois (procidens) externa a procidentia ani.

Haemorrhois ab exania, S. sp. 4. Ed. Ess. Liter. et Phys. ii, 27. Morg. de caus. ep. xxxiii.

3. Haemorrhois (fuens) interna absque tumore externo, vel procidentia ani.*

4. Haemorrhois (caeca) cum dolore et tumore ani, sine profusione sanguinis.

Haemorrhoides caecae, Junck. 12. Alberti, pag. 274.†Proctalgia inflammatoria, Macb. p. 540.

\section{G. XXXIX.-MENORRHAGIA.}

Dorsi, lumborum, ventris, parturientium instar, dolores;

- Stahlius, et ejus sequaces, distinxerunt hæmorrhoides in internas, quæ sanguinem ex ramis venae portarum, et externas, quæ sanguinem ex ramis venae cavae profundunt; sed, quantum video, scaturigo sanguinis profusi nullo modo dignosci queat. Huic itaque distinctioni nullus locus esse videtur, nec, si esset, quicquid ad distinguendam morbi naturam, vel effectum, conferre posset ; nam arteriae et venae, ad inferiorem intestini recti partem pertinentes, ortu utcunque diversæ, tam frequenter inter se invicem communicantur et conjungantur, ut uniuscujusvis affectio ad alteram statim communicari, et utriusvis effectus fere idem semper esse oporteat. Non ideo haemorrhoidem internam et externam ad mentem Stahlianorum distinxi, sed prout scaturigo sanguinis profluentis magis intra vel extra anum fuerit.

+ Hæmorrhois sæpe corporis universi morbus est, sive a quodam universi corporis statu pendens ; sed sæpe etiam morbus est mere localis. Non tamen hujusmodi species ad morbos locales referre volui ; quoniam hæmorrhois quævis, etiamsi initio mere localis sit, si sæpius, ut solet, redierit, tandem, sicut hæmorrhagia alia quævis sæpe rediens, necessario fit universi corporis morbus. Affines itaque -separare nolui, et hæmorrhoidem quidem omnem hic inter morbos universales recensendam esse censul. 
menstruorum copiosior, vel sanguinis, e vagina praeter ordinem fluxus.

Menorrhagia, Sauv. gen. 244. Lin. 202. Vog. 96.Metrorrhagia, Sag. gen. 179.-Uteri haemorrhagia, Hoffm. ii. 224.-Haemorrhagia uterina, Junck. 14. - Leucorrhoea, S. gen, 267. Lin. 201. Vog. 119. Sag. gen. 202. Cachexia uterina, sive fluor albus, Hoffm. iii, 348. Fluor albus, Junck. 133.*-Abortus, S. gen. 245. Lin. 204. Sag. gen. 180. Junck.92. Abortio, Vog. 97.-Fluor uterini sanguinis, Boerh. 1303.-Convulsio uteri, sive abortus, Hoffm. iii, 176.

Species sunt,

1. Menorrhagia (rubra) cruenta in non gravidis nec puerperis.

Menorrhagia immodica, S. sp. 3. a Pasta Dissert. sopra i menstrui.-Menorrhagia stillatitia, S. sp. 2.

2. Menorrhagia (abortus) cruenta in gravidis.

Menorrhagia gravidarum. S. sp. 6. Pasta intorno al flusso di sangue dall' utero nelle gravide.

Abortus effluxio, S. sp. 1.

a. subtrimestris.

b. subsemestris.

c. octimestris.

Abortus ab uteri laxitate, S. sp. 2.

3. Menorrhagia (lochialis) cruenta in puerperis.

Menorrhagia lochialis, S. sp. 8. Pasta diss. supra citat.

4. Menorrhagia (vitiorum) cruenta ex vitio locali.

Menorrhagia ex hysteroptosi, S. sp. 5.-Menorrhagia ulcerosa, sp. 9.

5. Menorrhagia ( $a l b a$ ) serosa sine vitio locali in non gravidis. $†$

Leucorrhœe, gen. ut supra.-Menorrhagia decolor, S.sp. 7.

- Leucorrhœam, sive fluorum album auctorum, hic ad menorrhagiæ genus retuli, et leucorrhœam sine vitio locali omnem huc omnino referendam esse censeo. Plerumque enim, et fere semper, leucorrhœa vel cum menorrhagia conjuncta est, vel eam mox secuta, et a temporibus quando apparet, leucorrhœea serum sub hac effusum $a b$ iisdem vasis, quæ menses profundant, provenire, et sæpe leucorrhœam ab iisdem causis ac menorrhagiam rubram oriri, satis verisimile est.

+ Hic fortassis lector eruditus mirari possit, quod ad clariss. medici Parisiensis Raulin, de fluore albo librum, Traite des fleurs blanches, neque studiosos relegaverim, neque species fluoris albi varias ab illustri auctore indicatas ipse recensuerim. Recte an secus fecerim, peritis dijudicandum relinquo; sed quod non fecerim hæc causa fuit. Plura in libro illo ingenioso subtilius, quam verius dicta, mihi videntur; et vel quomodo fluor albus, ab aliarum præter uteri partium affectione oriri possit, non intelligo, vel quod revera oriantur, ex meis saltem observationibus, forsan paucioribus, mihi nequaquam constat. Lectores itaque nostros potius ad auctorem ipsum relegare, quam doctrinam ejus male intellectam, et forsan minus recte traditam, hic exhibere velim. 


\section{(MENORRHAGIA.)}

-Leucorrhoea Americana, sp. 5.-Leucorrhoea Indica, sp. 6.

6. Menorrhagia (Nabothi) serosa in gravidis.

Leucorrhoea Nabothi, S. sp. 9.-Leucorrhoea gravidarum, sp. 8.

Menorrhagiam serosam, sive leucorrhoeam ex vitio locali, ad morbos locales referendam esse censeo.

Haemorrhagiae sequentes plerumque, si non semper, symptomaticae sunt.

Stomacace,

S. gen. 241. Lin. 175. Vog. 85. Sag. gen. 176.

Vel scorbuti, vel vitii oris, vel injuriae externae, symptoma est. Cum autem species Sauvagesii fere omnes recensere idoneum duco nunc sequenti serie recensitas velim.

Haematemesis plethorica, S. sp. 1.-catamenialis, sp. 5.haemorrhoidalis, ex splene, sp. 7.- ex hepate, sp. 13. ex pancreate, sp. 6. -atra, sp. 12.-scorbutica, sp. 8.ex aneurysmate, sp. 2.-carnoso cruenta, sp. 11. - traumatica, sp. 3.-ab hirudine, sp. 4.-cholerica, sp. 9. a veneno, sp. 14. - simulata, sp. 10.

Apud Sauvagesium species sunt,

1. Stomacace scorbutica.-2. universalis. -3 . haemorrhoa. 4. purulenta.

\section{Haematemesis.}

S. gen. 242. Lin. 184. Vog. 89. gen. 177.

Plerumque haemorrhagiae solitae vicaria est, vel vitii ventriculi localis, vel morbi nigri, vel denique vis externae, symptoma est.

1. Haematemesis plethorica. 2. ex aneurysmate. 3. traumatica. 4. ab hirudine. 5. catamenialis. 6. ex pancreate. 7. ex splene. 8. scorbutica. 9. cholerica. 10. simulata. 11. carnoso-cruenta. 12. atra. 13. ab hepate. 14. a veneno.

Haematuria

S. gen. 243. Lin. 198, Vog. 92. Sag. gen. 178. Edin. ess. v. 72.

Plerumque calculi renalis, aliquando febrium putridarum symptoma est.

Species secundum Sauvagesium sunt,

Haematuria spontanea, S. sp. 1.-catamenialis, sp. 12.calculosa, sp. 5.-nigra, sp. 11.-purulenta, sp. 4.- 
(HAEMORRHAGIAE.)

violenta, sp. 2.-traumatica, sp. 14.-haemorrhoidalis, sp. 9.-ejaculatoria, sp. 7.- stillatitia, sp. 8. -in exanthematicis, sp. 6.-spuria, sp. 10.-deceptiva, sp. 3.a transfusione, sp. 13. - a verme, sp. 15.

Cystirrhagia,

Vog. 93.

Plerumque calculi vesicalis, rarius alius morbi vesicae, symptoma.

\section{ORDO V. PROFLUVIA.*}

Pyrexia cum excretione aucta, naturaliter non sanguinea.

Profluvia, Vog. cl. ii.

\section{G. XL.-CATARRHUS.}

Pyrexia saepe contagiosa ; muci, ex glandulis membranae narium, faucium, vel bronchiorum, excretio aucta ; saltem hujus excretionis molimina.

Catarrhus, S. gen. 186. Vog. 98. Sag. gen. 145.-Coryza, Lin. 174. Vog. 100. Sag. gen. 196.-Rheuma, S. gen. 149. Sag. gen. 255.-Tussis, S. gen. 142. Lin. 155. Vog. 205. Sag. gen. 249. Junck. 30.-Tussis catarrhalis et rheumatica, Hoffm. iii. 109.

Genera sequentia ad hunc titulum referenda, plerumque symptomatica sunt.

Anacatharsis, S. gen. 262. Sag. 198. Expectoratio,'Lin. 178. - Raucedo, Lin. 146. Raucitas, Vog. 252.-Gravedo, Lin. 42.

Species sunt,

1. Catarrhus a frigore.

Catarrhus benignus, S. sp. 1.-pectoreus, sp. 6.-Coryza catarrhalis, sp. 1.-phlegmatorrhagia, sp. 2. Salmuth. obs. cent. 1. 37. Junck. 28.-Morgagn. de sed. xiv. 21.-febricosa, S. sp. 6. - Tussis catarrhalis, S. sp. 1. N.Rosen diss.apud Haller. disput. pract. tom.ii.-Rheuma catarrhale, S. sp. 1.-Amphimerina catarrhalis, sp. 2. Febris remittens catarrhalis, Macb. p. 359.-Amphimerina tussiculosa, S. sp. 13.-Cephalalgia catarrhalis, sp. 10.

- Ob eandem rationem qua Haemorrhagias, Profluvia etiam febriiia quaedam hic in classe Pyrexiarum collocavi. Catarrhus quidem, pyrexia et diathesi phlogistica, phlegmasiis maxime affinis est ; et dysenteria non solum pyrexia semper comitatur, sed in multis etiam a fluxibus aliis quibusvis differt.

vOL. $X$. 
(CAtakrhus.)

2. Catarrhus a contagio.

Catarrhus epidemicus, S. sp. 3.-Rheuma epidemicum, sp. 2. -Synocha catarrhalis, sp. 5.

Hujus exempla sunt :

Anno. 1323. Cronica meteorologica della Toscana, apud Targioni Tozzetti Osservaz. med. raccolta i. p. 175.

1328. Ibid.

1358. Ibid.

1387. Ibid.-Valescus de Taranta apud Morgagn. de sedibus, xiii. 4.-Marchesi ibid.

1510. Valleriola append. ad loc. com. cap. 2.

1575. Valleriola, ibid.-Riverii obs. in biblioth. vet. repert. obs. ix.-Foresti, lib. vi. obs. 1.

1580. Haenischius in Aretaeum, p. 315, 396.-Wieri obs. lib. ii.-P. Sal. Diversus, de febre pestilenti, cap. xi.Forestus lib. vi. obs. 3.-Riverii obs. in biblioth. vet. repert. obs. x.-Sennertus de febre, lib. iv. cap. 17.

1591. Sennertus, ibid.-Pechlin, lib. ii. obs. 17.

1658. Willis de febribus, cap. 16 .

1675. Sydenham, sect. v. cap. 5.-Raygerus in Ephem. Germ. dec. I. ann. vi. et vii. obs. 213.-P. de Sorbait.

1679. Sydenham, Ep. resp. ad R. Brady.-Zod. med. Gall. ann. I. dec. obs. 6.

1708. Schroeckius in Ephem. Germ. cent. i. et ii. in append. p. 14.

1709. Hoffm. ii. 47.

1712. Camerarius in Ephem. Germ. cent. iii. et iv. obs. 58. Schroeckius, ibid. in append. p. 26.

1729 et 1730. Hoffm. ii. 109.-C. Fr. Loew, in append. ad A. N. C. vol. iii.-Scheuchzer, obs. meteorol. in app. ad A. N. C. vol. iv.-Morgagni de sed. et. caus. xiii. 3, 4.-Beccarius, A. N. C. iii. obs. 48. p. 142--Hillary on weather and diseases, p. 35.-Winteringham, Comm. Nosol. p. 319.-Rutty's hist. of weather, p. 17.-Perkin's Soc. Royale, i. hist. p. 209.

1732 et 1733. Commerc. lit. Norimb. 1733. pag. 6, 52, 108, 267.-Detharding de febr. Eyderostad. apud Halleri disputat. pract. vol. v.-Edin. Med. Ess. vol. ii. art. 2. Hillary of weather, \&c. p. 47.-Winteringham, Comm. Nosol. p. 354.-Huxham de aëre, \&c. 1733. Mens. Febr.-Rutty's history of weather, p. 30.

1737. Jan. Rutty, ibid. p. 60.-Octob. Rutty, ibid. p. 60. -Julio. Comm. lit. Norimb. 1737, pag. 347.

1742. 1743. Comm. lit. Norimb. 1743, pag. 106, 188, 313, 336. - Juch et Zuberbuhler de febre catarrhali, apud Haller. Disp. pract. tom. v.-Huxham de aëre 1743, Mens. April.-Rutty, hist. of weather, p. 99.-Targioni Tozzetti Raccolt. i. di osservaz. med. p. 176. 
(CATARRHUS.)

Anno. 1748. Cleghorn, Minorca, p. 132.-Perkin's Soc. Royale i. hist. p. 210. \&c.

1758. Rutty, hist. of weather, p. 211.-Whytt, Lond. Med. obs. vol. ii. art. 13.

1762. Baker de catarrho, Lond. 1764.-Monro, Dis. of mil. hosp. pag. 137.-Macb. p. 333.

1767. Heberden, Med. Transact. i. art. 18.-Macb. p. 334. 1775. Lond. Med. Obs. vi. 30.

1779. et 1780. Soc. Royale, iii. mém. p. 16. - Saillant Tableau des Epidémies catarrhales, 1780.

Inter catarrhi, coryzae, et tussis species apud Sauvagesium, symptomaticae sunt,

Catarrhus rubeolosus, S. sp. 5.-Tussis exanthematica, sp. 20.-Coryza variolosa, sp. 4. virulenta, sp. 3. purulenta, sp. 5.-Tussis accidentalis, sp. 4. gutturalis, sp. 8. xerolaryngea, sp. 23. verminosa, sp. 21.-Tussis sicca, sp. 3. calculosa, sp. 22. phthisica, sp. 17. haemoptoica, sp. 15. a polypo, sp. 16. - Tussis a dentitione, sp. 12. stomachalis humida, sp. 6. stomachalis sicca, sp. 7. hysterica, sp. 2. gravidarum, sp. 14. hepatica, sp. 9. rheumatica, sp. 18. arthritica, sp. 19. metallicolarum, sp. 4.

\section{G. XLI.-DYSENTERIA.}

Pyrexia contagiosa ; dejectiones frequentes, mucosae, vel sanguinolentae, retentis plerumque faecibus alvinis; tormina ; tenesmus.

Dysenteria, S. gen. 248. Lin. 191. Vog. 107. Sag. 183. Hoffm. iii. 151. Junck. 76.

Non nisi speciem unicam novi cujus nomina apud Sauvagesium sunt,

Dysenteria epidemica, S. sp. 6. Sydenham, sect. iv. cap. 2. Helwich. in hist. morb. Wratislav. ann. 1699. Degner de Dysenteria Neomagensi 1736. Huxham de aëre, \&cc. ad. ann. 1743. Cleghorn's Minorca, chap. 5. Grimm. hist. dysenteriae malign. 1759, \&c. in append. ad A. Nov. N. C. vol. iii. Baker de catarrho et dysenteria, Lond. 1762. Akenside de dysenteria, Lond. 1762. Stark, hist. et sectiones dysenteric. diss. inaug. L. B. 1766. Zimmermann von der Ruhr. Zurich. 1767. Soc. Royale, iii. mém. p. 32.

Dysenteria castrensis, S. sp. 7. Ramazzini de morb. artific. cap. 40. Illust. Pringle, Dis. of the army, p. 224. Cl. Monro, Dis. of mil. hospitals, pag. 57.

Dysenteria aequinoctialis, S. sp. 14. Bont. de med. Ind. p. 3. 
(DYSENTERIA.)

Variat,

1. Vermibus comitata.

Dysenteria verminosa, S. sp. 15. Huxham de aëre, \&c. ad ann. 1743. M, Maio. IIl. Pringle, pag. 225. Monro p. 62.

2. Rejectis moleculis carnosis, vel sebaceis.

Dysenteria carnosa, S. sp. 16.-Diarrhoea carnosa, sp. 5 . Wedelius Ephem. Germ. dec ii. ann. ii. obs. 182. Wagner, ibid. dec. ii. ann. iii. obs. 187. cum scholio Schroeckii. Morgagn. de sed. Ep. xxxi. 17-24. Ill. Pringle, p. 237. Monro, p. 62.

3. Febre intermittente comitata.

Dysenteria intermittens, S. sp. 17. Morton in append. ad. exerc. ii. Cleghorn's Minorca, p. 236. Lautter. hist. med. bienn. cas. 17, 20.

4. Sine sanguine,

Dysenteria alba, S. sp. 10. Willis, Pharm. rat. pars i, sect. iii, cap. 3. Sydenham, ed. Leid. p. 179. Morgagn. de sed. ep. xxxi, 11. Ill. Pringle, p. 225. Cl. Monro, p. 61. Roederer de morbo mucoso, Gotting. 1762.

5. Miliaria comitata.

Dysenteria miliaris Gruberi, S. sp. 2. Febris exanthematico-dysenterica Tiguri epidemica, 1747.

Symptomaticae sunt,

Dysenteria benigna spontanea, S. sp. 1.-catamenialis, sp. 2.-gravidarum, sp. 4.-atrabilaria, sp. 5.-syphilitica, sp. 13. -scorbutica, sp. 18.-Polonica, sp. 19.a mesenterii vomica, sp. 11 .

Ad diarrhoeam pertinent,

Dysenteria Parisiaca, S. sp. 3.-a catharticis, sp. 12. 


\section{CLASSIS II.-NEUROSES.*}

Sensus et motus laesi, sine pyrexia idiopathica, et sine morbo locali.

\section{ORDO I. COMATA.}

Motus voluntarii imminuti cum sopore sive sensuum feriatione.

Comata, Sauv. cl. vi, ord. ii. Sag. cl. ix, ord. v.-Soporosi, Lin. cl. vi, ord. ii.-Adynamiae, Vog. cl. vi.-Nervorum resolutiones, Hoffm. iii, 194.-Affectus soporosi, Hoffm. iii, 209.-Motuum vitalium defectus, Junck. 114.

\section{G. XLII.-APOPLEXIA.}

Motus voluntarii fere omnes imminuti, cum sopore, $\uparrow$ plus minus profundo, superstite motu cordis et arteriarum.

Apoplexia, Sauv. gen. 182. Lin. 101. Vog. 229. Boerh. 1007. Junck. 117. Sag. gen. 228. Wepfer. hist. apoplecticorum.-Carus, S. gen. 181. Lin. 100. Vog. 231. Boerh. 1045. Sag. gen. 287. Macb. p. 561.+Cataphora, S. gen. 180. Lin. 99. Vog. 232. Boerh. 1048. Sag. gen. 286. Coma, Vog. 232. Boerh. 1048. Haemorrhagia cerebri, Hoffm. ii, 240 ,

Ad hoc genus pertinere mihi videntur,

Catalepsis, S. gen. 176. Lin. 129. Vog. 230. Sag. gen. 281. Boerh. 1036. Junck. 44.-Affectus cerebri spasmodico-ecstaticus, Hoffm. iii, 44.-Ecstasis, S. gen. 177 . Vog. 333. Sag. gen. 283.

- A tempore celebris quondam apud Anglos medici Thomæ Willis, morbos quosdam ab aliis quibusvis, sub nomine Nervosorum, distinxerunt Britanni ; sed, ni fallor, parum accurate, dum affectiones hystericas et hypochondriacas fere solas sub nomine Nervosarum indigitarunt. Quantum ego quidem video, motus morbosi fere omnes a motibus in systemate nervorum ita pendent, ut morbi fere omnes quodammodo Nervosi dici queant. Inter eos tamen distinctionem satis commode adhibere licet, et morbi isti qui nervorum systema fere solum, vel saltem primario, neque simul vel sanguinis circuitum, vel humorum naturam, nisi secundario afficiunt, Nervosi a nobis dicendi sunt. Classem itaque tam a classe Pyrexiarum, quam a classe Cachexiarum diversam, sub nomine Neurosium recte instituisse mihi videor.

+ Apud Linnaeum vocabulum Sopor, tam pro motuum, quam pro sensuum feriatione ponitur; sed sensu vulgari, tantum pro somno, vel pro statu somno simili, eodem utimur.

‡ Cum genera Carus, \&c. hic subjuncta non nisi apoplexiae leviores esse videntnr, nec vel respiratione vel excitatione satis accurate distinguenda sint, ad unum genus omnia referenda esse censui. 
(APOPLEXIA.)

\section{Symptomatica esse videntur,}

Typhomania, S. gen. 178. Lin. 97. Vog. 23. Sag. gen. 284. -Lethargus, S. gen. 179. Lin. 98. Vog. 22. Sag. gen. 285.

Apoplexiae idiopathicae species sunt,

1. Apoplexia (sanguinea) cum signis plethorae universalis, et praecipue capitis.

Apoplexia sanguinea, S. sp. 1. Morgagn. de causis, \&c. ii, iii, lx. Preysinger, sp. 1.-Carus spontaneus, S. sp. 2. Boerh. 1045.-Asphyxia* spinalis, Du Hamel, Hist. Acad.ann.1688, cap. 2. Boerh. praelect. ad Instit.401.Morgagn. de caus. \&c. iii, 3.-Cataphora coma, S. sp. 2.

2. Apoplexia (serosa) in corpore, plerumque senum, leucophlegmatico.

Apoplexia pituitosa, S. sp. 7. Apoplexia serosa, Preysinger, sp. 4. Morg. de causis, \&c. iv, lx. - Carus a hydrocephalo, S. sp. 16.-Cataphora hydrocephalica, sp. 6. Cataphora somnolenta, sp. 1.-Lethargus literatorum, sp. 7. Van Swieten in aph. 1010, $2 \gamma$ et $3 \alpha$.

3. Apoplexia (hydrocephalica) $\dagger$ paulatim adoriens; infantes et impuberes, primum lassitudine, febricula, et dolore capitis, dein pulsu tardiore, pupillae dilatatione, et somnolentia afficiens.

Hydrocephalus interior, S. sp. 1.-Hydrocephalus internus, Whytt's Works, p. 725. Lond. Med. Observ. vol. iv. art. 3, 6, and 25. vi, 6, 7, 8. Gaudelius de hydrocephalo, apud Sandifort Thesaur. vol. ii. Ed. Med.

Genus Asphyxiae ad Syncopen olim retuli, et ejusdem species quasdam non nisi gradu a syncope differre, adhuc censeo. Plures vero asphyxiae species ad apoplexiae genus pertinere olim et nunc etiam persuasum habeo; ideoque eas hic recensui. Asphyxia spinalis quidem hic apposita, utpote signis nullis externis dignoscenda, male inter species recensetur; si vero ad causas internas unquam respicere liceret, certe inter apoplexiae species recensenda esset. Cum autem ad causas externas et evidentes omnino respicere liceat, ab his satis perspectis plures asphyxiae species a Sauvagesio recensitas inter apoplexiae species enumeravi; nec huic obstare velim quod, in quibusdam asphyxiae speciebus, tum pulsus arteriarum tum respiratio, vita etiam superstite, tandem penitus cessant; nam in harum plerisque sub initio, cum motus et sensus fere penitus abolentur, superest adhuc motus quidam tum cordis tum pulmonum; et si tandem omnino cessant, idem aliquando apoplecticis ante mortem accidit. In apoplexia porro, cerebrum, in syncope autem cor, primario affectum esse opinor; et asphyxiae species itaque, prout cerebrum vel cor primario afficiant, quatenus id fieri, ex causis earum externis, conjicere liceat, ad apoplexiae vel syncopes genus refero.

+ In opere nosologico, morbos qui sub suo decursu variam induunt formam, rite collocare, ideoque apoplexiae hydrocephalicae locum maxime idoneum assignare, difficile est. Hunc morbum autem sub apoplexiae potius quam hydrocephali titulo ponere vellem : primo, quia hydrocephalus, qui hic subest, nequaquam sensibus evidens est; dein quia hic morbus symptomatis ab hydrocephalo evidente plurimum differt; denique, quia causa proxima et tandem symptomatis, apoplexisc quam maxime affinis est. 
Ess. ii, 18. iii, 23. Soc. Royale, iii, mém. p. 194.-Hydrocephalus acutus, Quin, diss. de hydrocephalo, Edinb. 1779. - Asthenia a hydrocephalo, S. sp. 3.

4. Apoplexia (atrabiliaria) in corpore melancholico. Apoplexia atrabiliaria, S. sp. 12. Preysinger, sp. 6. Carus traumaticus, $\mathbf{S}$. sp. 5.

5. Apoplexia (traumatica) a vi externa mechanica capiti illata.

Apoplexia traumatica, S. sp. 2. Ed. Ess. v, 52.

6. Apoplexia (venenata) a potentiis sedantibus interne vel externe adhibitis.

Apoplexia temulenta, S. sp. 3.-Carus a narcoticis, sp. 14. -Lethargus a narcoticis, sp. 3.-Carus a plumbagine, sp. 10.-Apoplexia mephitica, sp. 14. Ed. Ess. v, 55.Asphyxia a mephitide, S. sp. 9.-a musto, sp. 3.-Catalepsis a fumo, sp. 3.-Asphyxia a fumis, sp. 2.- a carbone, sp. 16.-foricariorum, sp. 11.-sideratorum, sp. 10. -Carus ab insolatione, sp. 12.- a frigore, sp. 6.-Lethargus a frigore, sp. 6.-Asphyxia congelatorum, sp. 5.

7. Apoplexia (mentalis) a pathemate mentis.

Carus a pathemate, S. sp. 11.-Asphyxia a pathemate, sp. 7.-Ecstasis catoche, sp. 1.-Ecstasis resoluta, sp. 2.

8. Apoplexia (cataleptica)* musculis, sub artuum a vi externa motu, contractilibus.

Catalepsis Sauv. gen. 176. Lin. 129. Vog. 230. Sag. gen. 281. Boerh. 1036. Junck. 44.

Species apud Sauvagesium sunt,

Catalepsis hysterica, S. sp. 1.-verminosa, sp. 2.-a fumo, sp. 3.-a menostasia, sp. 4.-melancholica; sp. 5.-delirans, sp. 6.

9. Apoplexia (suffocata) a potentia externa suffocante.

Asphyxia suspensorum, S. sp. 4.-Asphyxia immersorum, sp. 1. M'Donel de submersis, Edin. 1784.

Asphyxia flatulenta, sp. 12. ad apoplexiae genus pertinet, si a signis externis dignosci posset.

Apoplexia saepe symptomatica est,

1. Febris intermittentis.

Apoplexia febricosa, S. sp. 9.-Carus febricosus, sp. 3. Cataphora timor, sp. 8.-Typhomania febricosa, sp. 1.Catalepsis quartanaria, sp. 7 .

- Catalepsin nisi simulatam ipse nunquam vidi, et ab aliis visam frequenter simulatam fuisse credo. De morbo itaque rarius viso, varie descripto, et sæpe prorsus simulato, pro certo statuere mihi vix liceret; sed genere ab apoplexia neutiquam diversum esse, bene persuasus sum ; ideoque hic inter Apoplexiae species 
(APOPLEXIA.)

2. Febris continuae.

Carus febrilis, S. sp. 2.-Typhomania continua, sp. 2.Typhomania agrypnocoma, sp. 3.-Lethargus a febre, sp. 1.

3. Phlegmasiae.

Apoplexia inflammatoria, S. sp. 13.-Lethargus cephaliticus, sp. 4.-pulmonicus, sp. 2.

4. Exanthematis.

Carus variolosus, S. sp. 8.-Apoplexia metastatica, sp. 6.Carus exanthematicus, sp. 18.-Cataphora exanthematica, sp. 5.

5. Hysteriae.

Apoplexia hysterica, S. sp. 4.-Carus hystericus, sp. 9.Asphyxia hysterica, sp. 8.-Carus mystagmus, sp. 17.hypochondriacus, sp. 13.

6. Epilepsiae.

Apoplexia epileptica, S. sp. 8. - suspiriosa, sp. 10.

7. Podagrae.

Apoplexia arthritica, S. sp. 5.-Carus arthriticus, sp. 6 . Cataphora arthritica, sp. 4.-Lethargus arthriticus, sp. 5.

8. Vermium.

Apoplexia verminosa, S. sp. 15.-Carus verminosus, sp. 7 . Typhomania verminosa, sp. 4 .

9. Ischuriae.

Carus Ischuriosus, S. sp. 4.

10. Scorbuti.

Cataphora scorbutica, S. sp. 3.

\section{G. XLIII.-PARALYSIS.}

Motus voluntarii nonnulli tantum imminuti, saepe cum sopore.

Paralysis, Boerh. 1057.-Hemiplegia, S. gen. 170. Lin. 103. Vog. 228. - Paraplexia, S. gen. 171.-Paraplegia, Lin. 102. Vog. 227.-Paralysis, S. gen. 169. Lin. 104 . Vog. 226. Junck. 115. - A tonia, Lin. 120.

I. Species idiopathicae sunt,

1. Paralysis (partialis) quorundam musculorum tantum.

Paralysis, S. gen. 169. Lin. 104. Vog. 226. Junck. 115. Paralysis plethorica, S. sp. 1.-serosa, sp. 12.-nervea, sp.11.-Mutitas a glossolysi, sp.1.-Aphonia paralytica, sp. 8. 
(FARALYsis.)

2. Paralysis (hemiplegica) alterius corporis lateris.

Hemiplegia, S. gen. 170. Lin. 103. Vog. 228. Sag. gen. 276.

Variat ratione habitus corporis.

a. Hemiplegia in habitu plethorico.

Hemiplegia ex apoplexia, S. sp. 7.-Hemiplegia spasmodica, sp. 2.

b. Hemiplegia in habitu leucophlegmatico.

Hemiplegia serosa, S. sp. 10.

Male recensetur Hemiplegia apostematodes, S. sp. 11. quae signis externis dignosci non potest; et Hemiplegia ex epilepsia, S. sp. 12. recenseri vix meretur.

3. Paralysis (paraplegica) dimidii corporis transversim sumpti.

Paraplexia, S. gen. 171. Sag. gen. 277.-Paraplegia, Lin. 102. Vog. 227.-Paraplexia sanguinea, S. sp. 2.a spina bifida, sp. 3.-rheumatica, sp. 1 .

4. Paralysis (venenata) a potentiis sedantibus externe vel interne adhibitis.

Paralysis metallariorum, S. sp. 22.-Hemiplegia saturnina, sp. 14.

II. Species symptomaticae sunt,

Hemiplegia intermittens, S. sp. 8.-Paraplegia intermittens, sp. 6.-Paralysis febrilis, sp. 8.-Hemiplegia exanthematica, sp. 6.-Paralysis rheumatica, sp. 3.Hemiplegia arthritica, sp. 5.-Paralysis rachialgica, sp. 2.-Paraplegia rachialgica, sp. 5.-Paralysis biliosa, sp. 9.-Hemiplegia transversa, sp. 1.-Paralysis scorbutica, sp. 6.-scrophulosa, sp. 5.-Hemiplegia scrophulosa, sp. 4.- -syphilitica, sp. 3.-Paraplegia syphilitica, sp. 7.-Paralysis Polonica, sp. 7.-a vomica, sp. 10.traumatica, sp. 4.-Hemiplegia traumatica, sp. 9.-Paraplegia traumatica, sp. 4 .

Tremorem, utpote semper symptomaticum, in numerum generum recipere nollem; species autem a Sauvagesio recensitas, prout mihi Astheniae vel Paralysios, vel convulsionis symptomata esse videntur, hic subjungam.

Tremor.-Artus alterna per itus et reditus frequentes motitatio.

Tremor, S. gen. 129. . Lin. 139. Vog. 184. Sag. 236.

vor. I. 
(PARALYSis.)

1. Species asthenicae.

Tremor a debilitate, S. sp. 1. senilis, sp. 2. a coffea, sp. 4. a pathemate, metu, ira, gaudio, libidine, sp. 14. typhodes, sp. 19.

2. Species paralyticae.

Tremor paralytodes, S. sp. 11. vertiginosus, sp. 7: a plethora, sp. 13. a saburra, sp. 12. a hydrocephalo, sp. 8. scorbuticus, $\mathrm{sp} .10$. rheumatismalis, $\mathrm{sp} .18$. temulentus, sp. 3. metallurgorum, sp. 5. traumaticus, sp. 17.

3. Species convulsivae.

Tremor coactus, S. sp. 6. tendinum, sp. 9. palpitans, sp. 16.

\section{ORDO II.-ADYNAMIAE.}

Motus involuntarii, sive vitales sive naturales, imminuti.

Adynamiae, Vog. cl. vi.-Defectivi, Lin. cl. vi. ord. i.Leipopsychiae, S. cl. vi. ord. iv. Sag. cl. ix. ord. iv.

G. XLIV.-SYNCOPE.

Motus cordis imminutus, vel aliquamdiu quiescens.

Syncope, 8. gen. 174. Lin. 94. Vog. 274. Sag. 280. Junck. 119.-Leipothymia, S. gen. 173. Lin. 93. Vog. 273. Sag. 279.-Asphyxia, S. gen. 175. Lin. 95. Vog. 275. Sag. 281.-Virium lapsus et animi deliquia, Hoffm. iii. 267 .

I. Idiopathicae.

1. Syncope (cardiaca) sine causa manifesta saepe rediens, cum palpitatione cordis vehementi in intervallis: Ex vitio cordis vel vasorum vicinorum.

Syncope plethorica, S. sp. 5. Senac, Tr, du coeur, p. 540. - Syncope a cardiogmo, S.sp.7. Senac, du coeur, p.414. Morgagn. de sed. xxv. 2. 3. 10.-Syncope a polypo, S. sp. 8. Senạc, p. 471. -Syncope ab hydrocardia, S. sp. 12. Senac, p. 533. Schreiber Almag. 1. iii, sect. 196.Syncope Lanzoni, sp. 18. Lanzon. op. ii. p. 462.-Asphyxia Valsalviana, sp. 13.

2. Syncope (occasionalis) a causa manifesta oriens : Ex affectione totius systematis.

Leipothymia a pathemate, S. sp. 1. Senac, p. 544.-Syncope pathetica, S. sp. 21.-Asphyxiae a pathemate, S. sp. 7.- Syncope ab antipathia, S. sp. 9. Senac, p. 554. -Syncope a veneno, S. sp. 10. Senac, p. 546.-Syncope ab apostematis, S. sp. 11. Senac, p. 554.-Syncope a sphacelo, S. sp. 14. Senac, p. 553. - Syncope ab inani-
tione, S. sp. 1. Senac, p. 536. - Syncope a phlebotomia, 
(SYNCOPE.)

S. sp. 4. - Syncope a dolore, S. sp. 2. Senac, p. 583.Asphyxia traumatica, S. sp. 14.-Asphyxia neophytorum, S. sp. 17.

II. Symptomaticae, sive symptomata morborum vel totius systematis, vel aliarum praeter cor partium.

Syncope febrilis, S. sp. 3. Senac, p. 548.-Syncope febricosa, sp. 17. Torti Therap. spec. L. iii, cap. 1.Syncope exanthematica, sp. 19.-Syncope metastatica, sp. 20.-Leipothymia stomachica, sp. 2.-Syncope stomachica, sp. 13. Senac, p. 545.--Syncope hysterica,sp. 6 . Senac, p. 547.-Syncope arthritica, sp. 16. Musgrave de arthr. anomala, cap. 8.-Syncope scorbutica, sp. 15. Lind Treat. on scurvy. P. ii. chap. 11. Senac, p. 552.

\section{G. XLV.-DYSPEPSIA.}

Anorexia, nausea, vomitus, inflatio, ructus, ruminatio, cardialgia, igastrodynia, pauciora saltem vel plura horum simul concurrentia, plerumque cum alvo adstricta, et sine alio vel ventriculi ipsius, vel aliarum partium, morbo.

Dyspepsia, Vog. 277. Lond. Med. Obs. vi, 11.-Apepsia, Vog.276.-Diaphora, Vog. 278.-Anorexia, S. gen. 162. Lin. 116. Sag. gen. 268.-Cardialgia, S. gen. 202. Lin. 48. Vog. 157. Sag. gen. 160.-Gastrodynia, S. gen. 203. Sag. gen. 161.-Soda, Lin. 47. Vog. 161.-Nausea, S. gen. 250. Lin. 182. Vog. 159. Sag. gen. 185. Vomitus, S. gen. 251. Lin. 183. Vog. 214. Sag. 186. Flatulentia, S. gen. 271. Lin. 165. Vog. 127. Sag. gen. 207.

I. Idiopathicae.

Anorexia pituitosa, S. sp. 2. a saburra, sp. 9. exhaustorum, sp. 8. paralytica, sp. 1.-Nausea ex cacochylia, sp. 11.-Vomitus pituitosus, sp. 26. ruminatio, sp. 6 . a saburra, sp. 2. a crapula, sp. 1. lacteus, sp. 3.Flatulentia infantilis, sp. 5. acida, sp. 1. nidorosa, sp. 2.-Cardialgia bradypepta, sp. 9. a saburra, sp. 2. lactantium, sp. 11. flatulenta, sp. 3. paralytica, sp. 7 . Gastrodynia saburralis, sp. 1. flatulenta, sp. 2. periodynia; sp. 7 . astringens, sp. 9. atterens, sp. 10. a frigore, sp. 18.

II. Symptomaticae.

1. A morbo ipsius ventriculi.

Cardialgia scirrhosa, S. sp. 6. Vomitus a pyloro calloso, sp. 8. a steatomate ventriculi, sp. 11. - Nausea ab apostemate circa cardiam, sp. 9. Gastrodynia ulcerosa, sp. 5. Vomitus ab ulcere ventriculi, sp. 10,-Cardialgia a veneno, sp. 2. Gastrodynia a veneno, sp. 4. Vomitus a 
(DYSPEPSIA.)

veneno, sp. 7.-Flatulentia accidentalis, sp. 4. Gastrodynia a peregrinis, sp. 11. Anorexia biliosa, sp. 6 . Nausea biliosa, sp. 6. Gastrodynia biliosa, sp. 3.-Vomitus urinosus, sp. 27.-Gastrodynia calculosa, sp. 8. Vomitus bezoarticus, sp. '13.-Cardialgia'verminosa, sp. 10. Nausea taenia, sp. 1. Vomitus verminosus, sp. 5.-Gastrodynia a xiphoide, sp. 12. Gastrodynia Americana, sp. 6. Nausea ex compresso ventriculo, sp. 5. Vomitus a cartilagine xiphoidea, sp. 22.-Vomitus $\mathrm{ab}$ oesophago obstructo, sp. 21. Gastrodynia gastrocelica, sp. 20. Nausea ex gastrocele, sp. 4. Vomitus gastrocelicus, sp. 18.-Cardialgia sputatoria, sp. 5.Cardialgia inflammatoria, sp. 13.-Nausea ex gastritide, sp. 10. Vomitus gastriticus, sp. 19.

2. A morbo aliarum partium vel totius corporis.

Vomitus cephalalgicus, S. sp. 23.-Vomitus a dentitione, sp. 4.-Vomitus iliacus, sp. 24.-Nausea ex pancreatis schirro, sp. 3.-Vomitus a pancreate, sp. 17.-Vomitus ab hepate obstructo, sp. 16.-Anorexia melancholica, sp. 5.-Gastrodynia hypochondriaca, sp. 16.-Flatulentia hypochondriaci, sp. 3.-Flatulentia convulsiva, sp. 7 . Vomitus hypochondriacus, sp. 6.-Vomitus atrabilarius, sp. 15.-Anorexia mirabilis, sp. 10.-Gastrodynia hysterica, sp. 14.-Gastrodynia pulsatilis, sp. 13.-Gastrodynia chlorotica, sp. 15. - Nausea a semine corrupto, sp. 8.-Nausea gravidarum, sp. 2.-Vomitus gravidarum, sp. 12.-Flatulentia lochialis, sp. 6.Anorexia arthritica, sp. 12.-Cardialgia arthritica, sp. 8.-Nausea nephritica, sp. 7.-Vomitus nephriticus, sp. 20.-Anorexia Stewartiana, sp. 13.-Cardialgia a cardiogmo, sp. 12.-Anorexia plethorica, sp. 3.-Anorexia cachectarum, sp. 7.-Anorexia febrilis, sp. 4.Cardialgia febricosa, sp. 4.-Gastrodynia febricosa, sp 17.-Vomitus febricosus, sp. 29.-Vomitus rabiosus, sp. 14.-Vomitus haemorrhagicus, sp. 28.-Gastrodynia metastatica, sp. 19.-Nausea marina, sp. 12.-Vomitus marinus, sp. 25.-Anorexia neophytorum, sp. 11.

\section{G. XLVI.-HY POCHONDRIASIS.}

Dyspepsia cum languore, moestitia, et metu, ex causis non aequis, in temperamento melancholico.

Hypochondriasis, S. gen. 220. Lin. 76. Vog. 218. Sag. 332.-Morbus hypochondriacus, Boerh. 1098.-Malum hypochondriacum, Hoffm. iii. 64. Junck. 36.

Speciem idiopathicam unicam tantum novi.

Hypochondriasis melancholica, S. sp. 3.

De Hypochondriasi algida, S. sp. 10. mihi non liquet. 


\section{(HYPOCHONDRIASIS.)}

Species sequentes ex Fraecassino, ex theoria parum certa confictae mihi videntur :

Hypochondrisias biliosa, S. sp. 1. sanguinea, sp. 2. pituitosa, sp. 4.

Symptomaticae sunt,

Hypochondriasis hysterica, S. sp. 5. phthisica, sp. 6 . asthmatica, sp. 7. calculosa, sp. 8. tympanitica, sp. 9.

\section{G. XLVII-CHLOROSIS.}

Dyspepsia, vel rei non esculentae desiderium; cutis pallor vel decoloratio; venae minus plenae, corporis tumor mollis; asthenia ; palpitatio ; menstruorum* retentio.

Chlorosis, S. gen. 309. Lin. 222. Vog. 305. Sag. 135. Boerh. 1285. Hoffm. iii. 311. Junck. 86.

Chloroseos unicam speciem veram agnoscimus, scilicet,

Chlorosis virginea, S. sp. 1. a. Chlorosis amatoria.

Caeterae Sauvagesii species spuriae, sive decolorationes, symptomaticae sunt :

Chlorosis a menorrhagia, S. sp. 2. gravidarum, sp. 3. infantum, sp. 4. verminosa, sp. 5 . viridis, sp. 6 . ab hydrothorace, sp. 7. maculosa, sp. 8. Carthagenica, sp. 10. Bengualensis, sp. 11. rachialgica, sp. 12.

\section{ORDO III.-SPASMI.}

Musculorum vel fibrarum muscularium motus abnormes. $\dagger$

Spasmi, S. cl. iv. Vog. cl. v. Sag. cl. vii--Motorii, Lin. cl. vii.-Morbi spasmodici et convulsivi, Hoffm. iii. 9.-Spasmi et convulsiones, Junck. 45. 54.-Epilepsia, Boerh. 1071. 1088.

\section{SECT. I.-IN FUNCTIONIBUS ANIMALIBUS.}

\section{G. XLVIII.-TETANUS.}

\section{Plurium musculorum rigiditas spastica.}

- In hoc charactere antea dixi menstruorum saepe retentio; quasi species chlorosis esset in qua menses rite fluxerunt, et cogitavi de specie qualam cachexiae huc referenda. Sed nune dubito an species cachexiae quaevis sub titulo Chlorosis, vel sub charactere hic dato, reete poni possit; et nullam Chlorosis speciem veram, praeter illam quae retentionem menstruorum comitatur, agnoscere vellem. Hinc vocabulum saepe in charactere nunc omissum est; sed simul fateri cogor, chlorosin ideo hic minus recte pro genere recensitam esse, cum pro symptomate amenorrhoeae semper haberi possit.

+ Motus abnormes sunt, qui ex causa praeternaturali oriuntur, et qui violentia, frequentia, vel diuturnitate contractionis praeter modum fiunt; et nullus dubito quin hujusmodi motus, in morbis omnibus sub hoc ordine a nobis collocatis, revera fiunt; plures licet morbi sint, quam sub eodem or dine ab aliis nosologis recensiti fuerunt. 


\section{(TETANUS.)}

Variat gradu.

I. Tetanus; dimidium vel totum corpus spasmis affectum.

Tetanus, S. gen. 122. Lin. 127. Vog. 180. Sag. gen. 228.-Catochus, ${ }^{*}$ S. gen. 123. Lin. 128. Vog. 183. Sag. 229.-Opisthotonos, Vog. 181.-Episthotonos, Vog. 182.

Hujus species, ut solet, plures recensuit Sauvagesius; sed omnes, quae idiopathicae dici queunt, ad unam, ni fallor, referri possunt, et cujus forma praecipua apud Sauvagesium appellatur,

Tetanus Indicus, S. sp. 5. de quo plura haberi possunt apud Hillary, Barbadoes, p. 219. Chalmers, Lond. Med. Obs. vol. i. art. 12. D. Monro, Edin. Phys. and Liter. Essays, vol. iii. art. 30. A. Monro, art. 31. et sub nomine Spasmi apud Bontium de med. Ind. meth. med. cap. 2.

Nec alius morbus est,

Convulsio Indica, S. sp. 9.

Variat autem Tetanus ratione causae remotae, quatenus nimirum vel a causa interna, vel frigore, vel a vulnere oriatur. A vulnere, varietas frequentissima apud Sauvagesium appellatur

Tetanus traumaticus, S. sp. 11 .

Variat etiam quavis causa ortus Tetanus ratione partis corporis affectae; hinc,

Tetanus tonicus, S. sp. 1.-Tetanus holotonicus, sp. 4.Catochus holotonicus, sp. 1. M. Febr. in Ephem. German. dec. ii. ann. i. obs. 1.-Catochus cervinus, S. sp. 2. Storck. Ann. Med. 1758. M. Julio.-Tetanus opisthotonicus, S. sp. 3. Tetanus emprosthotonicus, sp. 2.

Tetani species symptomaticae sunt,

Tetanus febricosus, S. sp. 10. Storck. Ann. Med. 1759. p. 166. hemiplegicus, sp. 9. lateralis, $\nmid$ sp. 6. Morgagn. de caus. et sed. x. 2, et 3 . Pleurosthotonos, Strack. Tetanus syphiliticus, sp. 7. verminosus, sp. 8 . hystericus, sp. 12. mirandus, sp. 13. Catochus diurnus, sp. 6.

* Catochus apud Sauvagesium genus cst minime naturale, cum genus illud conjunxerit species natura prorsus diversas; ideoque ejusmodi genus omisi, sed species sub eo recensitas, quae a rigiditate spastica pendeant, ad Tetani genus huc retuli.

+ In exemplo Tetani lateralis quod profert Sauvagesius, adfuit in eodem aegro alterius lateris paralysis; ideoque hic mox post Tetanum hemiplegicum posui; sed si Tetani lateralis exemplum a Morgagnio allatum, sine alterius lateris paralysi fuit, de quo tamen mihi non constat, idem inter varietates idiopathicas recenseri potuisset. Interim, donec haec res certius determinaretur, post Tetanum lateralem posui Pleurosthotonon Strackii. 
(TETANUS.)

II. Trismus; praecipue maxillae inferioris spastica rigiditas.

Trismus, S. gen. 117. Lin. 124. Sag. 223.-Capistrum, Vog. 208.

a. Species verae sunt,

1. Trismus (nascentium) infantes intra duas primas a nativitate septimanas corripiens.

Trismus nascentium, S. sp. 1.-Gallis Americanis mal de machoire.-Heister, comp. med. pract. cap. xv. sect. 10. -Cleghorn's Minorca, Introd. p. 33.-Hofer in Act. Helvet. tom. i. p. 65.-Macb. p. 459.

2. Trismus (traumaticus) omnium aetatum, a vulnere vel frigore.

Trismus traumaticus, S. sp. 2. Lond. Med. Obs. vol. i. art. 1. 7. vol. ii. 8. 34. vol. iii. 31. vol. iv. 7. vi. 13. Med. Trans. ii. 4.-Angina spasmodica, S. sp. 18. Zwingeri act. Helvet. tom. iii. p. 319.-Convulsio a nervi punctura, S.sp. 2.-Trismus catarrhalis, S. sp. 15. Hillary's Barbadoes, 221. Lond. Med. Obs. vol. iv. 7.

b. Sequentes apud Sauvagesium Trismi species, spurias, vel falsas dicimus; vel quod non a spasmo, sed ab alio musculorum vitio pendeant, vel quod convulsivae potius quam spasmodicae sint, vel, denique, quod faciei potius quam maxillae inferioris, musculorum affectiones sint.

Trismus inflammatorius, S. sp. 3. scorbuticus, sp. 4. capistratus, sp. 11. arthriticus, sp. 9. verminosus, sp. 5 . hypochondriacus, sp. 8 . febrilis, sp. 10. crepitans, sp. 7. occipitalis, sp. 12 . maxillaris, sp. 13 . dolorificus, sp. 14. cynicus, sp. 16. cynogelos, sp. 17. sardonicus, sp. 18. diastrophe, sp. 19.

\section{G. L.-CONVULSIO.}

Musculorum contractio clonica, abnormis, citra soporem.

Convulsio, S. gen. 128. Lin. 142. Vog. 191. Sag. 235.

1. Idiopathicae.

Convulsio universalis, S. sp. 11.-Hieranosos, Lin. 144. Vog. 190.-Convulsio habitualis, S. sp. 12. intermittens, sp. 16. hemitotonos, sp. 15. abdominis, sp. 10 . ab inanitione, sp. 1. ab onanismo, sp. 13.-Scelotyrbe festinans, sp. 2 .

2. Symptomaticae.

Convulsio a capitis ictu, S. sp. 4. nephralgica, sp. 6 . gravidarum, sp. 14. - Scelotyrbe verminosa, sp. 5.-Convulsio febrilis, sp. 3 . 


\section{G. LI.-CHOREA.*}

Impuberes utriusque sexus, ut plurimum intra decimum et decimum quartum aetatis annum adorientes, motus convulsivi ex parte voluntarii, plerumque alterius lateris, in brachiorum et manuum motu, histrionum gesticulationes referentes; in gressu, pedem alterum saepius trahentes quam attollentes.

Scelotyrbe, S. gen. 136. Sag. gen. 243.-Chorea, Lin. 139.

Speciem unam tantum videre mihi contigit, quae apud Sauvagesium audit

Scelotyrbe chorea Viti, S. sp. 1.-Chorea St. Viti Sydenham. Sched. monitoria.

Nec alia est,

Scelotyrbe instabilis, S. sp. 3.-et fortassis, Scelotyrbe intermittens, sp. 4.-Hieranosis, Macb. 558.

\section{G. LII.-RAPHANIA.}

Articulorum contractio spastica, cum agitatione convulsiva, dolore violentissimo, periodico.

Raphania, Lin. 145. Vog. 193. Linn. Amoen. acad. vol. vi.-Convulsio raphania, S. sp. 7.-Germanis Die Kriebel-Krankheit._Eclampsia typhodes, S. sp. 1.-Sennert. de febr. lib. iv. cap. 16.-Gregor. Horst. oper. tom. ii. lib. viii. obs. 22.-Willis de morb. convuls.-Muller. apud Haller. disp. tom. i.-Waltschmied apud Haller. disp. tom. vii.-Wedel, ibid.-Hoffm. tom. i. 231.Kannegiesser, A. N. C. tom. vii.-Brunner in Ephem. Germ. dec. iii. ann. ii. obs. 224. -Willisch. ibid. Cent. vii. obs. 13. - Wepfer de affect. cap. obs. 120.-Breslauer Sammlung 1717. Julio, Septembri, et Decembr. ibid. 1723. Januar.-A. N. C. vol. vii. obs. 41.-Bruckmann. Comm. Norimb. 1753, p. 50.-Soc. Royale, i. mém. p. 303. ii. mém. p. 587.-Convulsio ab ustilagine, S. sp. 8.

An Convulsio Soloniensis vel Necrosis ustilaginea sit idem morbus?

Vide Salerne in Mémoires présentés à l'acad. des sciences, tom. ii.

In priore hujus libri editione, Choream pro convulsionis specie habui; cum autem ratione tum aetatis quam adoritur, tum motuum quos exhibet, a quavis alia convulsionis specie plurimum differat, hic pro genere diverso posui. 


\section{G. LIII.-EPILEPSIA.}

\section{Musculorum convulsio cum sopore.}

Epilepsia, S. gen. 134. Lin. 143. Vog. 188. Sag. 24. Boerh. 1771. Hoffm. iii. 9. Junck. 54. Lond. Med. Obs. vi. 9.-Eclampsia,* S. gen. 133. Vog. 189. Sag. 240.

\section{Species idiopathicae sunt,}

1. Epilepsia (cerebralis) sine causa manifesta subito adoriens; praegressa nulla sensatione molesta, nisi fortassis vertiginis vel Scotomiae alicujus.

Epilepsia plethorica, S. sp. 1.-Eclampsia plethorica, sp. 7. -Epilepsia cachectica, $\uparrow$ sp. 2 ?-a terrore, Macb. p. 556.

2. Epilepsia (sympathica) sine causa manifesta; sed pracgressa sensatione aurae cujusdam a parte corporis quadam versus caput assurgentis.

Epilepsia sympathica, S. sp. 8.-Epilepsia pedisymptomatica, sp. 6.

3. Epilepsia (occasionalis) ab irritatione manifesta oriens, et ablata irritatione cessans.

Variat pro diversitate irritamenti, et hinc

a. Ab injuria capiti illata.

Epilepsia traumatica, S. sp. 13.-Eclampsia traumatica, sp. 9.

b. A dolore.

Epilepsia a dolore, S. sp. 10. Ed. Ess. v. 61.-Epilepsia rachialgica, sp. 14.

Eclampsia a doloribus, sp. 4.

a. Rachialgica.

b. Ab otalgia.

c. A dentitione.

Eclampsia parturientium, sp. 3 .

c. A vermibus.

Eclampsia verminosa, S. sp. 2.

- Eclampsiam quasi morbum acutam, ab Epilepsia, quam pro Chronico habent, distinguere, et pro diverso genere ponere, voluerunt Sauvagesius et Vogelius; sed, cum inter morbos acutos et chronicos limites accuratos ponere ubique difficile sit, et cum Eclampsia Sauvagesii, tum causis tum phaenomenis plerumque cum Epilepsia exacte congruat, illam genere ab Epilepsia diversam esse nequaquam habere possum. Ejusmodi genus prorsus omisit Linnaeus, et quae plura ad Eclampsiam ab Epilepsia distinguendam protulit Sagarus, speciem tantum, non genus, distinguere possunt.

+ Epilepsia, apud Sauvagesium Cachectica dicta, revera duarum specierum esse videtur. Primo enim hanc cachecticam cum Serosa Hoffmanni et Pisonis eandem esse auctor statuit; deinceps, utpote acri repulso orta, eandem esse cum specie sua undecima, nimirum Exanthematica, statui potest. Hinc est quod hanc epilepsiam cachecticam utroque loco posui, sed dubius, et aliis dijudicandum relinquens.

VOI. I. 
(EPILEPSIA.)

d. A veneno.

Eclampsia ab atropa, S. sp. 11. ab oenanthe, sp. 12. a cicuta, sp.13. a coriaria, sp. 14.

$e$. A scabie, vel' alia acris humoris effusione, retropulsa. Epilepsia exanthematica, S. sp. 11.-Epilepsia cachectica, sp. 2.

f. A cruditate ventriculi.

Epilepsia stomachica, S. sp. 3.-Eclampsia a saburra, sp. 5.

g. A pathemate mentis.

Epilepsia a pathemate, S. sp. 7 .

$h$. Ab haemorrhagia nimia.

Eclampsia ab inanitione, S. sp. 8.

i. A debilitate.

Eclampsia neophytorum, S. sp. 15.

Epilepsiae symptomaticae sunt:

Epilepsia febricosa, S. sp. 9.-Eclampsia febricosa, sp. 17. exanthematica, sp. 10.-Epilepsia uterina, sp. 4. syphilitica, sp. 12.-Eclampsia ab hydrocéphalo, sp. 16. ab ischuria, sp. 6 .

SECT. II.-IN FUNCTIONIBUS VITALIBUS.

$$
\text { A.-In actione cordis. }
$$

\section{G. LIV:-PALPITATIO.}

Motus cordis vehemens, abnormis.

Palpitatio, S. gen. 130. Lin. 132. Vog. 213. Sag. 257. Hoffm. iii, 83. Junck. 33. Macb. 569.

Si palpitationis causae variae externis signis dignosci possent, plures ejus species cum Sauvagesio pro diversitate causae statuere potuissemus; sed, cum causae diversae nullo modo, quantum novimus, distingui queant, non nisi speciem unicam idiopathicam statuere possum, et quam charactere sequenti insigniri vellem :

Palpitatio (cardiaca) fere constans, saltem saepe rediens, sine alio morbo evidente.

Pro synonymis sequentes Sauvagesii species recensere placet.

Palpitatio ab aneurysmate cordis, sp. 1. Senac, Mal. du coeur. p. 284. Lancisi de aneurysm.-Palpitatio tremula, sp. 4. Senac du coeur, p. 504.-Palpitatio apostemato- 
(Palpitatio.)

sa, sp. 7. Lancisi de aneurysmate, lib. 1. prop. 5. Senac du coeur, 485.-Palpitatio a corde ossificato, S. sp. 2. Senac du coeur, p. 484. Morgagn. de sed. Ep. xxiv. 17.-Palpitatio calculosa, S. sp. 3.-Palpitatio a polypo, S. sp. 5. Senac du coeur, lib. iv. cap. 16. p. 484.-Palpitatio polyposa, S. sp. 6.-Palpitatio ex aortae aneurysmate, sp. 8.-Palpitatio ex aortae angustia, sp. 9. Lancisi de aneurysm. prop. 53. Senac, p. 484.

Palpitationis species symptomaticae sunt,

Palpitatio a pancreate, S. sp.10. Storck, Ann. med. i. p. 245. - Palpitatio arthritica, S. sp. 11.-Palpitatio chlorotica, sp. 12.-Palpitatio hysterica, sp. 13.-Palpitatio melancholica, sp. 14. Lancisi de aneurysm. prop. 44. Palpitatio febricosa, S. sp. 15. Storck, Ann. med. i. p. 75.

$$
\text { B.-In actione pulmonum. }
$$

\section{G. LV.-ASTHMA.}

Spirandi difficultas per intervalla subiens; cum angustiae in pectore sensu, et respiratione cum sibilo strepente; tussis sub initio paroxýsmi difficilis, vel nulla, versus finem libera, cum sputo muci saepe copioso.

Asthma, S. gen. 145. Lin. 161. Vog. 268. Sag. 252.Asthma convulsivum et spasmodico-flatulentum, Hoffm. iii. 94.-Asthma spasticum, Junck. tab. 51.

Species idiopathicae sunt,

1. Asthma (spontaneum) sine causa manifesta, vel alio morbo comitante.

Asthma humidum, S. sp. 1. flatulentum, Floyer on the asthma, chap. 1.-Asthma convulsivum, S. sp. 2. Willis, Pharm. rat. p. 2. sect. 1. cap. 12. Med. Tr. i. 7.Asthma hystericum, S. sp. 3. Floyer on the asthma, chap. 1. - Asthma stomachicum, S.sp. 8. Floyer, Scheme of the species of asthma. Periodic asthma, 6.-Orthopnoea spasmodica, S. sp. 3.-Orthopnoea hysterica, S. sp. 4.

2. Asthma (exanthematicum) a scabie vel alia acris effusione retropulsa.

Asthma exanthematicum, S. sp. 11.-Asthma cachecticum, sp. 13.

3. Asthma (plethoricum) a suppressa evacuatione sanguinis antea solita, vel a plethora spontanea:

Asthma plethoricum, S. sp. 15. 
(ASTHMA.)

Species symptomaticae sunt,

Asthma hypochondriacum, S. sp. 4.-Asthma arthriticum, sp. 5.-Asthma venereum, sp. 14. Junck. tab. 32.

\section{G. LVI.-DYSPNOEA.}

Spirandi difficultas perpetua, sine angustiae, et potius cum repletionis et infarctus in pectore, sensu. Tussis per totum morbi decursum frequens.

Dyspnoea, S. gen. 144. Lin. 160. Vog. 267. Sag. 251. Junck. 32.-Orthopnoea, S. gen. 146. Lin. 162. Vog. 269. Sag. 253. Macb. 574 .

Species idiopathicae sunt,

1. Dyspnoea (catarrhalis) cum tussi frequente, mucum viscidum copiosum ejiciente.

Asthma catarrhale, S. sp. 16.-Asthma pneumonicum, Willis, Pharm. rat. P. ii. sect. 1. cap. 12.-Asthma pituitosum, Hoffm. iii. sect. ii. cap. 2. sect. 3.-Asthma pneumodes, S. sp. 17. Ed. Ess. iii. 25.

2. Dyspnoea ( $\operatorname{sicca}$ ) cum tussi plerumque sicca.

Hujus causae variae haud facile dignoscendae sunt; sequentes autem Sauvagesii species huc referri possunt.

Dyspnoea a tuberculis, S. sp. 2. a steatomatis, sp. 5.Orthopnoea a lipomate, sp. 18.-Dyspnoea a hydatidibus, sp. 4 . polyposa, sp. 20 .

3. Dyspnoea (aërea) a minima quavis tempestatum mutatione aucta.

Dyspnoea a pneumatia, S. sp. 12.

4. Dyspnoea (terrea) cum tussi materiem terream vel calculosam ejiciente.

Dyspnoea calculosa, Macb. 574 .

5. Dyspnoea (aquosa) cum urina parca et oedemate pedum, sine fluctuatione in pectore, vel aliis characteristicis hydrothoracis signis.

Dyspnoea pituitosa, S. sp. 1.-Orthopnoea ab hydropneumonia, sp. 12.

6. Dyspnoea (pinguedinosa) in hominibus valde obesis. Orthopnoea a pinguedine, S. sp. 6.

7. Dyspnoea (thoracica) a partibus thoracem cingentibus laesis, vel male conformatis.

Dyspnoea traumatica, S. sp. 15.-Orthopnoea traumatica, sp. 14.-Dyspnoea Galenica, sp. 16-rachitica, sp. 10.Asthma a gibbo, sp. 9.

8. Dyspnoea (extrinsecu) a causis externis manifestis.

Asthma pulverulentorum, S. sp. 7. metallicum, sp. 12.- 
(DYSPNOEA.)

Orthopnoea a vaporibus, sp. 16. a deglutitis, sp. 9. a fungis, sp. 25. ab antipathia, sp. 15 . a bronchocele, sp. 10.

\section{Dyspnoeae species symptomaticae sunt symptomata,}

1. Morborum cordis vel vasorum majorum.

Dyspnoea a corde, S. sp. 11.-Asthma a polypo cordis, sp. 6.-Orthopnoea cardiaca, sp. 2. polyposa, sp. 26. Dyspnoea aneurysmatica, sp. 19.-Orthopnoea ab aneurysmate, sp. 8.-Dyspnoea ab aortae angustia, sp. 22.

2. Tumoris abdominalis.

Dyspnoea a physconia, S.sp. 7. a graviditate, sp. 8. tympanitica, sp. 9. a liene, sp. 14. a stomacho, sp. 13. a gastrocele, sp. 17.-Orthopnoea a gastrocele, sp. 11.

3. Variorum morborum.

Asthma febricosum, S. sp. 18 . Orthopnoea febricosa, sp. 20. Orthopnoea peripneumonica, sp. 1. pseudo-peripneumonica, sp. 21. variolosa, sp. 24.-Dyspnoea scorbutica, sp. 18. Orthopnoea scorbutica, sp. 22. Orthopnoea a hydrothorace, sp. 5 . ab empyemate, sp. 13. a vomica, sp. 7. Dyspnoea a vomica, sp. 6.-Orthopnoea a hydrocephalo, sp. 23 . a vermibus, sp. 17 .

\section{G. LVII.-PERTUSSIS.}

Morbus contagiosus ; tussis convulsiva, strangulans, cum inspiratione sonora, iterata; saepe vomitus.

Pertussis Sydenham. Ed. Leid.p. 200.311.312. Huxham de aëre, ad ann. 1732. Lond. Med. Obs. iii, 27. 30. Soc. Royale, iii. hist. p.'268. - Tussis convulsiva, sive ferina, Hoffm. iii, 111.-Tussis ferina, S. sp. 10. Sag. sp. 10.Tussis convulsiva, sp. 11. Sag. sp. 11.-Amphimerina tussiculosa, S.sp. 13.

SECT. III-IN FUNCTIONIBUS NATURALIBUS.

\section{G. LVIII.-PYROSIS.}

Epigastrii dolor urens, cum copia humoris aquei, plerumque insipidi, aliquando acris eructata.

Pyrosis, S. gen. 200. Sag. gen. 158.-Soda, Lin. 47. Vog. 154. - Scotis the Water-Brash.

Species unica vera,

Pyrosis Suecica, S. sp. 4.-Cardialgia sputatoria, sp. 5. Macb. p. 515. 
(PYROSIS.)

Caeterae Sauvagesii species symptomaticae sunt ;

Pyrosis vulgaris, S. sp. 1. a conceptione, sp. 6. biliosa, sp. 2. a phlogosi, sp. 3. ulcerosa, sp. 5 .

\section{G. LIX.-COLICA.}

Dolor abdominis, praecipue circa umbilicum torquens; vomitus; alvus adstricta.

Colica, S. gen. 204. Lin. 50. Vog. 160. Sag. 162. Junck. 106.-Colica spasmodica et flatulenta, Hoffm. ii, 284. Macb. p. 519.-Rachialgia, S. gen. 211. Sag. 168.-Ileus, S. gen. 252. Vog. 162.' Sag. 187.-Iliaca, Lin. 185.-Dolor et spasmus iliacus, Hoffm. ii, 263. Passio iliaca, Junck. 107.

Species idiopathicae sunt,

1. Colica (spasmodica) cum retractione umbilici et spasmis musculorum abdominalium.

Colica spasmodica, S. sp. 7. flatulenta, sp. 1.-nleus physodes, sp. 9.-Colica biliosa, sp. 5.* Edin. Med. Ess. iii, 27.-Colica pituitosa, S. sp. 2.†-Ileus a colo pituita infarcto, sp. 5. Ileus Indicus, sp. 8.

Variat symptomatis superadditis; hinc,

a. Colica cum vomitu stercoris, vel rerum per anum injectarum.

Ileus spasmodicus, S. sp. 3. Lond. Med. Obs. iv. 17. Edinb. Phys. Ess. ii. 27. 28.-Ileus volvulus, S. sp. 7.

b. Colica inflammatione superveniente.

Ileus inflammatorius, S. sp. 1.-Colica phlogistica, sp. 6.

2. Colica (pictonum) praeeunte ponderis vel molestiae in abdomine, praecipue circa umbilicum, sensu; accedente dolore colico, primum levi, non continuo, et praecipue post pastum aucto; tandem graviore et fere perpetuo; cum dolore brachiorum, et dorsi, in paralysin demum abeunte.

* Cum vomitus quivis vehemens et saepe repetitus bilem plerumqne eliciat, colicam quam, ex vomitu bilis solo, biliosam appellarunt medici, omnino spasmodicam esse censeo.

+ Pituita in parte quadam intestinorum congesta non nisi a spasmo obruente fieri videtur; idenque tum colicam pituitosam, tum ileum, a colo pituita infarcto, hic apposui.

\# Ileus gradu tantum, nequaquam natura, a Colica differre mihi videtur ; ideoqueileum pro varietate colicae tantum hic recensui. Multi quidem medici ileum omnem inflammatorium esse putarunt. Dolorem autem abdominis, sive colicam, cum vomitu stercoris sine inflammatione adesse, ipse aliquoties vidi. Sin autem inflammatio aliquando adsit, si eadem colicae supervenerit, colicam phlogisticam dari recte monuit Sauvagesius; nec ideo epigenomeno genus mutari; et si quidem cum dolore abdominis et vomitu ab initio'adfuerit inflammatio, morbum pro enteritide habere vellem. Denique, quovis modo dari ileum genus a colica diversum concedere non possum. 
Rachialgia pictonum, S. sp. 1.*-Colica pictonum Citesii et succedentium Auctorum. Med. Trans. i. 12, 13, 14, 15, 20. Edinb. Phys. Ess. i. 22. Chalmers's Carolina, p. 187.-Colica nervosa, Macb. p. 524.

Variat ratione causae remotae; et hinc

a. A veneno metallico.

Rachialgia metallica,S. sp. 3.-Colica pictonum, Tronchin, sp. 2. sive a causa remota secunda.

b. Ab acidis ingestis.

Rachialgia pictonum, S. sp. 1: Tronchin, sp. 3 .

c. A frigore.

Rachialgia ab adiapneustia, Tronchin, sp. 5.

d. A contusione dorsi.

Rachialgia traumatica, S. sp. 7 .

3. Colica (stercorea) in hominibus alvi tardae, post diuturnam alvi obstipationem.

Colica stercorea, S. sp. 3.-Ileus a faecibus induratis, S. sp. 2.

4. Colica (accidentalis) a materie acri ingesta.

Colica accidentalis, S. sp. 18.-Colica Japonica, sp. 10. Colica lactentium, sp. 20.-Ileus a veneno, sp. 14.Cholera sicca auriginosa a fungis venenatis, sp. 2.

5. Colica (meconialis) neophytorum a meconio retento.

Colica meconialis, S. sp. 19.

6. Colica (callosa) cum sensu in quadam intestinorum parte stricturae, et saepe ante eam collecti flatus cum aliquo dolore, qui flatus etiam per eandem paulatim transiens evanescit ; alvo tarda, et tandem non nisi faeces paucas liquidas egerente.

Ileus a callosa coli strictura, S. sp. 11. Edin. Med. Ess. iv. 31.

7. Colica (calculosa) cum duritie in quadam parte abdominis fixa ; calculis quondam per anum dejectis.

Colica calculosa, S. sp. 15. Edin. Med. Ess. i. 32. Edin. Phys. Ess. ii. 26.-Ileus calculosus, S. sp. 10.

- Rachialgiam a Colica genere diversam esse nequaquam video. Symptomata enim colicae essentialia in utroque morbo semper adsunt; et si forte alteri quaedam superaddita sint, haec speciem non genus diversum constituere possunt. Ut medici igitur nuperi fere omnes, ita ego quam rachialgiam appellarunt Astrucius et Sauvagesius, pro specie colicae habui ac recensui. 
(COLICA.)

Colicae symptomaticae sunt :

Colica febricosa, S. sp. 21. Rachialgia febricosa, sp. 2. Tronchin, sp. 1.-Colica hysterica, S. sp. 17.-gravidarum, sp. 16. - Colica verminosa, sp. 4. Ileus verminosus, sp. 13.-Colica Lapponica, sp. 9.-Rachialgia arthritica, sp. 4. Tronchin, sp. 4.-Colica scorbutica, S. sp. 6.-Ileus herniosus, sp. 6. Colica enterocelica, sp. 22. Ileus ab intestino compresso, sp. 4.-Colica schirrosa, sp. 12. pancreatica, sp. 13. mesenterica, sp. 11.-Rachialgia ab osteosarcosi, sp. 8.-Colica plethorica, sp. 8. $a$. haemorrhoidalis. $b$. catamenialis.-Colica pulsatilis, sp. 14.-Ileus imperforatorum, sp. 12.

\section{G. LX.-CHOLERA.}

Humoris biliosi vomitus, ejusdem simul dejectio frequens; anxietas; tormina ; surarum spasmata.

Cholera, S. gen. 253. Lin. 186. Vog. 110. Sag. gen. 188. Hoffm. ii. 165.-Diarrhoea cholerica, Junck. 112.

Species idiopathicae sunt,

1. Cholera (spontanea) tempestate calida, sine causa manifesta oboriens.

Cholera spontanea, S. sp. 1. Sydenh. sect. iv. cap. 2. Cleghorn's diseases of Minorca.-Cholera Indica, S. sp. 7.

2. Cholera (accidentalis) a rebus acribus ingestis.

Cholera crapulosa, S. sp. 11. a venenis fossilibus, sp. 4 . a veneno animali, sp. 5 .

Cholerae symptomaticae sunt,

Cholera intermittens, S. sp. 6. inflammatoria, sp. 8. arthritica, sp. 10 . verminosa, sp. 9 .

\section{G. LXI.-DIARRHOEA.}

Dejectio frequens; morbus non contagiosus; pyrexia nulla primaria.

Diarrhoea, S. gen. 253. Lin. 187. Vog. 105. Sag. 189. Junck. 112.-Hepatirrhoea, S. gen. 246.-Cholerica, Lin. 190.-Coeliaca, S. gen. 255. Lin. 189. Vog. 109. Sag. 199.-Lienteria, S. gen. 256. Lin. 188. Vog. 108. Sag.191.-Pituitaria, Vog.111.-Leucorrhois, Vog.112. -N. Lambsma, ventris fluxus multiplex, Amst. 1756.

Species idiopathicae sunt,

1. Diarrhoea (crapulosa) qua stercora naturalibus liquidiora et majori copia dejiciuntur.

Diarrhoea stercorosa, S. sp. 1. vulgaris, sp. 2. 
(DIARRHoEA.)

2. Diarrhoea (biliosa) qua faeces flavae magna copia dejiciuntur.

Diarrhoea biliosa, S. sp. 8.

3. Diarrhoea (mucosa) qua vel ab acribus ingestis, vel a frigore, praecipue pedibus applicato, mucus copiosus dejicitur.

Diarrhoea lactentium, S. sp. 19.-Dysenteria Parisiaca, sp. 3.-Diarrhoea ab hypercatharsi, sp. 16-Dysenteria a catharticis, sp. 12.-Pituitaria, Vog. 111.-Leucorrhois, Vog. 112.-Diarrhoea pituitosa, S. sp. 4.-Coeliaca mucosa, sp.3.-Diarrhoea serosa, sp. 10.-Diarrhoea urinosa.

4. Diarrhoea (coeliaca) qua humor lacteus specie chyli dejicitur.

Coeliaca chylosa, S. sp. 1. lactea, sp. 4.

5. Diarrhoea (lienteria) qua ingesta parum mutata celeriter dejiciuntur.

Lienteria spontanea, S. sp. 2.

6. Diarrhoea (hepatirrhoea) qua materies seroso-cruenta, sine dolore dejicitur.

Hepatirrhoea intestinalis, S. sp. 2.

Symptomaticae.

Diarrhoea febrilis, S. sp. 3. febricosa, sp. 20.-Hepatirrhoea intermittens, sp. 7.-Diarrhoea variolosa, sp. 6. pleuriticorum, sp. 21. arthritica, sp. 9. colliquativa, sp. 13. choleriodes, sp. 17.-Hepatirrhoea vera, sp.1. a vulnere, sp. 3. mesenterica, sp. 4.-Dysenteria a mesenterii vomica, sp. 11.-Diarrhoea purulenta, sp. 11.-Coeliaca purulenta, sp. 2.-Hepatirrhoea scorbutica, sp. 5.-Dysenteria scorbutica, sp. 18. - Lienteria scorbutica, sp. 3. ex ulcere ventriculi, sp. 1. aphthosa, sp. 4. secundaria, sp. 5.-Diarrhoea verminosa, sp. 14. a dentitione, sp.15. chiliensis, sp. 12. acrasia, sp. 7 .

\section{G. LXII.-DIABETES.}

Urinae plerumque praeternaturalis, copia immodica, profusio chronica.

Diabetes, S. gen. 263. Lin. 197. Vog. 115. Sag. 199. Junck. 99. Dobson, Lond. Med. Observations, vol. v, art. 27. Home's Clinical Experiments, sect. xri.-Diuresis, Vog. 114.

Species idiopathicae sunt,

1. Diabetes (mellitus) cum urina odoris, coloris, et saporis mellei.

- Diabetes Anglicus, S. sp. 2. Mead on Poisons, Essay 1.

voL. I.

2 A 
(DIABETES.)

Ejusdem Monita Med. cap. ix, sect. 2. Lond. Med. Obs. iii,26. Dobson, in Lond. Med.Obs. vol.v, art.27. Myers, Diss. inaug. de Diabete, Edinb. 1779.—Diabetes febricosus, S. sp. 7. Sydenh. Epist. resp. ad R. Brady.*

2. Diabetes (insipidus) cum urina limpida non dulci. $†$

M. Lister Exerc. medicin. ii. de Diabete.-Diabetes legitimus, S. sp. 1. Aretaeus de Morb. diuturn. Lib.ii. cap. 2. Diabetes ex vino, S. sp. 5. Ephem. Germ. dec. i, ann.ii, obs. 122.

Symptomaticae sunt,

Diabetes hystericus, S. sp. 3. Sydenh. diss. epist. ad G. Cole.-Diabetes arthriticus, S. sp. 6. Sydenh. de podagra.-Diabetes artificialis, S. sp. 4. Malpigh. de liene.

\section{G. LXIII.-HYSTERIA.}

Ventris murmura; sensus globi in abdomine se volventis, ad ventriculum et fauces ascendentis, ibique strangulantis; sopor ; convulsiones; urinae limpidae copia profusa ; animus, nec sponte, varius et mutabilis. +

Hysteria, S. gen. 135. Lin. 126. Vog. 219. Sag. 242.Malum hystericum, Hoffm. iii, 50. Junck. 36.-Affec-

* Quaeri potest, an diabetes febricosus Sydenhami ad diabetem mellitum, an ad insipidum referri debeat? Et simul quaeri potest, an urina in diabete hysterico vel arthritico sit mellita, an insipida, cum Sydenhamus ipse nullibi de sapore urinae, in diabete quovis, quicquid dixerit? Ad posterius respondere possum, quod, ex propria observatione, novi, urinam in diabete hysterico et arthritico nequaquam dulcem, et sere prorsus insipidam, esse, et diabetem febricosum Sydenhami ad diabetem mellitum ideo retuli ; quod in simili senis, post diuturnam febrem, diabete, urinam omnino dulcem inveni.

+ An hujusmodi diabetis species sit frequens, incertum est. In ommibus exemplis, iisque non paucis, quae viderat Willisius, urinam mellitam semper invenit; et observationes nuperae frequentes fere omnes ejusmodi urinam exhibuerunt; ita ut dubium sit, an alia diabetis idiopathicae et permanentis species revera detur.-Affirmat autem Listerus; et ego ipse inter plures mellitas unicam insipidam observavi. Ejusmodi itaque speciem dari concedo, ideoque recensui; sed an recte legitima dici queat, vel an diabetes Aretaei et plurium auctorum ejusmodi fuissent, multum dubito. Ante Willisium quidem, vel prioris aevi, vel extra Angliam, medicorum nemo urinam dulcem annotaverat; sed ejusmodi urinam temporibus Willisii primo et in Anglia tantum comparuisse, minus verisimile est; omnesque ante Willisium de urina in diabete dulci prorsus siluisse, potius observationi perfunctoriae, vel omnino neglectae, imputarem. Quod observatores, quibus diabetis exempla antea occurrebant, vel negligentes vel malae fidei fuissent suspicor ; cum plurimi eorum tam incredibilia, de potu in diabeticis per renes transeunte prorsus immutato, narrant. Sydenhamum autem ipsum, adeo perspicacem et diligentem, de statu urinae nihil monuisse, mirum videtur; sed cum scripta Willisii, Sydenhamo satis nota, urinam semper esse dulcem asseruerint, si diversam invenisset Sydenhamus, quin id indicasset, nullus dubito.

‡ Novit quisque, in Nosologia et in Medicina facienda quadantenus exercitatus, quam difficile sit inter dyspepsiam, hypochondriasin, et hysteriam semper accurate distinguere. Genera autem vera, et satis a se invicem distincta, indicasse mihi videor: Recte autem, an secus, fecerim, aliis dijudicandum relinquo. Morbos affines facile inter se misceri probe novi; et in quocunque casu, quisnam eorum pro primario sit habendus, per characteres nosologicos indicare, mihi difficillimum fore agnosco. Posteri, ut spero, hanc rem luculentius expositam dabunt. 
(HYSTERIA.)

tio hysterica, Willis de morb. convuls. cap. 5, 10, 11. Sydenh. diss. epist. ad G. Cole. Whytt on nervous disorders.

Quatenus specie differat hysteria idiopathica, dicere non possum, nec ut opinor, recte dixit Sauvagesius. Species enim ab eo recensitae non, nisi ratione causae remotae, varietates esse mihi videntur. Ita habet,

a. Ab emansione mensium,

Hysteria chlorotica, S. sp. 2. Raulin, cap. 13.

b. A menorrhagia cruenta.

Hysteria a menorrhagia, S. sp.3. Raulin. Sydenham, diss. epist. ad G. Cole.

c. A menorrhagia serosa, sive fluore albo.

Hysteria a leucorrhoea, S. sp. 4. Raulin, cap. 14.

d. A viscerum obstructione.

Hysteria emphractica, S. sp. 5. Raulin, cap. 5.

e. A vitio stomachi.

Hysteria stomachica, S. sp. 7. Raulin, cap. 4.

f. A salacitate nimia.

Hysteria libidinosa, S. sp. 6.*

Manifesto symptomaticae sunt,

Hysteria verminosa, S. sp. 1. Delii Amoenit. Academ.Hysteria febricosa, S. sp. 8. Wedel. in Ephem. German. dec. 1, ann. 11. obs. 193.

\section{G. LXIV.-HYDROPHOBIA.}

Potionis cujuslibet, utpote convulsionem pharyngis dolentem cientis, fastidium et horror; plerumque e morsu animalis rabidi.

Hydrophobia, S. gen. 231. Lin. 86. Vog. 30. Sag. 343. Boerh. 1138. Junck. 124.-Mead on poisons.-Desault sur la rage.-Sauvages diss. sur la rage.-James on canine madness.-Dalby, Virtues of cinnabar and musk against the bite of a mad dog.-Nugent on the hydrophobia.-Choisel, Nouvelle méthode pour le traitement de la rage._Journal de Médecine, passim.-Lond. Med.

- In speciebus hystériae recensendis, egregius Sauvagesius Raulinum, theoriae subtili et fallaci, potius quam observationi, innitentem, secutus fuisse videtur. Nec quidem inter caeteras hysteriae species indicasse videtur Sauvagesius, illam quam maxime exquisitam et frequentissimain, censeo, illam, scilicet, quae foeminas adultas laute viventes, sanguineas, plethoricas, valentiores, et a morbo alio quovis immunes, saepe ex pathemate mentis solo adoritur. 


\section{(HYDROPHOBIA.)}

Obs. and Inquir. vol. iii, art.34. vol. v. art. 19, 26, and App.-Lond. Med. Trans. vol. ii, art. 5, 12, and 15.Edinb. Med. Ess. v. art. 51.-Heysham, Diss. inaug. de rab. canin. Edinb. 1777.-Parry, Diss. inaug. de rab. contagiosa sive canina, Edinb. 1778.-Soc. Royale, i. Mém. p. 105, 146.-Andry, Recherches sur la rage, 1780. -Soc. Royale, ii. Hist. p. 229, 230, 456, iii. Hist. p. 167.-Vaughan, Cases of hydrophobia, second edit. 1778.

Species sunt,

1. Hydrophobia (rabiosa) cum mordendi cupiditate ex morsu animalis rabidi.

Hydrophobia vulgaris, S. sp. 1.

2. Hydrophobia (simplex) sine rabie, vel mordendi cupiditate.

Hydrophobia spontanea, S. sp. 2. Edinb. Med. Ess. i. 29.

\section{ORDO IV.-VESANIAE.*}

Mentis† judicantis functiones laesae sine pyrexia vel comate.

Paranoiae, Vog. cl. ix.-Deliria, S. cl. viii, ord. iii, Sag. cl. xi, ord. iii.-Ideales, Lin. cl. v. ord. i.

\section{G. LXV.-AMENTIA.}

Mentis judicantis imbecillitas, qua homines rerum relationes vel non percipiunt, vel non reminiscuntur.

Amentia, S. gen. 233. Vog. 337. Sag. 346.-Morosis, Lin. 106. - Stupiditas, morosis, fatuitas, Vog. 336.Amnesia, $\neq$ S. gen. 237. Sag. 347.-Oblivio, Lin. 107. Vog. 338.-Memoriae debilitas, Junck. 120.

- Vesaniarum ordinem hic instituere velim, quae cum classe Vogelii nona, Paranoiae inscripta, eadem omnino sit ; sed a classe Vesaniarum Sauvagesii et Sagari in eo diversa, quod horum auctorum ordines Hallucinationum et Morositatum prorsus excludat; itidemque a classe Mentalium apud Linnaeum diversa, quod hujus auctoris ordines Imaginariorum et Patheticorum etiam omittat.

A classe Vesaniarum autem tum Hallucinationes tum Morositates exclusas esse velim; quia neque causis, neque symptomatis cum Vesaniis stricte dictis ullo modo congruant. Quis enim Syrigmum vel Bulimiam, vel aliam Hallucinationem sive Morositatem, quae a mentis judicio non pendeat Vesaniam appellare vellet? Verum quidem est, tum hallucinationes, tum morositates, cum vesaniis aliquando conjunctas esse, sed simul adsunt alia vesaniae indicia, quae ejusmodi hallucinationes et morositates affectionis magis generalis symptomata tantum esse ostendunt. $\mathrm{Ob}$ has rationes, hallucinationes Sauvagesii et imaginarios Linnaei, ad morbos locales olim detuli; et nune morositates Sauvagesii et patheticos Linnaei, antea a nobis omnino omissos, ad locales etiam deferendos esse censui.

+ In priore Nosologiae nostrae editione, has functiones simpliciter mentis esse dixi, minus autem recte; nam perceptio falsa sive hallucinatio, et appetitus erroreus sive morositas, quodammodo ad mentis functiones pertinent; cum vero utrasque ab hac classe exclusas esse velim, oportebat classis characterem nune mutare, et mentis judicantis functiones dicere.

¥ Amnesiam cum amentia ad unum genus retuli, tum quia plerumque conjungantur, tum quia ab iisdem causis plesumque oriantur, et amnesia ingravescens ad amentiam semper ducat. 
(AMENTia.)

Species sunt,

1. Amentia (congenita) a nativitate constans.

Amentia morosis, S. sp. 8. microcephala, sp. 6.

2. Amentia (senilis) ex perceptione et memoria, ingravescente aetate, imminutis.

Amentia senilis, S. sp. 1. Amnesia senilis, sp. 2.

- 3. Amentia (acquisita) a causis externis, evidentibus, in hominibus sanae mentis superveniens.

Amnesia febrisequa, S. sp. 8.-Amentia a quartana, sp. 11. -Amnesia cephalalgica, sp. 6. plethorica, sp. 4.Amentia rachialgica, sp. 10.-Amnesia a venere, sp. 1 . a pathemate, sp. 5.-Amentia a venenis, sp. 3. a temulentia, sp. 7.-Amentia ab ictu, sp. 9. Amnesia traumatica, sp. 3.

Sèquentes Sauvagesii species in Nosologia methodica nequaquam recenseri debent, cum causae internae a quibus characteres desumuntur, per signa externa nequaquam dignosci possint.

Amentia serosa, S. sp. 2. a tumore, sp. 4. a hydatidibus, sp. 5. a siccitate, sp. 7. calculosa, sp. 12.

\section{G. LXVI.-MELANCHOLIA.}

Insania* partialis sine dyspepsia.†

Melancholia, S. gen. 234. Lin. 71. Vog. 332. Sag. 347. Boerh. 1089. Junck. 121. Daemonomania, S. gen. 236. Sag. 348. Daemonia, Lin. 69. Vesania, Lin. 70. Panophobia, Lin. 75. Athymia, Vog. 329. Delirium melancholicum, Hoffm. iii. 251. Erotomania, Lin. 82. Nostalgia, S. gen. 226. Lin. 83. Sag. 338. Junck. 125. Melancholia nervea, Cl. Lorry de melancholia, P. 1.

Variat pro varietate rerum de quibus homo delirat. Ita est,

a. Cum hallucinatione de statu corporis sui a levibus causis periculoso ; vel de statu rerum suarum tristi metuendo.

Melancholia vulgaris, S. sp. 1. argantis, sp. 4.-Panophobia phrontis, sp. 3.

b. Cum hallucinatione de statu rerum suarum jucundo.

- Insaniam adesse dicimus, quando rerum relationes omnino falsae mente concipiuntur, ita ut hominis vel pathemata vel actiones praeter rationem excitentur.

+ Hypochondriasis et Melancholia non semper distingui possunt. Si quando autem possint, nullo alio signo quam per dyspepsiam in hypochondriasi semper praesentem, in melancholia saepe absentem rite, quantum video, distinguendi sunt. 
(MELANCHOLIA.)

Melancholia moria, S. sp. 5. enthusiastica, sp. 13. saltans, sp. 8.

c. Cum amore vehementi sine satyriasi vel nymphomania. Melancholia amatoria, S. sp. 2.-Erotomania, Lin. 82.

d. Cum superstitioso futurorum metu.

Melancholia-religiosa, S. sp. 3.

$e$. Cum aversione motus et omnium vitae officiorum.

Melancholia attonita, S. sp. 6.

$f$. Cum inquietudine et status cujusvis impatientia.

Melancholia errabunda, S. sp. 7.

g. Cum taedio vitae.

Melancholia Anglica,* S. sp. 11. phrontis, sp. 14.

$h$. Cum hallucinatione de suae speciei natura.

Melancholia zoanthropica, S. sp. 12. hippanthropica, sp. 9. Scytharum, sp. 10.

Daemonomaniam nullam veram esse censeo, $\nmid$ et nostra sententia species sub hoc titulo a Sauvagesio recensitac sunt, vel,

1. Species melancholiae sive maniae, uti

Daemonomania fanatica, S. sp. 5. Indica, sp. 7.

Vel, 2. Morbi ab adstantibus ad daemonum potentiam falso relati, uti

Daemonomania a vermibus, S. sp. 4. Polonica, sp. 8. a cardiogmo, sp. 9.

Vel, 3. Morbi omnino simulati, uti

Daemonomania sagarum, S. sp. 1. Demonomania vampirismus, sp. 2. simulata, sp. 3 .

Vel, 4. Morbi partim veri, ut 2., partim simulati, ut 3. recensiti, uti

Daemonomania hysterica, S. sp. 6.

\section{G. LXVII.-MANIA.}

Insania universalis.

Mania, S. gen. 235 . Lin. 68. Vog. 331. Sag. 349. Boerh. 1118. Junck. 122. Battie on madness.-Para-

- Apud Anglos fortassis taedium vitae non semper a morbo pendet.

+ Ita censeo, quia daemonum in nostra corpora, vel hisce temporibus in mentes nostras, potentiam ullam esse pauci jam credunt. 
(MANIA.)

phrosyne, Lin. 66.-Amentia, Lin. 67.-Delirium maniacum, Hoffm. iii. 251.

Species sunt,

1. Mania (mentalis) omnino a pathemate mentis.

Mania a pathemate, S. sp. 1. Exempla inveniuntur ex melancholia apud Preysingerum de diagnosi morborum capitis ; ex terrore, apud Locherum de mania ; ex amore apud Ephem. Nat. Cur. dec. ii. ann. x. obs. 126. ; ex ira apud Hoffm. iii. p. 263.

2. Mania (corporea) a vitio corporis evidente.

Variat pro varietate vitii corporis; hinc

Mania metastatica, S. sp. 4.

ab ulcere siccato, Amat. Lusitan. Cent. ii. cur. 67.-a resécta plica, Hoffm. tom. iii. p. 263. Ephem. N. Cur. Cent. i. Obs. 35. - a represso herpete, Locher de mania.-a menstruis retentis, Schenck. Obs. p. 142.a semine retento, Schenck. ibid.-a repressa scabie, Lorry de melancholia, tom. ii. p. ii. cap. 3.-a graviditate, Lorry, p. i. cap. 7.-a parciori lactis et lochiorum fluxu, Puzos, 3. Mém. Mania lactea, S. sp. 2.

Mania hysteralgica, S. sp. 8.- ab hemicrania, sp. 3.-a venenis, sp. 5.-Phrenitis inanitorum, sp. 16.

3. Mania (obscura) praegresso nullo vel pathemate mentis, vel vitio corporis evidente.

Mania vulgaris, S. sp. 7.-Phrenitis apyreta, sp. 10. Mania periodica, sp. 6 .

Maniae species symptomaticae sunt,

Paraphrosyne, S. gen. 332. Sag. 345.

1. Paraphrosyne a venenis,

Paraphrosyne temulenta, S. sp. 1. a datura, sp. 2. ab opio, sp. 3. a conio, sp. 4. magica, sp. 5.

2. Paraphrosyne a pathemate, S. sp. 7.

Paraphrosyne hysterica, S. sp. 12.

3. Paraphrosyne febrilis.

Delirium, Lin. 65. Vog. 330.-Paraphrosyne febricosa, S. sp. 10. critica, sp. 11. calentura, sp. 9. puerperarum, sp. 8.

\section{G. LXVIII.-ONEIRODYNIA.}

In somno imaginatio vehementior vel molesta.

Somnium, Vog. 339.--Somnambulismus, S. gen. 221.

Lin. 77. Sag. 333.-Hypnobatasis, Vog. 340.-Noctambulatio, Junck. 124.-Ephialtes, S. gen. 138. Lin. 163. Sag. 245.-Incubus, Vog. 211 . Junck. 50. 
(ONEIRODYNIA.)

1. Oneirodynia (activa) excitans ad ambulationem, et motus varios.

Somnambulismus vulgaris, S. sp. 1. cátalepticus, sp. 2 .

2. Oneirodynia (gravans) ex sensu ponderis alicujus incumbentis, et pectus praecipue comprimentis.

Ephialtes plethorica, S. sp. 1. stomachica, sp. 2. hypochondriaca, sp. 6 . tertianaria, sp. 5 . verminosa, sp. 4. ex hydrocephalo, ${ }^{*}$ sp. 3.

\section{CLASSIS III.CACHEXIAE.}

Totius vel magnae partis corporis habitus depravatus; sine pyrexia primaria vel neurosi.

Cachexiae, Sauv. cl. x. Vog. cl. viii. Sag. cl. iii.-Deformes, Lin. cl. $\mathrm{x}$.

\section{ORDO I.-MARCORES.}

Corporis totius macies.

Macies, Sauv. cl. x. ord. i. Sag. cl. iii. ord. i.-Emaciantes, Lin. cl. $x$. ord. $\mathbf{i}$.

\section{G. LXIX.T TABES.}

Marcor; asthenia ; pyrexia hectica.

Tabes, S. gen. 275. Lin. 209. Vog. 309. Sag. 100. Species sunt,

1. Tabes (purulenta) ex ulcere externo vel interno, vel ex vomica.

Tabes a vomica, S. sp. 15. ulcerosa, sp. 12. apostematodes, sp. 3.

" Cum hydrocephalus per signa externa non semper dignosci possit, haec species minus apte recensetur; sed ideo apposui, quod ephialtes in is qui simul anasarca crurum, et hydrothorace vel ascite laborant, ad hydrocephalum utcunque referri possit. 
(TABES.)

Variat sede; hinc

Tabes renalis, S. sp. 2.-hepatica, sp. 8.-a stomacho, sp. 14.-a pericardio, sp. 7.-catarrhalis, ${ }^{*}$ sp. 13.

2. Tabes (scrophulosa) in corporibus scrophulosis.

Tabes glandularis, S. sp. 10. mesenterica, sp. 9.Scrophula mesenterica, sp. 4.-Atrophia infantilis, sp. 13. rachitica, sp. 8.-Tabes rachialgica, sp. 16.

3. Tabes (venenata) a veneno ingesto.

Tabes a veneno, S. sp. 17.

Atrophia febrisequa, S. sp. 16. ad tabem purulentam, vel ad genus atrophiae pertinet.

G. LXX.-ATROPHIA.

Marcor et asthenia, sine pyrexia $\uparrow$ hectica.

Atrophia, S. gen. 277. Lin. 210. Vog. 308. Sag. 102. Junck. 41.-Marasmus, Lin. 211.

- Species sunt,

1. Atrophia (inanitorum) ex evacuatione nimia.

Atrophia a ptyalismo, S. sp. 7. a vomitu, sp. 9. ab alvi fluxu, sp. 6.-Tabes nutricum, sp. 4.-Atrophia nutricum, sp. 5. a leucorrhoea, sp. 4.-Tabes dorsalis, sudatoria, sp, 6. a sanguifluxu, sp. 2.-Sanguinis inopia, Ill. Lieutaud. synops. univ. med. vol. i. p. 52.

2. Atrophia (famelicorum) a nutrimento deficiente.

Atrophia lactentium, S. sp. 3.

3. Atrophia (cacochymica) a nutrimento corrupto.

Atrophia scorbutica, S. sp. 12.-Tabes syphilitica, sp. 11. a hydrope, sp. 5.

4. Atrophia (debilium) a nutritionis functione depravata, praegressa nulla vel evacuatione nimia, vel cacochymia. Atrophia nervea, S. sp. 1. lateralis, sp. 15. senilis, sp. 11.

\section{ORDO II.-INTUMESCENTIAE.}

Totum vel magna corporis pars extrorsum tumens.

Intumescentiae, S. cl. x. ord. ii. ; Sag. cl. iii. ord. ii.Tumidosi, Lin. cl. x. ord. ii.

- Si cum catarrho diuturno adfuerit tabes, cum Pyrexia hectica, nihil dubitarem ejusmodi tabem catarrhalem ad purulentam referre.

+ Atrophia fortassis nunquam sine Pyrexia, saltem sine pulsu solito frequentiori, adest; sed absentia Pyrexiae hecticae, (pag. 259.) fere semper atrophiam a tabe distin guit.

¥ An ad atrophiam inanitorum, an ad atrophiam debilium, pertinet tabes dorsalis, duhium mihi videtur, at ad posteriorem referire mallem.

VOL. I. 
SECT I.-ADIPOSAE.

\section{G. LXXII.-POLYSARCIA.}

Corporis pinguedinosa intumescentia molesta.

Polysarcia, S. gen. 279. Lin. 213. Vog. 540. Sag. 106. -Steatites, Vog. 390.

Species unica,

Polysarcia adiposa, Sauv. sp. 1. Lond. Med. obs. iii. 9. v. 21, 22. Med. Trans. ii. 18.

\section{SECT. II.-FLATUOSAE.}

\section{G. LXXII.PNEUMATOSIS.}

Corporis intumescentia tensa, elastica, sub manu crepitans.

Pneumatosis, S. gen. 280. Vog. 391. Sag. 107.-Emphysema, S. gen. 13. Lin. 228. Vog. 392.-Leucophlegmatia, Lin. 214.-Combalusier, de flatulentis corporis humani affectibus.

Species sunt,

1. Pneumatosis (spontanea) sine causa manifesta.

Emphysema spontaneum, S. sp. 1.-Pneumatosis febrilis, S. species aliquot. Lond. Med. obs. vi. 19.

2. Pneumatosis (traumatica) a vulnere thoracis.

Pneumatosis a vulnere, S. sp. 2. Lond. Med. obs. ii. 2. iii. 4. 35. 36. Cheston, chap. 1.-Pneumatosis febrilis, S. species aliquot.

3. Pneumatosis (venenata) a veneno injecto vel applicato.

Pneumatosis a veneno, S. sp. 1 .

4. Pneumatosis (hysterica) cum hysteria.

Pneumatosis hysterica, S. sp. 3.

\section{G. LXXIII.-TYMPANITES.}

Abdominis intumescentia tensa, elastica, sonora ; alvus adstricta; caeterarum partium macies.

Tympanites, S. gen. 291. Lin. 219, Vog. 316. Sag. 118. Boerh. 226. Junck. 87.-Affectio tympanitica, Hoffm. iii. 339.-Meteorismus, S. gen. 292.

Species sunt,

1. Tympanites (intestinalis) cum tumore abdominis saepe inaequali, et cum rejectione aëris frequenti, tensionem et dolorem levante.

Tympanites intestinalis, S. sp. 1. Edinb. Med. Ess. i. 31. enterophysodes, S. sp. 3 . verminosus, sp. 7. $\quad$ spasmodi- 


\section{(TYMPANITES.)}

cus, sp. 5.-Meteorismus ventriculi, S. sp. 1. abdominis, sp. 2. hystericus, sp. 3. a manzanilla, sp. 4.

2. Tympanites (abdominalis) cum resonitu evidentiore, tumore magis aequabili, et emissione flatuum rariori et minus levante.

Tympanites abdominalis, S. sp. 2 . asciticus, sp. 4.-Ascites flatulento-peritoneus, sp. 28.-Tympanites Stewartii, sp. 6.

\section{G. LXXIV.-PHYSOMETRA.}

Tumor levis, elasticus, in hypogastrio figuram et sedem uteri referens.

Physometra, S. gen. 290. Sag. 117-Hysterophyse, Vog. 317.

Species sunt,

Physometra sicca, S. sp. 1. Tympanites uteri sicca, Astruc, sur les malad. des femmes, 1. ii. cap. 9.

Physometra humida, S. sp. 2.-Tympanites uteri humida, Astruc, loc. cit.

SECT. III.-AQUOSAE, SIVE HYDROPES.

\section{G. LXXV.-ANASARCA.}

Corporis totius vel partis ejus intumescentia mollis, inelastica.

Anasarca, S. gen. 281. Lin. 215. Vog. 313. Sag. 108. Boerh. 1225. Hoffm. iii. 322. Junck. 87.-Monro on the dropsy, 1765. Milman, Animadversiones de hydrope, 1779.-Phlegmatia, S. gen. 282.-Angina aquosa, Boerh. 791.

Species sunt,

1. Anasarca (serosa) a retento sero ob evacuationes solitas suppressas, vel ab aucto sero ob ingestam aquam nimiam.

Anasarca metastatica, S. sp. 1.

a. Anasarca periodica.

b. Anasarca ab adiapneustia.

Phlegmatia lactea, S. sp. 3. a menostasia, sp. 5. Anasarca urinosa, sp. 11. hydropotarum, sp. 10.

2. Anasarca (oppilata) a compressione venarum.

Anasarca gravidarum, S. sp. 5.-Phlegmatia gravidarum, sp. 4. cruralis, sp. 9.-Angina Loweriana, sp. 2. Angina oedematosa, Boerh. 791.

3. Anasarca (exanthematica) post exanthemata, et praecipue post erysipelas, suborta.

Anasarca exanthematica, S. sp. 3.-Phlegmatia exanthe- 
(ANASARCA.)

matica, sp. 10. ulcerosa, sp. 6.-Malabarica, sp. 7. elephantina, sp. 8.-Anasarca Americana, sp. 7.

4. Anasarca (anaemia) a tenuitate sanguinis per haemorrhagiam producta.

Anasarca a fluxu, S. sp. 2.

5 Anasarca (debilium) in debilibus a morbis longis, vel ab aliis causis.

Phlegmatia vulgaris, S. sp. 1.-Anasarca a febribus, sp. 4. hysterica, sp. 2.-Phlegmiatia hysterica, sp. 2.-Anasarca rachialgica, sp. 8.-Phlegmatia Deliensis, sp. 11.

Anasarca purulenta, S. sp. 9. vix ad anasarcam referri potest.

\section{G. LXXVI.-HYDROCEPHALUS.}

Capitis intumescentia mollis, inelastica, hiantibus cranii suturis.

Hydrocephalus, S. gen. 285. Lin. 216. Boerh. 1217.Hydrocephalum, Vog. 384.

Species unica,

Hydrocephalus exterior, S. sp. 2.* Lond. Med. obs. v. 13. Med. Trans. ii. 18. Edinb. Med. Ess. iii. 22.

\section{G. LXXVII.-HYDRORACHITIS.}

Tumor supra vertebras lumborum, mollis, exiguus, hiantibus vertebris.

' Hydrorachitis, S. gen. 287. Morgagn. de sed. xii. 9. et seq.-Spinola, Lin. 289.-Spina bifida, Vog. 386.

\section{G. LXXVIII.-HYDROTHORAX.}

Dyspnoea; faciei pallor; pedum sedemata ; urina parca ; decubitus difficilis; subita et spontanea ex somno cum palpitatione excitatio; aqua in pectore fluctuans.

Hydrothorax, Sauv. 150. Vog. 311. Boerh. 1219.

Species sunt,

Hydrothorax vulgaris, S. sp. 3. febrisequens, sp. 4. a scabie, sp. 9.

Sequentes minus recte recensentur, cum liquidi intus contenti neque natura, neque fons; neque sedes, per signa externa dignosci queat :

Hydrothorax chylosus, S. sp. 1. Willis, Pharm. rat. p. i.

\footnotetext{
* Hydrocephalum internum, antea hic recensitum, ad Apoplexiam, sub nomine Apoplexiae Hydrocephalicae, jam supra retuli.
} 


\section{(HYDROTHORAX.)}

sect. i. cap. 13.-Hydrothorax hydatidosus, S. sp. 5. Morgagn. de sed. xiv. 33.-Hydrothorax mediastini, S. sp. 6.-Hydrothorax pleurae, S. sp. 7. Hoffm. iii. p. 337.-Bergeron, diss. de hydrope pectoris.-Hydrothorax pericardii, S. sp. 8. Morgagn. de sed. xvi. 34, 36. Senac du coeur, tom. ii. p. 349 . Edinb. Med. Ess. v.' 56 , $58,59$.

Minus recte a Sauvagesio hic recensetur

Hydrothorax ab omento, S. sp. 2.

\section{G. LXXIX.-ASCITES.}

Abdominis intumescentia tensa, vix elastica, sed fluctuosa.

Ascites, S. gen. 288. Lin. 217. Vog. 314. Sag. 115. Boerh. 1226. Hoffm. iii. p. 322. Junck. 87. D. Monro on dropsy, 1765. Med. Trans. ii. 17.-Milman, Animadversiones de hydrope, 1779.

Species sunt,

1. Ascites (abdominalis) cum tumore totius abdominis aequali, et cum fluctuatione satis evidente.

Ascites vulgatior, S. sp. 1.

Variat ratione causae.

$a$. Ab oppilatione viscerum.

Ascites ab oppilatione, S. sp. 2. ab hepate, sp. 3. a liene, sp. 4. scrophulosus, sp. 5. artificialis, sp. 10 .

\section{b. A debilitate.}

Ascites ab exanthematis, S. sp. 8.-arthriticus, sp. 9febrilis, sp. 12. Ascites ex quartana, Werlhof.-scorbuticus, S. sp. 11.

c. A tenuitate sanguinis.

Ascites a sanguifluxibus, S. sp. 7 .

Variat etiam ratione liquidi effusi.

Ascites purulentus, S. sp. 21.*-urinosus, sp. 22. chylosus, sp. 23. -oleosus, sp. 24.

2. Ascites (saccatus) cum tumore abdominis, saltem initio, partiali et cum fluctuatione minus evidente.

Ascites ab ovariis, S. sp. 6.-ovarii, sp. 19. a. Ascites saccatus.-Hydrometra ovarii, sp. 6.-Ascites cruentus, sp. 20. Edinb. Med. Ess. v. 63. - stomachi, S. sp. 16. omentalis, sp. 18.-intercus, sp. 14.-sanguineo-inter- 
(ASCITES.)

\section{G. LXXX.-HYDROMETRA.}

cus, sp. 26.-sanguineo-peritonaeus, sp. 27.-purulentoperitonaeus, sp. 29.

Hypogastrii in mulieribus paulatim crescens tumor, uteri figuram referens, pressui cedens fluctuansve; citra ischuriam et graviditatem.

Hydrometra, S. gen. 289. Sag. 116. Boerh. 1224.

Species per externa signa distinguendae vix dari possunt; sed sequentes ex Sauvagesio recensentur.

Hydrometra ascitica, S. sp. 1. gravidarum, sp. 2. hydatica, sp. 4.-Ascites uterinus, sp. 15.-Hydrometra sanguinea, sp. 3.-Ascites sanguineo-uterinus, sp. 25.- Hydrometra puriformis, sp. 5 .

\section{G. LXXXI.-HYDROCELE.}

Tumor scroti non dolens, paulatim crescens, mollis, fluctuans, pellucidus.

Oscheocele, S. gen. 41. Vog. 388.-Oscheophyma, Sag. gen. 44.-Hydrops scroti. Vog. 389.-Hydrops testium, Boerh. 1227.

Species sunt,

Oscheocele aquosa, S. sp. 2. Monro in Edinb. Med. Ess. vol. v. art. 21. Sharp's operations and critical inquiry. Douglas on the hydrocele. Pott's remarks on the hydrocele. Le Dran, Operations de Chirurgie. Bertrandi in Mém. de l'acad. de chirurgie, vol. iii.-Oscheocele hydatidosa, S. sp. 3.-Oscheocele Malabarica, S. sp. 4. Kempfer, Amoen. exot. fasc. iii. ob. 7 .

\section{SECT. IV.-SOLIDAE.}

\section{G. LXXXII.-PHYSCONIA.}

Tumor quandam abdominis partem potissimum occupans, paulatim crescens, nec sonora, nec fluctuans.

Physconia, S. gen. 283. Vog. 325. Sag. 110.-Hyposarca, Lin. 218.

Species sunt,*

Physconia hepatica, S. sp. 1.

* Species sequentes nequaquam ad normam Nosologiae methodicae recensentur, sed accuratius physconiae species indicare difficile foret, saltem plus exegisset, quam quo nunc fruor otil. Interim Cussoni doctissimi opus summae ad pathologiam utilitatis repetere hic placuit. 
(PIIYSCONIA.)

Cl. Cusson. Bonet. Sepulchr. de graviditate falsa, obs. 3 . n. 8. 9. 10. - Verduc, Patholog. du rachitis.

a. Schirrhodea.' G. Segeri M. Nat. Cur. dec. 1. ann. iv. obs. 142.

b. Calculosa. Bonet. Sepulchr. de hypochondrii tumore, obs. 5 .

c. Hydatidosa. Winckeri Disp. de hydatidibus apud Bonet. Med. septent. T. ii. p. 313.

Physconia splenica, S. sp. 2.

a. Emphractica. Haller. Disp. pathol. T. iv. Disp. 109. Tulp. obs. med. lib. ii. cap. 30 .

b. Scirrhodea. Bonet. Sepulchr. de ventris tumore, obs. 34 . n. 3.

Physconia renalis, S. sp. 3.

Bonet. Sepulchr. de gravid. falsa, obs. 3. n. 7.

a. Calculosa. Plater. Obs. 1. ii. p. 449 . River. cent. iv. obs. 34.

b. Sarcomatico-cystica. Haller. Disp. pathol. Disp. 114.

Physconia uterina, S. sp. 4.

Bonet. sepulchret. de gravid. falsa, obs. 2. n. 5. 6. 7 .

Physconia ab ovario, S. sp. 5 .

Monro, Edinb. Med. Ess. tom. vi. art. 74. obs. 1.

Physconia mesenterica, S. sp. 6.

a. Hydatidosa. Greg. Horstii. lib. v. obs. 33 . Tulp. obs. lib. ii. c. 34 .

b. Strumosa. Bonet. ex Rudnicio de ventr. tumore, obs. $x$. n. 15.-Ambr. Paré, lib. vii. c. 21.-Scultet. Armam. chirurg. obs. 38.

c. Scirrhodea. Marc. Donat. Hist. med. mir. lib. vii. c. 15. -Claudin. consult. sect. ii. consult. 134.-Trincavell, lib. iii. cons. 22.-Riolan. Anthropogr. lib. ii. c. 26. Morgagni de sedib. ep. 39. n. 2.

d. Sarcomatica. Bonet. Sepulchr. de falsa gravid. obs. 3. n. 1 .

e. Steatomatosa. Barth. Cabrol. Obs. 33. et 35.-Tulp. obs. lib. ii. c. 32. et 33.-J. Lechelii apud Bonet. Med. sept. T. ii. p. 486.-Idem Collect. acad. tom. iii. p. 585. -Hierne Collect. acad. tom. iii. p. 226. obs. 166.

f. Fungosa. Bonet. Sepulchr. de gravid. falsa, obs. 3.

Physconia intestinalis, S. sp. 7.

a. Concreto-glomerata. Morgagn. de sedib. epist. 39. n. 21. 25.

b. Sarcomatica. Fontan. Obs. med. selectiores, obs. 11.

Physconia omentalis, S. sp. 8.

Frid. Reebman Diss. de omento. 


\section{(PHYSCONIA.)}

Physconia polysplachna, S. sp. 9.

a. Scirrhodea. Fabr. Hildani cent. ii. obs. 45.

b. Scirrhodea et sarcomatica. Huldenreich, Misc. Nat. C. dec. 1. ann. vi. et vii. p. 321 .

c. Scirrhodea et adiposa. T. Bartholin. cent. iii. obs. 6 .

d. Hydatidosa. Volch. Coiter. obs. anat. p. 117.-Maur. Cordaei comm. 5. ad lib. priorem Hippocr. de muliebr.

e. Hydatidosa et scirrhodea. Halleri Disp. pathol. tom. v. p. 256.

Physconia visceralis, S. sp. 10.

Bonet. Sepulchr. de gravid. falsa, obs. 3.-Megalosplanchnos Hippocrat.-Marc. Donat. Hist. med. mir. lib. vi. c. 2.-Schenck. ex Garnero de liene. lib. iii. obs. 9. Salmuth. cent. i. obs. 21.-Bonet. Med. sept. lib. vii. p. 487.-Bianchi Hist. hepat. t. i. p. 130.

Physconia externa lupialis, S. sp. 11.

Graham Trans. philos. compend. v. ix. cap. 4. p. 187.

Physconia externa scirrhodea, S. sp. 12.

Ger. Blas. obs. med. vi.

Physconia externa hydatidosa, S. sp. 13.

Bonet. Sepulchr. de gravid. falsa, obs. 3. \$. 2, 3, 4, 11, \&c.-Achotzii apud Schenckium, lib. iii. de hydrope, obs. 4. et apud Scholtzium cons. 339.-Solenandri cons. 15. sect. v.-Riverii obs. communicat. ultim.

Physconia ab adipe subcutaneo, S. sp. 14.

a. Simplex. Cl. Lieutaud. Comp. med.-J. Mich. Fehr. apud Bonet. Med. sept. T. i. p. 636.

b. Syphilitica, observante celeb. Fizes.

Physconia ab excrescentia, S. sp. 15.

\section{G. LXXXIII.-RACHITIS.}

Caput magnum anterius maxime tumens; genicula tumida ; costae depressae; abdomen tumidum ; caetera marcescentia.

Rachitis, S. gen: 294. Lin. 212. Vog. 312. Sag. 120. Boerh. 1480. Hoffm. iii. 487.-Zeviani della rachitide. Variat,

a. Simplex, sine alio morbo.

Rachitis nodosa, S. sp. 1.-Rachitis Britannica," sp. 2. Glisson de rachitide.

- Minus recte pro diversis speciebus ponuntur Rachitis Nodosa et Britannica, cum non differant aliter quam morbus incipiens et confirmatus. 
(RACHITIS.)

b. Aliis morbis conjuncta.

Rachitis strumosa, S. sp. 3. Polonica, sp. 5 elephantiaca, sp. 6.

Neque Rachitis Calmucana, S. sp. 4. neque Rachitis a castratione, sp. 7. pro morbo haberi potest.

\section{ORDO III.-IMPETIGINES.}

Cachexiae, cutem et externum corpus pracipue deformantes.

Impetigines, S. cl. x. ord. v. Sag. cl. iii. ord. v.

\section{G. LXXXIV_-SCROPHULA.}

Glandularum conglobatarum, praesertim in collo, tumores; labium superius et columna nasi tumida; facies florida, cutis levis; tumidum abdomen.

Scrophula, S. gen. 295. Vog. 367. Sag. 121.-Struma, Lin. 284.

Species sunt,

1. Scrophula (vulgaris) simplex, externa, permanens.

Scrophula vulgaris, S. sp. 1.

2. Scrophula (mesenterica) simplex interna, cum pallore faciei, inappetentia, tumore abdominis, et foetore faecum insolito.

Scrophula mesenterica, S. sp. 4.

3. Scrophula (fugax) simplicissima, et tantum circa collum, plerumque a resorptione ex ulceribus capitis proveniens.

Scrophula fugax, S. sp. 2.

4. Scrophula (Americana) cum framboesia conjuncta.

Scrophula Americana, S. sp. 5.

Neque scrophula periodica, S. sp. 6. neque scrophula Moluccana, sp. 8. ad genus Scrophulae pertinere mihi videtur.

\section{LXXXV.-SYPHILIS.}

Morbus contagiosus, post concubitum impurum et genitalium morbum, ulcera tonsillarum ; cutis, praesertim ad marginem capillitii, papulae corymbosae, in crustas et in ulcera crustusa abeuntes; dolores ostocopi ; exostoses.

Syphilis, S. gen. 300. Lin. 6. Vog. 319. Sag. 126.-Lues Venerea, Boerh. 1440: Hoffm. iii, 413. Junck.96. Astruc de lue venera.

voL. I. 
(sYPHILIS.)

Species unica,

Syphilis venerea, S. sp. 6 .

Syphilis Polonica, S. sp. 2. nequaquam ad hoc genus pertinet, et de Syphilide Indica, sp. 3. nobis non satis constat, ut pro diversa specie ponatur.

\section{G. LXXXVI.-SCORBUTUS.}

In regione frigida post victum putrescentem, salitum, ex animalibus confectum, deficiente simul materia vegetabili recente; asthenia; stomacace; in cute maculae diversicolores, plerumque livescentes, praesertim ad pilorum radices.

Scorbutus, S. gen. 301. Lin. 223. Vog. 318. Sag. 127. Boerh. 1148. Hoffm. iii. 369. Junck. 91. Lind on the scurvy. Hulme de scorbuto. Rouppe de morbis navigantium. Med. Trans. ii, 18, 21. Milman on the scurvy. Ferris de sanguinis per corpus virum circulantis putredine, Ed. 1784.

Variat gradu,

a. Scorbutus incipiens. b. crescens. c. inveteratus.

Variat etiam symptomatibus,

d. Scorbutus lividus, Nitzsch, Theoret. pract. Abhandlung des Scharbockes, \&c. e. petechialis, Nitzsch, ibid. f. pallidus, Nitzsch, ibid. g. ruber, Nitzsch, ibid. h. calidus, Nitzsch, ibid.

\section{G. LXXXVII.-ELEPHANTIASIS.}

Morbus contagiosus; cutis crassa, rugosa, aspera, unctuosa, pilis destituta ; in extremis artubus anaesthesia ; facies tuberibus deformis; vox rauca et nasalis.

Elephantiasis, S. gen. 302. Vog. 321. Sag. 128.-Elephantia Arabum, Vog. 322.

\section{Varietates sunt,}

Elephantiasis Orientalis, S. sp. 1. E. legitima, sp. 2. Lond. Med. Obs. vol. i, art. 19. Soc. Royale, i. mém. p. 161. E. leonina, S. sp. 3. E. Tyria, sp. 4. E. Alodecia, sp. 5. E. syphilitica, sp. 6. E. Javanensis, sp.7. E. Indica, S. sp. 9.

\section{G. LXXXVIII_LEPRA.}

Cutis escharis albis, furfuraceis, rimosis, aspera, aliquando subtus humida, pruriginosa.

* De Elephantiasi, Lepra, Framboesia, et Trichomate, utpote morbis a meipso nunquam visis, amplius statuere non ausus sum. 
(LEPRA.)

Lepra, S. gen. 303. Lin. 262. Sag. 129.-Lepra Graecorum, Vog. 320.

Varietates sunt,

Lepra Graecorum, S. sp. 1. ichthyosis, sp. 2. Indica, sp.3. Asturiensis, sp. 4. herpetica, sp. 5. malum mortuum, sp. 6.

\section{G. LXXXIX.-FRAMBOESIA.}

Fungi, mori, vel rubi idaei fructus referentes, in variis cutis partibus enati.

Framboesia, S. gen. 229. Sag. 125.

Varietates sunt,

Framboesia Guineensis, S. sp. 1. Edinb. Essays, vol. vi, art. 71.-Framboesia Americana, sp. 2.

G. XC.-TRICHOMA.

Morbus contagiosus, capilli solito crassiores, in cirrhos et funiculos inextricabiles implicati.

Trichoma, S. gen. 311. Sag. 137.-Plica, Lin. 313.Plica sive Rhopalosis, Vog. 323.

Species sunt,

Trichoma cirrosum, S. sp. 1. villosum, sp. 2.

G. XCI.-ICTERUS.

Flavedo cutis et oculorum; faeces albidae ; urina obscure rubra, immissa colore luteo tingens.

Icterus, Linn. 224. Vog. 306. Boerh. 918. Junck. 90. -Aurigo, S. 306. Sag. 132-Cachexia icterica, Hoffm. iii, 301.

Species idiopathicae sunt,

1. Icterus (calculosus) cum dolore in regione epigastrica acuto, post pastum aucto, et cum dejectione concretionum biliosarum,

Aurigo calculosa, S. sp. 9. Edin. Ess. i. 33. ii. 28, 29. Med. Trans. ii. 10.

2. Icterus (spasmodicus) sine dolore, post morbos spasmodicos et pathemata mentis.

Aurigo hysterica, S. sp. 13.

Species quaedam, quae a Sauvagesio sub titulo Auriginis a venenis, recensentur.

3. Icterus (hepaticus) sine dolore, post morbos hepatis.

Aurigo hepatica, S. sp. 11. ab obstructione, sp. 6. purulenta, sp. 7 . 
(ICTERUS.)

4. Icterus (gravidarum) sub graviditate oriens, et post partum abiens. Van Swiet. ad Boerh. Aph. 950.

Aurigo plethorica, S. sp. 5.

5. Icterus (infantum) in infantibus haud diu post natales oriens.

Aurigo neophytorum, S. sp. 12.

Icteri sequentes, siquidem recte icteri dicantur, ${ }^{*}$ symptomaticae sunt,

Aurigo febrilis, S. sp. 1. febricosa, sp. 8. accidentalis, sp. 2. typhodes, sp. 10. rachialgica, sp. 14. a venenis, sp. 4.

Aurigo Indica, S. sp. 3. sive quorundam gentium color naturalis, nequaquam pro morbo haberi potest.

\section{CLASSIS IV.-LOCALES.†}

Partis, non totius corporis, affectio.

Vitia $₫$ † S. cl. i. Lin. cl. xi. Vog. cl. x, Sag. cl. i.-Plagae, S. cl. i. ord. vii. Sag. cl. ii.-Morbi organici Auctorum.

\section{ORDO I.-DYSAESTHESIAE.}

\section{Sensus depravati aut deleti, ex organorum externorum vitio.\$}

* Flavedo cutis oriri potest, vel a bile non, ut solet, excreta, sed in vasa sanguifera excepta, et inde subter cuticulam effusa; vel a sero sanguinis saepe flavescente, subter cuticulam, ut in ecchymosi, effuso. In quibusdam casibus, an flavedo cutis ob una vel alters illarum causarum oriatur, apuil medicos non semper constat; sed tantum ex prima icterum genuinum, et ab altera morbum diversum, quique, quantum fieri possit, distingui debet, oriri censemus.

+ Fortassis aliquando ambiguum esse potest, utrum ad universales, vel ad locales, morbi quidam referri debent; de plerisque vero, utri classi referendi sint, satis obvium erit; et ex institutione hujus Localium classis, quamplurimos morbos rectius et facilius, quam in aliorum nosologorum systematibus, disponi posse contendimus.

+ Vitia hic dixi, utpote sub hac classe Localium comprehensa; morbos vero multo plures comprehendit hic classis, ita ut vox Vitia pro hujus synonymo nequaquam haberi potest, et idem de Plagis Sagari dictum velim.

$\$$ Vereor ne in sequentibus aliquando species recensuerim quae characteri ordinis non prorsus respondeant, quasdam, scilicet, dysaesthesias quae ex organorum externorum vitio non pendeant. Hajusmodi species quidem aliquando recensui ; sed paucae omnino sunt, et feci, quia species affines, et hic loci expectandas, separare nolui. 
Dysaesthesiae, S. cl. vi. ord. i. Sag. cl. ix. ord. i.-Hallucinationes, S. cl. viii. ord. i. Sag. cl. xiii. ord. i.-Privativi, Lin. cl, vi. ord. iii.

\section{CXII.-CALIGO.}

Visus imminutus, vel prorsus abolitus, ob repagulum opacum, inter objecta et retinam, oculo ipso vel palpebris inhaerens.

Caligo, S. gen. 153. Vog. 288. Sag. 259.-Cataracta, Lin. 109.

\section{Species sunt,}

1. Caligo (lentis) ob maculam opacam pone pupillam. Cataracta,* S. gen. 152. Lin. 109. Vog. 239. Sag. 26.

Apud Sauvagesium species sunt, verae vel spuriae :

Verae varietates sunt,

Cataracta vera, S. sp. 1. Glaucoma Woolhousii.-C. antiglaucoma, sp. 2.-C. glaucoma, sp. 3. Glaucoma, Maitre Jan et St. Ives.

Spuriae varietates sunt,

Cataracta membranacea, S. sp. 4. secundaria, sp. 5.

2. Caligo (corneae) ob corneam opacam.

Achlys, Vog. 242.

Variat pro varietate morbi opacitatem facientis.

Caligo a nephelio, S. sp. 5. a leucomate, sp. 6. ceratocele, sp. 10. a staphylomate, sp. 11. a pterygio, sp. 12. hyposphagma, sp. 13.

3. Caligo (papillae) ob obstructam pupillam.

Variat pro varietate causae.

Caligo ab ectasi, S. sp. 18. Amaurosis a synchysi, sp. 6 . Amaurosis a myosi, sp. 7. Caligo a synizesi, sp. 19. Synizesis, Vog. 240.

4. Caligo (humorum) ob vitium vel defectum humoris aquei.

Glaucoma, Vog. 241.

Variat pro diverso humoris statú.

Caligo hypoaema, S. sp. 15. lactea, sp. 16. a rhytidosi, sp. 17.-Amblyopia hydrophthalmica, sp. 7:

5. Caligo (palpebrarum) ob vitium palpebris inhaerens. Variat pro varietate vitii palpebrarum.

Caligo a symblepharosi, S. sp. 1. ab ancyloblepharo, sp. 2. a blepharoptosi, sp. 3. a pacheablepharosi, sp. 4. a lupia, sp. 7. a sarcomate, sp. 8. a cancro, sp. 9.

* Non video quod, cum Sauvagesio, Cataracta pro diverso a Caligine genere haberi possit ; et judicent periti, annon ideo recte Caliginis characterem mutaverim, et cataractam pro ejus specie posuerim. 


\section{G. XCIII-AMAUROSIS.}

Visus imminutus vel prorsus abolitus, sine vitio oculi evidente; plerumque cum pupilla dilatata et immobili.

Amaurosis, S. gen. 155. Lin. 110. Vog. 238. Sag. 261. -Mydriasis, Vog. 237.-Amblyopia, Lin. 108. Vog. 236.

Species sunt,

1. Amaurosis (compressionis) post causas et cum signis congestionis in cerebro.

Variat pro varietate causae remotae.

Amaurosis pituitosa, S. sp. 2. scrophulosa, sp. 3. plethorica, sp. 4. intermittens, sp. 13. exanthematica, sp. 11. venerea, sp. 10. traumatica, sp. 1.

2. Amaurosis (atonica) post causas et cum signis debilitatis.

Amaurosis congenita, S. sp. 5. exhaustorum, sp. 16 . rachialgica, sp. 14. arthritica, sp. 17. Amblyopia absoluta, sp. 6.

3. Amaurosis (spasmodica) post causas et cum signis spasmi.

Amaurosis a spasmo, S. sp. 8. hysterica, sp. 15.

4. Amaurosis (venenata) a veneno ingesto vel applicato. Amaurosis a narcoticis, S. sp. 12. foricariorum, sp. 9.

\section{G. XCIV.-DYSOPIA.*}

Visus depravatus, ita ut non nisi certa luce, vel ad certam distantiam, vel in certa positura, objecta clare videantur. Amblyopia, S. gen. 154. Sag. 258.

Species sunt,

1. Dysopia (tenebrarum) in qua non nisi n magna luce objecta videntur.

Amblyopia crepuscularis, S. sp 1.

2. Dysopia (luminis) in qua non nisi in obscura luce objecta videntur.

Amblyopia meridiana, S. sp. 2.

3. Dysopia (dissitorum) in qua longe distantia non videntur.

- Verbum Amblyopia, quo pro hujus generis nomine utitur Sauvagesius, alio sensu intelligunt tum veteres, tum Linnaeus et Vogelius; ideoque hujus generis nomen mutare necessarium duxi. 
(DYSOPIA.)

Amblyopia dissitorum, S. sp. 3.-Myopia, Lin. 309.-Myopiasis, Vog. 515.

4. Dysopia (proximorum) in qua proxima non videntur.

Amblyopia proximorum, S. sp. 4.-Presbytia, Lin. 308.

5. Dysopia (lateralis) in qua non nisi oblique posita videntur.

Amblyopia luscorum, S. sp. 5.

\section{G. XCV.-PSEUDOBLEPSIS.}

Visus depravatus, ita ut quae non existant homo se videre imaginatur, vel quae existunt aliter videt ac revera se habeant.

Suffusio, S. gen. 217. Sag. 329._Phantasma, Lin. 73. Vog. 289.

Species sunt,

1. Pseudoblepsis (imaginaria) in qua quae non existunt conspici videntur.

Variat pro varietate phantasmatis.

a. Suffusio myodes, S. sp. 1.-b. reticularis, sp. 2.-c. scintillans, sp. 3. Marmaryge, Vog. 290.-d. Suffusio coloris, sp. 4. Haemalopia, Vog. 239.

2. Pseudoblepsis (mutans) in qua objecta revera existentia aliquomodo mutata apparent.

Variat pro varia objectorum mutatione.

a. Suffusio metamorphosis, S. sp. 5.-b. nutans, sp. 7.-c. dimidians objecta, sp. 6.-d. multiplicans. Diplopia, S. gen. 218. Sag. 330. Dysopia Vog. 291.

Diplopia iterum variat pro varietate causae remotae.

«. Diplopia pyrectica, S. varietas 1. - $\beta$. a spasmo, var. 2.$\gamma$. a paralysi, var. 3. ab ancyloblepharo, var. $4 .-8$. a catarrho, var. 5.- - . a debilitate, var. 6. - n. a contusione, var. 8.- 0 . a terrore, var. 9. - 6 a temulentia. var. 10. $-x$. remotorum, var. 7 .

\section{G. XCVI.-DYSECOEA.}

Auditus imminutus vel abolitus.

Dysecoea, S. gen. 158. Vog. 246. Sag. 264.-Cophosis, S. gen. 160. Lin. 112. Sag. 266.-Surditas, Vog. 247.

Species sunt,

1. Dysecoea (organica) ob vitium in organis sonos ad internam aurem transmittentibus.

Variat pro varietate vitii et partis affectae.

a. Dysecoea monotonon seu malcorum, S. sp. 1.-b. a mea- 
(DYSECOEA.)

tu obstructo, sp. 2. Cophosis a meatu sp. 1.-c. Dysecoea a myringae atonia, sp. 3. Cophosis a tympano, sp. 4.-d. Dysecoea a myringae duritie, sp. 4.-e. a myringa perforata, sp. 5.-f. a tympani fistula, sp. 6.-g. a hydrotympano, sp. 7.-h. a tuba obstructa, sp. 8. Cophosis a tuba, sp. 3.-i. Dysecoea syphilitica. Cophosis syphilitica.-Cophosis congenita, sp. 8.

2. Dysecoea (atonica) sine organorum sonos transmittententium vitio evidente.

Variat pro varietate causae.

a. Cophosis critica, sp. 2. Dysecoea febrilis.-b. Cophosis a comate, sp. 7.-c. serosa, sp. 6.-d. a steatomate, sp. 9.

\section{G. XCVII.-PARACUSIS.}

Auditus depravatus.

Paracusis, S. gen. 159. Sag. 265.-Syrigmus, S. gen. 219. Sag. 231.

Species sunt,

I. Paracusis (imperfecta) in qua soni ab externis venienentes percipiuntur, non autem accurate vel cum solitis conditionibus.

Variat,

a. Cum auditu gravi.

Paracusis barycoea, S. sp. 1.

b. Cum auditu nimis sensibili.

Paracusis oxycoea, S. sp. 2.

c. Cum sono unico externo a causis internis duplicato.

Paracusis duplicata, sp. 3.

d. Soni quos homo audire velit, nisi sono alio vehementi simul excitato non audiuntur.

Paracusis Willisiana, S. sp. 4.

2. Paracusis (imaginaria) in qua soni extrinsecus non existentes ab internis causis excitantur.

Syrigmus, Sauv. gen. 219. Sag. 231.-Syringmos, Lin. 72.-Susurrus, Vog. 292.

Variat pro varietate soni percepti.

a. Syrigmus sibilus, S. sp. 4.-b. susurrus, sp. 5.-c. bombus, sp. 11.

Variat etiam pro varictate causae remotac.

d. Syrigmus a debilitate, S. sp. 1.-e. criticus, sp). 2.-f. plethoricus, sp. ${ }^{\circ}=$ g. vertiginosus, sp. 10. -h. cephalal- 
(PARACUSIS.)

gicus, sp. 6. -i. a ventriculo, sp. 7.-k. catarrhalis, sp.

8.- - l. ab oxycoea, sp. 9.

\section{G. XCVIII.-ANOSMIA.}

Olfactus imminutus vel abolitus.

Anosmia, S. gen. 156. Lin. 113. Vog. 248. Sag. 262.

Species sunt,

1. Anosmia (organica) ob vitium in membrana nares inter nas investiente.

Variat pro varietate vitii.

Anosmia catarrhalis, sp. 1. a siccitate, sp. 6. a polypo, sp.

3. ab ozaena, sp. 2. syphilitica, sp. 4. verminosa, sp. 5 .

2. Anosmia (atonica) sine vitio membranae narium evidente.

Anosmia paralytica, S. sp. 7 .

G. XCIX.-AGEUSTIA.

Gustus imminutus vel abolitus.

Ageustia, S. gen. 157. Sag. 263.-Ageustia, Lin. 114. Apogeusis, Vog. 449.

Species sunt,

I. Ageustia (organica) ob vitium in membrana linguae, a nervis sapida arcens.

Ageustia febrilis, S. sp. 1.

2. Ageustia (atonica) sine vitio linguae evidente.

Ageustia paralytica, S. sp. 2.

\section{C.-ANAESTHESIA.}

Tactus imminutus. vel abolitus.

Anaesthesia, S. gen. 161. Lin. 118. Sag. 267--Anodynia, Vog. 274.

Sequentes species a Sauvagesio recensentur, et, cum de iis mihi non satis liqueat, nihil mutare volui.

Anaesthesia a spina bifida, S. sp. 1. plethorica, sp. 2. nascentium, sp. 3. melancholica, sp. 4.

\section{ORDO II.-DYSOREXIAE.*}

Appetitus erroneus vel deficiens.

- Morositates Sauvagesii minus recte ad Vesaniarum classem referri, supra dixi, et nunc ad Locales detuli. Recte, ni fallor, cum Dysorexiae fere omnes manifesto partis sin. gularis, potius quam totius corporis, affectiones sint. Nostalgia sola, si quidem revera morbus sit, minime pro locali haberi potest; sed morbum incertum a caeteris dysorexiis separare non bene potui. 
SECT. I.-APPETITUS ERRONEI.

Morositates, S. cl. viii. ord. ii. Sag. cl. xiii. ord. ii.-Pathetici, Lin. cl. v. ord. 2.-Hyperaestheses, Vog. cl. vii.

\section{G. CI.-BULIMIA.}

Appetitus esculentorum majori copia quam quae digeri possit.

Bulimia, S. gen. 223. Lin. 79. Sag. 335-Bulimus, Vog. 296.-Addephagia, Vog. 297.-Cynorexia, Vog. 298.

Species idiopathicae sunt,

1. Bulimia (helluonum, sine morbo ventriculi, cibi majorem solita copiam appetens.

Bulimia esurigio, S. sp. 4.-Addephagia, Vog. 297.

2. Bulimia (syncopalis) cibum frequenter appetens, ob sensum famis syncopen minitantis.

Bulimia cardialgica, S. sp. 2.-Bulimus, Vog. 296.

3. Bulimia (emetica) cibum magna copia appetens, et mox per vomitum rejiciens.

Bulimia canina, S. sp. 1.-Cynorexia, Vog. 298.

Species symptomaticae sunt,

Bulimia verminosa, S. sp. 3. addephagia, sp. 5. convulsorum, S. sp. 6. ab acidis, sp. 7 .

\section{G. CII.-POLYDIPSIA.}

Appetitus majoris solita copiae potulentorum.

Polydipsia, S. gen. 224. Lin. 80. Vog. 275. Sag. 336.

Polydipsia fere semper symptomatica est, et variat tantum pro varietate morborum quos comitatur; hinc

Polydipsia febrilis, S. var. a.-hydropica, var. b.-fluxuum, var. c. - a venenis, var. $d$.

\section{G. CIII.-PICA.}

Desiderium non esculenti ingerendi.

Pica, S. gen. 222. Sag. 334.-Citta, Lin. 78.-Allotriophagia, Vog. 299.-Malacia, Vog. 300.

Cum de speciebus Picae mihi non satis liqueat, sequentes ex Sauvagesio recenseo.

Pica infantilis, S. sp. 1. chlorosiantium, sp. 2. mcia, sp. 3. antiscorbuticorum, sp. 4. voluntaria, sp. 5 . 


\section{G. CIV.-SATYRIASIS.}

In maribus effraenis veneris cupiditas.

Satyriasis, S. gen. 228. Lin. 81. Sag. 340.-Satyriasim pro specie maniae habet Vogelius.

\section{Species sunt,}

1. Satyriasis (juvenilis) cum veneris cupiditate praeter modum vehementi, corpore simul parum perturbato.

Satyriasis neogamorum, sp. 5.

2. Satyriasis (furens) cum veneris cupiditate effraeni, corpore simul multum perturbato.

Satyriasis acuta, S. sp. 1. Aretaei Cappad. Acut. lib. ii. cap. 12.

Satyriasis chronica, S. sp. 2. ad priapismum pertinet; et Satyriasis venerea,' sp. 3. necnon Satyriasis hydrophobica, sp. 4. manifesto symptomatica est.

\section{G. CV.-NYMPHOMANIA.}

In foeminis effraene veneris desiderium.

Nymphomania, S. gen. 229. Sag. 341.-Satyriasis, Lin. 81.-Furorem uterinum pro specie maniae habet Vogelius.

Nymphomaniae species unica est, et gradu tantum varians, hinc

Nymphomania salacitas, S. sp. 1. furibunda, sp. 2. fervor uteri, sp. 3. pruriginosa, sp. 4.

\section{CVI.-NOSTALGIA.}

In absentibus a patria, vehemens eandem revisendi desiderium.

Nostalgia, S. gen. 226. Lin. 83. Sag. 338.-Nostalgiam pro specie melancholiae habet Vogelius.

Species sunt,

1. Nostalgia (simplex) sine alio morbo.

Nostalgia simplex, S. sp. 1.

2. Nostalgia (complicata) aliis morbis comitata.

Nostalgia complicata, S. sp. 2.

SECT. II.-APPETITUS DEFICIENTES.

Anepithymiae, S. cl. vi. ord. ii. Sag. cl. ix. ord. ii.-Privativi, Lin. cl. vi. ord. iii.-Adynamiae, Vog. cl. vi.

G. LVII.-ANOREXIA.

Appetitus esculentorum deficiens.

Anorexia, S. gen. 162: Lin. 116. Vog. 279. Sag. 268. 
(ANOREXIA.)

Omnis Anorexia mihi symptomatica esse videtur, et variat tantum pro varietate morbi quem comitatur. Species quidem omnes, quotquot a Sauvagesio fuerant recensitae, ad genus dyspepsiae supra retuli; sed fortassis utile hic erit easdem seorsum, et rectius ordinatas, iterum recensere.

Species sunt,

1. Anorexia (humoralis) ab humore ventriculum gravante.

Anorexia pituitosa. sp. 2. biliosa, sp. 6. a saburra, sp. 9.

2. Anorexia (atonica) ob tonum fibrarum ventriculi amissum.

Anorexia paralytica, S. sp. 1. exhaustorum, sp. 8. melancholica, sp. 5. cachectarum, sp. 7. arthritica, sp. 12. febrilis, sp. 4.

Incertae naturae sunt,

Anorexia plethorica, S. sp. 3. mirabilis, sp. 10. neophytorum, sp. 11. Stewartiana, sp. 13.

\section{G. CVIII.-ADIPSIA.}

Appetitus potulentorum suppressio, sive sitis feriatio.

Adipsia, S. gen. 163. Lin. 117. Vog. 281. Sag. 269. Adipsiam sine alio morbo, qualis est

Adipsia primaria, S. sp. 1.

pro morbo habere nollem; omnemque adipsiam pro sympathica, sive symptomate morbi cujusdam, sensorium commune afficientis, habeo; ideoque non ad Locales referendi.

\section{G. CIX.-ANAPHRODISIA.}

Libidinis defectus, vel veneris impotentia.

Anaphrodisia, S. gen. 164. Sag. 270.-Atechnia, Lin. 119.-Agenesia, Vog. 283.

Species verae sunt,

Anaphrodisia paralytica, S. sp. 1. gonorrhoica, sp. 2.

Species spuriae, sive veneris rite exercendae impedimenta, sunt,

Anaphrodisia a mariscis, S. sp. 4.-Anaphrodisia ab urethrae vitio, sp. 5.*

Pro ficta et falsa habenda est Anaphrodisia magica, sp. 3 . 


\section{ORDO III.-DYSCINESIAE.}

Motus impediti vel depravati ex organorum vitio.

Dyscinesiae, S. cl, vi. ord. iii. Sag. cl. ix. ord. iii.

\section{G. CX.-APHONIA.}

Vocis plena suppressio, citra coma aut syncopen.

Aphonia, S. gen. 166. Lin. 115. Vog. 253. Sag. 272.

Species sunt,

1. Aphonia (gutturalis) a tumefactis faucibus et glottide.

Aphonia catarrhalis, S. sp. 4. Anginosa Morgagni.

2. Aphonia (trachealis) a compressa trachea.

Aphonia aneurysmatica, S. sp. 5. pulmonica, sp. 9.

3. Aphonia (atonica) a nervis laryngis resectis.

Aphonia traumatica, S. sp. 6.

Sequentes symptomaticae esse videntur.

Aphonia melancholica, S. sp. 1. paralytica, sp. 8. temulentorum, sp. 3. hysterica, sp. 7. ab antipathia, sp. 2.

\section{G. CXI.-MUTITAS.}

Verba articulandi impotentia.

Mutitas, S. gen. 165. Vog. 257. Sag. 271.

Species sunt,

1. Mutitas (organica) ex lingua ablata vel vitiata.

Mutitas elinguium, S. sp. 4 . a siccitate, sp. 5.

2. Mutitas (atonica) ex nervis linguae laesis.

Mutitas traumatica, S. sp. 2.

3. Mutitas (surdorum) ex surditate congenita, vel puerilibus annis aborta.

Mutitas surdorum, S. sp. 8.

Sequentes symptomaticae sunt,

Mutitas a glossocele, S. sp. 1. a narcoticis, sp. 3. spasmodica, sp. 6. proaeretica, sp. 7 .

\section{G. CXII.-PARAPHONIA.}

Vocis sonus depravatus.

Paraphonia, S. gen. 168.-Cacophonia, Sag. 274.-Raucedo, Lin. 146.-Raucitas, Vog. 252.-Asaphia, 250. -Clangor, 251.-Leptophonia, 254.-Oxyphonia, 255. -Rhenophonia, 256.

Species sunt,

1. Paraphonia (puberum) in qua, circa pubertatis tempus, vox ab acutiori et suavi in graviorem et ingratam mutatur. 


\section{(PARAphonia.)}

Paraphonia puberum, S. sp. 1.

2. Paraphonia (rauca) in qua, ob siccitatem vel tumorem faucium flaccidum, vox fit rauca et scabra.

Paraphonia catarrhalis, S. sp. 3.-Raucedo, Lin. 146. Raucitas, Vog. 250.

3. Paraphonia (resonans) in qua, ob obstructas nares, vox fit rauca, cum sonitu per nares sibilante.

Paraphonia nasalis, S. sp. 2. a polypo, sp. 8. Rhenophonia, Vog. 256.

4. Paraphonia (palatina) in qua, ob deficientem vel divisam 'uvulam, plerumque cum labio leporino, vox fit rauca, obscura, et ingrata.

Paraphonia gutturalis, S. sp. 5.-Asaphia, Vog. 250.

5. Paraphonia (clangens) in qua vox in acutam, clangentem, et exilem mutatur.

Paraphonia ulcerosa, S. sp. 4. sibilans, sp. 7.-Clangor, Vog. 251.-Leptophonia, 254.-Oxyphonia, 255.

6. Paraphonia (comatosa) in qua, ob laxatum velum palati et glottidem, sonus inter inspirandum editur.

Paraphonia stertens, S. sp. 6.

\section{G. CXIII.-PSELLISMUS.}

Verba articulandi vitium.

Psellismus, S. gen. 167. Lin. 138. Sag. 273.-Psellotis, Vog. 259.-Traulotis, 258.-Ischnophonia, 260.-Battarismus, 261.

Species sunt,

1. Psellisums (haesitans) in quo sermonis verba, praesertim prima, non facile proferuntur, et non nisi prima syllaba saepius repetita.

Psellismus ischnophonia, S. sp. 1.-Ischnophonia, Vog. 201.-Battarismus, 261.

2. Psellismus (ringens) in quo sonus literae $\mathbf{R}$ semper aspiratur, et quasi geminatur.

Psellismus rottacismus, S. sp. 2.-Traulotis sive blaesitas, Vog. 258.

3. Psellismus (lallans) in quo sonus literae $\mathbf{L}$ fit liquidior, vel loco literae $\mathbf{R}$ pronunciatur.

Psellismus lamdacismus, S. sp. 3.

4. Psellismus (emolliens) in quo literae durae in molliores mutantur, et litera $\mathbf{S}$ multum usurpatur.

Psellismus traulotes, S. sp. 4. 
(PSELLISMUS.)

5. Psellismus (balbutiens) in quo, ob linguam magnam vel tumefactam, literae labiales magis audiuntur, et saepe loco aliarum proferuntur.

Psellismus balbuties, S. sp. 5. a ranula, sp. 11.

6. Psellismus (acheilos) in quo literae labiales vix vel non omnino pronunciari possunt.

Psellismus mogilalia, S. sp. 6.

7. Psellismus (lagostomatum) in quo, ob divisum palatum, literae gutturales minus recte pronunciantur.

Psellismus lagostomatum, S. sp. 10. cotacismus, sp. 8.

\section{G. CXIV.-STRABISMUS.}

Oculorum axes optici non convergentes.

Strabismus, S. gen. 116. Lin. 304. Vog. 514. Sag. 222. Species sunt,

1. Strabismus (habitualis) a consuetudine prava oculo tantum uno utendi.

Strabismus vulgaris, S. sp. 2.

2. Strabismus (commodus) ab unius oculi, prae altero debilitate vel mobilitate majori, adeo ut uterque oculus non commode adhibeatur.

Strabismus Buffonii, S. sp. 3. spasmodicus, sp. 4. paralyticus, sp. 5 . catarrhalis, sp. 6 .

3. Strabismus (necessarius) ob mutatum $\backslash$ situm vel figuram partium oculi.

Strabismus a luscitate, S. sp. 1. caligantium, sp. 11. a crystallino, sp. 12 . myopum, sp. 9 .

De Strabismo aequinoctiali mihi non liquet.

Minus recte, ut mihi videtur, ad Strabismum referuntur sequentes: Strabismus symptomaticus, S. sp. 7. lagophthalmos, sp. 8.

\section{CXV.-DYSPHAGIA.}

Molestia deglutitionem impediens sine respirationis laesione vel phlegmasia.

Dysphagia, S. gen. 199. Sag. 216. Acataposis, Vog. 149.

Species a Sauvagesio recensitae sunt,

1. Dysphagia, spasmodica. 2. hysterica. 3. paralytica, 4. pharyngea. 5. œesophagea. 6. lactentium. 7. tussiculosa. 8. hydrophobica. 9. nauseosa. 10. a deglutitis. 11. a datura: 12. a sarcomate. 13. ab scirrho. 
(DYSPHAGIA.)

14. canina. 15. valsalviana. 16. aneurysmatica. 17. a labario. 18. a siccitate. 19. ab hypostaphyle.

\section{G. CXVI-CONTRACTURA.}

Artus unius vel plurium contractio diuturna, rigida.

Contractura, S. gen. 119. Lin. 299. Sag. 225.-Obstipitas, S. gen. 11. Caput obstipum, Vog. 513.-Digitium Vog. 221.

Species sunt,

1. Contractura (primaria) a musculis contractis, rigidis. a. A musculis ab inflammatione rigidis.

Contractura dolorifica, S. sp. 2. arthritica, sp. 7. catarrhalis, sp. 8.-Obstipitas catarrhalis, sp. 3.

b. A musculis a spasmo rigidis.

Contractura spasmodica, sp. 11.-Obstipitas spasmodica, sp. 6. renuens, sp. 1. lateralis, sp. 5.-Contractura hypochondriaca, sp. 1 .

c. A musculis, ob antagonistas paralyticos, contractis.

Contractura paralytica, S. sp. 4. rachialgica, sp. 5. Bohemica, sp. 6.-Obstipitas annuens, sp. 2.

d. A musculis ab acrimonia irritante contractis.

Contractura scorbutica, S. sp. 3.

2. Contractura (articularis) ob artus rigidos.

Contractura anchylosis, S. sp. 9. syphilitica, sp. 10.-Obstipitas gibbosa, sp. 4 .

\section{ORDO IV.-APOCENOSES.}

Fluxus sive sanguinis, aut humoris alii, solito uberius profluens, sine pyrexia impetuve fluidorum aucto.

Apocenoses, Vog. cl. ii. ord. ii.-Fluxus, S. cl. ix. Sag. cl. v.-Morbi evacuatorii, Lin. cl. ix.

\section{G. CXVII.-PROFUSIO.}

Fluxus sanguinis.

Profusio, Lin. 239.-Haemorrhagia, Vog. 81. Boerh. 218.

Ex sanguifluxibus, a Sauvagesio recensitis, sequentes, ad hunc locum pertinere videntur.

Haemorrhagia passiva, S. sp. 1.-Haemoptysis traumatica, sp. 12. Haematemesis traumatica, sp. 3.-Haematuria violenta, sp. 2.-Haemorrhagia ab hirudine, sp. 2. Haemoptysis ab hirudine, sp. 13. Hematemesis ab hirudine, sp. 4. 


\section{G. CXVIII.-EPHIDROSIS.*}

Sudoris praeter naturam evacuatio.

Ephidrosis, S. gen. 258. Sag. 194.-Sudor, Lin. 208.Hydropedesis, Vog. 121 .

Species idiopathica unica est,

Ephidrosis spontanea, S. sp. 1.

Ephidroses symptomaticae variant, pro varietate morbi quem comitantur, et simul pro varietate sudoris eliciti, et quodammodo pro varietate partis maxime sudantis. Hinc

Ephidrosis febrilis, S. sp. 3. febricosa, sp. 7. hectica, sp. 5. exanthematum, sp. 6. syncoptica, sp. 4 . scorbutica, sp. 2. a saburra, sp. 18. lactea, sp. 9. mellea, sp. 10 . vinosa, sp. 11. viridis, sp. 12. nigra, sp. 13. lutea, sp. 14. urinosa, sp. 15. cruenta, sp. 16. caerulea, sp. 17. acida, sp. 19. arenosa, sp. 20. lateralis, sp. 8.

\section{G. CXIX.-EPIPHORA.}

Fluxus humoris lachrymalis.

Epiphora, S. gen. 259. Lin. 172. Vog. 99. Sag. 195.

Ex speciebus a Sauvagesio recensitis, nulla pro idiopathica haberi potest, nisi

Epiphora frigida, S. sp. 8.

\section{Caeterae omnes symptomaticae sunt,}

Epiphora a pathemate, S. sp. 1. ophthalmica, sp. 3. calida, sp. 9. ex variolis, sp. 4. arthritica, sp. 12. cruenta, sp. 10. ex. rhyade, sp. 2. ex aegilope, sp. 5. ab ectropio, sp. 6. ab anchylope, sp. 7. sebacea, sp. 11. lactea, sp. 13.

\section{G. CXX.-PTYALISMUS.}

\section{Fluxus salivae.}

Ptyalismus, S. gen. 261. Lin. 176. Vog. 103. Sag. 197.

Si quis ptyalismus idiopathicus sit, pro eo habere vellem

Ptyalismum a laxitate, S. sp. 4.

\section{Sequentes omnes symptomaticae sunt,}

Ptyalismus nauseosus, S. sp. 1. hypochondriacus, sp. 8. gravidarum, sp. 14. a pyrosi, sp. 2. Lapponicus, sp. 3. febrilis, sp. 18. variolosus, sp. 6. arthriticus, sp. 9 . phthisicus, sp. 10. scorbuticus, sp. 7. catarrhalis, sp. 15 . aphthosus, sp. 13. a carie, sp. 16. purulentus, sp. 12.

- Ephidrosin minus recte ad morbos locales relatam esse agnosco; cum autem huic morbo nullum in systemate nostro locum idoneum invenerim, ne prorsus omitteretur, hic cum aliis quodammodo affinibus morbis evacuatoriis recensui.

voL. I. 
(PTYALISMUS.)

a calculo, sp. 17. syphiliticus, sp. 19. viridis, sp. 11 . urinosus, sp. 20. mercurialis, sp. 5.

\section{G. CXXI.-ENURESIS.}

Urinae e vesica fluxus involuntarius non dolens.

Enuresis, S. gen. 264. Lin. 195. Vog. 113. Sag. 200. Species sunt,

1. Enuresis (atonica) post morbos sphincterem vesicae laedentes.

Enuresis herniosorum, S. sp. 3. puerperarum, sp. 5. calculosa, sp. 7. a fistula, sp. 8.

2. Enuresis (irritata) a compressione vel irritatione vesicac.

Enuresis gravidarum, S. sp.4. catamenialis, sp. 6. a sparganosi, sp. 9.

Symptomatica est

Enuresis paralytica, S. sp. 2.

Pro morbo non haberi potest

Enuresis infantum, S. sp. 1.

\section{G. CXXII.GONORRHOEA.}

Humoris ex urethra, in maribus, cum vel absque libidine, praeter naturam fluxus.

Gonorrhoea, S. gen. 268. Lin. 200. Vog. 118. Sag. 204. Species sunt,

1. Gonorrhoea (pura) in qua, praegresso concubitu impuro nullo, humor puriformis, sine dysuria vel libidine, ex urethra subinde fluit.

Gonorrhoca pura, sp. 1. G. benigna Auctorum.

2. Gonorrhoea (impura) in qua, post concubitum impurum, humor puriformis cum dysuria ex urethra fluit.

Gonorrhoea syphilitica, S. sp. 4. maligna Auctorum.

Hujus sequela est,

Gonorrhoea $(m u c o s a)$ in qua, post gonorrhoeam impuram, humor mucosus, cum minima vel nulla dysuria, ex urethra subinde fluit.

Anglis $a$ Gleet.

3. Gonorrhoea (laxorum) in qua, humor plerumque pellucidus, sine penis erectione sed cum libidine, in vigilante, ex urethra subinde fluit.

Gonorrhoea libidinosa, S. sp. 2.

4. Gonorrhoea (dormientium) in qua liquor seminalis cum 
(GONORRHOEA.)

erectione et libidine, in dormientibus, ex somnio libidinoso, ejicitur.

Gonorrhoea oneirogonos, S. sp. 3.

Species spuriae, utpote in quibus ex urethra fluxus non fit,

Gonorrhoea spuria, S. sp. 5. Astruc, lib. 3. cap .3. sect. 2. -G. balani, sp. 6.

\section{ORDO V.-EPISCHESES.}

Excernendorum suppressiones.

Épischeses, Vog. cl. iii.-Suppressorii, Lin. cl. viii. ord. ii. Suppressiones, Sag. cl. vi.

\section{G. CXXIII-OBSTIPATIO.}

Dejectio faecum nulla vel solito rarior.

Obstipatio, Lin. 166. Vog. 128. Sag. 221.

\section{Species sunt,}

1. Obstipatio (debilium) in hominibus laxis, debilibus, et plerumque dyspepticis.

2. Obstipatio (rigidorum) in hominibus fibrae rigidae saepe hypochondriacis.

3. Obstipatio (obstructorum) cum symptomatis Colicae 1mae, 2dae, 4tae, vel 7 mae supra dictac.

\section{G. CXXIV.-ISCHURIA.}

Urinae suppressio absoluta.

Ischuria, S. gen. 293. Lin. 167. Vog. 129. Sag. 212. Home's Clinical Experiments, sect. xv. Lond. Med. Obs. v. Append.

\section{Species sunt,}

1. Ischuria (renalis) praegresso renum morbo, cum dolore vel molesto gravitatis sensu in regione renum, et sine hypogastrii tumore, vel ad mingendum stimulo.

Variat pro varietate causae; hinc

a. Nephritica, a renum inflammatione, S. sp. 1.-Schacht. Institut. med. pract. lib. viii. cap. i. et viii. Gort. syst. prax. med. de Ischur. Fabric. Hildan. de Lithot. cap. iv. Leal a Fonte, cons. 59. Bonet. sepulchr. lib. iii. sect. xxiv. obs. iv. \$ 3 . Schenck. lib. iii. de Ischur. fals. cap. iv. et v. Etmuller coll. pract. pag. 994.

b. Nephrolithica, a renum calculo, sp. 2.-Schacht. lib. citat. cap. ii. et viii. Gort. lib. citat. de morb. renum. 
(ISCHURIA.)

Car. Pison. de morb. a colluv. seros. Schenck de Ischur. Bonet. sepulchr. de urin. suppress. et Medic. septentrional. de Ischur. Edinb. Ess. ii. 31.

c. Nephroplethorica, a plethora, sp. 3.-River. cent. i. obs. 1. et 89. Etmull. Schacht. loco citat. Gaub. Pathol. de vit. mict. Ludwig. instit. med. clin. Seb. Nasii specul. method. med. pag. 167.

d. Lunatica, periodica, sp. 4.-Tulp. obs. med. lib. ii. cap. 49.

e. Nephrospastica, spasmodica, sp. 5.-Schacht. Gort. lib. citat. Sydenh. dissert. epist. tom. i. Raulin des malad. vapor. Mém. de l'Acad. R. des Sc. ann. 1715.

f. Nephrelmintica, verminosa, sp. 6.-Gort. lib. citat. Gaub. pathol. de vit. mict.

g. Nephrothromboides, a sanguine congrumato, sp. 7.Mercat. de morb. intern. cur. cap. 12. Etmull. de laes. urin. secret. pag. 314. Fernel. pathol. lib. vi. cap. 13. Gort. Gaub. loc. citat.

$h$. Nephropyica, a renum purulentia, sp. 8.-Schenck. lib. iii. de ren. tumorib. Gort. Gaub. loc. citat. Bonet. sepulchr. de urin. suppress. Sal. Divers. de affect. particularib. cap. 14.

i. Nephrophlegmatica, pituitosa seu mucosa, sp. 9.-Sal. Divers. loc. citat. Grasii Ephem. natur. cur. ann. iii. obs. 212. Schacht. Gort. Gaub. Mercat. loc. citat. Varand. de ren. affect. Bonet. sepulchr. de Ischur. obs. iv. $v$.

k. Nephroplegica, paralytodaea sp. 10.-Sal. Divers. Mercat. loc. citat. Horst. tom. ii. lib. iv. obs. 49. Marcel. Donat. Histor. med. mirab. lib. iv. cap. 28. Barthol. cent. iv. epist. 18, 38, 39 . Hoechstet. obs. dec. v. cas. 20. Claud. Chaptal. pract. Monspel.

l. Suppleta, ab alia evacuatione suppleta, sp. 11 .

a. A diarrhoea et sudore, Marcell. Donat. hist.med.mirab. lib. iv. cap. 27. Schenck. lib. iii. de diabete, obs. 9. $\beta$. Ab otorrhagia, Sennert. ex plat. pract. lib. iii. part. viii. sect. ii. cap. 9. $\gamma$. A perspiratione, Vandermond. Journ. de méd. tom. iv. $\delta$. A sudore, Vandermond. Journ. de méd. tom. $x$.

2. Ischuria (ureterica) praegresso renum morbo, cum doloris vel molestiae sensu in quadam ureteris parte, et sine hypogastrii tumore, vel ad mingendum stimulo.

Variat pro varietate causae; hine

a. Ureteritica, inflammatoria, sp. 12.-Sal. Divers. loc. citat. Ludwig. Instit. clin. Gaub. pathol. de vit. mict. Schacht. Instit. pract. de Ischur. spur.

b. Ureterolithica, calculosa, sp. 13.-Tulp. obs. med. lib. ii. cap. 45. Mouro Ess. d' Edinb. tom. vi. art. 68. Bonet. 
(ischuria.)

Sepulchr. lib. iii. de urin. suppress. Schenck. lib. iii. de ureterib. Sal. Divers. loc. citat.

c. Ureterothromboides, a sanguinis grumo, sp. 14.-Sal. Divers. Schacht. Gaub. loc. cit. Bonet. Sepulchr. de urin. suppress. obs. i.

d. Ureterophlegmatica, a pituita, S. sp. 15.-Gort. Prax. med. syst. Schacht. Sal. Divers. loc. citat. Bonet. Sepulchr. de urin. suppress. Sebast. Nas. Spec. meth. med. loc. citat. Epiphan. Ferdinand. hist. 97.

e. Ureteropyica, purulenta, sp. 16.-Gort. lib. cit. de ureter. morb. Schacht. Sal. loc. cit.

$f$. Ureterostomatica, $a b$ orificii ureterum inferioris clausura, sp. 17.-Theod. Eller. Miscell. Berolin. tom. iv. pag. 381. Verdier ex Noel. Mém. de l'Acad. de Chirurg. tom. ii. obs. 17. Lieutaud. Mém. de l'Acad. R. des Sc. ann. 1753. Franc. Sylv. Deleboe, Prax. med. lib. ii. Saltzmann. obs. anat. pag. 62.

3. Ischuria (vesicalis) cum tumore hypogastrii, dolore ad cervicem vesicae, et frequenti ad mingendum stimulo.

Variat pro varietate causae; hinc

a. Cystitica, a vesicae inflammatione, S. sp. 18.-Schacht Instit. Practic. Mém. de l'Acad. R. des Sc. ann. 1704, 1753. Boneti Sepulchr. lib. iii. de urin. suppress. Forest. lib xxv. obs. 27, 28. Zacuti Lusitani ex Galeno, Hist. med. princ. lib. ii. hist. 150.

b. Cystolithica, a vesicae calculo, sp. 19-Tulpii observ. med. lib. iv. cap. 37. Essais d'Edinburg, tom. iv. Foresti lib. xxv. obs. 23. Fabric. Hildani, cent. iii. observ. 67. Car. Pisonis de morb. a colluv. seros. Boneti Sepulchr. de urin. suppress. obs. x. \&c.

c. Cystospastica, a sphincteris vesicae spasmo, sp. 20.Mercati de morb. intern. cur. lib. iv. cap. 22.-Sebast. Nasii Specul. method. med. part. ii. pag. 267. Nenteri de Ischur. Schachtii, Gorterii, Gaubii, Varendaei, loc. citat.

d. Cystoplegica, a vesicae paralysi, sp. 21.-Lieutaud. Compend. medic. de Ischur. Zacut. Lusitan. ex Galeno hist. princ. hist. 140. Mangeti Biblioth. medic. pract. tom. iv. Serane Thes. pro Reg. Cathedr. Hollerii de morb. intern. cap. 47. Amat. Lusitan. cent. iv. cur. 10. Pringle Essais d'Edinburg, tom. i. artic. 32. Lond. Med. Obs. i. 10.

e. Polyurica, a vesica distenta lotio diutius cohibito, sp. 22.-Halleri praelect. in Boerhaave, tom. ii, $\S 384$. pag. 336. Forest. lib. xxv, observ. 14. Nenter de Ischuria. Paraei, lib. xvi, cap. 48. Sennert. pract. lib. iii, part viii, sect. 1. cap. 4. Etmulleri, Riverii, Varandaei de Ischur. \& 
(ISCHURIa.)

f. Cystopyica, purulenta, sp. 23.-Felic. Plateri Mantis. obs. 29. Camerar. Ephem. natur. curios. tom. ii. Zacuti Lusitani ex Galeno, Hist. medic. princip. hist. 148. Hollerii de morb. intern. cap. 47. Sebast. Nasii Specul. method. med. part xi, pag. 167. André des maladies de l'urethre, obs. 16. Vandermonde, Journ. de méd. tom. ix.

g. Cystothromboides, a sanguinis grumo, sp. 24.-Claudini consult. 141. Fabric. Hildani, cent. iii, obs. 66. Foresti, lib. 25. obs. 20. Zacuti Lusitani ex Galeno Hist. med. princip. hist. 145. et ejusdem prax. med. admirab. lib. ii, cap. 65. Mercati de morb. intern. cur. cap. 12.

h. Cystophlegmatica, a muco, sp. 25.-Mercati loc. citat. Sclacht. Instit. medic. pract. lib. viii, cap.9. Etmulleri de Ischur. vesicat. Amati Lusitani, cent. vi, cur. 12. Zacuti Lusitani, prax. med admirab. lib. ii, observ. 64. Boneti ex Thom. Bartholin. de urin.suppress. obs. 17.

i. Ectopocystica, a vesicae ectopia, sp. 26. $-\alpha$. Herniosa, seu a cystocele, Verdier, Mém. de l'Acad. de Chirurg. tom. ii, observ. 4, 6, 9, 10, 14. Felic. Plater. obs. pag. 830. - $\beta$. Proptoica, seu ab exocyste, Verdier ex Noel. lib. citat. obs. 17 .

$k$. Cystoproctica, ab intestino recto scybalis, calculo, flatibus, inflammatione, abscessu, haemorrhoidibus, turgente, sp. 27.-Dodonaei Observ. medic. cap. 47. Schenck. lib. iii. de Ischur. observ. 6. Wepferi Dissert. de Apopl. pag. 391. Vandermonde, Journ. de méd. tom. ix, pag. 261. Boneti, Sepulchret. de urin. suppress. observ. 18. sect. 4. Schacht. Graubii, loc. citat.

l. Hysterocystica, ab utero, sp. 28. - . gravido, Nordman. Dissert. de Ischur. gravidar. Mauriceau, lib. i, cap. 15. Roderici a castro, lib.ii, cap. 15. - $\beta$. parturiente, Boneti ex Riolani de urin. suppress. Vandermonde ex Duran. Journ. de Méd. tom.v.- $\gamma$. hydropico, Hippocrat. de morb. mulier. lib. xii. Sennerti, Pract. lib. iv, part. i, sect. 2. cap.10,11. Schenck. lib.iv, de mol. fals. Schachtii, Gaubii, loc. citat.- $\delta$. tumoribus distento, Gaubii, Schachtii, loc. citat. Sennerti, ibidem, ac supra, cap. 13. Knoeffelii, E. N. C. ann. iv. $\rightarrow$ prociduo, Nordmann, Dissert. citata. Sabatier, Mém. de l'Acad. de Chirurg. tom. iii. Sennerti, ibid. ac supra, cap. 16.

m. Atretarum, a menstruis in vagina retentis, sp. 29.Amyand, Transact. philos. ann. 1732 , No. 442, art. 8. pag. 45. Schenck. lib. iv, de part. genit. mulier. observ. 9. Heister. Chirurg. tom. ii, pag. 951. Astruc. de morb. mulier. tom. $i$, lib. i, cap. 5.

n. Paradoxa, sp. 30.-Morgagni, Epistol. Le Clerc, Journ. de méd. Juill. 1755, p. 11 .

4. Ischuria (urethralis) cum tumore hypogastrii, frequenti ad mingendum stimulo, et dolore in aliqua parte urethrae. 
(ISCHURIA.)

Variat pro varietate causae; hinc

a. Perinaealis, a perinaei tumore, sp. 31.-Galen. lib. de affect. loc. et ex eo Zacuti Lusitan. Hist. 149. Vandermonde ex Daran. Journ. de méd. tom. v. pag. 291. Tulp. observ. med. lib. iii. cap: 10. Forest, lib. xxvi. observ. 2.

b. Urethrolithica, a calculo urethrae impacto, sp. 32.Schmid. Eph. nat. cur. decad. i, ann. viii. obs. 89. Winckler. ibid. tom. vi. obs. 34. Zacut. Lusitan. Prax. med. admirab. lib. ii. observ. 66, 67, 68. Tulp. observ. med. lib. iii. cap. viii. Bonet. Sepulchr. de urin. suppress. obs. 14. Barthol. cent. iv. epist. 5. Heister. Chirurg. tom. ii. pag. 839.

c. Urethrophlegmatica, a muco urethram infarciente, sp. 33.-Bonet. Sepulchr. et medic. septent. Manget. Biblioth. med. pract. Amat. Lusitan. cent. v. observ. 71. Forest. libr. xxv. observ. 25. Varand. de Ischur. Lud. Apin. Eph. nat. cur. decad. iii. ann. iii. observ. 69.

$d$. Urethrothromboides, a sanguinis grumo urethram opplente, sp. 34.-Bonet. med. septentrion. de Ischur. et sepulchr. de urin. suppres. obs. xi. Forest. lib. 25. obs. 25:

e. Urethropyica, a pure urethram obstruente, sp. 35.-Bonet. med. septentr. de Ischur. Forest. Scholio modo citat. Boerhaave praefat.

$f$. Urethrohymenodes, a membrana in urethram impacta, sp. 36.-Hist. de l'Acad. R. des Sc. ann. 1714, hist. pag. 22.

g. Urethrelmintica, verminosa, sp. 37.-Joan. Petr. Albreicht. vid. Bonet. medicinam septentrionalem de urin. cap. 31. et Manget. Biblioth. med. pract. tom. iv. de morb. urin. spectant. Vandermonde, Journ. de méd. Septembre 1758, pag. 245. Joan. Rhodii centur. 3. observ. 36. Collect. Acad. tom. iii. pag. 497. observ. 77.

$h$. Urethritica, ab urethrae inflammatione, sp.38.-Hildan. cent. iv. obs. 54. Forest. de incert. urin. judiciis, lib. iii. cap. 3. versus finem. Varand. de affectib. ren. et vesic. cap. 7. Bonet. medic. septentr. de Ischuria, cap. 1. no. v. Goulard. des malad. de l'urethre. Heister. Chirurg. tom. ii. pag. 838. Lond. med. obs. vi. 24.

i. Carunculosa, a morbis ut vocant urethrae, sp. 39.-Amat. Lusitan. cent. iv. cur. 19. cent. v. cur. 48. Sharp Recherches critic. cap. 4. Goulard. des malad. de l'urethre. Heister. chirurg. tom. ii. pag. 834. André des maladies de l'urethre, passim.

$k$. Hydrocelodes, ab urethrae ruptura in scrotum hiante, sp. 40.-Joan. Lud. A pin. Miscel. nat. cur. decad. iii. ann. iii. obs. 68.

l. Cryptopyica, a penis intra corpus retractione, sp. 41.Fred. Hoffm. Consult. de morb. abdom. cas. 105. 
(ISCHURIA.)

m. Peridesmica, a vinculo strictiori peni injecto, sp. 42.Bonet. med. septentr. de Ischuria, cap. 1. no. viii.

n. Phimosica, a phimosi, sp. 43.-Horst. tom. ii. lib. iv. pag. 274. Goulard. des malad. de l'urethre. Heist. Chirurg. tom. ii. pag. 818. Bonet. Sepulchr. de Ischur. obs. xv.

o. Aspadialis, ab urethrae clausura, sp. 44. - Heister. Chirurg. tom. ii. pag. 818 et 951 . Horst. tom. ii. obs. 55. Joan. Wieri obs. pag. 221. Bonet. Sepulchr. de urin. suppres. observ. xv. schol. et med. septentr. de Ischur. cap. 2. sect. 6.

\section{G. CXXV.-DYSURIA.}

Dolorifica et quodammodo impedita urinae emissio.

Dysuria, S. gen. 265. Lin. 57. Vog. 164. Sag. 213. Stranguria Auctorum.

Species sunt,

1. Dysuria (ardens) cum ardore urinae sine morbo vesicae evidente.

Dysuria primaria, S. sp. 5. Dysuria a cantharidibus ingestis vel applicatis. arsura, sp. 7. neonympharum, sp. 14.

2. Dysuria (spasmodica) a spasmo ex aliis partibus cum vesica communicato.

Dysuria hysterica, S. sp. 1. rachialgica, sp. 11. diabetica sp. 17. nephralgica, sp. 3.

3. Dysuria (compressionis) a vicinis partibus vesicam prementibus.

Dysuria gravidarum, S. sp. 13. a cystocele, sp. 8. ab hysteroloxia, sp. 10. atretarum, sp. 16.

4. Dysuria (phlogistica) a vicinis partibus inflammatis.

Dysuria ab hysteritide, S. sp. 9. haemorrhoidalis, sp. 6 . venerea, sp. 4 . a caruncula, sp. 5.

5. Dysuria (irritata) cum signis calculi vesicalis.

Dysuria calculosa, S. sp. 12.

6. Dysuria (mucosa) cum excretione muci copiosa.

Glus, Lin. 200.-Pyuria mucosa, S. sp. 6.-Pyuria viscida, S. sp. 5.-Rarus vesicae morbus, Hoffm. T. iv. Consult. Med. Centur. ii. cas. xciii.-Catarrhus vesicae Lieutaud. Syn. p. 272.-Pyuria arthritica, S. sp. 9.

De dysuria ab infectis, S. sp. 15. mihi non satis liquet. 
G. CXXVI.-DYSPERMATISMUS.

Seminis in actu venereo tarda, impedita, et ad generationem insufficiens emissio.

Dyspermatismus, S. gen. 260.-Sterilitas, Lin. 171. Sag. 211.-Agenesia, Vog. 283.

Species sunt,

1. Dyspermatismus (urethralis) a morbis urethrae, S. sp. 1.

2. Dyspermatismus (nodosus) a nodis corporum cavernosorum, S. sp. 2.

3. Dyspermatismus (praeputialis) ab angustiori praeputii orificio, S. sp. 3.

4. Dyspermatismus (mucosus) a muco urethram infarciente S. sp. 4.

5. Dyspermatismus (hypertonicus) a validiori penis erectione, S. sp. 5. Edinb. Ess. i, 35.

6. Dyspermatismus (epilepticus) ab epilepsia spasmodica in coitu adveniente, S. sp. 6.

7. Dyspermatismus (apractodes) a genitalium ignavia, $\mathrm{S}$. sp. 7 .

8. Dyspermatismus (refluus) in qua nulla seminis in coitu emissio, ob refluxum ejusdem ex urethra in vesicam, S. sp. 9.

De dyspermatismo seroso, S. sp. 8. mihi non satis constat. G. CXXVII.-AMENORRHOEA.

Menses tempore quo fluere solent, vel solito parciores, vel non omnino fluentes, citra graviditatem.

Amenorrhoea, Vog. 130.-Dysmenorrhoea, Lin. 168. Sag. 218.

\section{Species sunt,}

1. Amenorrhoea (emansionis) in puberibus quibus post fluxus tempus solitum, menses non jam prodierint, et cum simul variae affectiones morbidae adsint.

2. Amenorrhoea (suppressionis) in adultis, quibus menses, quae jam fluere solebant, suppressae sunt.

3. Amenorrhoea (difficilis) in qua menses parcius et cum dolore fluunt.

Menorrhagia difficilis, S. sp. 1 .

\section{ORDO VI.-TUMORES.}

Partis magnitudo aucta sine phlogosi.

Tumores protuberantes, Lin. cl. x. ord. v. 


\section{G. CXXVIII-ANEURYSMA.}

Tumor mollis, pulsans, supra arteriam.

Aneurisma, S. gen. 32. Lin. 282. Vog. 408. Sag. 35.

\section{G. CXXIX.-VARIX.}

Tumor mollis, non pulsans, supra venam.

Varix, S. gen. 33. Lin. 282. Vog. 407. Sag. 36.

\section{G. CXXX.-ECCHYMOMA.}

Tumor diffusus, parum eminens, livescens.

Ecchymoma, S. gen. 6. Vog. 451. Sag. 5.

Sugillatio, Lin. 230.

\section{G. CXXXI.-SCIRRHUS.}

Partis, plerumque glandulae, tumor durus, non dolens, aegre suppurans.

Schirrus, S. gen.14. Lin.283. Vog.374. Sag.19. Boerh. 392.

\section{G. CXXXII.-CANCER.}

Tumor scirrhodeus dolens, in ulcus mali moris abiens.

Cancer, S. gen. 20. Vog. 375. Boerh. 492. Sag. 25.

\section{G. CXXXIII.-BUBO.}

Glandulae conglobatae tumor suppurans.

Bubo, Lin. 271.-Bubo, S. gen. 16. Vog. 368. Sag. 21. Parotis, S. gen. 17. Vog. 370. Sag. 22.

\section{G. CXXXIV.-SARCOMA.}

Extuberatio mollis, non dolens.

Sarcoma, S. gen. 23. Lin. 294. Vog. 376. Sag. 28.Polypus, Vog. 377.-Porrus, Vog. 427.

\section{G. CXXXV.-VERRUCA.}

Extuberatio durior, scabra.

Verruca, S. gen. 25. Lin. 277. Vog. 426. Sag. 30.

\section{G. CXXXVI.CLAVUS.}

Cuticulae crassities dura, lamellata.

Clavus, Lin. 278. Vog. 428.-Callus sive Tylloma, 429.

G. CXXXVII.LUPIA.

Extuberatio subter cutem mobilis, mollis, non dolens.

Lupia, S. gen. 37. Sag. 40.-Atheroma, Lin. 285.-Encystis, Vog. 420. 
G. CXXXVIII.-GANGLION.

Extuberatio durior, mobilis, tendini insidens.

Ganglion, Lin. 287. Vog. 379.

G. CXXXIX.-HYDATIS.

Vesicula cuticularis humore aqueo plena.

Hydatis, S. gen. 34. Sag. 38.-Phlyctaena, Lin. 273. Vog. 442.

G. CXL.-HYDARTHRUS.

Articulorum, genu potissimum, tumor, parum primo eminens, cuti concolor, dolentissimus, et mobilitatem imminuens.

Hydarthrus, S.' gen. 38.-Anglis a White Swelling.Reimarus de fungo articulorum, Leid. 1757.-Cheston, Path. Inq. chap. v.-Edinb. Ess. iv, 18, 19, 20.

G. CXLI.-EXOSTOSIS.

Tumor durus ossi insidens.

Exostosis, S. gen. 29. Lin. 290. Vog. 417. Sag. 34.Hyperostosis, Vog. 418.

\section{ORDO VII.-ECTOPIAE.}

Pars ex sua sede dimota tumorem faciens.

Ectopiae, S. cl. i, ord. vi. Sag. cl. i, ord. vi.

G. CXLII.-HERNIA.

Partis mollis ectopia, cute et aliis integumentis adhuc tecta.

Hernia, Lin. 291. Gaub. Pathol.

Enterocele.

Hernia intestini.

EPIPlocele.

Hernia omenti.

Gastrocele. Ign. la Chausse.

Ventriculi trans laxatos, diductos regionis epigastricae parietes, imo et per umbilici annulum elapsi ectopia herniosa.

Hernia ventriculi, Fabr. Hildani resp. ad Doring. pag. 915. Blegney Zod. med. Gall. ann. i. Febr. obs. 2. pag. 44. Gunzii libell. de hern. cap. 20. Ign. la Chausse Diss. de hernia ventrali, et Kirschbaum Diss. de hern. ventriculi in Disp. Chir. Haller. tom. 3. Diss. 68. 69.-Hernies de l'estomac. La Faye sur Dionis, p. 121. Arnaud Tr. des hernies, préface, p. 81. Sharp Rech. Crit. chap. i. Garengeot, Mém. de PAcad. R. de Chir. tom. i. p. 703. 
(HERNIA.)

Hepatocele, Gunzii Libell. de hern. p. 4.

Hepatis trans laxatos, diductos in umbilici vicinia abdominis parietes, vel per ipsum umbilicum ectopia herniosa.

Hernia hepatis, E. N.C. dec. i. ann. ii. et dec. ii. ann. vii. Bohnii Chir. rational. pag. 230.-Hernie du foye, Arnaud des hern. tom. i.

Splenocele, Gunzii Libell. de hern. p. 4.

Lienis trans laxatos, diductos abdominis in latere sinistro parietes, vel per inguinalem ejusdem lateris annulum elapsi ectopia herniosa.

Hernia lienis, Fabr. Hildani, Epist. Iv. pag. 999. Spigelii de corp. hum. fabric. lib. 8. cap. 14. Ruyschii Advers. Anat. dec. ii. pag. 23.-Hernie de la Rate, Arnaud des hern. tom. i. pag. 29.

\section{Hysterocele.}

Uteri trans annulos inguinales, aut laxatos, diductos inferne abdominis medietatis parietes elapsi ectopia herniosa.

Hernia uteri, Sennert. lib. 4. Med. Pract. part ii. sect. 2. cap. 17. Doringii Epist. ad G. Fabr. Hildanum de hernia uterina, p. 893 . Graaf. de mulier. organ. cap. 8. Ruysch. Adv. Dec. ii. pag. 23.-Hernie de la Matrice, Arnaud des hernies, tom. i. pag. 29. Sabatier Mém. de l'Acad. R. de Chir. tom. iii.

Cystocele, Platner. Inst. Chir. Ign. la Chausse de hern. ventrali.

Vesicae urinariae trans annulos inguinales, sub arcubus cruralibus, et per laxatos, diductos perinaei, hypogastrii, necnon vaginae parietes elapsae ectopia herniosa.

Hernia cystica quorundam.-Hernia vesicae urinariae, Salzmanni Diss. inter Halleri Disp. Chir. tom. iii. Diss. 72. Gunzii Libell. de hern. \&c.-Hernie de la vessie urinaire, Mery Mém. de l'Acad. R. des Sc. 1713. Garengeot Oper. de Chir. Levret Obs. sur les Polypes; Sharp. Rech. Crit. Verdier Mém. de l'Acad. R. de Chir. tom. ii.

Encephalocele, Corvini Diss. de hern. cerebri inter Halleri Disp. Chir. tom. ii. Diss. xlvi.

Cerebri, cerebelli, aut utriusque, verbo Ençephali, per ossium cranii non penitus ossificatorum hiatum prorumpentis, ectopia herniosa.

Hernia cerebri, Reiselii Eph. N. C. Dec. ii. ann. ii. obs. 115. Lechelii, ibid. obs. 158. Treu Comm. Litt. 1738, Hebd. 52. n. 3. Taconi Diss. de humano monstro Bononiae nato. Corvini, ibidem.-Hernie du Cerveau, Le Dran Obs. de Chir. Obs. 1. 
(HERNIA.)

Hysteroloxia.

Uteri, saepius gravidi, et contra quam in hysterocele fit, abdominis claustris contenti situs in obliquum errans.

Obliquitas uteri Roedereri, Elem. art. Obstetric. § 449, 509. Levret Observ. sur les Accouch.-Inclinatio, reclinatio, obliquitas uteri, Deventer, cap. 46, 47, 48. Ruyschii, Observ. xcviii.-Inclinaison, obliquité de la matrice, Sabatier, Mém. de l'Acad. de Chir. tom. iii. \&c.

Parorchidium.

Testiculi unius, vel utriusque, cujus post nativitatem sedes naturalior scrotum est, situs in alienum aberrans.

Testiculorum serior descensus, vel retractio, Felic. Plateri Mantiss. Observ. Ambr. Paraei lib. vii. Thom. Bartholini Hist. Anat. Morgagn. Advers. iv. Dionis Oper. de Chir. dem. iv. Quelmalzii inter Halleri Disput. Anatom. tom. v. Dissert. i. Verdier sur la Hernie de la Vessie, Mém. de l'Acad. R. de Chir. tom. ii.

\section{G. CXLIII.PROLAPSUS.}

Partis mollis ectopia nuda.

Prolapsus, Lin. 292._Prolapsus et procidentia, Gaub. Pathol.

\section{Exophthalmia.}

Bulbi oculi procidentia, aucta, vel non mutata sensibiliter nativa ipsius magnitudine.

Magnitudo nimia, Prolapsus, Expressio oculi, Lat.-Hygrophthalmia, Elephantiasis oculi, Boerh. de Morb. Ocul. pars ii. cap. 5.-Exophthalmia, Hydrophthalmia, Buphthalmia, Ophthalmoptosis, Ecpiesmus Graecorum. Mauchart, diss. de Hydrophthalmia, et oculi paracentesi inter Haller. Disput. Chir. tom. i.-Grosseur contre Nature, Hydropisie, Cancer, Chute de l'oeil. MaitreJan, part ii. chap. 6. Saint Yves, part ii. chap. 1 .

Blepharoptosis Mauchartii.

Palpebrae utriusque vel alterutrius elongatae, retractae, introversae, vel extroversae ectopia.

Blepharoptosis, Lagophthalmus, Ectropium, Entropium, Gr.-Palpebrae superioris casus, Retractio, Palpebrarum introversio, extroversio, Lat.-Chute, relaxation de la paupière superieure, eraillement des paupières, trichiase avec introversion des tarses. Vide Platner, Instit. Chirurg. §. 577. §. 584. Maitre-Jan, part iii. chap. 18, 19, 20, 21. Saint Yves, part. i. chap. 8, 9, 10, Dionis, Dem. 6. Boerh. de Morb. ocul. part. i. cap. 5. Heister. Chir. tom. i. part. ii. §. 2. cap. 45, 46, 48. Gorter. Chir. repurg. lib. v. cap. 10. 
(PROLAPSUS.)

HYPOSTA PHYLE.

Gurgulionis seu uvulae laxatae, inflammatae, ulceratae, incrassatae, attenuatae, bicornis, procidentia.

Staphyle, Kion, Imantium, Craspedon, Columella bicornis, Aretaei, Acut. lib. i. cap. 8. de affectibus columellae.Uvulae prolapsus, Nenter. tab. xxv. cap. 8.-Uvula nimium producta, Heister, Chir. tom. ii. p. 659.-Uvulae inflammatio, catarrhus, paralysis, casus, Gorter, Praxis Med. Syst. pag. 165.-Relachement de la luette, Dionis Dem. v.

Paraglosse.

Linguae motu deglutitorio in fauces revolutae vel ore extrusae, exundantis, exertae ectopia.

Linguae extumescentia, magnitudo, inflatio, Galen. Method. med. libr. xiv. Valesci de Taranta Philon. lib. ii. cap. 59. Marc. Donat. Med. Histor. Mirab. lib. iii. cap. 4.-Linguae retractio, exertio, Gorteri-Glossocele, Gaubii Pathol. § 242. Linguae extrusio, idem §. 254.--Renversement de la langue dans le gosier, Petit. Mém. de l'Acad. des Sc. 1742.

Proptoma.

Partis externae, etiam ante lapsum conspicuae, et a palpebris, bulbo oculi, lingua, ac gurgulione, diversae procidentia.

Exania.

Sphincteris ani, et intestini recti inversi, procidentia.

Proctocele Pathol. Meth. edit. 3.-Morbus poenalis Gerhamensium, lib. Regum i. cap. 5.-Prolapsus ani Auctorum, ut Plateri, Prax. lib. ii. cap. 2. Nenteri, tab. 126, \&c.-Chute de fondement Dionis, Dem. iv. p. 392. Arnaud des Hernies, tom. i. cap. 28. Levret obs. sur les polypes, sect. 3 . p. 165.

Exocyste.

Membranae tum vesicae corpus, tum ipsius collum, interne obvestientis procidentia, per meatum urinarium facta.

Prolapsus, inversio vesicae urinariae Auctorum; Salzmanni diss. de Hern. vesicae inter Halleri Disp. Sel. tom. iii. Solingen de mulierum et infant. morb. chir. p. 741 .

Renversement de la vessie urinaire, Noel obs. Recherches sur la Hernie de la vessie par Verdier. Mém. de l'Acád. R. de Chir. tom. ii.

Hysteroptosis.

Uteri vel vaginae procidentia.

Hysteroceles nudae, Sauv. Pathol. Method. edit. 3. 
(PROLAPSUS.)

Relaxatio, prolapsus, procidentia, inversio uteri vel vaginae Auctorum, ut Heisteri Inst. Chir. tom. ii. Gunzii de Herniis, \&c.

Relachement, chute, descente, renversement de la matrice ou du vagin. Arnaud, tom. i. cap. 24, \&c. Puzos sur les maladies de la matrice. Sabatier, mém. de l'Acad. R. de Chir. tom. iii.

\section{G. CXLIV.-LUXATIO.}

Os ex sua in juncturis sede dimotum.

Luxatio, Gaub. 226. Vog. 472-Exarthrema, S. 60. Sag. 67.-Diastasis, S. 61. Sag. 68.-Luxatura, Lin. 237.

\section{Exarthrema, Graec.}

Separatio contigui in ossium articulis diarthrosice, non vero immobiliter combinatis.

Exarthrosis, Parathrema, eorund.-Luxatio, subluxatio, distortio, Heister. Inst. Chir. tom i.-Boerh. Aph. comment. tom. i.; Gorter. Chir. repurgat. cap. 6. Platner. Instit. Chir. § 1090. 1213. - Luxation, entorse, Petit Malad. des Os, tom. ii. Coll de Villars Chir. tom. v. de la Faye Princ. de Chir. Duverney Malad. des Os, tom. ii.

Diastasis, Graecorum.

Ossium, catilaginum, immobiliter combinatorum, separatio, seu recessus ab invicem partialis vel totalis.

- Ossium recessus, Latin. Petit. tom. ii. chap. 8. et 13. La Faye Princ. de Chir. pag. 474. Duverney, tom. ii. ch. 1. et 5 .

\section{LOXARTHRUS.}

Ossium cum motu sensibili articulatorum situs relativus in alienum constanter mutatus, aut obliquitas respectiva persistens citra exarthrema et spasmum.

Perversio capitis ossium ac musculorum appensorum, $\mathrm{Du}$ verney, tom. ii. cap. 2.-Artis vari Duverney, tom. ii. cap. 22.-Gibbositas scapularis Auctorum.

\section{ORDO VIII.-DIALYSES.}

Solutio continui visu tactuve manifesta.

Plagae, S. cl. i, ord. vii, Sag. cl. ii.-Vitia dialytica et solutiones continui, Lin. cl. xi, ord. ii.-Exulcerationes, Lin. cl. xi, ord. iii.-Dissolutiones, Vog. cl. $x$, ord. vi. 
G. CXLV.-VULNUS.

Soluta unio recens, cruenta, partis mollis, a corpore duro moto.

Vulnus, S. gen. 63. Lin. 241. Vog. 465. Sag. 78. Boerh. 145.-Punctura, S. gen. 64. Lin. 243. Sag. 79.-Laceratura, Lin. 242.-Amputatura, S. gen. 70. Lin. 243. Sag. 86.

\section{G. CXLVI.-ULCUS.}

Partis mollis solutio purulenta vel ichorosa.

Ulcus, S. gen. 71. Lin. 250. Sag. 89.-Exulceratio, S. gen. 72. Sag. 90.-Sinus, S. gen. 73. Vog. 492. Sag. 92.-Fistula, S. gen. 74. Lin.254. Vog. 491. Sag. 91 . Boerh. 413.-Cacoethes, Lin. 250. Vog. 485.-Phagedaena, Vog. 488.-Moma, Lin. 251. Vog. 489.-Carcinoma, Lin. 252. Vog. 487.-Ozaena, Lin. 253. Vog. 500.-Achor, Lin.265. Vog.494.-Crusta, Vog.494.Therioma, Vog. 486.-Sycosis, Vog. 390.

\section{G. CXLVII.-HERPES.}

Phlyctaenae vel ulcuscula plurima, gregalia, serpentia, dysepuleta.

Herpes, S. gen. 7. Lin. 269. Vog. 443. Sag. 11.

G. CXLVIII.-TINEA.

In cute capillata ad radices capillorum ulcuscula, humorem, in crustam albam friabilem abeuntem, fundentia.

Tinea, Lin. 263. Vog. 497. Sag. 131.

G. CXLIX.-PSORA.

Pustulae et ulcuscula pruriginosa, contagiosa, manus male habens.

Psora, Lin.266.-Scabies, S. gen.304. Vog.444. Sag.130.

G. CL.-FRACTURA.

Ossis partes a cohaesione in magna fragmenta vi solutae.

Fractura, S.gen.67. Lin.235. Vog.468. Sag.98. Boerh. 339.

\section{G. CLI.-CARIES.}

Ossis exulceratio.

Caries, S. gen. 77. Lin. 493. Sag. 94. 


\section{CATALOGUS}

Morborum a nobis omissorum, quos omisisse fortassis non oportebat.-(Vide Prolegomena, p. 235.)

Aglactatio, Lin. Agalaxis, Vog.

Alopecia.

Anchylops,

Aegilops.

Anchylosis, Lin.

Anchylosis, Vog.

Angina, Sag. et Lin.

Angina pectoris.

Antipathia.

Beriberia.

Cardiogmus.

Cephalaea.

Cephalalgia.

Distortio.

Aglutitio.

Acataposis.

Oesophagismus.

Dystocia.

Parturitio, et alii gravidarum Elcosis. et parturientium morbi.

Essera.

Hemicrania.

Leucorrhoea ex vitio locali.
Lithiasis in organis uropoeticis, et in aliis corporis partibus.

Malis.

Cocyta, Lin.

Melaena.

Melaficterus.

Melanchlorus, Vog.

Myocoilites.

Necrosis.

Odaximus.

Dentitio, et alii infantum morbi.

Paralysis partis singularis ex vitio musculorum.

Physocephalus.

Pnigma.

Catarrhus suffocativus.

Pyuria.

Puoturia.

Sterilitas utriusque sexus.

Acyisis, Vog.

Stymatosis.

Tarantismus.

Venena.

Vermes. 


\section{(n)}

(2)

,

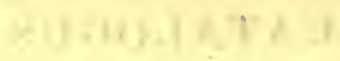

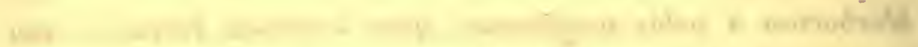

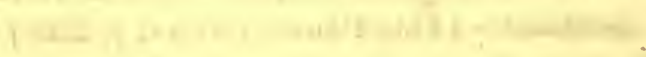

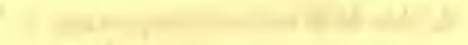

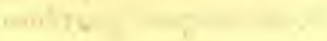
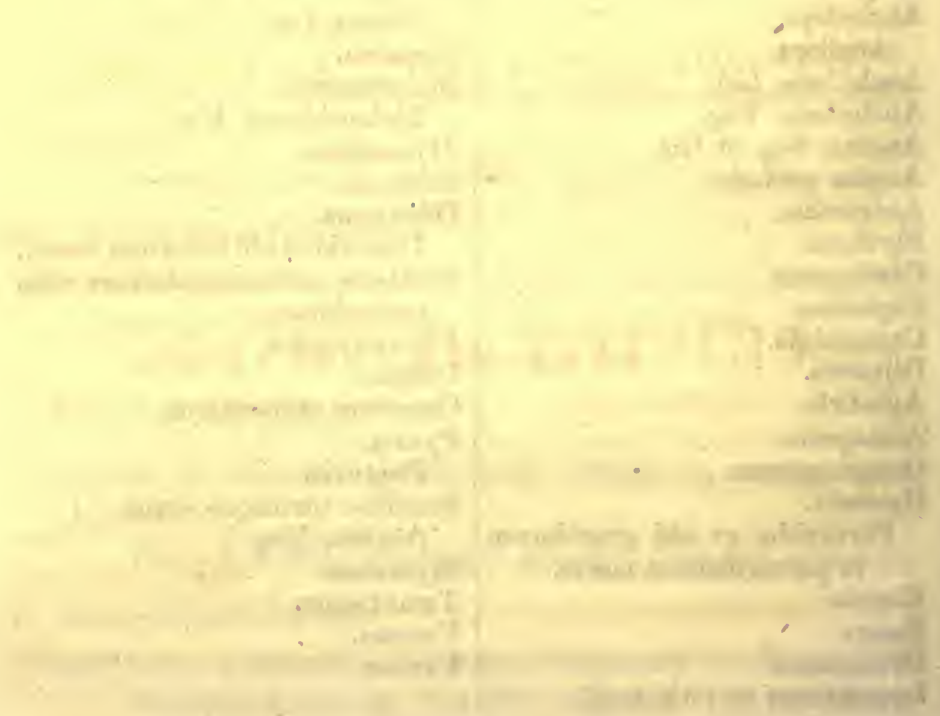

-

$x^{1}-2$

- 


\section{LECT URES}

INTRODUCTORY TO THE COURSE

ON THE

\section{PRACTICE OF PHYSIC.}


.

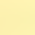




\section{LECTURES INTRODUCTORY, \&c.}

66 Gentlemen,

Oun business here is to give a course of lectures on the practice of physic; and I am now to give you the ordinary introduction to it, that is, to deliver the literary history of this branch of science.

It has been commonly thought that this part of our work is of little importance, and it is therefore very carelessly attended to; but I am well persuaded, that this is a great and hurtful mistake. It is indeed the business of students at college to learn one system only, and that as delivered by their professor, without attempting, to read many books; but, in the time allowed to our courses, it is impossible to deliver even one system very completely, so that a great deal must be left to be learned afterwards from books. And though the system taught were more complete than it commonly is, I should have a mean opinion of the genius of a student, who, being satisfied with that alone, should implicitly receive the opinions of his master without inquiring after those of others, without considering what doubts might be raised, or what objections might be made to the system he had first imbibed. I maintain that such a student would never understand even his own system sufficiently. In short, gentlemen, how much so ever we may teach you in college, your knowledge will be incomplete without a great deal of after reading and study. For this purpose, it is absolutely 
necessary to study the literary history of physic, to learn the character of authors, and therefore the periods at which they lived, the schools they belonged to, and the systems in which they were engaged. But these particulars are only to be learned from a history of physic; and it is a study, therefore, which I earnestly recommend to all my pupils. At the same time, I must own, that this study cannot be entered upon to any length till a man has acquired a good deal of knowledge in the art, or at least of one system of it. It may, therefore, seem very improper for me to offer this history at present to you, who may be supposed to be but beginning the study of medicine. It is certainly true, that, at present, to enter into any minute detail on this subject, would be very preposterous; but I am aware of this, and I mean not to do so. I am to give you a slight and general view only; and $\mathrm{I}$ hope it is proper to give that general view even at the very beginning of your studies. There will be some use in carrying it along with you, as it may engage you to mark many particulars relating to it, which, in the course of your studies might otherwise pass unheeded.

My proper business, on this occasion, is to give you the history of the branch I am to teach-that is, of the practice of physic only; but both for understanding that history better, and for prosecuting hereafter the history of physic more generally, I shall give you, as I can do it very shortly, a plan for the general history of physic; and I think it the more necessary here, as it is a plan peculiar to myself, and you cannot have it elsewhere. I propose now to give you a short account of what I think may be called the Revolutions of physic-that is, an account of the changes which, from time to time, have happened in the method of cultivating the art, with the general effects of these as well as history will allow us to discern them.

With these views, therefore, I divide the history of physic by marking in it seven periods, at each of which I suppose a considerable revolution to have happened; and in every different interval between these, I suppose the art to have been in a different state.

The first period extends from the beginning of the art till the time at which philosophy was formally introduced into the schools of physic. During this period, the art was cultivated 
by experience and observation almost alone ; and the first revolu. tion therefore to be marked, is, from the joining of philosophy and theory to experience. The persons who produced this change, from the speculative opinions on which they founded their system, were called Dogmatists, and their sect the Dogmatic. The second period of our history extends from the time that practical physicians attempted to lay the foundation of their art in reasoning on the human body; that is, from the rise of a Dogmatic sect, to the time when a sect of physicians arose, who professedly opposed all reasoning in physic, declared against philosophy and anatomy, the foundations of such reasoning, and maintained that experience alone was the only proper guide. The method of these physicians was called Empiricism; and the followers of it formed a sect which was called the Empiric. The third period of our history extends from the rise of the Empiric to the rise of the Methodic sect, which, as we shall afterwards explain, was distinguished by a particular modification of Dogmatism. The fourth period extends from the rise of the Methodic sect to the time of Galen, by whom both the Empiric and Methodic sects were in a great measure suppressed, and the ancient Dogmatism restored. The fifth period extends from the time of Galen to the time that Chemistry was introduced into the schools of physic, during which long period, the system of Galen had alone prevailed; but, by the introduction of chemistry, not only a new mode of Dogmatism was introduced, but also an occasion given to a return of much Empiricism. The sixth period extends from the time of the introduction of chemistry to the discovery of the Circulation of the Blood, which promised a more solid and durable foundation of Dogmatism, than had been laid before. During this period physicians were divided into the two sects of Chemists and Galenists; the first, pretendedly Dogmatists, but for the most part truly Empirics; and the others not rejecting experience, but making little use of it. The seventh and last period of our history of physic extends from the time of the discovery of the circulation of the blood to the present day. 'During this last period, physic has been constantly taught upon a Dogmatic plan, and most generally practised upon an Empiric one. But a more particular explanation of this will be given afterwards. 
I have thus divided the history of physic into seven periods by marking the several revolutions which have given occasion to that division; and, if I mistake not, these are the revolutions which chiefly, perhaps those which only, deserve to be taken notice of. I shall, in the next place, mark the same periods by the persons who gave occasion to, or were the authors of the several revolutions mentioned; and who have thereby been, as they deserved to be, the most remarkable persons in the history of physic. Our several periods of the history of physic may be distinguished by the persons concerned in them as follows:-

The first period is from the beginning of the art, or if you please, for the sake of a name, from the time of Asculapius, to the time of Hippocrates, who is supposed to have been the first who united in himself the philosophical and practical physician, and therefore to have been the first founder of a Dogmatic system. The second period is from the time of Hippocrates to the time of Serapion, most commonly supposed to have been the author of the Empiric sect. The third period is from the time of Serapion to that of Themison, the undoubted author of the Methodic sect. The fourth period is from the time of Themison to that of Galen, who, as we have said, contributed a great deal to the suppression of the Empiric and Methodic sects, and to the restoring of the ancient Dogmatism of Hippocrates. The fifth period is from the time of Galen to that of Paracelsus, who first introduced Chemistry into the schools of physic. The sixth period is from the time of Paracelsus to that of Harvey, undoubtedly the first who established the knowledge of the circulation of the blood. The seventh and last period is from the time of Harvey down to the present, or, if you will, to the time of Dr. Boerhaave, for though his system seems to be passing away, there is still no other name by which we can so properly distinguish the present age. Observe, therefore, that if you can but remember these eight names of Asculapius, Hippocrates, Serapion, Themison, Galen, Paracelsus, Harvey, and Boerhaave, you will easily recollect our division of the History of Physic.

But still it is not quite enough to remember these divisions thus generally, either by the revolutions produced, or by the persons producing them. It is further necessary to mark their relation to time more exactly, to mark the date and durations 
of each period, that all the several events in the history of physic may be still more easily remembered, by being referred to their proper date, and thereby connected with other events in the history of mankind. With this view, therefore, you will be pleased to observe that the first period of the history of physic extends from the first beginnings of society, perhaps from the creation of the world, to about 400 years before the Christian era, that is about fifty-six years before the birth of Alexander the Great, when Hippocrates, who was the cotemporary of Democritus, is supposed to have lived. The second period extends from the 400 th to the 287th year before Christ, when Ptolemy Philadelphus began to reign in Egypt, under whom Serapion is supposed to have flourished. The third period extends from the time of Ptolemy Philadelphus to the birth of Christ, under the celebrated reign of Augustus Cæsar, so well noted for the refined state of literature that then prevailed, when Themison flourished. The fourth period extends from the birth of Christ to the middle of the second century after, when Galen flourished, the cotemporary and favoured physician of the Emperor Marcus Aurelius. The fifth period is a very long one; it extends from the end of the second to the beginning of the sixteenth century after Christ, when Paracelsus lived cotemporary with the Emperor Charles V., and when the reformation of religion made a very considerable change in the affairs of Europe. The sixth period extends from the time of Paracelsus to the middle of the seventeenth century, about which time the discovery of the circulation of the blood by Harvey came to be very generally understood and received over Europe. This period needs hardly any other mark to distinguish it; but it was sufficiently remarkable by the civil war that then disturbed Britain, and by the changes that then and immediately after took place in the state of natural knowledge. The seventh and last period extends from the middle of the seventeenth century to the present time, which you are in a condition to distinguish as well as $\mathrm{I}$ am, whether you will do it by the age of Frederick II. king of Prussia, or as the period in which the ancient Republic of Poland was arbitrarily divided among its neighbours.

One other means of fixing your idea of the history of physic, 
I shall still give, and that is by marking the general state of it at the several periods mentioned. During the first it was chiefly Empirical, but from necessity rather than design. During the second it was very entirely Dogmatical, at least in the schools of physic; for at all times there must be empirics among practitioners. During the third period a professed and designed Empiricism prevailed. The fourth period was that in which the Methodic set flourished. During the fifth, Dogmatism again took entire possession of the schools, and has contrived to keep it ever since. But in the sixth period that Dogmatism took a particular form, which we call the Chemical. In the seventh and last period, a Dogmatism which may be called Mechanical, has chiefly prevailed, though it would be as fair to say that in this age it has been upon a general plan employing both the chemical and mechanical philosophies, and every other means that can be employed in the cultivation of science.

We have thus, gentleman, given you a plan, and the great outlines of the history of physic, which we hope you will remember, as it may be a foundation for your future inquiries into the subject. I shall now return to my proper businesș, which is to give you the history of the practice of physic, or the general state of it during the several periods we have mentioned.

First Period.-In considering the first period of our history, the subject which first presents itself to our inquiry is the origin of the art. This some would impute to a revelation made to the first man; but at any period we know of, the art has never shown the proper marks of such divine original. The heathens commonly imputed it to a particular inventor among men; but in this they were no better fcunded, for it was not possible for any single man, by the force of his own genius alone, to acquire so much knowledge as might deserve the name of an art.

It is sufficiently probable, that very soon after the first beginnings of human society, some art of physic and some knowledge of remedies arose among men: and, accordingly, no country has been discovered, among the people of which, however rude and uncultivated in other respects, an art of physic and the knowledge of a great number of remedies have not been found. The invention of remedies amongst the rudest people 
may in a great measure be accounted for, from the instincts arising in certain diseases-from the observation of spontaneous cures effected by the powers of the animal economy-from accidental errors in the choice of aliments-and even from those random trials, to which pain and uneasiness often lead.

These four sources-instinct, spontaneous cures, accidental errors, and random trial, afforded the first materials of observation, and must soon have led to an imitation, which formed an art of physic. The observations which had been made, continued indeed for some time scattered in different hands, so that in some countries men were obliged to expose their sick on the highways that every passenger might be consulted for a remedy: and there are some vestiges of this state of physic still remaining in Britain, where we consult every gossip that comes into a sick person's house, and every gossip is as ready to advise, as if there was no established art or profession of it in the country. This state, however, could not long continue in any country, as a turn for observation, and especially a turn of this sort in a person who had lived long at the head of a family would enable him to collect the facts of physic for himself; and the reputation of possessing such a collection would soon give many opportunities of increasing it, and thus establish one person as a practitioner. It would be easy to shew that many circumstances must have concurred to form such practitioners, and that the communication of their knowledge to their children or other disciples, would continue and increase their number. Thus the art and particular practitioners of it were established in every country; and there is no country we have heard of, or know at present, that is altogether without an art of physic. This we call the natural state of physic; but it did not subsist long in certain countries till it put on a more artificial form.

There never have been wanting in any society men cunning enough to take advantage of the superstition natural to mankind, and to lead and govern them by it; and the same persons have also taken hold of every passion that strongly engaged men, and therefore of their love of health and fear of death. Hence it is that in early ages we find physic and religion combined, and the priests the only physicians.

The most ancient account we have of arts is that of their 
being cultivated in Egypt, but we know few particulars of the state of them there that deserve to be taken notice of; and with respect to medicine in general, it is needless to inquire, as it is known to have been under such regulations as must have been a certain obstacle to its progress and improvement.

The first distinct accounts of the art of physic, as exercised by a particular class of men, are those we have of it in Greece among the priests of Esculapius. It would seem, that for some time these priests, if not the sole, were at least the chief practitioners of physic in that country; and as the trade was lucrative, it is to be presumed that these practitioners would endeavour to become knowing in it, and consequently to extend and enlarge their knowledge of remedies. In the temples of Esculapius, therefore, it is probable that a stock of knowledge was preserved and transmitted down from one set of priests to their successors; and at the same time, these temples afforded a particular means of preserving the knowledge of the materia medica; for we know it to have been then common for persons who had been cured of diseases by the remedies prescribed to them in the temple, to hang up there votive tables, on which was written some account of their disease, and of the remedies by which it had been relieved.

By this means these temples became the repositories of the records of physic, and the priests became the most knowing in the art. We find farther, that some of these temples became the most celebrated schools of physic in ancient times. The art the priests were possessed of could hardly be any other than the natural physic we have spoken of, perhaps a little farther cultivated, but still upon the same footing of observation and experience. But they were not on the best footing for this purpose, as they readily found that a charm answered the purpose of the trade as well as a genuine remedy; and it is to be suspected that many of these temples were scenes of knavery and craft. They were not even, at best, on a footing to gain much by experience, while they kept to their temples, and saw no sick persons but such as could be brought thither. But this state of practice could not serve the purpose of a country advancing in policy and arts, as Greece then was; and we accordingly find that either the priests themselves or their disciples 
issued forth from their temples, and spread over the country as clinical practitioners. One of these was the celebrated Hippo. crates, who, after having been instructed in all the knowledge of the school of Cos, and probably being also well acquainted with that of Cnidus, became an itinerant and clinical practitioner. At first they practised in a circumforaneous manner, but at length they became settled, and practised in the same manner as the physicians of Europe have done ever since.

Very few accounts remain of the medicines employed in the temples of Esculapius; and it will readily occur, that the first correct information must be expected and sought for in the most ancient medical writings now remaining; and which are those commonly ascribed to Hippocrates. These writings, however, at least for the purposes of history, afford a precarious and uncertain information; for, as we now have them collected together, they are certainly the works of many different persons, as well as of different ages ; insomuch, that it is impossible, with any clearness, to judge what was the true state of the materia medica in the time of Hippocrates. Besides, if we reflect in how many instances the nomenclature is entirely unknown, and in how many it is very doubtful and uncertain, we shall be satisfied how idle it is in modern writers to quote the authority of Hippocrates for the virtues of almost any medicine. Indeed laying, aside our partiality for that celebrated person, there can be no just ground for supposing, that at the period in which he lived, much discernment in the materia medica could have prevailed; and it is hardly necessary to add, that even although the substances named in those writings were known to us with more certainty than they are, yet the distinction of diseases, and of their circumstances, are so seldom given, that at present we can hardly be guided by them in employing any of the medicines they suggest.

This is the substance of what we have to point out to you as remarkable in the first period of our history. It is obvious with regard to the whole, that the art, whether we consider it in its natural state, or in the form of a particular superstition, must have been chiefly dependent upon experience. But while practitioncrs were thus dependent on experience, we have no reason to presume that they were strongly attached to it-that 
there was no other means of conducting the practice of physic. On the contrary, we presume it is natural to man to inquire into causes, and even to form theories with regard to the arts he practises. Theory came afterwards, and this is what must happen in every country advancing in refinement. Many persons are disposed to admire the natural state of physic among men, and to suppose that it proceeds to a wonderful degree in the number and efficacy of the remedies it has discovered; but I imagine that this is founded rather in a love of the ancient and marvellous and a partiality to empiricism, than on the real value of any such art. Upon a close inquiry, I think it will be found, that in this state of physic there is no exact discernment of diseases or of their different circumstances ; and, without both of these, we maintain that the application of the most efficacious remedies is extremely precarious. The value of the art, therefore, in this state, consists alone in the power of the remedies it has become acquainted with; but even in this way it does not seem to gó so far as has been commonly imagined.

From the time of Thales, who founded the Ionic, and of Pythagoras, who founded the Italic school of philosophy, Greece enjoyed a series of philosophers who were constantly making advances in natural knowledge. The human body could not fail to become the object of their study; and we find that the Pythagorean school was actually employed in dissection for the sake of learning the structure of animal bodies. We find farther, that the philosophers, upon many occasions, entered upon the practice of physic. We find that these philosophers were liable to despise the ordinary practitioners of physic for want of science; while, on the other hand, the practical physicians upbraided the philosophers with their impotency in practice. The physicians were probably in the right, as it is very certain that the philosophy of those days could go but a little way without an acquaintance with the facts which are to be learned cnly from actual practice. It was the combination of philosophy with a knowledge of the facts of physic, that could make any considerable change in the state of the art; and it was such a combination therefore that gives us occasion to mark the second period in the history of physic.

Second Period.-In a country advancing at the same time 
in philosophy and arts, the combination of these must necessarily take place. The inquiry into causes is natural to mankind : we are easily pleased with our conjectures, and sufficiently presumptuous in pushing them. The language of philosophy and an air of science are very imposing; and therefore, if any one thought of applying this to physic, it was no wonder if it was received with applause and admiration. This happened in Greece ; and it is commonly imputed to the celebrated Hippocrates. This perhaps is owing to his being the most ancient writer whose works we now possess; but it is sufficiently probable, from the history of literature in Greece, that it happened about his time. He is certainly the first in whom we find marks of a dogmatic system, and it is also sufficiently certain, that from his time forwards a dogmatic system of physic was cultivated without being referred to any more ancient author. The history of this celebrated person makes always a considerable part in the history of physic. At what precise period, however, or how long he lived, what books he truly wrote, and what was truly his system, are, I think, particulars not known with any certainty. That he was, indeed, a great man, we believe from the testimony of all antiquity; but the superstitious veneration of modern times appears to me to be founded often in ignorance and fashion. From the writings of Hippocrates, we find the state and practice of physic to be then considerably advanced. We find the most part of diseases distinguished, and names given to them; many of them had been carefully observed, and, with regard to them, many aphorisms or general conclusions had been formed; many nice and difficult operations of surgery had been practised; a great variety of medicines, and even compound ones, were employed ; and with all this we observe also a considerable attention to the management of exercise and diet.

After all my inquiries, my judgment concerning Hippocrates is this:-he was a man of parts, sagacity, and knowledge superior to the age in which he lived, and he probably laid a just foundation for the reputation he then acquired. But we know well, that no man can go much farther than the state of science at his particular period allows him, and that this is especially true with regard to those arts which depend upon an experience that 
can only be the produce of the industry of many ages, and upon sciences that are only the fruits of much cultivation. Hippocrates might have a great deal of the first, but with regard to the last his period was that of the infancy of philosophy; and though his genius might be of the highest kind, his real merit could not be so great as to deserve the superstitious veneration which has been bestowed upon him in latter ages. His writings, or those imputed to him, are extremely dissimilar and unequal; they present us sometimes with reflections which discover much sagacity, but they are hardly any where well connected or digested, and very often discover a childish frivolity. They certainly contain many useful facts, and some just conclusions drawn from them; but both these are probably disfigured by such chasms and interpolations as have happened to every ancient writing, and at best they are chiefly local, relative to Greece only. Though it does not very properly enter into my plan, I could not omit this stricture with regard to the celebrated father of physic, as he is commonly called.

But to return to our present purpose. It was either by Hippocrates himself, or by some person about his time, that a dogmatic system was introduced into the proper schools of physic, which has continued in most of the schools ever since. What was the condition of it in the hands of Hippocrates or of his family, as I have said, we do not exactly know; nor do we know any better what additions or alterations were made to it by Diocles, Chrysippus, Praxagoras, or the other celebrated physicians of Greece who came after; and it is the general state only that we are at present to take notice of. In respect of this we can say, that from the want of anatomy, and from the small progress that had been made in natural knowledge, we must think that the theory of those days could do little service to the art, and might do harm. But the dogmatists of those days had the apology to make, which is the only good apology for the dogmatism of any age, that, while they cultivated theory, they neglected nothing which experience had taught or could teach them; and that, in the mean time, it was very proper, nay, it was very necessary to cultivate the theory of the art. With this view they had long thought that anatomy was necessary ; they had long practised the dissection of animals, and at length 
they set about the dissection of the human body itself. This was especially done by Erasistratus and Herophilus, considerable names in physic. Both of them were Dogmatists, but they pursued different courses.

Erasistratus made many discoveries in anatomy, and many new attempts in physiology, but he was not judicious enough to perceive that these last were conjectures only, and not yet so well founded as to admit of application to practice. Erasistratus, however, unhappily overlooked this, and even founded upon his conjectures the general rules of his practice. Thus from theory he avoided bleeding and purging, two of the most powerful remedies then known, and at all times since acknowledged to be such. This is always a strong mark of the abuse of theory, when it excites too strongly either attachments or prejudices with regard to general remedies. Most of these have been established, by repeated experience, as useful in certain cases, and it is at the same time certain that none of them can either be admitted as universally useful, or be rejected as universally hurtful. Botallus, many ages afterwards, went to the opposite extreme in making bleeding an universal remedy; but the chemists, his cotemporaries, went into a more considerable error still, in universally rejecting it as altogether hurtful. The same was the error of Erasistratus, and it was a very manifest abuse of theory. Erasistratus, however, has given us another example of the abuse which theory is capable of, seemingly more innocent in the physician, but perhaps not less pernicious to patients. To understand it you must observe that theory, in general, is more fitted to raise doubts and fears than to remove them, and therefore it very often gives irresolution and timidity in practice; it takes away from our confidence in experience, and makes us avoid all the most powerful remedies, all those whose operation is sometimes violent, and whose effects, therefore, may upon some occasions be very bad, though upon others very good. It is probable that it was this theoretical timidity which made Erasistratus entirely avoid purging, as it had been then common to use purgatives of a violent kind; and it was probably the same temper that induced Erasistratus to employ very few drugs of any kind ; 
but, at the same time, as remedies cannot be entirely avoided, he used those of the gentle and dietetic kind. The "Saltem non nocere," was a maxim in the writings of Hippocrates, and it will always be the maxim of humanity and prudence; but it is especially supported by theory, and sometimes goes so far as to enervate the whole practice of physic. This seems to have been the case with Erasistratus. He, indeed, avoided all the mischiefs of physic, but at the same time missed almost all the advantages that art can give.

Herophilus, the cotemporary of Erasistratus, was equally industrious with him in dissection and the study of anatomy, and, without doubt, also in the study of physiology. In evidence of this he seems to have taken pains in cultivating, with great subtlety, the study of the pulse and some other parts of pathology; and there is no doubt that, in the main, he was a dogmatist; but we must also believe, that he was sensible of his physiology not being fit to go far or to be of any very useful application in practice, and that he had therefore a general distrust of his dogmatic system; for we find he was very busy in seeking everywhere for efficacious remedies. In all these respects, Herophilus seems to have been the pattern of the present age. The inquiry after remedies is certainly proper and useful; but it is very liable to abuse, as it leads men to neglect the study of diseases, and to what is of as bad consequence, to the neglect of any general system. Some system; indeed, and a great deal of theory also, our present practitioners cannot possibly avoid, as we shall hereafter explain; but by their general disparagement of it, they become incapable of employing it to any good purpose. They fancy themselves the disciples of experience, but they have truly a great deal of theory; and that of the worst kind, because it is such as arises occasionally without being digested or corrected by a system. Such a plan throws the bulk of physicians into a purely impotent and random empirical practice.

Third Period.- We have formerly remarked, that physic originally depended upon experience; and what we have called the natural state of it is, for the most part, entirely empirical ; but, at the same time, it is not absolutely without reasoning. The state of science at this period indeed, does not admit of 
much reasoning, but, at the same time, it does not absolutely reject this. Men are forward in reasoning, and while they are uncultivated by philosophy, they have few doubts, and are never professedly sceptical; but at the period we are now to mention, a more formal empiricism arose, and a sect of men appeared who opposed the dogmatists we have been speaking of, and professedly rejected all reasoning in physic; they even declared against all means of improving it as a science, and therefore against the study of anatomy. Who was properly the first author of this sect, is disputed among historians. Many impute it to Philinus of Cos, a disciple of Herophilus ; and it is very probable that the pursuit of efficacious remedies, in which we have said Herophilus was engaged, might determine Philinus to follow the same plan, which in all ages has degenerated into pure empiricism ; but it is not probable that any disciple of Herophilus should entertain that hatred of anatomy which was so remarkable among the empirics. We must seek, therefore, for some other source of empiricism besides the school of Hero'philus, and the following account of it is to me very probable : Herophilus practised his dissections of the human body at Alexandria, under the protection of the first Ptolemys who governed Egypt. But it is well known that the Egyptians had the utmost aversion and horror at the touching of a dead body; and they must have conceived the same with regard to the Greek physicians, who laid the foundation of their study in anatomy. It was therefore an obvious piece of policy in Sera pion, a physician, and probably a native of Alexandria, to raise himself in the favour of his countrymen by declaring against anatomy; and, at the same time, it was unavoidable that he should declare against dogmatism, from which anatomy is inseparable. For avoiding both, Serapion found many specious pretences; and he is commonly held to be founder of the empiric sect, which, immediately after his time, spread over Greece, and continued for a long time in some credit. Serapion did not at once give the system its finished form: that was reserved to Glaucias, who finished it by establishing what was called the Tripod of Medicine, that is, laying the foundation of the whole in these three particulars-Observation, History, and Analogy. It is not our business here to explain more particularly this em- 
piric system. We shall only observe in general, that their plan was sufficiently specious, and promised much; but, so far as we know, had no considerable effects. There were several men of reputation who belonged to the sect, but in what they particularly contributed to the improvement of medicine is not known. Heraclides of Tarentum was of the empiric sect, and is said to have been a person of judgment and diligence in the study of the materia medica; but neither his writings, nor those of any other of the same sect, produced what was to be expected from them, that is, a more complete and accurate history of diseases and remedies; for, if any such had ever existed, it must have been so much more valuable than any of the other writings of physicians, that it would probably have been transmitted to posterity; but we have not any one writing of the ancient empirics now remaining. We have this further proof of the fruitlessness of the empiric plan, that it had little effect in suppressing the dogmatism which, in those days, was certainly very frivolous; but, in spite of the empirics, the dogmatists were still very numerous, and men of considerable rank and practice were daily appearing amongst them. We have now no evidence remaining that the empirics either made any improvement in the history and discernment of diseases, that they found out more efficacious remedies than had been known before, or that, with more precision, they ascertained and limited the use of those formerly employed. The scheme of the empirics was sufficiently specious, but the accomplishment of it was only to be attained in the course of many ages ; and, therefore, while men constantly found it incomplete and imperfect, as it is even at the present day, practitioners were ever ready to desert it, and to seek for those aids which were promised by the other plans of physic. In short, in this account of the different states of the practice, the mention of the empiric sect was not to be omitted; but the effects of it, so far as I have been able to discover, are not at all known. I have always thought that their plan was more specious than solid; and I think we have a certain proof of it in this, that it had no effect either in exploding theory or in establishing practice. It might be supposed that their works have perished by the lapse of time, but that is improbable. For, if the effects had not been inconsiderable, they were likely also to have been 
permanent, and at least we might have expected some testimony in their favour to have remained. The noted sceptic Sextus was a physician, and has commonly been called Empiricus; but he was not truly such in his own profession, which he certainly would have been if the sect had produced any fruits suitable to their sceptical pretences.-(See Hypotyp. Pyrrhon: Lib. i. cap. 34.)-We have nothing more to observe with regard to the history of the empiric sect.

Fourth Period.-The dogmatists were still the most considerable party among physicians; and now, after the period we have mentioned, the only revolutions in the state of physic to be taken notice of, are the different modifications of dogmatism. As an instance of this kind, we have marked the rise of the sect called Methodic. This happened at Rome; and to introduce our account of the methodic sect, we must take some notice of the progress of physic in that city and its dominions. The illustrious commonwealth of Rome was for more than five hundred years without arts of any refinement; and they had that of physic only in its rude and natural state. At length, however, Rome received the arts of Greece, and amongst the rest their physic. When the first Greek practitioner, Arcagathus, presented himself at Rome, he was very kindly received; but his employment of many chirurgical remedies appeared severe and cruel to the Romans, and occasioned a general disgust of the Greek physic. Accordingly we do not find that it made any progress among them for a long time after, nor till Asclepiades, an elegant and artful man, and the friend and physician of Cicero, introduced the mild practice of Erasistratus. Asclepiades attached himself to none of the sects of physicians among the Greeks, which were but little known at Rome. He formed a plan and system of his own, but very nicely adjusted it to the manners and philosophy that then prevailed at Rome. His philosophy was that of his cotemporary Lucretius the Epicurean; and he readily framed a theory of the human body adapted to his purpose. He observed that fluids were in constant motion in the body; and he supposed that these fluids consisted of particles of different sizes and figures, and consequently that there must be as many different pores and passages adjusted to the transmission of these in different parts of the system. Upon 
this he readily formed a pathology, or an account of diseases. A stoppage of the pores formed one kind of disease, and the laxity, or a too free transmission, on the other hand, produced another set of diseases. He extended each of these genera to a great variety of species, according as the disease was seated in one part or other of the body, as it respected one fluid or another; but all this required so many subtle distinctions, that his system was not easily managed by persons of less acuteness and eloquence than he himself possessed.

Erasistratus, as we have said, employed few medicines, and particularly avoided all those of a harsher or more violent operation. This was extremely suitable to the temper of the Romans, who had not been by degrees accustomed to the disagreeable practices of the Greek physicians, and were now arrived at a degree of luxury which would not allow them to bear any thing harsh or painful. Asclepiades suited himself exactly to this temper: he professed to cure tuto, celeriter, et jucunde; and it was the last he especially studied. His practice consisted chiefly in the different kinds of gestation, in frictions, and in the proper management of wine. But it is not our business here to give the particulars of his practice; we mention him only as an example of that state of practice which is likely to prevail at all times in very great cities, in ages of great refinement, and among the luxurious part of mankind. Erasistratus fell into a mild practice from the errors and general timidity of theory. Asclepiades entered upon the same plan, and the physicians of great men will commonly do so, because it is agreeable to their patients. In such a state of physic, Placebos will abound and when these support the routine of the physician, there will be less anxiety about finding out or employing more efficacious remedies.

Themison, a physician at Rome, who came soon after Asclepiades, finding the subtleties of Asclepiades difficult, and judging them superfluous, proposed to abridge the system, and maintained that it was enough to consider what was common in diseases, and that the particulars might be neglected. He, therefore, reduced all diseases to three kinds only, the Strictum, the Laxum, and the Mixtum; the last consisting of the strictum in one part of the body, and of the laxum in another. 
He maintained that it was enough to refer any particular disease to one or other of these three heads, in order to form the proper indications of cure. This easy plan was, by way of eminence, called the Method, and the persons who followed it the Methodics. As this method derived reputation from Asclepiades, and was in itself so simple and easy, it was generally received at Rome; and the physicians of this sect continued there in fashion, and in the highest favour, for some time after. But Rome was a great and populous city, which supported a great number of physicians, and there was a conflux to it of these from every part of Greece. There were, accordingly, physicians of every sect there, methodics, empirics, and a great variety of dogmatists, so that physic was in a very divided state, when Galen came there about the middle of the second century after Christ.

Upon this occasion it is proper to take notice of the elegant Celsus, who lived at this period, and was the only native of Rome who ever became distinguished in the line of physic. Though perhaps not strictly of the profession, he was undoubtedly often engaged in practice; and in his writings we have many proofs of his discernment and good judgment. He was not attached to a particular school, or imbued with the prejudices of a sect. He had indeed more general views than those of any sect; but it was hardly possible for him to disengage himself from the prejudices of his age and country. He, therefore, in the main, follows the practice of Asclepiades, and of the Methodici; but we can at the same time perceive, that he was well acquainted with Hippocrates and the other noted physicians of Greece; and frequently, guided by them and his own good judgment, he gets beyond the narrowness of any sect, and is as much an eclectic as human nature commonly allows of. In his works, we find a great deal more relating to the materia medica than in those of any former author, many medicines being enumerated by him, and a judgment given with respect to them. Unfortunately, however, we are under such uncertainty with regard to his nomenclature, that we cannot always be determined in our judgment respecting the propriety of his doctrines. He is particularly' full in his account of alimentary substances; so that it is with respect to these we can best judge 
of his opinions; and it is there we find some singularities which we can hardly approve of.

Aretæus Cappadox either lived in the time we have mentioned, that is before Galen, or we have in his writings what probably was produced at that period. He was of a sect called the Pneumatic; but that affected only his reasoning, and we can hardly perceive its effect upon his practice. This in Aretrus, with respect to its general character, seems to have been in the best condition. Diseases are well described and distinguished, many remedies are proposed, and that without attachment to, or prejudice against any one in particular. His practice is in general bold and firm ; and every means, dietetic, pharmaceutical, or chirurgical, are employed in it.

These are the remarks we have to make on the state of physic before the time of the celebrated Galen; but we are now come to a remarkable period of our history, when we are to consider it in the hands of this great leader and those of his followers. Hitherto we have found the face of physic frequently changing and sometimes hurt by the extravagance of sectaries; now we are to find it more steady, but perhaps not less hurt by being in the hands of servile followers,

Fifth Period.-Galen was born of wealthy parents, and enjoyed all the advantages of a liberal education; these he improved by uncommon diligence, and he became conversant with every kind of literature. He devoted himself to medicine, and did not spare any pains in acquiring the knowledge of it; he studied under various masters, and applied to every branch of the science. He particularly studied anatomy, and undertook journeys in different countries to ascertain the state of medicine. Thus prepared he appeared at Rome, and was immediately considerable enough to be an object of envy; and the artifices of that envy were sufficient to prevent his progress in business, and even to make him retire again to his own country. However, he had acquired so much reputation at Rome as to attract the notice of the Emperor Marcus Aurelius ; and, after some time, by the invitation of that illustrious prince and philosopher, he was again brought back to Rome, and established there with great credit. He soon gained the favour of the Empress Faustina, and therefore that of the ladies of the 
Court, which is always sufficient to render a physician of much less merit than Galen, of the first fashion. In this manner established, he became the physician of greatest fame and highest authority at Rome.

In forming his system of physic, as it was usual to assume a leader, Galen pretended to follow Hippocrates, as perhaps still the greatest name among ancient physicians ; and it is probably owing to this that the name and writings of Hippocrates have been transmitted to us with more fame than they otherwise might have been. What he precisely borrowed from his acknowledged master, it is not easy to ascertain, but he certainly gave the whole of his system a new model, employing chiefly the principles of the Aristotelian or Peripatetic philosophy. But by pretending to follow the doctrines and practice of Hippocrates, he seemed to deliver the practice free from the partialities of any sect. He admitted every kind of remedy to a share in it, he employed those of the most efficacious kind, he studied remedies without becoming empirical, and he admitted every improvement which experience afforded. In all these respects he put the practice of physic on the best footing possible, but, at the same time, he gave a system of theory which was on a very narrow basis, but too far extended and too much detailed in the application. This tended to limit, rather than improve, the practice. Accordingly the practice experienced little improvement whilst the theory of Galen subsisted in the schools of physic which was for $\mathbf{1 4 0 0}$ years after his time. Galen was fond of writing, and composed many books, scarcely leaving any part of the medical system untouched; and it is presumed, that he gave a much more extensive, complete, and consistent system than had appeared before his time. This was sufficient to procure for him many readers and many followers. His writings gave lustre to the high rank he held in the profession, and that high rank must also have contributed to give authority to his doctrines. It is no wonder, therefore, that his system should have generally prevailed, and he endeavoured to support it by every means. In his writings, and probably in his conversation, he vilified and railed at all the other sects; and, by the authority which he assumed, and which his rank and reputation supported, he was probably the 
means of destroying their credit, in so much that there is no appearance of their having had any after his time.

What we know of Greek or Roman physic after the time of Galen, was entirely upon his model. There were three or four physicians of some note, and whose writings still remain. In these we find some particulars of practice added, but the general scheme of theory and plan of practice were exactly the same as those of Galen. It happened, indeed, unluckily for physic, that Galen lived at a period when literature and science were beginning to decline; -and when, at such a period, any system happens to acquire considerable credit, it has the effect of checking the further progress of science. The ingenious are often too modest to attack established characters, though they may perceive their blemishes, while the great horde of physicians are always servile imitators, who can neither perceive nor correct the faults of their system, and are always ready to growl at, and even to worry the ingenious person that could attempt it. Thus was the system of Galen secured in the possession of the schools of physic, till soon after the irruption of the Goths and Vandals destroyed every vestige of literature in the western parts of Europe, and drove all that remained of it to seek a feeble protection at Constantinople. Here we know that literature subsisted for a long time, but in such a sickly and languishing state as to be incapable of any improvement. Accordingly physic received none; the system of Galen-and that even in a mean state and condition-continued to be there the only physic known. For some time the only science that subsisted in the whole of Europe, or the western parts of Asia, was the little that was to be found in the territories of Constantinople; but, in the ninth century, it had some revival in another quarter, of which we must take notice.

In the seventh century, a new religion and a new empire had arisen in Arabia. This empire arose amongst a rude illiterate people; and it arose from superstition, which is always fatal to literature. Accordingly, as it first extended itself, it destroyed, wherever it came, all the remains of art and science. But at length, becoming a great empire, it happily acquired some monarchs disposed to the arts of peace and the cultivation of learning. For this, however, the Saracens had no funds of 
their own, but had to seek them from the Greeks. They did so; and obtaining many translations from the Greek into their own language, they laid a foundation for the pursuit of science in several branches. In doing so, they were, by certain accidents, led to receive the Aristotelian system of philosophy, and of course to take the medical system of Galen, which is founded upon that philosophy. For some time they were busied in this, but still, labouring under various disadvantages with respect to physic, they never attained to any great proficiency in the science; they made few corrections, and hardly any additions to the system they had received.

Under the dominion of the Saracens various schools of physic were established, and they produced many writers; all of whom, from the language that they wrote in, are called Arabians. They are all of one cast. From their living in countries which had not before been the seats of sciences, they give us accounts of some new diseases; and, from the same circumstances, they give some new articles of materia medica. They made also some improvements in surgery, and they were casually the inventors of chemistry; but the general system was still the same as that of Galen, with very little new illustration.

Nor did the knowledge of some new diseases and new remedies make any change in the general state and character of the practice; on the contrary, it appears that we may suppose it to have happened among them as has been observed on other occasions, that a system or theory implicitly followed, gave a more timid and inert practice, than even that of its original author. The cultivation, therefore, of physic by the Arabians did not amount to a great matter, but it was otherwise essentially useful, as it was the means of preserving the knowledge, and of reviving the study of it in the western parts of Europe, in which, as we have said before, it had been almost entirely extinguished. If the Arabs themselves, however, did so little, we have still less to expect from the physicians of Europe, who, from the twelfth to the sixteenth century, were servile imitators, and weak followers of the Arabians; and properly, on this account, denominated by Haller, the Arabistue.

The Saracens, extending their conquests to the western parts of Africa, from thence came over into Spain, and took pos- 
session of that kingdom. There they established schools, and revived a taste for study, which also spread, in some degree, into the different countries of Europe. In the eleventh century, the Arabians, or their disciples, founded schools of physic at Salernum in Italy, and at Montpellier in France, from whence some knowledge of it diffused itself in various directions. In the course of the twelfth century, the university of Paris was established; and not long after, similar institutions were founded in other countries; and by these means some degree of study prevailed, and, with other parts of it, physic had its share. But in this, all the knowledge that appeared was derived from the Arabians; and we have said that among them it was in a very imperfect state; and in being brought into Europe, physic was by no means brought into a soil favourable to its culture. By their learning from the Arabians, the European physicians necessarily became acquainted with the names of Hippocrates and Galen; but being entirely unacquainted with the Greek language, they could not consult their original writings, and could only read them in the very imperfect translations that were made of them, and of such too from the Arabic. Rhazes and Avicenna were the names of most repute among them; and their writings afforded the ordinary text for the lectures of the professors in all the universities of Europe; but it was still the system of Galen that they entirely followed; and the state of all literature and science was so low, that the physicians of those days were in no condition to make either alterations in, or additions to it.

Europe, overrun by the brave but barbarous nations of the north, had fallen into a state of the grossest ignorance and want of literature. War was the sole occupation of the people; and the practice and knowledge of civil arts were almost entirely obliterated. By the Christian religion still subsisting, some remains of literature connected with it were preserved; but the religion itself had degenerated into a gross superstition, which had no tendency to promote peace, or any of its arts. It had rather corrupted the policy of states; and from this and other causes, that policy was every where in a barbarous condition, very unfavourable to study and to the progress of knowledge. Though study, therefore, by the establishment 
of schools, as we have said, had the appearance of being revived, it made a slow progress. Physic in particular had been learned from the Arabian masters of little ability; and it came into the hands of students no ways qualified to surpass their masters. It continued, therefore, in the same state in which it had been acquired, and suffered no sensible improvement till after the middle of the fifteenth century.

About this time, several accidents happened which produced a change in the state of literature. In the beginning of this century some measures had been taken to improve the study of the Latin language, and even some attempts had been made in the study of Greek; when in 1453, the Turks getting possession of Constantinople, obliged many Greeks to seek refuge in Italy. These Greeks were still in possession of their ancient language, and of many writings in it, which they brought with them into Italy; and they contributed greatly to restore and diffuse the study of them in Italy, and by degrees to spread it into other parts of Europe. Where study was in any measure begun, there was no surer means of improving the taste in it than acquainting men with the models of ancient Greece and Rome. This actually took place towards the end of the fifteenth century, and this is properly enough marked as the date of the restoration of letters in Europe. Several other circumstances contributed to favour this progress. The art of printing, so suitable to this purpose, appeared soon after the middle of this century, and before the end of it, had become very general. At the same time, the policies of the most considerable states in Europe having taken on a more steady form, gave more tranquillity to the governments, and more leisure for study. Towards the end of the fifteenth century, also, the discovery of America, and of the new passage to the East Indies by the Cape of Good Hope, gave a new spur to industry in arts and commerce. The sixteenth century therefore, opened with every circumstance favourable to the progress of study, which accordingly proceeded with great vigour. The physicians, in becoming acquainted with the Greek writers, immediately perceived that these were the original sources from whence the Arabians themselves had drawn their information; and it was justly presumed that the most pure knowledge was to be thence obtained. They accord- 
ingly set about the study of the original writings of Hippocrates and Galen. Early in the sixteenth century, the Greek physic was diligently studied; and in the course of that century much pains was bestowed in illustrating the Greek writings, particularly at Paris. A Hippocratic school, as Haller properly enough calls it, was established by Jacobus Sylvius, Fernelius, Hollerius, Duretus, Ballonius, and others. In studying these Greek writings, they imagined that the doctrines had been often corrupted by the Arabians, and therefore they decried these Arabians, and would admit of no knowledge but what was immediately derived from the Greeks. Such, however, is the attachment of mankind to what they have been accustomed to, that some schools, as they had been long in the habit of taking their knowledge from the Arabians, retained some respect for their first masters, and even maintained that in many cases they had improved upon the Greeks. Some physicians, therefore, strictly adhered to the Arabians, upon whose doctrines they had been formed; whilst others were for restoring the practice of the Greeks. Such was the occasion of the famous controversy about blood-letting in pleurisy, in the beginning of the sixteenth century. The Arabians had been accustomed to bleed on the side opposite to the part affected, but it was found that the Greeks bled on the same side. Brissot and several others proposed to follow the Greek physicians, as the higher authority; but many even of considerable rank in those days, defended and followed the practice of the Arabians. The controversy rose so high that the authority of Charles $\mathrm{V}$. was necessary to compose it. This, with other such disputes, contributed to promote the study of the practice, and to make some considerable changes in it.

The Arabians continued to have many followers for a long time after; and even in the middle of the seventeenth century, Rolfinck, a professor of Jena, prelected upon Rhazes, and Plempius of Leyden published and commented upon a work of Avicenna : yet, during the course of the sixteenth century, the party of the Greeks prevailed more and more, and the Arabians came to be entirely neglected.

However, whether physicians pretended to follow the Greeks or the Arabians, the system was fundamentally the same, to wit, that of Galen. Fernelius attempted improvements both in 
the theory and practice, and Argenterius criticised the system of Galen very freely, but they had little effect towards promoting any general change. Something more violent was necessary to shake an authority which had been so long established. This at length happened; and a considerable schism was formed in the schools of physic, which very soon gave occasion to a considerable difference in the state of practice.

Sixth Period.-It has been already remarked, that chemistry appeared first among the Arabians, and it is probable that some of their first operations were upon metallic substances. Accordingly we find a preparation of mercury mentioned in Rhazes; and the invention of distillation, which gave occasion to distinguish an art by the name of Chemistry, happened much about the time that the Arabians first appeared in literature, and they are commonly considered as the inventors of this art. Why the name of chemistry was imposed, or how several different practices were associated under this term, we do not certainly know. It is sufficient to our purpose, that an art under that name was employed in various practices which have at all times since distinguished it, that this art appeared first among the Arabians, and was applied by them to the preparation of medicines. These practices, and the application of them to medicine we have mentioned, were communicated by the Arabians to their disciples in Europe, and spread there with their physic. We find however that the application of chemistry to physic was not considerable, and, probably, from the violence and danger that often attended the operation of chemical medicines, they were little employed by the regular practitioners, that is, by those who were in possession of the schools of physic; and therefore it is that we hear but little of them in the writings of the physicians of the thirteenth, fourteenth, and fifteenth centuries. There is, however, reason to believe that there were then, as at all times since, some more hardy empirical practitioners, who employed chemical remedies, and who were occupied in cultivating the art of preparing them. For, about the end of the fifteenth century, when there was little or no appearance of chemistry in the schools of physic, a chemical writing was published under the feigned name of Basil Valentine, said to have been composed by different persons, but who are yet unknown. 
This book was called the Currus Triumphalis Antimonii ; and from this it appears that chemical pharmacy had been privately cultivated and had made considerable progress. The medicines: now proposed were generally of a violent kind, very different from the mild remedies which the Galenists generally employed, and therefore not likely yet to be received by this sect. But another circumstance at the same time happened to bring chemical remedies into some credit. The Lues Venerea had been lately introduced from America, and yielded to none of the ordinary remedies of the Galenists. It was soon found to yield to nothing so readily as to mercury, which was a remedy hitherto only employed by the chemists. This discovery of the power of mercury, and the publication $I$ have mentioned, recommending the virtues of antimony, prepared the public for favouring chemical remedies; and these might have been received without any alteration in the system of medicine, had it not been that Paracelsus, who came soon after, took advantage of the power of chemical remedies to make a considerable revolution both in the theory and practice of medicine. This noted person was born about the year 1492. He does not appear to have studied in any of the established schools of those days; but, having determined to follow his father's profession, which was that of physic, he seems to have travelled about in quest of remedies amongst all sorts of people, and particularly among the chemical practitioners of those times. From these he learned the use of mercury and antimony ; and from some hardy empirics, the use of opium ; at least, a more free use of this than was then common. By employing these remedies, he was enabled to cure many diseases which had baffled the inert remedies of the Galenists ; and being of a bold and boastful disposition, he made the most of these accidents, while at the same time the partiality of mankind to empiricism soon contributed to give him great fame.

Paracelsus was so far more fortunate than any former chemical practitioner had been in acquiring a general reputation, that he was called to a professor's chair in the university of Basle. In this situation he found it necessary to become systematic; and making use of such theories as he could derive from his predecessors in chemistry, he upon these grounds attempted a system of 
physic, blended with most extravagant and visionary doctrines, supported and covered by a great deal of new and meaningless jargon of his own. His lectures were chiefly employed in recommending his own chemical remedies, and declaiming in the most outrageous manner against the established schools of physic.

$\mathrm{He}$ had the books of Galen and Avicenna brought into his school, and publicly burnt as useless lumber. He thus formed a sect which soon became considerable, in so much that from that time to the middle of the seventeenth century, physicians were divided between the two sects of Chemists and Galenists ; and, as they adhered to one party or the other, they exhibited a different state of the practice of physic. The chemical sect was founded upon an attachment to particular remedies, supposed to be of uncommon power and efficacy. This made them neglect the study and discernment of diseases, and rendered them pure empirics; employing the remedies at random, and solely intent upon multiplying these, they introduced a great number of the inert and purely superstitious kind. On the other hand, the Galenists were more attached to the study of their system than to the invention of remedies; they practised, therefore, with more discernment, and made some progress in the knowledge of diseases; but, while they opposed the new remedies of the chemists, their practice, as far as it depended upon pharmaceutical remedies, was feeble and inert.

It was indeed a symptom of the most absolute languor in the pursuit of science, that the system of Galen had now subsisted for 1400 years, unrivalled and undisturbed, and it was a lucky incident, that the noise and bustling of Paracelsus occurred to rouse the schools of physic from the lethargy they were then under, and to shake off that exclusive attachment to one system, which is always a check to the progress of science. It happened fortunately, at the same time, that the study of anatomy was revived, and that its chief improver, Vesalius, was industrious in marking the errors of Galen, and thereby lessening his general credit and authority. Galen however was still supported by the slavish attachment that is liable to prevail among men with regard to those opinions, with which they have

vor. $\mathrm{I}$. 
been first imbued, and particularly by this, as we have observed, that his philosophy was the same with that of Aristotle.

In the fiftenth and sixteenth centuries, the philosophy of Aristotle had the entire possession of the public schools; but at this period a spirit of inquiry necessarily began to produce some doubts and differences of opinion, and accordingly some efforts were made to introduce other systems. These efforts, however, were feeble; and no change was produced till the time when Galileo and Bacon introduced, if not a new philosophy, at least a new method of philosophizing. This method, consisting of mathematical reasonings and experimental inquiries, had so many advantages over that which was followed by the schools, and so readily discovered the errors and frivolities of these, that it soon weakened the authority, and at length entirely exploded the system of the Aristotelians.

The system of Galen in physic was upon the same foundation, and therefore necessarily shared in the same ruin. At the same time the dogmatic system of the chemists, though neither better founded, nor more applicable than that of the Galenists, yet as being in opposition to the tyranny of establishment, was received with some degree of favour by the followers of the new method of philosophizing; and, as the arts of the chemists afforded and promised many assistances to the new scheme of experimental philosophy, the chemists in general were cherished and protected.

It was in Germany that the chemical practitioners especially prevailed; and there was hardly a sovereign court in that country in which an alchymist and a chemical practitioner of physic were not retained. Even the Galenical practitioners there came soon to employ the remedies of the Chemists; and Sennertus, one of the most eminent Galenists of Germany, endeavoured to reconcile the two opposite parties.

Linacre and Kay, the restorers of physic in England, were zealous Galenists; but as no regular school of physic was ever well established in this country, the persons destined to physic chiefly resorted to the schools of Italy and France, where they generally became Galenists; and although the London college 
showed some disposition to oppress the chemical practitioners in the person of Francis Antony, it was more under the pretence of checking quackery than of opposing chemistry.

Very early in the seventeenth century, Sir Theodore Mayerne, who as a chemical physician had been much opposed and oppressed by the Galenists of France, was called over into England, where he was appointed first physician to the king, and continued to hold that office for more than thirty years after. His theory and his prescriptions were very like those of the Galenists; but he was a greater favourer of chemical medicines, and particularly of antimony, the medicine with regard to which the two sects were most especially divided. It does not however appear, that upon this account he met with any opposition from the physicians of England; and, indeed, on the contrary, we find him becoming a member of, and acquiring great authority in the London, college. It is probable that this great credit put an end, in England, to all distinction between the Galenic and chemical practitioners; and the year 1666, when the faculty of physic of Paris, by a solemn decree restored the use of antimony, which, just a hundred years before, they had condemned, may be considered as the period of the downfall of Galenism, and of the full establishment of chemical remedies. The philosophy of Des Cartes also, which adopted so much from the doctrines of the chemists, united readily with their general system, and established its credit.

The Chemists, thus in possession of the schools, became less empirical, and necessarily more systematic; but this was attended with no improvement in their medical practice. They never were men of liberal and comprehensive views, and they formed only a short imperfect system of acid and alkali, which neither led to the study of diseases, nor to the improvement of remedies. They were still within the very narrow bounds of a sect, almost entirely neglected blood-letting, and in many respects they corrupted or limited the practice. The Cartesians, as we have said, adopted the chemical system, and made some additions to it in so far as they considered the consistence as well as the quality of the fluids. They were accordingly the first who introduced the consideration of viscidity or lentor, which has since made such a figure in our systems. 
Chemistry now prevailed, and the authority of Aristotle in philosophy, and that of Galen in physic, were destroyed, or very much weakened; and, in general, men were prepared for new opinions, when Dr. Harvey, by publishing his doctrine of the circulation of the blood, finished the ruin of the whole Galenic fabric, and at the same time made way for the correction of the chemical system, or at least obviated the bias that then ran too strong in its favour.

For a hundred years before Dr. Harvey, anatomists had been daily improving in the knowledge of the human body, but they had not yet acquired any proper or just view of the connexion of the whole, and therefore were not in a condition to reason properly concerning its functions. The chemists hardly contributed to any improvement in this respect; they only afforded a different language with regard to the different states of the fluids. It was the knowledge of the circulation of the blood, which had been aimed at by several physicians before but only completed by Dr Harvey, that gave some view of the connexion of the whole system. About six years before the publication of Dr. Harvey's work, Asellius had discovered the Lacteals; and about the time that the doctrine of the circulation came to be generally received in Europe, (1650) Pecquet had discovered the receptacle of the chyle and thoracic duct, so that, the true course of the blood and of the chyle being now known, it was very properly observed by Gassendi, that it was upon these two poles that the system of physic must afterwards turn. They had immediately the effect of removing the liver from the important function it had held so long in the system of Galen.

It was to be expected, that the discovery of the circulation of the blood would have considerable effects on the system of physic, which must be always correct and complete in proportion to our knowledge of the structure and mechanism of the human body. Hitherto the anatomists had laboured, as we may say, only on the detached and separate parts of the economy, without perceiving the connexion of the whole, or, what was worse, they had the false view which Galen had given of it. It was the discovery of the circulation of the blood, and of the course of the chyle, that first gave the pro- 
per view of the hydraulic apparatus in the animal economy, and seemingly of the whole of the system of animal bodies. From this period only, therefore, physicians were in a condition of attempting a system. They have since made considerable progress in it; and it is now our business to give you some account of the general state of medicine, from that time to the present.

When, after many ages of darkness, which had destroyed almost the whole of ancient literature, learning was again restored in the fifteenth century, so, from causes which are well known, it was the system of Galen alone that the physicians of those days became acquainted with ; and during the course of the sixteenth century, the study of physicians was almost solely employed in explaining and confirming that system. Early, indeed, in the sixteenth century, the noted Paracelsus had laid the foundation of a chemical system which was in direct opposition to that of Galen; and, by the efficacy of the medicines employed by Paracelsus and his followers, their system came to be received by many : but the systematic physicians continued to be chiefly Galenists, and kept possession of the schools till the middle of the seventeeth century. It is not, however, necessary to enter here into any further detail respecting the fate of those two.opposite sects; for the only circumstance concerning them which I would wish at present to point out, is, that in the writings of both, the explanations they severally attempted to give of the phenomena of health or sickness, turned very entirely upon the state of the fluids of the body.

Such was the state of the science of physic till about the middle of the seventeenth century, when the circulation of the blood came to be generally known and admitted; and when this, together with the discovery of the receptacle of the chyle, and of the thoracic duct, finally exploded the Galenic system. About the same period, a. considerable revolution had taken place in the system of natural philosophy. In the course of the seventeeth century, Galileo had introduced mathematical reasoning; and Lord Bacon having proposed the method of induction, had thereby excited a disposition to observe facts, and to make experiments. These new modes of philosophizing, it might be supposed, would soon have had some influence on the 
state of medicine; but the progress of this was slow. The knowledge of the circulation did indeed necessarily lead to the consideration as well as to a clearer view of the organic system in animal bodies; which again led to the application of the mechanical philosophy towards explaining the phenomena of the animal economy; and it was applied accordingly, and continued, till very lately, to be the fashionable mode of reasoning on the subject. Such reasoning, indeed, must still in several respects continue to be applied: but it would be easy to shew that it neither could, nor ever can be, applied to any great extent in explaining the animal economy; and we must therefore look for other circumstances which had a greater share in modelling the system of physic.

With this view it may be remarked, that, till the period just now mentioned, every physician, whether Galenist or chemist, had been so much accustomed to consider the state and condition of the fluids, both as the cause of disease and as the foundation for explaining the operation of medicines, that what we may term an Humoral Pathology, still continued to make a great part of every system. In these circumstances, it was soon perceived that chemistry promised a much better explanation than the Galenic or Aristotelian philosophy had done; and, therefore, while the latter was entirely laid aside, a chemical reasoning was every where received. Lord Bacon, with his usual sagacity, had early observed that chemistry promised a great number of facts, and he thereby gave it credit; whilst the Corpuscularian philosophy, restored by Gassendi, readily united with the reasonings of the chemists; and the philosophy of Des Cartes readily united with both. From all these circumstances, an humoral, and chiefly a chemical pathology, came to prevail very much till the end of the last century; and has indeed continued to have a great share in our systems down to the present time.

We have thus deduced the history of physic through many ages, in the course of which it has appeared under various forms. It has had the fate of literature in general, to be sometimes neglected and sometimes cultivated, and when the last took place, it has always followed the fate of natural philosophy, from which it is inseparable. For it is difficult for me to be- 
lieve, that any man is so well determined to empiricism, as to abstain from reasoning altogether; and while most men indulge in it, the reasoning in physic must be good or bad in proportion to the knowledge of nature, the mode of philosophizing, and the ardour in the culture of philosophy, that prevails for the time, or which the particular physician possesses. Now, if we view physic as dependent on these circumstances, we shall not be surprised if it is yet far from perfection. Consider how little of the time which this world is supposed to have existed, has been employed in the culture of science; or, if it is alleged that some culture has always appeared, consider to what small portions of the earth that has been commonly confined, how often it has been checked by premature regulations, how often perverted by superstition, and interrupted by wars, and how often the little accumulation that has been made by polished nations, in many ages, has been destroyed in a moment by the irruptions of barbarians. Whoever considers the history of mankind, will perceive why physic and science have been so long of attaining the heights they aim at. But.we must observe farther, that a moderate degree of refinement, even in a small nation, might produce poets, orators, historians, and every thing that depends on the general culture of the human mind, but it is Experience only that can produce natural knowledge; and experience can only be acquired by many hands and repeated labours; it can only be the uninterrupted work of many ages, secured in leisure, provided with its proper instruments, and admitted to a communication with all the parts of the globe,-and all this under the conduct of the abstract sciences of mathematics and metaphysics.

Consider all this, and compare the state of Europe for a hundred years past with any former period, and you will perceive why physic has not yet made the progress it may be expected to make, why hitherto its experience has been little, imperfect, and incorrect, and why its reasonings have been conjectural, and for the most part frivolous. The history which we have already delivered, therefore, is more a matter of curiosity than of use; but we are now to enter upon our seventh and last period, which is more interesting; and if it does not exhibit medicine near to perfection, yet, by the progress already 
made, and the plan that is pursued, it gives us encouragement to aspire, and great hopes of high attainment.

Seventh Period.-You must here observe, that the effects of a dogmatic system depend not so much upon the peculiarity of its being dogmatic, as upon its being limited in its views with respect to the animal system, and thereby becoming attached to certain remedies, whilst it absolutely rejects others, and, by doing so, shuts out experience, which should correct both. But this had been the fault of all the systems hitherto. The Methodics were too limited in their view of the organic system; and although Galen cannot be said to have absolutely neglected the organic system, since he admitted the doctrine of Plethora, yet it is sufficiently evident that his system turned almost entirely upon the intemperies of the fluids, and his followers were very properly, by Van Helmont, called Humoristae. The Chemists who succeeded the Galenists, neglected still more the view of the organic system, and even that which Van Helmont, who was of their own sect, had opened to them. In short, the chemists generally became more strictly humorists than their predecessors, especially as they absolutely rejected the doctrine of Plethora. All this produced a limited state of practice; and it was such among the Galenists, Chemists, and Cartesians, till after the middle of the seventeenth century. But now the doctrine of the circulation, coming to be fully understood, in some measure forced the attention of physicians towards the organic system. The study of mathematics prevailing at the same time, the mathematical physicians appeared, who were necessarily attached to the study of the hydraulic system; and the Cartesians became blended by degrees with these mathematical physicians, so that the system of physic became gradually enlarged. The state of it, however, in the hands of Sylvius de le Boe, Willis, and Etmuller, the chief systematics for some time after the middle of the seventeenth century, was still much limited.

The effect to be especially expected from the discovery of the circulation, was, that physicians should immediately apprehend that interruptions and irregularities in the motion of the fluids have a very large share in the diseases of the body, and perhaps much more than changes in the condition of the fluids, to which diseases had hitherto been imputed by the chemists 
and Cartesians. It was, I say, to be expected that, upon the discovery of the circulation; physicians should immediately study the laws of the hydraulic system; but matters were hardly yet prepared for this purpose. The mathematicians had not as yet ascertained the general principles ; and, though they had, most of the data necessary for their application were still wanting, and only to be obtained from the farther labours of anatomists. However, the mathematical school of Galileo, as in other matters took also the lead in this, and Borelli gave his ingenious work " de motu Animalium." Borelli, however, wàs no physician, but his disciple Bellini was; it was he who extended the application of mathematics to both the physiology and pathology of medicine. His system had such a specious appearance, promised so much more certainty than the former reasonings of physicians, and coincided so exactly with the reigning taste in philosophy, that it immediately prevailed in Italy ; and being brought to this side of the Alps by Dr. Pitcairn, it soon became the prevailing system in Holland, England, France, and even in Germany. In all these countries the mechanical physic came into fashion, and was the prevailing system till very lately; but with what advantage, is still disputed, and we shall consider it in another place. In the mean time we must observe, that whether it was with advantage or not, many improvements have been derived from mathematics to the system of physic: they have certainly contributed to put physic in the good condition in which it is at present. For, agreeably to the plan that was laid down by the mechanical philosophers, the mechanical physicians were always declared friends to observation and experiment, and they were highly useful both in detecting the falsehood of particular hypotheses, and in exploding the use of them in general. This established the innocence of the theories of the present age, that the particular inferences drawn from them are always subjected to the test of experience; and with regard to the most noted leaders in them, and particularly the mechanical philosophers, it can hardly be alleged that their speculations have ever slackened their diligence in observation and experiment, or rendered them averse to those presented by others. We have a particular confirmation of this in one re- 
markable event in the history of modern physic. Soon after the middle of the last century, when the cultivation of the modern philosophy was begun, but had made little progress, Sydenham appeared.

Dr. Sydenham was bred in physic rather by his own industry than by any instruction, and therefore entered upon it free from the attachments and prejudices of any sect. He certainly looked into the writings of many, but, imbued with the taste of philosophy then prevailing, he could not be satisfied with any of the theories he met with. Setting out, therefore, with the doctrine of the Autocrateia, he proposed upon that plan, by his own observation alone, to form a system of practice for himself. On this account he was more employed in observation, than any person had been that we know of from the time of Hippocrates; and though he had always some theory in view, he was less governed by it than any of his predecessors. This is the peculiarity of his conduct, that he sought rather for theory to unite his observations under some general heads, than for facts to confirm his theory. (See the Dissert. de Hydrope, p. 493.) He had also this particular advantage, that he had no general $\mathrm{Pa}$ thology, but endeavoured to form a particular one for every particular disease, or at least for every genus of disease. It is thus that Sydenham has given a model for the cultivation of the practice of physic, which has been followed by every good practitioner since. Every one nowadays pretends to neglect theory, and to stick to observation. But the first is in talk only, for every man has his theory, good or bad, which he occasionally employs; and the only difference is, that weak men who have little extent of ability for, or have had little experience in reasoning, are most liable to be attached to frivolous theories; but the truly judicious practitioners, and good observers, are such as have the most extensive views of the animal economy, and know best the true account of the present state of theory, and therefore know best where to stop in the application of it. Such persons may have formed to themselves a general scheme of Pathology; but admitting it as general only, they are never so much attached to it, but that they can form one for a particular disease by itself, as they may be directed by observation. It is a leading prin- 
ciple with them that, whatever theories they form, they are al ways ready to allow experience to correct every part of their system. Thus it is, that, from the example of Sydenham, and the general mode of philosophizing now established, the practice of physic is now, for the most part, cultivated free from the attachments or prejudices of any sect. Even our systems ar e much enlarged in their general plan; and however they may differ from each other in particulars, this affects only weak minds with the spirit of a sect.

In pursuing, with wonderful steadiness and sagacity, the scheme of studying medicine which he had adopted, Sydenham has given us a work that is allowed, so far as it goes, to be more useful in the practice of physic, than any that had ever appeared before; and certainly it was more valuable than any that we know to have been produced by a single person. It was the result of experience and observation alone; and though on many occasions he had a theory in view, it was without ever admitting its undue influence. He was, perhaps, the first that shewed us that there might be a great deal of theory in a man's head without its affecting his practice. Dr., Sydenham's countrymen and contemporaries, Willis, Morton, and Lister, who were in the old way of philosophizing, and very much in the Chemical and Cartesian systems, neglected and opposed him ; while the mechanical physicians, Pitcairn and Boerhaave, received his facts themselves, and first recommended them to the attention of others.

It is proper now, however, to observe, that about the beginning of the present (eighteenth) century, when every part of science came to be on a more improved and correct footing, there appeared in the writings of Stahl, of Hoffmann, and of Boerhaave, three new and considerably different systems of physic, which have ever since had a great share in directing the practice of it. In order, therefore, to give a nearer view of the present state of physic, I shall offer some remarks upon these different systems, endeavouring to point out the advantages as well as the disadvantages of each, and how far they still prevail, or, according to my judgment, deserve to do so.

I shall begin with considering that of Dr. Stahl, which I 
think appeared first, and for a long time after was the prevailing system in Germany.

The chief and leading principle of this system is, that the rational soul of man governs the whole economy of his body. At all times physicians have observed, that the animal economy has in itself a power or condition, by which, in many instances, it resists the injuries which threaten it ; and by which it also, on many occasions, corrects or removes the disorders induced or arising in it. This power physicians very anciently attributed, under a vague idea, to an agent in the system, which they called NATURE; and the language of a vis conservatrix et medicatrix naturae, has continued in the schools of medicine from the most ancient times to the present.-(Physiology CXX.)

Numberless phenomena lead to the notion of a primary moving power in the animal economy. We perceive that in the Timaeus of Plato notice is taken of an anima presiding over the functions of the material part of our bodies, and even Hippocrates marked toे ह่ofereãy or impetum faciens, in his system. The operation of this power is so often suited to the obviating of injuries and correcting the deviations of the system, that it is no wonder if men supposed it to be directed by some intelligence. Hence the notion of a sensitive soul among the philosophers of the last age. Van Helmont supposed an intelligent Archaeus, governing many of the functions of the body; Dolaeus, making use of a singular jargon, proposed his Microcosmetor, and Wepfer a Proses systematis nervosi, all on the same footing. But Dr. Stahl went beyond them all, referring the government of the body directly and entirely to the rational soul ; and by this he established the notion of a complete intelligence and absolute power in the soul with respect to the body.

Dr. Stahl has explicitly founded his system on the' supposition, that the power of nature, so much talked of, is entirely in the rational soul. He supposes, that, upon many occasions, the soul acts independently of the state of the body ; and that, without any physical necessity arising from that state, the soul, purely in consequence of its intelligence, perceiving the tendency of noxious powers threatening, or of disorders anywise arising in the system, immediately excites such motions in the 
body as are suited to obviate the hurtful or pernicious consequences which might otherwise take place. Many of my readers may think it was hardly necessary for me to take notice of a system founded upon so fanciful an hypothesis; but there is often so much seeming appearance of intelligence and design in the operations of the animal economy, that many eminent persons, as Perrault in France, Nichols and Mead in England, Porterfield and Simpson in Scotland, and Gaubius in Holland, have very much countenanced the same opinion; and it is therefore certainly entitled to some regard. It is not, however, necessary for me here to enter into any refutation of it. Dr. Hoffmann has done this fully in his Commentarius de differentia inter Hoffmanni doctrinam medico-mechanicam et G. E. Stahlii medico-organicam; and both Boerhaave and Haller, though no favourers of materialism, have maintained a doctrine very opposite to that of Stahl.

In my Physiology, (XXXI., CXXII.) I have offered some arguments against the same; and I shall only add now, that whoever considers what has been said by Dr. Nichols in his Oratio de Anima Medica, and by Dr. Gaubius in some parts of his Pathology, must perceive that the admitting of such a capricious government of the animal economy, as these authors in some instances suppose, would at once lead us to reject all the physical and mechanical reasoning we might employ concerning the human body. Dr. Stahl himself seems to have been aware of this ; and therefore, in his Preface to Juncker's Conspectus Therapeiae Specialis, has acknowledged, that his general principle was not at all necessary; which is in effect saying, that it is not compatible with any system of principles that ought to govern our practice. Upon this footing, I might have at once rejected the Stahlian principle : but it is even dangerous to bring any such principle into view ; for, after all Dr. Stahl had said in the passage just now referred to, I find, that, in the whole of their practice, both he and his followers have been very much governed by their general principle. Trusting much to the constant attention and wisdom of nature, they have proposed the Art of curing by expectation; have therefore, for the most part, proposed only very inert and frivolous remedies; have zealously opposed the 
use of some of the most efficacious, such as opium and the Peruvian bark; and are extremely reserved in the use of general remedies, such as bleeding, vomiting, \&c.

Although these remarks upon a system which may now be considered as exploded or neglected, may seem superfluous, I have been willing to give these strictures on the Stahlian system, that I might carry my remarks a little farther; and take this opportunity of observing, that, in whatever manner we may explain what have been called the operations of nature, it appears to me, that the general doctrine of Nature curing diseases, the so much vaunted Hippocratic method of curing, has often had a very baneful influence on the practice of physic, as either leading physicians into, or continuing them in a weak and feeble practice, and at the same time superseding or discouraging all the attempts of art. Dr. Huxham has properly observed, that even in the hands of Sydenham it had this effect. Although it may sometimes avoid the mischiefs of bold and rash practitioners, yet it certainly produces that caution and timidity, which have ever opposed the introduction of new and efficacious remedies. The opposition to chemical medicines in the sixteenth and seventeenth centuries, and the noted condemnation of antimony by the medical faculty of Paris, are to be attributed chiefly to those prejudices which the physicians of France did not entirely get the better of for near an hundred years after. We may take notice of the reserve it produced in Boerhaave, with respect to the use of the Peruvian bark. We have had lately published, under the title of Constitutiones Epidemicae, notes of the particular practice of the late Baron Van Swieten, upon which the editor very properly observes, that the use of the bark in intermitting fevers appears very rarely in that practice; and we know very well where Van Swieten learned that reserve.

I might go further, and shew how much the attention to the Autocrateia, allowed of in one shape or other, by every sect, has corrupted the practice among all physicians, from Hippocrates to Stahl. It must, however, be sufficiently obvious; and I shall conclude the subject with observing, that although the vis medicatrix natura must unavoidably be received as a fact, yet, 
wherever it is admitted, it throws an obscurity upon our system; and it is only where the impotence of our art is very manifest and considerable, that we ought to admit of it in practice.

To finish our remarks upon the Stahlian system, I shall shortly observe, that it did not depend entirely upon the Autocrateia, but also supposed a state of the body and diseases that admitted of remedies, which, under the power and direction of the soul, acted upon the organization and matter of the body so as to cure its diseases. Upon this footing the Stahlian pathology turned entirely upon Plethora and Cacochymy. It was with respect to, the former that they especially applied their doctrine of the Autocrateia in a very fanatical manner; and, with respect to the latter, they have been involved in a humoral pathology as much as the systematic physicians who had gone before them, and with a theory so incorrect as not to merit now the smallest. attention. After all, I ought not to dismiss the consideration of the Stahlian system without remarking that, as the followers of this system were very intent upon observing the method of nature, so they were very attentive in observing the phenomena of diseases, and have given us in their writings many facts not to be found elsewhere.

While the doctrines of Stahl were prevailing in the university of Halle, Dr. Hoffmann, a professor in the same university, proposed a system that was very different. He received into his system a great deal of the Mechanical, Cartesian, and Chemical doctrines of the systems which had appeared before; but, with respect to these, it is of no consequence to observe in what manner he modified the doctrines of his predecessors, as his improvements in these respects were nowise considerable, and no part of them now remain; and the real value of his works, beyond what I am just now going to mention, rests entirely on the many facts they contain. 'The merit of Dr. Hoffmann and of his work is, that he made, or rather suggested an addition to the system, which highly deserves our attention. Of this I cannot give a clearer account than by giving it in the author's own words. In his Medicina Rationalis Systematica, tom. iii. sect. i. chap. 4. he has given his "Genealogia morborum ex turbato solidorum et fluidorum mechanismo ;" and in the 46th and last paragraph of this chapter, he sums up his doctrine in the following words :- 
' Ex hisce autem omnibus uberius hactenus excussis, perquam dilucide apparere arbitror, quod solus spasmus et simplex atonia, aequabilem, liberum, ac proportionatum sanguinis omnisque generis fluidorum motum, quibus excretionum successus et integritas functionum animi et corporis proxime nititur, turbando ac pervertendo, universam vitalem oeconomiam subruant ac destruant; atque hinc universa pathologia longe rectius atque facilius ex vitio motuum microcosmicorum in solidis quam ex variis affectionibus vitiosorum humorum, deduci atque explicari possit, adeoque omnis generis aegritudines internae, ad praeternaturales generis nervosi affectiones sint referendae. Etenim laesis quocunque modo, vel nervis per corpus discurrentibus, vel membranosis quibusvis nervosis partibus, illico motuum anomaliae, modo leviores, modo graviores, subsequuntur. Deinde attenta observatio docet, motus quosvis morbosos principaliter sedem figere et tyrannidem exercere in nervosis corporis partibus, cujus generis praeter omnes canales, qui systaltico et diastaltico motu pollentes, contentos succos tradunt, universum nimirum intestinorum et ventriculi ab oesophago ad anum canalem, totum systema vasorum arteriosorum, ductuum biliariorum, salivalium, urinariorum et subcutaneorum, sunt quoque membranae nerveo-musculares cerebri et medullae spinalis, praesertim haec, quae dura mater vocatur, organis sensoriis obductae, nec non tunicae illae ac ligamenta, quae ossa cingunt artusque firmant. Nam nullus dolor, nulla inflammatio, nullus spasmus, nulla motus et sensus impotentia, nulla febris aut humoris ullius excretio, accidit, in qua non hae partes patiantur Porro etiam omnes, quae morbos gignunt causae, operationem suam potissimum perficiunt in partes motu et sensu praeditas, et canales ex his coagmentatos, eorum motum, et cum hoc fluidorum cursum, pervertendo ; ita tamen, ut sicuti variae indolis sunt, sic etiam varie in nerveas partes agant, iisdemque noxam afficent. Demum omnia quoque eximiae virtutis medicamenta, non tam in partes fluidas, earum crasin ac intemperiem corrigendo, quam potius in solidas et nervosas, earundem motus alterando ac moderando, suam edunt operationem. De quibus tamen omnibus, in vulgari usque eo recepta morborum doctrina, altum est silentium.'

The dissection of the nervous system was, I think, the part 
most neglected by the restorers of anatomy, and by almost all the anatomists who followed, till the middle of the last century. About that time Dr. Willis, with the assistance of Lower, applied more earnestly to the dissection of the brain and nerves; and, perhaps from thence, was led to study the pathology of the nervous system. He accordingly made a beginning on this subject; but his labours generally were so unsuitable to the taste then arising in philosophy, that he was not likely to become a leader, and his doctrine of the nerves made no progxess. The doctrine, however, was in general of such importance, that it could not fail to be cultivated; and accordingly, soon after, it was so by Baglivi in Italy, and by Hoffmann in Germany. Baglivi gave his excellent specimen de fibra motrici et morbosa; but although possessed of a theoretical genius, he was much attached to observation, and his practical labour occupied so entirely the short life he enjoyed, that he could not complete his theoretical system; this, with some considerable errors in anatomy which he had committed, prevented his making any party beyond the bounds' of Italy - at least Hecquet is the only one I know of elsewhere, who followed him. It is true, that Dr. Willis had laid a foundation for this doctrine, in his Pathologia Cerebri et Nervorum; but by him as well as Baglivi, it was either not extensively applied to diseases, or was still so involved in many physiological errors, that it had attracted little attention; and Dr. Hoffmann was the first who gave any tolerably simple and clear system on the subject, or pointed out any extensive application of it to the explanation of diseases.

By these, and many other means, the doctrine of the nerves has now a full share in our general system. And as it appears to me certain, that the human system can only be viewed in these three respects, that is, as a chemical mixt, as a hydraulic machine, and as an animated nervous frame, I consider our system of physic to be now complete as to the parts which it ought to comprehend.

There can be no sort of doubt, that the phenomena of the animal economy in health and in sickness, can only be explained by considering the state and affections of the primary moving powers in it. It is to me surprising, that physicians were so long of perceiving this, and I think we are therefore particularly

vol. I. 
indebted to Dr. Hoffmann for putting us into the proper train of investigation; and it every day appears, that physicians perceive the necessity of entering more and more into this inquiry. It was this, I think, which engaged Dr. Kaaw Boerhaave to publish his work, entitled Impetum faciens; as well as Dr. Gaubius to give the Pathology of the Solidum vivum. Even the Baron Van Swieten has, upon the same view, thought it necessary, in at least one particular, to make a very considerable change in the doctrine of his master, as he has done in his Commentary upon the 755th Aphorism. Dr. Haller has advanced this part of science very much by his experiments on irritability and sensibility. In these, and in many other instances of some progress in the study of the affections of the Nervous System, (particularly in the writings of Mr. Barthez of Montpellier,) we must perceive how much we are indebted to Dr. Hoffmann for his so properly beginning it. The subject, however, is difficult: the laws of the Nervous System, in the various circumstances of the animal economy, are by no means ascertained; and, from want of attention and observation with the view to a system on this subject, the business appears to many as an inexplicable mystery. There is no wonder, therefore, that on such a difficult subject, Dr. Hoffmann's system was imperfect and incorrect, and has had less influence on the writings and practice of physicians since his time than might have been expected. He himself has not applied his fundamental doctrine so extensively as he might have done; and he has everywhere intermixed an Humoral Pathology, as incorrect and hypothetical as any other. Though he differed from his colleague, Dr Stahl, in the fundamental doctrines of his system, it is but too evident that he was very much infected with the Stahlian doctrines of Plethora and Cacochymy, as may be observed throughout the whole course of his work ; and particularly in his chapter, 'de morborum generatione ex nimia sanguinis quantitate et humorum impuritate.'

But it is needless for me to dwell any longer upon the system of Hoffmann : and I am next to offer some remarks on the system of Dr. Boerhaave, the cotemporary of both the other systematics, and who, over all Europe, and especially in this part of the world, gained higher reputation than either of the others. 
Dr. Boerhaave was a man of general erudition; and, in applying to medicine, he had carefully studied the auxiliary branches of anatomy, chemistry, and botany, so that he excelled in each. In forming a system of physic, he seems to have studied diligently all the several writings of both ancient and modern physicians; and, without prejudice in favour of any former systems, he endeavoured to be a candid and genuine Eclectic. When Dr. Boerhaave came into the school of Leyden, about the begining of this century, he found that school divided between the chemical system of Sylvius de le Boe, and the mechanical one of Bellini and Pitcairn. His sagacity perceived the general utility of both, and his diseernment selected very properly from each. From Bellini he took the doctrine of obstruction, and at the same time the doctrine of lentor, which Bellini had taken from the Cartesians. From the chemists Boerhaave took the doctrine of acid and alkali, but he very properly limited and corrected it. He thus combined the doctrines of the Mechanicians, Cartesians, and Chemists, and also admitted the doctrine of Plethora, the only remains of the Galenical theory, which the discovery of the circulation of the blood tended to support. Possessed of an excellent systematic genius, he gave a system superior to any that ever before appeared. As in the great extent, and seemingly perfect consistency of sys tem, he appeared to improve and refine upon every thing that had before been offered, and as in his lectures he explained his doctrines with great clearness and elegance, he soon acquired a very high reputation, and his system was more generally received than any previous one had been since the time of Galen. Whoever will consider the merits of Dr. Boerhaave, and can compare his system with that of former writers, must acknowledge that he was very justly esteemed, and gave a system which was at that time deservedly valued.

But, in the progress of an inquisitive and industrious age, it was not to be expected, that any system should last so long as Boerhaave's has done. The elaborate Commentary of Van Swieten on Boerhaave's system of practice, has been only finished a few years ago; and though this commentator has added many facts, and made some corrections, he has not, except in the particular mentioned above, made any improvement in the 
general system. It is even surprising, that Boerhaave himself, though he lived near forty years after he had first formed his system, had hardly in all that time made any corrections of it or additions to it: the following is the most remarkable:-In aphorism 755, the words ' forte et nervosi, tam cerebri quam cerebelli cordi destinati inertia,' did not appear in any edition before the fourth; and what a difference of system this points at every physician must perceive.

- When I first applied to the study of physic, I learned only the system of Boerhaave; and even when I came to take a professor's chair in this university, I found that system here in its entire and full force; and as I believe it still subsists in credit elsewhere, and that no other system of reputation has been yet offered to the world, I think it necessary for me to point out particularly the imperfections and deficiencies of the Boerhaavian system, in order to shew the propriety and necessity of attempting a new one. To execute this, however, so fully as I. might, would lead me into a detail that can hardly be admitted of here; and I hope it is not necessary, as I think that every intelligent person, who has acquired any tolerable knowledge of the present state of our science, must, in many instances, perceive its imperfections. I shall therefore touch only upon the great lines of this system; and from the remarks I am: to offer, I trust that both the mistakes and deficiencies which run through the whole of his works will appear.

Dr. Boerhaave's treatise of the diseases of the simple solid has the appearance of being very clear and consistent, and was certainly considered by him as a fundamental doctrine : but, in my apprehension, it is neither correct, nor extensively applicable. Not to mention the useless, and perhaps erroneous notion of the composition of earth and gluten; nor his mistake concerning the structure of compound membranes; nor his inattention to the state of the cellular texture-all of them circumstances which render his doctrine imperfect-I shall insist only upon the whole being very little applicable to the explaining the phenomena of health or sickness. The laxity or rigidity of the simple solid does indeed take place at the different periods of life, and may perhaps, upon other occasions, occur as the cause 
of disease ; but I presume, that the state of the simple solid is, upon few occasions, either changeable or actually changed; and that, in ninety-nine cases out of an hundred, the phenomena attributed to such a change, do truly depend on the state of the solidum vivum, a circumstance which Dr. Boerhaave has hardly taken notice of in any part of his works. How much this shews the deficiency and imperfection of his system, I need not explain. The learned work of Dr. Gaubius, above referred to, as well as many other treatises of late authors, point out sufficiently the defects and imperfections of Boerhaave on this subject.

After Dr. Boerhaave has considered the diseases of the solids, he, in the next place, attempts to explain the more simple diseases of the fluids; and there, indeed, he delivers a more correct doctrine of acid and alkali than had been given before: but, after all, he has done it very imperfectly. We have, indeed, since his time, acquired more knowledge upon the subject of digestion; and so much as to know, that a great deal more is yet necessary, to enable us to understand in what manner the animal fluids are formed from the aliments taken in. And although Dr. Boerhaave has fallen into no considerable error with respect to a morbid acidity in the stomach, he could not possibly be complete upon that subject; and his notion of the effects of acidity in the mass of blood seems to have been entirely mistaken, and is indeed not consistent with what he himself has delivered elsewhere.

His doctrine of alkali is somewhat better founded, but it is probably carried too far; and the state of alkalescency and putrefaction, as well as all the other changes which can take place in the condition of animal fluids, are particulars yet involved in great obscurity, and are therefore still subjects of dispute.

There is another particular, in which Boerhaave's doctrine concerning the fluids appears to me imperfect and unsatisfactory; and that is, in his doctrine de Glutinoso spontaneo. The causes which he has assigned for it are by no means probable, and the actual existence of it is seldom to be proved. Some of the proofs adduced for the existence of the phlegma calidum, are manifestly founded on a mistake with respect to what has been called the inflammatory crust, (See Van Swie- 
ten's Commentary, page 96.) ; and the many examples given by Boerhaave, of a glutinosum appearing in the human body, (Aph. 75.) are all of them nothing more than instances of collections or concretions found out of the course of the circulation.

If, then, we consider the imperfection of Dr. Boerhaave's doctrine with respect to the state and various condition of the animal fluids; and if, at the same time, we reflect how frequently he and his followers have employed the supposition of an acrimony or lentor of the fluids, as causes of disease, and for directing the practice; we must, as I apprehend, be satisfied, that his system is not only deficient and incomplete, but fallacious and apt to mislear. Although it cannot be denied, that the fluids of the human body suffer various morbid changes; and that, upon these, diseases may primarily depend; yet I must beg leave to maintain, that the nature of these changes is seldom understood, and more seldom still is it known when they have taken place; that our reasonings concerning them have been, for the most part, purely hypothetical; have therefore contributed nothing to improve, and have often misled the practice of physic. In this, particularly, they have been hurtful, that they have withdrawn our attention from, and prevented our study of the motions of the animal system, upoil the state of which the phenomena of diseases do more certainly and generally depend. Whoever, then, shall consider the almost total neglect of the state of the moving powers of the animal body; and the prevalence of an hypothetical humoral pathology, so conspicuous in every part of the Boerhaavian system, must be convinced of its very great defects, and perceive the necessity of attempting one more correct.

After giving this general view, it is not requisite to enter into particulars : but, I believe, there are very few pages of his aphorisms in which there does not occur some error or defect; although, perhaps, not to be imputed to the fault of Boerhaave so much as to this, that since his time a great collection of new facts has been acquired by observation and experiment. This, indeed, affords the best and most solid reason for attempting a new system : for, when many new facts have been acquired, it becomes requisite that these should be incorporated into a sys- 
tem, whereby not only particular subjects may be improved, but the whole may be rendered more complete, consistent, and.useful. Every system, indeed, must be valuable in proportion to the number of facts that it embraces and comprehends; and Mons. Quesney could not pay a higher compliment to the system of Boerhaave, than by saying that it exhibited la Médecine collective.

Having now given you some account of the state of the practice of physic at different times, and particularly as it is at present cultivated in Europe, I propose, in the next place, to say on what footing we are to take it up, and upon what general plan we propose to teach this important art.

In entering upon the study of physic, it is of importance to choose a proper plan; but students, beginning the study, are not well qualified to do this for themselves: They are indeed hardly left to do it, as they are in some measure obliged to follow the plan of their professor; and this must in a great measure be the case with most of you. But as you will follow with more steadiness and ardour in proportion as you are more persuaded of the propriety of my plan, I shall here endeavour to satisfy you in this matter, as far as the present state of your knowledge can be supposed to allow.

Whether the study of physic is to be pursued on an Empiric or. a Dogmatic plan, that is, whether we are to be guided by experience alone, or if we must have recourse to reasoning, and may derive some advantage from it, is a question which was long ago agitated among physicians, has often since been discussed, and still subsists among them. From the history of physic, it appears that, for two thousand years past, there have been two plans proposed, to wit, the dogmatic and empiric; and, as upon different occasions both of these have had their free course and seemingly full trial, it might be supposed, that, before now, experience had decided in the choice of these two plans by their several effects. This however has not happened; and physicians are still much divided upon the question. The schools are every where dogmatical, and seemingly from neces- 
sity, while the most part of practitioners at least profess empiricism. The last have occasion so often to declare against theory, that they either influence the opinion of students on this subject, or at least render them undetermined. I consider this as of the worst consequence, that is, for students to be prejudiced against a measure they are obliged to pursue, and it must lead to a pernicious inattention and negligence; for it must.very often happen, that in neglecting what they think is theory, they must miss or neglect the most important facts. It is necessary, therefore, that you should be determined on this subject; but a full discussion of it would require more time than we can bestow upon it. I must suppose that you have in some degree considered it at first entering upon the Institutions of Medicine. If any of you have not had that opportunity or some other such of considering it, I would still earnestly recommend it to your particular attention. The subject has hitherto been treated as a controversy between parties, who always embrace or reject. without distinction; but it will appear that there are advantages in either plan, and that therefore we should endeavour to employ the useful parts, while we carefully avoid and reject the: faults, of both. It is not necessary to determine absolutely either for experience or against reasoning, but it is extremely. necessary to know that both have their imperfections, that both are exposed to fallacies, and that, in the present state of. science, either of them is by itself insufficient. Every body ad" mits the use of experience, and the necessity of consulting it, but few are aware of its being exposed to much fallacy, and that we have more false facts obtruded upon us, and subsisting in: our books, than false reasonings; so that the stock of true facts is very small, and far short of the demands which the practice of physic has for them. On the other hand, no body pleads for the use of theory alone, and every body knows, that it is fallacious, and has often corrupted our art; but few perceive that at the same time it is unavoidable, necessary, and to a certain extent useful. Almost every body can perceive the errors of theory in general, but very few are aware, that, while they declare against it in general, they themselves employ it on particular occasions too freely, and with more mischief, because of their general prejudices. For perceiving and understanding all 
these circumstances more fully, it is very proper that the subject be seriously considered, that these inconsistencies be avoided, and that the true use and proper limits, both of experience and reasoning, be well understood. In assisting you in this matter, I cannot at present go so far as I could wish, but I suspect that the notions of many of you may be too loose and undetermined for my plan, and I shall now say so much as may be necessary to reconcile you to this, and, what is of more consequence, to fix your attention to it with some earnestness.

The object of my present discourse, therefore, shall be to show, that the empiric plan, when employed by itself, is difficult, fallacious, and insufficient; and that the addition of the dogmatic is not only really useful but necessary and unavoidable.

To this purpose I could produce many arguments, but at present I shall confine myself to three only. The first is, that reasoning in physic is unavoidable, and that, to render it safe, it is necessary to cultivate theory in its full extent.

The second argument is, that supposing the chief object of our study to be the acquiring of facts, the study of a dogmatic: system has been the chief means of obtaining those which we have already acquired, and is also the most certain means of acquiring those we still want : it is therefore useful and necessary.

Our third argument is, that there is no tolerable foundation for the study of physic upon an empiric plan, and that all attempts hitherto made towards delivering it upon that footing, have been not only fruitless but pernicious.

These arguments require to be explained more fully; and I: now proceed to do it.

The first argument then is, that reasoning in physic is unavoidable, and that, to render it safe, it is necessary to cultivate theory in its full extent. I maintain this by observing, that there is in human nature a strong propensity to seek for causes, and to assign them also on the slightest grounds; and mankind are very generally guided in their affairs by their judgment of causes and effects. I must own, indeed, that there is nothing: more weak or false than their reasonings often are; but I imagine the propensity is irresistible. Sceptics and Academics may demonstrate the fallacy, or the rash presumption of human reasoning, but thev will never persuade men to give it up, nor even. 
to be restrained, in the use of reasoning. The only remedy for the abuse, that we know of, is the making men better reasoners, the exercising them much on the particular subjects they are to be employed in, and directing their attention to every consideration that may influence their determinations. A physician will sometimes reason in matters of law, but in doing so he gives occasion to the lawyer to smile at his weakness, and I know that a lawyer, in like manner, may be ridiculous in his turn. In this case each profession will perceive the abuse in the other; but, to correct it, neither the lawyer nor the physician will think of persuading his neighbour to give up reasoning in general, but may very properly advise him, to give it up with regard to a subject in which he has not been sufficiently exercised. But it is still doubtful if the advice would be followed; and if there were any propriety in the physician's attempting to reason in law, the only means of rendering it safe, would be to engage him in the study of that science in its full extent.

Now all this applies to physic, and, as I judge, very exactly. Such is the general propensity I have mentioned, that I have not, in all my life, known a single person belonging to the profession who did not, upon many occasions, use reasoning concerning it; and what may fairly be called theory. Every practitioner has daily proofs of the propensity and presumption of his patients in this respect; and among the practitioners themselves, though they can declare that Paracelsus was a knave, that Helmont was a madman, and Des Cartes a fool, and that all theory is nonsense, yet I find that they constantly employ it themselves. This man is plethoric, and therefore must be blooded; that man's stomach is foul, and he must be vomited; a third man's blood is full of acrimony, and he must be purged. Every body acquainted with practitioners must be familiar with reasonings of this kind. The persons who employ them may not perhaps perceive that they are using theory; but I know that they are using it, and that of a bad kind too. I have known a man deemed plethoric who was only fat; I have known a stomach supposed foul, when it was only sympathetically affected; and I have known an acrimony of the blood often concluded from what was merely a cutaneous affection. In short, so far as my observation goes, there is not any one practitioner, even 
the most professed empiric, who does not, upon many occasions, use theory, from a tincture of the school in which he was bred, or from the books he has read. The abuse is indeed often very great, but I take the propensity to be irresistible; and, in my opinion, the only possible means of correcting the abuse is, by engaging men in the study of the theory in its full extent. Every function of the human body is connected with the others, and all of them with some general laws of the whole economy. The whole makes but one system whose parts are connected and mutually depending on one another. The application of particulars to a whole will often discover their fallacy, and the applying any general system to particulars will often discover its true limits. A man who studies the theory of physic with any discernment, will indeed often find errors to be rejected; but, by the same means, he will also avoid many errors that he himself might have been exposed to ; and I will venture to affirm, that he only is provided with a due restraint upon his own reasoning who is best acquainted with the fallacy of that of others. From the whole therefore of what we have now said, I think it is evident that reasoning, and what is called theory in physic, is unavoidable, and that the errors or abuses of it are only to be guarded against by studying it in its full extent, and, therefore, by studying physic on a dogmatic plan. In all this I have argued only from its necessity to enable us to avoid error; that it is directly useful I shall say in another place. But, in the meantime; I proceed to the second argument I proposed to consider here. Our second argument is, that it has been the study of a dogmatic system which has given the facts of physic which have been already acquired, and that the cultivation of a dogmatic system is the most probable means of acquiring the knowledge of those facts upon which we would found an empiric system. The first part of this proposition is easily maintained; for, though chance and accident have given us many facts, it is the dogmatists only who have preserved these, and who have given us many more from the experiments which their system suggested; and they have given many from observations which would neither have been attended to nor preserved, but for their application to a system. I have said before, that the labours of the ancient empiries are now entirely lost, and I shall have occasion to say hereafter, that 
any modern labours upon the same plan are of very little value: It is therefore the dogmatic systems to which we are chiefly obliged for the facts we have already acquired; but it is not quite so obvious that the same study must still conduct us in our farther collection; I shall however endeavour to prove it in this manner.

In the course of my history, in giving an account of the Empiric sect, I took occasion to explain the nature of their plan, and told you that it consisted of three parts, Observation, History' and Analogy; and I can now say that if this plan could be as easily executed, and as successfully applied as the Empirics supposed, we should have few objections to its being the sole plan in physic. But it is more specious than practicable. Observation is extremely difficult : it requires the knowledge of a number of circumstances that are not always obvious, that are very often hidden in their nature, or concealed of design. Even with regard to the more obvious circumstances, observations require an attention to such a variety and series of facts, as few men are equal to ; and, from both considerations, they are so difficult to make, that but few good or complete observations have at any time been collected. It is plain that they can be rendered complete only by opportunities of being frequently repeated. If we consider this, with the prodigious number of subjects upon which observation is required, we shall readily perceive that the work of one man's life can go but a little way in this business ; and the empirics properly enough perceived, that the stock of facts necessary to their plan must be the work of ages, accumulated in History. But this does not relieve their plan of its difficulties ; for history must partake of all the imperfections and inaccuracies of observation, and might continue these faults by seeming to supersede the repetition which was necessary to correct them. But further, when we consider that the particulars of observation are often sensations difficult to be communicated; that they are often estimates made by our senses, very different in different persons ; that all these, as conveyed in history, are under the imperfections of language and men's different interpretations of this; we shall perceive that history not only carries on the inaccuracy of observation, but also greatly increases its imperfection. If to all this we add what we are well assured of, 
that, partly from mistake and partly from design, history, at all times, has in many particulars been absolutely false, we shall, upon the whole, perceive that observation and history afford a very precarious foundation for the practice of physic on an empiric plan. You know that the advocates of this plan propose that the practice of physic should be, as much as possible, conducted by a simple imitation; that is, when a disease occurs, they should, either in their own observation or in the history of those of others, find a case exactly like to the present, and that therefore they should in the present case employ the remedy which had been experienced useful in the former. But if we only consider the number and diversity of diseases, the difficulty of observation, and the fallacy of history, we shall be led to expect what has actually happened, that simple imitation has seldom been practised:

The empirics themselves foresaw all this, and therefore proposed to provide against it by having recourse to Analogy. This proceeds upon a supposition that there is in many diseases seemingly different, somewhat of a common nature; so that the remedies found useful in one of these, may be presumed to be useful also in others resembling it. This common nature of diseases the empirics presumed to learn entirely from hence that they had some symptoms in common with each other; and this supposes the study of a Nosologia methodica. The proceeding must be in this way: Among the concurring. symptoms of diseases, we find some more constant, others more accidental, and thus distinguish species from varieties; and still proceeding farther, we may find that even amongst the symptoms constituting species, there are some more constant than others, and may thereby unite several species into one genus. Now, if we may presume that the treatment of one species of disease is applicable to all those of the same genus, we have a foundation from an analogy tolerably extensive, and entirely independent of theory. The scheme is probable, but the execution is difficult. The Nosologia methodica required is not yet obtained. What the ancient empirics might contribute to this purpose we have not learned. We find that in modern times, till very lately, no attempt was made in this way but by Felix Plater, who, however, did not propose to separate this study 
from a dogmatic system, and, either with a view to the one or to the other system, he has given us a very imperfect work. It has only been within these forty years, that M. de Sauvages has renewed the attempt, and carried it somewhat farther; but even in a fourth edition his work is very imperfect and incomplete; and what others have attempted in the same way, neither corrects his errors nor supplies his defects. Whoever shall apply to the same work, will, I presume, find it very difficult ; and though, with Dr. Gaubius, we might suppose that by repeated attempts it may at length be brought to perfection, we must, at the same time, think, that many repeated attempts will be required, and that the perfection desired cannot be hoped for but in a great length of time.

I know it may be alleged here by the Empirics, that, though the facts they are possessed of are not displayed with all the pedantry of method, nor can yet be united into a whole, that is, a tolerably. perfect system, yet they have truly so many facts as may be an ample foundation for an Empirical practice. That this is in part true, I shall admit, and of what applications it is capable I shall afterwards say; but, in the mean time, that it is to any extent accurate and useful-any ways answerable to the demands of practice, I must absolutely refuse; and I do it on this single ground, that these facts are not yet capable of method and system, as this to me is a certain proof that the particulars are neither accurately determined nor their number sufficient. For, not to insist on the abstract and general argument, that nothing is accurately defined that is not defined by its genus and species, the matter may be well illustrated by what has happened with regard to natural history, which has now for some time been treated by method. When the first attempts were made in this subject, they came out very imperfect; and plainly because the particulars of which the system was to be formed, were either not observed or not accurately enough defined. They had remained so from the beginning of time till within these hundred years past; and it was then only an attempt towards system, that discovered the imperfection, or directed the supplying of it. In short, in natural history it is the attempts towards system that have produced the knowledge of particulars; and although these, in their turn, are necessary 
to render the system perfect, it has however been especially every new effort in system, that has excited a new industry in completing and rendering more accurate the knowledge of particulars. The system of Tournefort, for example, led to a more exact observation of the form of flowers, that of Rivinus directed to the number and disposition of the leaves; a slight attempt in Vaillant and Boerhaave led to the studying of stamina and pistilla, and the system of Linnæus has carried the same much farther. In short, I think, every body acquainted with the progress of Natural History must know, that the attempts in system, and the study of particulars, have mutually promoted and supported ${ }^{-}$each other.

It is certainly the same with regard to the study of diseases. If a system, a Nosologia methodica, cannot just now be rendered tolerably perfect, it is a certain proof that the particulars of which it should be formed are at present neither accurate nor complete, and it is equally probable that they cannot be rendered so, till our attempts in system have been repeated, and have made some farther progress. In proof of this, every one that looks into the work of Sauvages, will find many imperfections in system which can only be corrected by farther observation. This, however, would hardly have been suggested but by our perceiving the imperfection of system. Our attempts in system therefore are necessary to enlarge our stock of facts.

But let us in the next place consider how attempts in Nosological system may be best conducted.

It is, I think, now agreed, that the dissection of morbid bodies is one of the best means of improving us in the distinction of diseases. Sauvages indeed has rejected the employment of the internal seat of diseases as a means of distinguishing them; but he has, in an hundred instances, tacitly employed it; and under the ambiguity that often occurs in external symptoms, it is evident that dissection, by showing the parts singly or jointly affected, shows the real and steady changes in the system, upon which the external symptoms depend, and therefore must lead to the proper limiting of genera and species; and if in no other way, at least in this-by leading us to observe more exactly the external appearances connected,with the internal observed from dissection. This, therefore, like the attempts in system which 
we montioned before, must lead to a more accurate observation of particulars. But if dissection is necessary and useful to a Nosological system, let us next consider what this farther implies : plainly, our judgment of morbid bodies must depend on our knowledge of the healthy state; and accordingly we know well that the dissection of morbid bodies has become more accurate exactly in proportion to the progress in anatomy; and, indeed, that in this and other ways, a minute and accurate anatomy is necessary to the Nosology every one now perceives. But every one does not perhaps perceive that anatomy owes its progress to the study of the use of the parts, and the study of the use of the parts is inseparable from the study of the whole economy. It is, however, pretty certain that the study of physiology, by proposing inquiries, has contributed as much to direct the knife and microscope of the anatomist, as the facts produced by these have contributed to give us a system of physiology. Here is again the connexion between the study of system and that of particular facts; and while gentlemen pretend to distinguish the provinces of reasoning and experience, and to determine with regard to the importance of the one or of the other, I wish they would, once for all, observe, that they are truly inseparable; or at least that, separately, they have never been of much service. On the present subject, I think it must now appear evident, that the distinction of diseases must be often guided by the dissection of morbid bodies-must be constantly guided by anatomy, physiology, and pathology united together; and, therefore, that the discernment and accurate distinction of external symptoms will be most effectually obtained by the cultivation of a Dogmatic system. In farther proof of this, we may observe, that, as we have above explained, it appears from the history of physic, that it has not been Empiricism, but Dogmatism, or the study of the proximate causes of diseases, which has collected for us the facts of physic, and has led to the systematic and useful application of them. From the nature of things, this must continue to be the case ; and as on the one hand we have shewn that there exists no proper foundation for an Empirical system, and as we shall presently farther shew that no such system is yet formed, so on the other hand, as from the constitution of mankind a Dogmatic system has always prevail- 
ed, it will upon the whole appear, that at present we can have no other foundation for the regular study of the practice of physic. But observe, that my argument here is, that the study of a dogmatic system is necessary to the acquiring and ascertaining the facts of physic; I might maintain this by many other considerations, but shall mention only two. The first is, that if the observation of remote causes is useful in physic, as every one allows, and particularly towards establishing the proper distinction between diseases, as might be easily shewn, I say that a dogmatic system is absolutely necessary to ascertain the facts on this subject. For when the human body, as often happens, has been at the same time exposed to the action of many different powers, it is difficult to determine which of them, how many of them, or what concurrence of them, has operated in producing diseases. It cannot indeed be determined without the philosophy of those several powers; and as it is also well known that these powers operate very variously, according to the different condition of the subject they operate upon, so not only the philosophy of external powers, but the philosophy also, that is the physiology and pathology, of the human body, is necessary to determine most of the facts with regard to remote causes. In the second place, a dogmatic system is necessary to ascertain the facts with regard to remedies. This proceeds upon reasoning of the same kind; and while the consideration of remedies often leads to the distinction of diseases, and is otherwise a head to which most of the facts of empiricism are to be referred, it is at the same time well known that most of the facts with regard to remedies have been involved in the greatest uncertainty; and I think it can be shown, that they never can be tolerably ascertained but by the cultivation of all the several parts of a dogmatic system. But now to conclude, I hope you are in general aware that we do and must assume that the facts of physic are more frequently the inferences of reason than the simple objects of sense, and therefore that the bringing out the facts that are necessary, and the ascertaining them to be such, will always proceed in proportion to our advances in the knowledge of system, and that truly an empiric system can hardly be perfect till the dogmatic is nearly so.

But there is a still greater difficulty in the way of analovor. I. 
gy. There is great reason to doubt if the general principle upon which the whole plan of analogy is founded, be solid and just, that is, whetler the treatment applicable to one species of disease be also applicable to every other species of the same genus, or even whether the treatment applicable to a species is also applicable to the same species under all its varieties. It is indeed true, that, upon a purely empiric plan, this principle is the only one that can guide us upon the occasion of a new variety or species presenting itself, and it may be taken as a guide where we have no better; but every empiric must acknowledge that it is extremely fallacious. Dr. Sydenham is the only physician who has employed this empirical analogy to any purpose; but at the same time he has expressed his sense of its fallacy in the strongest manner. He has expressly told us that every new Epidemic required a particular treatment, not to be learned from any analogy, but by particular experience. Indeed both experience and theory concur in rendering the principle of empirical analogy extremely doubtful; and to conclude, however the empirics may boast of the general use of their analogy, it is sufficiently obvious that it can never be of certain application, till, by many and repeated observations, every case shall be brought to be nearly a case of simple imitation : and hence analogy is involved in all the difficulties, exposed to all the fallacies, and liable to all the imperfections of imitation.

I proceed now to my third argument, which is to show that any attempt which has been made to teach physic upon a purely empiric plan has been hitherto fruitless, and, in some instances, pernicious. Sydenham seems to have made an attempt in this way, and, so far as he goes, he is truly useful; but he goes but a small way only, and is far short of the demands for practice. But I would say farther, that I deny that Sydenham proceeds upon an empiric plan. His "Processus Integri" may seem to be such; but whoever looks into his larger work, must perceive that it was the result of dogmatic studies and conclusions. But it is not necessary to say more on this subject, for his practical conclusions are adapted to particular cases, or to cases only a little generalized; he is far from aiming at a system, and, as I have said, falls very far short of it; so 
that a practitioner, who had no other instruction, would find himself on a very narrow bottom. But even such as it is, it is the only tolerable approach that has been made towards an empirical system : what can be farther done in that way, we may judge from the works of Dr. Shaw in England, and of M. Lieutaud in France, which are upon this plan. The first is now so much neglected, that it requires no criticism to point out its frivolity; the last has been produced more lately by a man of reputation; and his work has been generally well received, and is now very common in the hands of practitioners.

But here it will, perhaps, be suggested to me, that the only useful work on the subject of physic, is the making a collection of all the facts that relate to the art, and therefore of all that experience has taught us with respect to the cure of diseases. I agree entirely in the opinion; but I doubt if it can ever be properly accomplished, without aiming at some system of principles, by a proper induction and generalization of facts ; at least I am persuaded that it can be done not only very safely, but most usefully in this way. This, however, must be determined by a trial. I know that the late Mr. Lieutaud has attempted a work on the plan of collecting facts without any reasoning concerning their causes: and while I am endeavouring to give some account of the present state of physic, I cannot dismiss the subject without offering some remarks upon the promising Synopsis universa medicina, composed by the first physician of a learned and ingenious nation.

In this work there are many facts and much observation from the author's own experience, which may be useful to those who have otherwise acquired some knowledge and discernment; but, throughout the whole work, there is such total want of method, arrangement, system, or decision, that, in my humble opinion, it can be of little use, and may prove very perplexing to those who have yet to learn. The distinction of the genera of diseases, the distinction of the species of each, and often even that of the varieties, I hold to be a necessary foundation of every plan of physic, whether dogmatical or empirical. But very little of this distinction is to be found in the work of Mr. Lieutaud; and, in his preface, he tells us, that he meant to neglect 
such arguta sedulitas. And indeed his method of managing his subject must certainly interrupt and retard all methodical nosology. His arrangement of diseases is according to no affinity, but that of the slightest and most uninstructive kind, the place of the body which they happen to affect. His Generalia et incertae sedis, have hardly any connexion at all ; the titles, Rheumatismus, Hypochondriasis, Hydrops, follow one another. When he does attempt any general doctrine, it is not till long after he has treated of the widely scattered particulars. Under each particular title which he assumes, he has endeavoured to enumerate the whole of the symptoms that ever appeared in a disease under that title; and this without aiming at any distinction between the essential and accidental symptoms, or marking the several combinations under which these symptoms do for the most part steadily appear. From the concurrence of accidental symptoms, the variety of the same disease is frequently considerable, a circumstance necessarily perplexing and distracting to young practitioners; but it seems strange to me, that an experience of thirty years, in considerable practice, could do nothing to relieve them.

Mr. Lieutaud has, at the same time, increased the confusion that must arise from this want of distinction, by his considering as primary diseases, what appear to me to be the symptoms, effects, and sequels of other diseases only. Of this I think the Astus morbosus, Virium exolutio, Dolores, Stagnatio sanguinis, Purulentia, Tremor, Pervigilium, Raucedo, Suffocatio, Vomica, Empyema, Singultus, Vomitus, Dolor stomachi, Tenesmus, all treated of under separate titles, are examples. A general Symptomatologia may be a very useful work, with a view to a system of Pathology; but, with a view to practice without any system, it must have bad effects, as leading only to a palliative practice, and diverting from the proper efforts towards obtaining a radical cure. Mr. Lieutaud, indeed, has endeavoured to exhibit the symptoms above mentioned as so many primary diseases : But he has seldom succeeded in this; and, in delivering the practice, he commonly finds it necessary to consider them as symptoms, and that not without some theory, implied or expressed, with respect to their proximate causes. 
His title of Dolores may be taken as an example of this; and from which it may be readily perceived how far such treatises can be really useful.

In establishing a proper Pathology, there is nothing that has been of more service than the dissection of morbid bodies. $\mathrm{Mr}$. Lieutaud has been much and most commendably employed in this way, and in this Synopsis he has endeavoured to communicate his knowledge on the subject; but, in my humble opinion, he has seldom done it in a manner that can be useful. In the same way that he has delivered the symptoms of diseases without any instructive arrangement; so, on the subject of the appearances after death, he has mentioned every morbid appearance that had ever been observed after the disease of which he is then treating: But these appearances are strangely huddled together, without any notice taken of those which belong to one set of symptoms or to another; and, with regard to the whole, without any attempt to distinguish between the causes of diseases and the causes of death; although the want of such distinction is the well-known ground of fallacy upon this subject. I take, for an example, the appearances mentioned as having been observed after dropsy. Here morbid appearances, found in every part of the body, in every cavity of it, and in every viscus contained in these cavities, are enumerated; but which of these morbid states are more frequent or more rare, and which had been more particularly connected with the different causes, or with the different state of symptoms previously recited, we are not informed, nor has he enabled us to discover. In short, the dissection of morbid bodies has been, and may be, highly useful ; but in order to be so, it must be under a different management from what we find, either in M. Lieutaud's synopsis, or even in the Historia anatomico-medica.

I cannot dismiss this subject without remarking, that the dissection of morbid bodies is chiefly valuable upon account of its leading us to discover the proximate causes of diseases: And the great and valuable work of the illustrious Morgagni, is properly intituled de sedibus et causis. It may well seem surprising, then, that Lieutaud should find the whole of proximate causes atra caligine mersas; and that he should never have 
thought of applying his dissections towards the ascertaining at least some of these.

But let me now proceed to consider the important part of every practical work, and of this Synopsis universa medicina; that is, the method of curing diseases.

Here, again, upon the same plan as in giving the histories of disease, the method of cure is delivered by enumerating the whole of the remedies that have ever been employed in a disease under the title prefixed, without assigning the species, or the circumstances to which the remedies, though of a very different and sometimes opposite nature, are peculiarly adapted. On the subject of asthma, he very justly observes, that physicians have been to blame in confounding, under this title, almost all the species of dyspnoea; and he himself very properly considers asthma as a disease distinct from all the other cases of dyspnœa. Still, however, he considers asthma as of many different species, arising from many different causes, which, till we understand better, we cannot attempt to remove.' Notwithstanding all this, he proceeds to deliver a very general cure. Parum abest, says he, quin specifici titulo gaudeant pectoralia, vulneraria, et incidentia! But from such language I receive no clear idea; nor can I obtain any clear direction from the enumeration of his medicines. Bacca juniperi, gummi tragacanthum vel ammoniacum, sapo, aqua picea, terebinthina, \&c. quo tamen haud indiscriminatim sunt usurpanda, sed pro re nata; delectu opus est. Very justly indeed, delectu opus est; but here, as in many other instances, he gives us no sort of assistance.

From his endeavours, though not always successful, to neglect all system, his practice is generally delivered in a very indecisive manner; or, what has the same effect, in a way, so conditional as will render it always difficult, and often impossible, for a young practitioner to follow him. Let us take, for example, his cure of dropsy. "The cure may be begun by blood-letting in certain conditions; but, in others, it cannot

- be employed without danger. It gives relief in difficult breathing; but, after it is practised, the symptoms are aggravated, and rendered more obstinate. It is not to be concealed that 
some persons have been cured by repeated blood-lettings, or spontaneous hæmorrhagies; but it is at the same time known, that such a remedy, inopportunely employed, has in many instances hastened on the fatal event."

In the same manner he treats of vomiting, purging, sweating, and the use of mineral waters. But I must confess, that he has no where removed any of my doubts or difficulties, and indeed he has sometimes increased them. He says, that hepatics, or aperients, such as the lingua cervina, herba capillares, \&c. deserve commendation; but that, when the disease has arisen to a certain degree, they have been, for the most part, found to be useless. He observes, that the powder of toads given in wine, to the quantity of a scruple or more, has succeeded with several.

Such are, commonly, the methods of cure delivered by Mr. Lieutaud, longiori et forte felicissima praxi edoctus.

It would be tedious to enter further into that detail, which a criticism of this immethodical and uninstructive work might lead me into; but, if the limits of these lectures did not prevent me, I would particularly shew that the work is far from being free from those reasonings which the author pretends to avoid, and would affect even to despise. He still holds the doctrines of the concoction and critical evacuation of morbific matter; doctrines depending upon subtile theories, and which, in my opinion, can in nowise be ascertained as matters of fact. Mr. Lieutaud likewise is still very much upon the old plan of following nature, and therefore gives often what I consider as a feeble and inert practice. The humectantia, diluentia, demulcentia, et temperantia, are with him very universal remedies, and often those which alone are to be employed.

The mention of these medicines might lead me to take notice of Mr. Lieutaud's second volume, in which, ab insulsa remediorum farragine alienus, he promises a great reformation upon the subject; but this falls so far short of the idea of British physicians, that I need not make any remarks upon it. With respect to his list of simples, or Emporetica, as he is pleased to. term them, an English apothecary would smile at it; and with respect to his Officinalia, I believe they are to be found nowhere but in the Codex Medicamentarius of Paris ; and in his 
Magistralia his doses are generally such as the most timid practitioner of this country would hardly descend to, and such as none of our practitioners of experience would depend upon. In short, the whole of the work, both with respect to the theories with which it abounds, and to the facts which it gives, will not, in my apprehension, bear any serious criticism. But I must conclude, and shall only say further, that such as I have represented it, is this work, executed by a man of the first rank in the profession. It is indeed for that reason I have chosen it as the example of a work, upon the plan of giving facts only, and of avoiding the study, or even the notice of the proximate causes of diseases : and with what advantage such a plan is pursued, I shall leave you to consider.

These are the arguments I would employ to prove that an empirical system is at present impossible, and that a dogmatical plan in the study of physic is absolutely necessary. I could employ many more to the same purpose, but I hope they are not necessary at present; and I shall conclude the subject with obviating one argument that is often produced on the other side of the question. It has been said that physicians not only of the same time, but even those of different ages, however widely they may differ in their theoretical systems and modes of reasoning, have very often come to the same conclusion, and agreed in the same rules and modes of practice; it is, therefore, they would say, very superfluous to discuss the questions about proximate causes, it is enough to deliver the established practice; but I presume the fact is false, and therefore the conclusion.

It is indeed true that at one time, and in one country, one particular system of physic generally prevails, and therefore a mode or fashion of practice; but as at all times there are few persons who think for themselves, it is no wonder that all those who have learned that theory from the same master, should seem to agree and establish the same practice. I remember since there was hardly any practitioner in this country, but who had been bred in the school of Boerhaave, and all of them who understood their system were wonderfully uniform both in their theory and practice; but there are always a great number incapable of any theory, and who can therefore follow no other 
measure but that of imitating, as well as they can, the fashion established by others. It is not, therefore, that by different theories men come at the same practical conclusions, but it is because they either have the same theory or none at all. I know, however, also from much acquaintance with physicians, that though indolence, modesty, or good nature may produce a harmony in consultation, yet every man who has a peculiar theory, will also have a peculiar practice whenever he acts by himself.

With regard to physicians of different ages, nothing appears to me more false than the supposition of their agreement in practice. The superstitious admirers of antiquity can find in Hippocrates every piece of theory or practice that has been started since; but the slightest acquaintance with the history of physic must inform us how much different ages and sects have differed from each other. Observe only since the beginning of the sixteenth century, how much the Galenists, the Chemists, the Cartesians, the Mechanicians, and the Stahlians, have differed in practice from each other, and all of them, in many particulars, from the Ancients. There are, indeed, diseases of frequent occurrence and very similar appearance in all ages, and it is to be supposed, that with regard to these, time and experience have established some general rules of practice, which no new system will venture to contradict; but with regard to the application of these rules, and with regard to practice in diseases of less uniform appearance, we shall constantly find that, according to the difference of theory, the practice is widely different and even directly opposite. I wish, indeed, it could be said for the theory of physic, that it is harmless, because it absolutely rests on speculation; but indeed while I plead for it so much, I cannot do it on this ground, and I must own that it has often done harm; and we cannot think of the physician that Lieutaud mentions who bled his patient one hundred times in a year, and thereby occasioned his death, without horror; nor can we think, without smiling, of the death of Van Helmont, who, from theory, refused to be blooded in a pleurisy, and endeavoured to cure it with a little sanguis hirci; nor can we think of many other such instances without being convinced of the baneful influence of false theory. But I spend your time 
in proving what nobody doubts of, and what the same gentlemen, on another occasion, would urge very strongly, that theory, whatever it is, may influence practice. But if the opinion we spoke of has any meaning at all, it is indeed this, that there is no use or propriety in the study of proximate causes, because nobody employs or applies them; and that, in the study of physic, we have nothing to do but to study the distinction of diseases, and to learn the established practice. This is, indeed, the opinion and the language of the common run of practitioners : they have neither the capacity nor application, nor, therefore, the erudition that is necessary to the study of a system, but they can learn somewhat of the routine that is for the time in fashion; and this, they think, is learning the established practice. But I maintain that the established practice has been established by dogmatism, and cannot be understood or applied without understanding its dogmatical foundation; for, with regard to particular cases, it is always imperfect, requiring both correction and extension, neither of which can be given to it, but by the most diligent study of a dogmatic system.

Upon the whole, it appears that an empiric plan cannot at present carry us any length in the practice of physic; nor is it likely to be soon fit for this purpose. We would wish, therefore, to find a shorter course; and it may perhaps be obtained by another plan of analogy, or means of establishing a common nature in different diseases. This may be by finding their proximate causes, or that state of the body upon which the external symptoms depend; and this is shortly the plan of the dogmatists. It requires a cultivated state of anatomy and philosophy; and while these were formerly in a very imperfect condition, it is no wonder that the dogmatists made many very fruitless attempts towards the execution of their plan; and not only offered many frivolous conjectures, but also, in trusting to these, introduced errors into the practice of physic. It is in this subject that the empirics have a fund for specious declamation; and it is upon these grounds that they not only object to the futilities of the theory that has been formerly offered, but they can also fairly allege that the state of anatomy and philosophy are to this hour so imperfect as to render a theoretical system of physic still impossible; and that while the attempts towards it are liable to a 
great deal of fallacy, the rendering it complete must still be at a great distance. But while the dogmatists ought to acknowledge all this-and the present dogmatists readily do so-they, however, still plead, that such is the difficulty and fallacy of establishing a common nature of diseases upon an empirical footing that an attempt to do it by ascertaining their proximate causes, may give much assistance, and is almost unavoidable. This is the true state of the dogmatic plan: 'while it takes advantage of every part of the empiric, it proposes to make an addition to it from the lights of anatomy and philosophy; and while it does so with the same accuracy and circumspection that observation and history require, it is supposed to be done with sufficient safety. A dogmatist knows well, that anatomy and philosophy are not so absolutely perfect as to give a complete system of physic, but he thinks that they give an useful and applicable illustration of many parts of it, and that he may safely study it with respect to the whole, while he takes care to distinguish what is tolerably palpable and applicable, from what is purely speculative and uncertain. Now, to prove certainly that a dogmatist may make some useful additions to our empiric plan, it is only necessary to prove that anatomy and philosophy have thrown light upon the nature of diseases. With regard to the first, it is not a question at present ; for nobody doubts that anatomy, in many cases, has informed us very exactly with regard to the more obvious organic affections; and even where these are in the internal parts, anatomy has often explained their connexion with external symptoms, so as to establish a common nature in different diseases more certainly than any observation of the symptoms alone. There is no doubt, therefore, that anatomy was very improperly rejected by the ancient empirics; and I doubt if the present writers on the Nosologia methodica are right in so universally rejecting the distinction of diseases by their internal seat. With regard to philosophy, the use and application of it must have a considerable effect in explaining the proximate causes of diseases, and at the same time the nature of remote causes is entirely the business of philosophy, mechanical or chemical. It will be equally evident, that the nature of experienced remedies must in many cases contribute.to explain the nature of diseases. But the nature of remedies is also the business of philosophy, 
chemical or mechanical. Philosophy, therefore, by explaining the nature of remote causes and remedies, must be useful in physic, and the only doubt with regard to its general utility that can remain, is, with regard to its explaining the actions of the animal body itself. With regard to this, indeed, it has often failed, and what is more, has often made great mistakes; nay, I would go farther, and say, that it is still liable to mistake: but with all this, I shall be surprised if any body acquainted with the subject should affirm that it has done no good; or that, guided by anatomy, mechanics, and chemistry, we have not explained many of the actions of the animal body, so as to throw light upon the nature of its diseases. I believe nobody will affirm this; and, therefore, the question turns entirely upon the extent we shall give to the application of philosophy. Upon this subject I shall quarrel with nobody. I shall allow any body to urge the fallacy to which the application of philosophy is in general liable. I shall allow them to examine any particular application of it with the utmost rigour, and particularly to show the utmost diffidence and jealousy with regard to every general system upon this plan. In all such cases I shall join them with the utmost zeal. I wish to carry an empirical plan as far as it will go. I only plead, that a dogmatic plan can make useful additions to it; and if this is allowed it is enough to my purpose. For if it is allowed to be useful in any degree, I can maintain that it must be studied in its whole extent. There is, in animal bodies, such a connexion of parts, that every action is, with regard to some other, both a cause and an effect, and the whole turns, in this respect, as in a circle; so that there is no understanding one part without studying in some measure the whole. There is nothing more evident than that hitherto the defects and errors of our theory have been owing to the animal system being studied too much by parts, whereby some of the powers concurring, and their effects, have been overlooked and neglected. It is true, indeed, that great difficulties arise from our being obliged to view every part as a part of a whole; and from this too, some difficulties are likely long to remain; but whatever may be the effect of more comprehensive views, they are the surest means of discovering error and preventing the improper application of theory. 
This, therefore, is our first and principal argument for the study of theory in its whole extent, that there is no examining the theory of any single part or action, without examining its relation to others; and where that relation lies, nobody can know, but from a study of the whole; which is farther recommended by this, that the study of the whole is the surest means of discovering the errors in particulars.

Another argument for the same, is, that there is a similarity between several functions, and by knowing one we can, by analogy, very often safely apply that knowledge to another.

A third argument to the same purpose, is, that as the theory of an animal body requires often an acute and subtle reasoning, there is no becoming dexterous in this without a frequent exercise of it in all the different circumstances in which it may be applied.

The fourth and last argument I shall mention is this: If the empiric system of physic were so tolerably complete as seldom to require assistance from the dogmatic, I should readily agree to the neglecting of this altogether. But as that is not the case, and it can be demonstrated that an empiric plan at present can give only a very limited or a random and dangerous practice, and that therefore the demands of assistance are necessary and must be frequent, so there is hardly any man so well determined in rejecting dogmatism as, on such occasions, not to have recourse to it. In the course of a large and long acquaintance with practitioners, I have never met with one who could resist it. But see what is the consequence of this: When a man unprepared and unexercised, and occasionally only, attempts the theory of physic, he can form only crude and random conjectures, that can be no better nor less dangerous than random experiments. If this, therefore, be true, that where experience fails, every man will have recourse to theoretical conjectures, even on this account is the extensive study of theory necessary. It is only by this that the real assistance it affords can be obtained; and it is only by the same thorough acquaintance with, and frequent exercises of it, that the abuses of it can be prevented. The theory of physic has often been erroneous; it is still, in many parts, weak and imperfect; but it is only a person well acquainted with the whole that can properly discern the faults 
of it. It is only the acute, the exercised, the extensively and well informed philosopher that can be really a sceptic. If any one, from a thorough acquaintance with the subject, and by arguments drawn from the nature of it, reject dogmatism altogether, I shall allow him to be entitled to do so. But the case is otherwise with the bulk of physicians. Many of them have not had the opportunity of studying anatomy, chemistry, mechanics, and the study of nature, to the extent that is necessary to the theory of physic. Many, from laziness and dissipation, have neglected the opportunities they had; and many more still, with opportunity and application, are incapable of acquiring or applying the necessary knowledge. If all these shall lay down a weapon they cannot wield, I shall not be surprised at it; but if they shall impute to the weapon the blame that is entirely in their own arm, I shall have no regard to their opinion. If such at any time attempt to employ an instrument they have not previously acquired the use of, I shall think the attempt ridiculous, as the execution must be awkward and blundering.

Upon the whole, I plead for a dogmatic plan as a necessary addition to the empiric, and as, on many occasions, a very useful means of rendering the empirical analogy more certain. I plead also for a dogmatic plan, in its utmost extent, because it cannot be studied by parts, and because, without knowing the whole, we cannot select the useful nor guard against the erroneous. I go still farther and say, that in studying a dogmatic plan we must often try hypotheses. I hold the practice safe and useful ; safe if it is only employed for leading to facts and experiments, and highly useful if it does so. Hypotheses have been much decried, and justly, because they have been abused; but, at the same time, I am persuaded they are almost unavoidable, and have been more useful than is commonly believed; for they have often produced facts that would otherwise have passed unheeded, and experiments that would otherwise not have been made. I mentioned before, how little has been done in physic by professed empirics, and I maintain that the great stock of facts we are at present provided with, has been collected chiefly, and almost only by dogmatists. I finish the whole controversy with this : that at present every sensible 
dogmatist takes every advantage he can possibly derive from an empiric plan, but endeavours to render the analogy more certain, to catch the view of it more quickly, and determine his practice more firmly, by all the lights he can draw from anatomy, chemistry, mechanics, and the general study of nature. We conclude, therefore, that medicine is to be studied upon a dogmatic plan that will also comprehend the whole of the empiric. I propose to teach it upon this footing; and I have no doubt of forming, thereby, not only more ornate, but also more skilful physicians.

I presume you are now well persuaded of the propriety, and even necessity of studying physic upon a dogmatic plan, and that therefore you will patiently follow me while I endeavour to deliver a course on such a plan.

- But I cannot enter upon it without saying, that, when I thus argue for the propriety and necessity of a dogmatic plan, I must, at the same time, own that it is pursued with difficulty, and exposed to fallacy. I am very desirous that you should be aware of the last, and that you should receive with diffidence, and examine rigorously every piece of reasoning that is not of the most clear and simple kind. I am sufficiently sensible, that in attempting a system it is extremely difficult to avoid, on some occasions, reasonings that are subtle, incomplete in their parts, and therefore fallacious and hazardous in their application; but, as well as I can, I shall always give you warning of such. I shall myself direct your doubts, and, on some occasions, I shall tell you candidly that our system is entirely defective, and must remain so till further experience and reflection shall enable us to supply it. In this way I expect to render my theoretical labours absolutely safe; and that, upon the whole, we may depend upon theory no more than is absolutely necessary, I shall endeavour to order my plan so as to collect and communicate to you every fact in physic which experience has afforded, and take care that no conclusion I form shall be contradicted by any known fact or experienced rule in practice. I should be ashamed if any course gave you more facts or results of experience than I shall do. But by giving them, on a dogmatic plan, I hope I shall give you them so much generalized as to be more applicable, and so arranged as to be more 
easily recollected, and applied in the best manner your judgment may direct.

I have thus given you my general plan for treating the practice of physic; I hope you know what a dogmatic plan implies, and what it supposes necessary on your part. I shall however say this here, shortly. It supposes then, First, That from the mechanical and chemical philosophy you know the nature of all the several powers that can act upon the human body ; Second, That from anatomy you know minutely the structure of the human body itself; Third, That from Physiology you know the general laws by which the animal economy is governed, and these detailed in explaining the function of each particular part ; Fourth, That from Pathology you know the deviations from a healthy state which the body is capable of; Fifth, That from a comparison of Physiology and Pathology you know the several changes that are necessary to be made for restoring the body from a morbid to a healthy state, that is, the several indications as they are called; and, Lastly, That from the knowledge of the powers which act upon the human body, you know the means by which diseases may be avoided, or health restored when it is wanting. All this, in other words, is the knowledge of the Institutions of Medicine; and the teaching of the Practice is the directing the application of these general doctrines to particular diseases. The better you are prepared in this way you will learn the more from my course; but I am sensible that from various circumstances there are many gentlemen come here, not so well prepared as I could wish; but I hope to accommodate myself to such, so as that they may acquire some foundation for the practice of physic, as good a one at least as their state of knowledge will admit of, and as good a one as they could acquire on any other plan. In this hope I enter now on teaching the practice. The several diseases to be treated of are determined by the Nosology. What I call a genus is every where to afford to me a particular subject of discourse; and under each of these heads I shall treat the following subjects.

First, I am to give what may be called the History of the Disease - of the genus, and perhaps of the species, when it may seem necessary; that is, an account of all the several phenomena which constantly attend the appearance of such a disease, 
as they are severally combined together, or occur in succession. You will soon perceive, that although for a hundred years past it has been proposed to obtain proper histories of diseases, and much pains have been bestowed on the attempt, yet we have few histories such as we could wish. Among the first good writers on this subject was Dr. Sydenham, who is still the most sensible, perfect, and judicious. We have but few performances equal to his; the symptoms are too often not delivered in the order of succession, and their combinations are not properly marked. Here you will attend to my exécution of this subject, and mark the defects, in order to supply them yourselves.

Secondly, The investigation of the proximate cause, on the knowledge of which the cure of the disease is chiefly and almost unavoidably founded. This is the nicer part of our work, and to you the most difficult. But, whatever may be said of the obscurity of this subject, still, in many diseases, the proximate causes may be assigned in a very clear and complete manner, so that they. will apply to the explanation of all the different phenomena. This I shall make good, therefore, with respect to many diseases ; and, wherever it can be done, the advantages resulting from it will be very considerable, and will afford much satisfaction in the practice of physic, particularly in the method of cure. But I must own, that in this respect; my whole course cannot be complete, as I cannot pretend to give such simplicity and clear explanation in regard to all diseases. It depends, however, on the extensive knowledge and judgment of the physician, to discern the different degrees of probability in medical doctrine; to admit those reasonings only as a foundation for practice, which are simple, obvious, and certain; and, for the most part, to adopt as proximate causes those only, which are established as matters of fact rather than as deductions of reasoning. This must either be learned from the dissection of morbid bodies, which is the surest method of judging of internal diseases, and for which purpose Bonetus and Morgagni must necessarily be studied ; or we must, from the most cautious reasoning, deduce what is the proximate cause of the disease, and what is the particular state of the solids and fluids at the time. This is the foundation of the whole dogmatic system; , even where we cannot go the whole length; we can point out the way, and trace it so far as will serve us to

vor. I.

2 k 
discover many errors. After this I must still say, that there are a few diseases in which we cannot proceed any length in investigating the proximate cause; and here I can only offer conjectures, of which I mean to be very sparing: I shall then always give you due warning, and let you know when we can hardly rise above a total empiricism. For, when the proximate cause cannot be established with sufficient certainty, the judicious and prudent physician will have recourse to experience alone, always, however, aware of the hitherto incomplete and fallacious state of empiricism.

This then is what you are to expect with regard to the doctrine of proximate causes. There has not been greater blundering in the schools of physic than with regard to this subject. In your systematic writers you find every thing explained, but upon very slight grounds; and these being given without any warning, students were led into gross mistakes by theory. But I shall be the first to express my doubts and diffidence in all such circumstances; I will be always impartial with respect to opinions; I will acquaint you where you may expect our reasoning to be weak, and where $I$ advise you to give up reasoning till we have further experience: and my business will not be so much to explain how this and the other phenomenon is produced, as to examine what is truly matter of fact; we shall therefore follow the dogmatism of Dr. Sydenham, and we shall only proceed so much further than he has done, as the improvement of Anatomy and Pathology, since his time, will allow us; and I hope to give a specimen of dogmatism, (I avoid the word theory, which, however, you may use if you please,) which is very little known in the schools of physic. It rests so much on matters of fact, that it will not be refused by the most professed empiric.

Thirdly, From the phenomena of the disease, and with a view to the conclusion respecting the proximate cause, I am next to enter into a critical disquisition with regard to the proper character and limits of every genus, and its division into species and varieties. Here I must necessarily take notice of the attempts which have been made by others upon this head, and give the reasons for my deviating from them. I propose to do this so as to instruct you in a critical Nosology, and so that you 
may be able to correct and improve what I have attempted under many disadvantages, and with several imperfections.

In the fourth place, we shall proceed to the consideration of the remote causes, upon which the prevention of diseases chiefly depends. To this subject all sects of physicians, except some of the Methodici, have attended. While the proximate causes were considered as obscure or latent, the remote causes being those most evident, have been very much valued. These causes, however, such as they generally appear in medical writings, are far from being simple, and, in some cases, unavoidably lead to reasoning; and I am sorry that physicians have too often delivered them upon that footing. It shall be my business to ascertain the matters of fact with clearness and certainty, and to avoid the inferences of reasoning, which are to be found in most works on this subject. At the same time I shall keep in view the proximate causes, and endeavour to establish a connexion between them and the remote. This will often lead us to correct the doctrine of the proximate causes; as, by shewing that many of the remote causes are doubtful facts, we will raise doubts with respect to the proximate cause.

Fifthly, We shall proceed to the Prognostic. I should have remarked before, that what is called the Diagnostic will be delivered by us under the third article, where we point out the means not only of determining the place of each disease, but also those of distinguishing it from all others. The Prognostic I shall also deliver upon a dogmatical plan, and as connected with the doctrine of the proximate cause; but as a great part of what has been delivered upon this subject may not be capable of being rendered consistent with the proximate cause, I shall also mention that Prognostic which has been alleged from experience. The ancients have been more full upon this than upon any other part of medicine.

The sixth and last article is that for which all the others are intended, viz. the method of cure, commonly termed methodus medendi. Here also I shall follow the dogmatic plan, forming the indications from the proximate cause. I hope to persuade you, that a Practice of physic formed upon any other footing, stands upon the most uncertain grounds, and is the most inconvenient for use. At the same time, however, I shall use the 
greatest caution, which indeed is here especially necessary. I shall then deliver the remedies adapted to our several indications; and from the Materia medica I shall show how the remedies are adapted to produce these effects. I hope to be able to say, that these indications, although taken from the proximate cause, are also approved of by experience. In many cases indeed, I will not be able to assign the method of cure upon this dogmatical foundation, and then it must rest entirely upon experience. Remedies of this kind have been proposed, and in some measure vouched for, by persons of great credit. Thus, Dr. Gilchrist has ascertained the use of warm bathing in many cases of fever; and I think Dr. Hahn has ascertained with as much certainty the use of cold bathing. I know not whether I shall be able to reconcile either the one or the other of these remedies with my idea of the proximate cause of fever; but I shall always tell you that such modes of practice have been established by persons of credit. And even if a secret remedy is alleged, such as James's Powder; supposing that I should not know its nature and therefore cannot adapt it to my theoretical doctrine, I should think myself not to be excused, if I did not tell you what has been learned by experience with regard to it.

You have thus, gentlemen, my plan for treating the several heads-the several genera of diseases which are to enter into my course; and I have only to add, that though every genus that might be assigned will not enter into it, I hope to comprehend all that are necessary as a foundation for your study, and all those that will be the chief objects of your practice.

We have attempted an arrangement of diseases according to their genera and species, founded on their obvious symptoms, or a Methodical Nosology; and as we shall have frequent occasion to refer to this work, and as the several diseases to be treated of are determined by it, I think it necessary to give you a short introduction to the Nosology.

I wish to make you critics in Nosology; but this I shall perhaps find a difficult task. Perfect division and definition is 
the summit of human knowledge in every part of science, and requires not only the clearest but the most comprehensive views, such as, with respect to diseases, we can arrive at only by oftenrepeated exercises and much study. The attempt may at first appear uncouth and difficult, but by repetition it will become more familiar and easy, and you must be content to make such a progress. When, a little more than thirty years ago, I first got a sight of the botanical system of Linnæus, it appeared to me to be a piece of the most uncouth jargon and minute pedantry ; but, by length of time, it is now as familiar to me as my mother tongue: and with whatever difficulty it was first received in most parts of Europe, it has now surmounted these, and its utility has reconciled every person to the study of it.

I might begin by giving a general idea of what we call method, and illustrate it by examples taken from Natural History; but I cannot suppose this necessary. Natural history, in which method is so generally applied, is now so universally studied, and there is such a propensity to it among all ranks of men, and all kinds of students over the whole of Europe, that to give an account of it here to gentlemen advanced in their several studies, would be equally absurd and unnecessary. I can hardly think it necessary even to take much pains in illustrating the necessity of this study in general, and in pointing out to you that it is applicable to a certain length in every part of science, when we aim at an accurate distinction of one thing from another: it has been long ago established in logic, that nothing is distinguished, till it is distinguished by its genus and species. I hardly think it necessary to inform you, that it is impossible to convey any accurate knowledge of things from one country to another, or from one age to another, without more or less of this method; and it is certain that we have lost much of the knowledge which the ancients had acquired in the animal, vegetable, and fossil kingdoms, because they wanted the means of conveying it by those proper characters which method only teaches; but this is an accident which, we can plainly perceive, will not happen to learning again, as long as this methodical distribution is anywhere cultivated among men. In the last place, I hardly think it necessary to speak of the necessity of method in order to the investigation of the productions 
of nature, or to the acquiring a knowledge of any enumeration of things that are to be distinguished from one another.

There have been objections to the application of method to some parts of science, but even those who make such objections, agree that, wherever the species of any kind are very numerous, it is impossible to prosecute the investigation with clearness and advantage without method. If there are ten thousand different species of plants, or five thousand different species of insects to learn, to acquire the knowledge of all these separately, as was the state of things among the ancients, is next to impossible; and we know that we can now acquire the knowledge of ten thousand more easily than we could formerly the knowledge of five hundred.

But, from the same' means by which we have acquired the knowledge of method in general, I must suppose you to have acquired it for the purpose I speak of; and I go on to say, that the distinguishing of things by genus and species is universally applicable to every two things that are to be distinguished from one another, and, therefore, among others, to the distinguishing of diseases; and this distinction only requires that we should know what is peculiar to one thing, and at the same time common to no other; it is, to know every thing by something peculiar to it and in common to no other. These several circumstances or particulars, and the combination of them that serve this purpose, is what in natural history we call a character; in physic we call it a Pathognomonic. These were long ago marked, indeed, as the great desiderata in the history of diseases: they long talked of pathognomonics in the history of diseases, in order to their accurate distinction, but it was, however, in very few diseases that such pathognomonics were found out ; where a single symptom proved pathognomonic, it was perhaps observed, but this does not occur once in a hundred cases : it is a combination of several symptoms that is necessary to constitute such a character or pathognomonic, and this was not attempted.

In all the ancient systems, and I may say till very lately, they had a title, the Diagnostic, which should liave given the pathognomonics the strict and proper characters; but if you consult these you will find, or a little acquaintance with physic will 
show you how far they have fallen short of establishing such characters. Within these hundred years, neglecting the study of pathognomonics, we have thought of another measure; we have aimed at what we thought somewhat more complete, at what we call a history of diseases, that is, the enumeration of the whole of the symptoms that at any time accompany a disease, collected under the title we employ; a very useful work, to be sure,-nothing is more so; but unluckily it happens that as yet we have very few good descriptions or histories of diseases, and, what is worse, the best descriptions do not answer our purpose; they contain a great deal of superfluous matter; they assume a certain title of a genus or species, and enumerate every single symptom which, at any time or upon any occasion, has appeared to accompany that disease, but they have taken no proper pains to distinguish between what are the essential and what the accidental symptoms, between the simple and the complicated ones.

This is a state of physic that requires very much to be amended; and this can be done in no other way but by the application of methodical Nosology.

If Nosology could be rendered tolerably perfect, nobody would doubt of what I have been saying; but several have refused to admit that this is possible, and have objected to it upon different considerations. The first and most considerable is this, that at present the distinction of diseases lies in so much confusion, that Nosology must certainly be difficult; and some carry it so far as to assert. that the attempt is impossible. But certainly this is going a great deal too far. I acknowledge very readily, that many difficulties occur in methodical Nosology, and the difficulties are sometimes such as I cannot pretend to surmount; but this is not universal; if it were so, what would be the consequence? it would make the study of physic absolutely impossible, for if we cannot arrive at some distinction of diseases, we must act at random; nay, this is as much as to say, that there are greater difficulties in our way in ascertaining facts than all that have been urged against false theories. But if on the contrary, we can distinguish diseases, surely we can likewise say in what manner we do distinguish them. Now Nosology only tells you in what manner any person does distinguish dis- 
eases; I expect that nobody will refuse that we can go a certain length in this; and certainly no objection can be urged against going a certain length though we can go no farther; but how far we can go, nobody will say till he has tried.

Within these hundred years some of the most experienced men in natural history, and particularly in botany, for instance Mr. Ray, imagined when a methodical arrangement was first proposed to them, that it was impossible; and Mr. Ray's first attempts were extremely feeble; but every body will now readily acknowledge, that they have truly gone a great deal farther, than was, not long ago, thought possible; and I know that within these fifty years some of the most considerable botanists still thought that the classification and distribution of plants was a pure fancy. But these difficulties with respect to natural history are now entirely removed, and so they ought to be with respect to Nosology. The principal objection is derived from the imperfection of the trials that have actually been made; and I must own that our nosologists have begun at the wrong end. 'Their first attempt was to form the classes and orders with which they began their system, whereby they have made very odd work; and they do, to be sure, associate diseases that are in themselves very dissimilar, and separate many that are nearly related. We may take for an example of this the most systematic author in Europe, the celebrated Linnæus. It appears to every body else extremely ridiculous when he arranges syphilis among fevers, merely from the circumstance of their being both contagious; and when he separates diseases so nearly related as the gout and rheumatism; especially the last, from other inflammatory diseases; and indeed many similar absurdities occur in his system. But it will be plain that this is not the fault of method in general ; it is a fault arising from this, that nosologists have been more studious of the general system than of particulars; and this is a mistake which we must be liable to, till every species has been fully taken notice of.

I am so far from thinking that we ought to enter anxiously into the general systematic arrangement, that in my prolegomena I have insinuated a contrary notion. Because $I$ think there is use in making the attempt, I have with others attempted to form classes and orders; but for the accuracy of these I would by 
no means vouch. I have many difficulties still with regard to them, and I think I could still point out considerable improvements; but my labour is to study genera in the first place, and, when I can arrive at them more certainly, species.' If I can distinguish these with tolerable accuracy, I am not concerned with my distribution into orders and classes being more or less exact. It is from our accuracy with respect to the species that all the rest of the divisions can be properly established; and in nosology indeed we are very much relieved from a more particular attention to classes and orders.

Wherever the species of things are very numerous, as in the case of plants and insects, it is absolutely necessary to attempt classes and orders. But there are many departments, even of natural history, where this is not so necessary, because the species are not so numerous; or if the species are, because the genera at least are not, so that we need not go higher. The first is the case with respect to quadrupeds; and the second, perhaps the first, with respect to Nosology. Nobody has inveighed more vehemently against the use of method than M. de Buffon. He says the whole known species of quadrupeds on the face of the earth are not above two hundred : now, we can easily remember the separate characters of two hundred things or species without uniting them into genera, far less throwing them into orders and forming classes. I say the same will apply in the case of Nosology, where the species are not very numerous, as appears pretty clearly from the abridgment of what have been called genera in my synopsis; and without laying down characters of either classes or orders, it is easy to remember the separate characters of a hundred and fifty genera which I have there marked; (and this is what I meant by the passage in my prolegomena, to which I have already referred.) But I do not mean to push this too far; for there are advantages which, I say, may, and I hope will arise from the study of even classes and orders; and M. de Buffon is much in the wrong in rejecting such an attempt with regard to his quadrupeds. The attempt to establish genera and species is always the study of the affinities between different things ; and though we distinguish them by their differences only, we arrive at a great deal of know. ledge also from their affinities; and we would never have marked 
and attended to their common relations, if we had not brought them into genera, orders, and classes; and without that, we would miss a great many particulars which relate to our subject. Now, I say the same is true with regard to diseases: the characters of a class, order, and genus, must enter more or less into the character of every species; they all relate to facts which are of consequence in our Pathology.

I shall have occasion to acknowledge that I have myself not kept free of every doubtful matter in my present execution of this subject; although I still maintain that I have with great propriety prosecuted the attempt upon this plan. I have, for instance, made one general division of diseases into Systematic and Local ; my first three classes (Pyrrexiae, Neuroses, Cachexiae,) comprehend the former, and the fourth (Locales) the latter; but I own that the constitutional or local nature of a disease is not easily determined by what the nosological character should be determined by, viz. the external marks; and, therefore, doubts will arise with regard to it on many occasions. Thus the lex systematis made me place among local diseases the Amenorrhoea, though I own that it is but seldom local, and much more frequently a systematic disease. And so with respect to other diseases several doubts may arise; but these will always lead to questions in Pathology.

Another difficulty which has been suggested with respect to Nosology, and which is indeed the greatest, is the difficulty of distinguishing between what we call Species and what we call Varieties of diseases. I shall not at present undertake to shew that this difficulty may be easily obviated; but, in the mean time, I say, that it affords no proper objection to a methodical arrangement. The very same difficulty occurred in Botany; and it goes so far, that even in the system of the very elegant and accurate Tournefort, one third at least, and probably more, of the plants which he enumerates under the title of species, are truly now allowed to be merely varieties. The botanists were early aware of this, and saw the impropriety of prosecuting the distinction of varieties, which are infinitely variable and no ways steady, and for the knowledge of which there is very little use, as in extremely few instances can we say that the qualities or virtues of plants are different in consequence of the variety. The bo- 
tanists found that, on many occasions, they could trace the production of the variety to a different culture; but the matter was not so easily determined with respect to plants found in their natural places, where the variety was not the effect of culture or soil; and here they had recourse to a much more effectual means, which was by observing, whatever was the variety, if the seed taken from one plant brought up a plant of the same kind, and without these varieties; all such as they found to be thus steadily propagated, they considered as varieties of the same species only; but what did not follow the seed, they considered as different species: and by this means the species of plants, which amounted to twenty thousand, have now been reduced to nearly half that number. 'The same difficulties occur in Zoology, and the same means, viz. the propagation in the way of generation, applies there also with advantage, but not as yet so perfectly and with such ease as in botany. In the third kingdom, Mineralogy, we have no such means of distinguishing between species and varieties, and therefore the matter is left in much uncertainty. We can in mineralogy go a certain length in establishing distinctions of genera, and, in many cases, those of species ; but beyond these there is a very considerable number of varieties which we still need to enumerate. The taking notice of the varieties in fossils, and prosecuting them, is of much more use than it is with respect to plants; for in plants a change of virtue does not follow a variety; but in fossils the quality of the ore, and the quantity of the metal, are often connected with certain varieties; and, therefore, we allow the study of varieties to remain here, because it is in a great measure of use.

Now, with regard to Nosology, which is our province, we can go somewhat farther than in Mineralogy; for we can there find something analogous to the propagation of seed in living bodies. We observe this in the case of all contagions, particularly in those we call specific contagions ; and, as far as my observation goes, even in those which are not strictly specific, when we can trace a disease to its contagion, we can, in some measure, fix its species. Thus, in the case of smallpox, a great many varieties have been marked, but they are varieties only of one species : a proof of which is, that from 
the same contagion, that is, from the same seed, all the essential circumstances of the disease are produced; thus a confluent smallpox may arise from a contagion of the mildest kind, and vice versa. We believe, therefore, that the seed is but one, and that the changes, the varieties, are analogous to those produced in plants by culture, that is, that they depend on the state of the body into which the contagion is conveyed. This is the solid foundation of inoculation, that we have now learned to modify the body in such a manner that the contagion, when applied, will not give rise to those varieties and anomalies. I shall add here what I think a curious corollary, viz. that the specific nature of contagions, and the dependance of the variety of the disease upon the nature of the body, are presumptions in favour of the inoculation of all specific contagions. When we shall have acquired some more experience with regard to the manner of fitting the body, and of conducting the inoculation in other diseases, as well as we now do in the smallpox, I am persuaded that the practice will be equally applicable. I must own, however, that this manner of ascertaining the species of diseases, by the propagation of a contagion, does not go a great length, as there are not many diseases which we can limit in this manner. But I obviate the whole objection in the same manner as in the case of fossils, by saying, that there is use for prosecuting the study of the varieties of diseases; and it is not of very great consequence to ascertain the limits between species and varieties; for some steady conditions and circumstances, which accompany variety, require different practice, and therefore are equally to be studied as what may be more strictly called species.

I have to add a curious particular with regard to this matter. Diseases are distinguished by a single symptom, or by a concourse of symptoms, and more frequently by the latter. But wherever we find a concourse of symptoms constantly and very frequently occurring together, and where this fixed concourse does upon occasion occur, with a variety of other symptoms added to it - these additional symptoms, however, being sometimes absent and sometimes present-I maintain that it is the first concourse only which establishes the character of the disease. There may be some use in taking notice of the occasional varieties, 
but they do not form either genera or species of a disease. Thus, to give an example, a tertian has the general character of an intermittent fever, with the particular circumstance of an interval of forty-eight hours between the beginning of one paroxysm and that of the following. This is a steady and very frequent recurrence; but we know that besides this, a number of other circumstances frequently accompany it ; particularly a number of intermediate fits of considerable variety. A fit may happen every day, or twice in one day and once in another; but the prevailing character is steady and constant, while the other circumstances of the intermediate fits are more unsteady, constantly shifting and changing, very often in the same patient, and much more frequently in the same epidemic.' Hence it is, that in my synopsis I have made a genus of Tertiana, and have marked all the other subdivisions merely as varieties. But here you may be stumbled, as I have given the Tertiana as a genus, and at the same time speak of it as a species; for, according to the principles of method, that is strictly called a species, which is a concourse of symptoms or a character, not admitting of a subdivision of steady and constant particulars. The species therefore is the ultimate character; and whenever you come to this, it ought, in strict propriety, to be called a species. But no system has avoided this difficulty; and it has often been found necessary to confound genus and species. Thus, in the animal kingdom, Elephas, Noctilio, and many other animals in the other classes, are entered into the system of Linnæus as genera, whereas they are truly species, admitting of no subdivision that is at all steady. The Rhinoceros, also, is a species, although marked as a genus. You will find that in every system, the name of genus and species are in this respect confounded. But the thing to be remarked is, that it is the ultimate concourse, the ultimate character, which you are to have in view; that this is the character in Nosology-no matter whether you call it species or genus-which is the fixed object of our practice, and the chief object; for, though I know that the study of the variety may be necessary, it is by no means universally so. And I would here observe, that, though I say we can only aim at a generic practice, I now say more clearly; that such genera may be 
considered as species. It has happened, I think accidentally, that in Nosology, by beginning with the characters of the class, and from thence descending to orders and genera, the term genus has been very universally applied to species; and, in short, by imitation, I have been led to do the same thing; for, of the one hundred and thirty-three genera which $I$ established (in my first edition), a hundred are properly species, and admit of no farther division except into varieties: the genera, as there claracterized, have the ultimate concourse, the steady character of species, and admit of no other subdivision but what are truly - varieties. In the other systems the number of genera has amounted to a great many more ; but I maintain that the same proportion holds, that three-fourths, perhaps, of their genera are species in the strict language of method. I have come to my conclusion, that the chief business in Nosology is the formation of the character of what I have called genera, and which you truly, for the most part, may consider as species.

I now proceed to give you some remarks upon the conduct of our Nosology in forming these characters.

I have said that the characters of diseases are formed by a concourse of various symptoms, every particular of which, in the language of methodical writers, is a nota or mark : now, in forming the character, the first, and the most important rule is, that these marks should be neither more nor fewer than are absolutely necessary. If we have more, we pass from characters or definitions to descriptions; we leave persons in doubt when they find a disease with the strictly essential character, by adding other circumstances which they may suppose to be equally necessary. The Nosologists have been faulty in giving both superfluous and deficient characters. As an instance of superfluous character, take Vogel's definition of 'Febris pestilentialis:' Febris pandemia, contagiosa, acutissima, in qua bubones aut certe carbunculi aut vesiculae oriuntur cum leipyria, ingenti siti, spiritu, sudore et dejectionibus foetidis, delirio, nausea, vomitu, pulsuque parvo ac obscuro.' The most part of this character is superfluous. The plague may appear without being 'pandemia ;' it is often not 'acutissima ;' and the only necessary circumstances are the contagion and eruption. The ' carbunculi, leipyria, ingen's sitis,' etc. are in common to the plague with 
many other fevers, and therefore no ways characteristic. This, however, is not so common a fault as the contrary, a deficient character from an accidental imitation of the botanists, who, when they have a number of species to name, study to make the specific character as short as possible; but, in Nosology, by endeavouring to be short, we have become obscure and imperfect. For however long the character may be, if all its parts are necessary marks, they are to be admitted. Let us take the ninety-second genus of Sauvages, and see what character he has given to Variola: 'Eruptio pustularum phlegmonodearum in suppurationem tendentium.' This applies to the itch as well as to the smallpox, and the imperfection will scarcely be supplied by his general character of Exanthemata- Eruptiones cutaneae cum pyrexia saepius maligna, quandoque lenta, - unless the duration and fixed time of the eruption is mentioned to distinguish it from other exanthemata. Through the whole of Linnaeus' arrangement particularly, these deficient characters are evident ; and as hardly any disease is distinguished by a single pathognomonic symptom, but all by a concourse of symptoms, I would undertake to shew, that whenever a character is very short, it is artificial and not natural.

A second rule is, that the marks which we employ should be sufficiently evident, and, if possible, constantly present at every period of the disease; these at least are the most characteristic. Nosologists transgress against this rule when the marks are taken from the duration, and much more from the event of the disease. To affirm that a disease is a fatal one, is indeed a part of its history, but cannot serve as a character when we first view the disease. Of this fault we have examples in the character of fevers, by Sauvages and Linnæus, who have distinguished the genera entirely by their duration. Thus the Ephemera terminates within three or four days, the Synochus in two or three weeks; the Typhus extends to three weeks, \&c:; but these are no characters at all ; and, in order to distinguish these diseases, it was absolutely necessary to employ other characters. In general, therefore, the terms acuta, chronica, lenta, are improper and insufficient in Nosology. I must observe, however, that it is impossible to avoid contravening this rule, in some measure, in certain cases in which the diseases are dis- 
tinguished as much by their series of symptoms in succession as by the concourse present at any time. Thus fevers in general, and particularly intermittents, are distinguished by the series and succession of the different stages of each paroxysm, and especially by the repetition of several paroxysms; nor can the exanthemata be in general distinguished but by joining the fever and the eruption. In such cases, therefore, we must submit to the nature of things, and allow the character of the disease for some days to be uncertain.

The third rule is, that the notce chosen should be expressed in clear and very intelligible terms, and, if possible, in terms the meaning of which is already fixed in the science. Vogel; in characterizing Pruritus, has the term 'dolorifica voluptas ;' such an expression certainly ought not to be employed. In like manner; Linnæus characterizes a fracture as 'ossis solutio citra substantiae dissolutionem,' which expression is repeated in the three characters of Luxatura, Ruptura, Contusura, following this. Now it is difficult to see a meaning here, or to find one which is proper.

Linnæus has been remarkable for inventing useful terms, and for fixing the precise meaning of others; but having been successful in many instances, he has been too bold in others, and has introduced many terms which we perhaps can never explain. Thus, in the definition of Miliaria, he says, 'febris synocha sudore fœetido punctorio :' understanding by these words, a sweat which comes out with a sensation of pricking, like the points of pins, in various parts of the skin; but the meaning certainly is not sufficiently clear. Again, in his definition of Lassitudo, we find, 'debilitas ponderosa;' in that of Hysteria 'verticis pressio,' instead of what has been otherwise called clavus hystericus; in that of Scurvy 'opacitas faciei,' a darkness of the countenance. But among other obscure expressions is particularly the term Dolor, which is in all the systems taken for every uneasy sensation arising from diseases, all the 'sensationes molestre ex morbo.' Thus the class Dolores, of Sauvages, comprehends Anxietas, Lassitudo, Stupor, \&c., all of which are considered as genera, whereas they certainly are, in strict propriety, only certain uneasinesses, but not strictly pain.

The want of due precision of language affects many parts of 
all our systems. It is well known, that the want of precision in the use of terms for a long time retarded the perfection of Botany'; and it has been the greatest merit of Linnæus, that he gave much more precision, and a determined meaning to almost every word employed in that science, which he did by forming a delineatio plantae, by fixing a term for every part of the plant, and for all the circumstances in which these parts can vary. But we will not bring Nosology to a good condition till something of the same kind, a delineatio morbi, be attempted, which ought to consider all the symptoms that enter into a specific character in another view than that taken by the Pathologists, who only trace them to their causes. Here we ought to examine the symptoms more minutely with respect to the variety of their appearance, and affix terms to them accordingly. This however is a difficult task; which my time has not allowed me to accomplish. In the mean time, to avoid, as much as possible, the faults which I mentioned, I have adhered to the terms which have been employed formerly; and I have been as careful to avoid new terms of symptoms as of diseases.

The fourth rule is, that the characters should be absolutely free and independent of all theory and hypothesis. Sauvages, in his Prolegomena, mentions ten or twelve definitions of Pleurisy, all taken from some view of the proximate cause; but all of them would now be entirely rejected. By looking into the systems, however, you will perceive that physicians have gone on in the same track of defining diseases by their proximate causes, which are in many cases disputable, and may long be so. Thus, in Juncker's conspectus, eyery definition is taken from the proximate cause; and I believe most physicians of this country will be of opinion, that nine out of ten of these definitions are fallacious and good for nothing. This, therefore, we must avoid hereafter: we must endeavour to distinguish diseases independently of every theoretical view; for the theory which we employ, however specious, however we may be persuaded of its truth, will not appear in the same point of: view to others, and must therefore occasion endless disputes.

On this subject it may be proper to observe, that I have formerly expressed myself as if the internal seat might, in consequence of our now knowing it so well from dissections, frequently be at-

VoI. I. 
tended to in forming our characters; but I am satisfied that though this internal seat is, with much propriety, added to the history of the disease, it is better not taken into the specific character, as it never is directly evident, and only such marks ought to be employed as are evident. Linnæus in the characters of his class Dolorosi, always employs the internal seat; and he is sufficiently cautious with regard to some of them, as when he says, ' Gastrica, ventriculi dolor epigastrii;-Colica, intestini dolor umbilicalis;' but the definitions of Hysteralgia and Nephritica, by ' uteri dolor,' and 'renis dolor,' are inadmissible, because it is not always certain when the uterus and when the kidneys are the seat of pain.

These are a few rules with respect to what diseases should be comprehended in our system, or what diseases we should attempt to characterize. I must mention another, however, which may appear sufficiently obvious, but which it is not unnecessary to advert to, viz. that every disease which can be distinguished from another, ought to be taken into our system. It has been long objected to the Botanists, that they have loaded us with a number of useless plants; for of the ten thousand of which they possess the knowledge we do not know any use for more than two hundred. This objection may be still farther urged against those who have described insects. But those who make such objections have not been aware of the purpose of method in any part of science. Supposing that we wish to know about three hundred plants, we cannot do this completely till the whole ten thousand have been also characterized : for we shall not be able to separate and distinguish the former, till the whole are compared. In order, therefore, to render our arrangement perfect and complete, we require to distinguish not only what is absolutely necessary and useful, but, even that which may happen to be of no use. The Nosologists who have gone before me, accordingly have done well in attempting to bring every kind of disease into their system. - But I find it extremely difficult to do the same thing: and both from want of confidence in my own knowledge, and from want of leisure, I have not attempted to comprehend the whole of diseases, because I think it better to leave the general plan imperfect than to introduce into it any thing about which I am uncertain. While I only attempt 
subjects of which I have a knowledge, I stand a better chance of escaping many errors than those who have grasped at every thing, trusting not only to their own knowledge, but more commonly to that of others. Upon this footing they have committed many faults, taking in many diseases of which we have as yet very imperfect accounts-such às those of distant foreign countries-and admitting certain trifling aberrations from the standard of human perfection which are not diseases. What shall we think of. Vogel's genus Seline, 'Macula alba in ungue?' Linnæus makes me smile when, at the end of his Nosology, after the definition of many trifling spots of the skin, and at least of Ephelis-the brown spots which appear on the face in delicate complexions in summer-there follows immediately-

' Heu mihi, tot mortes homini, quot membra, malisque

Tot sumus infecti, Mors ut Medicina putetur.'

It appears, therefore, gentlemen, that the very good general rule of comprehending in the system every species of disease, may be pushed to excess; and this is the first error of Nosologists.

The second error is that by which chiefly the genera and species have been multiplied, viz. enumerating every single symptom as a distinct and separate disease. In the system of Sauvages and others, Pandiculatio, Rigor, Sternutatio, Oscedo, Anxietas, Lassitudo, Stupor, Algor, Ardor, and many similar symptoms, form distinct and separate genera, although it is certain that they are neither genera nor species, that they never are the principal or leading symptoms of any disease with which we are acquainted, but are always combined with others. Errors of this kind may be useful in leading to pathological discussions of the symptoms, but they confound the Nosology.

A third error of our Nosologists is, their enumerating different degrees of the same genus as totally different, forgetting the rule that 'majus et minus non variat speciem.' Thus they have marked Carus, Cataphora, \&c. as different diseases', although they are only different degrees of apoplexy. Many similar instances of this you will find alluded to in my Synopsis, where I have comprehended șeveral genera in one.

A fourth error is, enumerating what are obviously only spe- 
cies or varieties of other diseases as distinct genera. Thus the Amphimerina and Tritæophyia are mere varieties of the tertian and quartan fevers; and in like manner Erythema is to be considered as a species of Phlogosis, and Lumbago and Ischias both as species of the genus Rheumatismus.

A fifth error relates to what I call the Sequelæ, the immediate and constant consequences of a disease. Though these are to be viewed in a different light, they are not to be considered as forming separate genera in the Nosology. Thus the older systems have made genera of Apostema, Abscessus, Gangræna, and Sphacelus, totally separating them from the Phlegmone or Inflammatio : I have marked them, as I think, in their proper place, as sequela of this disease. It must be obvious, that many diseases in their course change their form to such a degree, as to require a practice altogether different from that which was necessary in their former state. Thus, in the fever which I call Synochus, the practice in the end is totally different from that at the beginning; but nobody would say that these states ought to form two different genera or even species of diseases. So much for explaining the few cases in which I have introduced this new mode of Nosology; but I own that in many cases there is some difficulty in admitting this practice. We would do it only when one disease is very constantly a consequence of another, and on no occasion arises otherwise. But in many other cases a disease changes to one which is different, and to which it is only conjoined by certain external circumstances, and in this last case they ought to be kept as separate genera. I have seen Hydrothorax, for instance, as the consequence of Peripneumony; but it would not have been proper to introduce it as the sequela of Peripneumony. So the fistula in ano is frequently the consequence of hæmorrhois, but by no means a constant and necessary one: hence it is to be considered in another place, and under a separate head. The case of most importance where this difficulty occurs, is Phthisis, which I have placed as a sequela of Hæmoptoe. I own that I am not clear of being right in doing so ; but it will be of little consequence in the system, and will lead to little error.

The sixth and last error of Nosologists which I shall mention, is that of enumerating diseases of which we have no external symp- 
toms or certain marks. There are many internal affections which, could we discern them, we should certainly mark, but we know them only in consequence of dissection. Vogel, in the conclusion of his system, remarks, ".6 nec silentio prorsus transeunda sunt vitia quædam occulta, quxe non nisi in càdaverum sectione se offerunt :" they are, indeed, to be remembered; but the Nosologist is to be conducted by what are the objects of his senses; he is never to insert any thing which is the consequence of inference; and however imperfect this may leave our art in this respect, yet this is an imperfection which cannot be remedied. Where these internal affections are to be considered as primary, we mention them as possible causes of the external phenomena; and our experience very often enables us to say with probability when they take place. But they very rarely admit of being inserted in our Nosology. Sauvages, and particularly Vogel, have trespassed against this rule, mentioning diseases which indeed exist, but which were not to be noticed, as being without external marks. Thus Vogel, in establishing the genus Pancreatica, observes, " notae desunt," and thus assigns himself the reason why it should not have been mentioned.

These are the several errors which, I think, Nosologists have committed; and you will perceive how, in consequence of them, the genera have been too much multiplied, as has particularly been done by Vogel; and you will also observe how I had an opportunity of greatly diminishing the number of genera. I know of some, indeed, which in my synopsis are omitted, as I either had no clear or accurate knowledge of them, or could not. work them into any part of my system. When Ray attempted his system of Botany, he found that a number of plants would not incorporate; and hence he found it necessary to add at the end a list of anomalous plants, or, as Linnæus has it, "incertx sedis." I once thought of subjoining a few of that kind; but, as I am not sufficiently acquainted with many of them, I have omitted them.

All that I have now said, refers to the important article of distinguishing what in our system are called Genera, but, strictly speaking, are Species, or that ultimate concourse of symptoms which does not, for the most part, admit of a farther division but into Varieties. I have still to observe, that as I have 
adopted Classes and Orders, the character of these must always enter as a part of the character of the genus. Sometimes I have left it out, at other times I have inserted in the character of the genus that of the class or order.

Every species ought to have the character of its genus; and in naming or distinguishing species in Nosology or in Botany, it is supposed sufficient merely to name the genus, and to add such marks as distinguish the species. Take this remark, however, that in Botany as well as in Nosology, though the character of the genus is always supposed to be in the species, yet every part of the character of the genus is not always in the species. As an example from Botany, I may mention the Ranunculus, which stands in the class Polyandria : one species of Ranunculus, however, the $\mathbf{R}$. hederacea, never has more than five stamina, and ought therefore to have belonged to the class Pentandria; but the generic character of Ranunculus does not solely depend upon the number of stamina, but upon many other particulars, all of which occur in this species. If, accordingly, the character of a genus consists of five parts, and any given plant contains four out of the five, the want of the fifth does not separate it from the genus which it most resembles. Nor will the want of a single part of the character in the definition of a disease separate one individual from a species; it may happen that in a case of jaundice the "faeces albae" may be wanting, yet if the yellow colour of the face and eyes, and certain circumstances about the precordia, are present, there will be no doubt that the case is jaundice.

After these remarks, I must add, that nosologists and other systematists have in view two things, viz. Distinction and Denomination; that is, a part of the methodical system consists in affixing proper terms to the distinctions established. This last part is hardly less necessary than the other, as we, by applying fixed terms, in a manner realize our distinctions, and give precision to our ideas. I can refer you to very full information on this subject in the Philosophia Botanica of Linnæus; for what he says as to the appellation of plants will apply to every other department of science, and in particular to Nosology. And it is the less necessary to insist on this subject, because, in the attempt $\mathrm{I}$ have made towards a method, I have nowhere 
introduced one single new name of a disease, but have adopted names which had been employed by one or other of the systematics before. I found it absolutely necessary indeed to make a choice, and upon many occasions $I$ have not taken the name which was formerly the most common; and in other instances, where I have adopted names, I am not quite satisfied that they are the most proper; but rather than occasion any sort of confusion by new names, I have avoided every thing of that kind.

Now, after this introduction to Nosology in general, I return to consider the Synopsis, and shall add a very few words explanatory of my plan. The purpose of the whole is to fix the characters of diseases with somewhat more precision than has been hitherto done. As I thought it necessary for you to make a constant comparison, I have every where, under the title of the genus which I have chosen, given you an opportunity of comparing the systems of my predecessors, Sauvages, Vogel, Linnæus, and Sagar, by constantly referring to their corresponding places. I have farther referred to the most considerable systems of these days-to Boerhaave, to Hoffmann, and to Juncker, in place of Stahl; as also to the best monographists where the single subjects are more particularly and usefully treated.

You must begin by studying what we call genera; but you must not stop there : it is absolutely necessary to proceed to the study of species, and even to that of varieties; and I have therefore thought it proper to attempt giving you some assistance in this particular. I wished indeed to have assigned and characterized the several species, as I have done under the genus Cynanche, according to my own view of the matter; but I found that, owing to various insuperable difficulties, particularly as I had no other assistance but the Nosology of Sauvages, this was impossible. I have attempted, however, to make my arrangement more convenient and useful than that of Sauvages. He has gathered a great many particulars relating to species and varieties of diseases; he seems to have marked down whatever he found in his reading, so that his work has more the appearance of a common-place book, afterwards to be arranged by the author, than of any thing else : there is every 
where a want of arrangement, a want of digestion into order. But it is very probable that Sauvages. would have done a great part of what I have done, if time and leisure had allowed him.

I have taken pains to distinguish the species of Sauvages by arranging them under the two heads of idiopathice and symptomatica. Wherever he found a principal or very remarkable symptom of a disease, which likewise appears as a symptom in another disease, he has repeated it as a species in both places, and therefore multiplied the species, to the confusion"of the student, to an unreasonable number. Thus, among the tertian fevers, we have very properly the Tertiana hysterica as a variety of the tertian; but, afterwards, in the genus Hysteria, you find among the species symptomaticæ, the Hysteria febricosa ; by comparing these in the work of Sauvages, you will find that they are the same affection; the primary disease is a tertian, of which the hysteria is an accidental symptom. The same remark applies to Sauvages' Tertiana epileptica and Epilepsia febricosa, which are one and the same affection-a variety of tertian fever. Thus, I have separated the idiopathic and symptomatic species of Sauvages; but $I$ have further given to both quite a new arrangement : I have placed those togethe which I suspect to be one and the same, and next those which have the nearest affinity to one another. In many places again, where Sauvages has multiplied the genera, and repeated the same species under each of them, I have taken care to put these together, so that you may know the real number of species. The effect of all this, I think, must be to lessen considerably the whole number; and it must be comfortable to the student to see that his object, which was seemingly so large, may be comprehended in a narrower view. 


\section{FIRST LINES}

OF THE

\section{PRACTICE OF PHYSIC.}

(FROM THE EDITION 1784.) 


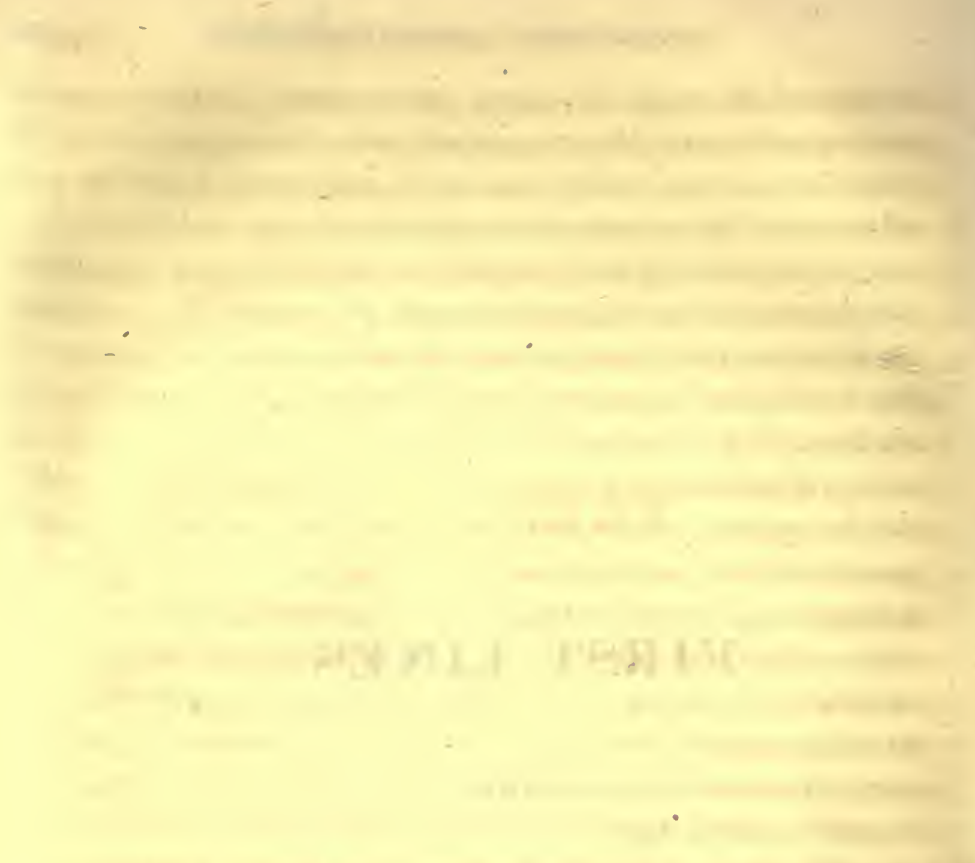

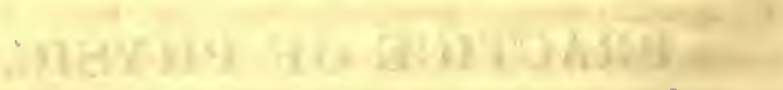

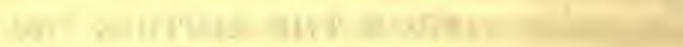
10

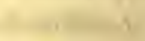

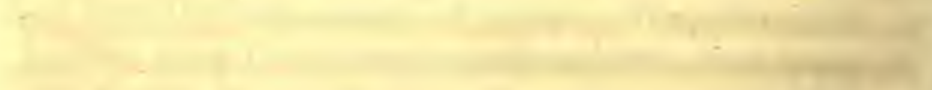

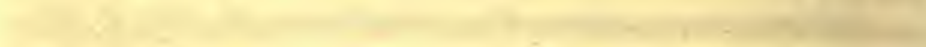

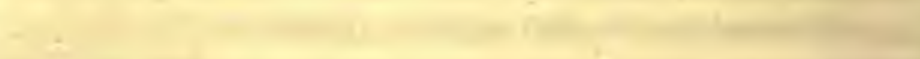
10 1

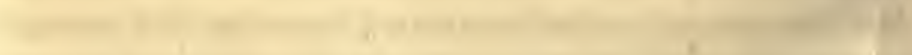
1 (2) (

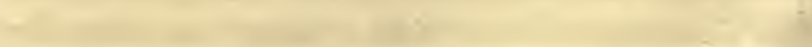

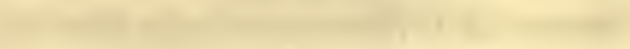

- 


\section{P REFA C E.}

To deliver a System of the Doctrines and Rules proper for directing the Practice of Physic, is an undertaking that appears to me to be attended with great difficulty; and, after an experience of more than forty years in that practice, as well as after much reading and reflection, it was with great diffidence that I ever entered upon such a work. It was, however, what seemed to be my duty as a professor, that induced me to make the attempt; and I was engaged in it by the same sentiments that the illustrious Dr. Boerhaave has expressed in the following passage of the Preface to his Institutions: 'Simul enim docendo admotus eram sensi, propriorum cogitatorum explicatione docentem plus proficere, quam si opus ab alio conscriptum interpretari suscipit. Sua quippe optime intelligit, sua cuique prae caeteris placent, unde clarior fere doctrina, atque animata plerumque sequitur oratio. Qui vero sensa alterius exponit, infelicius saepenumero eadem assequitur; quumque súo 'quisque sensu abundat, multa refutanda frequenter invenit, unde gravem frustra laborem aggravat, minusque incitata dictione utitur. $\because$ It is well known, that a text-book is not only extremely useful, but necessary to students who are to hear lectures: and from the same considerations that moved Dr. Bocrhave, I also wished to 
have one for myself; while, at the same time, from some peculiar circumstances in my situation, I had some additional inducements to undertake such a work.

Before I was established as a Professor of the Practice of Physic in this University, I had been employed in giving Clinical Lectures in the Royal Infirmary; and upon that occasion had delivered, what, in my opinion, seemed most just with regard to both the nature and the cure of the diseases of which I had occasion to treat. But I soon found that my doctrines were taken notice of, as new and peculiar to myself; and were accordingly severely criticised by those who, having long before been trained up in the system of Boerhaave, had continued to think that that system neither required any change, nor admitted of any amendment. I found, at the same time, that my doctrines were frequently criticised by persons who either had not been informed of them correctly, or who seemed not to understand them fully; and, therefore, as soon as I was employed to teach a more complete system of the Practice of Physic, I judged it necessary to publish a text-book, not only for the benefit of my hearers, but that I might also have an opportunity of obtaining the opinion of the public more at large, and thereby be enabled either to vindicate my doctrines, or be taught to correct them. These were the motives for my attempting the volumes I formerly published; and now, from many years' experience of their utility to my hearers, as well as from the favourable reception they have met with from the public, I am induced to give a new edition of this work, not only, as I hope, more correct in many parts, but also more complete and comprehensive in its general extent.

At the first publication of this work, it was intended chiefly for the use of those gentlemen who attended my lectures; although, even then, for the reasons I have mentioned, it was rendered more full than text-books commonly are; and, in the repeated editions I have since had occasion to give, I have been constantly endeavouring to render it more full and comprehensive. In these respects, I hope the present edition will appear to be rendered more fit for general use, and better calculated to afford satisfaction to all those who think they may still receive any instruction from reading on this subject. 
While I thus deliver my work in its now more improved state, with the hopes that it may be of use to others as well as to those who hear my lectures, I must, at the same time, observe, that it presents a system which is in many respects new ; and, therefore, I apprehend it to be not only proper but necessary, that I should explain here upon what grounds, and from what considerations this has been attempted.

I apprehend that in every branch of science with respect to which new facts are: daily acquired, and these consequently giving occasion to new reflections, which correct the principles formerly adopted, it is necessary, from time to time, to reform and renew the whole system, with all the additions and amendments which it has received and is then capable of. That at present this is requisite with regard to the science of medicine, will, I believe, readily occur to every person who at all thinks for himself, and is acquainted with the systems which have hitherto prevailed.

In the following treatise I have endeavoured to collect the facts relative to the diseases of the human body, as fully as the nature of the work, and the bounds necessarily prescribed to it would admit; but $I$ have not been satisfied with giving the facts, without endeavouring to apply. them to the investigation of proximate causes, and upon these to establish a more scientific and decided method of cure. In aiming at this, I flatter myself that I have avoided hypotheses, and what have been called theories. I have, indeed, endeavoured to establish many general doctrines, both physiological and pathological; but I trust that these are only a generalization of facts, or conclusions from a cautious and full induction : and if any one shall refuse to admit, or directly shall oppose my general doctrines, he must do it by shewing that I have been deficient or mistaken in assuming or applying facts. I have, myself, been jealous of my being sometimes imperfect in these respects; but I have generally endeavoured to obviate the consequences of this, by proving that the proximate causes which I have assigned are true in fact, as well as deductions from any reasoning that I may seem to have employed. Further, to obviate any dangerous fallacy in proposing a method of cure, I have always been anxious to suggest that, which, to the 
best of my judgment, appeared to be the method approved of by experience as much as it was the consequence of system.

- Upon this general plan I have endeavoured to form a System of Physic that should comprehend the whole of the facts relating to the science, and that will, I hope, collect and arrange them in better order than has been done before, as well as point out in particular those which are still wanting to establish general principles. This which I have attempted, may, like other systems, hereafter suffer a change; but I am confident, that we are at present in a better train of investigation than physicians were in before the time of Dr. Hoffmann. The affections of the motions and moving powers of the animal economy, must certainly be the leading inquiry in considering the diseases of the human body. The inquiry may be difficult; but it must be attempted, or the subject must be deserted altogether. I have, therefore, assumed the general principles of Hoffmann. And if I have rendered them more correct, and more extensive in their application, and, more 'particularly, if I have avoided introducing the many hypothetical doctrines of the Humoral Pathology which disfigured both his and all the other systems that have hitherto prevailed, I hope I shall be excused for attempting a system, which, upon the whole, may appear new.

Edinburah, Nov. 1783. 


\section{INTRODUCTION.}

I. IN teaching the Practice of Physic, we endeavour to give instructions for discerning, distinguishing, preventing, and curing diseases, as they occur in particular persons.

"I say that we teach to cure diseases as they occur in particular persons, in order to distinguish the course of the Practice from that of the Institutions. In the Institutions certain general doctrines with regard to health, disease, and remedy, are delivered; whereas what we call the Practice is the medical art applied to particular diseases and persons."

II. The art of Discerning and Distinguishing diseases may be best attained by an accurate and complete observation of their phenomena, as they occur in concourse and in succession; and by constantly endeavouring to distinguish the peculiar and inseparable concurrence of symptoms, to establish a Methodical Nosology, or an arrangement of diseases according to their genera and species, founded upon observation alone abstracted from all reasoning. Such an arrangement $I$ have attempted in another work, to which, in the course of the present, I shall frequently refer.

"It is certain that there is a peculiar congeries of phenomena or symptoms constituting diseases in individuals. It must also 
be allowed that these depend upon certain causes; but these causes are by no means evident, and physicians are therefore by no means agreed with regard to them. I cannot therefore think, that Boerhaave and Gaubius have been happy in their manner of defining a disease. Gaubius ( $\$ 34$. ) says, ' status ille corporis humani viventis quo fit, ut actiones hominis propriae non possint apposite ad leges sanitatis exerceri, morbus dicitur.' In like manner, Boerhaave (Instit. $\$$ 696.) says, ' status corporis viventis tollens facultatem exercendae actionis cujuscunque, vocatur morbus.' In both these definitions the sense is precisely the same; in the one it is 'status quo fit,' in the other 'status tollens.' But we learn nothing by taking the cause into the definition: you will observe also, that these definitions embarrass both Boerhaave and Gaubius in distinguishing between the disease itself and its proper cause.

In order to explain this a little, I must remark, that the doctrine of disease has formerly been sometimes named Pathology, and sometimes more accurately Nosology. The terms were considered as of the same import till of late, when they have. been applied to different meanings and differently limited. When we speak of the pathology of a disease, we consider the disease in its causes and effects; whereas, when we speak of a disease in nosology, we abstract from its cause, and consider it only as evident from certain external appearances; and we then distinguish diseases only by their differences in these external appearances. But if this is allowed to be a proper foundation for the study of the Practice of Physic, we must give a suitable definition of disease, we must define it as consisting of a certain concourse of phenomena, and without any/reference whatever to the cause. We must therefore desert the definition of Gaubius; the language of which we can however easily convert into a proper definition by reading 'in quo,' instead of the words "quo fit."'

III. The prevention of diseases depends upon the knowledge of their remote causes; which is partly delivered in the general Pathology, and partly to be delivered in this treatise.

"We observe, that almost every event may be considered as a part of a chain or series of causes which have in that series produced one another, and which, therefore, have produced the 
last effect, which we consider ; and in so far every part of the chain may be considered as a cause of the last effect. Thus, a man on board a ship takes up a lighted match, and applies it to the touch-hole of a loaded cannon; this kindles the gunpowder; this produces an explosion, which pushes on with great rapidity the bullet; this bullet strikes on a piece of timber, divides it into splinters, and these splinters happen to hit with great force the head of a man standing by, so that he is instantly killed. Now this death may be traced through this whole series of causes. The same applies to most other events in nature; and hence it is common to apply the term cause to each of these actions or motions. There is often, however, a necessity for distinguishing them into the more immediate and the more remote causes: in the above case, the stroke of the splinter was the immediate cause of the man's death; the whole series besides this were the remote causes. This very necessary distinction has been much employed in physic, and has given rise to the terms proximate and remote causes.

"Disease, considered as the effect of a particular state of the body, may be traced backwards in a series of causes, all of which may be called remote; but physicians have not been thus limited in taking the very immediate as the proximate cause. This will be best understood by an example. Pain in a joint constitutes the disease we call Rheumatism; in tracing the causes, we find that the pain is owing to an overdistention of the sensible fibres, in consequence of an overstretching of the blood-vessels. We further find, that this overstretching is caused by the increased impetus of the blood from a stimulus applied to those vessels, often to the whole system, but often to particular vessels; and that this has for its cause the application of cold. Now the application of cold may be said to be the only proper remote cause; but the stimulus, the increased impetus, the overdistention, may all be considered as proximate causes.

"That the meaning of this may be better understood, we take another example. A fluctuating swelling of the abdomen forms the disease which we call Ascites. We find that it has for its cause a quantity of water collected in the cavity of the abdomen, which may be further traced to an increased exhalation

$$
\text { vor. I. }
$$


or effusion of the halitus that is constantly thrown into the cavity of the abdomen. This we may trace to some resistance to the motion of the blood in the veins of the abdomen, which may have for its cause a congestion of blood in the liver, arising, again, from a suppression of the hæmorrhoidal flux; and this, lastly, may have been produced by the external application of cold. Every link of this chain, again, may be said to be a cause of the ascites, some links being more immediate, and others more remote; yet physicians would, in this case, consider only the cold applied as the remote cause, and the suppression of the hæmorrhoidal flux, the congestion of blood in the liver, \&c. as parts of the proximate cause.

"A scientific practice of medicine is founded upon the knowledge of the indications, of the changes to be produced in the body in order to cure a disease: whatever gives this indication is a part of the proximate cause; whether there be a series of causes and effects, or whether they act concurrently at the same time, if they continue to operate, and have a share in the effects observed, they are all considered as the proximate cause. It is often very difficult to assign the limits between remote and proximate causes. A remote cause may continue to form a part of the proximate cause: thus the splinter which I formerly mentioned, may strike a man's body, and then be still fixed in the body, so as not to be easily extracted; and so long as it continues to be an irritant, and prevents the healing of the wound, it is to be considered as a part of the proximate cause. A plethora also is commonly considered, and may be considered as the remote cause of a hæmorrhagy that afterwards arises; but whenever that plethora continues, it is to be considered as a part of the proximate cause of such hæmorrhagy. The only good definition of a proximate cause, is that given by Boerhaave, (Institut. \$. 740.) ' Causa proxima morbi appellatur, tota_illa simul quæ totum jam præsentem directe constituit; hæc semper est integra, sufficiens, præsens, totius morbi, sive simplex fuerit, sive composita. Hujus prosentia ponit, continuat morbum. Hujus absentia eum tollit.' He adds, 'Est fere eadem res ipsi integro morbo ;' and indeed this definition of the proximate 
cause is scarcely to be distinguished from his definition of disease.

"It has been common to divide causes into external and in. ternal, the last comprehending whatever has subsisted for some time before it produced its effects upon the body, the first, whatever is applied from without, and immediately operates its effects. There is often some use in this distinction of causes; but it is a great mistake to suppose that it implies the same as that between remote and proximate causes; there may be internal causes which are not proximate, and external causes which may be considered as a part of the proximate.

"Another distinction with regard to causes, viz. that into the predisposing and exciting causes, deserves attention; as, when an effect is produced by a particular agent acting upon a particular subject, which agent would not have acted upon every subject. Thus to recur to my former illustration, in order to kindle gun-powder, a spark can be produced only by the collision of two certain bodies, flint and steel. The collision of flint with soft iron will not produce the effect, which will therefore depend upon a concurrence of causes. Now the human body is at different times in different conditions with regard to its fitness for being acted upon by particular agents; such a condition is called a Predisposition, and the causes which produce it are termed predisposing causes. All those agents, on the other hand, which produce their effects only under certain conditions, are called occasional or exciting causes."

IV. The cure of diseases is chiefly, and almost unavoidably, founded in the knowledge of their proximate causes. This requires an acquaintance with the Institutions of Medicine; that is, the knowledge of the structure, action, and functions of the human body; of the several changes which it may undergo; and of the several powers by which it can be changed. Our knowledge of these particulars, however, is still incomplete, is in many respects doubtful, and has been often involved in mistake and error. The doctrine, therefore, of proximate causes founded upon that knowledge, must be frequently precarious and uncertain. It is however possible for a judicious physician to avoid what is vulgarly called theory, that is, all reasoning founded upon hypothesis, and thereby many of 
the errors which have formerly taken place in the Institutions of medicine. It is possible also for a person who has an extensive knowledge of the facts relative to the animal economy in health and in sickness, by a cautious and complete induction, to establish many general principles which may guide his reasoning with safety ; and while, at the same time, a physician admits as a foundation of practice those reasonings only which are simple, obvious, and certain, and for the most part admits as proximate causes those alone that are established as matters of fact rather than as deductions of reasoning, he may with great advantage establish a system of practice chiefly founded on the doctrine of proximate causes. But when this cannot be done with sufficient certainty, the judicious and prudent physician will have recourse to Experience alone; always, however, aware of the hitherto incomplete and fallacious state of Empiricism.

"That experience is to be consulted, is the vulgar cry of every one who has. not had the opportunity of studying, or the capacity: of understanding theory, and it is too generally the cry of those who know not the hitherto incomplete and fallacious state of Empiricism.-Hæc nosse salus est adolescentix.They ought to know the difficulty of Observation;-from the number of circumstances to be observed,-from the difficulty of estimating these exactly, even by the physician's senses, and still more so by the patient's feelings; - from the number of observations necessary, while exact resemblance is never found, in order to distinguish the constant from the accidental, the specific from the generic;-from the difficulty of guarding against the fallacy of temperaments, age, sex, climate, \&c. in the condition and administration of remedies, and many other circumstances; - from the difficulty of guarding against the mistakes and deceit of patients.

"The uncertainty of observations is thus shown, and all that uncertainty must pass into history, which must be further uncertain from the inaccuracy; and therefore the fallacy, of language in general, and from that of particular persons.

6 These considerations point out the uncertainty of facts; but, what is worse, there are false facts, - from the design of supporting favourite theories, - from the design of supporting par- 
ticular remedies, - from the desire of giving marvellous stories, from the vanity "of giving facts and observations; in short, I have often said there are more false facts than false opinions in physic, and the latter are more easily avoided than the former."

V. With a strict attention to these considerations in the whole of the following treatisé, I proceed to treat of particular diseases in the order of my Methodical Nosology.

"I have distributed all diseases into four classes, which we title, Pyrexiæ, Neuroses, Cachexiæ, and Locales. From the term applied to the last, you must perceive that I mean to make a general division of diseases into Universal and Local, or into those which affect the whole system at once, and those which affect one part only. Here there are some difficulties, but it is enough to give what is very general." 


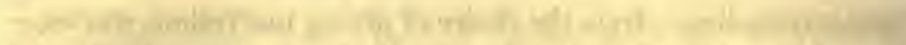

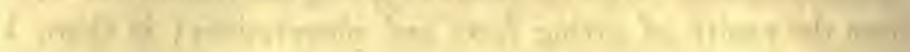

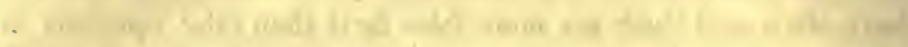

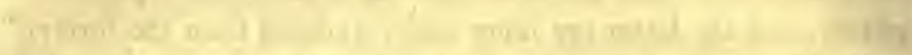

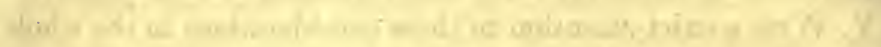

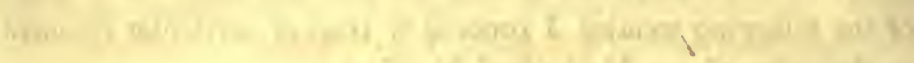

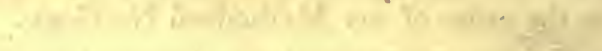

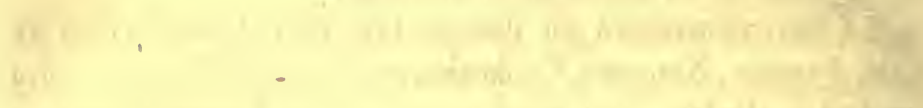

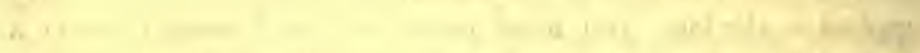

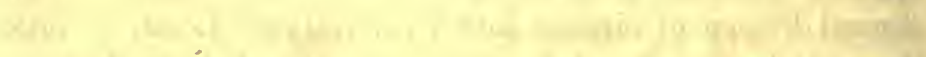

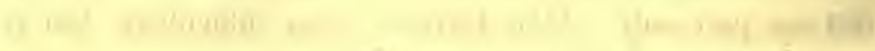
7. - 


\section{FIRST LINES}

OF THE

\section{PRACTICE OF PHYSIC.}

\section{PART I.}

OF PYREXIAE, OR FEBRILE DISEASES.

VI. Pyrexiae, or febrile diseases, are distinguished by the following appearances. After beginning with some degree of cold shivering, they shew some increase of heat, and an increased frequency of pulse, with the interruption and disorder of several functions, particularly some diminution of strength in the animal functions.

VII. Of these Pyrexiae I have formed a class, and have subdivided it into the five orders, of Fevers, Inflammations, Eruptions, Hæmorrhagies, and Fluxes. (See Synopsis Nosologice Methodicae.) 


\section{BOOK I.-OF FEVERS.}

\section{CHAP. I.-OF THE PHENOMENA OF FEVERS.}

VIII. Those diseases are more strictly called Fevers which have the general symptoms of pyrexia, without having along with them any topical affection that is essential and primary, such as the other orders of the Pyrexiæ always have.

"'The 'pulsus frequens,' of the moderns, which they have made the sole character of the pyrexia, or the ' calor major,' which was the sole character of the ancients, is not sufficient to constitute a disease of this class; and they only do so when these symptoms accompany horror, and more or less of debility. Such is the description of fever in every book, and I would not need to have insisted upon it, were it not for an observation of Boerhaave's, that the horripilatio is confined to diseases from internal causes, while the other symptoms are universal, so that, according to him, there is a hot fit which is not preceded by a cold one. But here to apply a general rule, I say that a fever of this sort does not occur once in ten thousand times. It is possible, that merely violence of exercise and excessive heat may bring on a fever without any excessive horror; but even in such cases $I$ have always observed that the horror did take place before any permanent fever came on; and if it does not, in no case does the fever come on, unless there is some topical affection, some congestion which is the cause of inflammation. And however we may speak in common language with respect to our being in a fever, upon many occasions this has been owing to the mistake of physicians with respect to its nature; and the character must remain as it stands, that the horror is universally the prelude to all kinds of fever.

"The term Pyrexia has no word of the same import in our language, but it has been common to apply the term Fever in the same extensive way. Thus, in common language, we say that the smallpox is a fever; but I only call one order of the 
class pyrexia by the name of fever, the character of which is, 'praegressis languore, lassitudine, et aliis debilitatis signis, pyrexia sine morbo locali primario.'

66 This part of the character, 'praegressis languore, lassitudine, et aliis debilitatis signis,' is left for introducing the other marks of debility; and we might add here the anorexia, nausea, and vomiting, as some part of the same; and further, that the pulse will be found to be always weaker before the attack. But whatever be in that, let me observe that these symptoms of debility are particularly to be found in the beginning of all intermittents, and in the most part of those continued fevers which we would arrange under the Typhus or Synochus. But with respect to the Synocha, we have not an opportunity of observing the debility that precedes. But further, in giving the character of a disease, it never rests on a single symptom, but on a concourse of them; so that whatever difficulty there may be in applying the ' praegressis languore, lassitudine, et aliis debilitatis signis' in Synocha, we are relieved by what is subjoined, ' sine morbo locali primario;' and indeed this last would have been sufficient. Here you will take notice of the phrase ' morbo locali primario,' for if every word be not of importance, it ought not to be in the definition. The ' morbus localis' may be very universal, but here $I$ use it in the common acceptation. Thus, in a fever, an inflammation may supervene, or, from some concurrent cause, may attend a fever, but then it is not the primary disease upon which the fever depends; so that the import of the whole definition is what constitutes fever."

IX. Fevers, as differing in the number and variety of their symptoms, have been very properly considered as of distinct genera and species. But we suppose, that there are certain circumstances in common to all the diseases comprehended under this order, which are therefore those essentially necessary to, and properly constituting the nature of fever. It is our business, especially, and in the first place, to investigate these; and I expect to find them as they occur in the paroxysm or fit of an intermittent fever, as this is most commonly formed.

"The beginning the class Pyrexia with the 'Febres strictius dictr,' is evidently proper, as their characters run through those of all the other orders. So we shall begin with considering 
these fevers more fully, and with. enumerating all the phenomena belonging to them.

"But this has always been a difficult matter; and the rock upon which the classical writers have always split has been in giving the history of a class instead of that of a genus, a method which is attended with much fallacy. We are not safe in delivering the history of even the lowest genus; but we are in still greater danger when attempting a higher genus. We can never be secure in our description unless we constantly keep in view a particular species, or what truly and certainly happens in nature, as in every artificial combination there is danger of our being misled. We therefore keep in view the Febres strictius dictæ, their first section, the intermittent, and of this the first genus, the tertian, which I consider as single. And now that we are to enter upon the first article, and to give its history or description, you will attend to the difference between a definition and a description. A definition or character implies so many particulars, or mentions so many phenomena as are just necessary to distinguish one disease from other diseases. But besides these, every disease is accompanied by a great number of other phenomena, which are variously combined, and appear in different orders of succession. The enumeration of both these kinds of phenomena is necessary to form a proper description. From a neglect of this distinction, and from taking the genus for the species, authors have thrown a great many superfluous circumstances into their definitions, and these not well arranged. But here I hope to give an example of a history or description, which is at once full and sufficiently distinct in its several parts."

$\mathbf{X}$. The phenomena to be observed in such a paroxysm are the following:-The person is affected, first, with a languor or sense of debility, a sluggishness in motion, and some uneasiness in exerting it, with frequent yawning and stretching. At the same time, the face and extremities become pale, the features shrink, the bulk of every external part is diminished, and the skin over the whole body appears constricted, as if cold had been applied to it. At the coming on of these symptoms, some coldness of the extremities, though little taken notice of by the patient, may be perceived by another person. At length the - patient himself feels a sensation of cold, commonly first in his 
back, but from thence, passing over the whole body ; and now his skin feels warm to another person. The patient's sense of cold increasing, produces a tremor in all his limbs, with frequent succussions or rigors of the trunk of the body. When this sense of cold and its effects have continued for some time, they become less violent, and are alternated with warm flushings. By degrees, the cold goes off entirely, and a heat greater than natural prevails, and continues over the whole body. With this heat, the colour of the skin returns, and a preternatural redness appears, especially in the face. While the heat and redness come on, the skin is relaxed and smoothed, but for some time continues dry. The features of the face and other parts of the body recover their usual size, and become even more turgid. When the heat, redness, and turgescence have increased and continued for some time, a moisture appears upon the forehead, and by degrees becomes a sweat, which gradually extends downwards over the whole body. As this sweat continues to flow, the heat of the body abates; the sweat, after continuing some time, gradually ceases; the body returns to its usual temperature, and most of the functions are restored to their ordinary state.

"Is the patient, when he feels the cold, really colder? At one period he is, but afterwards the thermometer and the feelings of the bystanders shew the heat to be increased, even whilst the patient is trembling.

"Now this is one series of phenomena which characterizes an intermittent fever. Indeed, there are not perhaps in nature two things which are absolutely uniform, but this is the series that commonly and almost universally characterizes a paroxysm of intermittent fever."

XI. This series of appearances gives occasion to divide the paroxysm into three different stages, which are called the Cold, the Hot, and the Sweating Stages or Fits.

In the course of these, considerable changes happen in the state of several other functions, which are now to be mentioned.

"In the foregoing description I have chosen those phenomena that are especially perceived by the person himself, and are most obvious to the bystanders; but there is a series of phenomena 
with respect to other functions which accompanies those already mentioned, and which is more frequently to be observed by the physician. Thus,"

XII. Upon the first approach of languor, the pulse becomes sometimes slower, and always weaker than before. As the sense of cold comes on, the pulse becomes smaller, very frequent, and often irregular. As the cold abates, and heat comes on, the pulse becomes more regular, hard, and full; and in these respects increases till the sweat breaks out. As the sweat flows, the pulse becomes softer and less frequent, till the sweat ceasing altogether, it returns to its usual state.

XIII. The respiration also suffers some changes. During the cold stage, the respiration is small, frequent, and anxious, and is sometimes attended with a cough. As the hot stage comes on, the respiration becomes fuller and more free, but continues still frequent and anxious, till the flowing of the sweat relieves the anxiety, and renders the breathing less frequent and more free. With the ceasing of the sweat, the breathing returns to its ordinary state.

XIV. The natural functions also suffer a change. Upon the approach of the cold stage, the appetite for food ceases, and does not return till the paroxysm be over, or the sweat has flowed for some time. Generally, during the whole of the paroxysm, there is not only a want of appetite, but an aversion from all solid, and especially animal food. As the cold stage advances, there frequently comes on a sickness and nausea, which often increase to a vomiting of a matter that is for the most part bilious. This vomiting commonly puts an end to the cold stage and brings on the hot. As the hot stage advances, the nausea and vomiting abate; and when the sweat breaks out, they generally cease altogether.

$\mathrm{XV}$. A considerable degree of thirst is commonly felt during the whole course of the paroxysm. During the cold stage, the thirst seems to arise from the dryness and clamminess of the mouth and fauces; but during the hot stage, from the heat which then prevails over the whole body; and as the sweat flows, the mouth becomes moister, and the thirst, together with the heat, gradually abates.

$\mathrm{XVI}$. In the course of a paroxysm, there is often a consider- 
able change in the state of the secretions. The circumstances just now mentioned shew it in the secretion of the saliva and mucus of the mouth; and it is still more remarkable with respect to the urine. During the cold stage the urine is almost colourless, and without cloud or sediment. In the hot stage it becomes high coloured, but is still without sediment. After the sweat has flowed freely, the urine deposites a sediment commonly lateritious, and continues to do so for some time after the paroxysm is over.

XVII. Excepting in certain uncommon cases, which are attended throughout with a diarrhœa, stools seldom occur till towards the end of a paroxysm, when commonly a stool happens; and which is generally of a loose kind.

XVIII. Analogous to these changes in the state of the secretions, it frequently happens that tumours subsisting on the surface of the body, suffer, during the cold stage of fevers, a sudden and considerable detumescence; but generally, though not always, the tumours return to their former size during the sweating stage. In like manner, ulcers are sometimes dried up during the cold stage, and return again to discharge matter during the sweating stage, or after the paroxysm is over.

XIX. Certain changes appear also in sensation and thought. During the cold stage the sensibility is often greatly impaired; but when the hot stage is formed, the sensibility is recovered, and often considerably increased.

XX. With respect to the intellectual functions, when the cold stage comes on, attention and recollection become difficult, and continue more or less so during the whole paroxysm. Hence some confusion of thought takes place, and often arises to a delirium, which sometimes comes on at the beginning of the cold stage, but more frequently not till the hot stage be formed.

XXI. It belongs also to this place to remark, that the cold stage sometimes comes on with a drowsiness and stupor, which often increase to a degree that may be called comatose or apoplectic._" With the tremor, too, other symptoms sometimes occur, as spasmodic and convulsive affections, all of which gradually decrease with the hot fit."

XXII. We have still to add, that sometimes, early in the cold stage, a headach comes on; but which, more commonly, is not 
felt till the hot stage be formed, and then is usually attended with a throbbing of the temples. The headach continues till the sweat breaks out; but as this flows more freely that gradually goes off. At the same time with the headach, there are commonly pains of the back, and of some of the great joints; and these pains have the same course with the headach.

XXIII. These are nearly the whole, and are at least the chief of the phenomena which more constantly appear in the paroxysm of an intermittent fever; and we have pointed out their ordinary concourse and succession. With respect to the whole of them, however, it is to be observed, that, in different cases, the several phenomena are in different degrees; that the series of them is more or less complete; and that the several parts or stages, in the time they occupy, are in a different proportion to one another._" This variety is founded in this, that all these several symptoms are in various degrees in different cases, and the whole series occupies a shorter or longer space of time, which may be said to extend from five hours, which is the shortest, to twenty hours, which is the longest; and we also find that the three different stages occupy different portions of time with respect to one another; thus the cold fit is sometimes hardly observable; at other times it occupies several hours; sometimes there is no hot fit, the sweat breaking out along with the heat ; and, lastly, there is sometimes a hot fit formed without any sweating.

"Here it is particularly worthy of the notice of practitioners, that the cold fit frequently proves fatal to the patient without any hot fit being formed in consequence of it. Before the person dies, however, some degree of hot fit is frequently formed, which has not been attended to by writers; indeed few persons appear to die in the paroxysm of an intermittent after the hot fit is fully formed.-So far with respect to the phenomena as they refer to a single paroxysm."

XXIV. It is very seldom that a fever consists of a single paroxysm, such as we have now described; "so that we must go further, and speak of the relation of different paroxysms to one another, and thereby approach nearer to the history of the whole of fever." And it more generally happens, after a certain length of time has elapsed from the ceasing of the paroxysm, that the 
same series of phenomena again arises, and observes the same course as before; and these states of FEVER and APYREXIA often continue to alternate with one another for many times. In such cases, the length of time from the end of one paroxysm to the beginning of another, is called an INTERMission; and the length of time from the beginning of one paroxysm to the beginning of another next succeeding, is called an INTERvar.

XXV. When the disease consists of a number of paroxysms, it is generally to be observed that the intervals between them are nearly equal ; but these intervals are of different lengths in different cases. The most usual interval is that of forty-eight hours, which is named the TERTIAN period. The next most common is that of seventy-two hours, and is named the QUARTAN period. Some other intervals also are observed, particularly one of twenty-four hours, named therefore the QuotidiaN; and the appearance of this is pretty frequent. But all other intervals longer than that of the quartan are extremely rare, and probably are only irregularities of the tertian or quartan periods.- With respect to these intervals, or periods, it often happens that they continue exactly the same; but this is not universally the case; nay, on the contrary, it is perhaps more common for the interval to be a little more or a little less than the time mentioned, which has given occasion to the terms postponing and anticipating paroxysms. This, however, when duly considered, will be found not to disturb our general position, that the tendency of nature is to form intervals of 48,72 , or 24 hours."

XXVI. The paroxysms of pure intermittent fevers are always finished in less than twenty-four hours; and though it happens that there are fevers which consist of repeated paroxysms, without any entire intermission between them, yet in such cases it is observed, that though the hot and sweating stages of the paroxysm do not entirely cease before the twenty-four hours from their beginning have expired, they suffer, however, before that time, a considerable abatement or REMission of their violence; and, at the return of the quotidian period, a paroxysm is in some shape renewed, which runs the same course as before. This constitutes what is called a REM ITTENT FEVER.

$\mathrm{XXVII}$. When in the remittents the remission is considerable, 
and the return of a new paroxysm is distinctly marked by the symptoms of a cold stage at the beginning of it, such fevers retain strictly the appellation of REMitTents. But when it happens, as it does in certain cases, that the remission is not considerable, is perhaps without sweat, and that the returning paroxysm is not marked by the most usual symptoms of a cold stage, but chiefly by the aggravation or EXACERBATION of a hot stage, the disease is called a conTINUEn FEver.- "When the pulse falls from 120 to 100 or 90 , this is reckoned a considerable remission ; when from 120 to 110 only, it is inconsiderable."

XXVIII. In some cases of continued fever, the remissions and exacerbations are so inconsiderable as not to be easily observed or distinguished; and this has led physicians to imagine that there is a species of fever subsisting for several days together, and seemingly consisting of one paroxysm only. This they have called a CONTINENT FEVER; but, in a long course of practice, I have not had an opportunity of observing such a fever.

XXIX. It is, however, to be observed here, that the fevers of a continued form are to be distinguished from one another; and that, while some of a very continued form do still belong to the section of intermittents, there are others which, though still consisting of separate and repeated paroxysms, yet, as being different by their causes and circumstances from intermittents, are to be distinguished from the whole of these, and are more strictly to be called and considered as contrnued. Such are most of those which have been commonly supposed to be ConTINenT; and those which by most writers have been simply named coNTINUED, and which term I have employed as the title of a section, to be distinguished from that of INTERMITTENT.

I shall here add the marks by which, in practice, these different continued fevers may be distinguished from one another.

Those fevers of a continued form, which, however, still belong to the section of intermittents, may be distinguished by their having passed from an intermittent or remittent form, to that of a continued; by their shewing some tendency to become intermittent, or at least remittent; by their being known to have been occasioned by marsh miasmata ; and, for the most part, by their having but one paroxysm, or one exacerbation and remission, in the course of twenty-four hours. 
On the other hand, continued fevers, to be more strictly so called, may be distinguished by their showing little tendency to become intermittent or remittent in any part of their course, and especially after the first week of their continuance; by their being occasioned by human contagion, at least by other causes than the marsh miasmata; and by their having pretty constantly an exacerbation and remission twice in the course of every twenty-four hours. In both cases, the knowledge of the nature of the epidemic for the time prevailing, may have a great share in determining the nature of the particular fever.

XXX. With respect to the form, or TYPE, of fevers, this further may be observed, that the quartan, while it has the longest interval, has, at the same time, the longest and most violent cold stage; but, upon the whole, the shortest paroxysm : that the tertian having a shorter interval than the quartan, has, at the same time, a shorter and less violent cold stage, but a longer paroxysm : and, lastly, that the quotidian, with the shortest interval, has the least of a cold stage, but the longest paroxysm.

XXXI. The type of fevers is sometimes changed in their course. When this happens it is generally in the following manner: Both tertians and quartans change into quotidians, quotidians into remittents, and these last become often of the most continued kind. In all these cases, the fever has its paroxysms protracted longer than usual, before it changes into a type of more frequent repetition.

XXXII. From all this a presumption arises that every fever consists of repeated paroxysms, differing from others chiefly in the circumstances and repetition of the paroxysms; and, therefore, that it was allowable for us to take the paroxysm: of a pure intermittent as an example and model of the whole.

"From this view I say, we may presume that fevers of all sorts are not only of one order, but nearly of what may be called one genus; and the particular application I would make of this consideration, is, that I had good grounds for taking the paroxysm of an intermittent fever as a proper example and model of the whole of fevers. And I would add farther, that if we can arrive at a tolerable explanation of that fit, we will go far to. explain the whole. In order to this, you must attend to the

vor. I. $2 \mathrm{~N}$ 
facts I have delivered; and as I have only mentioned the leading circumstances, for the whole variety you must go to your books."

CHAP. II.-OF THE PROXIMATE CAUSE OF FEVER.

XXXIII. The proximate cause of fever seems hitherto to have eluded the research of physicians; and I shall not pretend to ascertain it in a manner that may remove every difficulty; but. I shall endeavour to make an approach towards it, and such as I hope may be of use in conducting the practice in this disease : while, at the same time, I hope to avoid several errors which have formerly prevailed on this subject.

"In the first place, it must be observed, that a great difficulty arises from this, that the phenomena are very numerous and various, and that therefore it cannot be easy to refer the whole to one simple and common cause; at the same time, from these phenomena being so constantly combined together, and occurring in a regular train of succession, a strong presumption arises that there is one common cause of the whole. In some cases indeed, perhaps in all, the cause may be of a compound nature; and it is certainly true, that of the many phenomena commonly enumerated, several may depend upon accidental causes concurring with the common cause of fever. But we must first take the matter upon the most simple footing, and must not embarrass ourselves with the supposition of a compound cause; we must find out a common and simple cause of those phenomena that are most commonly combined together; so it is proper to attend here merely to the Symptomata morbi, leaving out what are called the Symptomata causæ, and the Symptomata accidentalia."

XXXIV. As the hot stage of fever is so constantly preceded by a cold stage, we presume that the latter is the cause of the former; and, therefore, that the cause of the cold stage is the cause of all that follows in the course of the paroxysm. (See Boerh. Aph. 756.)

" It is at present nearly agreed, that the frequency of the pulse must follow a cold fit in order to constitute a fever. Sauvages has founded his character entirely upon this opinion; but it is Dr. 
Hoffmann who has laid the proper foundation of this, and has pointed it out in the most express terms : (Opera, v. i. p. 301.) His words are these:- Nulla enim ejus species est, sive sit intermittens, sive continua, eaque vel benigna, vel maligna, acuta sive lenta, inflammatoria, sanguinea, aut lymphatica vel biliosa, item cum vel sine exanthematibus, sive denique symptomatica, in qua non sub accessione, progressu, immo tempore exacerbationis, partium extimarum refrigeratio, summa cutis ejusque pororum constrictio, vasorum detumescentia, horripilatio, partium rigor, sudoris suppressio alvique constipatio, observentur.' Here it is asserted in the most positive terms, that a fever has constantly as a part of it, a cold fit appearing always once, or occurring more or less frequently.

"Here I must observe, that by Bellini and Boerhaave the symptoms of the cold fit are supposed to be produced by a viscidity of the blood occasioning its stagnation in the extreme vessels, and this has continued to be the doctrine of all the Boerhaavians till lately; and M. Quesnay in particular has adopted it. But it would not be difficult to shew that it is without foundation, and even Boerhaave himself perceived the incompleteness of it. In the first edition of his Aphorisms, he only mentions the ' viscositas liquidi arteriosa ;' but he has since added, ' forte et liquidi nervosi inertia ;' and Van Swieten has entirely deserted the former supposition, and says, that the phenomena depend upon a change in the state of the nervous power. Dr. Whytt has expressed himself to the same purpose, that the sense of cold seems not to be owing to viscid fluids stagnating in the extreme vessels, but to a spasmodic constriction occasioned by that irritation which the nervous system suf fers. Wherefore, from the hint which Boerhaave throws out, from the opinion of his commentator, Van Swieten, from Dr. Hoffmann, and from Dr. Whytt, we lay it down, that the cold fit of fevers is an affection of the nervous system, which I am now to make out more fully."

XXXV. To discover the cause of the cold stage of fevers, we may observe, that it is always preceded by strong marks of general debility prevailing in the system. The smallness and weakness of the pulse, the paleness and coldness of the extreme parts, with the shrinking of the whole body, sufficiently show that 
the action of the heart and larger arteries, is, for the time, extremely weakened. Together with this, the languor, inactivity, and debility of the animal motions, the imperfect sensations, the feeling of cold, while the body is truly warm, and some other symptoms, all shew that the energy of the brain is, on this occasion, greatly weakened; and I presume that, as the weakness of the action of the heart can hardly be imputed to any other cause, this weakness also is a proof of the diminished energy of the brain.- "So I conclude, that a debility of the nervous power forms the beginning of the cold fit, and lays the foundation of all the other phenomena."

XXXVI. I shall hereafter endeavour to shew, that the most. noted of the remote causes of fever, as contagion, miasmata, cold, and fear, are of a sedative nature, and therefore render it probable that a debility is induced. Likewise, when the paroxysms of a fever have ceased to be repeated, they may again be renewed, and are most commonly renewed by the application of debilitating powers. "This is illustrated in the case of tertians, which cease for a period or two, and then recur, commonly after the application of some debilitating power, as cold, fatigue, \&c. Celsus remarked the power of habit in such cases, and founded. a prudent precept on it." And farther, the debility which subsists in the animal motions and other functions through the whole of the fever, renders it pretty certain that sedative or debilitating powers have been applied to the body.

XXXVII. It is therefore evident, that there are three states which always take place in fever; a state of debility, a state of cold, and a state of heat; and as these three states regularly and constantly succeed each other in the order we have mentioned them, it is presumed that they are in the series of cause and effect with respect to one another. This we hold as a matter of fact, even although we should not be able to explain in what manner, or by what mechanical means these states severally produce each other.

"Dr. Hoffmann's system goes no further than the spasm and hot fit, not taking in debility at all ; and I own that I have assumed the existence of this state of debility previous to the spasm before it has been clearly proved to exist in every case. When we come to consider the cases of inflammation and hromorrhagy, 
it will be the proper place for considering this matter more fully; and I hope I shall be able to make it evident, that the production of spasm, even there, will admit of an explanation upon the system I have laid down, and will be found to depend upon a previous debility, or something analogous to it."

XXXVIII. How the state of debility produces some of the symptoms of the cold stage, may perhaps be readily explained; but how it produces all of them, I cannot explain otherwise than by referring the matter to a general law of the animal economy, whereby it happens, that powers which have a tendency to hurt and destroy the system, often excite such motions as are suited to obviate the effects of the noxious power. This is the vis medicatrix natura, so famous in the schools of physic; and it seems probable, that many of the motions excited in fever are the effects of this power.

XXXIX. That the increased action of the heart and arteries, which takes place in the hot stages of fevers, is to be considered as an effort of the vis medicatrix naturce, has been long a common opinion among physicians; and I am disposed to assert, that some part of the cold stage may be imputed to the same power. I judge so, because the cold stage appears to be universally a means of producing the hot; because cold externally applied has very often similar effects._- If a man is suddenly plunged into cold water, taken out trembling and shaking, and put to bed, he will become preternaturally hot and even sweat, and hence have all the symptoms of an ague."-And more certainly still, because it seems to be in proportion to the degree of tremor in the cold stage, that the hot stage proceeds more or less quickly to a termination of the paroxysm, and to a more complete solution and longer intermission. (See XXX.)

XL. It is to be particularly observed, that during the cold stage of fever there seems to be a spasm induced every where on the extremities of the arteries, and more especially of those upon the surface of the body. This appears from the suppression of all excretions, and from the shrinking of the external parts; and although this may perhaps be imputed in part to the weaker action of the heart in propelling the blood into the extreme vessels, yet as these symptoms often continue after the action of the heart is restored, there is reason to believe that 
a spasmodic constriction has taken place, that it subsists for some time, and supports the hot stage; for this stage ceases with the flowing of the sweat, and the return of other excretions, which are marks of the relaxation of vessels formerly constricted: Hoffmann, Med. rat. System. tom iv. p. i. sect. i. cap. 1. art. 4. - After Hoffmann, almost every one who has read his writings has acknowledged that a spasm of the extreme vessels takes place in the beginning of fevers; that the subsistence of this gives their continuance; and that upon the yielding of this the fever has its solution. This is his system; and the doctrine is in a great measure perfectly well founded. It explains well the purpose of the increased action of the heart and arteries, which is to overcome the spasm, and thereby give a solution of the disease, rather than for the concoction of morbific matter, \&c. ; and it also explains well why evacuations do accompany and seem to be the means of the solution of fevers, as the relaxation of spasm must be attended with more or less evacuation."

XLI. The idea of fever then, may be, that a spasm of the extreme vessels, however induced, proves an irritation to the heart and arteries; and that this continues till the spasm is relaxed or overcome. There are many appearances which support this opinion; and there is little doubt that a spasm does take place, which proves an irritation to the heart, and therefore may be considered as a principal part in the proximate cause of fever. It will still, however, remain a question what is the cause of this spasm; whether it be directly produced by the remote causes of fever, or if it be only a part of the operation of the vis medicatrix naturc.

XLII. I am disposed to be of the latter opinion, because, in the first place, while it remains still certain that a debility lays the foundation of fever, it is not obvious in what manner the debility produces the spasm, and, what seems to be its effect, the increased action of the heart and arteries; and, secondly, because, in almost all the cases in which an effort is made by the vis medicatrix naturc, a cold fit and a spasm of the extreme vessels are almost always the beginning of such an effort. (See Gaub. Pathol. Medicin. art. 750.)

XLIII. It is therefore presumed, that such a cold fit and 
spasm at the beginning of fever, is a part of the operation of the vis medicatrix; but, at the same time, it seems to me probable, that during the whole course of the fever there is an atony subsisting in the extreme vessels, and that the relaxation of the spasm requires the restoring of the tone, and action of these.

XLIV. This it may be difficult to explain, but I think it may be ascertained as a fact by the consideration of the symptoms which take place with respect to the functions of the stomach in fevers, such as the anorexia, nausea, and vomiting, (XIV.)

From many circumstances it is sufficiently certain, that there is a consent between the stomach and surface of the body; and in all cases of the consent of distant parts, it is presumed to be by the connexion of the nervous system, and that the consent which appears between the sentient and moving fibres of the one part with those of the other, is such, that a certain condition prevailing in the one part occasions a similar condition in the other.

In the case of the stomach and surface of the body, the consent particularly appears by the connexion which is observed between the state of the perspiration and the state of the appetite in healthy persons ; and if it may be presumed that the appetite depends upon the state of tone in the muscular fibres of the stomach (See Physiology, CCIII.), it will follow, that the connexion of appetite and perspiration depends upon a consent between the muscular fibres of the stomach and the muscular fibres of the extreme vessels, or of the organ of perspiration, on the surface of the body.

It is further in proof of the connexion between the appetite and perspiration, and at the same time of the circumstances on which it depends, that cold applied to the surface of the body, when it does not stop perspiration, but proves a stimulus to it, is always a powerful means of exciting appetite.

Having thus established the connexion or consent mentioned, we argue, that as the symptoms of anorexia, nausea, and vomiting, in many cases manifestly depend upon a state of debility or loss of tone in the muscular fibres of the stomach, so it may be presumed that these symptoms, in the beginning of a fever, depend upon an atony communicated to the muscular fibres of 
the stomach from the muscular fibres of the extreme vessels on the surface of the body.

That the debility of the stomach which produces vomiting in the beginning of fevers, actually depends upon an atony of the extreme vessels on the surface of the body, appears particularly from a fact observed by Dr. Sydenham. In the attack of the plague, a vomiting happens which prevents any medicine from remaining on the stomach; and Dr. Sydenham tells us, that in such cases he could not overcome this vomiting but by external means applied to produce a sweat, that is, to excite the action of the vessels on the surface of the body.

The same connexion between the state of the stomach and that of the extreme vessels on the surface of the body, appears from this also, that the vomiting, which so frequently happens in the cold stage of fevers, commonly ceases upon the coming on of the hot, and very certainly upon any sweat coming out, (XIV.). It is indeed probable, that the vomiting in the cold stage of fevers, is one of the means employed by nature for restoring the determination to the surface of the body ; and it is a circumstance affording proof, both of this and of the general connexion between the stomach and surface of the body, that emetics thrown into the stomach and operating there, in the time of the cold stage, commonly put an end to it, and bring on the hot stage.

It also affords a proof of the same connexion, that cold water taken into the stomach produces an increase of heat on the surface of the body, and is very often a convenient and effectual means of producing sweat.

From the whole we have now said on this subject, I think it is sufficiently probable, that the symptoms of anorexia, nausea, and vomiting, depend upon, and are a proof of an atony subsisting in the extreme vessels on the surface of the body; and that this atony, therefore, now ascertained as a matter of fact, may be considered as a principal circumstance in the proximate cause of fever.

XLV. This atony we suppose to depend upon a diminution of the energy of the brain; and that this diminution takes place in fevers, we conclude, not only from the debility prevailing in so many of the functions of the body, mentioned above 
(XXXV.), but particularly from symptoms which are peculiar to the brain itself. Delirium is a frequent symptom of fever; and as from the Physiology and Pathology we learn that this symptom commonly depends upon some inequality in the excitement of the brain or intellectual organ (See Physiology, p. 146.), we hence conclude, that in fever it denotes some diminution in the energy of the brain. Delirium, indeed, seems often to depend upon an increased impetus of the blood in the vessels of the brain, and therefore attends phrenitis. It frequently appears also in the hot stage of fevers, accompanied with a headach and throbbing of the temples. But as the impetus of the blood in the vessels of the head is often considerably increased by exercise, external heat, passions, and other causes, without occasioning any delirium, so, supposing that the same impetus in the case of fever produces delirium, the reason must be, that at the same time there is some cause which diminishes the energy of the brain, and prevents a free communication between the parts concerned in the intellectual functions. Upon the same principles, also, I suppose there is another species of delirium, depending more entirely on the diminished energy of the brain, and which may therefore arise when there is no unusual increase of the impetus of the blood in the vessels of the brain. Such seems to be the delirium occurring at the beginning of the cold stage of fevers, or in the hot stage of such fevers as shew strong marks of debility in the whole system.

"I have observed, that in the hot fit of an intermittent a delirium frequently arises, and that then it is attended with headach and throbbing of the temples, and with a full and strong pulse; here we do not at all hesitate in ascribing the delirium to the increased impetus of the blood in the vessels of the brain ; and we know that persons have been freed from it by raising their head a little higher, and even by changing sides the headach and delirium have been found sensibly to abate; besides, every inflammation of the brain consists in such an increased impetus, and is constantly accompanied with increased delirium. So there is no doubt that one species of delirium depends upon an increased action of the vessels of the brain. "But pathologists have gone too far, and supposed this to 
be constantly the case, and have referred all cases of delirium to the state of the circulation in the brain. See, for instance, Boerhaave's 701st aphorism. In the whole tenor of it, notwithstanding his ' plurimae causae,' he has chiefly in his view the state of the circulation of the brain, and that, too, confined to increased impetus, as we may see from the method of cure which he gives in the next aphorism; for nine out of ten of his remedies are plainly means of diminishing the determination to the brain, and tend only to remove increased impetus in its vessels. It is true, Van Swieten, his commentator, thinks we must go farther, and says, that there are sympathetic deliria, depending upon affections of distant parts of the system, particularly of the stomach, so that the functions of the brain may be affected independently of the circulation. But he had no conception of delirium being cured by the application of powerful stimulants, of which there are cases, I am told, on very good authority; as that of a gentleman in a fever, who had his delirium removed by the use of wine, and was obliged to drink eight bottles a-day, because, as soon as the effects of the wine were over, he became delirious. Now, this is one species of delirium which, I imagine, neither Boerhaave nor Van Swieten ever thought of. In short, the motions of the nervous power can on many occasions be changed without any alteration in the state of the circulation; and it is only in the state of the motions of the nervous fluid that we are to seek for the cause of any change in the state of our intellectual faculties. Some even think that these changes are independent of any change whatever in the circulation of the blood; and that if they are connected with any changes in our corporeal part, it must be with those of the nervous power.

"Now, with respect to its depending upon the state of the circulation, I have said that no delirium, perhaps, depends upon this alone ; for we know, that in violent exercise the impetus is greatly increased above what it is in many fevers, and in many cases of exhaustion the impetus is greatly diminished, and yet the intellectual faculties are no ways affected. We are therefore to seek for the cause of such changes in the state of the nervous system alone.

"But an inequality of the state of the brain may explain delirium more particularly, as we may look upon. the whole doc- 
trine of Fever as depending upon the inequality of the Excitement or Collapse of the brain.

"6 We know that in fevers a debility lays the foundation of the disease, or that there takes place a diminution of the excitement or energy of the brain, which even sometimes proceeds to a deeper state of collapse, or to what is termed Coma, or a disposition to sleep. This collapse is unequal with respect to the different parts, and continues great with respect to the animal functions, while it is recovered from with respect to the vital. At the same time, the impetus of the blood is increased, which is a powerful cause of excitement; and some degree of collapse at the same time taking place, this will readily give a delirium, such as occurs in the hot fit of fevers. Or when there is an excess of collapse from the sedative operation of the remote causes, and some cause of excitement is applied, as the impetus of the blood in the ordinary course of circulation, such a delirium may occur as that which frequently takes place in the cold fit of fevers. It appears, then, that the inequality of the excitement of the brain lays the foundation of delirium, which therefore may be of two kinds, as it may either depend upon the excess of excitement, or upon the excess of collapse.

"Now there is no doubt that this explanation will sufficiently account for all the transitory deliria that happen in the cold fit of intermittent fevers; and I do think that it will also account for many of the instances of it which occur in continued fevers, where it takes place without any topical affection of the circulating organs of the brain, and where it appears to depend entirely upon the states of excitement and collapse of the nervous system, and to proceed from causes immediately affecting it, independently of the state of the sanguiferous system.

"But indeed these causes producing delirium, whether more durable or more transitory, are not the only causes which produce this effect; for in fevers the brain may also be topically affected, which may be the cause of excitement and collapse greater than would arise from the general state of fever. Thus a degree of topical inflammation of the brain may take place, so as to give more or less of the phrenitic delirium, from which circumstance it will be more obstinate, or there may be other 
causes of increased excitement of the brain which will have a similar effect.

"It belongs to this place to observe, that other symptoms occur in fevers, such as those of sleep and watching; which are to be explained on the same footing. If you consult the writings of Boerhaave, more especially as they are explained by Van Swieten, on Coma and Pervigilium, you will find that these symptoms are explained by the state of the organs of circulation in the brain; and this may be often the cause, but if the physiology I have delivered with respect to sleep and watching (Physiology, CXXVII.) be well founded, nothing is more evident than that the Coma may depend upon the affection of the nervous system, and upon this alone independently of the sanguiferous, viz. upon its state of excitement and collapse ; the cause of fever inducing that state in which the coma consists. The deliria being transitory, is a strong proof of their depending upon the nervous system ; for what vanishes in an hour or two cannot depend upon any considerable topical affection, or any considerable derangement of the circulating fluids, but rather must depend upon the diminished excitement of the brain, or on the exercise of its energy.

"We here also take notice of the horror and tremor which commonly form so considerable a share of the cold fit of fever. These symptoms are upon other occasions the effect of cold applied to the body, and therefore may be considered as the effects of the sense of cold, which always precedes and accompanies the beginning of fever. It will be of use to consider this matner still farther, and to inquire more particularly in what tremor consists.

6'Tremor occurs in a state of debility, and is an effort arising from a constant alternation of the debility and the effort of the will. And here it is to be observed, that there are many instances of motions produced by the effort of the energy of the brain, without any consciousness of the concurrence of the will to produce such motion. Thus, the chattering of the teeth, or tremor of the lower jaw, arises from its tendency to fall down in consequence of its weight, and the constant effort of the energy of the brain to raise it.

" Tremor then occurring in fever is to be considered as a mark of the reaction of the system, not as a part of the direct 
effect of the debilitating cause, not purely as a mark of debility, as it might at first appear, but of the effort of the system towards a reaction to overcome the state of debility. This reaction, I have remarked, is the effect of the spasm; now in proportion to the greater degree of tremor, the reaction proves more considerable, and the spasm is more speedily resolved. This is exactly agreeable to observation, for we find that in the beginning of a paroxysm of an intermittent, the tremor is more considerable than in the beginning of a continued fever; and intermittents admit of a solution more quickly, in proportion as the reaction and the tremor are more violcnt. Senac (de recondita febrium natura), in treating of the febrile cold, observes, that where the rigor and tremor in the beginning are very violent, there the fever certainly turns out an intermittent, and the paroxysms then admit of a quick solution. He also remarks; that the cold fit is sometimes extended to hours; but whenever the paroxysms are long protracted, the cold fit in the beginning is in proportion less remarkable. But we also find, that the cold stage is most considerable in those intermittent fevers which have the shortest paroxysms. The horror, tremor, and rigor, are always most considerable in quartans-less so in tertians-least of all in quotidians-and continued fevers are marked by almost no tremor at all.

"It appears from this, that the horror and tremor are not to be considered as purely symptoms of the first operating debilitating cause, but that they are the consequence of the reaction of the system which follows: hence, from their condition, we are enabled to judge of the condition of the disease that is to follow, which is of great consequence in distinguishing fevers."

XLVI. Upon the whole, our doctrine of fever is explicitly this: The remote causes (XXXVI.) are certain sedative powers applied to the nervous system, which, diminishing the energy of the brain, thereby produce a debility in the whole of the functions (XXXV.), and particularly in the action of the extreme vessels, (XLIII. XLIV.). Such, however, is, at the same time, the nature of the animal economy (XXXVIII.), that this debility proves an indirect stimulus to the sanguiferous system; whence, by the intervention of the cold stage and spasm connected with it (XXXIX. XL.), the action of the 
heart and larger arteries is increased (XL.), and continues so (XLI.) till it has had the effect of restoring the energy of the brain, of extending this energy to the extreme vessels, of restoring therefore their action, and thereby especially overcoming the spasm affecting them; upon the removing of which, the execretion of sweat, and other marks of the relaxation of excretories take place.

"Now this is what I call the theory of fever; but take notice what kind of a theory it is ; for it is no more than saying that there are certain states of the body which are combined together in a certain order if succession, and that from this constant combination they are to be considered as a series of causes and effects. This I assume as a fact, and I suppose that this connexion is determined by a certain mechanism of the body; but I do not explain the nature of this; I might offer conjectures upon the subject, but while they are only conjectures, I shall not trouble you with them, so shall go no further."

XLVII. This doctrine will, as I suppose, serve to explain not only the nature of fever in general, but also the various cases of it which occur. Before proceeding, however, to this, it may be proper to point out the opinions, and, as I apprehend, the mistakes, which have formerly prevailed on this subject.

XLVIII. It has been supposed, that a lentor or viscidity prevailing in the mass of blood, and stagnating in the extreme vessels, is the cause of the cold stage of fevers and its consequences. But there is no evidence of any such viscidity previously subsisting in the fluids; and, as it is very improbable that such a state of them can be very quickly produced, so the suddenness with which paroxysms come on, renders it more likely that the phenomena depend upon some cause acting upon the nervous system, or the primary moving powers of the animal economy. (See Van Swieten apud Boerh. Aph. 755.)

XLIX. Another opinion, which has been almost universally received, is, that a noxious matter introduced into, or generated in the body, is the proximate cause of fever; and that the increased action of the heart and arteries, which forms so great a part of the disease, is an effort of the vis medicatrix naturce to expel this morbid matter; and particularly to change or 
concoct it, so as to render it either altogether innocent, or, at least, fit for being more easily thrown out of the body. This doctrine, however, although of as great antiquity as any of the records of physic now remaining, and although it has been received by almost every school of medicine, yet appears to me to rest upon a very uncertain foundation. There are fevers produced by cold, fear, and other causes, accompanied with all the essential circumstances of fever, and terminating by sweat, but, at the same time, without any evidence or suspicion of morbific matter.

There have been fevers suddenly cured by a hæmorrhagy, so moderate as could not carry out any considerable portion of a matter diffused over the whole mass of blood; nor can we conceive how the morbific matter could be collected or determined to pass by such an outlet as in that case is opened.

Even supposing a morbific matter were present, there is no explanation given in what manner the concoction of it is performed; nor is it shown that any such change does in fact take place. In certain cases indeed it is evident, that a noxious matter is introduced into the body, and proves the cause of fever: but even in these cases, it appears that the noxious matter is thrown out again, without having suffered any change; that the fever often terminates before the matter is expelled; and that upon many occasions, without waiting the supposed time of concoction, the fever can be cured, and that by remedies which do not seem to operate upon the fluids, or to produce any evacuation.

L. While we thus reason against the notion of fever being an effort of nature for concocting and expelling a morbific matter, I by no means intend to deny that the cause of fever frequently operates upon the fluids, and particularly produces a putrescent state of them. I acknowledge that this is frequently the case; but, at - the same time, I maintain, that such a change of the fluids is not commonly the cause of fever; that very often it is an effect only; and that there is no reason to believe the termination of the fever to depend upon the expulsion of the putrid matter.

LI. Another opinion which has prevailed remains still to be mentioned. In intermittent fevers, a great quantity of bile is commonly thrown out by vomiting; and this is so frequently 
the case, that many have supposed an unusual quantity of bile, and perhaps a peculiar quality of it, to be the cause of intermittent fevers. This, however, does not appear to be well founded. Vomiting, by whatever means excited, if often repeated, with violent straining, seems to be powerful in emulging the biliary ducts, and commonly throws out a great deal of bile. This will happen especially in the case of intermittent fevers; for as, in the state of debility and cold stage of these fevers, the blood is not propelled in the usual quantity into the extreme vessels, and particularly into those on the surface of the body, but is accumulated in the vessels of the internal parts, and particularly in the vena portarum; so this may occasion a more copious secretion of bile.

These considerations will, in some measure, account for the appearance of an unusual quantity of bile in intermittent fevers; but the circumstance which chiefly occasions the appearance of bile in these cases, is the influence of warm climates and seasons. These seldom fail to produce a state of the human body, in which the bile is disposed to pass off, by its secretories, in greater quantity than usual, and perhaps also changed in its quality, as appears from the disease of cholera, which so frequently occurs in warm seasons. At the same time, this disease occurs often without fever; and we shall hereafter render it sufficiently probable, that intermittent fevers, for the most part, arise from another cause, that is, from marsh effluvia; while, on the other hand, there is no evidence of their arising from the state of the bile alone. The marsh effluvia, however, commonly operate most powerfully in the same season that produces the change and redundance of the bile; and, therefore, considering the vomiting, and other circumstances of the intermittent fevers which here concur, it is not surprising that autumnal intermittents are so often attended with effusions of bile.

1. "It has been very universally observed, that warm seasons and warm climates produce some change in the state of the bile in human bodies; and, in particular, that the cholera morbus, as it is called, that is a copious afflux of some acrid bile to the intestines, is the particular effect of warm seasons and warm climates. Sydenham has said, that the spontaneous cholera continued to the month of August in the south of England where 
he practised; and Cleghorn observes, that in Minorca it is the produce of every warm season, and appears there sooner or later as the heat is sooner or later in setting in, though, in general, it extends from the middle of July to the middle of September.

"Such is the fact; but the theory is uncertain, in as much as it is a question, whether the bile is secreted in greater quantity, or in a more acrid state, occasioning a more copious afflux by its stimulus. But it is a fact, also, that the cholera may happen without any fever either preceding or accompanying it : whatever therefore may be the change affecting the bile, it does not of itself necessarily produce fever. We observe, indeed, when this state of the bile takes place, and a cause of fever occurs at the same time, that then such fever will probably be attended with an evacuation of bile. But this afflux is an effect of fever rather than a cause. For it is obvious that the attack of fever is attended with a constriction of the extreme vessels ; which, together with the debility of the body, certainly prevents the blood from being sent in its usual quantity to the extreme vessels : it will therefore be accumulated in the body, and particularly in the venous system, where the circulation is more languid, and the dilatation greater. Thus it will be more copiously determined to the vessels of the internal parts, and especially to what I call the hypochondriac system, or system of the vena portarum, in which there is not only the largest proportion of venous blood, but where it is more difficultly transmitted; for wherever the blood is not determined to the surface of the body, we find that it is in consequence collected in the vena portarum. And that such congestions are in fact formed in fevers, is well known; the dissections of such as have died of intermittents, have shewn a great increase in the bulk of the liver and spleen. But if the blood is thus collected in that region, it must produce a more copious secretion of bile.

"Another circumstance, also, which attends the beginning of fevers, has a share in producing this bile, viz. the vomiting, which, when forcibly exerted, does emulge the biliary ducts, and therefore produces-a more copious afflux of bile into the intestines, which, in consequence of the inverted peristaltic motion, is thrown into the stomach.

"Now, I think this unusual discharge of bile may appear withvoln I. 
out affording us any grounds for imagining that the bile had any concern in the case of fever. Notwithstanding this opinion has been adopted both by ancients and moderns, and Bilious fever is a term still subsisting in every body's mouth. I, for my part, cannot admit of the bile as a cause; for it is by no means adequate to the effect of producing any fevers; not even those which we call bilious fevers, and which may be properly enough so named from the redundancy of bile which occurs in them. Fevers are owing to something else taken from without; and I shall prove that autumnal fevers are owing to what I call marsh effluvia: and I maintain, that whatever be the heat of the climate, and, consequently, the acrimonious state of the bile, if marsh effluvia be avoided, no intermittent fever will arise. The whole of Dr. Lind's ingenious work on the preservation of health of Europeans in warm climates, turns upon this, that the heat of the climate will not bring on fevers if the marsh effluvia be avoided."

This view of the subject does not lead us to consider the state of the bile as the cause of intermittents, but merely as a circumstance accidentally concurring with them, from the state of the season in which they arise. What attention this requires in the conduct of the disease, I shall consider hereafter.

LII. From this view of the principal hypotheses which have hitherto been maintained with respect to the proximate cause of fever, it will appear, that fevers do not arise from changes in the state of the fluids; but that, on the contrary, almost the whole of the phenomena of fevers lead us to believe, that they chiefly depend upon changes in the state of the moving powers of the animal system. Though we should not be able to explain all the circumstances of the disease, it is at least of some advantage to be led into the proper train of investigation. I have attempted to pursue it, and shall now endeavour to apply the doctrine delivered towards explaining the diversity of fevers.

CHAP. III.-OF THE DIFFERENCE OF FEVERS, AND ITS CAUSES.

"6 I must repeat that in the order Febres there occur the three states of Debility, Spasm, and increased Reaction. These I have hitherto considered as succeeding each other, but you 
must now attend to this, that though I have constantly spoken of them as separate and successive, this is not strictly true; for during each paroxysm, except in the beginning and at the end of it, all the three different states are, in some measure, present at one time. Thus, to take the most intermediate part: while the hot fit continues without any sweating taking place, and while, at the same time, the urine continues thin and limpid, we conclude that the extreme vessels are still under a state of constriction, that is, the spasm does still remain so long; but while the spasm remains, the debility which occasioned it must remain also; we must infer, therefore, that the energy of the brain is not restored to the extreme vessels. That this state of debility does subsist for a great part of the time which a paroxysm occupies, is not only evident from this reasoning, but manifestly appears from the extreme debility of the animal functions, and of those of the senses, and, therefore, from the symptoms relative to the stomach, the want of appetite, nausea, \&c. which may be considered as marks of the same debility. It is consequently the continuance of the debility and spasm which properly regulates the duration of fever. Now, if we can suppose that these states subsist in a different degree and proportion in different fevers, it will be a considerable step, as we will then have found, in general, upon what the difference of fever especially turns. But, before we proceed further, I must observe what differences of fever are established by just observation, so that our theory, as we call it, may correspond to matter of fact.

"All physicians are agreed that fevers are of two kinds, the Intermittent and Continued, constituting the first and second sections in our synopsis. The intermittents, in the most common acceptation of the word, are such fevers as consist in a repeated number of paroxysms, between which an intermission or apyrexia intervenes. The second kind, the continued fevers, are with more difficulty explained. They consist also of repeated paroxysms, but without the intervention of any perfect intermission. When I say that they consist of paroxysms, it necessarily follows that if they are not separated by an intermission or state of apyrexia, they are at least distinguished by times of exacerbation and remission, which are particularly to be observed with respect to the hot fit. As these remissions have been more 
or less observable, some fevers have been farther distinguished as Remittent, which, in practical writers, have formed a third section. Authors have, however, for the most part. applied the term Continued in the same manner as I do, to those fevers in which the alternate state is observable, but less so; but to those fevers in which the remission is very considerable and evident, they have given the name of Remittent, and so they have made the meaning of the term Continued less comprehensive. But let us see what these multiplied distinctions have further led to. There are certain fevers in which these remissions are very difficultly observed, and from this, I suppose, physicians have conceived that there are fevers consisting of one paroxysm of several days' duration, or that there is a kind of fever which continues in the same state during its whole course; and to such a fever they have given the name of Continent; supposing that but one hot fit, or that only, one reaction takes place through the whole course of the fever. Now, whether there is any such fever as this Continent in the language of the schools, may be disputed; and, as the question is of great consequence, and its determination will have a great influence on the doctrine I am delivering, I propose to consider it more particularly."

LIII. To ascertain the difference of fevers, I think it necessary to observe, in the first place, that every fever of more than one day's duration consists of repeated, and, in some measure, separate paroxysms; and that the difference of fevers taken notice of above (from XXV. to XXX.) appears to consist in the different state of paroxysms, and in the different circumstances of their repetition.

LIV. That fevers generally consist of distinct, and, in some measure separately repeated paroxysms, I have alleged above to be a matter of fact; but I shall here endeavour to confirm it, by assigning the cause.

LV. In every fever in which we can distinctly observe any number of separate paroxysms, we constantly find that each paroxysm is finished in less than twenty-four hours; but as I cannot perceive any thing in the cause of fevers determining to this, I must presume it to depend on some general law of the animal economy. Such a law seems to be that which subjects the economy, in many respects, to a diurnal revolution. Whether 
this depends upon the original conformation of the body, or upon certain powers constantly applied to it, and inducing a habit, I cannot positively determine: but the returns of sleep and watching, of appetites and excretions, and the changes which regularly occur in the state of the pulse, shew sufficiently that in the human body a diurnal revolution takes place.

"The course, I say, of every intermittent fever which I know, consists of repeated paroxysms, with a distinct apyrexia, and is always finished in less than twenty-four hours, whether the fever be quotidian, tertian, or quartan; and there is not one observation in physic in contradiction to this. The same applies to every fever which physicians have been pleased to call Remittent; and it also holds with regard to those which are called Continued, in which the alternate states are the least distinctly observed; but if they can be observed at all, they are found to occur once in the twenty-four hours.

"I do not pretend to know so much of the nature of fevers or of the operation of those causes which determine the paroxysm to run such a course, as to be able sufficiently to clear up this matter; but I presume, that this phenomenon of the paroxysm of fever being always finished in less than twenty-four hours, does not depend on the cause of the fever, but rather on the nature of the economy itself, or upon some law of the system determining it to a diurnal revolution which modifies fevers in this respect. It may proceed from those causes which are produced by the diurnal revolution of the earth, viz. the regular alternation of heat and cold, light and darkness, which must greatly influence our constitution. We know, indeed, that the mere alternation of light and darkness alters the routine of all common business, and influences the whole train of our affairs; and from this we can see sufficient cause for establishing such a law, or for disposing a system so readily used to habit as ours is, to diurnal revolutions. It must be allowed, however, that this is still but reasoning; I would add, therefore, that, whatever the cause may be, I presume from facts, that in the human system, whether from its original constitution or from the cause just mentioned, a diurnal revolution takes place. The phenomena of sleep and watching, the return of hunger, and of the excretions, which all recur in the space of twenty-four 
hours, shew that our body is subject to a diurnal revolution steadily and regularly. But in none of the functions is this revolution more remarkable and more regular than in that of the pulse, as may be seen from Dr. Bryan Robinson's Observations. When a man first awakens out of sleep, suppose he be free from any disease, his pulse is at the slowest; but he has no sooner stirred a little, than it becomes quicker, and continues to be so during the forenoon. And this quickness is not altogether owing to exercise, \&c. for although this be used, or stimuli applied, instead of farther increasing in frequency the pulse decreases about noon: then it, rises in the afternoon about dinner time; but, in spite of stimuli, diet, and all the irritations the system is exposed to during all this time, it suffers a remission about seven or eight o'clock; then it suffers another exacerbation, and continues to increase till midnight, when it rises higher than ever; remitting again from about two o'clock in the morning, till it arrives at its state of greatest slowness in the morning.-But there is hardly a function in the animal economy which is not a proof that our system, by a certain law or habit, is subject to diurnal revolutions."

LVI. It is this diurnal revolution, which, I suppose, determines the duration of the paroxysms of fevers; and the constant and universal limitation of these paroxysms (as observed in $\mathrm{LV}$.), while no other cause of it can be assigned, renders it sufficiently probable that their duration depends upon, and is determined by, the revolution mentioned. And that these paroxysms are connected with that diurnal revolution, appears further from, this, that though the intervals of paroxysms are different in different cases, yet the times of the accession of paroxysms are generally fixed to one time of the day; so that quotidians come on in the morning, tertians at noon, and quartans in the afternoon.

LVII. It remains to be remarked, that as quartans and tertians are apt to become quotidians, these to pass into the state of remittents, and these last to become continued; and that even in the continued form, daily exacerbations and remissions are generally to be observed; so all this shows so much the power of diurnal revolution, that when in certain cases the daily exacerbations and remissions are with difficulty distinguished, we 
may still presume, that the general tendency of the economy prevails, that the disease still consists of repeated paroxysms, and, upon the whole, that there is no such disease as that which schools have called a continent fever.- " We observe, I say, the most distinct intermittent changing into the most continued form. Thus, the quartan, which has an interval of seventytwo hours, gradually changes its form : it first has its paroxysm lengthened out, then it becomes a double quartan, having a paroxysm on one of the intermediate days which was free before; then it changes into a triple quartan, appearing every day, yet being still a quartan, because it arises out of that form and often passes back into it, and because the fits of every fourth day only are similar. Then it changes into a remittent, the exacerbations of which gradually become more obscure, till it puts on the form of continued fever. So that a quartan may take the form of what is called a continent, in which little exacerbation or none at all can be observed; yet, by its cure and by its frequent return back again, we know that it was originally an intermittent.

"The same is the case with respect to the tertian, which, doubling, has a fit every day, thus putting on the appearance of a quotidian, and afterwards goes into the form of a remittent, and into that of a continued fever.

"Now, I say, from this change which so frequently occurs in practice, I think it is to be suspected that, if in any case physicians can allege that the exacerbations are not observed, this is rather to be imputed to the difficulty of observing them, or to the inaccuracy of physicians, than to any deviation of the disease from what is so universal and so strongly determined by a general law of our system. I would say, therefore, that there is no such fever as the schools have agreed to call a Continent; but that every fever which runs out to more days than one, is formed of repeated paroxysms, recurring in the course of twentyfour hours, or truly consists of diurnal exacerbations.

"' I think I have rendered this conclusion very probable, but it may still remain doubtful, as being a question about a matter of fact, which has not been sufficiently attended to. And, to excuse myself in giving this against the testimony of physicians in all ages-for few have admitted of the very doubt-I must beg leave to observe, that the observations of the greater number are but little to be regarded, being generally guided by 
authority rather than by their own observation; and that the servility in believing, and bigotry in maintaining, what has been delivered by ancient authority, govern physicians as well as the rest of mankind. So the great mass of testimony against us will be greatly diminished as to the number of witnesses, and will not have such weight as might be imagined.

" The physicians of all ages have, however, concurred in the opinion, that the greater number of fevers, at least ninetynine out of an hundred, are such as we have supposed, in which the exacerbations of every twenty-four hours may be distinguished. I must, indeed, say that there are varieties here in some respects, for there is no setting bounds to the exceptions of nature; but still if they are only varieties they deserve less attention; and while there is seemingly such an endless variety, there is indeed a wonderful uniformity in nature's works, which is now every day more attended to. Every fact, accordingly, which is in strong contradiction to the general tenor of nature, is always a suspicious fact, and in most instances owing to superficial observation, which accuracy and attention bring back to its true point of view.

"To all this I would add, if it can have any weight, that in the course of nearly forty years' pretty extensive practice, I have never observed what I could call a continent fever, but could always discern, by attentive observation, more or less of exacerbation and remission. And I have some authorities on which you will lay more weight, - such as De Haen, a physician of much practice, and also an attentive observer, who grants the same thing. I think since this subject has been started, physicians have come more into the opinion; and indeed there is no applying to practice with any steadiness without attending to it."

I expect that this doctrine will be confirmed by what I shall say hereafter concerning the periodical movements observed in continued fevers.

LVIII. It being thus proved, that every fever of more than one day's duration consists of repeated paroxysms, we, in the next place, remark, that the repetition of paroxysms depends upon the circumstances of the paroxysms which have already taken place. From what was observed in XXX. and XXXI. it appears, that the longer paroxysms are protracted they are 
the sooner repeated; and therefore that the cause of the frequent repetition is to be sought for in the cause of the protraction of paroxysms.

"Having formed this conclusion, I proceed upon it, that every fever consists of repeated paroxysms, and that the funda. mental division of fevers is that into intermittent and continued, or into such as have separate paroxysms, and such as have them most obscurely formed; between which there are all the intermediate states.

"We next proceed to inquire into the cause and nature of these different forms of fever; and, first, as there are intermittents which occur at different intervals at first, particularly the three forms of Quotidian, Tertian, and Quartan, there immediately occurs to us the notice of a particular fact, viz. that the paroxysm of a quartan is constantly of shorter duration than that of a tertian; and the paroxysm of a tertian is of shorter duration than that of a quotidian. From this it appears, that the shorter the paroxysm is, the longer is the interval, and, consequently, the longer is the intermission with respect to the paroxysms. Thus, if the paroxysm of the quartan be five or six hours, it leaves for the intermission about sixty-six hours, which is a great part of the seventy-two; and thus the tertian leaves about forty hours of an intermission.

" This suggests to us, what perhaps is an important conclusion, that the recurrence of paroxysms does not so much depend upon the nature of the fevers as upon the duration of those paroxysms which do occur; and therefore that the particular form which the fever takes, one being tertian, another quartan, and a third quotidian, may be considered as depending upon the duration of the paroxysms once formed; the longest intervals being universally connected with, and evidently depending upon, the shortest paroxysms, and vice versa. This, I say, is a considerable step in the doctrine of fevers, as it establishes the general difference between intermittents and continued fevers. As every fever whose paroxysm is protracted eighteen hours or longer, must necessarily have a quotidian recurrence, if this fever again, from any cause, be protracted to twenty-four hours, it can have no intermission, as it must have a recurrence at this period; and therefore such a fever must 
continue in the form of a remittent or continued, and that merely from the duration of the paroxysms; and this is further confirmed from what we observed before with respect to the change of the form of fevers; for it is by the prolongation of paroxysms that quartans and tertians change into quotidians, and these again into, remittent and continued fevers.

"We conclude accordingly, that the form of fevers depends upon the duration of the paroxysms themselves; hence, in order to account for the different forms of fevers, we must look out for the cause of the duration of the paroxysms, or inquire into the circumstances that determine their nature. This is a difficult problem, but I hope we shall be able to make some progress here with probability and clearness."

LIX. Agreeably to what is laid down in XLVI., and to the opinion of most part of physicians, I suppose, that in every fever there is a power applied to the body, which has a tendency to hurt and destroy it, and produces in it certain motions which deviate from the natural state; and, at the same time, in every fever which has its full course, I suppose, that, in consequence of the constitution of the animal economy, there are certain motions excited which have a tendency to obviate the effects of the noxious power, or to correct and remove them. Bóth these kinds of motion are considered as constituting the disease.

But the former is perhaps strictly the morbid state, while the latter is to be considered as the operation of the vis medicatrix naturce, of salutary tendency, and which I shall hereafter call the REACTION of the system.

LX. Upon the supposition that these two states take place in every paroxysm of fever, it will appear to be chiefly in the time of the hot stage that the reaction operates in removing the morbid state; and, therefore, as this operation succeeds more or less quickly, the hot stage of paroxysms will be shorter or longer. But as the length of paroxysm depends chiefly upon the duration of the hot stage, so the longer duration of this and of paroxysms, must be owing either to the obstinacy of resistance in the morbid state, or to the weakness of the salutary reaction; and it is probable, that sometimes the one, and sometimes the other of these circumstances takes place.

LXI. It seems to be only by the state of the spasm, that we 
ean judge of the resistance of the morbid state of fever : and with respect to this spasm, I observe, that either the cause exciting it may be different in different cases, or, though the cause should be the same in different persons, the different degree of irritability in each may give occasion to a greater or lesser degree of spasm; and, therefore, the reaction in fever being given, the continuance of the hot stage, and of the whole paroxysm, may be longer or shorter, according to the degree of spasm that has been formed.

LXII. One cause of the obstinacy of spasm in fevers may be clearly perceived. In inflammatory diseases there is a diathesis phlogistica prevailing in the body, and this diathesis we suppose to consist in an increased tone of the whole arterial system. When, therefore, this diathesis accompanies fever, as it sometimes does, it may be supposed to give occasion to the febrile spasms being formed more strongly, and thereby to produce more protracted paroxysms. Accordingly we find, that all inflammatory fevers are of the continued kind; and that all the causes of the diathesis phlogistica have a tendency to change intermittent into continued fevers. Continued fevers, then, being often attended with the diathesis phlogistica, we conclude, that, in many cases, this is the cause of their continued form.

LXIII. In many fevers, however, there is no evidence of any diathesis phlogistica being present, ' nor of any other cause of more considerable spasm; and, in such cases, therefore, we must impute the protraction of paroxysms, and the continued form of the fever, to the weakness of the reaction. That this cause takes place we conclude from hence, that, in many cases of fever, wherein the separate paroxysms are the longest protracted, and the most difficultly observed, we find the most considerable symptoms of a general debility; and therefore we infer, that in such cases the protracted paroxysms, and continued form, depend upon a weaker reaction; owing either to the causes. of debility applied having been of a more powerful kind, or from circumstances of the patient's constitution favouring their operation.

LXIV. Upon these principles, we make a step towards explaining in general, with some probability, the difference of fevers; but we must own, that there is much doubt and difficulty 
in applying the doctrine to particular cases. It applies tolerably well to explain the different states of intermittents, as they are more purely such, or as they approach more and more to the continued form. But several difficulties still remain with respect to many circumstances of intermittents; and more still with respect to the difference of those continued feyers which we have distinguished in our Nosology as different from intermittents, and as more especially entitled to the appellation of Continued (See Syn. Nos. Meth. cl. i. ord. i. sect. 2.), and explained more fully above.

"With respect to the division into intermittent and continued fevers, a great difficulty still remains. Under the title of Intermittents, I comprehend what other systems call Remittents. I am led to this conclusion by these considerations :-first, in the same seasons and climates, we generally find the Intermittents and the Remittents epidemic at the same time: we never find in any case, except in a few springs of northern climates, intermittents without remittents; at the same time, we can observe that the remote causes of each are truly the same, viz. Marsh Effluvia;-secondly, the remittents very generally arise out of the intermittents, and vice versa;thirdly, the remittents, when they appear, very universally observe the periods of the intermittents; and, fourthly, they are cured by the same remedy, viz. the Peruvian bark: which, if I mistake not, never is a remedy to the same extent in continued fevers.

"Now, from all these considerations, I would infer that the intermittents and remittents are the same disease; and although this is discussing a seemingly trifling question in Nosology, this and every other such question is important in Pathology, and deserves to be settled."

LXV. From the view given (LXII. and LXIII.) of the causes of the protraction of paroxysms, and therefore of the form of continued fevers, strictly so called, it seems probable, that the remote causes of these operate by occasioning either a phlogistic diathesis, or a weaker reaction; for we can observe, that the most obvious difference of continued fevers depends upon the prevalence of one or other of these estates.

LXVI. Continued fevers have been accounted of great di- 
versity; but physicians have not been successful in marking these differences, or in reducing them to any general heads. The distinctions made by the ancients are not well understood; and, so far as either they or the modern Nosologists have distinguished continued fevers by a difference of duration, their distinctions are not well founded, and do not apply in such a manner as to be of any use.

"Sauvages has five genera of continued fevers-Ephemera, Synocha, Synochus, Typhus, and Hectica, which he has distinguished by their respective durations. The Ephemera extends to half a week; the Synocha to about a week; the Synochus to two or three weeks; the Typhus frequently to three weeks; and the Hectica beyond a month. In like manner the Continentes of Linnæus are divided into four genera-Diaria, which is the same with the Ephemera of Sauvages, Synocha, Synochus, and Lenta.

"With respect to these distinctions, I have to remark, first, that they are not founded in the nature of fevers; and, secondly, that though they were, they do not apply as characters. (See Introductory Lectures, page 455.) The inflammatory fever indeed is generally shorter than the nervous; but it has no certain limits to its duration. It is sometimes protracted beyond a week, whilst the nervous fever sometimes is within that period. We seek, therefore, for some other distinctions ; and now almost all physicians have agreed to divide continued fevers into the inflammatory and the nervous."

We think it agreeable to observation, and to the principles above laid down (LXII. LXIII.), to distinguish continued fevers according as they shew either an inflammatory irritation or a weaker reaction.

LXVII. This distinction is the same with that of fevers into the Inflammatory and Nervous, the distinction at present most generally received in Britain. To the first, as a genus, I have given the name of Synocha; to the second, that of Typhus; and little studious whether these names be authorized by the ancient use of the same terms, I depend upon their being understood by the characters annexed to them in our Nosology, which I apprehend to be founded on observation.

"This distinction is founded in nature and observation, and 
if we may trust to our conclusions with respect to the proximate cause, it follows most naturally, from the view there given, that the continued fever is always owing to an excess of spasm, or to an excess of debility : as the one or other of these prevails, it will give one or other of the two forms, either the Synocha or inflammatory fever, or the Typhus or nervous fever. And the other genus, which can be marked, but of the propriety of which I have some doubts, is that which I have given under the title of Synochus, which is a fever where the inflammatory and nervous symptoms are intermixed,-the inflammatory occurring first, and the nervous last. We neglect the consideration of this last genus at present, as it depends upon that of the two first.

66 With respect to Synocha and Typhus, you will perceive that the characters are industriously contrasted, and must be constantly had in view together; for neither of them will be fully understood without attending to this opposition. I say the character of Synocha will be best understood when we review the other character of Typhus. I have rejected here ' morbus contagiosus,' for the proper synocha is probably without contagion, and is owing to cold and other external causes; but as it is dangerous to employ negative propositions, I have not said, 'morbus non contagiosus.' In the character of typhus, on the other hand, I have said, ' morbus contagiosus,' because contagion is for the most clearly observed in it. We sometimes indeed see a nervous or petechial fever in an individual, and the rest of the family not contract it. But this I impute to the ventilation and cleanness of the houses, and perhaps to some other circumstances.

" The opposition of the two characters further appears in the circumstances of the heart and pulse. In synocha, 'pulsus frequens, validus et durus,' this is more constantly the case; but in the typhus we can only say, 'plerumque frequens,' for there are cases in which the pulse 'parum a naturali recedit:' these instances, however, are rare, and commonly it is frequent even to a greater degree than in the synocha. In the character of synocha I have added the state of the urine, because it holds universally; but in the typhus it is by no means so steady. Most commonly it keeps its natural colour; sometimes it 
changes to a paler wheyish colour, and sometimes it has the red colour. But the most considerable difference between the two forms is, 'sensorii functiones parum turbatæ' in synocha; and 'plurimum turbatæ' in typhus. Delirium does accompany the inflammatory fever very frequently, as well as the typhus; but in general the functions of the sensorium are both soon and considerably affected in the latter. If I could give in proper terms the characters of the two different deliriums, the ferox and mite, I would make such a distinction: (See Physiology, page 148.) The furious phrenitic delirium which depends upon the increased impetus of the blood, attends the synocha; whereas typhus is generally accompanied by the mild calm delirium which is without any thing furious or impetuous, but often appears by the patient muttering to himself in an incoherent manner, and sometimes becomes more considerable, so that he loses attention to every thing about him, but is without much ferocity of phrenitic appearance. But this difference is not sufficiently established in our Pathology, and there is nothing steady in this respect, as in typhus also the delirium is sometimes phrenitic. I have therefore only said, that the functions of the sensorium or nervous system are more considerably altered and disturbed in the nervous than they are in the inflammatory fever. I have added in the character of typhus, ' vires multum imminutre' as the prostration of strength is more considerable in this genus. In synocha a debility occurs to a certain extent, but it never appears so early as in the former case."

LXVIII. By these characters I think continued fevers may in practice be distinguished; and if that be the case, the principles above laid down will be confirmed.

"Now this is the principal distinction of continued fevers, into the inflammatory and nervous; it is now some time since physicians have agreed in it, and I hope I have explained it in a consistency with our general system. But I have only given you what I call a theoretical idea of these fevers, wherefore it will be proper now to give you a series of facts, that we may take care to keep in view what happens in nature. I proceed, therefore, to point out in what manner these two cases of fever may be distinguished by their external symptoms. This is very different from the definition, in which we select the chief 
and distinguishing marks only, while here we relate the full phenomena.

"What physicians commonly call the Inflammatory Fever is arranged in our Nosology under the title of Synocha. It is a disease more frequent in cold climates and cold seasons, not however but that there are certain vicissitudes of heat and cold in every climate which may produce somewhat of the same effect. Every constitution may be liable to it, but the robust and sanguineous are more commonly affected with it. It comes on suddenly, without much previous warning, and without being preceded by any very considerable degree of lassitude, loss of appetite, \&c. which we would attribute to a preceding degree of debility. It frequently comes on from the application of cold, seldom from contagion; but we are apt to be mistaken here, for every application of cold does not bring on a Synocha, and the cold may be only an exciting cause where miasmata were formerly lodged. The attack is commonly with little horror, and without any degree of tremor ; the cold fit is always without vomiting and delirium, and, in general, in whatever shape it appears, it is always short. There are, during the attack of these inflammatory fevers, seemingly repeated attacks or frequent returns of chilliness with hot flushings, but they are less remarkable, and the repetition of what we call the cold fit is always shorter. In the hot fit there is a considerable degree of heat, with redness and turgescence of the face, and the appearance of an approaching sweat. The heat is soon diffused over all the body to the extremities. The pulse is soon full, strong, and commonly hard; very often its throbbing may be perceived in the temples; and the fever is accompanied with considerable headach, which is relieved by the head being bound tight. There is commonly pain in the small of the back, and more or less in the joints of the limbs. The breathing is frequently attended with some degree of anxiety, but it is neither small nor laborious; nor is the anxiety generally great, whether it proceeds from the stomach or thorax. The appetite for food is seldom taken away, and the anorexia seldom rises to the degree of nausea. The thirst is considerable; it arises from a sense of heat in the fauces, and may be referred even to the more internal parts. The belly is bound, the urine high coloured, but, in the begin- 
ning, without sediment. The disease generally is of short duration: I would not pretend to limit it to days, but it is agreed that its most common termination is in seven days. In the course of it the exacerbations and remissions, which we maintain do truly happen, are here obscure in their symptoms. The termination is c(mmonly by a sensible evacuation, as hæmorrhagy or sweat; the urine, at the same time, while these evacuations take place, depositing a considerable sediment.

"Now these are shortly the phenomena of inflammatory fever, always supposing that it is of the most pure kind. But I must observe that the character as I have given it purely is seldom to be found, but it is generally accompanied with more or less of catarrh, rheumatism, or some other inflammatory affection. I have found the symptoms of inflammatory fever nowhere so fully enumerated, or its combinations so distinctly marked, as I have described it; but it has been best done by Dr. Hoffmann (Opp. tom. ii. p. 105.), where he treats of this fever under the title of ' Febris acuta sanguinea, Graecis Synocha.'

"I next proceed to relate the phenomena of that fever which is called the Nervous, named in our Nosology, Typhus. It very commonly arises from contagion or effluvia, or some foreign matter introduced into the body. It most readily affects persons of weak constitutions, or those who were lately before exposed to particular causes of debility, and also to the action of cold, which generally appears as the exciting cause. The disease generally comes on by degrees and slowly, and before it appears formally, the person is previously affected with loss of appetite, languor, and lassitude. Then there is a sense of cold, or rather a sensibility to the coldness of the air, especially towards the evening, with a slight degree of horror ; in the course of the night a slight degree of heat comes on, with uneasy and restless sleep, which, however, is relieved in the morning; but at this time the chilliness returns, and towards the erenirty the same symptoms recur again, accompanied with a more considerable hot fit during the following night. This becomes more constant during the following day; and after two or three such days as this, the hot fit becomes more and more remarkable and constant, with a frequent, but neither full, nor strong, nor hard

vor. I. 
pulse. With these seemingly mild appearances, there is a great prostration of strength, the force of the animal functions is suddenly diminished, and the mind is affected with a remarkable despondency. The appetite is lost, a nausea takes place, and a vomiting arises. There is little sleep and it is much disturbed, and very soon a delirium occurs, or a typhomania, that is, a delirium between sleeping and waking. The heat of the body is often moderate, and commonly not equal, and is remarkably less in the lower extremities. The face is seldom flushed, and never much so. The belly is irregular, frequently bound, but sometimes there are rather frequent and loose stools. The urine is nearly in its natural state, but rather paler and without sediment. The exacerbations and remissions which take place here are at first very observable, but by degrees they become more obscure. And thus the disease, with respect to its whole duration, draws out to two or three weeks, and then terminates without any sensible evacuation or crisis.

"In the whole course of this disease the chief symptoms are such as shew some considerable interruption of the functions of the brain. Thus, the delirium which is of the milder kind, the typhomania, or degree of coma, the carphologia, subsultus tendinum, \&c. are all evident symptoms of the debility of the nervous power; and this has given occasion to call the disease a nervous fever. I think that the whole phenomena may be explained by a prevailing debility in the energy of the functions of the brain.

"It has been supposed that Typhus has been observed only in modern times, and that the Febris lenta nervosa arose first in England: it is true indeed that in the years $1720-21$, Winteringham gave the first account of the Febris putrida nervosa; and Dr. Huxham is perhaps the first who gave us a full account of this disease; yet the disease was known long before, for early in the sixteenth century the learned Fracastorius published a treatise on the Febris pestilens, in which we find just as clear an account of the disease as we do in Huxham. It is a matter of curiosity to point this out. In his second book, Fracastorius, treating de Febribus contagiosis, and particularly de pestilentibus Febribus, after some general remarks, says-

" ' Jam de accidentibus earum dicatur, quatenus signa, et no- 
titia earum haberi possint: primum ergo, quod per se has febres sequitur, est esse contagiosas, quod et maximum est earum signum quod, quoniam non semper datur videre posse, alia signa attendenda sunt : commune autem est omnibus ferè, per initia, omnia videri valde placida, ita, ut vix febrire existimetur, qui nihilominus latentem pestem concepit : pulsus urinæque id attestantur, sed ita tamen, ut peritum non lateant: per processum porro tametsi sua sunt argumenta, tota tamen per se febris placida videtur, et lenis : calor tactu mordax non est, introrsum vero morosus magis, quam acris percipitur, quippe interrogati, qui agrotant, num febriant, non febrire se dicunt, sed tamen in angustia quadam versari : decubitus supinus est, dorsum perinde ac verberati, anhelitus solito longior, pulsus non citatus, non altus, sed pressus magis, et vix audens se attollere : hæc ergo signa per primos morbi processus apparent, quæ quum vides, tum ad alios spectato, quos febres tenent, ac quum multos emori vides e consimili morbo, tunc time pestilentem inesse febrem, cui astas : in dies autem certiora se ostendent signa, nam vel maculæ in cute apparebunt, vel abscessus circa emunctoria vocata: oculi ad hæc subrubent, et vacillant, anhelitus extraneo quodam fotore gravis est, quo solo signo periri sæpe pestilentem febrem deprehendunt; verba sæpe dicuntur multa, et interdum incomposita, caput gravescit, et modo vigiliæ, modo somnus nimius ægrum tenet. Hypochondria tenduntur, egestiones liquidæ sunt, corruptæ, longinquæ a forma fæcum et humorum, fœetentes urinæ aut subjugalium similes, aut quasi vinum granatorum, aut tenues, crudæ, aut confusæ, rubræ, quandóque et similes sanis sunt: lingua sordescit, virtus omnis labat et fatiscit: mala autem signa sunt, delirium perseverans, urinæ detentio, fluxus sanguinis narium, ad quem levatio nulla consequitur, ventris dejectio multa per initia oborta, vel e levi pharmaco facta, apparitio macularum, et abscessuum, ac subita mox delitescentia.'-Fracastorii Opera, tom. i. p. 150.

"The particular character is "virtus omnis labit et fatiscit." Now, when we compare this with Dr. Huxham's account, we find here as exact a nervous fever as in these later times. I must own indeed that he did not distinguish between the fevers; they are all comprehended under the title of ' febris pestilens ;' 
but we observe that they may be separated in his sixth chapter ; and he is the first author who describes Petechiæ."

LXIX. Besides these differences of continued fever now mentioned, I am not certain of having observed any other that can be considered as fundamental. But the most common form of continued fevers in this climate seems to be a combination of these two genera; and $I$ have therefore given such a genus a place in our Nosology under the title of Synochus. At the same time, I think that the limits between the Synochus and Typhus will be with difficulty assigned; and I am disposed to believe, that the Synochus arises from the same cause as the Typhus, and is therefore only a variety of it.

"Were the two principal forms of continued fever, Synocha and Typhus, tolerably understood in their appearance, we should have much less difficulty in their theory; but the phenomena of fevers are greatly diversified and variously combined; and the symptoms of inflammatory and nervous fevers are so often intermixed with one another, that it is necessary to explain this more particularly.

"For this purpose, we observe that the causes of fever, whatever they are, do not preserve and continue in the same tenor and condition in which they operate on their first attack, but as the fever proceeds in its course, the repetition of the paroxysms makes a considerable change in its circumstances. It is probable, on the one hand, that the power of reaction does increase, so that by repeated paroxysms it overcomes the spasm to which it was not equal at the first ; and it is from this consideration that we account for the spontaneous solution of fevers : and on the other hand, it appears that the repetition of paroxysms diminishes the power of reaction, and so increases the force of the spasm; or from other causes the force of the spasm is increased, so that the disease is protracted, and a greater number of paroxysms is required to restore the energy of the brain for overcoming the spasm, and so obtaining a solution of the disease; or the force of the spasm may be increased to such a degree, that it is not capable of being resolved at all, but subsists, till at last the reaction ceases, and death ensues. And here it is difficult to say what bad symptoms are to be imputed to de- 
bility, and what good symptoms to increased action. But in general we can see a foundation for a great part of the various combinations of the circumstances of fever, and particularly for that combination which is the most frequent in this very climate, where fevers put on at first the appearance of the inflammatory, but in their continuance assume the nervous form. For when the cause of debility is not very strong, as in northern climates is frequently the case, and the vigour of the constitution inclines it to the inflammatory diathesis, the fever takes the inflammatory form; but when, by the repetition of the paroxysms, both the force of the spasm, and in some measure the energy of the sensorium are diminished, the fever subsists in a nervous form; and it is to such a combination and form of fever that we have appropriated the term of Synochus. My aversion to change terms has made me employ this ancient word, which implies $a$ continued fever.

"Synochus probably universally arises from a contagion of a particular nature, and this may be often traced. It is to be considered as a disease arising from the same contagion as Typhus, but which, merely from the accidental circumstances of climate or temperament, puts on the appearance of an inflammatory fever in the beginning. The disease most commonly puts on this form in young persons of a robust habit, so that in an army consisting of men in the prime of life, it appears in the form of synochus, that is, it begins with a considerable degree of inflammatory symptoms."

LXX. The Typhus seems to be a genus comprehending several species. These, however, are not yet well ascertained by observation; and in the meantime we can perceive, that many of the different cases observed do not imply any specific difference, but seem to be merely varieties, arising from a different degree of power in the cause, from different circumstances of the climate or season in which they happen, or from different circumstances in the constitution of the persions affected.

LXXI. Some of the effects arising from these circumstances require to be particularly explained.

One is, an unusual quantity of bile appearing in the course of the disease. This abundance of bile may possibly attend some continued fevers, strictly so called; but for the reasons 
above explained, it more commonly attends intermittents, and we believe it might have been enumerated (XXIX.) among the marks distinguishing the latter kind of fevers thom the former. But though an unusual quantity of bile should appear with continued fevers, it is considered in this case; as in that of intermittents, to be a coincidence only, owing to the state of the season, and producing no different species or fundamental distinction, but merely a variety of the disease. I think it proper to observe here, that it is probable that the most part of the continued fevers named Bilious, have been truly such as belong to the section of intermittents.

LXXII. Another effect of the circumstances occasionally varying the appearance of typhus, is a putrescent state of the fluids. The ancients, and likewise the moderns who are in general much disposed to follow the former, have distinguished fevers as putrid and non-putrid. But the notions of the ancients on this subject were not sufficiently correct to deserve much notice; and it is only of late that the matter has been more accurately observed and better explained.

From the dissolved state of the blood, as it presents itself when drawn out of the veins, or as it appears from the red blood being disposed to be effused and run off by various outlets, and from several other symptoms to be hereafter mentioned, I have now no doubt, how much soever it has been disputed by some ingenious men, that a putrescency of the fluids, to a certain degree, does really take place in many cases of fever. This putrescency, however, often attends intermittent, as well as continued fevers; and, of the continued kind, both the synochus and typhus, and all of them in very different degrees; so that, whatever attention it may deserve in practice, there is no fixing such limits to it as to admit of establishing a species under the title of Putrid.

LXXIII. Beside differing by the circumstances already mentioned, fevers differ also by their being accompanied with symptoms which belong to diseases of the other orders of pyrexix. This sometimes happens in such a manner as to render it difficult to determine which of the two is the primary disease. Commonly, however, it may be ascertained by the knowledge of the remote cause, and of the prevailing epidemic, or by observing 
the series and succession of symptoms.- " Thus, fever is often joined with phlegmasia, as in the Synochus pleuritica, or the Febris pleuritica of Sydenham, in which it was plain that the fever was the primary disease, upon which the other supervened, and which passed away when the original fever subsided. This was a clear instance of a fever in its own nature free from phlegmasia, yet combined with it ; but frequently cases occur where it is difficult to distinguish which is the idiopathic or primary disease; and as this is a matter of importance we shall pay some attention to it.

"Whether the phlegmasia, then, is the primary disease may be determined from the following considerations: First, By the season of the year; in the spring phlegmasiæ are frequent and commonly epidemic; in the autumnal season pure fevers prevail. At the same time this will not entirely determine the question, because towards the end of autumn, when the cold sets in, the phlegmasiæ re-appear. Secondly, By the symptoms which first appear; thus, if the inflammatory symptoms first take place, we have reason to look upon the phlegmasia as the primary discase, if otherwise, we conclude the fever to be pure. In the case of a pleurisy, for instance, it may perhaps be said that there is often some fever subsisting for twenty-four hours before the characteristic symptoms appear; but this will not be found to be the case if we look carefully. If the fever subsists for several days without the inflammation, it will be reasonable to suppose that the fever is the primary disease. Thirdly, Where the fever and the inflammatory symptoms are simultaneous, it may be determined by the knowledge of the epidemic which prevails. Thus the symptomatic pleurisy of Sydenham was ascertained by this, that the inflammatory symptoms, as he says, supervened, being no part of the original epidemic; and further, these accidental symptoms disappeared while the original febrile epidemic constitution remained. Fourthly, By the exacerbations and remissions being more regular and evident, when the fever is idiopathic. I have mentioned as a fact, that fevers attended by inflammations are always continued. So, in the Synochus pleuritica of Sauvages, he had that mark that the pleurisy sometimes was removed when the fever ran its course.These marks may serve to determine the nature of such com. 
plications, especially whenever the diseases are epidemic; in sporadic cases it may be more difficult.

" Proper fever is also combined with the Exanthemata. Thus the fever frequently depends upon a contagion, and the eruption appears during the fever, as in the smallpox and measles. But there are cases where it is supposed that certain exanthemata are produced merely in consequence of a state of fever, independent of any peculiar specific contagion, as in the case of Petechiae, or the eruptions which come out in some fevers, and which we do not admit into the number of the exanthemata. There are miliary eruptions, too, which are to be considered in the same light; these, indeed, sometimes depend upon a specific contagion, but more commonly they are merely symptomatic."

LXXIV. Most of our systems of physic have marked, as a primary one, a species of fever under the title of Hectic; but as it is described, I have never seen it as a primary disease. I have constantly found it as a symptom of some topical affection, most commonly of an internal suppuration, and as such it shall be considered in another place.

LXXV. The distinction of the several cases of intermittent fever I have not prosecuted here; both because we cannot assign the causes of the differences which appear, and because I apprehend that the differences which in fact occur may be readily understood from what is said above (XXV., XXVI., and XXVII.), and more fully from our methodical Nosology, cl. i. ord. i. sect. 1 .

"The TERTIAN is the most universal of all the forms of intermittent fevers; it occurs in nine out of ten cases, nay, it may be considered as the fundamental and most natural form of fevers. I have defined it thus : ' Paroxysmi similes intervallo quadraginta octo circiter horarum : Accessionibus meridianis.' In this definition I differ from Sauvages and Linnæus, whose ' tertio quovis die' is somewhat ambiguous. Thus, in common language, we would say that a Tertian returns every second day, but in medical language, the first day in which the disease begins is also reckoned; so when a Tertian begins on Monday, the fit returns on Wednesday. But as these expressions lead to ambiguity, I have, in my character, mentioned the interval 
alone. I have added ' circiter,' for though the interval is generally forty-eight hours, nature is by no means so exact or uniform in this particular, the fits coming frequently a little sooner or later than that period.

"Of the Tertian there is but one species; all the other subdivisions are merely varieties, and I have been even doubtful in what manner to lay out these; I proceed therefore by subdivisions, which I find generally useful. The difficulties in tracing the varieties arise from this, that the forms of nature are various, and these varieties are constantly interchanging with one another; therefore we do not pretend to establish any thing as universal but only as more general.

"I I divide each genus of intermittent fevers into two heads, those admitting of a perfect intermission (interposita apyrexia); and those which admit of a remission only (interposita remissione tantum.)

"I. Tertians with a perfect intermission, vary in five different ways; 1. Paroxysmi duratione; 2. Paroxysmorum recursu; 3. Symptomatis; 4. Aliis morbis complicatae; 5. Ratione principii.

" 1 . By the duration of its several paroxysms (paroxysmi duratione); the more universal form of tertians is that which $I$ have set down first, "Tertiana paroxysmis haud ultra horas duodecim extensis.' The fit always lasts more than six hours; and where it has an intermission, it is finished in ten, though sometimes it extends to nearly twelve hours. As this is the most usual form, it has been called Tertiana legitima. When the paroxysms are longer, the fever is called spurious: hence the division into the 'legitima,' and the 'spuria sive notha;' the latter of which is defined 'Tertiana paroxysmis ultra horas duodecim extensis.' These tertians never go the length of eighteen hours, or if the paroxysms are longer, the fever becomes actually a remittent.

66 2. By the recurrence of the paroxysms being more frequent (paroxysmorum recursu); and of this there are again several varieties. The first : ' Tertiana quotidie revertens, paroxysmis inæqualibus, alternis similibus ;' a Tertian which has a fit every day, those on the two successive days being unequal, - but these on the alternate ones equal; the double tertian of Sau- 
vages. What we call the principal fit, as that on the Monday and Wednesday, and so on every other day, comes on about twelve o'clock at noon, sometimes a little sooner, sometimes a little later; it is never much earlier, however, without putting on the Quotidian form, nor much later without assuming the appearance of a Quartan ; and this paroxysm is as much connected with noon as the character is with forty-eight hours; it is attended with horror and tremor, and has its solution with a copious sweat; it goes off for the most part about eight o'clock in the evening, or a little sooner; the other paroxysm goes off about six o'clock, or even later, and is accompanied with less horror and tremor, and with less sweat in its solution.-A second form is the 'Tertiana alternis diebus revertens, paroxysmis eodem die binis.' This, like the proper tertian, comes only every other day, but the fit occurs twice upon the same day; hence it is called ' 'Tertiana duplicata ;' but it is of very rare and uncommon appearance, and is mentioned only as an addition to the history of fever.-The third variety, which is defined ' Tertiana quotidie revertens, paroxysmis altero die binis; altero unico tantum,' differs from the former two in the following circumstances: It has a paroxysm every day, in which it agrees with the 'Tertiana duplex,' and the single paroxysm is precisely in the circumstances of the double tertian; but it differs from it in having two paroxysms on one of the days, in which it is like the 'Tertiana duplicata.' These Tertians, by their doubling in their recurrence, come nearer and nearer to the remittent form. They have been named by practitioners, Semitertians; but I give you a name which is more easily understood, the ' Tertiana triplex.'-The fourth variety is thus defined : ' Tertiana quotidie revertens, interposita remissione inter diem imparem et parem magis, inter parem et imparem minus, notabili.' I shall explain this variety by marking the days of the week, as Cleghorn endeavours to do ; but I hope to do it more clearly. Suppose a patient affected with a Tertian whose paroxysm recurs on Monday at noon; he has remission towards the evening, and on the same evening there is a new exacerbation; next day the patient is free from fever till the evening; when a new fit comes on, which has its intermission and remission again on the. Wednesday morning; but the same course returns, he has a fit on 
the forenoon of that day, which remits towards the evening, when there is a new exacerbation. Or you may take it very distinctly from Cleghorn; there is a Tertian to be met with, he says, in which there are three different periods, three fits, and as many intervals. Here you will observe that what Cleghorn calls a period, I call an interval, and what he calls an interval, I call a remission. As I have explained it, this form of Tertian fever returns every day, but with a remission that is more considerable on the one day, and hardly to be noticed on the other. This is the Hemitriteus of Celsus, and the Semitertiana of Cleghorn. It is true $\mathrm{I}$ have the testimony of most authors, that what has been called the Semitertian is a remittent fever only ; but there is something undetermined in that matter, which is not very easily avoided, for there are truly no bounds or limits to be set to these forms, as they are constantly running from a true tertian, consisting of a fit of eight or ten hours, to a spurious tertian whose fit extends to eighteen hours, then to a double tertian having a fit every day, then to two fits in one day, then to one fit in one day, and to two in another; then truly and entirely to a remittent.

"After these observations, you must not be surprised at my mentioning the varieties so accurately here. Whether it is of importance to attend to such, I cannot say; but it would be dangerous to say that these varieties are of no importance. We must first take the facts as they are, and even the superfluous ones, least we should miss any that are of consequence. It were to be wished that we had the species of diseases in a nosological method with some accuracy; I am aiming at it, but it is a task I am not able to perform. It requires more experience and actual practice than I have had, and also an immense erudition in looking into the facts, which is a circumstance that my own parts, and my own library, or any other I can be supplied with, are not sufficient for, so that I might proceed on my own bottom. Sauvages is the only person who has attempted this; but what a mass of confusion there is in this part of his work! It is of importance that I point out his mistakes, and endeavour to correct them.

"3. By their symptoms; the principal varieties of tertians 
in this respect are, first, those accompanied with soporose affections (Tertiana affectibus soporosis stipata), such as Lethargy, Coma, Carus, or Apoplexy ; secondly, those accompanied with spasmodic and convulsive affections, 'Tertiana spasmis et motibus convulsivis stipata,' such as Asthma, Hysteria, and Epilepsy; thirdly, those accompanied with cutaneous eruptions, 'Tertiana efflorescentia cutis stipata,' such as Petechiæ, Urticaria, and Miliary eruptions ; and fourthly, those accompanied with inflammatory affections, "Tertiana phlegmasia stipata,' such as Pleurisy, Gout, and Rheumatism. With regard to this last variety, I must remark, that intermittent fevers, particularly those of the vernal season, are very frequently accompanied with manifest symptoms of phlogistic diathesis; and this is in proof at what degree that diathesis may arrive : but even when merely the diathesis is present, it requires a particular attention in treatment. In several of these cases it may be a question how far these affections are symptomatic, or whether they concur only from their own separate cause and constitute a complicated disease.

"6 4. As complicated with other diseases. I have here marked the complications mentioned by Sauvages. To the " Tertiana scorbutica,' I have given a place, but more from theory than from distinct observation. The ' $T$. syphilitica' may be a true complication, though it but seldom occurs. By far the most considerable of these complications, is the ' $T$. verminosa, which I have set down in pure complaisance to certain authorities. The author I have chiefly in view is Van der Bosch, who, in a treatise entitled Constit. Epid. Verminosa ann.1760, most fully details the symptoms of worms accompanying intermittent fevers, and makes the worms, in a great measure, the foundation of the disease, or at least of its principal symptoms. As I have had little opportunity of observing these remittent Tertians, I cannot correct him from my own experience, but I conceive that he is totally mistaken, and that worms are merely a symptom concurring in these autumnal remittent tertians. I have referred to the writers who are clearly in proof of this, viz. Lancisi, Ramazzini, and Sir John Pringle.

66 5 . By the remote cause (ratione principii): I should 
hardly have admitted this division, if Sauvages had not some species which could not have come in otherwise, the T. accidentalis, and the 'T. a scabie: these, however, would deserve more notice if they were established upon a better foundation, (See Synopsis, page 248 , note ${ }^{*}$.)

"II. We come now to the second head of Tertian fevers, those which admit of a remission only, ( $T$. interposita remissione tantum.) The Remittent fever destroys more men in southern climates than all other diseases taken together, particularly persons from the north of Europe, when they go into warm climates. Although it is more peculiar to those climates, it frequently occurs in England, but more rarely as you recede towards the north. It is therefore certainly an important object of our study, and it is of consequence to know all its different forms. I have attempted, with respect to the intermittent Tertians, to express the character to the extent $I$ have seen; and if $I$ had found any thing steady in the Remittents, I would have made an attempt of the same kind with respect to them; but they are every way so irregular, that we can make no determined distinctions whether they approach to the double, duplicate, and other forms, or with respect to their symptoms and the length of the paroxysms, and the remissions that occur; but they are various and anomalous even in the course of the same Epidemic, and the forms are constantly changing even in the same person. They may however be reduced to two heads of fevers; 1st, Those that begin very commonly intermittent, or very distinctly remittent; and, $2 \mathrm{~d}$, those in which the disease begins as a continued fever, and after some days discovers remissions, and afterwards intermissions. This occurs most commonly in the summer season, and young persons are most subject to it; but it is not attended with the same degree of danger as the former.

"6 Torti has described the Remittent Tertians with all their varieties, and with regard to these I follow him, because he refers to a great number of other writers, but especially because he himself is among the most valuable. His work ' De Febribus' contains a great deal of medical erudition, many particular observations with regard to fevers, and many good hints with regard to the method of cure. Though Torti is clear that there is nothing steady in the form of intermittent and remittent fe- 
vers, he has divided the latter into two kinds, the Tertiana solitaria aud Tertiana comitata. The solitaria is that fever which has distinctly the form of Tertian, and is not accompanied with dangerous symptoms; the comitata is the Tertian, accompanied with dangerous symptoms.

"I have endeavoured to reduce the species of Sauvages to those marked by Torti. But as I could not enter into a full description of all the varieties, I have given a long list of authors to consult on this subject, and that in a great measure in chronological order, by observing which you will find that the disease has been almost always the same. Dr. Sydenham is apt to mislead us in this respect : he saw a considerably variety, as he was more accurate in his observations; and though he did not suppose these variations endless, but believed them to return after a period of years, yet he has led us to a view of a greater variety than really exists. For from the middle of the sixteenth century, for more than two húndred years, this Epidemic has been wonderfully uniform. So we need not be distracted with the varieties which we may find in those authous, for several of them have seen things in a false light; and though there be an appearance of difference, when more nearly viewed these varieties are found to be the same.

"The series of authors to which I have referred, serves to point out the sameness of the disease, not only in different periods of time, but also in different countries and parts of the worldwhether it occurs in Germany and other parts of Europe, or in the East and West Indies: we are indebted to Dr. Lind for pointing out the strict uniformity of all these intermittent fevers.

"Such as do not incline to study, all those celebrated authors, may form a compendious library, and read first Morton and Willis. They were succeeded by Dr. Sydenham, who eclipsed their reputation : and in consequence of that age declaring so much against all theoretical writings whatever, they were thrown under reproach. Their writings contain a great deal of frivolous theory, which, however, I think, may easily be avoided at this day; but they both abound with facts of very great importance. After them you may take Torti, and after him Cleghorn, Senac, and Lind. 
"The QUARTAN is, next to the Tertian Intermittent, the most general form of intermittent fever, and like the Tertian, it has been subdivided into the Intermittent and Remittent; but remittent Quartans rarely occur.

"I. Intermittent Quartan fevers vary, First, With respect to the form or type, or the period of recurrence: 1. The Quartan which is pure and legitimate, 'Quartana paroxysmis quarto quoque die singulis, aliis diebus nullis,' that which has a fit every fourth day and none upon the intermediate days; 2 . The Quartana duplicata, 'paroxysmis quarto quoque die binis, aliis diebus nullis,' that which has its fits too upon the fourth day, but has always two fits on one day; 3. 'Quartana paroxysmis quarto quoque die tribus, intermediis diebus nullis,' that form in which three fits recur each fourth day. This form is admitted as a fact by Sauvages, who quotes it from a brother physician in Montpellier that had the disease himself. I cannot refuse the fact, but I am extremely doubtful of it, as it is the only fact on record of this kind of Quartan. I doubt all singular facts, contrary to the usual order of nature, and this one the more, because Sauvages has not described it with sufficient accuracy ; further, it is contrary to the whole analogy of fever, for a triple fit was never observed in any other of the tertian fevers. 4. A more frequent form of Quartan is what has been called the 'Quartana duplex,' ' quae ex quatuor diebus tertium tantum a febre vacuum habet, paroxysmis quarto quoque die similibus,' where the paroxysm returns upon the first and second. day, and there is none upon the third, but there is the usual return again upon the fourth day. 5. The last form is what we call the Quartana triplex, 'quotidie accedens, paroxysmis quarto quoque die similibus,' where there is a fit every day, but it is known to be a quartan by the fits corresponding every fourth day. With regard to both these double and triple quartan fevers, the difference is commonly in this way: The principal quartan fit, in the double quartan, comes on early in the afternoon, from three to five o'clock, whereby it is distinguished from the paroxysm of a Tertian Intermittent, which comes on at noon or before it, or at latest soon after. The triple quartan again has these fits still later in the evening. 
They are so many approaches to remittent and frequently to continued fevers.

" 'The other varieties of Intermittent Quartan fevers, depending upon their symptoms and upon their complication with other diseases, are precisely the same with those which have been enumerated under the head of Tertians, as varying by the same causes.

"II. Remittent Quartan Fevers.-Of these the first variety is the Tetartophya simplex (see Synopsis, page 252, note *), or a continued fever without any topical affection, observing the quartan form. Here you will observe that the quartan is the most remote in its nature from the continued form, so that it is a rare accident. But it is of more consequence to consider the variety described under the name of Tetartophya maligna, a disease similar to the malignant tertian. It begins as a quartan, but loses its proper form about the fourth day, and becomes fatal. I consider the Tetartophya carotica, which Sauvages has made a separate species, as the principal species of the Tetartophya maligna: it is attended with fits of coma, carus, and a degree of apoplexy.

"The next two instances of remittent quartan, the Tetartophya splenalgica and hepatalgica, are owing to the commonness of a fact which may give occasion to them, viz. the quartans producing congestions and topical affections in the spleen and liver.-(See Synopsis, p. 252, note †.)

"I have now gone through my remarks upon the different formis of the Tertian and Quartan fevers; but, besides them and the Quotidian, a fourth form of Intermittents is introduced by Sauvages under the title of Febres Intermittentes Erratic.s. These certainly are to be taken notice of as facts in the history of diseases; they are however so rare as not to be much worthy of our consideration, and they are not separate forms constituting genera, but only varietics of the Tertian and Quartan fevers, and from the universality of these forms nine out of ten of the Erraticæ must belong to them. We find that when, by art or nature, the disease has seemingly disappeared, and the person is in good health, these intermittents do very frequently return, perhaps after two weeks, and that, when this is the case' 
the paroxysms return always upon that day they would have kept to, if they had continued without any interruption, so that the tendency has still continued in the constitution; and Celsus gives it as a general caution to mark the days in which the Intermittent should return, and then to be cautious with respect to trespasses in the non-naturals. This is a certain proof that a disposition, a tendency, or certain motions analogous to the paroxysms, still lurk in the constitution.

" The Erratica quintana, coming on the Friday after the Monday, is only a tertian which has missed a fit on Wednesday. I will not be positive that it is always so, but there are instances of this kind, where obscure paroxysms have been observed on the Wednesday, which seems to favour this opinion. There are I confess cases that are not to be explained in this way, but they are supposed to belong to the quartan; and as we cannot certainly assign the reason why the quartan interval is in three days, the same power that protracts the tertian to the quartan, may protract the quartan to the quintan period.

"6 The Erratica septana may be explained in a double way, as the septan period belongs to both the tertian and quartan. For the tertian from the Monday returns upon the Sunday, the seventh day, and the quartan form from the Monday returns upon the Thursday, or, passing that, upon the Sunday; so it may be either a period of the tertian missing two fits, or of a quartan missing only one fit. Accordingly, there are frequent instances of both; and, according to the nature of the fever which subsisted before, we shall be able to determine whether a fever belongs to the tertian or quartan, and explain the septana in one way or other.

"Vogel is the only person who has given us an Erratica sextana. Were this fact proved, I must confess it would disturb, our whole doctrine. I never have any reserve upon that account; nature in her variety may play us tricks which we may not be able to account for; but this disturbs our theory very little when neither Sauvages nor I myself could find any instances of it in the history of physic.

"But there is another form which tends to disturb our theory, where the fit returns every eighth day, of which we have many instances. There is nothing, however, in which we are more liable vol. $\mathrm{r}$. 
to be mistaken than with respect to the days. We are in danger here of mistaking the eighth day for the scventh: thus, if the discase appears upon the Monday, the seventh is the Sunday; but if it attacks on the Monday evening, the next attack will not be till the Sunday evening; and this may be so obscure as not to be noticed till the eighth day.

" Erratica vaga.-I could find but few instances with respect to the fact, and they are so rare that we would not allow them to disturb any tolerable system. The probability is, that they will be always found within the tertian periods.

"I proceed to the Quotidian fevers.-Here I might first enter into the question, whether there is such a thing as a proper quotidian existing? This seems to be agreed upon, that, whenever repeated paroxysms are alike with respect to the time of their attack and other circumstances, they are considered as forming a particular genus or species of fever; but when the repetitions are alternately alike only, they are considered as not a distinct species, but an anomaly or irregularity of some of the other kinds, and are distinguished by the name of duplicata, duplex, \&c. ; wherefore, as the similarity of fits recurring every day is sufficient for forming a genus, we say, that although we allow the genuine quotidian to be very rare, still there is such a fever, especially in those countries where intermittents are more frequently epidemic. As I am not clear, however, that such a distinction leads to any difference in practice, I am not so solicitous about it.

"I divide Quotidians into the intermittent and remittent.

"1. The Intermittent I subdivide into the solitarioe and comitato, employing the terms of 'Torti. The solitariæ are without any unusual symptoms; the comitata with some unusual symptoms. Instead of subdividing the comitatæ I have thrown all the species together.

"6 With regard to the Solitaria, a curious subdivision is that into universal and partial; according as the fever occupies very evidently the whole system, or appears only in one part of the body, or at least is remarkably different in degree in one place from what it is in other parts.

"To the Universal I have added a little character, which, when 
there is but one species of the genus, is unnecessary; for the sake of an observation, however, I have defined it 'Quotidiana eadem hora matutina rediens.' This leads us to mention a fact not much taken notice of, but which I hinted at before (LVIII.), that intermittents, besides differing in the frequency of the recurrence of the fits, are also distinguished by the time of the day when they make their attack. This is variable, indeed, in the case of their postponing and anticipating; but in general the quartan does not make its attack till about four or five in the afternoon; the tertian again comes on at noon, or near to it; and the quotidian always in a morning hour, some hours before noon. This is a fact of some curiosity, and from its steadiness we would infer that it is connected with some laws of the animal economy. But I had another reason for setting down that character. There is a very frequent occurrence of a fever that either attacks entirely, or at least has a manifest exacerbation every evening. I have pointed out as the foundation of this, that, in consequence of a ccrtain law of our economy, the action of the heart, and what depends upon it, the state of the pulse, is variable through the day, increasing and subsiding at different times, but particularly in the evening acquiring its utmost frequency; and this sometimes sooner, sometimes later. In many cases this exacerbation appears early in the afternoon, there being some remission towards the evening, and then again an exacerbation. These quotidian fevers as they may be called, are very universally symptomatic of some other disease present in the system, so are to be distinguished from our intermittent and remittent quotidian, which has its exacerbation in the morning hour. I will not be positive that there are no quotidians that have this attack in the evening, but I believe when the quotidian is gone, it remains only in consequence of some other disease or topical affection which gives this evening paroxysm. So much for the Quotidiana universalis.

"With regard to the Quotidiana partialis, however unaccountable the fact may be, it is certainly true that every circumstance of a paroxysm may occur and affect a very inconsiderable portion of the body, or a single limb only. So in the German Ephimerides we have an instance in which one arm only became 
cold, and was seized with the shivering and hot fit, which did not go beyond the shoulder; and we have two instances in the Medical Essays where one leg and thigh were affected, and another instance, which is more ambiguous indeed, in which sometimes the arms, and sometimes the legs, and sometimes both together, were affected with the cold fit. I myself have seen it confined to the lower extremities; and Senac furnishes us with several examples to the same purpose.

" The other cases are different from this; they are merely a pain attacking only one particular part, and that pain occurs with the circumstance of a cold or hot fit; but it returns regularly at a certain hour, subsists for a certain number of hours, and is cured, like the genuine intermittents, by the use of the Peruvian bark. The most noted instance is the Quotidiana cephalalgica, or the Hemicrania, which is so frequently quotidian. It affects one side of the head only, commonly near to the eye, and sometimes takes in the orbit of the eye, and so is attended with ophthalmia:

"The chief varieties of the Quotidianæ comitatæ, are the Quotidiana ischiadica, the Quotidiana nephralgica, and the Quotidiana epileptica. There are also the Quotidianæ vespertinæ vel symptomaticæ, such as the hysterica, catarrhalis, and stranguriosa, which are manifestly owing to the presence of another disease, and are to be considered as symptomatic of it only .

"II. The only Remittent quotidian is the Amphimerina of Sauvages and Linnæus. I admit one species only, the Amphimerina latica; and even with regard to it I am doubtful; for if it is doubtful if even the intermittent quotidian be a genuine species, it is still more doubtful with respect to the Amphimerina."

CHAP. IV. - OF THE REMOTE CAUSES OF FEVER.

LXXVI. As fever has been held to consist chiefly in an increased action of the heart and arteries, physicians have supposed its remote causes to be certain direct stimulants fitted to produce this increased action. In many cases, however, there is no evidence of such stimulants being applied; and, in those in which they are applied, they either produce only a tempo- 
rary frequency of the pulse, which cannot be considered as a disease, or, if they do produce a permanent febrile state, it is by the intervention of a topical inflammation, which produces a disease different from what is strictly called fever (VIII.).

LXXVII. That direct stimulants are the remote causes of fever, seems farther improbable, because the supposition does not account for the phenomena attending the accession of fevers, and because other remote causes can with greater certainty be assigned.

LXXVIII. As fevers are so generally epidemic, it is probable that some matter floating in the atmosphere, and applied to the bodies of men, ought to be considered as the remote cause of fevers: and these matters present in the atmosphere, and thus acting upon men, may be considered either as ConTAGIONS, that is, effluvia arising directly or originally from the body of a man under a particular disease, and exciting the same kind of disease in the body of the person to whom they are applied; or miAsmatA, that is, effluvia arising from other substances than the bodies of men, producing a disease in the person to whom they are applied.

LXXIX. Contagions have been supposed to be of great variety; and it is possible this may be the case: but that they truly are so, does not appear clearly from any thing we know at present. The genera and species of contagious diseases, of the class of pyrexiæ, at present known, are in number not very great. They chiefly belong to the order of fevers, to that of exanthemata, or that of profluvia. Whether there be any belonging to the order of phlegmasiæ is doubtful; and though there should, it will not much increase the number of contagious pyrexiæ. Of the contagious exanthemata and profluvia, the number of species is nearly ascertained; and each of them is so far of a determined nature, that though they have now been observed and distinguished for many ages, and in many different parts of the world, they have been always found to retain the same general character, and to differ only in circumstances that may be imputed to season, climate, and other external causes, or to the peculiar constitutions of the several persons affected. It seems, therefore, probable, that, in each of these species, the contagion is of one specific nature; and that the number of 
contagious exanthemata or profluvia is hardly greater than the number of species enumerated in the systems of nosology.

LXXX. If, while the contagious exanthemata and profluvia are thus limited, we should suppose the contagious pyrexiæ to be still of great and unlimited variety, it must be with respect to the genera and species of continued fevers. But if $I$ be right in limiting, as I have done, the genera of these fevers (LXVII.-LXX.), it will appear likely that the contagions which produce them are not of great variety; and this will be much confirmed, if we can render it probable that there is one principal, perhaps one common source of such contagions.

LXXXI. To this purpose it is now well known, that the effluvia constantly arising from the living human body, if long retained in the same place, without being diffused in the atmosphere, acquire a singular virulence; and, in that state, being applied to the bodies of men, become the cause of a fever which is highly contagious.

The existence of such a cause is fully proved by the late observations on jail and hospital fevers: and that the same virulent matter may be produced in many other places, must be sufficiently obvious. And it is probable that the contagion arising in this manner, is not, like many other contagions, permanent and constantly existing, but that, in the circumstances mentioned, it is occasionally generated. At the same time, the nature of the fevers from thence upon different occasions arising, renders it probable that the virulent state of human effluvia is the common cause of them, as they differ only in a state of their symptoms; which may be imputed to the circumstances of season, climate, \&c. concurring with the contagion, and modifying its force.

LXXXII. With respect to these contagions, though we have spoken of them as of a matter floating in the atmosphere, it is proper to observe, that they are never found to act but when they are near to the sources from whence they arise; that is, either near to the bodies of men, from which they immediately issue; or near to some substances which, as having been near to the bodies of men, are imbued with their effluvia, and in which substances these effluvia are sometimes retained in an active state for a very long time. 
The substances thus imbued with an active and infectious matter, may be called Fomites; and it appears to me probable, that contagions, as they arise from fomites, are more powerful than as they arise immediately from the human body. "For the facts in proof of all this, which are now commonly known, I refer you particularly to Dr. Lind."

LXXXIII. Miasmata are next to be considered. These may arise from various sources, and be of different kinds; but we know little of their variety or of their several effects. We know with certainty only one species of miasma which can be considered as the cause of fever; and, from the universality of this, it may be doubted if there be any other.

LXXXIV. The miasma, so universally the cause of fever, is that which arises from marshes or moist ground, acted upon by heat. So many observations have now been made with respect to this, in so many different regions of the earth, that there is neither any doubt of its being in general a cause of fevers, nor of its being very universally the cause of intermittent fevers, in all their different forms. The similarity of the climate, season, and soil, in the different countries in which the intermittents arise, and the similarity of the diseases, though arising in different regions, concur in proving, that there is one common cause of these diseases, and that this is the marsh miasma.

What is the particular nature of this miasma, we know not; nor do we certainly know whether or not it differs in kind: but it is probable that it does not; and that it varies only in the degree of its power, or perhaps as to its quantity, in a given space.

"6 But it seems to be always a combination of moisture and heat which operates here, for the same moisture in cold seasons and cold climates has not the same effects, as is evident when we recede from the equator to the pole; nor is it occasioned by heat alone, for in the warmest climates, and in the warmest seasons of other climates, if they are dry, these fevers are not produced. It is upon this fact that Dr. Lind grounds his whole practice of preserving the health of Europeans in hot climates. But neither does the fever arise when the heat acts upon a pure moisture, such as that of a lake or of the sea, where the exhal- 
ation is so considerable. Dr. Lind, therefore, finds it advisable for people in warm countries to have their habitations upon dry grounds; but if that is not attainable, he recommends them to go aboard a ship. I once passed six months in a warm climate, in the harbour of Portobello, and I observed that the men who lay aboard the ships contracted no fever nor flux; whereas those who went ashore, and lay for a single night there, were attacked with the one or the other of these diseases. These effluvia, then, do not arise from pure moisture, but seem to be connected with a particular moisture arising from the earth. When the impurities of the ground are entirely covered with water, such exhalations are either not formed, or they are absorbed and rendered innocent by the incumbent water. We have an example of this in Egypt, where, from the time that the waters of the Nile recede, in consequence of which a certain mud is left, the Typhus Agyptiacus arises and continues, till again, at the returning season, the Nile rises to a certain height, and overflows the ground, when the disease disappears, though this inundation happens at the warmest season of the year. In like manner, Senac informs us of a town in Germany, surrounded by a considerable lake, where no disease was produced when there was an entire surface of water; but by length of time, some part of the filthy sediment emerging, the exhalation from it came to be of the same nature with that from every other marshy ground, and produced tertians, and other intermittents."

LXXXV. It has now been rendered probable, that the remote causes of fevers (VIII.) are chiefly contagions or miasmata, and neither of them of great variety. We have supposed that miasmata are the cause of intermittents, and contagions the cause of continued fevers strictly so named; but we cannot with propriety employ these general terms. For, as the cause of continued fevers may arise from fomites, and may, in such cases, be called a miasma; and as other miasmata may also produce contagious diseases, it will be proper to distinguish the causes of fevers, by using the terms Human or Marsh Effuvia, rather than the general ones of Contagion or Miasma.

"It is a very curious, but at the same time a difficult problem, to determine in what cases Contagion or Miasma does or does not act. It certainly happens, that during the prevalence of Epidemics, 
when every body is exposed, a certain, and sometimes a small number only are seized, and some always escape. I shall offer a few remarks upon this curious and important matter; and I observe, that the action of the effluvia will be according to their degree of power', or that fevers will arise more or less readily as the miasmata or contagions are more or less powerful. Now it may be presumed that as these effluvia are the product of a certain fermentation, this fermentation may be, upon different occasions, in different degrees of activity, which will be one source of the different matter produced; or, the same matter may be accumulated in different quantities, and possess a different degree of concentration in a given space; but the question still remains, what is it that increases the activity and quantity of this matter? To this I answer directly, that heat is to be considered as the chief instrument in giving activity to fermentation, and hence may be the chief means of supporting the action of the effluvia, by increasing not only their activity but their quantity. So, according to the heat of the season, the quantity and quality of the effluvia will be in proportion; and the observations which have been made will be found to be agreeable to this.

"6 Fevers arising from miasmata may indeed appear in northern climates, but their operation is more considerable in the warm climates; heat alone, however, is not sufficient; in dry bodies no fermentation takes place; moisture is a necessary circumstance, and it is only in humid grounds that noxious miasmata arise. But it is not yet determined whether every part of the earth's surface, properly acted upon, be a proper substance for producing this miasma, or if it must be impregnated with a particular putrescent matter in order to produce it. There are indeed cases of inundations which leave putrefying animal matter exposed to the air; but inundations from recent rains also, which do not afford such a matter, produce noxious effluvia in consequence of a certain degree of heat. And we have many instances of earth being merely kept moist by the neighbourhood of woods, which upon occasion prove noxious. All earth in a humid state accordingly contains a sufficient quantity of putrescent matter; or rather there is no part of the earth, from which, after it has been exposed to a certain degree of moisture 
and heat, $\mathbf{a}$ sufficient quantity of noxious matter may not be exhaled. In all great cities noxious effluvia arise from many putrescent bodies which we are constantly breathing, and yet we very rarely observe any persons affected with fever by them. But that putrefaction alone does not produce intermittent fever is evident from the case of scavengers, anatomists, the persons concerned about whales, \&c. among whom the disease in question is not commonly produced; and when putrid effluvia have been the cause of fever, as in the case of anatomists, it is always of the continued kind, and one of the varieties of typhus."

LXXXVI. To render our doctrine of fever consistent and complete, it is necessary to add here, that those remote causes of fever, human and marsh effluvia, seem to be of a debilitating or sedative quality. They arise from a putrescent matter; their production is favoured, and their power increased, by circumstances which favour putrefaction, and they often prove putrefactive ferments with respect to the animal fluids. As putrid matter, therefore, is always, with respect to animal bodies, a powerful sedative, so it can hardly be doubted, that human and marsh effluvia are of the same quality ; and it is confirmed by this, that the debility which is always induced, seems to be in proportion to the other marks that appear of the power of those causes.

LXXXVII. Though we have endeavoured to shew that fevers generally arise from marsh or human effluvia, we cannot, with any certainty, exclude some other remote causes, which are commonly supposed to have at least a share in producing those diseases. And I proceed, therefore, to inquire concerning these causes; the first of which that merits attention, is the power of cold applied to the human body.

LXXXVIII. The operation of cold on a living body is so different in different circumstances, as to be of difficult explanation; it is here, therefore, attempted with some diffidence.

The power of cold may be considered as absolute or relative.

The absolute power is that by which it can diminish the temperature of the body to which it is applied. And thus, if the natural temperature of the human body is, as we suppose 
it to be, that of 98 degrees of Fahrenheit's thermometer ; ${ }^{*}$ every degree of temperature liss than that, may be considered as cold with respect to the human body ; and in proportion to its degree, will have a tendency to diminish the temperature of the body. But as the living human body has in itself a power of generating heat, so it can sustain its own proper heat to the degree above mentioned, though surrounded by air or other bodies of a lower temperature than itself; and it appears from observation, that, in this climate, air, or other bodies applied to the living man, do not diminish the temperature of his body, unless the temperature of the bodies applied be below 62 degrees. From hence it appears, that the absolute power of cold in this climate, does not act upon the living human body, unless the cold applied be below the degree just now mentioned.

It appears also, that the human body's being surrounded by air of a lower temperature than itself, is necessary to its being retained in its proper temperature of 98 degrees : for, in this climate, every temperature of the air above 62 degrees, applied to the human body, though still of a lower temperature than itself, is found to increase the heat of it. And from all this it appears, that the absolute power of cold with respect to the human body, is very different from what it is with respect to inanimate bodies.

LXXXIX. The relative power of cold with respect to the living human body, is that power by which it produces a sensation of cold in it; and with respect to this, it is agreeable to the general principle of sensation, that the sensation produced is not in proportion to the absolute force of impression, but according as the new impression is stronger or weaker than that which had been applied immediately before. Accordingly, with respect to temperature, the sensation produced by any degree of this, depends upon the temperature to which the body had been immediately before exposed; so that whatever is higher than this feels warm, and whatever is lower than it feels cold; and it will therefore happen, that the opposite sensations of heat

* In every instance of our mentioning degrees of heat or cold, we shall mention them by the degrees in Fahrenheit's scale; and the expression of higher or. lower shall always be according to that scale. 
and cold may on different occasions arise from the same temperature, as marked by the thermometer.

With respect to this, however, it is to be observed, that though every change of temperature gives a sensation of cold or heat, as it is lower or higher than the temperature applied immediately before, the sensation produced is in different cases of different duration. If the temperature at any time applied is under $62^{\circ}$, every increase of temperature applied will give a sensation of heat; but if the increase of temperature does not arise to $62^{\circ}$, the sensation produced will not continue long, but be soon changed to a sensation of cold. In like manner, any temperature applied to the human body, lower than that of the body itself, gives a sensation of cold ; but if the temperature applied does not go below $62^{\circ}$, the sensation of cold will not continue long, but be soon changed to a sensation of heat.

It will appear hereafter, that the effects of the sensation of cold will be very different, according as it is more permanent or transitory.

XC. Having thus explained the operation of cold as absolute or relative with respect to the human body, I proceed to mention the general effects of cold upon it.

1. Cold, in certain circumstances, has manifestly a sedative power. It can extinguish the vital principle entirely, either in particular parts, or in the whole body; and considering how much the vital principle of animals depends upon heat, it cannot be doubted that the power of cold is always more or less directly sedative.

This effect may be said to take place from every degree of absolute cold; and, when the heat of the body has upon any occasion been preternaturally increased, every lower temperature may be useful in diminishing the activity of the system ; but it cannot diminish the natural vigour of the vital principle, till the cold applied is under $62^{\circ}$; nor even then will it have this effect, unless the cold be of an intense degree, or be applied for some length of time to a large portion of the body.

2. It is equally manifest, that, in certain circumstances, cold proves a stimulus to the living body, and particularly to the sanguiferous system.

It is probable, that this effect takes place in every case in 
which the temperature applied produces a sensation of cold; and this, therefore, as depending entirely on the relative power of cold, will be in proportion to the change of temperature that takes place.

It appears to me probable that every change of temperature, from a higher to a lower degree, will prove more or less stimulant; excepting when the cold applied is so intense as immediately to extinguish the vital principle in the part.

3. Beside the sedative and stimulant powers of cold, it is manifestly also a powerful, astringent, causing a contraction of the vessels on the surface of the body, and thereby producing a paleness of the skin and a suppression of perspiration; and it seems to have similar effects when applied to internal parts. It is likewise probable, that this constriction, as it takes place especially in consequence of the sensibility of the parts to which the cold is applied, will, in some measure, be communicated to other parts of the body; and that thereby the application of cold proves a tonic power with respect to the whole system.

These effects of tonic and astringent power seem to take place both from the absolute and relative power of cold; and therefore every application of it which gives a sensation of cold, is, in its first effect, both astringent and stimulant, though the former may be often prevented from being either considerable or permanent, when the latter immediately takes place.

XCI. It will be obvious, that these several effects of cold cannot all take place at the same time, but may, in succession, be variously combined. The stimulant power taking place obviates the effects, at least the permanency of the effects, that might otherwise have arisen from the sedative power. That the same stimulant power prevents these from the astringent, $I$ have said above; but the stimulant and tonic powers of cold are commonly, perhaps always, conjoined.

XCII. These general effects of cold now pointed out are sometimes salutary, and frequently morbid; but it is the latter only I am to consider here, and they seem to be chiefly the following.

1. A general inflammatory disposition of the system, which is. commonly accompanied with rheumatism or other phlegmasix. 
2. The same inflammatory disposition accompanied with catarrh.

3. A gangrene of particular parts.

4. A palsy of a single member.

5. A fever, or fever strictly so called (VIII.), which it often produces by its own power alone; but more commonly it is only an exciting cause of fever, by concurring with the operation of human or marsh effluvia.

"I will not positively determine that cold may not of itself produce fever, but it very seldom does so ; and I am persuaded that if ever it produces any thing like fever, it is the simple and mild Synocha. To this opinion on a point which is of some consequence I am determined by the following considerations.

"In those cases where we can distinctly observe the operation of cold, and where nothing else can be supposed to have operated, we find that it universally produces topical affections of the inflammatory or catarrhal kind. In those cases, therefore, in which neither the one nor the other, but proper fever is produced, it may be doubted whether that is the effect of cold alone. It is to be observed, that though miasma and contagion are not always evident, they may operate more frequently than has been imagined. In proof of this I say, that every country of which I have got a physical history, has upon certain occasions heat enough to generate the marsh effluvia; and from any accounts $I$ have had of these countries, they have been affected with those epidemics which are the particular effects of marsh effluvia. These effluvia may be more or less virulent; and though not in such quantity as to produce a remarkable epidemic, yet they may attack persons in particular circumstances, and operate with the concurrence of cold and other causes; so that though they are not always evident, it would be rash to put a negative upon their existence. With respect to the human effluvia, the matter is still more evident. The poor people in all countries wear the same body and bed clothes for a considerable time, and live frequently in close unventilated places, where putrid excrements are produced. This is particularly the case in jails and hospitals, where the effluvia are accumulated in a remarkable degree; and when we consider that the cause exists so frequently, we may indeed wonder that the 
effects are not more remarkable; and this wonder can only be taken off by considering that the effluvia are frequently not accumulated in sufficient quantity to operate by themselves, but are ready to operate with the concurrence of other causes,-so that proper fever then is hardly to be imputed to the sole operation of cold, but it is probably owing to miasma or contagion, even when it appears affecting a single person only. Agreeably to this you will observe that the poor people are more subject to fevers, and in Epidemics are always sooner affected than those of the higher ranks.

"But although we deny that cold is the proper cause of fever, we must allow that it is one of the most general exciting causes, and that it has areat share in modifying and determining thefever to be more or less of the inflammatory kind. Thus Dr. Willis, one of the earliest writers in England upon the nervous fever (1661), observes that, in certain cases, the fever took a determination to the breast, and produced cough and other catarrhal affections ; which only amounts to this, that cold may mix itself with every disease so as to produce these effects. It is owing to the power of cold that so many fevers are in their beginning seemingly of this nature, and that Synochus is so frequent, more especially in cold climates and cold seasons; and it is in consequence of this that fevers are more frequently of the continued kind here.-So much with regard to the important consideration of the share which cold has either in producing or in modifying the forms of fever."

XCIII. Cold is often applied to the human body without producing any of these morbid effects, and it is difficult to determine in what circumstances it éspecially operates in producing them. It appears to me, that the morbid effects of cold depend partly upon certain circumstances of the cold itself, and partly on certain circumstances of the person to whom it is applied.

XCIV. The circumstances of the cold applied, which seem to give it effect, are, 1. The intensity or degree of the cold; 2. The length of time during which it is applied; 3 . The degree of moisture at the same time accompanying it; 4 . Its being applied by a wind or current of air ; 5 . Its being a vicis- 
situde, or sudden and considerable change of temperature from heat to cold.

$\mathrm{XCV}$. The circumstances of persons rendering them more liable to be affected by cold, seem to be, 1. The weakness of the system, and particularly the lessened vigour of the circulation, occasioned by fasting, by evacuations, by fatigue, by a last night's debauch, by excess in venery, by long watching, by much study, by rest immediately after great exercise, by sleep, and by preceding disease. 2. The body, or its parts, being deprived of their accustomed coverings. 3. One part of the body being exposed to cold, while the rest is kept in its usual or a greater warmth.

XCVI. The power of these circumstances (XCV.) is demonstrated by the circumstances enabling persons to resist cold. These are, a certain vigour of constitution, exercise of the body, the presence of active passions, and the use of cordials.

Besides these, there are other circumstances which, by a different operation, enable persons to resist cold acting as a sensation, such as, passions engaging a close attention to one object, the use of narcotics, and that state of the body in which sensibility is greatly diminished, as in maniacs. To all which is to be added, the power of habit with respect to those parts of the body to which cold is more constantly applied, which both diminishes sensibility, and increases the power of the activity generating heat.

XCVII. Beside cold, there are other powers that seem to be remote causes of fever; such as fear, intemperance in drinking, excess in venery, and other circumstances, which evidently weaken the system. But whether any of these sedative powers be alone the remote cause of fever, or if they only operate either as concurring with the operation of marsh or human effluvia, or as giving an opportunity to the operation of cold, are questions not to be positively answered : they may possibly of themselves produce fever; but most frequently they operate as concurring in one or other of the ways above mentioned.

"I shall not decide whether fear of itself ever produces fever ; but, concurring with miasmata and contagion, it may certainly prove a very powerful exciting cause. Thus, it may act in 
epidemics as the occasional cause of the fever in particular persons, as in those cases the persons most under the impression of fear are most easily affected.

"The presence of food in the stomach has not only the power of exciting that particular organ, but from the connexion of the stomach with the system, it may affect the whole body; and though we should not be able to explain the manner of its operation, it is a fact that affections of the stomach are accompanied with a frequency of pulse and horror, which form a kind of fever; and this is more or less according to the quantity and quality of the food introduced; so that certain aliments alone may be the cause of fever, or, as having a tendency this way themselves, they may act in concurrence with other causes which give them more power and activity. Thus, we observe, that indigestion frequently excites and renews intermittent fevers. Here, however, we cannot answer several questions which might be started; as why vegetables of difficult digestion, such as cucumbers, melons, \&c. or why milk and fish together act this way? But the fact is enough to our purpose, and our not being able to give the theory of the operation does not affect it."

XCVIII. Having now mentioned the chief of the remote causes of fevers, it may be further observed, that these will arise more or less readily, according as miasmata and contagions are more or less favoured by the concurrence of cold and other sedative powers.

"Contagion, like other powers acting upon our system, is subject to the power of habit, and by repetition becomes ineffectual; and it is in this way that men who have been some time in jail frequently resist it, while others upon their coming in are readily affected. In this way also, the West Indians escape the yellow fever with which the Europeans on going among them are frequently attacked. This circumstance has given occasion to doubt if it is a contagious disease; but the fact is, it only does not attack the persons who are habituated to it, and we also find that it is contagious when it is carried to other places. I am willing to believe that physicians escape contagion because they are at first exposed to it in small quantity, and by habit become capable of resisting it ; and it is to voL. I. 
be imputed to the same power of habit also, that most epidemics are most violent on their first appearance."

" Having ascertained the different species of fevers, and assigned the remote causes of them, we are now in a condition to enter upon the consideration of that variety which does truly occur in epidemic fever.

"This is one of the most important problems in physic, whether we consider the violence and mortality of these diseases, or the great number of persons affected with them. But there is besides another reason for attempting it: hitherto.it has been represented as inexplicable, an opinion of which Dr. Sydenham in particular has laid the foundation. After the observation of many years, he concluded, that the epidemics of every year differ essentially, so that according to his experience he could not judge if the same kind of epidemic might appear again in the same rotation, and consequently he could not pretend to ascertain the species and to fix the general method of cure. Dr. Boerhaave, who was not a practitioner when he drew up his aphorisms, but whose sagacity led him to follow Dr. Sydenham, has unfortunately followed him in this respect also, and he even carries the matter still farther than Sydenham had done.

"Now there is no foundation for ascribing the variety of epidemics to changes in any of the six non-naturals, although perhaps it might be referred to the atmosphere; . but the inexplicable variety of the exhalations going on there, would give us hardly any hopes of attaining our purpose; and the changes we do see occurring in the sensible qualities of the atmosphere leave us without hopes of ascertaining this variety. If this doctrine accordingly be true, that the varieties of exhalations in the atmosphere are truly inexplicable, and that diseases do so immensely differ, we cannot hope to be able to reduce epidemics to any common or general nature, and to find out a general plan of cure; we must then be obliged to remain attached to Dr. Sydenham's plan of studying every epidemic as it arises, and perhaps must destroy several lives before. we can learn the method of treating the remainder. But this opinion is not sufficiently, well founded, for, from the observations I 
have delivered, the variety of these epidemics is not nearly so great, and we may determine nearly to what length the variety proceeds, reduce them to certain genera, and thereby attain a general method of cure.

"Now, to do this, we must again consider epidemic diseases more generally, and also the causes which can produce them. Epidemics or diseases affecting a great number of persons at the same time, must arise from certain causes in common to all these different persons. What variety there may be here, I will not negatively determine, but we must seek for them either in the state of the diet or in the state of the air.

"The Diet I may discuss very shortly, not however following the many conjectures which have been offered about the qualities of certain waters which are common like the air. Much has been said about the qualities of waters producing rarious epidemic diseases. I could shew the opinion to be ill-founded, but it would lead me too far from my present subject to insist upon this, more especially as it is not alleged that the waters are the cause of epidemic fevers. We will not, therefore, suppose the cause of epidemic diseases to be in the common drink. But, with regard to our solid food, this may be alleged to be the cause with more foundation. Of this there are two noted cases.

" First, The Raphania, a convulsive disorder which arises from a particular corruption of certain grains, particularly the rye; but as this does not produce epidemic fever, or at least the fever is only symptomatic, I shall take no farther notice of it here. Besides this, I do not know of any remarkable poisonous matter being mixed with our common aliment. So the only other case worthy of our attention here, is,

"Secondly, That of diseases arising in consequence of great famine. I might have taken notice of this among the remote causes of fever, but it was proper to reserve it to this place, for we have no particular species of diseases arising from famines; and it is pretty obvious that on such occasions a variety of causes act, some of which may produce particular diseases: but the most part of these causes are such as only favour the production, operation, and propagation of miasma and contagion, and so give no particular diseases, but only have a spe- 
cial effect in concurring with and exciting the power of miasma and contagion. The circumstances which operate especially in times of famine, are, 1. The corruption of the common grain. It is not well got in, it is spoiled, and less pains are taken by the farmer to separate the corrupted part from the sound, so that the grain comes to the market in a more corrupted condition. 2. The corruption of animal food. It has been observed by Senac, that the air or the moist exhalations which produce miasmata increase extremely the putrefaction of animal food"; and the poor in times of scarcity are often obliged to make use of corrupted food which they would at other times reject. 3. Want of animal food; not only the corruption, but the want of animal food makes the bodies of men more sensible of contagion; in all cases, weakness, and therefore the want of aliment also renders the bodies of men more susceptible of contagions. 4. As the poor are obliged to spend their whole funds upon the scanty aliment, they are less able to procure a change of raiment, which gives rise to the corruption of the effluvia; the great cause of contagion, and the want of clothes and fuel occasions more exposure to cold. 5. In such cases, great numbers commonly resort to great cities, where by begging or otherwise they can procure a living, whereby the more noxious effluvia are more certainly generated; and this circumstance, in a great measure, gives occasion to the putrid, malignant, and petechial fevers which so constantly arise in these cities.-These are the circumstances then which may generate or favour the application of contagions ; but I cannot find any reason to suppose that any different disease is produced by famine from what is produced on other occasions.

"So much for the epidemics, which we suppose to arise from the state of the diet. I will not say what accidental poisons may be infused into our waters or other aliments, whether they may not be affected at times by exhalations from apertures in the earth, \&c.; but these instances are so very rare that they cannot be considered as a general cause. I proceed therefore to inquire after those epidemics which arise from the state of the air, to which they have been imputed by all physicians. 
"The effects of the air may be referred either to its sensible qualities, viz. Heat and Cold, Moisture and Dryness; or to its insensible qualities, viz. the various matters with which the air may, as a menstruum, be impregnated. I refer to the air, accordingly, whatever is applied to our bodies in the way of vapour, effluvia, \&c. It may be expected, that I should have here mentioned the essential properties of the air, as its gravity and elasticity; and these no doubt have their effects, but I cannot perceive that there is any suspicion that fevers do arise from any change in these properties. I go on therefore upon my limited plan with respect to the influence of the sensible or insensible qualities of the air. Physicians have been much divided with respect to the question, how much ought to be attributed to the one, and how much to the other of these, and whether they are to be considered as acting separately or in conjunction.

" Hippocrates, our greater leader in this subject, in enumerating the epidemics of the time in which he lived, has taken such pains with respect to the sensible qualities, that he has been understood by modern authors as if he had attributed all the effects to them. In like manner, Wintringham, sen. has endeavoured to ascribe the epidemic diseases of Britain, for twenty years, to the sensible qualities of the air. And Huxham also has done much the same; but his situation at Plymouth led him to see that contagions had a great share, so that he is not determined to conclude positively with respect to either supposition. Neither was Hippocrates entirely of one system. He speaks of the rò 'Tes, or somewhat from above, which may be understood as the insensible qualities of the air. Sydenham again has, upon the other hand, taken up this view

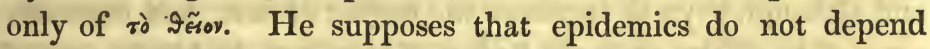
for their origin upon the sensible qualities of the air, but upon certain miasmata, as he terms it. For my own part, I have no doubt in declaring, that both Wintringham and Sydenham are in extremes. I have already endeavoured to shew, that miasmata and contagion are the chief causes of fevers, and so far I support Sydenham; but the sensible qualities of the air also, may, in certain circumstances, produce Epidemic Pyrexix, and may modify fever, strictly so called, which properly arises from the insensible qualities. 
"I proceed, accordingly, to point out the effects of the sensible qualities of the air, viz. heat and cold, dryness and moisture, with respect to epidemic diseases. I refer diseases chiefly to the first two, viz. the powers of heat and cold; for, though dryness and moisture seem to have a considerable share, I think they will be found to be concerned only in as far as they increase or diminish the other two. Thus it is generally observed, that in Britain the moist summers, being the coolest, are most free from disease; and Wintringham and Huxham observe, that moist winters, being the warmest, are also the most healthy, providing they be free from contagion, which the moisture seems to propagate and to continue. This then is the general view, but there are some seeming exceptions to it; for though moisture certainly always moderates the intensity or the degree of heat, if it does not moderate it so as take it down below the putrefactive degree, it does mischief. Thus, if the ordinary temperature of a climate is at $60^{\circ}$, miasmata may be produced in autumn; but if the season is moist, and the heat brought down to $50^{\circ}$, which is hardly compatible with any degree of putrefaction, such seasons will escape epidemic diseases. But if the heat of the climate is at $80^{\circ}$, and the moisture takes down the heat below $70^{\circ}$, this is still sufficient to bring on putrefaction, and more miasmata will be produced in consequence of the moisture. Heat is the chief cause of the production of miasmata, but a degree of moisture is necessary for their accumulation, and the moisture also contributes in applying them to the bodies of men. And though in winter moist seasons be warmer than dry, they may, by favouring evaporation, increase the force of miasmata. So much for the effect of moisture in modifying the operation of heat; and although dryness increases the degree of heat, and increases some of its effects upon the bodies of men, yet, by binding up of the surface of the earth, by diminishing the exhalations, and by diffusing them through the atmosphere, it prevents the generation and application of the noxious effluvia, and so may give the most healthy season. The particular effect of the dry northeast wind, on the contrary, upon men in most of the countries of Europe, in exciting intermittent fevers, is not owing to any particular matter that it brings along with it, but to its being extremely dry, and, therefore, by a more sudden evaporation from 
our bodies, generating a möre considerable degree of cold, which shews its effects in the innermost recesses, in so much that persons in their beds in the morning are sensible of the blowing of such a wind.

"Having thus explained the several modifications of heat and cold by moisture and dryness, I am now brought back to the more particular consideration of these agents.

"I begin with the effects of cold; this; by increasing the tonic powers of the simple solids and of the moving fibres, gives a tendency to the Diathesis phlogistica; and it is in this way that it modifies epidemic diseases, and that inflammatory diseases are constantly the produce of these circumstances in cold climates and seasons. It is indeed a certain vicissitude of heat and cold which has the most considerable share, so that these diseases are not so much the production of the winter as of the spring, and are therefore called vernal diseases ; it is the preceding cold, however, which gives the diathesis and the characteristic form of the epidemic.

"Heat does manifestly relax the arterial system, and, contrary to the popular notion, makes it less susceptible of the diathesis phlogistica ; and not only does that modification of fever which depends upon the inflammatory diathesis occur more rarely in warm climates, but when it is produced in the spring to a great degree, the heat of the summer takes off the inflammatory diathesis. But heat also increases the tendency to putrefaction, which is always present in the animal fluids; so that if the causes of fevers act, a hot state of the air determines them to be of a more putrid kind; and as heat is the leading circumstance in the production of marsh effluvia, and in 'giving force and activity to human contagion, it may be considered as the chief cause which gives occasion to fevers; and from the septic nature of the cause, and a concurring state of the fluids, it determines fevers in warm climates to be of the putrid kind, which accordingly is the character of all summer and autumnal diseases.

"We have thus two classes of epidemics, which comprehend a great number, and will explain Sydenham's division of fevers into vernal and autumnal; the first of these being considercd as inflammatory, and the last as putrid. With regard to this 
division, another effect of heat which is to be particularly observed, is that of increasing the putrid tendency of the fluids. It has been supposed to act upon some of them more than upon others, and particularly upon the bile; hence the opinion that the bile is the most putrescent of the animal fluids; but without discussing that matter, we may allow the fact, that hot-seasons have an effect upon the bile, and that it is liable to be poured into the intestines in larger quantity; and from its effects it would also appear to be possessed of unusual acrimony. (See LI.)

"With regard to this division, Sydenham has supposed that the seasons varying those states of diseases, commence with the solstices, and not with the beginning of spring or of autumn; that the vernal diseases commence at the winter solstice, and the autumnal at the summer; or the one at the shortest, the other at the longest day. This is perhaps the only term that can be fixed, but it may be considerably different in different places; and it is perhaps more applicable to Britain than to other countries; for in warm countries, the season of putrid diseases comes on before the summer solstice, and in cold climates the season of inflammatory diseases comes on sooner than the winter solstice, or towards the end of harvest.

"So far with respect to the influence of the sensible qualities of the air upon the general causes of epidemics, but the same qualities have also an influence upon the type. Thus, the winter season generally determines fevers to be continued, and very often to be of the inflammatory kind; the same fevers in the spring, upon the cold diminishing, become intermittents; and in summer, the heat, acting as a stimulus, brings them back again into the continued form ; for though I have said, according to general observation, that long continued heat takes off the inflammatory diathesis, yet by the stimulus it affords, it has analogous effects to every other stimulus; and so may modify diseases by producing the inflammatory diathesis. U'pon the remission of the heat in autumn, the fevers will again become intermittent; and in the form which has the most distant periods, the quartan.

"Now, these effects of the sensible qualities of the air will 
agree with our general doctrine of fevers; and they are particularly well illustrated by Wintringham, in his Commentarium Nosologicum; or if there is any doubt with regard to their influence, Sydenham himself, who supposes fevers to arise from miasmata, has given us the fullest proofs of the facts I have mentioned. For though he supposes an epidemic constitution independent of the sensible qualities of the air, he observes that it suffers various modifications according to the states of the weather; and although this difference will not go so far as he alleges, yet it is sufficient to establish this point, that the sensible qualities have a share in modifying that state of the air which arises from miasmata and contagion."

CHAP. V.-OF THE PROGNOSIS OF FEVERS.

"With regard to the event of fevers, this is the fundamental principle : in fevers nature cures the disease; that is, certain motions tending to death continue the disease, but in consequence of the laws of the animal economy, other motions are excited by these, which have a tendency to remove it."

XCIX. As fevers (by LX.) consist of both morbid and salutary motions and symptoms, the tendency of the disease to a happy or fatal issue, or the prognostic in fevers, has been established by marking the prevalence of the morbid or of the salutary symptoms ; and it might be properly so established, if we could certainly distinguish between the one and the other of these kind of symptoms. But the operation of the reaction, or salutary efforts of nature in curing fevers, is still involved in so much obscurity that I cannot explain the several symptoms of it so clearly as to apply them to the establishing prognostics; and this I think may be done better by marking the morbid symptoms which shew the tendency to death in fevers.

C. This plan of the prognostics in fevers must proceed upon: our knowledge of the causes of death in general, and in fevers more particularly.

The causes of death in general, are either direct or indirect.

The first are those which directly attack and destroy the vital principle as lodged in the nervous system; or destroy the or-. 
ganization of the brain immediately necessary to the action of that principle.

The second, or the indirect causes of death, are those which interrupt such functions as are necessary to the circulation of the blood, and thereby necessary to the due continuance and support of the vital principle:

CI. Of these general causes, those which operate more particularly in fevers seem to be-

First, The violence of reaction, which either, by repeated violent excitements, destroys the vital power itself, or; by its violence, destroys the organization of the brain necessary to the action of that power ; or, by the same violence, destroys the organization of the parts more immediately necessary to the circulation of the blood.

Secondly, The cause of death in fevers may be a poison, that is, a power capable of destroying the vital principle; and this poison may be either the miasma or contagion which was the remote cause of the fever ; or it may be a putrid matter generated in the course of the fever. In both cases, the operation of such a power appears either as acting chiefly on the nervous system, inducing the symptoms of debility, or as acting upon the fluids of the body, inducing a putrescent state in them.

CII. From all this it appears, that the symptoms shewing the tendency to death in fevers, may be discovered by their being either the symptoms

Of violent reaction,

Of great debility,

Or of a strong tendency to putrefaction in the fluids.

And upon this supposition I proceed now to mark those symptoms more particularly.

CIII. The symptoms which denote the violence of reaction, are, 1. The increased force, hardness, and frequency of the pulse. 2. The increased heat of the body. 3. The symptoms which are the marks of a general inflammatory diathesis, and more especially of a particular determination to the brain, lungs, or other important viscera. 4. The symptoms which are the marks of the cause of violent reaction; that is, of a strong sti- 
mulus applied, or of a strong spasm formed, the latter appearing in a considerable suppression of the excretions.

"' Marks of determinations of blood to the brain, lungs, or other important viscera.' - The excess of stimulant power may be dangerous merely by the repeated and violent excitement, and the state of debility that necessarily follows. But while the increased action is very general, and without any particular determination, I doubt if it ever by itself proves mortal; and while it is thus general, nothing is more in our power than to reduce it, as in practice we find nothing easier than to diminish the powers of the system. But we find that in the case of an inflammatory diathesis to a very considerable degree, if it is not so determined as to destroy an organ whose function is of importance to life, the body will not only sustain it for a long time, but it is curable. I infer this from the consideration of the case of : Rheumatism, where the inflammatory symptoms are as high as in any case whatever; but the determinations are changeable, and are made into parts, the functions of which are not of immediate importance to life; and this disease is so generally curable, and, while thus general, so seldom mortal, that $\mathbf{I}$. conclude that the excess of stimulant power, while general with respect to the system, is of no dangerous consequence.

"We therefore consider the excess of stimulant power as chiefly dangerous, when it is attended with topical determinations to organs whose functions are of immediate consequence to life. And we proceed to consider when the determination is to the brain, to the lungs, or to the abdominal viscera.

"With respect to all of these, when the determination is accompanied with considerable topical inflammation of a particular part, i.e. when it is attended with Phrenitis, Peripneumony, Gastritis, Hepatitis, or any other of the Phlegmasia affecting these parts, it is commonly sufficiently obvious, by the peculiar symptoms accompanying these inflammations. Now, if that was all, we would only have to refer to what are the symptoms of these particular phlegmasia for what we have in view. But very often, on dissection after fevers, we find the marks of inflammation, gangrene and sphacelus in these several parts, when, during the course of the fever, the symptoms of each are by no 
means evident. What we are therefore to attend to, are those symptoms which, though not decisive, give a presumption of a determination to these parts.

" $A$. Of the symptoms which mark out the Determination to the Head and Brain.-With respect to these we must observe, that, in Intermittent fevers, where the cold fits are attended with a very considerable constriction, which is continued for a long time, we find that the determination is chiefly to the abdominal viscera; whereas, in the case of that application of cold, which produces cough and catarrh, the determination is to the lungs; but, abstracting from these circumstances, the chief and most common determination is to the Brain. The cause, however, is not very evident. We may, however, observe that the arteries carrying the blood to the head are in larger proportion than those sent to the other parts of the system, and the course of the blood through them is more potent and direct, which may be partly owing also to the heart being nearer to the brain than to the inferior parts. Whether it is owing to these circumstances, or to this, that the cause of fevers operates in a more especial manner upon the brain and produces a reaction there, 'I shall not determine, and you may, at your leisure, consider the several circumstances, which may lead to an explanation, why, in continued fevers, most commonly and universally the increased impetus of the blood is towards the head and brain. But we have the proofs of the fact, first, in the pulsation or throbbing of the temporal arteries, or of the principal arteries of the brain and carotids as they lie upon the os petrosum. The internal carotid artery communicates a vibration which may affect the organ of hearing, but which, in consequence of custom, is not perceived in ordinary health; it may be perceived, however, by applying the fingers to the temporal arteries ; ${ }^{7}$ it is readily observed when the head is pressed upon a pillow, whereby the vibration being resisted, it is more directly felt; and it becomes very evident when there is a stronger impulse in these arteries leading to the brain.

"In all fevers that are not of the lowest nervous kind, we commonly have another symptom of this determination, viz. a considerable redness and turgescence of the face. This may, however, be produced either by the increased impetus of the 
blood, or by a resistance in the right ventricle of the heart to the return of the blood from the veins of the head. The turgescence of the face, therefore, is a mark only when no difficult respiration or obstruction to the transmission of the blood through the lungs is present. This redness, suffusion, and turgescence is not always remarkable over the whole face, for a less degree will produce a particular suffusion, as where the vessels of the eye only are affected, where a redness, or a number of red vessels, appear in the adnata; but more especially where a fulness of the ball of the eye occurs, sometimes even with a considerable protuberance. When these marks occur without any obstruction through the lungs, they are strong marks of the increased impetus and of obstruction in the head.

"Sometimes these external marks do not appear, but we perceive the increased impetus and tension most obvious in the finer vessels of the organs of sense, and we find no difficulty in explaining in what manner they take place; the nerves of the senses, as those of the eye and ear, derive their tension from a mixture of blood vessels which nature has industriously connected with them, and the sensibility of the organ is in proportion to this tension, so that the light and noise which were formerly not in the least disagreeable, now become painful. This may arise from a particular irritation of the nervous system without any increased impetus of the blood vessels. But, undoubtedly, that sensibility to light and noise which occurs in fevers, may. be commonly taken as a mark of some increased impetus of the blood in the vessels of the head, affecting especially those extremities that are mixed with the nerves of sense.-(See Physiology, LVI. 3.)

"To these symptoms I would next join Headach. This is commonly considered as the first of the marks of increased impetus ; but no morbid symptom is of more difficult theory than headach. Thus I find that sometimes it appears with a slow pulse and palid countenance; and there certainly it cannot depend upon an increased impetus; headach therefore is to be considered as an expression of the increased impetus only when it occurs with more or less of the symptoms which I have mentioned. Headach is very often the first symptom in the attack of fevers of the nervous kind, and it is no where more remark- 
able than through the course of this disease; but here the impetus of the blood does not appear to be considerably increased; so that I consider this headach as a little uncertain. But when, after the cold fit of an Intermittent is over, the heat has returned, then the suffusion of the face, the throbbing of the temples, the sensibility to light and noise, all shew the headach to be certainly a mark of the increased determination of the blood towards the head.

66 Another symptom which is to be put upon the same footing, is a constant waking, perhaps for some nights and days together. There may be irritations which affect the nervous system purely, and may be the foundation of this; but as the ordinary impetus of the blood in the vessels of the head is the chief means of supporting the excitement of the brain, so an increase of it may oppose the collapse which is necessary for sleep. I do not know, accordingly, of a more certain symptom than constant waking, as expressing a particular determination to the vessels of the head.-(Physiology, CXXIX.)

"To this head we refer also a phrenitic delirium. This, like headach and waking, has various and seemingly opposite causes; but I kave hinted (Physiology, p. 146, First Lines, XLV.) that it always depends upon an inequality of the excitement in the different parts of the sensorium, and may therefore either be from the increased impetus in the vessels of the brain, or it may occur with the ordinary impetus of the blood, the causes of collapse taking place at the same time. But we may determine this case pretty well, as our courage, active passions, anger in particular, and a disposition to it, or an irascibility of temper, are commonly in proportion to the impetus of the blood in the vessels of the brain; therefore the determination to the brain is attended with impetuosity, with anger or direct furor, or what all authors call phrenitic delirium. Ambiguities do occur upon this subject; for, when I can observe this delirium to a certain degree, with great restlessness, quick talking, an angry tone, easily provoked, and this amounting often to outrage and violence, I must suspect that there is an increased impetus of the blood; but when I see, at the same time, that this passes off very soon, and that the disease goes on with more tranquillity, and ends with health in no long time after, I cannot believe that any 
particular topical inflammation has been formed. In short, we must admit of degrees in this matter; where the delirium has been long continued, and attended with constant waking, \&c. it gives the strongest presumption of a topical inflammation of the brain; but every such appearance must not lead us to consider the disease as desperate or irrecoverable. These are the observations which I had to make with respect to the increased impetus of the blood in the vessels of the brain.

"6 $B$. Symptoms of the increased Determination to the Lungs. The symptoms of Peripneumony are perfectly well known, and I need not insist here upon those cases in which it is known to be present; but there are obscure degrees of it, which at length prove dangerous and fatal, and over the appearances of which we must watch in fever. The symptoms are,

"1. A sensation of Anxiety in the thorax. The disagreeable sensations in diseases are all reducible to the two heads of pain and anxiety. Anxiety is an uneasy sensation, which is never fixed in any confined spot as pain always is; it occupies, as I may say, a whole member, or the whole parts relating to a particular function. It arises universally, I think, from some difficulty which attends the exercise of certain functions. (See Physiology, page 59.) So the anxiety of the thorax also arises from a considerable debility affecting the organs of respiration; or, the ordinary vigour of these functions being given, it may proceed from a difficulty. in the transmission of the blood through the lungs, some degree of obstruction affecting its vessels. It is rendered further evident by the frequent, laborious, and difficult respiration. There is an ambiguity here, as the difficulty of transmission may arise either from debility or from increased impetus; but I suppose that you carry along with you the marks of the excess of the stimulant power in general. If each ordinary inspiration does not transmit the due quantity of blood through the lungs, we endeavour to compensate that by large inspirations. Ordinary inspiration depends upon the contraction of the diaphragm downwards, and the motion of the ribs is hardly observable; but when the respiration is difficult, the sternum and ribs are elevated to a greater degree, which is therefore a mark of the larger respiration, and of its being more difficult; we may increase it still more by stretching out the 
head to give more freedom to the thorax, and particularly to give an opportunity to the action of those muscles which fix the upper ribs and pull the inferior ones upwards.

6 2 . The determination to the lungs is especially expressed by any Pain about the thorax ; if that pain is fixed in one side, as between the sternum asd scapulæ, it is an evident symptom of pleurisy, or peripneumony; but where there are only wandering pains about the thorax, though they are not sufficiently expressive of these phlegmasiæ, they are marks of such determinations to the lungs as nearly approach to them.

"3. The uneasiness of a recumbent or horizontal posture is especially a mark of some difficulty in the transmission of the blood through the lungs. The position of the body has a considerable effect upon various internal functions, but upon none more considerable than upon that of respiration, so that a reclining, recumbent, or horizontal posture of the body makes a considerable difference in this. For the particular enlargement of the diaphragm is favoured by the erect posture, while in all other positions it is more difficult; and there is hardly any posture of the body which does not, in some measure, impede the action of the intercostal muscles. In ordinary cases this does not affect the respiration much; but when it does so to a considerable degree, this is a mark of still more considerable obstruction taking place. When this merely amounts to an uneasiness in lying, and is removed by change of posture, it is not so considerable, but when it is not so easily removed, and is fixed in one particular place, it is a still more clear mark, not only of a general difficulty in the passage of the blood through the lungs, but of that difficulty being more considerable in one portion of them than in another. This is the decubitus difficilis of authors, when the affection is thus particular, and especially when it increases the anxiety and perhaps the cough which happens to accompany it. This leads me to consider, 66 4. The principal mark of an increased determination to the lungs, viz. the presence of Cough and of catarrhal symptoms. How an application of cold gives a determination to the mucous glands in general, and to those of the bronchia more especially, it is not my business here to explain; but certain it is in fact, that the application of cold operates in this way. This may be 
attended with some degree of infarction, and therefore with some degree of resistance to the free motion of the blood in the lungs; and as all the inflammatory states of the lungs have constantly more or less of cough attending as a symptom, this is to be considered as a chief mark of the particular determination of blood to the lungs.

"I have only to add, that the degree of this determination is especially expressed by the state of the countenance, by a turgid, bloated, and purple suffusion of the face, which is only to be explained by a resistance in the lungs preventing the return of the venous blood to the right ventricle of the heart. This may have its effects upon the Cava descendens as well as upon the veins of the head; but we may doubt if ever it is considerable in the former, as there are no external symptoms which discover it, as in the veins of the head."

" $C$. The symptoms of a particular determination to the other important viscera, those of the Abdomen. I have hinted that this does not so frequently occur in continued fevers as the other two determinations, but that it especially occurs in intermittent fevers. Neither is it so easily discernible; but for the most part we may conclude, that a determination has taken place to the abdomen, from the following circumstances :-

" 1 . From a fulness and tension of the Hypochondria, a symptom to which the ancients were extremely attentive, as we find from Hippocrates, and which is more seldom attended to by the moderns. We are sometimes, however, attentive to it, and we can feel a manifest difference with respect to the tension of the two hypochondria, which points out an accumulation of blood, and an increased determination of it to the hypochondriac system; and this must first depend upon an accumulation of blood in the system of the vena portarum, and some degree of inflammatory' diathesis in the corresponding arteries. We may join to this some degree of painful tension over the whole abdomen. - It is difficult to suppose that the blood is ever accumulated in such a manner as to give this external sensation of tension over the whole abdomen, but we may suppose that very trivial and local affections of the abdominal viscera may be communicated to the abdominal muscles, just as we find colics may depend upon a spasm of the abdominal muscles; if we voL. I. 
can therefore avoid the ambiguity of the distention being only flatulent, in which case it is not so painful, this tension gives a suspicion of an inflammatory affection in particular parts, and this will be still more clear if there are fixed pains in any particular part of the abdomen. We may join to the symptoms of an inflammatory determination to the abdomen, a decubitus difficilis occurring without any affection of the thorax. We have explained how this symptom may arise from affections of the thoracic viscera. Now, where the peculiar symptoms of these are absent, and the uneasy lying is considerable, this may, along with the other symptoms, be taken as a mark of the determination to the abdominal viscera. Where there is an anxiety without any affection of the thorax, we are in some measure conscious of a difficult transmission of the blood through the system of the vena portarum, from which the anxiety arises; and wherever it takes place without our suspecting nausea, or other particular affections of the stomach, we may consider it as a mark of the inflammatory determination to the viscera of the abdomen.

"The stomach is an organ which is liable to a very great. variety of states, and that from a great variety of causes, and the symptoms of it afford us only a very doubtful conclusion. Thus vomiting is very often no other than a symptom of a considerable state of debility; sometimes it is to be considered no otherwise than as a mark of considerable constriction affecting the state of other parts, as of the extreme vessels on the surface of the body, which may be considered as the chief of the causes of vomiting. But if the vomiting and nausea arise from the constriction upon the surface, they will very often yield more or less to the restoration of the determination to the surface, and to the use of medicines which have that effect, such as the saline draughts; or, when they are owing to irritation, they will yield to the use of opiates. But when a vomiting continues frequent, and does not yield to any of these remedies, it may be considered as a mark of some inflammatory affection in the abdominal viscera.

"To these marks we may add, that the determination to the abdomen is especially to be suspected to have occurred, whenever more or less of the nature of intermittent fever intervenes. In delivering the history of fever (LI.), I have. 
explained how that form of fever, in a particular manner, produces this determination. But we must acknowledge, that however we establish this distinction, doubts and difficulties will arise on particular occasions, as fevers constantly change their form from the one to the other. But whenever any thing of the intermittent has prevailed, and where there are other symptoms of it, we have to suspect a determination to the abdomen; and we have a great number of instances from Morgagni, Cleghorn, Lind, and Pringle, where marks of those affections in the abdominal viscera were found in persons who had died of intermittents.

"I must add to all this, as I said at first, that these symptoms are very often obscure, but that we have more reason to suspect them when the excess of stimulant power prevails, and when those remote causes have been applied which induce the inflammatory diathesis. Then it is to be suspected that this particular determination, the marks of inflammatory tendency, will be produced. Thus, the coldness of the climate and season gives a presumption; next to that the plethoric habit, and especially the more young and vigorous state of the system, which is more liable to the inflammatory diathesis, and therefore to the exertion of its particular effects. I need not repeat what I said before with respect to the special effects of this inflammatory tendency. They are in general the destroying an important organ, or producing effusions which stagnate and produce a poison.

" ' $A$ strong spasm formed,' \&c.-The spasm prevailing in the skin affects the extreme vessels, as is expressed by the dryness of the skin, there being no sweat and even very little perspiration. This dryness of the skin very often accompanies the symptoms of increased heat, which in this case we distinguish by the feeling of a scorched or dry heat. It is this state which distinguishes that part of the paroxysms of fevers which we more strictly call the hot fit, in distinction from the sweating fit which follows; and, as arising from the spasm, it is always understood as a mark of the excess of stimulant power.

"There is another circumstance we make more use of, viz. the dryness of the tongue, which we have no doubt in referring to a diminished secretion of mucus and saliva, and that to a 
constriction affecting the cxcretories of these secretions.-Another symptom depending on the same cause, is the preternatural thirst. Sometimes it arises purely from the spasm, as in the cold fit of fever where the constriction prevents the mouth and fauces from being supplied by saliva; but in the hot fit, there is joined to this cause, the state of heat in the system : thirst, therefore, is constantly a mark of the excess of stimulant power.

12 "The diminution of the secretions in general is a mark of spasm as giving occasion to the increase of stimulant power. We have not always an opportunity of judging of the degree of secretion on the skin, as it may be diminished even when the halituosus mador appears there. Sometimes we have evidence of it from the scantiness of the urine, and its high colour, which is an expression of the state of heat in the system. We observe the same thing in the state of the intestinal secretions; as they are more or less copious, we conclude that these parts are more or less dry ; a dry and bound belly, therefore, is to be considered as a mark of the excess of stimulant power.

"This finishes what I have to say with respect to the first prognosis, from the excess of stimulant power."

CIV. The symptoms which denote $a$ great degree of debility, are,

In the Animal Functions : 1. The weakness of the voluntary motions; 2. The irregularity of the voluntary motions, depending on their debility; 3. The weakness of sensation; 4. The weakness and irregularity of the intellectual operations.

In the Vital Functions: 1. The weakness of the pulse; 2. The coldness or shrinking of the extremities; 3 . The tendency to deliquium animi in an erect posture; 4. The weakness of respiration.

In the Natural Functions: 1. The weakness of the stomach, as appearing in anorexia, nausea, and vomiting; 2 . Involuntary excretions, depending upon a palsy of the sphincters ; 3 . Difficult deglutition, depending upon a palsy of the muscles of the fauces.

"I have said that the excess of stimulant power, with the concurrence of other causes, may be the cause of death in fevers, but it is especially the excess of sedative power that proves so; 
the symptoms, therefore, which we are now to mention, give the most certain presages of death in fevers.

"It is surprising that physicians have so long overlooked debility as a considerable part of the proximate cause of fevers. Nobody can fail to observe, that in the course of fevers many symptoms of debility appear, and go on constantly increasing with the disease till it proves fatal; it may perhaps be said to be the effect rather than any part of the cause; but I maintain that it has a considerable share, as arising immediately from the remote cause. Thus miasmata and contagion, which are the principal remote causes of fever, shew their sedative power not only in as far as we presume that they are more or less of a putrefactive nature, and, like all putrefactive powers, universally sedative and debilitating, but they are often so powerful as to kill the person immediately, of which we have frequent instances in most plagues; and, in other cases, the debility is truly among the first symptoms which appear; the body is languid, affected with lassitude, or with a sense of debility on motion, and an uneasiness in exerting it. The first symptoms are farther discovered in the weaker action of the heart and arteries. I have little doubt that repeated paroxysms may induce debility in consequence of the violent excitement; but this debility occurs in the very beginning of the disease also in a very strong degree; and however difficult we may find it to explain how debility produces reaction, yet we find that the one precedes and the other follows, and we presume from the order of their succession that they are connected as cause and effect.

" $A$. Of the Symptoms of Debility as they occur in the Animal Functions; and,

"6 1 . With regard to the voluntary motions; which we consider as affected in two ways, either with a state of debility giving merely a less degree of force in their several actions, or with the common effect of this debility by which the motions appear irregular in the intervals of their repetitions, and unequal in the degree of force in the several repetitions.

" $a$. With respect to the Debility of the voluntary motions, I have already remarked that one of the very first symptoms which appear in fevers, viz. Lassitude, or a sense of weariness like that which follows long continued or violent labour and exercise, 
is an expression of such a debility. But let us farther take notice of the several marks of debility in the voluntary motions of the body, as we will understand it best by tracing it in its several degrees. First the patient feels some uneasiness in walking about or in standing upright, and is obliged to sit down; perhaps he can still bear a sitting posture, but as the debility proceeds he is unable to exert so many muscles as are necessary to support the body even in a sitting posture, so that he is obliged to lie along in the horizontal posture, in which few muscles are exerted : thus he is unable to bear the erect posture in consequence of the sense of lassitude and the debility of the muscles; and the very raising the head from the pillow makes him uneasy, and is attended with giddiness. This proceeds still farther, and when the person lies down the debility appears still in his not being able to perform the motions which we can easily execute in that situation, such as turning the body from one side to the other; and at length even the lying upon one side being attended with the exertion of several muscles, becomes uneasy or impossible; the patient therefore tumbles upon his back, in which posture fewer muscles are exerted. But as the bed is sometimes made declining to the lower part, and the head a little raised, the exertion of some muscles is necessary to keep the body even in that posture; and it is observed that the person is constantly sliding down in bed lower and lower, till at length the limbs become flaccid and hang over the foot of the bed. These are clearly the symptoms of a constant progress of debility in fevers; and the higher degrees of it are mentioned among the bad presages of all the ancients.

" These symptoms are taken from the voluntary motions of almost the whole body; but there is a more compendious way of remarking this debility, that is, by observing the features of the face and the state of the eyes. The muscles of the face are almost in constant employment, are variously moved, and give different expressions of the countenance in consequence of every revolution of our thoughts; they are endowed with a remarkable power and alacrity of motion; and when they are flaccid and changing, this is considered properly as a sign of considerable debility. But it is especially in the quick and vivid motions of the eye that the state of health and vigour is to be per- 
ceived, and when these become languid, they express debility in a remarkable degree. Nothing is more obvious than the difference between the eye of health and the eye of disease.

"s $b$. The same debility is expressed by some irregularity in the state of these motions, as by tremor, which, I say, is always an expression of debility arising upon the attempt to exert any motion. Sir John Pringle marks the tremor of the hands as a principal sign of the coming on of typhus or jail fever; and this irregularity has proceeded to a considerable degree, when that active voluble member, the tongue, is liable to the same tremor. But not only this tremor, which is more decisively an expression of debility, but spasm also, or a fixed and more durable contraction in various muscles, is to be considered in the same light. A degree of tremor which is liable to end in spasm occurs in those parts especially which are liable to more vivid motions, as the eye; the eyes not only lose their accustomed alacrity of motion, but are absolutely fixed; and we can observe the spasm from the inequality of the motions of the two eyes, or from their squinting, or the ball of the eye is turned up within the upper eyelid; and the eyelids which have a share in the vivid motions are contracted and shut: hence the oculi conniventes are considered as a state of spasm. The irregularity of motion appears in a still more considerable degree, when such spasm affects the lower jaw, and some degree of locked jaw occurs.-I shall not at present inquire why the jaws in many cases remain free, while causes operate which have a tendency to produce spasm; why the locked jaw is sometimes the first, and very often the only effect; and why in certain circumstances the jaw is more liable to be affected by such causes.

"Convulsive motions also are marks of the same debility. Most of the muscles in different parts of the body are liable to be affected with momentary convulsions; when the tendons, as those of the wrist, which lie bare, are affected in like manner owing to the motions of the muscles themselves, such motions go under the title of Subsultus Tendinum. The lower jaw affected with convulsive motions gives the Stridor Dentium, or a seeming gnashing of the teeth, which arises readily in children from various irritations; we impute it commonly to worms, from which cause it frequently proceeds; but even in children it 
arises upon various other occasions, and in them it is not a symptom of so much importance, but occurring in adults it is a. strong mark of powerful convulsions; and it frequently arises to affect a considerable part of the system at once, and to give plainly epileptic fits.

66 With respect to convulsions, and even spasms, it may be doubtful, in many cases, whether they are properly mentioned here as marks of debility, and whether they ought not rather to be considered as marks of a particular irritation? They are no doubt marks of particular irritation, but we know that they are very often likewise the consequence of mere debility, and that irritation will not produce them but from a state of debility. When we come to consider these convulsive motions as a peculiar disease, we shall have occasion to shew the proof of their depending upon and concurring with debility, and that the marks of debility are at the same time present, so that these marks are but an expression of the same state of the system.-So much with respect to this part of the animal functions, the voluntary motions.

" 2. In the animal functions we also attend to the exercise of Sensations, external and internal, which are often variously affected and vitiated. We first take notice of the general tone or temper of mind, as I may express it. In consequence of the debility prevailing in fever, a languor of sense and thought appears very early, and is commonly increased in the course of fevers, forming what we call the dejected state of mind, which is a consequence of a degree of timidity; this is in a great measure involuntary and without any improper object; for while reflection remains, an object is constantly before the patients' eyes, viz. the event of the disease, with regard to which their temper of mind commonly arises to an actual despair; and the worst sort of fevers are remarkable in this that they immediately inspire the patient with despair with respect to the event. Whatever theory we assume with respect to the passions in general, we will find that these timid passions, as fear, dejection, and despondency, correspond with a state of debility in the energy of the brain, while the passions of a contrary kind are marks of vigour, and strength in this energy.

- $\quad$ ii "In like manner, the intellectual faculties are disturbed and 
interrupted in fevers. At first this appears only in what is called a confusion of the head, that is, we do not easily follow our ordinary train of thought, which is constantly liable to be broken off by something different and foreign to what we were employed about ; this difficulty and uneasiness of thought not only appears by the interruption of our ideas, but we cannot easily command the train we are intent upon, the memory fails us, and we find the greatest difficulty in recollecting what we were about. These are among the first symptoms of the disturbance coming on in our intellectual faculties. Another circumstance attending it, is this, that whatever slumbers are obtained are disturbed by constant dreaming. I call dreaming the delirium of our sleep, as delirium is the dreaming of our waking hours; they are both very analogous, and turbulent and confused dreams are commonly the prelude to the coming on of delirium. Now commonly delirium first comes on before we are fairly asleep, or when in coming out of sleep we are long in being quite awake; 'and this is the common account I get when I inquire whether patients have been affected with any delirium, that they talk a little incoherently only in coming out of sleep, or in falling into it. But when a patient speaks incoherently when he is evidently awake, then it is what we properly call delirium. In speaking of the causes of delirium (Physiology, p. 148, First Lines, XLV.), I have mentioned that it may be of two kinds, ferox or mite ; but it is the last only that I consider here as an expression of debility, and therefore the delirium we are to speak of now is not phrenitic; it is without any emotion or passion of the mind, without any thing like irascibility; it is placid and expressed by incoherent talk only; the patient has not his body agitated, and there is not any great degree of restlessness, and only a degree of low muttering incoherence, with a considerable degree of sleeping at first perhaps; this however is more remarkable when it comes to be accompanied with a very considerable degree of loss of memory. Delirium may be owing to certain powers operating upon the sensorium, and exciting false ideas that have no external object corresponding to them. But these would be often banished and driven away, if the memory were not lost and unable to recover the ordinary association of ideas which should be most powerful 
in removing the delirium; and I have known many instances of persons who, when no light and noise were present, were affected with considerable delirium, but whom light, and an accustomed voice speaking to them, brought back to their senses. We consider the delirium as going to the highest degree when the person is insensible to the most established associations, as when he mistakes his own bed or nearest relations. It may be considered as a mark of the same when the associations with respect to certain functions of the body, which have immediately and steadily governed us, such as the evacuations by stool and urine, are destroyed. We are particularly accustomed to the stimuli of these excretions, and to a particular manner of exercising them; and the greater part of mankind is affected with a particular sense of cleanliness and decorum with respect to them. When this is forgot, and the freces and urine voided insensibly, without taking notice of their stimuli, this is a proof that the ordinary associations are lost, and that a total obliteration of the memory has taken place. Some ambiguity may indeed arise when we consider that this may depend upon a paralytic state of the sphincter ani, or of the muscular fibres in the neck of the bladder; but with respect to the sphincter ani, when the patient retains clysters perfectly well, and when in voiding the faeces there are some marks of effort, though no signal is given, we know that it is not owing to a paralytic state of the parts, but merely to a state of the sensorium which has obliterated the ordinary sense with respect to the most common associations. But further, with respect to the exercise of those functions that relate to the states of sleep and waking, I have said (Physiology, CXXXI.) that sleep depends upon a state of collapse more or less prevailing in the sensorium, particularly with respect to the animal functions; every propensity therefore to sleep is a mark of the diminished energy of the system ; and drowsiness or coma indicate the same. Very often this drowsiness is not succeeded by sleeping, or is without a propensity to sleep, and the sleep is readily interrupted; this is what physicians call Coma vigil, or Typhomania, where nature proceeds the length of actual sleeping, but this is prevented by the operation of some internal cause. Any degree of this typhomania may be considered as a mark of collapse, and when it 
proceeds further to a state of actual somnolency or lethargy, it comes to be a mark of debility by itself; but for some reasons which I cannot well explain, the latter is not a mark of the worst kind; for it is universally observed, that except in a state of the utmost debility, a comatose state, in the more advanced state of fevers, is much safer than constant waking, or any degree of typhomania.

"6 I must mention another expression of Collapse, which might go to another head, but which is frequently connected with this, and may be explained upon the same footing; that is, a loss of hearing, a degree of deafness occurring in fevers. It is not easy to explain this from any topical affection of the organ; it must be referred to the sensorium, or to some degree of collapse with respect to the organ of hearing. At the same time it is for the most part a sign expressing no great danger; nay, upon the contrary, a degree of coma, and a loss of hearing coming on in some fevers are rather favourable symptoms. More danger, however, is to be apprehended from a loss of sense in the several organs of sense; as in the cold fit of fevers, when the insensibility to touch may proceed to such a degree, that the soles of the feet have been burnt, and an eschar produced, without the patient perceiving it. In the advanced state of fevers we have not an opportunity of observing this, but we observe it with respect to other senses, as that of thirst, which is a symptom almost inseparable from fevers; and yet though all the causes that we can assign for thirst occur, as dryness of the fauces, heat of the internal parts, and a great degree of putrescency going on in the stomach, which is a most powerful cause of thirst, the mind is very often insensible to it, and no thirst appears. Somewhat perhaps might be imputed to that crust which often covers the extremities of the nerves of these organs, but this is confined to the tongue alone, and I know no instance of its being extended to the fauces, which are the chief parts which gives this sensation. 'The want of taste therefore must be imputed to the loss of sensibility, and not to the crust upon the tongue preventing the communication of the sapient substances to that organ. This insensibility sometimes appears even in the loss of sight. No organ is more sensible to impression than the eye, so that when this occurs it is certainly 
a symptom of a strong degree of collapse of the sensorium. We observe that it occurs in various degrees; there is first an imperfect or erroneous sight, and frequent instances of false vision, and the imagining of objects really not present. It has been common to impute this to the state of the sensorium alone, but I have several facts to show that it may be in a great measure owing to the state of the organ itself, as it is affected by the collapse of the sensorium; as where blackish spots appear to cover every thing the persons look at, which they are employed in picking with their fingers in order to take them off. This gives occasion to the term Floccorum collectio, the Carphologia of the Greeks, which has been considered as a fatal sign; but as it occurs in an organ which is exquisitely sensible, it is not always to be considered so, and may be partly owing to the state of the organ as affected by the sensorium.-These are the several symptoms of Debility in the animal functions.

" $B$. Of the Symptoms of Debility, as they appear in the Vital functions; and,

" 1 . In the state of the Circulation, or the action of the heart. What it is which, in ordinary health, gives stability and permanency to the motion of the heart, is difficult to explain; but certainly this is one of the most stated and most constantly exerted functions of the system. W' $W^{\top}$ hen therefore a Deliquium animi occurs, it may be considered as a mark of very great debility; it does occur in fevers, but more especially on the attack of intermittent or remittent fevers; in continued fevers, either in their beginning or progress, it is a much more rare symptom. But even there various symptoms occur that show an approach to it, so that there is a tendency to the state of absolute debility.

" Approaching debility is announced by some degree of vertigo, dimness of sight, singing in the ears, or, as it is expressed, Tinnitus aurium, of which we have frequent instances even in continued fevers. This, however, commonly occurs only when the body is raised to more or less of an erect posture ; an entirely erect posture would in a number of instances bring on this symptom and Syncope itself; and in other cases it goes so far that the raising a small part of the body, as the head, from the pillow, brings on at least the first approach- 
es to it, as Vertigo and Tinnitus aurium. I need not repeat what is to be said in theory upon this subject; the action of the heart depends upon the constant energy of the brain, and the energy of the brain itself upon the action of the heart, or the impulse of the blood in its vessels. (See Physiology, CXXXIV.) It is a sign, therefore, that the heart is weak, when it is not able to propel the blood into the vessels of the head in an erect posture; and the brain also is thereby considerably weakened, the influence of these organs being mutual and reciprocal. 'But however you consider this matter, in every light these symptoms are expressions of considerable debility. As they however do not always occur, we generally judge of the force of the circulation by the state of the pulse; we suppose debility, where the pulse is weak, and, by a necessary consequence, frequent, and also in the same case unavoidably irregular. If the action of the heart is weak, the quantity of blood in the ventricles is not entirely evacuated, so that they are sooner filled and irritated to a new contraction. Perhaps there is a sense in us, though little perceived by the mind, that in consequence of any difficulty being presented, some degree of reaction arises, or some effort to exert the force of the circulation; and this will always account for the frequency of the pulse. In consequence of the weakness every muscular fibre is liable to be unsteady, and the heart itself is liable to tremor as any other muscle is ; when weak, therefore, it will likewise show an irregularity or inequality in the repetition of the pulse.

"When upon this subject, it is necessary to take notice of a slow pulse occurring in fevers. A frequent pulse is the very characteristic of fevers, but we know that we must not depend upon that single circumstance alone, and, accordingly, at present most physicians agree that there is a nervous fever where there is a pulse ' sanorum similis,' nay, where it is even slower than is usual in a state of health. I cannot refuse that the fact occurs, but I must say that I have not met with this circumstance in one out of an hundred of the nervous fevers that I have had occasion to observe. I have, however, in complaisance to the fact set down in the character of the typhus, ' pulsus plerumque frequens,' but I would say, that in general, nay, very universally, the pulse is, on the contrary, more frequent in nervous fevers than 
it is in any of the inflammatory, till the progress of the disease has induced that weakness which lays the foundation of the quick pulse. If the pulse, therefore, is less frequent than might be expected in proportion to the affection we perceive in the nervous system, as the thirst, heat, \&c. this may be considered as a mark of debility. One case of this kind, in which the pulse becomes slow, and sometimes remarkably so, occurs in the Tertiana comatosa or apoplectica, or a Tertian attended with stupor, coma, or apoplexy; but even in this case it is extremely. rare in the beginning, though, upon the coma coming on, I have found it slower than it was before, or was expected to be. Now, here also it is to be considered as a mark of debility, though, as I said before, it is a state of debility which is commonly attended with the least danger; as a comatose state, except when it is in very great excess, seldom portends half the evil that a constant waking, or a degree of typhomania, does.

"We judge likewise of the state of vigour in the circulation by the heat of the body. The ancients defined fever as being a preternatural heat; and this is indeed a common attendant upon every quicker motion of the blood, and commonly occurs in fever. But there are fevers in which increased heat does not occur, or is inconsiderable ; and this is considered as a mark of the nervous fever, in which debility prevails. Whenever, therefore, the heat is not in proportion to the frequency of the pulse, or to other circumstances of the fever affecting the sensorium, we consider it as a mark of prevalent debility, or at least of the want of irritation. We judge of the increased heat from its effects ; the first of which is a dryness of the tongue; the moistness of the tongue is a mark of the absence of heat, and is a sign of that fever to which this is most common, viz. the nervous fever. The absence of heat often goes so far as to give place to cold-I was going to say, what perhaps is not so accurate, an actual coldness. This, whenever it occurs in the system, may constantly be considered, I think, as a mark of a weaker circulation, or weaker impulse of the blood into those parts in which the cold is felt. I formerly (VIII.) gave some remarks with regard to cold occurring in the beginning of fever. Dr. Boerhaave has not been quite so accurate in representing this symptom. He has given a general Prognostic, that all the 
other following symptoms of fever are to be in a degree higher in proportion to the cold which occurs at the commencement. Now, where the coldness is without horror, tremor, and rigor, it may be considered as a mark of a strong sedative power producing the fever; but it must always be taken with this qualification, that the horror, tremor, and rigor are symptoms of a reaction taking place which is excited for the solution of the paroxysm. But there is no ambiguity when cold occurs in the advanced state of fevers, whenever it affects what we strictly call the extremities. It most readily occurs in the feet, as a mark of the weaker action of the heart ; still more so when it affects the hands, which are nearer to the heart; but it is to a greater degree still when it affects parts on the surface of the body which are only a little prominent, such as the tip of the ear, or tip of the nose, which are only a little more exposed to the air than other parts. This is considered as a mark of extreme debility, and very often is the symptom of approaching death.

"To these symptoms some others are frequently joined, which express in like manner the weaker action of the heart in propelling the blood to the different parts of the system, such as a pallid countenance, which is among the first expressions of the approach of fevers. When the reaction is vigorous, this symptom is not only absent, but a redness and turgescence of the face appears; and a paleness appearing instead of this, is certainly a clear symptom of an increasing debility in the system. In ordinary health, a number of red vessels appear upon the adnata of the eye; when they do no longer appear, this is a mark of the debility prevailing; very often, however, while the countenance is pale, and there are other marks of debility in the advanced state of fevers, we observe a considerable redness in the adnata of the eye, which may be considered as a mark of irritation in the brain; for even though the action of the heart is weak, there may be a considerable determination to the vessels of the head, with which the vessels of the eye have more communication than those of the face-Not only the paleness of the countenance is a mark of the weaker circulation, but at the same time there is a collapse of the features of the face, which become not merely languid but flat, and the face appears leaner. The turgescence of the skin in persons in health cer- 
tainly depends in a great measure upon the fulness of the vessels, so when the action of the heart is weakened, there is a remarkable shrinking of the whole external parts of the body; and as the determination is so readily to the face, the want of it there must be a bad mark. This, in the common language of authors, is termed the Hippocratic face. Hippocrates has, indeed, described the face of persons worn out by a highly colliquative fever, and it amounts to this, that there is a considerable collapse of the whole surface, the eyes are flat, the nose sharp, \&c. and we find that this when it appears in fever, and when not preceded by the causes of collapse and exhaustion, may be taken as a mark of the weak action of the heart in propelling the blood to the extremities.

" To this we may subjoin cold sweats occurring about the head and neck, particularly when they appear on the forehead, while at the same time the countenance is pale and the features collapsed; from experience we know that these cold sweats are a mark of debility, but how they are to be explained is not so evident; nay, I would have concluded that it is commonly the expression of the increased action. But though every exertion may be considered as a mark of the impulse of its vessels, the matter is not always so, for we know that certain excretions may appear increased from a relaxation of the extremities; while the impetus of the blood is no greater than usual. Whether the excretories every where are furnished with a kind of sphincter, we cannot determine, but where these are more evident, the relaxation of them does produce a seemingly increased excretion. If we can, therefore, suppose that the extreme vessels are under a considerable degree of atony or debility, a quantity of fluid may be squeezed out, with the smallest degree of force in the large vessels, or even by their contractile power. This to me is the most obvious and satisfactory account with respect to the symptom of cold sweats, which so frequently announces the deliquium animi itself, especially the cold drops of sweat standing upon the forehead.-These, upon the whole, are the marks of debility, as relating to the circulation of the blood.

" 2 . The marks of debility, expressed by the weakness of the Respiration.

"We may consider the respiration as under a state of debil- 
ity, where it is small and frequent, especially where it is frequent upon the smallest motion, and where at the same time it. is laborious. But the matter is ambiguous, because these same symptoms are often only marks of obstruction in the vessels of the lungs, which gives a resistance to the transmission of the blood; but where they are without any other symptoms of that obstruction or resistance, they are to be considered as marks of debility. How debility will produce them is I think obvious : A small respiration occurs when the muscles which perform it are weaker; but if the inspiration for one moment be smaller than it ought to be, transmitting a less quantity of blood through the lungs, then, in order to compensate that, either the exertion of a vis medicatrix naturæ, or the stimulus of that accumulation will immediately produce a more frequent inspiration. And though I have said that a laborious breathing is especially an attendant on an obstruction in the lungs, it will especially occur when the ordinary muscles of inspiration are weak. We observe that the breathing is laborious, when not only the diaphragm, which is chiefly employed in that function, is exerted, but when the intercostal muscles are likewise considerably employed; but not only the ordinary intercostal muscles, but all those which lie between the clavicle and scapula, and between the vertebræ and ribs, and farther, the muscles that give a fixed point to them, those extending the head and neck, are exerted. This constitutes the Respiratio sublimis of pathologists, and it occurs more frequently as a mark of debility than as a mark of resistance to the transmission of the blood. The two states, however, may occur together,-the weakness appearing first, but the accumulation which it occasions amounting to a state of obstruction. Dr. Boerhaave's saying, therefore; that almost every person dies of a peripneumony, amounts to little more than this, that every body dies for want of breath. It is very likely that before death happens, the accumulation of the blood in the lungs, in consequence of this weaker respiration, concurs as one of the means of putting a final end to life; but it would be improper to say that this was a peripneumony, though the weakness may be sometimes of the peripneumonic kind, or may approach to the peripneumonic degree.

"While we are uncertain in interpreting the state of the revoL. I. 
spiration, there is one mark which is much more decisive in pointing out the debility of the system, that is, a change of the tone of voice. This indeed almost always depends upon the state of the muscles of the glottis; it is properly an animal function, and is not necessarily connected with the vital, but the consideration of the respiration brings it especially to our minds. It is observed, that it is among the first marks of sickness attended with debility. This change in the tone of the voice goes on through various degrees till it amounts to an aphonia; and the common people of this country mention it as a sure mark of approaching death, that the patient is past speaking. I find some difficulty in explaining this; but I may observe, that however the formation of the voice is to be explained, it depends upon the muscles which move the membranes that form the glottis, and the cartilages with which those membranes are connected. Whether the voice is formed merely by the contraction of the parts concerned, our thorax being in that respect a wind instrument, or whether it depends on the different degrees of tension, according to the late and now common opinion, I would not determine here ; but it is certain that it depends upon some exquisitely nice and minute motions, which, like those of the eye and tongue, are first affected in fever; whether this change of the voice proceeds from a diminution of the tension, or of the aperture, we can see that it is a very proper mark of the state of debility in the system.-So much with respect to the case of debility as the symptoms of it occur in the vital functions, and what is immediately related to them."

" $C$. The marks of debility in the Natural Functions; and, First, as it appears in the Appetite and state of the stomach. Here the debility is in very different degrees; as it appears in the anorexia or want of appetite; in a manifest aversion to food; or still further, as that amounts to a nausea, which, when a little increased, frequently arises to actual vomiting. The symptoms, then, I speak of, are, anorexia, nausea, and vomiting. I formerly (XLIV.) explained upon what grounds all of these may be considered as marks of debility, and how they'generally appear more or less in proportion to the other marks of debility prevailing in the system; at the same time, I observed that they are in a particular manner connected with the state of the 
surface, and with the spasm that occurs there. I might add now that the nausea and vomiting may indeed be considered sometimes as depending in some measure upon substances in the stomach itself, and upon their being more or less in a putrid state. But whether these are to be considered as marks of spasm or putridity, they all resolve into a state of debility; and accordingly, I have no doubt in marking them here as symptoms of debility, because I say, that abstracting from some topical affection of the stomach which may happen to prevail, or some irritation from the abdominal viscera, the anorexia, nausea, and vomiting, are very generally in exact proportion to the other symptoms of debility. The most obstinate vomitings, accordingly, are those that occur in the worst kind of fevers, as the pestilential fever itself. In the natural functions, however, we cannot easily find many symptoms of fever. It is not easy for us to observe the state of the numerous secretions of the system, and with regard to the principal function of nutrition there is too short a space of time for any considerable change to occur. It is only certain motions that belong to the natural functions that we attend to, such as deglutition, which may be considered as a natural function; but we only take notice of its debility when it goes to a high degree, and the power of swallowing is lost. When this depends upon a topical affection of the fauces it is easily discerned; but when it is without any thing of this kind, it is to be considered as a paralytic affection of the fauces and cesophagus, the Dysphagia paralytica of Sauvages, so to be called when the symptoms of debility are so increased that the parts are completely paralytic, and the swallowing ceases entirely.

" Under the same head we would mention, that when in the natural functions the weakness affects those parts which are especially endowed with a very permanent contractile power and tone, as the bladder and rectum, and involuntary excretions follow, which depend upon the loss of tone in the sphincter; this is a symptom of extreme debility. I have mentioned (page 578) the ambiguity that occurs here, that the involuntary excretion sometimes is rather to be considered as a symptom of coma and insensibility than of the relaxation of the sphincter, and that we know this from the retention of clysters, while the fæces pass without the patient noticing them; but where the other marks of 
debility are plain, this is a mark of very extreme debility. We observe this most distinctly in the case of the excretion of the urine, not when it is voided at considerable intervals, but where there is a manifest incontinency. I should have observed that the very cold sweats are an instance of the same relaxation and debility.

"C This finishes the consideration of the state of debility ; and as with respect to the former set of symptoms, those of increased reaction, so here also a judgment is to be formed from the degree, or rather it is by the combination of symptoms that we determine - the event. It is not from debility appearing in one or other function alone that there is the most certain danger, but where it appears universally. In concluding this subject, however, I must observe, that Hippocrates has told us that the prognostics neither of life nor of death are to be trusted to absolutely ; it is certain that both are often liable to deceive us in the case of debility, and I have met with surprising deceptions in that respect; though undoubtedly the combination of the debility in all the functions commonly proves the most certain presage of death, yet there are cases where it has gone a great length, and yet the person has recovered, as in the advanced state of the typhus. Here there is certainly a reaction which produces a crisis or a change in the state of the disease, so that it ends in health, but so obscurely that we cannot explain it, and I have not observations sufficient to form any rule or to give any certain directions how to judge when we may still retain hopes in the case of debility. The only rule is this, where the heart continues to retain some degree of its force, and particularly where the pulse is not weak and frequent in proportion to the other symptoms, we hope for a favourable determination; when the pulse is under 120 in the minute, I do not lose my hopes in fevers; and I do not hold symptoms of debility alone to indicate the most certain danger, but when they are joined to marks of a topical affection of the brain, they are especially decisive. That some inflammatory state may be going on in the brain dissections very commonly shew ; it is therefore when there are, with the marks of debility, marks of a topical affection of the brain, or of an extreme degree of putrefaction, that we are to infer that the event will prove fatal. 
"I propose, therefore, here to mention what the circumstances are which express a combination of irritation and debility, particularly as the irritation occurs in the brain, and gives a suspicion 'of those topical affections which after death we find to have truly happened, though symptoms of the inflammatory state had not happened to appear before.

"Now, one symptom of such an irritation applied to the brain and nervous system, is an increased sensibility to light and noise, while other symptoms of irritation do not.appear to affect the sanguiferous system. Any degree of inflammation in the retina, by increasing the tension of the vessels which are so intimately connected with the medullary fibres, must give a very singular sensibility (Physiology, LVI. 3.). If, therefore, these marks of sensibility appear in the organs of seeing, and also in the organs of hearing, where I presume they are to be explained in the same manner, they are to be imputed to a preternatural fulness and distention, or to an increased impetus in the vessels of the eye, while at the same time there is no fulness or hardness of the pulse, nor any considerable redness or suffusion of the face, which point out a topical affection of the blood-vessels of the brain.

"I should have observed, that though inflammatory affections in any particular part of the system, do commonly produce the diathesis over the whole, this is not universally the case; for we have innumerable instances of topical affections merely, which do not affect the sanguiferous system more generally; and it is a dangerous state where this takes place with respect to the brain. This symptom will be rendered still clearer by many vessels appearing on the adnata of the eye, while in other parts there is no appearance of an inflammatory distention.

"Another symptom of irritation appearing in the system, and particularly applied to the brain, is interrupted sleep. The collapse of the brain, in which sleep consists, naturally recurs upon us every twenty-four hours; and, according to the health of the system, and the absence of irritations, the sleep for some time is quite profound and uninterrupted. If, therefore, dreams occur, or if sleep is any otherwise broken off; we can perceive that irritations are applied to some part or other: and disturbed sleep - 
has been agreed upon among physicians as a symptom of particular irritation in the brain, and very frequently of an inflammatory state of it. Another symptom is a constant waking, which is a stronger mark of the same condition; or, when not only constant waking, but a constant restlessness and inquietude takes place at the same time, when the patient frequently changes his posture, and can find no ease, which must be owing either to pain or uneasiness, the latter generally depending upon what we more strictly call anxiety. (Physiology, p. 59.). And we find that there is no cause of uneasiness so powerful in producing anxiety and restlessness, as an irritation applied in a particular manner to the brain. Uneasiness arises from the mind as well as from the body, and we join to this delirium. We have distinguished Delirium into two kinds, the placid and furious; the latter, when it is with more emotion and restlessness, and particularly when it is to the degree of a remarkable impatience to get out of bed, \&c. when it may be considered as a certain mark of a considerable irritation applied to the brain.

"We may add every degree of convulsive and spasmodic motions. These, for the reasons $I$ have given, may be reckoned under the head of debility, since, if they were not from debility, they would seldom take place; but I must add, that they are also marks of irritation, particularly as applied to the nervous system; and when they appear in the vital functions, as in the action of the heart, producing a very frequent, small, and contracted, or hard pulse, they are owing to some peculiar cause of debility and irritation joined together. I have explained how the weaker action of the heart gives a small and frequent pulse; but I am to add now, that when a stimulus is applied to the heart, and occasions a frequent contraction, so that it is imperfectly evacuated, it must also give a small pulse ; or, when it is more or less applied to the arterial system, it gives a contracted hard pulse; and where the symptoms of irritation are not very clear and evident, but are however taking place in the system in an obscure manner, I know of no more certain means of judging than from this small, frequent, hard, and contracted pulse.

" Lastly, Irritation may appear in the excretions, but I can mention it only in one particular instance, in the case of the 
urinary secretion. The urine, from being high-coloured, and seemingly saturated with matter, all at once is changed to a limpid, colourless, and inodorous state. This has been, since the time of Hippocrates, considered as a symptom of actually formed Phrenitis, or of a Delirium phreniticum. It shows that there is some constriction prevailing in the branches of the descending aorta, and hence a greater determination of blood towards the head; and it may in part be explained in this way, but I consider it more distinctly as a mark of some irritation applied to the nervous system more generally. I should not pass it over, however; without saying that the limpid urine is not always the bad symptom that Hippocrates supposed it to be. But I reserve the more particular consideration of this subject till I come to speak of the urine more fully.-These are the symptoms that express an irritation applied to a certain part of the system, the brain especially, while there are other powers of debility, acting in the same part, and preventing the effects of the irritation from appearing so considerable over the whole sanguiferous system; and wherever that is the case, the symptoms of debility that are joined are more full presages of death."

CV. Lastly, The symptoms denoting the putrescent state of the fluids, are,

1. With respect to the stomach; the loathing of animal food, nausea, and vomiting, great thirst, and a desire of acids.

2. With respect to the fluids; 1 . The blood drawn out of the veins not coagulating as usual. 2. Hæmorrhagy from different parts, without marks of increased impetus; 3. Effusions under the skin or cuticle, forming petechiæ, maculæ, and vibices; 4. Effusions of a yellow serum under the cuticle.

3. With respect to the state of the excretions; fetid breath, frequent, loose, and fetid stools, high-coloured turbid urine, fetid sweats, and the fetor and livid colour of blistered places.

4. The cadaverous smell of the whole body.

CVI. These several symptoms have very often, each of them singly, a share in determining the prognostic: but more especially by their concurrence and combination with one another, particularly those of debility with those of putrescency.

CVII. On the subject of the prognostic, it is proper to observe, that many physicians have been of opinion, there is some- 
thing in the nature of fevers which generally determines them to be of a certain duration; and therefore that their terminations, whether salutary or fatal, happen at certain periods of the disease rather-than at others. These periods are called the Critical Days; carefully marked by Hippocrates and other ancient physicians, as well as by many moderns of the greatest eminence in practice; while at the same time many other moderns, of no inconsiderable authority, deny their taking place in the fevers of these northern regions which we inhabit.

- CVIII. I am of opinion that the doctrine of the ancients, and particularly that of Hippocrates, on this stbject, was well founded; and that it is applicable to the fevers of our climate.

CIX. I am of this opinion, first, Because I observe that the animal economy, both from its own constitution, and from habits which are easily produced in it, is readily subjected to periodical movements. Secondly; Because, in the diseases of the human body, I observe periodical movements to take place with great constancy and exactness; as in the case of intermittent fevers, and many other diseases.

" Nothing is more obvious than that the animal economy is subject to periodical habits, and that we are of a nature which is easily affected by custom and habit. The animal economy is exposed to certain causes recurring regularly to act upon it; these in a body so disposed, therefore, must actually produce' such periodical movements : and further, we know that in fact they do take place in our ordinary health, in the business of sleep, appetite, and excretions ; and that particularly in many diseases they are distinctly to be observed, as in the various Intermittents. Let us take but the single instance of a quartan, which for the space of six months will return every fourth day, and that at stated hours as exactly as the clock strikes. In the smallpox, measles, epilepsy, asthma, \&c. we have occasion to observe the same exactness in the recurrence of the paroxysms or of their periodical movements. I would say that they occur particularly in continued fevers, from this consideration, that many of these do arise from intermittents in the manner I before explained, by shortening the intervals more and more till they come not only to a daily paroxysm, but to a paroxysm twice a-day; and there are few continued fevers in which the 
most heedless observer may not perceive such paroxysms, and though in the advanced state of the disease they are more obscure, we cannot doubt of their existence. I before took notice of the fact, with respect to intermittents, that when they seem to be at an end, yet there is some cause lurking in the system which gives a constant tendency to the repetition; and that on those days. which would have been the days of the paroxysm, had the disease constantly appeared without intermissions. Hence that particular rule of Celsus, that the patient ought to be attentive to the days upon which the fever would have returned, lest any trespass in the non-naturals should bring it on again. And we are readily led to believe, that where fevers are of the most continued kind, there is still a tendency to the same periods, which we see the system is constantly disposed to follow."

CX. These considerations render it probable that exact periodical movements may take place in continued fevers; and I think there is evidence of such movements actually taking place.

CXI. The critical days, or those on which we suppose the termination of continued fevers especially to happen, are, the third, fifth, seventh, ninth, eleventh, fourteenth, seventeenth, and twentieth. We mark none beyond this last; because, though fevers are sometimes protracted beyond this period, it is, however, more rarely; so that there are not a sufficient number of observations to ascertain the course of them; and further, because it is probable that, in fevers long protracted, the movements become less exact and regular, and therefore less easily observed.

CXII. That the days now mentioned are the critical days, seems to be proved by the particular facts which are found in the writings of Hippocrates. From these facts, as collected from the several writings of that author by M. De Haen, it appears, that of one hundred and sixty-three instances of the termination of fevers, which happened on one or other of the first twenty days of the disease, there are one hundred and seven, or more than two-thirds of the whole number, which happened on one or other of the eight days above mentioned; that none happened on the second or thirteenth day; and upon the eighth, 
tenth, twelfth, fifteenth, sixteenth, eighteenth, and nineteenth, there are but eighteen instances of termination, or one-ninth of the whole.

CXIII. As the terminations which happen on the seven days last mentioned, are, upon the whole, few, and, upon any one of them, fewer than those which happen on any of our supposed critical days, so there are therefore nine days which may be called Non-critical ; while, on the other hand, the many terminations which happened on the seventh, fourteenth, and twentieth days, afford a proof both of critical days in general, and that these are the chief of them. Hereafter I shall mention an analogy that renders the power of the other critical days sufficiently probable.

CXIV. It appears further, that as, of the terminations which were final and salutary, not a tenth part happened on the noncritical days, and of the terminations which were final and fatal, though the greater number happened on the critical days, yet above a-third of them happened on the non-critical, so it would appear, that the tendency of the animal economy is to observe the critical days, and that it is by the operation of some violent and irregular cause that the course of things is sometimes turned to the non-critical.

CXV. What has been said gives sufficient ground for presuming that it is the general tendency of the animal economy to determine the periodical movements in fevers to be chiefly on the critical days. At the same time, we must acknowledge it to be a general tendency only, and that, in particular cases, many circumstances may occur to disturb the regular course of it. Thus, though the chief and more remarkable exacerbations in continued fevers happen on the critical days, there are truly exacerbations happening every day; and these, from certain causes, may become considerable and critical. Further, though intermittent fevers are certainly very strongly determined to observe a tertian or quartan period, we know there are circumstances which prevent them from observing these periods exactly, and which render them either anticipating or postponing so much, that the days of the paroxysms come to be quite changed; 
and it is allowable to suppose that the like may happen with respect to the exacerbations of continued fevers, so as thereby to disturb the regular appearance of critical days.

A particular instance of this occurs with respect to the sixth day of fevers. In the writings of Hippocrates there are many instances of terminations happening on the sixth day; but it is not therefore reckoned among the critical days ; for of the terminations happening on that day, there is not one which proves finally of a salutary kind - the greater number are fatal, and all the rest are imperfect, and followed by a relapse.-All this shews that some violent cause had, in these cases, produced a deviation from the ordinary course of nature; that the terminations on the sixth day are nothing more than anticipations of the seventh, and therefore a proof of the power of this last.

CXVI. The doctrine of critical days has been much embarrassed by some dissonant accounts of it which appear in the writings imputed to Hippocrates. But this may be justly accounted for from these writings being truly the works of different persons, and from the most genuine of them having suffered many corruptions; so that, in short, every thing which is inconsistent with the facts above laid down, may be ascribed to one or other of these causes.

CXVII. This, further, has especially disturbed the doctrine of critical days, that Hippocrates himself attempted, perhaps too hastily, to establish general rules, and to bring the doctrine to a general theory, drawn from Pythagorean opinions concerning the power of numbers. It is this which seems to have produced the idea of odd days, and of a quaternary and septenary period; doctrines which appear so often in the writings of Hippocrates. These, however, are inconsistent with the facts above laid down; and, indeed, as Asclepiades and Celsus have observed, are inconsistent with one another.

CXVIII. Upon the whole, therefore, it is apprehended that the critical days above assigned are truly the critical days of Hippocrates, and may be consistently explained in the following manner.

CXIX. From the universality of tertian or quartan periods in intermittent fevers, we cannot doubt of there being in the ani- 
mal economy a tendency to observe such periods; and the critical days above mentioned are consistent with this tendency of the economy, as all of them mark either tertian or quartan periods. These periods, however, are not promiscuously mixed, but occupy constantly their several portions in the progress of the disease ; so that, from the beginning to the eleventh day, a tertian period takes place; and from the eleventh to the twentieth, and perhaps longer, a quartan period is as steadily observed.

CXX. What determines the periods to be changed about the eleventh day, we have not clearly perceived-but the fact is certain; for there is no instance of any termination on the thirteenth, that is, the tertian period next following the eleventh ; whereas, upon the fourteenth, seventeenth, and twentieth, which mark quartan periods, there are forty-three instances of terminations, and six only on all the intermediate days between these.

This prevalence of a quartan period leaves no room for doubting that the twentieth, and not the twenty-first, is the critical day marked by Hippocrates, though the last is mentioned as such in the common edition of the aphorisms, taken from an erroneous manuscript, which Celsus also seems to have copied.

CXXI. A consistency with the general tendency of the system renders the series of critical days we have mentioned probably the true one; and the only remaining difficulty in finding what we have delivered to be the same with the genuine doctrine of Hippocrates, is the frequent mention of the fourth as a critical day.

It is true, there are more instances of terminations happening on this day than on some of those days we have asserted to be truly critical. But its inconsistency with the more general tendency, and some other considerations, lead us to deny its being naturally a critical day; and to think that the instances of terminations which have really occurred on the fourth day, are to be reckoned among the other irregularities that happen in this matter.

CXXII. I have thus endeavoured to support the doctrine of critical days, chiefly upon the particular facts to be found in the writings of Hippocrates : and although I might also produce many other testimonies of both ancient and modern times, 
yet it must be owned, that some of these testimonies may be suspected to have arisen rather from a veneration of Hippocrates than from accurate observation.

CXXIII. With respect to the opinions of many moderns, who deny the prevalence of critical days, they are to be little regarded; for the observation of the course of continued fevers is known to be difficult and fallacious; and therefore the regularity of that course may have often escaped inattentive and prejudiced observers.

"Many of the physicians of modern times have that veneration for antiquity that they will not refuse these facts as delivered by Hippocrates, and therefore say, that they believe it to be true that such critical days occur in Greece; but they think that they do not happen with the same regularity in northern climates, especially now in the improved state of physic, when the power of our remedies is more capable of disturbing the course of nature. I maintain, however, that they do occur, though not in such a remarkable manner. I formerly gave you the reasons why the continued fever is more frequent in this country, and the intermittent less so ; but from the general tendency of the animal economy, there is no room to doubt that the power operates, and that the tendency subsists, though this is more obscure and undiscernible to us. In short, the chief argument that has arisen for refusing these days, is a negative one-that they are not observed. They have been observed formerly however. This, therefore, is no proof that they do not happen; their not being observed is owing to the physician, and not to any change in the state of nature.

"We have the authority of almost every eminent practitioner, from Hippocrates to Sydenham-the last of whom is as much an advocate for this doctrine as the first. Authorities, in matters of opinion, are of little value; but in matters of fact, as in this case, authorities are testimonies, and the testimonies with respect to these days in fevers are very numerous."

CXXIV. Our own observations amount to this, that fevers with moderate symptoms, generally cases of the synocha, frequently terminate in nine days, or sooner, and very constantly upon one or other of the critical days which fall within that 
period: but it is very rare in this climate that cases of either the typhus or synochus terminate before the eleventh day; and when they do terminate on this day, it is for the most part fatally. When they are protracted beyond this time, I have very constantly found that their terminations were upon the fourteenth, seventeenth, or twentieth day.

In such cases, the salutary terminations are seldom attended with any considerable evacuation. A sweating frequently appears, but is seldom considerable ; and I have hardly ever observed critical and decisive terminations attended with vomiting, evacuations by stool, or remarkable changes in the urine. The solution of the disease is chiefly to be discerned from some return of sleep and appetite, the ceasing of delirium, and an abatement of the frequency of the pulse. By these symptoms we can often mark a crisis of the disease; but it seldom happens suddenly and entirely: and it is most commonly from some favourable symptoms occurring upon one critical day, that we can announce a more entire solution upon the next following.

Upon the whole, I am persuaded, that if observations shall be made with attention, and without prejudice, I shall be allowed to conclude with the words of the learned and sagacious Gaubius, "Fallor, ni sua constiterit Hippocratr auctoritas, Galeno fides, Nature virtus et ordo."

"One general rule, both with regard to the event, and the duration of fevers, is taken from what has usually happened in like diseases formerly or for the time epidemic. This is the foundation of all observation, shewing the importance of every sign without regard to the reasoning a priori; and this the ancients understood as well or better than the moderns."

CHAP. VI.-OF THE METHOD OF CURE IN FEVERS.

"This is to be delivered as adapted to the genera; because the species are uncertain, because the supposed species may be only varieties, and because it is probable that the species are formed by combinations of the differences distinguishing the genera." 
Sect. I.-Of the Cure of Continued Fevers.

CXXV. As it is allowed, that in every fever which has its full course, there is an effort of nature of a salutary tendency, it might be supposed that the cure of fevers should be left to the operations of nature, or that our art should be only directed to support and regulate these operations, and that we should form our indications accordingly. This plan, however, I cannot adopt, because the operations of nature are very precarious, and not so well understood as to enable us to regulate them properly. It appears to me, that trusting to these operations has often given occasion to a negligent and inert practice; and there is reason to believe, that an attention to the operations of nature may be often superseded by art.

CXXVI. The plan which to me appears to be most suitable, is that which forms the indications of cure upon the view of obviating the tendency to death; while, at the same time, the means of executing these indications are directed by a proper attention to the proximate cause of fevers.

Upon this plan, in consequence of what has been laid down above on the subject of the prognostic, we form three general indications in the cure of continued fevers; and the one or other of these is to be employed according as the circumstances of the fever (CII.) shall direct.

The first, therefore is, to moderate the violence of reaction.

The second is, to remove the causes, or obviate the effects of debility. And,

The third is, to obviate or correct the tendency of the fluids to putrefaction.

CXXVII. The first indication may be answered, that is, the violence of reaction may be moderated,

1. By all those means which diminish the action of the heart and arteries.

2. By those means which take off the spasm of the extreme vessels, which we suppose to be the chief cause of violent reaction. 
CXXVIII. The action of the heart and arteriés may be diminished,

1. By avoiding or moderating those irritations, which, in one degree or other, are almost constantly applied to the body.

2. By the use of certain sedative powers.

3. By diminishing the tension and tone of the arterial system.

"6 The first title, then, is the means of avoiding irritation. By irritation I do not mean the various external, occasional, and, as I may say, accidental causes of irritation. I am not to speak of avoiding extremely warm and stimulating medicines, which belongs to another head, the preparatory indications; here we properly comprehend those irritations which occur from the ordinary business and actions of life, and which are in some degree unavoidably applied to the system. Our system is not a mere automaton, nor supported in its duration by any powers, whether of mind or body, subsisting within itself; it appears that we have constant need of some external assistance, of external impressions; and if these could be removed, we would not only certainly fall asleep, but we would very soon become dead. I have formerly said that the energy of the brain, that which is properly the vital substance, does depend upon certain other exercises and functions of the animal economy, and both together certainly depend upon the power of external impressions. In this respect, therefore, some degree of irritation applied to our system is unavoidable; but when it is attended with the excess of stimulant power in the case of fever, it is our business to render this unavoidable irritation as moderate as possible."

CXXIX. The irritations (CXXVIII. 1.) almost constantly applied, are the impressions made upon our senses, the exercise of the body and mind, and the taking in of aliments. The avoiding these as much as possible, or the moderating their force, constitutes what is rightly called the Antiphlogistic Regimen proper to be employed in almost every continued fever. "(A distinction ought to be made between Remedy and Regimen. Thus bleeding is an antiphlogistic remedy, but not a part of the antiphlogistic regimen.)"

CXXX. The conduct of this regimen is to be directed by the following rules and considerations. 
1. Impressions on the external senses, as being stimulant to the system, and a chief support of its activity, should be avoided as much as possible; those especially of more constant application, those of a stronger kind, and those which give pain and uneasiness.

No impression is to be more carefully guarded against than that of external heat; while, at the same time, every other means of increasing the heat of the body is to be shunned. Both these precautions are to be observed as soon as a hot stage is fully formed, and to be attended to during its continuance; excepting in certain cases, where a determination to sweating is necessary, or where the stimulant effects of heat may be compensated by circumstances which determine it to produce a relaxation and revulsion.

c. "Heat is the fundamental stimulus of our system; it is certainly the first vivifying power applied to it, and is necessary to a certain degree; and its influence through the whole of life shows its considerable powers, and that it may prove a stimulus indeed in any degree.

"The animal economy has a power within itself of generating heat to a certain determined degree which it always produces in ordinary circumstances, and which can neither be much diminished nor increased without some prejudice to the system. But we must always remember that the body unavoidably has a constant relation to the temperature of the atmosphere surrounding it. The heat of the body, therefore, is always in the ratio of the generating power within, and the external temperature. We find that in the temperature of $60^{\circ}$ to $62^{\circ} \mathrm{F}$. the proper heat of the body is preserved, so that one might go naked without feeling cold. But at a temperature lower than this, our heat would fall too low, if not preserved by means of clothes, houses, or other artificial heat. When, on the contrary, the external temperature is raised above that point, we have not so many means of obviating its effects, so that almost unavoidably the heat of the body is increased, especially when the change is made suddenly. But when the external heat is greater, the generating power must be diminished, and thus our due temperature is preserved.

"From all this it appears, that if in any case the power of vol. I. 
generating heat in the body is increased, the surrounding temperature ought to be somewhat lowered, in order to prevent a pernicious increase of heat. We shall afterwards consider to what degree external cold may in this case be applied; but when the reaction of the system has continued for some time, and when the hot fit is fully formed, the cold, which would have been pernicious at an earlier period, may now be a salutary, and even a necessary remedy.

- "It is to be observed, that the temperature of the air surrounding the body may be increased from various causes; and, moreover, the air remaining the same, that portion of it which is applied to the body, by remaining upon its surface, may accumulate the heat. The effects of the generating power, therefore, are different in proportion to the clothes which confine the heat produced by it, and in proportion to their being more or less proper for transmitting the heat to the external air. A garment, for instance, of linen, which transmits heat more readily, will not preserve the heat as well as the same weight of woollen. The covering, however, being given, the effects will be greater or less, as the effluvia from the body are more or less confined-as the effluvia are closed up, and the vapour not allowed to escape; or as, on the other hand, the clothes lie loose, and allow the heat and vapour to escape. Here, even the hand laid out, affords a spiraculum or outlet to the heat, which would otherwise be accumulated.

"There are two cases, however, which appear to be exceptions to this rule of avoiding external heat. The first of these is the application of remedies, which we shall mention hereafter, viz. warm bathing and warm fomentations; but such applications are allowable, because they are so conducted that they act more powerfully by relaxing the whole of the system, and by determining to the surface, than by heating the body. The other case happens when the determination to the surface, which is necessary for the solution of the fever, is already excited by the vis medicatrix natura, and where the flowing of the cutaneous secretion is to be supported by the application of warmth."

2. All motion of the body is to be avoided, especially that which requires the exercise of its own muscles; and that posture of the body is to be chosen which employs the fewest muscles, 
and which keeps none of them long in a state of contraction. Speaking, as it accelerates respiration, is particularly to be refrained from.

It is to be observed, that every motion of the body is the more stimulant in proportion as the body is weaker.

3. The exercise of the mind also is a stimulus to the body; - so that all impressions which lead to thought, and those especially which may excite emotion or passion, are to be carefully shunned.- " Not only impressions, but their consequences, sensation, thought, and emotions, are powerful stimuli, which commonly ought to be avoided. It will be obvious, that the exercise of thought proves more or less a stimulus to the system. It is next to impossible to suspend its operations; but we can avoid presenting such impressions as engage the attention, or draw necessarily into a train of thinking. If we cannot prevent thinking altogether, our next measure is to present such objects as require least attention, and lead least to any prosecution of thought.-These are seemingly small circumstances, but we must neglect none, and they are indeed of more consideration than may be readily imagined."

With respect to avoiding impressions of all kinds, an exception is to be made in the case of a delirium coming on, when the presenting of accustomed objects may have the effect of interrupting and diverting the irregular train of ideas then arising in the mind.- "We are disposed to fall into such a delirium when we are without the impression of external objects, as when we are in absolute darkness; and upon many other occasions, when our thoughts are liable to wander, the recalling them to usual and well accustomed objects interrupts the confused train. Frequently, when a delirium has come on, the patients having been kept in darkness and silence, they can be recovered by bringing them to the light, by a familiar voice speaking to them, and by presenting to them their more usual and accustomed objects. It must be left to the physician's discretion to judge of the circumstances when this is practicable; and when, on the contrary, objects exciting new and more forcible impressions may be dangerous in leading again to a train which increases the confusion."

4. The presence of recent aliment in the stomach always 
proves a stimulus to the system, and ought therefore to be as moderate as possible. A total abstinence for some time may be of service; but as this cannot be long continued with safety, we must avoid the stimulus of aliment by choosing that kind which gives the least. We suppose that alimentary matters are more stimulant, according as they are more alkalescent; and this leads to avoid all animal, and to use vegetable food only." Patients in fever are very rarely hurt by a very considerable abstinence. The ancients paid much attention to this subject, and carried abstinence farther than we do. For, if our patients retain their appetite, we are ready to indulge it; the ancients on the contrary enjoined a total abstinence during the three first days of fever. I entertain doubts, however, whether this is proper as a general rule in fevers, particularly in those which are attended with much debility in their advanced state, where it must be dangerous to lessen the vigour of the system so much at the commencement. If, therefore, the appetite remains, I would not think it very dangerous to gratify it in some measure; indeed, I think the appetite, if not gratified, might generate an uneasy impression which ought to be avoided; only I would make a proper choice in the quality of the food."

As our drinks also may prove stimulant, so all aromatic and spirituous liquors are to be avoided; and in answering the present indication, all fermented liquors, excepting those of the lowest quality, are to be abstained from._" There may be a particular exception to this rule, where custom has rendered the use of spirituous liquors habitual and in some measure necessary, some curious instances of which are on record, but they are rare and uncommon cases.

". In speaking of fermented liquors, we must constantly keep in view that they are to be avoided on account of their stimulus; hence, I doubt if it is necessary to forbid the use of those of a low quality, which form our common beverage. I believe that Sydenham was perfectly in the right in admitting the use of small beer."

CXXXI. Beside these stimulant powers more constantly applied, there are others which, although occasional only, yet, as commonly accompanying fevers, must be attended to and removed. 
One is, the sense of thirst, which, as a powerful stimulus, ought always, in one way or other, to be removed:

Another stimulus frequently arises from crudities, or corrupted humours in the stomach; and it is to be removed by vomiting, by dilution, or by the use of acids.- "The antiphlogistic regimen also implies the use of diluent fluids, which not only render all such acrimony inert, but promote the evacuation of the contents of the stomach in general. From this view, therefore, diluents are extremely proper, particularly when joined with acids, which will be considered under the head of sedative medicines."

A third stimulus often arises from the preternatural retention of frecs in the intestines; and ought to be removed by frequent laxative glysters._" The case of flatus broken downwards, shews the sense of distention over the whole system which commonly precedes, and the general relaxation which attends, their discharge. It is indeed difficult to explain the connexion of a small portion of the great guts with the whole system; but the fact is undoubtedly true, that the whole alimentary canal has a remarkable connexion with the whole nervous system. We can understand, therefore, to what the irritation of hardened freces will amount, and how necessary it is to avoid it. But the suppression of the usual secretions into the intestines in fever renders the fæces dry, and occasions costiveness. A part of the antiphlogistic regimen, therefore, consists in the frequent use of emollient and laxative glysters, or even stronger means for taking off this irritation."

A fourth stimulus to be constantly suspected in fevers, is a general acrimony of the fluids, as produced by the increase of motion and heat, joined with an interruption of the excretions. This acrimony is to be obviated or removed by the taking in of large quantities of mild antiseptic liquors.

CXXXII. The avoiding of irritation in all these particulars (CXXX. and CXXXI.) constitutes the antiphlogistic regimen absolutely necessary for moderating the violence of reaction : and, if I mistake not, is proper in almost every circumstance of continued fevers, because the propriety and safety of employing stimulants is often uncertain, and because several of 
those above mentioned, beside their stimulant powers, have other qualities by which they may be hurtful.

It appears to me that the supposed utility of stimulants, in certain cases of fever, has often arisen from a mistake in having ascribed to their stimulant, what really depended upon their antispasmodic power.

CXXXIII. A second head of the means (CXXVIII. 2.) for moderating the violence of reaction, comprehends certain sedative powers, which may be employed to diminish the activity of the whole body, and particularly that of the sanguiferous system.

The first of these to be mentioned is the application of cold.

Heat is the chief support of the activity of the animal system, which is therefore provided in itself with a power of generating heat; but, at the same time, we observe that this would go to excess, were it not constantly moderated by a cooler temperature in the surrounding atmosphere. When, therefore, that power of the system generating heat is increased, as is commonly the case in fevers, it is necessary not only to avoid all means of increasing it further, but it seems proper also to apply air of a cooler temperature, or at least to apply it more entirely and freely, than in a state of health.

Some late experiments in the smallpox, and in continued fevers, show that the free admission of cool air to the body is a powerful remedy in moderating the violence of reaction; but what is the mode of its operation, to what circumstances of fever it is peculiarly adapted, or what limitations it requires, I shall not venture to determine, till more particularly instructed by farther experience.- " A very singular observation has been made in modern times, that even cold bathing and washing the body all over with cold water is often a very useful remedy; and we are sure from facts, that the application of cold is not only a safe but a proper remedy."

CXXXIV, A second sedative power which may be employ'ed in fevers is that of certain medicines, known, in the writings on the Materia Medica, under the title of refrigerants.

The chief of these are acids of all kinds, when sufficiently diluted; and they are, in several respects, remedies adapted to 
continued fevers. Those especially in use are, the Vitriolic and Vegetable, and on many accounts we prefer the latter."It appears to me, that these refrigerants act very directly upon the arterial system, diminishing its activity, and thus the heat of the body also. The first and best known refrigerants are acid substances generally. Their sedative operation is indeed not easily explained, and I do not think it at all necessary to attempt any explanation, but their power is universally acknowledged by physicians, and they are generally used in hæmorrhagies and phlegmasix, where the heat of the system is increased in consequence either of increased reaction, or of particular changes in the state of the blood, in which case their effects are still more evident.

"I must, however, go still farther, and observe, that the acids have by experience been found the most powerful quenchers of thirst, which, indeed, occasions an especial appetite for acid drinks. They diminish the heat of the mouth and fauces, occasion some stimulus to the salivary and common secretions, and hence are useful in taking off both the heat and the dryness upon which thirst depends, and in obviating that clamminess of the mouth which so frequently arises in fever. Their operation extends still farther, to the stomach and intestines, where putrid matters are liable to abound: indeed we know nothing more immediately or strongly powerful in obviating that putrescency than the use of acids. They might even be supposed to exert their antiseptic power in the mass of blood as well as in the intestinal canal; but I doubt this antiseptic power, considering the small quantity in which they are taken, and their very general diffusion throughout the body. Acids have another power, however, in common with all saline substances, they are determined towards the several watery secretions, the perspiration and urine, and it is obvious that in this way they may be of considerable utility in fevers.

"I suppose, upon good grounds, that all these effects may be obtained by every kind of acid which is pure, and not combined with any other substance. Of the fossil acids, the vitriolic is now almost only employed; and I know no good objection against the nitric and muriatic acids.

"The vegetable acids, however, may be employed, perhaps, more properly and effectually than any of these fossil acids. 
We can throw them into the body in much larger proportion, and in consequence of this and of their suffering a more intimate mixture with our fluids, the vegetable acids are most powerful antisceptics. From their want of concentration they may fall short indeed in correcting putrescency in the primæ viæ; but as far as we want an alteration on the mass of the blood, it can be obtained by the vegetable acids only in large quantities. From their ready diffusion over the whole system they are the only acids which have been employed to promote sweating. The acid of vinegar has been so employed, and it is very favourable for promoting the determination to the surface which we so often desire to excite, and also the urinary secretion.

"It is proper here also to take notice of the native vegetable acid, as it occurs in acid and acescent fruits. These fruits combine the advantages of the acids, but may also be considered as alimentary, as entering into the composition of our fluids. They are moreover useful laxatives, and perhaps the only laxatives properly employed here, besides glysters, for obviating costiveness."

CXXXV. Another set of refrigerants are the Neutral Salts, formed of the vitriolic, nitrous, or vegetable acids, with alkalies, either fixed or volatile. All these neutrals, while they are dissolving in water, generate cold ; but as that cold ceases soon after the solution is finished, and as the salts are generally exhibited in a dissolved state, their refrigerant power in the animal body does not at all depend upon their power of generating cold with water. The neutral chiefly employed as a refrigerant is Nitre; but all the others, compounded as above mentioned, partake more or less of the same quality.

"Before I dismiss the consideration of acids and neutrals, I have a singular fact to mention. Both acids and neutrals are found, upon occasions, to irritate the lungs by being carried to the mucous glands of the bronchia and exciting cough. This is particularly observed from the muriatic acid, but also from the vegetable acid. Chenot observes, that he employed vinegar in large quantities in the plague, and that it was sometimes observed to have the effect of exciting cough. The fact is not so obvious with regard to neutrals, but from a.remarkable instance with which I have met, I cannot doubt of their general tendency also. I knew an asthmatic physician, who could not 
have the smallest quantity of nitre thrown in by way of glyster without a considerable irritation and uneasiness in the lungs. This gives a caution with respect to the use of both acids and neutrals in pulmonic affections."

CXXXVI. Besides these neutrals, some metallic salts also have been employed as refrigerants in fevers, and particularly the Sugar of Lead. But the refrigerant powers of this are not well ascertained, and its deleterious qualities are too well known to admit of its being freely used.

CXXXVII. Under the third general head (CXXVIII. 3.) of the means to be employed for moderating the violence of reaction, are comprehended the several means of diminishing the tension, tone, and activity of the sanguiferous system. As the activity of this system depends in a great measure upon the tone, and this again upon the tension of the vessels given to them by the quantity of fluids they contain (Physiology, p. 79.), it is evident that the diminution of the quantity of these must diminish the activity of the sanguiferous system.

CXXXVIII. The quantity of fluids contained in the sanguiferous system, may be diminished most conveniently by the evacuations of blood-letting and purging.

CXXXIX. Nothing is more evident, than that blood-letting is one of the most powerful means of diminishing the activity of the whole body, especially of the sanguiferous system, and it must therefore be the most effectual means of moderating the violence of reaction in fevers. Taking this as a fact, I omit inquiring into its mode of operation, and shall only consider in what circumstance of fevers it may be most properly employed.

CXL. When the violence of reaction, and its constant attendant, a phlogistic diathesis, are sufficiently manifest; when these constitute the principal part of the disease, and may be expected to continue throughout the whole of it, as in the cases of synoch $a$, then blood-letting is the principal remedy, and may be employed as far as the symptoms of the disease may seem to require, and the constitution of the patient will bear. It is; however, to be attended to, that a greater evacuation than is necessary may occasion a slower recovery, may render the person more liable to a relapse, or may bring on other diseases.

CXLI. In the case of synocha, therefore, there is little doubt 
about the propriety of blood-letting; but there are other species of fever, as the synochus, in which a violent reaction and phlogistic diathesis appear, and prevail during some part of the course of the disease; while, at the same time, these circumstances do not constitute the principal part of the disease, nor are to be expected to continue during the whole course of it; and it is well known, that, in many cases, the state of violent reaction is to be succeeded, sooner or later, by a state of debility, from the excess of which the danger of the disease is chiefly to arise. It is, therefore, necessary, that, in many cases, bloodletting should be avoided; and even though it may be proper during the inflammatory state of the disease, it will be necessary to take care that the evacuation be not so large as to increase the state of debility which is to follow.

CXLII. From all this it must appear, that the employing blood-letting, in certain fevers, requires much discermment and skill, and is to be governed by the consideration of the following circumstances :

1. The nature of the prevailing epidemic.- " If we know that a contagion prevails which forms a fever of a particular character, suppose that which we call of the nervous kind or typhus, then, even if it first appears like a synocha, I have less regard to the inflammatory symptoms, though they be strongly marked; as I know that they may soon disappear and be succeeded by the symptoms of debility. Sir John Pringle found, that one bleeding in the beginning of the jail fever was safe and attended with advantage; but the repetition certainly was extremely dangerous. Our knowledge, therefore, of the nature and tendency of the prevailing epidemic will determine our practice in bleeding."

2. The nature of the remote cause.- "When we know of no contagious fever prevailing, and have direct evidence of the operation of cold, bleeding is the proper remedy ; but where a contagion is present, it is to be employed with caution."

3. The season and climate in which the disease occurs." The inhabitants of northern climates sustain bleeding better than those of the southern; even those who are exposed to very cold winters, if they are also exposed to the vicissitude of very warm summers, do not bear bleeding so well as those who have 
cold winters and never warm summers. I learned from many practitioners, that in the late war in America the Europeans bore bleeding much better than the Americans. The winter and spring seasons chiefly produce inflammatory diseases, and we may therefore allow of blood-letting with more freedom from the winter to the summer solstice, than in the other half of the year. 6 A difficulty however occurs here: Dr. Cleghorn who practised in a warm climate, at Minorca, seems to have been extremely free in the use of the lancet, and the diseases bore bleeding better than might have been anticipated. But the cases were chiefly purely inflammatory, such as pleurisies and peripneumonies, and they occurred among soldiers from a northern climate. Practitioners in the West Indies accordingly also find, that people lately come from Europe will bear bleeding better than those who have for some time inhabited the warm regions."

4. The degree of phlogistic diathesis present.—" Where the pulse is full, hard, and quick, where the heat of the body is considerably increased, where there are most of the marks of turgescence, as a fulness of the countenance, a redness of the face, and a sense of distention in some measure over the whole body; but more especially where along with these symptoms there are marks of topical determinations, where there are fixed pains in other parts of the body different from those which accompany fever more generally, such as, for instance, the headach, which is not decisive, or a pain in the small of the back, which appears even in the typhus, or a general complaint of pains in the limbs or over all the body, which also are not always sufficient to establish the inflammatory state of the system; if these pains are, on the contrary, any where fixed about the thorax, or even occupy one particular joint of the body, or are attended with the circumstance of some tumor more distinctly and evidently of the rheumatic kind, these are circumstances which indicate venesection; and if they are accompanied with catarrhal symptoms, as a frequent, violent, and bound cough, they are still more distinctly the marks of synocha or inflammatory fever; and whatever be the nature of the fever, I would say, that so long as these symp- 
toms taken altogether, or the most part of them are present, venesection is strongly indicated."

5. The period of the disease.- "I have sufficiently explained why the synochus is the most common form of fever in this and in many other countries, that is to say, why our fever has more or less of the inflammatory nature in the beginning, so that venesection may be employed, but afterwards assumes more of the nervous character, with more of debility, when venesection is employed with more danger. The ancients were very nice in this matter, and in general they seldom admitted of venesection after the fourth day. A late writer in a treatise de vence sectione in acutis, \&c. shows that indeed such was the general rule, but at the same time attempts to show, very properly, that it is not to be limited by the number of days, and that we are rather to be guided by other circumstances, and particularly by the state of the symptoms. The ancients, even in inflammatory cases, as in peripneumony and pleurisy, did not bleed after the fourth day; and we say that venesection during the three or four first days, is especially effectual; but there are circumstances in which witlout all doubt venesection may be continued, while there are not evident symptoms of a tendency to suppuration or gangrene. But where we have no topical phlegmasiæ present, the difficulty is greater, and, in general, I have no doubt in agreeing with the ancients that venesection is only safe in the beginning, and that the danger is greater as the disease advances. But there is a great difference in the duration of the inflammatory state in the synochus, and there are instances of the good effects of venesection in the more advanced stages. The inflammatory symptoms which for some time, perhaps, were not very considerable, from certain accidents, sometimes come on with fresh vigour, when venesection may be the only remedy. It would therefore be extremely dangerous to establish any general rule; only we are sure that, as the disease advances, venesection is to be employed with more caution, and may be attended with more danger; but from several instances which I have met with, where we were obliged to swerve from the general rule, when inflammatory symptoms happened to recur with a hardness and tension of the 
pulse, and where venesection was practised with very evident relief, I am clearly of opinion that this matter is to be studied more anxiously than it has hitherto been done."

6. The age, vigour, and plethoric state of the patient.\$6 The fulness and vigour of the constitution may not be always distinctly marked, but we may presume its existence when the patient is young. The plethoric state of the system generally prevails from the age of puberty to that of manhood, commonly from fourteen to thirty-five years. In the course of these years a variety may again occur, as the solids increase in firmness and solidity; and I believe, that the inflammatory tendency is most frequent from twenty-one to thirty. The judgment from age, however, is not to be solely trusted to, as many persons even at that age are of a weak, relaxed habit."

7. The patient's former diseases and habits of blood-letting."There are constitutions of a peculiarly inflammatory tendency, which are exposed to Angina, to rheumatic and to peripneumonic affections; and although such symptoms do not evidently appear, if they have frequently occurred formerly, this must determine us to blood-letting. Another remarkable constitution is the hæmorrhagic. Thus, if a person has been frequently liable to bleeding at the nose, especially after the age of puberty, he will bear blood-letting better, and it will be the more necessary."

8. The appearance of the blood drawn out._" There is nothing in which physicians are more rash and presumptuous, than in ascertaining the condition of the blood from its appearance when extravasated. In one case the blood forms entirely one crassamentum, and is therefore easily in danger of obstructing every vessel of the body; in other cases they find various degrees of density in the crassamentum, and various proportions of serum, which gives occasion to the like sort of judgment. But we know now that these reasonings are fallacious, and that there is a great variety of circumstances from the condition of the orifice in the vein, the manner in which the blood flows, and the temperature of the air, and that the appearance is very much regulated by the vessels in which the blood is received. In the Royal Infirmary, the use of tin plates gives occasion to a particular appearance of the blood, which does not occur in private 
practice, where we generally employ porcelain cups. I hare frequently observed, that in the plates in this house, the size of the blood appears very considerable with respect to its thickness, and that it is expanded even over the whole of the surface; while in the porcelain cups it is generally contracted into an insula, forming a sort of hollow cup upon the surface of the crassamentum; and upon turning the whole mass from the tin plate, very little separate serum appears, whereas in the porcelain cups the serum is generally in considerable proportion.

" Many practitioners are too rash in concluding the absence of a phlogistic diathesis, when the blood shews no inflammatory crust, and on this account do not proceed in blood-letting: but this negative is of no weight at all, as it may depend on some of the circumstances in drawing the blood already mentioned; and it is therefore dangerous to conclude against the existence of a phlogistic diathesis from the absence of the crust. Where it is present, it determines us in favour of the repetition; but even this is not always certain ; for, even in fevers of the nervous kind, the inflammatory crust may be distinctly marked, and we are in danger of being led astray by it."

9. The effects of the blood-letting that may have been already practised.- " If the pulse is manifestly contracted and small in persons whose habit and time of life should allow of one more full; and if the venesection seems to strengthen it, as it by relaxing the system allows the pulse to become fuller and to appear stronger, we consider this as a proof of the propriety of the bleeding which we have practised, and repeat it with confidence.

" There is a question occurring in practice, which is indeed the most difficult, viz. when, in the advanced state of fevers, we have symptoms of topical determination, and at the same time there is a very general debility prevailing in the system, as in the case of typhus; when there are sometimes topical determinations to internal parts, particularly to the brain, which end in death; and when, with all the symptoms of debility, we find that actual inflammation and suppuration has taken place in the brain; when this appears in the advanced state of typhus, and when no so sort of irritation, not even the smallest quantity of wine, can be applied without ag- 
gravating the symptoms; and when, from the redness of the eye, the delirium, and other circumstances, these topical determinations are to be apprehended. It is a question of great difficulty also when such determinations appear to be in the lungs or other viscera; and the difficulty arises very much from hence, that these topical determinations do not appear distinctly marked, because though they have all the effects of inflammation in the particular part, yet the irritation is not communicated to the rest of the system, which renders the observation difficult; the general venesection has very little effect in removing these symptoms, and by having recourse to it, we increase the general debility without affecting the particular part. All this leads to the conclusion, that, while perhaps there is no other certain remedy but blood-letting, topical blood-lettings, which can act on the particular part affected, are the only ones admissible. Thus, a little scarification of the vessels of the eyes themselves, or a leech or two applied near them will have more effect than two pounds of blood taken from the arm in the general way.

"But even these topical venesections, if they are large, may produce considerable effects upon the whole system, so that even these are not to be admitted but with moderation, and where they can be directed so as to influence the vessels of the part affected; so topical blood-lettings are more useful in cases of topical determinations to the brain, than in the case of such a determination to the lungs or abdominal viscera. At the same time I would not neglect it, or exclude it in these last cases, especially as they are seldom accompanied with the same symptoms of debility, so that our cupping and scarifying may be practised with safety and advantage."

CXLIII. When, after the consideration of these circumstances, blood-letting is determined to be necessary, it should be observed, that it is more effectual according as the blood is more suddenly drawn off, and as the body is, at the same time, more free from all irritation, and, consequently, when in a posture in which the fewest of the muscles are in action.

"Before I dismiss this subject, I must advert to a difficulty which arises with respect to the practice of blood-letting at different times of the paroxysms of both intermittent and continued 
fever. While the cold fit exists, venesection, by weakening the system, increases the spasm which lays the foundation of the disease. We have been so much accustomed to inflammatory fevers, in which we universally have recourse to bleeding as a remedy, that this caution has been very much neglected; but I have often observed bleeding indiscreetly employed. Our fevers do not come to the hot fit for several days; and so long as the shiverings recur, I hold it dangerous to admit of bleeding. ' In ipso impetu sanguinem mittere hominem jugulare est,' says Celsus. His observation applies especially to intermittent fevers; but we can mark, in the first two days of continued fevers also, that a little after noon, and again towards the evening, when if not a cold fit, at least something analogous to it may be observed; and at these times, as long as the hot stage of the fever, or the increased reaction, has not entirely taken place, we should abstain from bleeding."

CXLIV. Another evacuation whereby the quantity of fluids contained in the body can be considerably diminished, is that of Purging.

CXLV. If we consider the quantity of fluids constantly present in the cavity of the intestines, and the quantity which may be drawn from the innumerable excretories that open into this cavity, it will be obvious that a very great evacuation can be made by purging; and if this be done by a stimulus applied to the intestines, without being at the same time communicated to the rest of the body, it may, by emptying both the cavity of the intestines, and the arteries which furnish the excretions poured into it, induce a considerable relaxation in the whole system; and therefore purging seems to be a remedy suited to moderate the violence of reaction in fevers.

CXLVI. But it is to be observed, that as the fluid drawn from the excretories opening into the intestines, is not all drawn immediately from the arteries, as a part of it is drawn from the mucous follicles only, and as what is even more immediately drawn from the arteries is drawn off slowly; so the evacuation will not, in proportion to its quantity, occasion such a sudden depletion of the red vessels as blood-letting does; and therefore cannot operate so powerfully in taking off the phlogistic diathesis of the system. 
CXLVII. At the same time, as this evacuation may induce a considerable degree of debility, so in those cases in which a dangerous state of debility is likely to occur, purging is to be employed with a great deal of caution, and more especially as the due measure of the evacuation is more difficult to be applied than in the case of blood-letting.

CXLVIII. As we shall presently have occasion to observe that it is of great importance in the cure of fevers, to restore the determination of the blood to the vessels on the surface of the body, so purging, as in some measure taking off that determination, seems to be an evacuation not well adapted to the cure of fevers.

CXLIX. If, notwithstanding these doubts (CXLVI. CXLVII. and CXLVIII.), it shall be asserted, that purging, even from the exhibition of purgatives, has often been useful in fevers, I would beg leave to maintain that this has not happened from a large evacuation, and therefore, not by moderating the violence of reaction, excepting in the case of a more purely inflammatory fever, or of exanthemata of an inflammatory nature. In other cases of fever, I have seen a large evacuation by purging, of mischievous consequence ; and if, upon occasion, a more moderate evacuation has appeared to be useful, it is apprehended to have been only by taking off the irritation of retained frecs, or by evacuating corrupted humours which happened to be present in the intestines, for both of which purposes frequent laxatives may be properly employed.

"I explain myself; the cases are of two kinds: first, where the contents of the stomach and intestines are manifestly copious, there having been a retention or suppression of the alvine excretion for some time; and secondly, where we have reason to suspect that the contents are acrid, which we presume not only from a want of evacuations, but also from the symptoms of the stomach in the fevers called Bilious, from their being attended with an increased effusion of bile. In the latter case, the evacuation by stool is indicated, because it is the natural course of the bile. Sometimes the bile may not appear very copious either upwards or downwards, and the actual effusion of it into the intestines is not evident, but a considerable determination of blood towards the abdominal viscera appears, in consequence of which congestions

vol. I.

$2 \times$ 
and accumulations are formed in these viscera. Such are especially to be relieved by opening the excretories into the intestines which immediately communicate with the affected viscera. I think I have formerly (LI.) sufficiently explained, that in most intermittents, especially the autumnal, this determination to the abdominal viscera, particularly to the hypochondriac system, the liver and spleen occurs; and certainly nothing can more effectually obviate these congestions than opening the excretories of the intestines. The indication of opening the bowels accordingly arises in all fevers which are bilious, or of the remittent kind, where we know that purging is required. I may farther observe that bilious and autumnal fevers are often of the putrid kind, and accompanied with petechiæ; in that case purging is not only useful, but in a great measure necessary; for in these diseases especially the contents of the intestines have a tendency to putrefaction. I do not apply this to the bile only, but in a particular manner to the reliquiæ of the aliment, and to all the fluids contained in the intestines."

CL. Another set of means (CXXVII. 2.) for moderating the violence of reaction in fevers, are those suited to take off the spasm of the extreme vessels, which we believe to be the irritation that chiefly supports the reaction.

Though I have put here this indication of taking off the spasm of the extreme vessels, as subordinate to the general indication of moderating the violence of reaction, it is however to be observed here, that as fever universally consists in an increased action of the heart, either in frequency or in force, which in either case is supported by a spasm of the extreme vessels, so the indication for removing this is a very general one, and applicable in almost every circumstance of fever, or at least with a few exceptions, to be taken notice of hereafter.

CLI. For taking off the spasm of the extreme vessels, the means to be employed are either internal or external.

CLII. The internal means (CLI.) are,

1. Those which determine the force of the circulation to the extreme vessels on the surface of the body, and, by restoring the tone and activity of these vessels, may overcome the spasm on their extremities.

2. Those medicines which have the power of taking off spasm 
in any part of the system, and which are known under the title of Antispasmodics.

CLIII. Those remedies which are fit to determine to the surface of the body, are,

1. Diluents.

2. Neutral Salts.

3. SUDorifics.

4. Emetics.

CLIV. Water enters, in a large proportion, into the composition of all the animal fluids, and a large quantity of it is always diffused through the whole of the common mass. Indeed, in a sound state, the fluidity of the whole mass depends upon the quantity of water present in it. Water, therefore, is the proper diluent of our mass of blood; and other fluids are diluent only in proportion to the quantity of water they contain.

"6 We may seek for various impregnations of water with a view to dilution, but the chief matter always is water in a large proportion; it is a substance especially fitted for dilution, being most bland and fluid, entering into the smallest vessels of our system, filling and dilating them, and thereby exciting their action."

CLV. Water may be said to be the vehicle of the several matters which ought to be excerned; and in a healthy state the fulness of the extreme vessels, and the quantity of excretions, are nearly in proportion to the quantity of water present in the body. In fever, however, although the excretions are in some measure interrupted, they continue in such quantity as to exhale the more fluid parts of the blood; and while a portion of them is, at the same time, necessarily retained in the larger vessels, the smaller and the extreme vessels, both from the deficiency of fluid and their own contracted state, are less filled, and therefore allowed to remain in that condition.

CLVI. To remedy this contracted state, nothing is more necessary than a large supply of water, or watery fluids, taken in by drinking, or otherwise ; for as any superfluous quantity of water is forced off by the several excretories, such a force applied may be a means of dilating the extreme vessels, and of overcoming the spasm affecting their extremities.

CLVII. Accordingly the throwing in of a large quantity of 
watery fluids has been at all times a remedy much employed in fevers; and in no instance more remarkably than by the Spanish and Italian physicians, in the use of what they call the diceta aquea.

"This was first thought of in Spain, from thence transported to Naples, and has been practised in Italy for many years. There is an account of it in the notes of Cirillo, a Neapolitan physician, to Etmuller's works (Genev. 1736. 4 tom. fol.). The practice has not been confined to Italy, but has extended to France; and it is a favourite remedy of Senac (de recond. feb. nat. p. 254.").

CLVIII. This practice consists in taking away every other kind of aliment and drink, and in giving in divided portions every day, for several days together, six or eight pounds of plain water, generally cold, but sometimes warm. All this, however, is to be done only after the disease has continued for some time, and at least for a week.

CLIX. A second means (CLIII. 2.) of determining to the surface of the body, is by the use of neutral salts. These, in a certain dose taken into the stomach, produce soon after a sense of heat upon the surface of the body; and, if the body be covered close and kept warm, a sweat is readily brought out. The same medicines, taken during the cold stage of a fever, very often put an end to the cold stage and bring on the hot; and they are also remarkable for stopping the vomiting which so frequently attends the cold stage of fevers. All this shows that neutral salts have a power of determining the blood to the surface of the body, and may therefore be of use in taking off the spasm which in fevers subsists there.

CLX. The neutral most commonly employed in fevers, is that formed of an alkali with the native acid of vegetables: but all the other neutrals have more or less of the same virtue; and perhaps some of them, particularly the ammoniacal salts, possess it in a stronger degree.

CLXI. As cold water taken into the stomach often shows the same diaphoretic effects with the neutral salts, it is probable that the effect of the latter depends upon their refrigerant powers mentioned above (CXXXIV.). What is the effect of the neutral salts, given when they are forming, and in a state of 
effervescence? It is probable that this circumstance may increase the refrigerant power of these salts, and may introduce into the body a quantity of fixed air; but for these purposes it would seem proper to contrive that the whole of the effervescence should take place in the stomach.

CLXII. A third means (CLIII. 3.) of determining to the surface of the body, and taking off the spasm subsisting there, is by the use of sudorific medicines, and of sweating.

CLXIII. The propriety of this remedy has been much disputed; and specious arguments may be adduced both for and against the practice.

In favour of the practice, it may be said,

1. That in healthy persons, in every case of increased action of the heart and arteries, a sweating takes place, and is seemingly the means of preventing the bad effects of such increased action.

2. That, in fevers, their most usual solution and termination is by spontaneous sweating.

3. That, even when excited by art, it has been found manifestly useful, at certain periods, and in certain species of fever.

CLXIV. Upon the other hand, it may be urged against the practice of sweating,

1. That as in fevers a spontaneous sweating does not immediately come on, so there must be in these some circumstances different from those in the state of health, and which may therefore render it doubtful whether the sweating can be safely excited by art.

2. That, in many cases, the practice has been attended with bad consequences. The means commonly employed have a tendency to produce an inflammatory diathesis; which, if not taken off by the sweat following their use, must be increased with much danger. Thus, sweating employed to prevent the accession of intermitting fevers, has often changed them into a continued form, which is always dangerous.

3. The utility of the practice is further doubtful, because sweating, when it happens, does not always give a final determination; as must be manifest in the case of intermittents, as well as in many continued fevers, which are sometimes in the 
beginning attended with sweatings that do not prove final; and, on the contrary, whether spontaneous or excited by art, seem often to aggravate the disease.

CLXV. From these considerations, it is extremely doubtful if the practice of sweating can be admitted very generally ; but, at the same time, it is also doubtful, if the failure of the practice, or the mischiefs said to have arisen from it, have not been owing to the improper conduct of the practitioner.

With respect to this last, it is almost agreed among physicians,

1. That sweating has been generally hurtful when excited by stimulant, heating, and inflammatory medicines; " by the force of such medicines as have the power of inflaming the particular part to which they are applied, from which we know their inflammatory nature. Such medicines, about one hundred and fifty years ago, were employed by the Chemists and Cartesians, under the title of Alexipharmics; and it was this very practice that Sydenham took so much pains to explode, in banishing the use of these heating medicines, from which some persons certainly did escape; but to use the expression we have in scripture, they escaped कंs dic̀ $\pi$ vẹ̀s, purged as by fire."

2. That it has been hurtful when excited by much external heat, and continued with a great increase of the heat of the body.

3. That it is always hurtful when it does not soon relieve, but rather increases the frequency and hardness of the pulse, the anxiety and difficulty of breathing, the headach, and delirium.

4. That it is always hurtful if it be urged when the sweat is not fluid, and when it is partial, and on the superior parts of the body only.

CLXVI. In these cases it is probable, that either an inflammatory diathesis is produced, which increases the spasm on the extreme vessels, or that, from other causes, the spasm is too much fixed to yield easily to the increased action of the heart and arteries; and, upon either supposition, it must be obvious, that urging the sweat, as ready to produce a hurtful determination to some of the internal parts, may be attended with very great danger. 
CLXVII. Though the doubts started (CLXIV.) are to be attended to, and although the practices (CLXV.) having been found hurtful, are therefore to be rejected, it still remains true,

1. That sweating has certainly often been useful in preventing the accession of fevers, when the times of this have been certainly foreseen, and a proper conduct employed.

2. That, even after fevers have in some measure come on, sweating, when properly employed, either at the very beginning of the disease, or during its approach and gradual formation, has often prevented their further progress.

3. That, even after pyrexiæ have continued for some time, sweating has been successfully employed in curing them, as particularly in the case of rheumatism.

4. That certain fevers, produced by a very powerful sedative contagion, have been generally treated, so far as we yet know, most successfully by sweating.

CLXVIII. These instances (CLXVII.) are in favour of sweating, but give no general rule, and it must be left to further experience to determine how far any general rule can be established in this matter. In the mean time, if the practice of sweating is to be attempted, we can venture to lay down the following rules for the conduct of it :

1. That it should be excited without the use of stimulant inflammatory medicines.

2. That it should be excited with as little external heat, and with as little increase of the heat of the body, as possible.

3. That, when excited, it should be continued for a due length of time, not less than twelve hours, and sometimes for twentyfour or forty-eight hours; always, however, providing that it proceeds without the circumstances mentioned (CLXV. 3. 4.).

4. That for some part of the time, and as long as the person can easily bear, it should be carried on without admitting of sleep.

5. That it should be rendered universal over the whole body; and therefore, particularly, that care be taken to bring the sweating to the lower extremities.

6. That the practice should be rendered safer by moderate purging, excited at the same time.

7. That it should not be suddenly checked by cold any how 
applied to the body._- "This is rendered difficult when the patients lie in linens, which are indeed sufficiently favourable for sweating but liable to become very cold upon being removed a little from the body. We must, therefore, avoid that cold moist linen be again applied to the surface of the body; nay, this consideration renders it more proper to give the patient a flannel shirt, or to put him into blankets only. If, however, he will not submit to lie in wool, we must obviate the inconvenience in any remission of the sweating by shifting the patient entirely. There has been much fear from the change of linen in fevers in general; but it may be practised with the utmost safety if the linen is warm and sufficiently dry."

CLXIX. When attention is to be given to these rules, the sweating may be excited, 1. By warm bathing, or a fomentation of the lower extremities. 2. By frequent draughts of tepid liquors, chiefly water, rendered more grateful by the addition of a light aromatic, or more powerful by that of a small quantity of wine. 3. By giving some doses of neutral salts. 4. Most effectually, and perhaps most safely, by a large dose of an opiate, joined with a portion of neutral salts, and of an emetic.

In what cases may cold water, thrown into the stomach in large quantities, be employed to excite sweating ?-(See Celsus, lib. iii. cap. vii.-ix.)

CLXX. The fourth means (CLIII. 4.) of determining to the surface of the body, and thereby taking off the spasm affecting the extreme vessels, is by the use of emetics.

CLXXI. Emetics, and particularly antimonial emetics, have been employed in the cure of fevers ever since the introduction of chemical medicines; but, for a long time, they were employed by chemists and chemical practitioners only; and although of late the use of them has become very general, their efficacy is still disputed, and their manner of operating is not commonly explained.

"Emetics, as remédies in fevers, make a very great figure in our present mode of practice; and their use is now become so general among the most eminent practitioners in all the countries of Europe, that either we can have no doubt of their efficacy, or the whole depends upon fashion.

"I am ready to acknowledge, however, that their effects are 
not so remarkable as might be wished, or as might have been expected, from the first account of James's powder. Nay, this goes so far, the effects are so seldom remarkable, that I know practitioners of some rank who pretend to say that they have never observed their good effects, and are willing to suppose that it is more from fashion than from any real foundation that they are so generally employed.

"Such negative observations, indeed, have no great weight with me. I, for my own part, in many instances, have seen their effects very evident, and very remarkable, in giving a decisive turn to the state of the fever; in many other instances they give some relief; though they do not put an end to the fever, they make matters go on with more safety to the usual time of the crisis ; and there is with me no doubt of their being remedies of the most useful kind."

CLXXII. Vomiting is in many respects useful in fevers; as it evacuates the contents of the stomach; as it emulges the biliary and pancreatic ducts; as it evacuates the contents of the duodenum, and perhaps also of a larger portion of the intestines; as it agitates the whole of the abdominal viscera, expedes the circulation in them, and promotes their several secretions; and lastly, as agitating also the viscera of the thorax, it has like effects there. All these several effects are, in many cases and circumstances of fever, procured with advantage; but do not properly fall under our view here, where we are to consider only the effect of vomiting in determining to the surface of the body.

CLXXIII. This effect we do not impute to the exercise of vomiting in agitating the whole frame, but to the particular operation of emetics upon the muscular fibres of the stomach, whereby they excite the action of the extreme arteries on the surface of the body, so as thereby effectually to determine the blood into these vessels, remove the atony, and take off the spasm affecting them.

CLXXIV. That such is the power of emetics will appear from the several considerations mentioned above (XLIV.); and, therefore, that they are remedies well suited to the cure of fevers.

CLXXV. Emetics, for that purpose, are administered in two 
different ways: that is, either in such doses as may excite full and repeated vomitings, or in such doses as may excite sickness and nausea only, with little or no vomiting at all.

CLXXVI. Full vomiting is best suited to the several purposes mentioned (CLXXII.); and is also well suited to determine to the surface of the body, so as thereby to obviate the atony and spasm which lay the foundation of fever. Thus vomiting, excited a little before the expected accession of the paroxysm of an intermittent, has been found to prevent the paroxysm altogether. And it has been observed also, that, when contagion has been applied to a person, and first discovers its operation, a vomit will prevent the fever, which was otherwise to have been expected. (See Lind on Fevers and Infection.)

CLXXVII. These are advantages to be obtained by exciting vomiting at the first approach of fevers, or of the paroxysms of fevers; and after fevers are formed, vomiting may also be employed to take off, perhaps entirely, the atony and spasm, or at least to moderate these, so that the fever may proceed more gently and safely.

CLXXVIII. It is seldom, however, that vomiting is found to produce a final solution of fevers; and, after they are once formed, it is commonly necessary to repeat the vomiting several times; but this is attended with inconvenience, and sometimes with disadvantage.

"If either the stomach itself or any of the contiguous abdominal viscera have a tendency to the inflammatory state, the exercise of vomiting must be a considerable aggravation of this ; and upon this is founded the prejudice against emetics. However, I agree with several practitioners in observing, that though this inflammatory state may sometimes occur, it commonly does so in the more advanced state of fevers, and that in the beginning it is a much more rare occurrence than has been imagined:

"Another objection may arise from the consideration of those congestions that may be formed in the several abdominal viscera, as in the liver and spleen particularly; for, by increasing the impetus of their vessels in vomiting, we might perhaps do harm, unless the emulgent effects compensate for that increased action. I have said indeed how emetics are fitted to this, but 
in many cases it may be uncertain, or not in the due proportion; and it is indeed necessary that it should be effectually executed, which is to be obtained either by directing the operations of the emetic to be at the same time purgative, or by its being accompanied with some purgative medicine at the same time."

The operation of full vomiting commonly soon ceases, and the exercise of vomiting is often a debilitating power ; and therefore when the vomiting does not remove the atony and spasm very entirely, it may give occasion to their recurring with greater force.

CLXXIX. For these reasons, after fevers are fully formed, physicians have thought proper to employ emetics in nauseating doses only. These are capable of exciting the action of the extreme vessels, and their operation is more permanent. At the same time, they often shew their power by exciting some degree of sweat, and their operation is rendered more safe by their commonly producing some evacuation by stool.

CLXXX. Such are the advantages to be procured by nauseating doses of emetics; and it only remains to mention, what are the medicines most fit to be employed in that manner, what are the most proper times for exhibiting, and what is the best manner of administering them.

CLXXXI. The emetics at present chiefly in use, are Ipecacuanha and Antimony.

The former may be employed for every purpose of emetics, particularly those mentioned (CLXXII.). It may likewise be employed, either in larger or smaller doses, for determining to the surface of the body; but even in very small doses, it so readily excites vomiting, as to be with difficulty employed for the purpose of nauseating only ; and however employed, there is reason to believe that its effects are less permanent, and less powerfully communicated from the stomach to the rest of the system than those of antimony.

CLXXXII. This, therefore, is generally preferred; and its preparations, seemingly various, may all be referred to two heads; the one comprehending those in which the reguline part is in a condition to be acted upon by acids; and therefore on meeting with acids in the stomach becomes active; and the 
other, comprehending those preparations in which the reguline part is already joined with an acid rendering it active.

CLXXXIII. Of each kind there are great numbers, but not differing essentially from one another. It will be enough for us to compare the Calx Antimonii Nitrata (Oxyd. Antim. cum Phosphate Calcis) of the Edinburgh Dispenșatory, with the Emetic tartar of the same. The former, as I judge, is nearly the same with what is called James's Powder. Which of these is best suited to the cure of fevers, as above explained, seems doubtful; but it appears to me that although the former may have some advantages from its slower operation, and may thereby seem to be more certainly sudorific and purgative, yet the uncertainty of its dose renders it inconvenient, and has often given occasion to the timid to be disappointed and to the bold to do mischief. On the other hand, the dose of the emetic tartar can be exactly ascertained; and I think it may be exhibited in such a manner as to produce all the advantages of the other.

CLXXXIV. Whichsoever of these preparations be employed, I judge the most proper time for exhibiting them to be the time of accessions; or a little before, when that can be certainly known. In continued fevers, the exacerbations are not always very observable; but there is reason to think that one commonly happens about noon, or soon after it, and another in the evening; and that these, therefore, are the most proper times for exhibiting emetics.

CLXXXV. With respect to the manner of administration, that of the Calx Nitrata is simple, as the whole of what is judged a proper dose is given at once, and no more can properly be given till the time of the next accession.

The administration of the emetic tartar is different. It is to be given in small doses not sufficient to excite vomiting; and these doses, after short intervals, are to be repeated for several times, till sickness, nausea, and some, but not much, vomiting come on. The difference of this administration must depend upon the dose, and the length of the intervals at which it is given. If it be intended that the medicine should certainly operate by stool, the doses are made small, and the intervals long. On the contrary, when vomiting is proper, or when much 
purging ought to be avoided, and therefore some vomiting must be admitted, the doses are made larger, and the intervals shorter.

CLXXXVI. With respect to both kinds of preparations, the repetition is to be made at the times of accession, but not very often: for if the first exhibitions, duly managed, have little effect, it is seldom that the after exhibitions have much; and it sometimes happens that the repeated vomitings, and especially repeated purgings, do harm by weakening the patient.

CLXXXVII. The other set of internal medicines (CLII. 2.), which I suppose may be useful in taking off the spasm of the extreme vessels, are those named Antispasmodic. How many of these may be properly employed, I am uncertain; and their mode of operation is involved in great obscurity. It is certain, however, that opium, camphor, musk, and perhaps some others, have been employed in fevers with advantage; but the circumstances in which they are especially proper and safe, I find difficult to ascertain, and therefore cannot venture here to lay down any general doctrine concerning them.

"Here, I say, my doubts and difficulties arise more considerably than with respect to any other part of my subject. The operation of the medicines we call Antispasmodics, is by no means yet fully explained. I can, in many cases, observe that the antispasmodics are truly sedative medicines; in other cases I can observe that they prove antispasmodic by means of a stimulus; and there are other cases again in which I can apply neither of these views, and, I own, it must be supposed, that there is in some medicines somewhat that we may call specifically antispasmodic, which is owing neither clearly to their sedative nor to their stimulant power, or at least to some peculiar combination of these, and that therefore they are truly antispasmodic. Our theory upon this subject is somewhat doubtful, and therefore we may be at a loss where to give them a place in this consideration of the remedies of fever ; but it is certain that they are remedies in fever, and several of them are means of removing the spasm which takes place in it."

Opium, as a stimulant to the heart and arteries, may be considered as a principal remedy in fevers, and as such we are 
disposed with the most part of our present practitioners to consider it. But that it is universally such, and, in every circumstance of fever, proper, we are very far from thinking.

In very many of the fevers of this climate, there appears in the beginning to be more or less of the inflammatory diathesis in the system; and, during this state, $I$ hold, and have often seen the use of opium to be hurtful. It does not then either induce sleep or relieve pain, but aggravates the inflammatory symptoms, and often determines to particular inflammations, which prove afterwards fatal.

In different fevers this inflammatory state is in different degrees, and of different duration. In some cases of the most powerful contagions it may appear very little, and such cases may very possibly admit the early use of opium; but, in most of the cases in which cold so frequently concurs in the production of the fever, we suppose that such inflammatory state commonly subsists for the first week of the disease ; and, therefore, for that space of time, we hold opium to be a dangerous, and at least an ambiguous remedy. $-M . M$.

" The principal writer upon this subject of opium, is Dr. Tralles, who has given two quarto volumes upon it. He has been at a great deal of pains to render it universal, that opium is extremely hurtful in fevers; but $I$ am not much influenced by his opinion; if it was fit to enter into a particular criticism, I could show that he forms most of his conclusions from a few instances of its abuse in the cases I speak of, where the disease was manifestly inflammatory; and I have the less regard to his judgment, that I think I could show certainly and clearly that his reasoning is upon a bad foundation. He has, indeed, in some measure, given us an useful work, by compiling such a prodigious number of opinions, and such a multitude of facts; but with regard to his more general theory, I think it universally wrong."

As the fever advances, the inflammatory state generally disappears in the second week, and the symptoms of debility become more evident. In this condition opium may be employed, and more or less as the symptoms of debility and irritation are more distinctly apparent; but even in this second stage it is an ambigu- 
ous remedy; and if it increases delirium, and does not readily induce sleep, it may be very hurtful, and is therefore to be employed with great caution.

In this advanced state of fevers, a circumstance occurs, that we are assured of as a fact, though we cannot well explain it. The cause of fevers gives an irritation to the brain, which is not of the inflammatory kind, but produces convulsive motions of the limbs to a considerable degree; and to this irritation it is that we impute the 'subsultus tendinum,' so much taken notice of as occurring in the advanced state of fevers.-The same irritation also frequently produces a delirium, which, as not of the phrenitic and inflammatory kind, we name the maniacal. Such an appearance of a maniacal delirium, and attended with some impetuosity and furor, is in fevers generally imputed to a phrenitic state, to more or less of inflammation in the brain; and it is to be acknowledged that it is very often justly so; but I have seen several instances of the impetuous or furious delirium, which, from their suddenly admitting of a solution, and from their being frequently cured by the use of opium, I conclude for certain, whatever were the appearances, were not truly of the inflammatory kind. I own, that though in general I can distinguish between the mild and furious delirium, and am pretty confident that it is proper in the one to reject, and in the other to admit of opium, there is an uncertainty attends the whole of this affair. Sir John Pringle marks out the phrenitic delirium, when the eye becomes wild and the voice quick; but he does not go further, and leaves us to this general view; and there he very properly avoids wine, and would certainly opiates too. But I have seen that wild look and quick voice, with a languid and weak pulse, and with a peculiar redness of the eyes, which were what I would call decisive marks of inflammation; and yet I have seen these cases pass away into a state of good tranquillity, and manifestly cured by inflammatory medicines, by wine and by opium: It appears therefore that there are such cases as will admit of these remedies, and I must leave you, in the course of practice, to find out other means of being more exact in this matter.

There is still another circumstance in the advanced stage of fevers that on this occasion requires to be taken notice of. 
Though the most part of our fevers arising from contagion may have little general inflammatory diathesis accompanying them, and be on the contrary attended with much debility, there occurs, however, from causes not easily assigned, some topical inflammation of the brain, which has frequently appeared upon dissection after fevers of the most nervous or putrid kind. These topical inflammations and the delirium depending upon them, do not admit of opium, and all their symptoms are much aggravated by it.- $M$. $M$.

"I pass on to a more general case in which the sedative power of opium may be more useful, where indeed all inflammatory diathesis is very manifestly gone, or perhaps never strongly subsisted, and where that spasm only which depends upon debility remains. Here, I imagine, the sedative power of opium may be employed more frequently than it is ; the combination of its stimulant power may be of use joined to the sedative power; if by the sedative power we can take off the spasm of the extreme vessels, by the stimulant power we excite to a considerable degree the heart and arteries more effectually to produce sweat; and we do this more safely by opium than by any stimulant power whatever, not combined with such a sedative."

These are the remarks I have to offer on the use of opium in continued fevers. Many are fond of a more free use of it than had been common before, and have believed that this was introduced by a certain noted teacher and author, but I assert that I myself was the first who freely and largely employed opium in fevers, under certain restrictions indeed, which, neglected by other practitioners, have occasioned much mischief.-M. $M$.

Camphor has been much employed in fevers of all kinds, particularly in nervous fevers attended by delirium and much watchfulness; and in such I have frequently employed it with advantage. Some time ago I have often seen it employed by my fellow practitioners in such cases; and that the good effects of it did not always appear, I imputed to its being used only in small quantities. Since we came into the free use of wine and opium, camphor has been little employed in the practice of this country. The use of it, however, has been very fully estab- 
lished by some of the most eminent physicians on the continent.-M. $M$.

"Musk is a medicine that has been very lately introduced into practice, but since it has, we have enough of facts to show that it is a powerful remedy; and were we a little more secure of its genuine condition, it would be very often employed with great advantage. But while $I$ have seen it of great service, I have in many instances been entirely disappointed, even in very large doses, such as 3 ss. repeated several times a day; whether from the medicine not being suited to the case, or from its being in a bad condition and suited to no case at all, which certainly often happens, I shall not determine. With regard to what we can observe of its operation when it is given in large doses, and operates sensibly, it commonly procures a sound sleep, and very. often a profuse sweat, removing the spasm which had taken place. From this operation it seems to approach in its nature to opium; and at present it appears that its use is, for the most part, to be regulated in the same manner as that of opium. We abstain from it in inflammatory cases, or in cases where the Diathesis phlogistica is supposed to subsist, and in topical affections of the brain; but I am disposed to say-and I should have said the same with regard to camphor-that even in these. cases it may be employed with more safety than opium, so far as my experience goes; and in fevers, without much nicety in discerning the circumstances, wherever the symptoms of strong spasm appear, where there is a delirium, subsultus tendinum and convulsive motions, without the irritation being remarkable, and where we presume the disease to be in the nervous system, there the musk has been of considerable service."

CXCI. The inflammation produced by the application of cantharides to the skin, affords a certain proof of their stimulant power: but, in many persons, the effect of that stimulus is not considerable; in many, it is not communicated to the whole system; and, even when the effect does take in the whole system, it seems to be taken off very entirely, by the effusion and evacuation of serum from the blistered part. I conclude, therefore, that neither much good is to be expected, nor much harm to be apprehended, from the stimulant power of blistering; and the certainty of this conclusion is established by the great benefit

voL. I. 
arising from the proper practice of blistering in inflammatory diseases.

CXCII. Much has been imputed to the evacuation occasioned by blistering; but it is never so considerable as to affect the whole system; and therefore can neither, by sudden depletion, relax the sanguiferous vessels, nor, by any revulsion, affect the general distribution of the fluids.

CXCIII. The evacuation, however, is so considerable as to affect the neighbouring vessels; and the manifest utility of blistering near the part affected, in inflammatory diseases, leads me to believe, that blistering, by deriving to the skin, and producing an effusion there, relaxes the spasm of the deeper seated vessels. I apprehend it to be in this manner that the tumour of a joint, from an effusion into the cellular texture under the skin, takes off the rheumatic pain affecting that joint.

CXCIV. Analogous to this, it may be held, that the good effects of blistering in continued fevers arise from its relaxing the spasm of the extreme vessels, by a communication of the blistered part with the rest of the skin; and this is illustrated by the effect of blistering in colic and dysentery.

CXCV. It appears to me, that blistering may be employed at any period of continued fevers; but that it will be of most advantage in the advanced state of such fever, when, the reaction being weaker, all ambiguity from the stimulant power of blistering is removed, and when it may best concur with other circumstances tending to a final solution of the spasm.

"Blisters may be used, I say, in the nervous fever strictly so called, at any period; but they are most properly used in the advanced state of the Synochus, when the inflammatory state is over and the debility prevails. Some persons may say in favour of their early application promiscuously, that if their power tends to take off the spasm, it is more desirable to obviate that spasm, than to remove it afterwards. But in spite of what may be said in favour of this, I am well persuaded that it is not in the power of any remedy whatever, to cut short the duration of certain fevers."

CXCVI. From the view of this matter given in CXCIII. and CXCIV. it will appear, that the part of the body to which blisters ought to be applied is indifferent, excepting upon the 
suspicion of topical affection, when the blistering ought to be made as near as possible to the part affected.

CXCVII. Whether Sinapisms, and other Rubefacientia, act in a manner analogous to what we have supposed of blistering, may be doubtful; but their, effects in rheumatism, and other inflammatory diseases, render it probable.

CXCVIII. The other external means of taking off the spasm of the extreme vessels, is warm bathing. This was frequently, and in various circumstances, employed by the ancients; but till very lately has been neglected by modern physicians. As the heat of the bath stimulates the extreme vessels, and, with the concurrence of moisture, also relaxes them, it seems to be a safe stimulus, and well suited to take off the spasm affecting them.

CXCIX. It may be applied to the whole body by immersion; but this is, in many respects, inconvenient; and whether some of the inconveniences of immersion might not be avoided by a vapour-bath, I have not learned from experience. I know, however, from much experience, that most of the purposes of warm-bathing can be obtained by a fomentation of the legs and feet, if properly administered and continued for a due length of time, which ought not to be less than an hour.

CC. The marks of the good effects of such a fomentation, are, the patient's bearing it easily, its relieving delirium, and inducing sleep.

" This practice seems well adapted to the advanced state of fever, when the phlogistic diathesis is abated or gone, and the application of heat thereby safe, but when there is still a resistance to the sensorium, appearing in delirium, stupor, subsultus, and such like affections, with a weak pulse. The bathing, by its heat stimulating the extreme arteries, and by its heat and moisture relaxing their extremities, seems well fitted for taking off both the atonia and spasm on the surface of the body, and thereby relieving the brain, as a part of both the nervous and the sanguiferous systems. It seldom gives an immediate solution, but by repetition for several days, it commonly contributes to an entire cure.

"It is to be observed here, that the consideration of both bathing and fomentations seems to show, that in fevers the action 
of the extreme vessels is greatly diminished; and that the cure often consists in restoring it."

CCI. Having now considered the several means of satisfying the first general indication in the cure of fevers, I proceed to the second (CXXVI.), which is, to remove the cause, or obviate the effects of debility.

CCII. Most of the sedative powers inducing debility cease to act soon after they have been first applied; and, therefore; the removing them is not an object of our present indication. There is only one which may be supposed to continue to act for a long time; and that is, the contagion applied: but we know nothing of the nature of contagion that can lead us to any measures for removing or correcting it. We know only its effects as a sedative power inducing debility, or as a ferment inducing a tendency to putrefaction in the fluids. The obviating the latter will be considered under our third general indication, and the former alone is to be considered here.

CCIII. The debility induced in fevers by contagion, or other causes, appears especially in the weaker energy of the brain; but in what this consists, or how it may be directly restored, we do not well know. As nature, however, does, seemingly for this purpose, excite the action of the heart and arteries, we ascribe the continuance of debility to the weaker reaction of the sanguiferous system; so that the means to be employed for obviating debility, are immediately directed to support and increase the action of the heart and arteries; and the remedies used are Tonics or Stimulants.

CCIV. In contagious diseases, both from the effects which appear and from dissections, it is known that the tone of the heart and arteries is considerably diminished; and that tonic remedies, therefore, are properly indicated.

These are to be considered as of two kinds; the first being the power of cold, the second that of tonic medicines.

CCV. The power of cold, as a tonic, I have mentioned above (XC.); and it is employed in fevers in two ways; either as the cold matter is thrown into the stomach, or as it is applied to the surface of the body.

CCVI. As it has been shown above, that the tonic power of cold can be communicated from any one part to every other 
part of the system; so it will readily be allowed, that the stomach is a part as fit for this communication as any other; and that cold drink, taken into the stomach, may therefore prove an useful tonic in fevers.

CCVII. This the experience of all ages has tonfirmed: but, at the same time, it has been frequently observed, that, in certain circumstances, cold drink, taken into the stomach, has proved very hurtful; and, therefore, that the use of cold drink in fevers requires some limitations. What these limitations should be, and what are all the circumstances which may forbid the use of cold drink, is difficult to determine; but it seems clearly forbidden in all cases where a phlogistic diathesis prevails in the system, and.more especially when there are topical affections of an inflammatory nature.

CCVIII. The other method of employing cold as a tonic, is by applying it to the surface of the body. The application of cold air to the surface of the body, as a refrigerant power fit to moderate the violence of reaction, I have spoken of above (CXXXIII.); but probably it may also be considered here as a tonic, and useful in cases of debility.

CCIX. Not only cool air, but cold water also may be applied to the surface of the body, as a refrigerant, and perhaps as a tonic. The ancients frequently applied it with advantage to particular parts, as a tonic ; but it is a discovery of modern times, that in the case of putrid fevers attended with much debility, the body may be washed all over with cold water.

CCX. This was first practised at Breslaw in Silesia, as appears from a dissertation, under the title of Epidemia verna qua Wratislaviam anno 1737 affixit, to be found in the appendix to the Acta Nat. Curios. vol. x. And from other writers we find, that the practice has passed into some of the neighbouring countries; although in this island, so far as I know, we have hitherto had no experience of it.

"The practice of applying cold externally to the surface of the body is attended with still more difficulty, and supported by fewer authorities than the former. But the practice was not unknown to the ancients, though not very common among them. (See Celsus, lib. iii. cap. 7. de curatione ardentis febris, and also several passages in the works of Galen.) An English 
writer, Sir John Floyer, gives instances of cold bathing applied accidentally, as where a person in the delirium of a fever escapes from his keepers, runs out of the room, plunges himself into a river or mill-pond, is brought back to his bed, recovers his senses, falls into a sweat, and obtains an entire solution of the fever.

"I could produce instances, particularly from several of the modern Galenists in the 16th century, where the application of cold to the head, hands, stomach, feet, \&c. was allowed, but cautiously. You know that about the middle of the last century physicians had got universally into the warm regimen, and kept the body covered very carefully, and the application of cold was not then thought of. About forty years ago a physician of this country took his patient's hands from the bedclothes, in the midst of a sweat, and put them into warm water, whereby the patient was relieved from his anxiety, the sweating was renewed with more vigour, and he soon after fell into a calm sleep; but this was considered as a bold and random practice, and was not thought of being imitated.

"The fever of the petechial and malignant kind, which prevailed at Breslaw, is mentioned by Sauvages in his Synopsis, under the title of the Tritæophya. The disease, in nine cases out of ten, proved fatal. Dr. Hahn, by the advice of his father, had his whole body washed over with cold water; it was probably in consequence of the difficulty of taking the patient out of bed, that only a cold washing was employed; and we have a strong proof of its being attended with considerable success, from its being employed upon the author himself. Hereafter the family became prejudiced in favour of the remedy, and a brother of Hahn's has published a dissertation upon the power of cold water : it is a small piece in the German language, which has never reached this country. The practice was received in some other places, as we learn from Dr. Schreiber, in the account of the cure of diseases which he wrote for the use of hospitals, and it has frequently been tried at St. Petersburg with advantage; but the difficulty and hazard attending the practice, is a check upon all regular physicians, and the practice has not yet spread far. Dr. Ludwig, in his Medicina Clinica, mentions it as a remedy, but at the same time doubts of its being gencrally safe. 
"From this historical detail, it will appear plainly that from both the ancients and the moderns, we have enough of facts to consider cold applied to the surface of the body as a remedy of some importance, and that it may have place wherever the use of cold water as a drink is admissible.

" The facts, however, are not so well ascertained in continued fevers as they are in smallpox, where the effects of cold air are very apparent. In continued fever the cold not only moderates reaction, but also acts as a tonic. There is wanted an experiment with the thermometer, to show first the increase of heat in an eruptive fever, and, secondly, the degree of diminution after exposure to cold.

"When the tonic power of cold is wanted, ought its application to be transient? A typhus patient has been relieved by washing him with cold water, but the same person continuing immersed would probably have been injured."

CCXI. The medicines which have been employed in fevers, as tonics, are various. If the Saccharum Saturni has been found useful, it is probably as a tonic rather than as a refrigerant; and the Ens Veneris, or other preparations of iron which have been employed, can act as tonics only. The preparations of copper, from their effects in epilepsy, are presumed to possess a tonic power; but whether their use in fevers be founded upon their tonic or their emetic powers, may be uncertain. The use of arsenic and of alum, in intermittent fevers, seems manifestly to depend upon their tonic power. And, upon the whole, there may occur cases of continued fevers, which may be cured by tonics taken from the fossil kingdom : but the use of these has been rare, as well as the effects uncertain; and physicians have employed, more commonly, the vegetable tonics.

CCXII. A great variety of these has been employed in the cure of intermittent fevers; but how many of them may be employed in continued fevers, or in what circumstances of these fevers, is not well ascertained; and I shall now only consider the question with respect to the most celebrated of these tonics, the Peruvian bark.

CCXIII. This bark has been commonly considered as a specific, or as a remedy of which the operation was not under- 
stood. But it is certainly allowable to inquire into this matter ; and I apprehend it may be explained.

CCXIV. To this purpose it is to be remarked, that as, in many cases, the effects of the bark are perceived soon after its being taken into the stomach, and before it can possibly be conveyed to the mass of blood, we may conclude, that its effects do not arise from its operating on the fluids; and must, therefore, depend upon its operating on the nerves of the stomach, and being thereby communicated to the rest of the nervous system. This operation seems to be a tonic power, the bark being a remedy in many cases of debility, particularly in gangrene : and, as the recurrence of the paroxysms of intermittent fevers depends upon a recurrence of atony (XXXV and XXXVI.), so probably the bark, by its tonic power, prevents the recurrence of these paroxysms ; and this is greatly confirmed by observing, that many other tonic medicines answer the same purpose.

CCXV. If the operation of the bark may be thus explained, from its possessing a tonic power, it is easy to perceive why it is improper when a phlogistic diathesis prevails; and, from the same view, we can ascertain in what cases of continued fever it may be admitted. These are either after considerable remissions have appeared, when it may be employed to prevent the return of exacerbations, on the same footing that it is used in intermittent fevers, or in the advanced state of fevers, when all suspicion of an inflammatory state is removed, and a general debility prevails in the system; and its being then employed is sufficiently agreeable to the present practice.

When Intermittent fevers have changed into Remittents, and these have become of a very continued form, or when either this transition has been manifestly perceived, or, from the place of the patient's habitation, from the season of the year, and from the nature of the prevailing epidemic, there is reason to conclude that a fever has arisen from the samemarsh effluvia which produce intermittent or remittent fevers in their ordinary form; in such cases, fcvers, however continued in their form they may then seem to be, may still be considered as of the intermittent kind, and be treated by the bark in the same manner that we have shown may be done in remittent fevers_of little distinct remission. $-M . M$. 
I can by no means concur with certain authors, who maintain that, in Synochus, as soon as the primæ viæ have been cleared by an emetic and purgative medicine, we may immediately employ the bark, and trust the cure of the disease entirely to it. I have frequently observed the mischievous consequences of such a practice, from its aggravating the inflammatory state of the system, and determining to local and fatal inflammations of the brain and lungs.-M.M.

CCXVI. With respect to the use of the bark, it is proper to add, that good effects are to be expected from it, almost only when given in substance and in large quantity.

"To be more precise, no less than 3 ss. of bark has any sensible effect in any disease, but particularly in the case of fevers; the smallest quantity that I have found to cure an intermittent is at least $3 \mathrm{vj}$. during an interval; but it is much more effectual if we go the length of $\xi \mathrm{i}$. or more; very often $\xi$ iss. or $\xi \mathrm{ij}$. only are to be depended upon; and when by the negligence of physicians in giving it, or the aversion of patients to take it, we fail, we attribute to the mischievous effects of the bark what was merely owing to the not giving it in sufficient quantity. For this practice I may refer you to Dr. De Haen; it is one of his corollaries that the bark only operates in large quantities; and I would observe, that it may be given in many cases in very large doses. I have known frequently 3 ss. given at one dose without any inconvenience felt in the stomach; I shall allow, however, that this is rare, that most stomachs will refuse such a quantity, few will bear $3 \mathrm{ij}$. at. once, but most stomachs will bear $3 \mathrm{i}$. at a dose. With regard to this circumstance, of the quantity necessary to be given, I must observe, that by the accounts we have of the first practice when it first appeared, much smaller doses answered the purpose than we now find to be necessary. They often cured intermittents with the quantity of $3 \mathrm{ij}$. or less; this is taken notice of by De Haen, but to what it is precisely owing he does not pretend to determine. I imagine it is owing to the different condition of the bark, for I can observe in different parcels of bark a difference in the quality, which very much depends upon the fineness of the grain; this is farther confirmed by the taste; for when there is a stypticity along with the bitter, even approaching somewhat to the aromatic, one drachm will 
go farther than $3 \mathrm{ij}$. of that which is more commonly in use. I mention this, because certainly whatever we may find some stomachs able to bear occasionally, we ought to be as tender of them as possible, and we cannot take too much pains in the choice of this medicine. Next, let me observe, that it is not perhaps desirable to exhibit the large doses that even the stomach will bear. Within these twelve years, it was a practice in the London hospitals to cure intermittents by giving a large dosethree or four or more drachms-immediately at the approach of the fit. I do not say that it is the best practice; upon the contrary, there are several objections attending it, and the stomach will seldom bear such large doses; but wherever we are obliged to divide the doses, we should take care that those which we give separately, be brought as near to one another as possible, that so the effects may be more considerable upon the system. So, if we give one drachm for a dose, we should give it at as short intervals as may be, as at the interval of one hour.

" There are cases of children, particularly, where neither in one form nor other can we exhibit the bark by the mouth; and it is of consequence to know that it may be exhibited in the form of glysters with advantage and efficacy. I say this, however, only from the accounts of other persons, for I have never been quite sensible of its effects when given in this form; but the experience of many others, whose accuracy I can have no doubt of, is in favour of this.

"W With regard to the exhibition of bark, in spite of its seeming astringency, it is most certain that it very often proves purgative. To what particular circumstance of the constitution this is owing I cannot explain; but so it is in fact, in certain persons it runs off by stool; and so often as it does so, it fails in the cure of intermittent fevers. It would perhaps be difficult to explain that; but I can say, that Sydenham and many other practitioners have cbserved, that we are in danger of bringing back intermittent fevers if we employ purgative medicines soon after we have stopped them with the bark; and we have the same observation in De Haen.

CCXVII. Another set of medicines to be employed for obviating debility and its effects, are the direct stimulants (CCIII.). These, in some measure, increase the tone of the 
moving fibres; but they are different from the tonics, as more directly exciting and increasing the action of the heart and arteries. This mode of their operation renders the use of them ambiguous; and when an inflammatory diathesis is present, as so often happens in the beginning of fevers, the effects of these stimulants may be very hurtful; but it still remains probable, that, in the advanced state of fevers when debility prevails, they may be useful.

CCXVIII. What are the stimulants that may be most properly employed, I am uncertain, as the use of them in this age has been rare; but I am disposed to believe that, of all kinds, wine is the best.

" It would be proper to consider the other remedies which at one time or other have been employed, but $I$ do not find myself in a condition for this ; for, during the time of my practice, these stimulants have been so carefully avoided, that I have not observations to conduct me in this matter; and with respect to former times, when physicians were strongly prejudiced for or against them, it is difficult to trust to any of their facts. I must therefore content myself with some general reflections upon one or two of these which are still retained in practice.

"The first I shall mention is the Radix Contrayervæ, which is somewhat stimulant in its sensible properties, but by no means considerably so ; and I am persuaded that at all times it has had more credit than it deserves : so far as I can observe, it has never been employed in a simple state, or without the combination of substances to which its powers may be imputed."

The Serpentaria, by its aromatic acrimony, proves a powerful stimulant to the system, and therefore may be useful also in some cases of continued fevers; but it is to be employed with much caution. The stimulant power of the serpentaria is especially suited to the low and advanced state of the typhus only; and even then it will be more safely joined with the bark than employed for its stimulant power alone.-M. $M$.

"6rom the rareness of nervous and low fevers in this country, and particularly from the Sydenhamian principles which I had early imbibed, I have never seen the serpentaria so frequently employed as to pass a confident judgment with regard 
to its powers, and where I have seen it employed, it showed stimulant effects that were hurtful."

CCXIX. Wine has the advantage of being grateful to the palate and stomach, and of having its stimulant parts so much diluted, that it can be conveniently given in small doses, so that it may be employed with sufficient caution; but it is of little service unless taken pretty largely.

"It is now generally agreed, that wherever a stimulus is necessary, the most safe, and perhaps the most effectual, is wine; with regard to this, however, the theory is not very clear. It contains alcohol, which possesses a sedative, narcotic, and an antispasmodic power, if you will, when used in a sufficient quantity, to a considerable degree; and whether its virtues are to be imputed to its sedative and antispasmodic, or to its stimulant power, is not a matter that is very clearly determined. But if it operates in the first way, it should not be a better remedy than opium, and some people do say so, but I will not, nor do I know of any practitioner who has learned to employ opium in the same manner and with the same effects that we do wine. In short, whatever are its other powers, in the way we employ it, by small and separate exhibitions, it may be considered as only acting as a stimulant. I take my system with regard to wine from Sir John Pringle, and nobody has illustrated the use of it more agreeably to my notions. He lays the foundation in this manner : he observes, that a delirium will arise from two different errors, the one from large and repeated venesection, and the other from wine and other cordial medicines given in the beginning of the disease. Now, this is the foundation which I also would wish to lay. I have formerly pointed out these two cases of delirium, and, I hope, fully enough explained them, the one depending upon a state of debility, the other upon a state of increased impetus, accompanied with the phlogistic diathesis; and this explanation is fundamentally connected with the whole doctrine of fevers. Now, in the first of these cases, where the debility prevails, and even a considerable degree of delirium in consequence of it, wine is certainly, as Sir John Pringle observes, a proper remedy. I have had instances myself of wine given to a considerable quantity removing a delirium entirely; and I have had other instances from other practitioners that were still more re- 
markable. Sir John Pringle relates the case of a gentleman in the smallpox, who drank above four bottles of wine in the day to obviate a delirium which recurred when the effects of the wine seemed to cease, and was again removed by returning to that remedy. Sir John Pringle observes, that in the low state of fevers, nothing is comparable to wine ; and, in the last account of his jail fever, so great were its virtues, says he, that he has known many recover from the lowest condition by nothing but a little panada and wine, and the volatile mixture, by turns.

"Where the symptoms are evident, we may presume upon the virtues of wine, and the state of the body that admits of it; but there is certainly another case, as Sir John Pringle observes, when the delirium is found to increase upon the use of wine, when the eyes look wild, the pulse quickens, and there is the presumption of a true phrenitis, then wine and all stimulant medicines aggravate all the symptoms.-This is a theory, as $I$ call it, with respect to the use and application of wine, which shews that we are mentioning it here in its proper place; and that it acts especially as a stimulant. Let me add a few practical directions with regard to it.

"I would give this general rule: When fevers manifestly arise from contagions, when they are known to be of the contagious kind, and so depend upon a sedative power prevailing in the system, when, at the same time, they have few or no inflammatory symptoms, we may, very early in the course of the disease, have recourse to the most safe of all our stimulants, wine.

"But there are many cases in which the circumstances are not sufficiently decisive, that is, where we are not secure of the absence of inflammatory diathesis; and in such cases it is proper to abstain from wine entirely till the symptoms of debility appear more evident; and they commonly appear even in the second, but more certainly in the third week of our continued fevers. But even when this period is arrived, and when debility very certainly prevails, whilst we cannot be secure against the presence of some topical determination, some inflammatory affection, we may, in this uncertainty, be determined by the following circumstances: First, By the former habit of the patient: if he has used every day a quantity of wine, as a part of his ordinary diet, and especially in larger doses, perhaps to the 
quantity of intoxication, which, when the fumes of the wine are dissipated, is followed by a prodigious languor and a state of great debility, respect may be had to the former habit, and we may be more bold, nay, it is almost necessary to exhibit wine.

"Secondly, where we have not that habit of the patient to guide us, there are few persons of any condition in life who have not frequently taken more or less wine, and that are not acquainted with its effects; and the most abstemious will feel a languor that will make them call for this cordial; and wherever the patient's own appetite makes the demand, there we may with some confidence gratify and indulge it; or at least we may make a trial, and then we are farther determined by the patient's feelings ; if he feels it a cordial without its proving heating or increasing the thirst, we may confidently repeat it, and more especially, if the frequency of the pulse, and the delirium which subsisted before, are not increased, but rather diminished.

"B But I must observe, that frequently among the poor people in this country, it is not uncommon to employ brandy or other such ardent spirits in place of wine, and sometimes, I believe, with great effect; but there is no sort of doubt, that though the stimulus of ardent spirits is of the same nature as that of wine, yet, from the difference arising from the other substances blended with the plain spirit, the latter by itself is always a more inflammatory stimulus, and is therefore employed with much more hazard. And I carry this so far as to observe, that even of the different wines, some are neat, and purely depend upon the fermentation of the grape, while in many others it is necessary to put in a quantity, sometimes a considerable quantity, of spirits on account of their exportation; and $I$ hold it a rule in all cases of fever to employ the first, the most genuine and neat wines, such as Claret and Rhenish wine, and to abstain from the Spanish and Portuguese wines, which have spirits put into them in their respective countries.

"As the danger from the use of wine arises chiefly from its stimulus, practitioners have thought it necessary to give it always considerably diluted; and in the main this is a safe and proper practice, especially in the first trials of it. We dilute it in the form of posset, wine-whey, or what we call negus. : The first has the most considerable effect, but it forms a mawkish 
liquor which is hardly refreshing, and the other form is more agreeable. But I am disposed to say that if we will dilute wine, it should be constantly with cold water ; for a little plain wine and water is a much more refreshing cordial than when it is any how warm and diluted at the same time. I am willing to make this remark, because I am strongly disposed to think that it is rare that wine is admissible but when cold water is so too at the same time; indeed where more or less of catarrh and cough, with some degree of angina, accompanies a nervous fever in the winter or spring season, the cold water may be improper; but this is more rarely the case, and never in the summer or autumnal fevers."

CCXX. It may be supposed, and on good grounds, that wine has an operation analogous to that of opium and some other narcotic medicines. It may indeed be said, that we can distinctly mark its stimulant power only, which renders its effects in the phrenitic delirium manifestly hurtful, and in the mild delirium depending on debility, as remarkably useful. But in all this, the analogy with opium is still obvious; and it is probable, that both wine and opium are more useful by their sedative and antispasmodic than by their stimulant powers.

CCXXI. These are the means of answering our second general indication (CXXVI. 2.); and I now proceed to the third, which is, To obviate or to correct the tendency of the fluids to putrefaction.

CCXXII. This may be done-

1. By avoiding any new application of putrid or putrescent matter.

2. By evacuating the putrid or putrescent matter already present in the body.

3. By correcting the putrid or putrescent matter remaining in the body.

4. By supporting the tone of the vessels, and thereby resisting further putrefaction, or obviating its effects.

CCXXIII. The farther application of putrid or putrescent matter may be avoided-

1. By removing the patient from places filled with corrupted air.

2. By correcting the air from which he cannot be removed. 
3. By preventing the accumulation of the patient's own effluvia, by a constant ventilation, and by a frequent change of bedclothes and body-linen.

4. By the careful and speedy removal of all excremental matters from the patient's chamber.

5. By avoiding animal food, or correcting it.

"It is not possible to have a number of men in the healthiest state shut up in a place, and their effluvia accumulated, without their becoming noxious, by retention, by the heat which is occasioned, and perhaps by some additional fermentation. And this tendency must be increased in the case of hospitals, where there is a particular ferment arising from every sick body ; therefore certainly the hospital destroys more than the disease would do ; and persons affected with contagious disorders ought if possible to be removed; and if that cannot be done, it is of the utmost consequence that a constant ventilation be provided, and every means of cooling and of correcting the air be employed, as fires, various fumigations, the frequent application of acids and other substances, which are found to change and correct it.

" Nay, from every single person labouring under a fever, noxious effluvia proceed, which, though not very virulent as they arise, by adhering to the bed-clothes in greater quantity, by being more concentrated, and probably by additional fermentation, acquire more virulent powers. From many observations, I am persuaded, that it is seldom that the effluvia directly arising from bodies, even in putrid fevers, are extremely poisonous. In the year 1750 the remarkable jail fever appeared and affected so many persons; and we know that when forty of these, who were persons of some note, were subjected to this fever in the court, their contagion did not spread to any one of their respective familiès; and it was certainly much more innocent from their bodies than as lodged in the garments and clothes of the. jail prisoners; and very possibly this was owing to their being no fomes provided; they were by themselves, in chambers which were kept well ventilated; and other measures were taken to prevent any faults of the air, and particularly the changing of the bed-clothes next the body. And I am so much persuaded that the danger arises from the contagious matter lodged in the garments for some length of time, that I think nothing is of 
more consequence in some diseases than the frequent changing of the patient's bed-clothes ; and it is one of the principal prophylactic means for preventing the spreading of such distempers. A person being near another labouring under a putrid fever, will very readily escape the contagion, if he is only exposed to the effluvia arising from the person, whereas, by a more close contact with the bed-clothes, he will catch the infection; and I know instances of persons catching it from remaining some time upon the bed of such a person, while many others, exposed to it from the air, escaped unhurt : there must be pains taken with our fellow-creatures, but such a contact should be avoided as much as possible.

"Not only in this way do we take care to avoid the effects of a contagion, but every other excretion must be attended to in the same manner. We know well, that in the case of Dysentery it is the fæces alvinæ that especially contain a contagion, and very readily communicate it; and it is therefore known to be a proper precaution, that in armies and hospitals, if possible, dysenteric persons should not be admitted to the common privies, and that their excretions be removed out of the chamber, and conveyed in such a manner as to prevent their doing mischief. And possibly the same attention is necessary with respect to other excretions, as that of the urine; it should not be allowed to continue too long in the patient's bed-chamber, and the vessels should be constantly well cleaned from the last effusion. Also, where there are excretions by spitting; \&c. the clothes which receive these should be changed and removed."

CCXXIV. The putrid or putrescent matter, already present in the body, may be evacuated, partly by evacuating frequently the contents of the intestines, and, more effectually still, by supporting the excretions of perspiration and urine by the plentiful use of diluents.

"It is probably an useful practice, which has now become so common, the keeping the belly open in putrid fevers. (See CXLIX.) Dr. Strack in Germany rests the whole cure upon this management. We do not agree with him entirely upon this subject, but it is on several occasions necessary to obviate vol. I. 
the stagnation in the primæ viæ, which would otherwise run on to putrefaction."

CCXXV. The putrid or putrescent matter, remaining in the body, may be rendered more mild and innocent by the use of diluents; or may be corrected by the use of antiseptics. These last are of many and various kinds ; but which of them are conveniently applicable, or more particularly suited to the case of fevers, is not well ascertained. Those most certainly applicable and useful, are acescent aliments, acids of all kinds, neutral salts, and fixed air.

CCXXVI. The progress of putrefaction may be considerably retarded and its effects obviated, by supporting the tone of the vessels. And this may be done by tonic remedies; the chief of which are, Cold, and Peruvian bark, both sufficiently treated of above (CCV. et seq.).

CCXXVII. I have now finished the consideration of the three general indications to be formed in the cure of continued fevers; and have mentioned most of the remedies which have been, upon any occasion, employed in this business. It was necessary, in the first place, to consider these indications and remedies separately, and to explain the operation of the latter more generally : but, from what has been now delivered, compared with what was said above concerning the difference of fevers, and the signification of their several symptoms in forming the prognostic, I expect it will not be difficult to assign the indication, and to select and combine the several remedies mentioned, so as to adapt them to the several species and circumstances of continued fevers.

I think it may be useful for my readers to have the whole of the cure of CONTINUED FEVERs brought under one view, as in the following table:

In the Cure of contrinued Fevers, the indications are, I.-To moderate the violence of reaction.

Which may be done, by

1. Diminishing the action of the heart and arteries, by A. Avoiding or moderating those irritations which are almost constantly applied to the body ; as, 
a. The impressions made upon our senses, particularly,

a. Increased heat, whether arising from

as. External heat, or

$\beta \beta$. The accumulation of the heat of the body.

b. The exercise of the body.

$c$. The exercise of the mind.

$d$. The taking in of aliment.

e. Particular irritations arising from

$\alpha$. The sense of thirst,

$\beta$. Crudities, or corrupted humours, in the stomach,

$\boldsymbol{\gamma}$. The preternatural retention of fæces,

ठ. A general acrimony of the fluids.

B. Employing certain sedative powers; as,

a. Cold,

b. Refrigerants; the chief of which are,

. Acids of all kinds,

$\beta$. Neutral salts,

$\boldsymbol{\gamma}$. Metallic salts.

C. Diminishing the tension and tone of the arterial system; by

a. Blood-letting,

b. Purging.

2. Taking off the spasm of the extreme vessels, by

$A$. Internal means; which are,

a. Those remedies which determine to the surface, as,

๙. Diluents,

ß. Neutral salts,

भ. Sudorifics,

8. Emetics.

b. Those remedies, named Antispasmodics.

B. External means; as,

a. Blistering,

b. Warm bathing.

II.-To remove the causes, or obviate the effects of debility, by,

1. Supporting and increasing the action of the heart and arteries, by

A. Tonics, as,

a. Cold, 
b. Tonic medicines, which are either,

«. Fossil, as, ax. Saccharum saturni, \&c. or

B. Vegetable, as, aж. Peruvian bark.

B. Stimulants, as,

a. Aromatics, \&c.

b. Wine.

III.-To obviate or correct the tendency of the fluids to putrefaction, by

1. Avoiding the application of putrid or putrescent matter, by

A. Removing the patient from places filled with corrupted air.

$B$. Correcting the air from which he cannot be removed.

$C$. Avoiding the accumulation of the patient's own effluvia, by

a. A constant ventilation,

b. Frequently changing the bed-clothes and body-linen.

$D$. Removing carefully and speedily all excremental matters.

$E$. Avoiding animal food, or correcting it.

2. Evacuating the putrid or putrescent matter already present in the body, by

$A$. Evacuating frequently the intestines.

$B$. Supporting the excretions of perspiration and urine, by a. Diluents,

b. Neutral salts.

3. Correcting the putrid or putrescent matter remaining in the body, by

A. Diluents,

B. Antiseptics,

C. Fixed air.

4. Resisting farther putrefaction, or obviating its effects, by Supporting the tone of the vessels, by Tonic remedies.

"If there is any imperfection attending this view of the treatment of continued fevers, I think it is this, that I have 
found it necessary to consider symptoms as well as remedies, as separated from one another, whereas they ought to be viewed as combined together, and as the symptoms and remedies are related.' I would hope, from the account I have given, that you are ready for this application; but it may be still useful to give you some examples, to state some cases of fever of different kinds, and to point out where indeed the difficulty occurs with respect to the employment of remedies. Had I my choice, I would state the cases, and put the questions to you, and it would be very useful for you to give me answers; but this would not perhaps be very readily received, and would take up too much time; I must therefore put the questions, and give the answers myself.

"The cases, then, which I am to put, are of three kinds, - as they respect either the approach of fever, or the first formation of fever, or various cases of the disease already formed.

"I. The approach of fever undoubtedly is marked by a variety of symptoms, which are not properly symptoms of the fever, but occur before the proper formation of it. Thus, a person feels a certain sluggishness, an aversion to motion, a want of that alacrity in the employment of his body or mind, which he usually had; with these symptoms, which are comprehended under the term of Lassitude, there is a want of appetite, with a sensibility to the coldness of the air, so that upon the approach of a stream of air he is apt to complain of cold, though without any shivering; at the same time, the vivacity of his eyes and features is disturbed.

"By these symptoms we very often discern the approach of a fever, and in the time of a contagion they are pretty certain presages; at other times, however, they are hardly to be regarded, especially if the patient can assign certain causes for them, such as perhaps some debauch or fatigue, or watching, or that he knows that such symptoms have formerly proceeded, and usually do proceed from some particular disorder of the stomach. But if the patient can assign no such cause, an approaching fever is to be suspected, and the question is, What are the proper measures to be taken in such circumstances? It is certainly of the utmost importance to know, if, by good luck, we can prevent a dangerous disease from coming on. I will not 
say how far we may succeed, but I know that a neglect of certain precautions and measures has been commonly of the worst consequence, and has been followed by fevers of the most fatal tendency. Where we have an opportunity of advising, I would insist upon the person keeping at home, and his not exposing himself to fatigue, and to the vicissitudes of temperature that would attend his going abroad. At home he should avoid cold by keeping to his chamber, for cold has very ready access to people at rest within doors; and when they are more sensible to cold, any stream of air is dangerous; such streams of air accordingly must be avoided, by not going through passages where they may take place. The individual should give up application to business, and should even avoid any fatiguing situation of the body, and lie much upon his bed; whether more or less covered, is a question I shall speak to presently. In proportion to his want of appetite, he should avoid taking food altogether, or at least should eat very moderately. With respect to drink, perhaps another rule is to be given; if a person has been used, in ordinary health, to take a few glasses of wine more or less daily, he is not to abstain from it altogether in his present situation, but ought to use it moderately according to his former habits, only keeping within narrower bounds. Such is the regimen that is to be attended to.

"With regard to remedies in this situation, there is hardly any doubt that a vomit may be useful. From the symptoms I have been mentioning, and the explanation I formerly gave, it appears that the part first affected is the stomach, that a retardation of its evacuation and a congestion of crudities are to be suspected. On account of this, and with a view of obviating the fever by restoring the determination to the surface, a vomit is very generally proper.

"Whether another remedy, that is moderate sweating, may be employed at this period, is certainly a question to be put. Most practitioners are of opinion that it is, and, from every view of the subject, it is very probable that it is very universally safe for the person, not only to lie in bed, but to be moderately covered, so that if any tendency to sweat comes on it may be encouraged; or, even without that, that some moderate 
degree of sweat should be raised, and that this diaphoresis should be supported for a due length of time, as I formerly explained (See CLXVIII). The practice of sweating is under a great deal of doubt and uncertainty, but that arises especially from the degree to which the sweat is excited, as the heat the patient is detained in, or any other stimulus may raise more or less of the inflammatory diathesis, and form a more obstinate spasm. I have no doubt, however, that if sweating is procured by gentle means, or rather if nature seems to yield spontaneously and assist, it is a very probable means of obviating the accession of fevers, especially if conducted in this way, and continued for a length of time. If a person enters upon that course of sweating, he ought to continue it for at least fortyeight hours; and any interruption is in hazard of converting the remedy into a poison.

"Now, what I have said with respect to this first case is very applicable to the inflammatory as well as to the nervous fever; and the same regimen will be as useful in every catarrh, especially in the beginning, or in any other state attended with some degree of pain ; for instance, in rheumatic affections, where an antiphlogistic regimen, and encouraging moderate sweating will be generally found useful.

"II. The case of the formal attack of the disease, when there is manifestly a cold fit in its different degrees, when there are not only the sensibility to cold, and some degree of coldness creeping along the surface, thrilling along the back like cold water, and spreading along the body with some degree of shivering, but when there is the more formal attack of horror, tremor, and rigor, and more distinctly when these are followed more immediately by heat and flushing, by a considerable frequency of pulse, and especially when they are attended with headach. The latter symptom indeed may be joined with the circumstances that mark the approach of fever, but when it amounts to some degree, it is rather to be considered as a mark of a formal attack.

"With regard to the management: Where we have an opportunity of being present at the very beginning of the formal attack, when the cold fit yet subsists, then, whatever may be the nature of the fever, we may treat it in the same manner as the 
cold fit of an intermittent, that is, a vomit is instantly to be exhibited (CLXXVI.). I have formerly referred to Dr: Lind with regard to this practice, which he has established in his hospital. I have spoken of its being applied more early upon certain feelings of a received contagion, as when some degree of sickness and nausea immediately, affects a person who has been near contagious diseases. The immediate exhibition of a vomit has been found of service, and, as I have said, it is probable that it operates by taking off the spasm. The patient should be put to bed, and some degree of sweat raised immediately after the operation of the vomit; or, if you will, let it be one and the same operation. The sweating is to be promoted by the means I mentioned, by the use of warm liquors or the milder neutrals, or it may be carried farther if to these more or less of the opiate is joined. If these measures are pursued, and continued to the time I mentioned, to forty-eight hours, or at least by the patient's keeping in bed so long, to support the determination to the surface; and if, at the same time, all farther irritations be carefully avoided; this probably is a measure that would cut short, if any can, a continued fever.

"I must observe that it is extremely common in this country as soon as there is any appearance of any formed fever, to fly to venesection. It is a very curious circumstance which Dr. Lind tells us, that where venesection was administered before the vomit, the vomit had not the effect that it would have had, if it had been administered first. I have marked the attack of fever very distinctly : it is not made simul et semel, but a coldness and shivering comes on; that is very often succeeded by some degree of heat, which is followed by a new shivering fit, and these continue to alternate for a day or two together. In many cases, the first cold fit of an evening is hardly observable, till the disease has renewed its attack in the succeeding evening, and is in all its circumstances more considerable. Now, in all these cases where the disease is forming, but not yet properly formed, I hold it to be always hurtful to administer venesection, and it is never admissible but when the hot fit is steadily formed; nay, if we have any foundation for alleging that it is a debility that lays the foundation of fevers, the blood-letting may be one of the several circumstances which serve to give 
a more violent disease (CXLIII.).-So much I have to say with regard to the management of the formal attack.

"III. Let us next attend to the several circumstances of the disease already formed - and these may be supposed:

" 1 . Cases of the second day of fever; and here, again, I may put,

" $a$. A first case, where the symptoms are remarkably moderate; some chilliness, some little horror, has occurred in the evening, followed by some preternatural heat in the course of the night; sleep takes place, but is more disturbed, and after such a night considerable lassitude and languor are felt in the morning: the mouth is clammy, the tongue foul, some thirst, but a small degree of headach, the pulse more frequent than natural, but to no great degree, from 80 to 90 , neither full nor hard. Such cases we have often seen; and when they occur the nature of the future disease is uncertain.

"W With regard to the practice, this is the first rule, and an universal one, that the antiphlogistic regimen is to be prescribed in all its parts; that it is universally proper to evacuate the stomach by a full vomit, the intestines by a glyster, both of which may be considered as a part of the antiphlogistic regimen. We are sure that these measures are proper; but whether or not venesection is proper in the circumstances I have described, may be doubted; and I imagine that it is improper. For, suppose the disease to be of the inflammatory kind,-to judge from the symptoms I have described, it will seemingly be a very moderate one, and urging no haste in the measures that may become necessary. But not only do we, from these symptoms, presume a moderate synocha, but there is, perhaps, more probability that a fever beginning in this way may turn out a typhus, and that pretty purely so, without any admixture of synocha, where, accordingly, venesection is improper. Another question may occur, whether or not sweating in the present circumstances may be employed? but I reserve that question till I can state it with respect to several other cases I am to mention.

" $b$. I put a second case, of the same period, viz. the second day of the disease; where the attack the evening before or the day before is much more distinctly marked, the horror more evi- 
dent during the night, when the sleep is extremely disturbed, much thirst, a more considerable degree of headach, the pulse more frequent, to 100 and upwards, and full, with some degree of hardness. In this case, after prescribing the antiphlogistic regimen in general, there is no doubt that venesection is the next remedy, and that without regard to the nature of the disease which may follow afterwards. This case, therefore, is to be considered as inflammatory or a synochus; but even supposing it to be known from the epidemic prevailing, that it is to be a typhus, there is no hesitation about a venesection, and a large venesection too, which, accordingly, is the first measure to be pursued.

" $c$. We put a third case, which is somewhat of a middle kind between the two former, where the symptoms are more violent than in the first $(a)$, and at the same time somewhat more moderate than in the last $(b)$, and therefore not so decisive with respect to the practice $I$ am speaking of. I say that in such cases it may be often very doubtful whether we ought to blood or not, and particularly to what degree we should do it; and it is not to be determined alone from the circumstances which we may call proper to the disease, as I have just now put them, but commonly from some other collateral circumstances with respect to the remote cause, the constitution of the patient, and some other accidental circumstances. With that view I would say, if such a fever as I speak of happens at a time when a common contagion is not known, or where there is none remarkably powerful; if, on the other hand, we can perceive that the application of cold had a considerable share in bringing it on ; if we have more reason to suspect this from the season of the year, either in the winter or spring, and if the attack of the disease was without those symptoms of approach that I first described, but was more sudden and sufficiently marked; if this happens in a young, full, and vigorous constitution; and which perhaps has been formerly liable to inflammatory disorders, and in consequence of this accustomed to venesection; if, to these several circumstances, there are farther joined catarrhal symptoms, rheumatic affections, or especially some topical determination, then there is no sort of doubt that venesection is the most proper remedy. 
"Though there be no proper variation in the symptoms peculiar to the disease, we may come to a very different determination merely from a different state of these collateral circumstances. If, in the time of a contagion generally prevailing, a person has had a manifest intercourse with those labouring under that disease ; if we know nothing of any particular application of cold inducing it, or at least can only see it concurring with the contagion; if the season is summer or autumn, if there are no catarrhal symptoms or rheumatic affections; if there is nothing particular to determine in the habit, young, full or vigorous, and nothing to be expected from hæmorrhagic evacuations, or artificial venesection; and, going back to the circumstances of the disease, if, though the pulse is frequent, to above $100^{\circ}$, though there are considerable heat, thirst and headach, yet the pulse at the same time not very full, nor very hard,- then there can be no doubt that that disease is probably to turn out a typhus or synochus at least, and venesection is improper, or at least we should practise it with much hesitation, and only in the case of a certain ambiguity, from a mixture of these circumstances; but though we know that a contagion is prevailing, and that we can trace the disease to it, if the system is remarkably full and vigorous, and the pulse remarkably hard, still some venesection may be allowed.

" $d$. I put a fourth case which differs considerably from all the others in its symptoms. The case occurs in the time of a contagion very generally prevailing, and where that contagion approaches to the worst kind, to a nervous or petechial fever ; where there is a manifest suspicion of an actual contagion caught from the person perhaps living in an hospital, or having a communication with a person labouring under the disease in a more violent degree; where the symptoms of the approach of the disease appear in the shape I have formerly described; where the attack is gradual and obscure, and even where we can see the disease is formed, yet the symptoms of the inflammatory appearance are not considerable, and the state of the thirst very moderate. In such cases, there most generally occurs a frequent pulse, but which, at the same time, is weak and soft ; there is some headach, not violent, with no suffusion of face, no redness of the eyes; but there are great complaints of a 
confusion of the head. These are the circumstances that occur early, and with these there are marks of considerable debility; the person perhaps is incapable of bearing any degree of the erect posture, the mind is affected with great fear and despondency, and with this, upon attempting any motion, tremor appears. I observe here, that $I$ am putting a case of the second day; but it is extremely difficult, nay impossible, where a disease comes on so gradually, to fix this to the case of a second day; the consideration applies to several of the first days. Now where the above combination of circumstances occurs, I say this is a case which more certainly than any does not admit of venesection. I own that some practitioners have such a practice of universal venesection upon the approach of fever, that they use it even in the circumstances I have mentioned. Accordingly, Sir John Pringle allows, that in such circumstances as I have described one venesection may be practised; but it is certainly in view of the particular circumstance of a constitution that is remarkably full and vigorous. A second venesection is always hurtful, and where that is the case, the first must be very doubtful, and be considered as an exception from the general rule, arising from a combination of some degree of inflammatory symptoms, and more especially with respect to the constitution or habits of the patient. Such a combination of inflammatory symptoms may concur; and just now we have a fever prevailing which is certainly of the nervous kind, but which has been very frequently accompanied with catarrhal symptoms, nay with some more pointed rheumatic affections; yet even in these, when we consider them as not belonging so much to the disease as they are to be imputed to accidental circumstances of the season, venesection is not proper, it has rather been of prejudice; and in several instances I have found, that even where otherwise the time of life, \&c. would seem to admit of it, the patients did not really bear it tolerably.

"Now, when I have stated these several cases, with respect to the second (b.), where the inflammatory symptoms are stronger, I have no doubt that sweating is not to be proposed; but in the other three ( $a . c . d$.), where the symptoms are moderate on the second day, and even where the symptoms are more considerable, but still, where the circumstances that seem to 
determine against venesection take place, and epecially in the fourth case $(d$.$) , that of typhus or petechial fever, the question$ may be put, if sweating is a remedy to be attempted, in order to cut short the course of the disease? I have given all the various considerations that can influence us. I have told you that practical authors themselves are undetermined. Sir John Pringle allows that very early in the disease it may be attempted, but not if the disease has been of any standing. He has not, however, been explicit enough in limiting that duration. But, I would say, as soon as the fever puts on its proper form, that sweating is always attended with hazard; and I would join to that, that in several cases where it has spontaneously occurred, or where especially it has been excited by art, it has been prosecuted and encouraged with very bad effects ; 'and, as the sudorific regimen is unavoidably attended with the application of a considerable degree of heat, which must prove a considerable stimulus to the system, it is certainly attended with hazard. But, at the same time, from the consideration of what is the successful practice in the case of the plague, and from the near approach of petechial fevers in their nature to the pestilential, I am still under a suspicion that sweating may be employed with advantage in order at once to cut short the disease. In the many instances where it has been attended with mischief, it is very doubtful if the measures employed were proper. I have mentioned the difficulty. The sweating is to be carried on without any additional stimulus, and must be protracted for a due length of time; and I dare not conclude, but that the attempting it by emetics, and the following these with the exhibition of neutrals, and perhaps even the addition of some quantity of opium may be of advantage. Many of you may come to be in a situation, where, in a disease that is frequently fatal, and where every other measure is of little consequence, you may be tempted to try it.

"We have now considered the variety in the cases of fever, which occurs during the first days of the disease. I consider these as very important cases. We do not pretend to cut short the course of fevers; the most we can pretend to do, is to conduct them safely to their destined termination, and that, I think, is chiefly to be done by the remedies we employ during 
the first days. It is very well known, that if, in inflammatory fevers, we neglect venesection during the first three days, the mischief of that neglect is not to be recovered afterwards; and as certainly in the nervous fever, the too liberal use of that remedy will induce a fatal debility that is not to be recovered. The same may be said of the different remedies we employ, as the Emetic Tartar, which is a medicine of considerable efficacy for conducting fevers with more safety to their salutary end, when given during the first week; but unless we obtain the effect of it in moderating the symptoms of fever, and in obtaining considerable remissions during this period, we seldom observe much benefit afterwards.

" 2 . I go on to consider some cases a little more advanced; and,

"A. A continuation of the second and third cases, that I stated before (b.c.) ; of the second (b.), which is more considerable, and manifestly a synochus, or of the inflammatory case of the third (c.), where the symptoms are at least dubious; and most of the collateral circumstances lead towards the inflammatory. The symptoms continued, especially those of an increased action of the heart and arteries, are not very violent indeed, or they would immediately lead to the repetition of the venesection; but one special circumstance happens to occur, of which I must take notice here. After one venesection, after a vomit and glysters, and the use of saline mixtures," a sweating has spontaneously come on, and this sweating, from a prejudice which has been common enough of expecting relief by it from all feverish disorders, has been very much encouraged; the patient has been kept close covered in bed-has been urged to take warm drink - to take the saline and other sudorific medicines. In these circumstances, we find that every symptom of the hot fit goes on increasing, the heat continues great, headach violent, the pulse increases in frequency, and, perhaps, in hardness - a very decisive proof, as I mentioned before, that the sweat is not operating properly; and, with all this, the sweat is not of a proper kind, is not universal, does not extend to the lower extremities; it frequently sinks in, as the language is, it is fluid, but clammy. This case frequently occurs in our practice, before the physician has had occasion to direct or advise; and where this happens, the measure is immediately to stop 
such sweating, to take away any additional bed-clothes, or even to diminish those that are usual-to take the fire out of the patient's chamber-to take out the patient's hands from under the bed-clothes-to take away his warm drink, and the use of the neutral salts or sudorific remedies, and to give those that are more cooling, by being acidulated. Where that has been neglected, and the accession of the fever allowed to go on for some time longer, a phrenitic delirium, and all the various symptoms of an inflammatory fever have come on.

${ }_{66}$ в. A case of a different kind, likewise frequently occurs on the third or fourth day, where no such sweating has occurred either by design or accident, and without any other circumstance of heating regimen; where blood-letting, vomiting, glysters, the saline mixtures, and even the emetic tartar, have been employed; and, notwithstanding all this, the pulse continues to increase in frequency - is still full-the headach violent-the febrile anxiety and restlessness considerable, and there is an almost total want of sleep. I mention these circumstances to say, that without regard to the nature of the disease, though it very certainly is a synochus which is to change into the form of a typhus, in the circumstances I mention another venesection or more is necessary; and if you join to the case the considerations that lead to give a presumption of the phlogistic diathesis, several venesections may be necessary. In the same case however, by changing the circumstances a little we come to have more doubt. If the pulse is somewhat more moderate, hardly above a hundred, neither hard nor full; if, at the same time, there is no particular evidence of the phlogistic diathesis; and particularly if we know certainly from the nature of the epidemic prevailing, that the disease is to turn out a synochus of three weeks duration, the question with regard to venesection is to be put and answered with some doubt and difficulty. Here it is especially that we are to have particular regard to the circumstances of the patient, his age, habit, \&c. which may diminish the difficulty; and where there is any doubt, we may, in repeating the venesection, be in some measure determined by the quantity of blood that has been previously taken, and by the appearance of the blood. If the size upon its surface is very considerable, this last mark, at the 
same time, will appear in fevers which are to turn out with considerable symptoms of debility : therefore, that alone, though it will have its weight, is but a fallacious mark, and I would be more influenced by the effect of the former venesection. We will be determined to repeat the venesection, if the pulse has been considerably relaxed, and rendered fuller, softer, and even less frequent by the former venesection, but relapses into the same circumstances, becomes again full, contracted and hard.

"I have thus stated a variety of cases in which the question whether we are to blood or not, is to be put: and I now go on to touch another question which occurs at the same period of the disease; viz. when the symptoms express an excess of stimulant power, whether evacuations' which may be supposed to take off that excess are always to be practised, particularly purging; to what length or degree it is to be employed in the beginning of fevers, particularly in the case of synocha, and in the case of synochus, where the excess of stimulant power is considerable. With regard to this I observe first, that I know of no practitioner who has proposed to make large evacuations by stool; I have formerly mentioned the uncertainty of the effect; I have said that purgatives may weaken the system in greater proportion than they affect the heart and arteries (CXLVII.), and when they do make considerable evacuations they change the determination of the system, deriving too much from the surface of the body (CXLVIII.). Where this has taken place, I have more frequently observed it of bad than of good consequences; and, as I concluded before, I must repeat now, that if it is the excess of stimulant power that gives the indication, this will be answered more effectually by venesection. The matter, I think, is properly digested by Sir John Pringle; he says that, in the beginning of inflammatory fevers, after the first venesection, he opens the belly with some lenient laxative; but he adds farther, that through the course of the fever he finds it sufficient to prevent costiveness by daily glysters; that he has observed a motion or two procured daily in this way to be one of the best and most general remedies in fever. But that is far from the indication of taking off the excess of stimulant power by it. As I marked (CXLIX.) the necessity of 
opening the excretories of the abdominal viscera in intermittent fevers; and, as the congestions take place more or less in every fever, it is very necessary to keep the belly open, and to take off these by glysters of one kind or other in the manner Sir John Pringle proposes, only giving one, or perhaps two, for one day.-In short, with respect to purging the conclusion is, that the state of the bowels is constantly to be attended to, and that the drying up of their excretories must always have a bad effect; and I find that there is not a more convenient practice, than to keep the bowels in their proper condition by the use of warm water glysters alone, thrown in in considerable quantity, without the danger of any stimulus. But large purging, from one means or other, $I$ have found of bad effect.

"Now, it is necessary for me to take our cases even in parts, as they lead to questions with respect to particular remedies. I have not mentioned all those which may be employed in the very cases I have been describing; let us go back therefore to the cases of the second day (III. 1.). After the antiphlogistic regimen is established, and full vomiting employed; after the bowels have been opened by glysters, and the evacuations by venesection are determined for the time, but the fever continues; then we say, what are the remedies necessary to render the course of the fever safe, or, if you will, to take it off entirely? They are such, I think, as have a power of taking off the spasm by which the fever subsists; and the chief of these is the employment of emetics, such as the James's powder, or Emetic Tartar, especially the last, in a particular manner; this is the practice, which we know much the best, and can direct with most steadiness. It is, I think, the most important of the remedies lately introduced into practice. While the other remedies we have been speaking of are intended to obviate other circumstances, and to moderate particular excesses, this may be considered as the most direct remedy in fever. During the first days, accordingly, and even during the whole of the first week of the fever, Emetic Tartar is to be employed with the effect of moderate vomiting, or rather not with vomiting; at least it is not to be encouraged by the drinking of warm water, and, if it is thus properly directed, it will operate more or less by stool;

voL. I. 
and if it does bring out some degree of sweat, that is to have its course during the time of the accession or paroxysm, without being urged. I need not repeat that the use of the Emetic Tartar is to be entirely confined to the times of accession; given at other times, at intervals through the course of the day, it is of no manifest effect but to keep open the belly, which is inconsiderable compared with the keeping up of nausea in the stomach. The use of this remedy is to be continued for some days at the time of the accessions. I have formerly chosen the evening accessions, but I have of late found that the accession a little after noon is almost equally certain, and that the Emetic Tartar is employed then with equally good effects. If it is not disposed to purge largely, I believe it may be used twice a day with some advantage; but where it gives a very great degree of uneasiness and sickness, and is disposed to purge, there is danger from such repetition. I have thus put the disease, as I may say, in a train.

"3. I now go on to consider cases somewhat more advanced, as towards the fifth, sixth, or seventh day. Supposing the practice I have described to be fully exhausted, the venesection carried as far as it was proper, the emetic tartar used, \&c.; and the disease still continuing much the same, at least without much diminution, the frequency of the pulse being commonly somewhat increased, the headach not so considerable, but at the same time with more confusion of head, and some degree of approaching delirium: what are the proper measures in these circumstances? We must not stand still if the disease does not yield. It has been a very common practice after the venesection, vomiting, \&c. immediately to have recourse to blistering. I have told you my doubts with regard to that practice; I will farther observe upon this subject, that if the fever is attended with any marks of topical determination; if a delirium is coming on with suffusion of face, redness of eyes, and phrenitic affections attacking the head, then there is no sort of doubt but blisters applied to the head are a proper remedy at any period of the disease. If the lungs are attacked, and the catarrhal symptoms point now more at a peripneumonic affection, the blisters are to be applied to the side. If there are rheumatic affections, and not the common pains in the joints, that are pretty obstinate, 
there too we may apply blisters. And in short, at any period of the disease where we have a topical affection, blisters near the affected part are proper; but without any topical affection, merely considering the general course of the fever, where there are symptoms of increased action, and danger from every stimulus, they are to be employod with a great deal of caution. But if the disease from the beginning puts on the form of a nervous fever or typhus, I am of opinion that we may begin blistering at any period of the disease without any hesitation. The case that gives difficulty is this; where the disease is a synochus, a general fever without topical determinations, but with marks of increased impetus, frequency of pulse, and some degree of fulness in it, with considerable heat and thirst, whether, when the other remedies are exhausted, we should have recourse to blistering? I am disposed to think that we should not. I do not say that the chief effect of blistering is by its stimulus; but it is a stimulus, and even the temporary stimulus may have effect in the case of a general phlogistic diathesis; and I see that Dr. Lind, who is so remarkable an advocate for blistering in contagious fevers, observes that he has known them to have done a great deal of harm in those fevers which approach near to the inflammatory form ; and Sir John Pringle also, who is certainly an advocate for their use, acknowledges his doubts with regard to them in such cases. I therefore say that their use is doubtful, at least so long as the fever keeps the form of the synochus; and it is agreed upon by all practitioners, that their most certain use in general fever is when the Diathesis phlogistica is very nearly gone, when only the febrile spasm properly remains, and when blisters may be of service even by their stimulus. But there is another consideration that weighs with me upon this subject. I have said that the duration of fevers is not absolutely determined, yet it commonly is by several circumstances very much so; and if it is so, if we cannot hope immediately to cut short the course of fevers by blisters, it would be desirable to put off their use till they become more necessary. There are not many places to which they can be well applied, and it is not proper to pre-occupy these, lest if a topical determination should occur, it might prevent their more convenient use in the time of the greatest need. 
66 4. I put another case of fever that only happens in the more advanced state; when, after the seventh or some of the following days, it appears pretty plainly that the synochus is changed to a typhus, that the inflammatory is changed to the nervous state. This we commonly ascertain more certainly when we find that though the pulse grows more frequent, and manifestly smaller and weaker; when the heat, which had been considerable in the beginning of the disease, becomes now more obscure, and is only felt on pressing deep on any part; when the thirst which had been considerable before, is now manifestly abated; when there is a manifest drowsiness without succeeding sleep; when the confusion of head is increased, and a low delirium has prevailed; when, with these symptoms, there is a considerable increase of debility in the whole of the animal functions; and when joined to that there is a tremor, and perhaps subsultus tendinum. There are two other symptoms that may be taken notice of-the state of the tongue and of the urine; but I cannot make use of them very properly. One would expect that the extreme dryness of the tongue would be a consequence of heat and the inflammatory diathesis; but I find that even in the most pure nervous fevers, it occurs to as great a degree as in the other; but if with the symptoms $I$ have been just now describing of considerable nervous affections, the tongue still suffers very little change, and retains its usual softness, it may be taken in as a mark of the diminution of the increased impetus or force of the system; but that is a very rare occurrence. The other symptom that I speak of, viz. the state of the urine, is as ambiguous. Taking the account of the ancients, they considered that the urine, from being very high coloured and turbid becoming clear and thin, was a mark of a translation to the brain, and of a more considerable affection of that part, but I do not find that it applies that way in this part of the world; and I do maintain that the urine, from thick, turbid, and high coloured, becoming more clear and pellucid, is generally a favourable appearance. But let us take the other symptoms that are sufficiently, characteristic, and then it is evident that other measures are to be pursued, viz. such as have the power of relaxing the febrile spasm. Here it is that blisters have their proper place; and it is commonly necessary to support a succession of such 
applications. The other remedy that is at the same time of the most efficacy in relaxing the spasm, is the use of warm-bathing or fomentations, as I have mentioned formerly. - At the same time, in view of the great debility, we are to attempt the use of wine, or volatile alkali, under all the cautions and restrictions I formerly mentioned. And I would add one other remedy: whatever doubt there may be with respect to the use of cold drink in the beginning of the disease, it is undoubtedly one of the most useful remedies at this period.

"Such is our practice in what I may call the second stage of fevers, which very commonly begins in the second, but more certainly in the third week of the disease. As the disease advances, however, it is commonly with more symptoms of debility, and an affection of the nervous system; and, therefore, some other remedies are to be thought of adapted to the variety of circumstances. Accordingly,

" 5 . I would here mention the case where petechix appear; or other symptoms of putrefaction are evident; then, without all doubt, our resource is immediately in the bark ; and it may be made a question whether or not it may be used more early in the disease. This is suggested by the practice of Dr. James, who, when by means of his powder, he has at any period of the disease obtained a considerable remission of the fever, then immediately employs the Peruvian bark; and I am very ready to believe, that in such remissions it is a proper and effectual practice, and perhaps it is the surest means of cutting short the duration of fevers, if they admit of it at all. But I have not had any proper experience of it; for though $I$ have frequently observed some degree of remission both by the use of James's powder and the emetic tartar, I have not had sufficient courage for the use of the bark.

"Another question is this, when in the advanced state, towards the end of the second or the beginning of the third week, there are considerable symptoms of debility, but without any direct symptom of putrefaction, whether, in this case, the bark is to be employed? We have not often practised it, but I believe it ought to be; and the further the disease is advanced before we come to this question, the more certainly I would determine it positively. Of this indeed I have had some more 
experience; since De Haen, and some others, inculcated this practice, I have had more courage to employ it, and not only with safety, but with remarkable success in some cases.

"6. I have now but one other case to state, and that is, where, in the advanced stages of the disease, there come on more considerable symptoms of a great irritation of the brain, where the delirium, which had hitherto been of the low kind, is more of the phrenitic, when the patient talks louder and with more rapidity, when he becomes heedless of what is about him, and insensible to his most accustomed impressions, when he is easily provoked, particularly when he is more restless and impatient, and desires to get out of bed, or becomes remarkably enraged and furious. In these circumstances, I have often seen patients from the lowest state of debility seemingly recover their strength; at the same time, in their exertions of strength, most considerable tremor, subsultus, and even convulsions occur; if, with these, the face is flushed and the eyes have an uncommon redness, we suppose that a phrenitic affection of the brain has taken place. One circumstance gives a greater degree of difficulty here, viz. that the inflammation is topical, and not remarkably communicated to the arterial system, so that there is little hope from the common remedies in inflammation, and the state of debility makes them inadmissible. In such circumstances, therefore, it is extremely difficult to determine what is to be done : one remedy we attempt, is topical bleeding, the applying leeches to the temples, or cupping-glasses, which is likely to have more effect upon a topical affection without considerably weakening the system. But even this can do mischief : it may induce a greater degree of debility; and I have to add, that all the symptoms I have mentioned, I now know, from frequent experience, not to be inflammatory. What other kinds of irritations can be applied to the brain, I do not pretend to explain; but from their transient state, from their yielding without any antiphlogistic remedies employed, and the disease, after all, taking a salutary issue, I entertain no doubt that they may occur without any inflammation. Under this ambiguity, the remedies to be employed are chiefly blisters and warmbathing; but further, the antispasmodics of the least doubtful kind are proper in these circumstances, such as large doses of 
camphor; and though I will not say that musk is not a more heating and inflammatory remedy, I would employ large doses of it. If I can be relieved from some part of the ambiguity, and find that the subsultus tendinum, tremor, and even the convulsions are considerable, while the pulse does not increase, either in frequency or fullness, while there is no flushing of the face, or suffusion of the eyes, I would also have recourse to the opium. I had occasion to say before, that I have seen instances of maniacal delirium entirely cured by the use of this remedy; and here we must follow the advice of Celsus, 'præstat anceps remedium quam nullum experiri ;' and, in giving musk and opium, we have this encouragement, that, if the case is truly inflammatory, it is almost as certainly fatal, so that we at least cannot blame ourselves for having killed the patient by the remedies we employed.

"I have thus finished all the particular cases that I think necessary to put, in order to illustrate our doctrine with respect to the treatment of Continued Fevers, and to give the particular application of it."

\section{Sect. II.-Of the Cure of Intermittent Fevers.}

CCXXVIII. It still remains to consider the cure of intermittent fevers; and, with respect to these, we form also three general indications :

1. In the time of intermission, to prevent the recurrence of paroxysms.

2. In the time of paroxysms, to conduct these so as to obtain a final solution of the disease.

3. To take off certain circumstances which might prevent the fulfilling of the two first indications.

CCXXIX. The first indication may be answered in two ways :

1. By increasing the action of the heart and arteries some time before the period of accession, and supporting that increased action till the period of the accession be over, so as thereby to prevent the recurrence of the atony and spasm of the extreme vessels which give occasion to the recurrence of paroxysms.

2. Without increasing the action of the heart and arteries, 
the recurrence of paroxysms may be prevented, by supporting the tone of the vessels, and thereby preventing the atony, and the consequent spasm.

CCXXX. For the purpose mentioned in CCXXIX. 1., the action of the heart and arteries may be increased,

1. By various stimulant remedies, internally given or externally applied, and that without exciting sweat.

2. By the same remedies, or others so managed as to excite sweating, and to support that sweating till the period of accession be for some time past.

3. By nauseating doses of emetics, given about an hour before the time of accession, thereby supporting and increasing the tone and action of the extreme vessels.

"Ipecaccianha has been particularly recommended in the cure of intermittent fevers; and I knew a practitioner who cured these by giving, an hour before the accession that was expected, five grains, or so much as would occasion a strong degree of nausea and sickness, without vomiting; and by one or two such practices he was frequently successful. It is true that this may be executed by tartar emetic, and I recommended the trial of this to the practitioner I speak of ; but he assured me, that in several trials he could not easily adjust the dose of this so as to produce the proper degree of sickness without vomiting, so well as he could do by the other."

CCXXXI. The tone of the extreme vessels may. be supported without increasing the action of the heart and arteries (CCXXIX.), by various tonic medicines; as,

\section{Astringents alone.}

2. Bitters alone.

3. Astringents and bitters conjoined.

4. Astringents and aromatics conjoined.

5. Certain metallic tonics.

6. Opiates.

Lastly, an impression of horror.

A good deal of exercise, and as full a diet as the condition of the patient's appetite and digestion may allow of, will be proper during the time of intermission, and may be considered as belonging to this head.

CCXXXII. Of all the tonic remedies mentioned (CCXXXI.) 
the most celebrated, and perhaps the most certainly effectual, is the Peruvian bark, the tonic power of which we have endeavoured to demonstrate above (CCXIV.), and have at the same time explained its use in continued fevers.

The same observation as made in CCXVI. is especially proper in the case of intermittents: and, further, with respect to these, the following observations or rules are offered here.

1. That the bark may be employed with safety at any period of intermittent fevers, providing that, at the same time, there be neither a phlogistic diathesis prevailing in the system, nor any considerable or fixed congestion present in the abdominal viscera.

2. The proper time for exhibiting the bark in intermittent fevers, is during the time of intermission; and where intermissions are to be expected, it is to be abstained from in the time of paroxysms.

3. In remittents, though no entire apyrexia occurs, the bark may be given during the remissions; and it should be given; even though the remissions be inconsiderable, if, from the known nature of the epidemic, intermissions or considerable remissions are not to be so soon expected, and that great danger apprehen ded from repeated exacerbations.

3. In the case of genuine intermittents, while a due quantity of bark is to be employed, the exhibition of it ought to be brought as near to the time of accession as the condition of the patient's stomach will allow.

5. In general, in all cases of intermittents it is not sufficient that the recurrence of paroxysms be stopped for once by the use of the bark; a relapse is commonly to be expected, and should be prevented by the exhibition of the bark, repeated at proper intervals.

"In case the bark is wanting, which happens in several parts of the world, to the great prejudice of physicans; or if it is not of good enough quality, I think is to be wished that we could find a substitute for it; and I think from a great number of experiments which are very certain, that bitters will very often answer the purpose. Before the bark was introduced, intermittents were often cured by such bitters, and particularly by Chamomile flowers, which were the remedy of Riverius; and Dr. Pitcairn alleged that they were equally efficacious with the bark.

vor. I. 
I have tried the chamomile flowers, gentian, and other bitters, and I have no doubt of their efficacy when exhibited in sufficient quantity, which must be as large at least as the quantity we commonly employ of the bark, agreeably to the method of Hoffmann, giving, several times during the intermission, from half a drachm to a drachm of the flowers in powder. But I find an inconvenience attending the use of these bitters, that, given in sufficient quantity, they, more certainly than the bark, prove purgative, which disappoints us of their effects. It is necessary, therefore, that with such bitters we should combine some astringent substance. Various kinds have been tried, and have upon occasion been found successful; and were we to enter into any theory with respect to the bark, we would be led very strongly to consider it as a combination of a bitter and an astringent. The German physicians seem at present to be well agreed in this, and do practice with such a combination of a bitter and an astringent. I have repeated the experiment; I have joined gentian and galls, one of the simplest bitters to one of the simplest astringents; but I have not made experiments enough in order to ascertain the best proportion of these two; but in the practice in our infirmary, I found that I could procure the effect of stopping the course of an intermittent by this combination. Now, are there any other combinations, or, what is more desirable, whether is there such a combination already formed by nature in any one substance? I am very ready to believe that there is some, as the hippocastanum, the ash bark, and particularly the willow bark, with regard to which, in the philosophical transactions, there is a pretty good voucher of its efficacy in the case of intermittents. The testimonies of Stone, Clossius, and Gunzius, also are very strongly in its favour; and although we have not had many opportunities of employing it in intermittent fevers, the few that have been made show that it may be in some cases an effectual remedy."

I have employed the oak bark in powder, giving it to the quantity of half a drachm every two or three hours during; the intermissions of the fever; and both by itself, and joined with Chamomile flowers, have prevented the return of the paroxysms of intermittents.-M. $M$.

The febrifuge virtues of gentian have, by some writers, been made equal to those of the Peruvian bark; but in many cases 
FEVER.

the gentian alone has fallen short of that; but joined with galls or tormentil, in equal parts, and given in sufficient quantity, it has not failed in any intermittents of this country in which I have tried it.-M. $M$.

CCXXXIII. Our second general indication for conducting the paroxysms of intermittent fevers, so as to obtain a final solution of the disease, may be answered,

1. By exhibiting emetics during the time of the cold stage, or at the beginning of the hot.

2. By opiates given during the time of the hot stage.

"We have, for these hundred years past or thereby, been in the humour of blaming the age before us, the age of Alexipharmics, when the stimulant and heating medicines were employed, and their principal stimulus was opium. Now, I cannot believe that the practitioners in these days were quite so blind as to go on in this practice, if they had found it universally hurtful ; and I must conclude, that in many cases, they found it safe and effectual; so that there are more cases where opium may be employed than might at first sight be supposed, but I cannot go back to proper enough facts, so must take thern as they are given us in later times. A very remarkable one to our present purpose is what we find in Dr. Lind's Appendix to his work on the Diseases of Europeans in Warm Climates. From frequent experience he has found that opium may be employed in the hot fit of intermittents, that it brings on more equally the sweats that are to give the solution, and gives a more complete apyrexia than is to be obtained by any other means, and he seems to recommend it as in general an useful practice. However, I must say, that in this, and in some other instances, I am suspicious that he may be a little prejudiced in favour of his remedies, and especially of those he seems to have discovered. $\mathrm{He}$ hardly admits of limits to this, though he allows it is not universal; he says it succeeds in every case where delirium, to a considerable degree, is not present. You may understand him that he means to say, that delirium which depends upon an increased impetus; and then to be sure we must admit his exception, and I apprehend his doubts are just, that such a delirium has been dangerously increased by the use of opium.

"I would willingly mark the limitations of the free use of opium, but I can observe that it is especially in intermittents 
where the inflammatory diathesis is absent, that it proves beneficial, and from some of our late trials here, from a good deal of our late hospital practice, we find that opium may be useful in all the different stages of intermittents with more safety than was imagined; we find that an opiate may be employed in the time of the hot fit, very soon after it has come on; and if that is a clear point there is no difficulty of transferring the analogy to continued fevers; there may be cases of these that admit of the same thing, but I have not experience enough to allow me to assign the cases more exactly."

We need hardly say here, that almost every practitioner has found it useful to join opium to Peruvian-bark, or other tonics, in the cure of intermittents. Not only in correcting the purgative quality of the bark, or other tonics which some times take place, but even where no such quality is to be apprehended, we know that a certain quantity of opium, joined with the bark, makes it sit easier on the stomach than, with some persons, it would otherwise do ; and that a portion of it, joined with two or three doses of the bark, which are given immediately before the time of accession, enables it in less quantity than it would otherwise do, to prevent the return of paroxysms.-M.M.

CCXXXIV. The circumstances which may especially prevent the fulfilling of those two indications, and therefore give occasion to our third, are, a phlogistic diathesis prevailing in the system, and congestions fixed in the abdominal viscera. The first must be removed by blood-letting and the antiphlogistic regimen; the second, by vomiting and purging.

Where these measures are not immediately effectual, I hold it safer to attempt the cure of the disease by the means pointed out in general in CCXXIX., rather than by those in article second of the same paragraph. 
$-4$

$y^{2}-2+2+2=0$

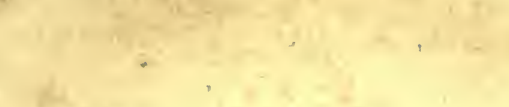
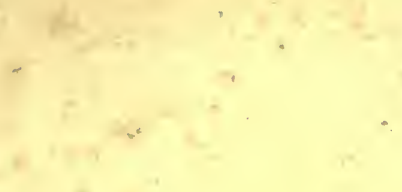

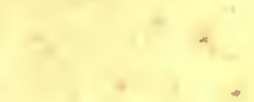

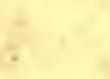

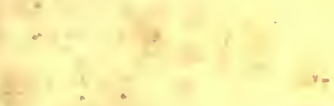

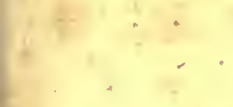

C. . . .

(

$-$

?

4

$+x+2=-x+2 y$

. 





BINDING Eニ-T. AUG 141973

$\begin{array}{lc}\text { MPR } & \text { Cullen, William } \\ \text { C } & \text { Works }\end{array}$

PLEASE DO NOT REMOVE

CARDS OR SLIPS FROM THIS POCKET

UNIVERSITY OF TORONTO LIBRARY 
